\title{
Functional analysis of Hypoxylon pulicicidum genes required for heterologous biosynthesis of nodulisporic acids
}

This thesis is presented in partial fulfilment of the requirements for the degree of

\section{Doctor of Philosophy (PhD)}

in

Biochemistry

at

Victoria University of Wellington,

Wellington, New Zealand

Kyle Cornelius Van de Bittner

2018 



\section{ABSTRACT}

Nature holds some of the greatest secrets in drug design and development and the ability to access these trade secrets has been revolutionised by modern bioengineering technologies. In order to exploit these technologies it is essential to understand what genes are involved in compound production and the enzymatic steps that limit flux to the desired product. This thesis describes the discovery of four secondary-metabolic enzymatic steps involved in the biosynthesis of a group of valuable natural products known as nodulisporic acids.

Nodulisporic acids are known for their potent insecticidal activities; however, biosynthesis of these compounds by the natural fungal producer, Hypoxylon pulicicidum (Nodulisporium sp.), is exceptionally difficult and has prevented the commercial development of novel nodulisporic acid-containing veterinary medicines and crop protects.

To discover how nodulisporic acids are biosynthesized:

1. the H. pulicicidum genome was sequenced

2. a gene cluster responsible for nodulisporic acid production was predicted

3. genes in the cluster were functionally characterised by pathway reconstitution in a common, fast growing mould, Penicillium paxilli

In turn, four genes involved in the biosynthesis of the nodulisporic acid core compound, nodulisporic acid $F$, have been functionally characterised. The four genes encode a geranylgeranyl transferase (NodC), a flavin adenine dinucleotide-dependent oxygenase (NodM), an indole diterpene cyclase (NodB) and a cytochrome P450 oxygenase (NodW). Two of the gene products (NodM and NodW) catalyse two previously unreported reactions that provide the enzymatic basis of the biosynthetic branch point unique to nodulisporic acid biosynthesis.

From here, future efforts will explore how these genes can be engineered to overcome flux bottlenecks and enable production of significantly increased, and even industrially relevant, product titres. 


\section{OUTPUTS FROM THIS THESIS}

For all of the below outputs:

Emily Parker, Matt Nicholson, and Barry Scott conceived the fungal project. Emily Parker sourced funding, provided the lab space and materials, and acted as the biochemistry expert to link the predicted gene functionality to individual biosynthetic steps. Matt Nicholson assisted in sourcing funding, provided expertise around fungal work and acted as primary bioinformatician to identify the NOD cluster and annotate the genes. Barry Scott provided fungal strains and fungal expertise. Leyla Bustamante acted as lead science and lab manager, ensuring the projects ran smoothly at all times, provided molecular biology expertise and teaching, and performed some experimental work. I, Kyle Van de Bittner, established the gene characterisation pipeline in our lab including method development for plasmid construction, fungal transformations, protoplast preparations, chemical extraction and compound analysis by TLC, LC-MS, NMR, and preparation of compound standards. Craig van Dolleweerd conceived MIDAS, constructed the MIDAS vector suite, and provided expert advice on using the MIDAS system. Sarah Kessans prepared some of the plasmid assemblies and fungal transformations.

Additional information that is specific to individual outputs is listed below.

\section{Publications:}

- Van de Bittner, K. C.; Nicholson, M. J.; Bustamante, L. Y.; Kessans, S. A.; Ram, A.; van Dolleweerd, C. J.; Scott, B.; Parker, E. J., Heterologous biosynthesis of nodulisporic acid F. J. Am. Chem. Soc. 2018, 140 (2), 582-585. ${ }^{1}$

I completed nearly all of the experimental work and wrote the manuscript (including generation of all figures and tables) with input from my supervisors, Emily Parker and Matt Nicholson. Emily Parker and Matt Nicholson edited the manuscript. Arvina Ram provided expertise around fungal transformation and protoplast preparation methods, prepared the $H$. pulicicidum DNA for genomic sequencing, and managed all of the material transfers from Barry Scott's lab. 
- Nicholson, M. J.; Van de Bittner, K. C.; Ram, A.; Bustamante, L. Y.; Parker, E. J., Draft Genome Sequence of the Filamentous Fungus Hypoxylon pulicicidum ATCC 74245. Genome A. 2018, 6 (2).²

Matt Nicholson wrote the manuscript, sourced the H. pulicicidum strain, identified the NOD cluster and annotated its genes. Arvina Ram, Leyla Bustamante, and I completed the experimental work.

- van Dolleweerd, C. J.; Kessans, S. A.; Van de Bittner, K. C.; Bustamante, L. Y.; Bundela, R.; Scott, B.; Nicholson, M. J.; Parker, E. J., MIDAS: A modular DNA assembly system for synthetic biology. ACS Synth Biol. 2018, 7 (4), 1018$1029 .^{3}$

Craig van Dolleweerd wrote the manuscript. Sarah Kessans, Leyla Bustamante, Rudy Bundela, and I performed the plasmid preparations and fungal transformations. I performed all of the LC-MS and NMR analysis and provided many figures and tables. Emily Parker, and I edited the manuscript.

\section{Patents:}

- Inventors: Parker, E. J.; Nicholson, M. J.; Van de Bittner, K. C.; Bustamante, L. Y.; Kessans, S. A.; van Dolleweerd, C. J., "Heterologous Biosynthesis of Nodulisporic Acids", A.U. Patent AU2017903956 filed 29/09/2017.

Emily Parker, Matt Nicholson, and I wrote the patent with input from our patent attorney, Jon Ashen. I generated all of the figures and tables and performed nearly all of the experimental work required for this patent.

- Inventors: van Dolleweerd, C. J.; Parker, E. J.; Nicholson, M. J.; Beneficiaries: Van de Bittner, K. C.; Bustamante, L. Y.; Kessans, S. A.; "Modular DNA Assembly System", A.U. Patent AU2017903955 filed 29/09/2017.

Craig van Dolleweerd conceived MIDAS, constructed the MIDAS vector suite, and wrote the patent with input from our patent attorney, Jon Ashen. Emily Parker, Matt Nicholson, and I edited the patent. 


\section{ACKNOWLEDGEMENTS}

To reach the point of writing the acknowledgments for this piece of work was no small undertaking. By no means can I say that this work is mine alone, and so luckily, I have a place to acknowledge many of those who have contributed - not only to this work, but to granting me all the resources and support I have required to get to this point in my life.

To start I would like to thank those who were directly involved in the work that brought this thesis into fruition. I will start with the person who gave me the opportunity to get amongst this amazing science endeavour, my primary supervisor Professor Emily Parker. Although she was not physically present for the majority of the labwork instead serving as what she deems the main "cheerleader"- this title she gives herself only highlights how humble she is; because without her, there would be no lab, no funding, and no past or future of this project. It comes with no surprise that when she announced she intended to relocate the lab from Christchurch to Wellington, nearly everyone followed. Secondly, comes my co-supervisor, Dr. Matthew Nicholson, who was 'the man with a plan' - the one who approached Emily with the initial idea to make a fungal factory for nodulisporic acids. In times of despair, Matt was my go-to father figure who consoled me. Thank you Emily and Matt for giving me the opportunity to work on a commercially relevant thesis project, which has inspired me to pursue future work in the biotech industry. I so look forward to what we can create together in the coming years. Next and most important to me, is my partner in crime, lab and science manager, super fancy scientist and extraordinary role-model, Dr. Leyla Bustamante. She has been with me nearly every step of the way, giving me support and love in the darkest of times and celebrating with me in the lightest. She is the main reason I decided to stay in New Zealand and pursue my PhD here rather than going back to the States. It is for her that I reserve my deepest gratitude for I cannot begin to explain all she has taught me over the years.

I would also like to thank all of our collaborators who have enabled this project to succeed. Namely, those in Professor Barry Scott's lab at Massy University in Palmerston North - in particular, Prof. Barry Scott himself, for his insightful discussions and providing the project with all of the heterologous expression hosts, as well as Arvina Ram, who taught me how to transform fungi and ran quality control on the genomic DNA samples used to sequence the Hypoxylon pulicicidum genome. 
Additionally, I would like to thank Dr. Craig van Dolleweerd from Callaghan Innovation who conceived the Modular Idempotent DNA Assembly System (MIDAS) used as our platform to functionally characterise genes and reconstitute metabolic pathways in $P$. paxilli and Dr. Sarah Kessans for assembling some of the MIDAS constructs used in this study. I am especially grateful to those who often go unnoticed; the technicians and mechanics who ensured that all of the labware was running properly, thereby preventing unneccesary disruptions in the flow of my research. In particular, I would like to thank Dr. Amelia Albrett from the University of Canterbury and Jan Vorster from Victoria University of Wellington for their technical service around liquid chromatography-mass spectrometry and nuclear magnetic resonance spectrometry, as well as Wayne Mackay who was the truly spectacular all-round handy man and goto Mr. Fix-It at Univeristy of Canterbury.

Of course the project would not be possible without our funding bodies, the New Zealand Ministry of Business, Innovation, and Employment who supplied the Smart Ideas grant to get this project off the ground, Fulbright New Zealand for supplying funding for my first year of research, the University of Canterbury for supplying me with a $\mathrm{PhD}$ scholarship for my second year of research, and Victoria University of Wellington for supplying me with $\mathrm{PhD}$ scholarship for my third.

Finally, thank you Mom and Dad, Gen and Great Mom, for giving me your deepest love and support from when I was a little munchkin all the way through the present. I feel privileged to have been born into such a loving environment - full of inspiring people who taught me to never regard anything as impossible. Thank you for giving me more than what the world could ever offer.

Love forever,

Kyle 


\section{TABLE OF CONTENTS}

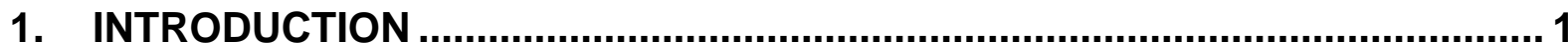

Fungal secondary metabolites....................................................................... 2

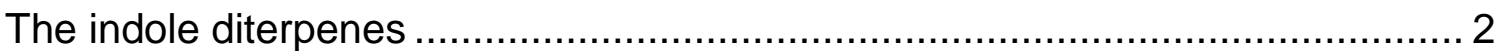

A review of indole diterpene biosynthesis: From early labelled substrate feeding studies to recent pathway reconstitution studies ............................... 4

Primary metabolites involved in indole diterpene biosynthesis ......................... 7

Secondary metabolism step 1: Formation of geranylgeranyl pyrophosphate (3) . 8

Secondary metabolism step 2: Geranylgeranylation of indole-containing

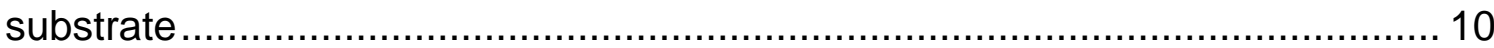

Secondary metabolism step 3: Epoxidation of 3-geranylgeranylindole .............. 14

Secondary metabolism step 4: Formation of cyclic indole diterpene scaffolds .. 21

Decoration of the cyclic scaffolds: Gene identification and elucidation of the decoration steps

Identification of gene clusters enables rapid confirmation of individual gene function

Identification of the $P A X$ gene clusters and functional analysis of $\operatorname{pax} P, \operatorname{pax} Q$ and $\operatorname{pax} D$

Identification of the ATM gene clusters and functional analysis of $\operatorname{atmP}$, atmQ and atmD

Identification of the $L T M$ gene clusters and functional analysis of $L T M$ genes .. 44 Identification of the TER gene cluster and functional analysis of terP and terQ 52 Identification of the PTM and PEN gene clusters and functional analysis of the genes

Identification of the $J A N$ gene cluster and functional analysis of the genes. 63 Identification and functional analysis of unclustered indole diterpene genes, which led to the identification of another indole diterpene cluster, ATS5 ........... 71

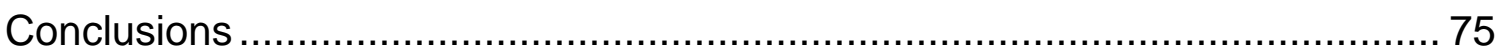

Nodulisporic acids: the target for this research............................................. 77

The discovery of nodulisporic acid A's (1) insecticidal activity 79 Nodulisporic acid A (1) selectively targets glutamate gated chloride ion channels 79

Nodulisporic acid A (1) is an orally safe, systemically active, ectoparasiticide .. 80 
Medicinal chemistry studies: elucidation of the nodulisporic acid A (1)

pharmacophore and development of nodulisporic acid $A(1)$ analogues with more desirable properties.

Analysis of the ideal nodulisporic acid A (1) analogue drug target: $N$-tert-butyl nodulisporamide (78)

Difficulties with nodulisporic acid A (1) production prevent drug development ...88

2. THE GENE CHARACTERISATION TOOLBOX 89

Bioinformatics: Identification of the NOD cluster .90

DNA assembly: The Modular Idempotent DNA Assembly System (MIDAS) ...98 Heterologous expression ............................................................................100

A library of expression hosts for confirming gene function ..........................101

Compound analysis ...................................................................................103

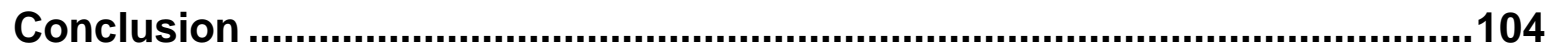

3. FUNCTIONAL CHARACTERISATION OF NodC .........................................105

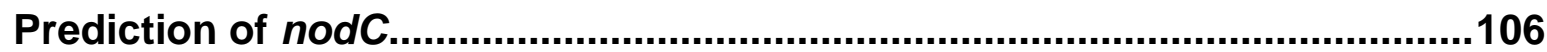

Functional analysis of NodC...........................................................................107

Preparation of MIDAS Level-1 plasmids.................................................. 109

Generation of PCR products ......................................................... 109

Assembly of Level-1 plasmids ............................................................. 110

Preparation of MIDAS Level-2 plasmids..................................................113

Preparation of MIDAS Level-3 plasmids..............................................117

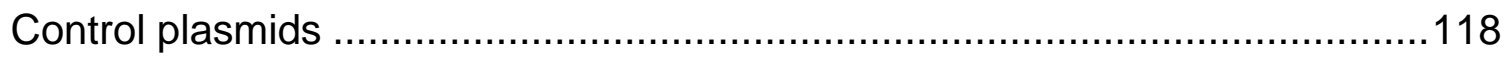

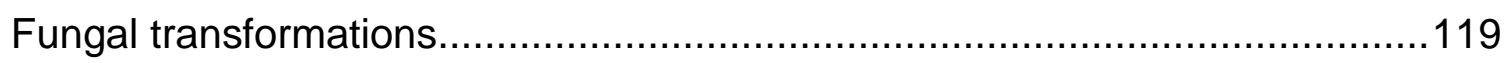

Resolution of MIDAS construct problem................................................. 120

Successful complementation of nodC into the $\triangle$ paxC $P$. paxilli deletion mutant 122

LCMS confirmation of paxilline (2) production ...........................................123

Conclusions

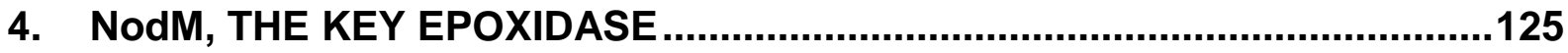

The indole diterpene epoxidases .................................................................126

Identifying the NOD epoxidase .................................................................127

Functional analysis of nodM...................................................................128

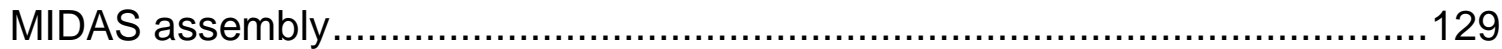




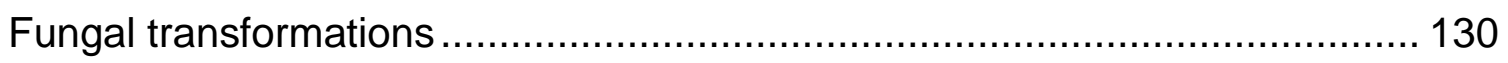

A note about promoter choice................................................................ 133

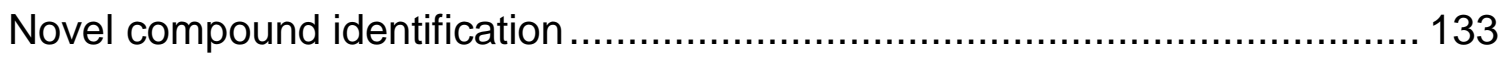

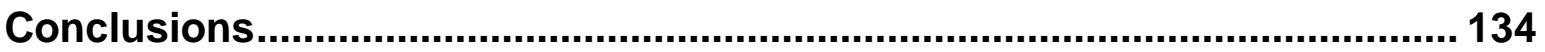

Explanation for emindole SB (18) production ........................................... 134

5. NodB, A PROMISCUOUS INDOLE DITERPENE CYCLASE ….....................136

Indole diterpene cyclases are notoriously promiscuous ............................. 137

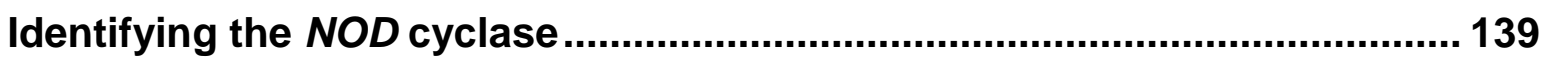

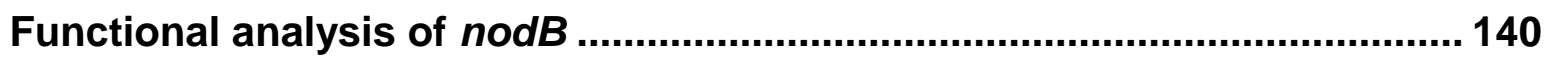

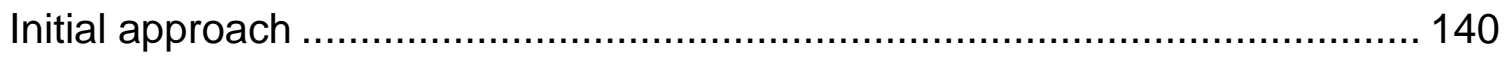

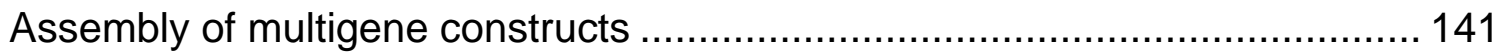

Transformations of the multigene constructs .......................................... 147

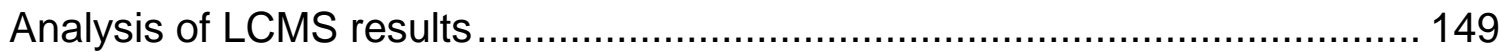

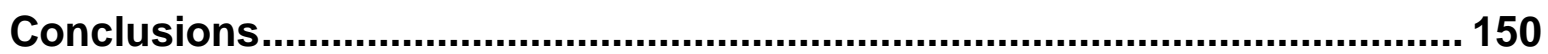

6. NodW, THE FUNNEL TO NODULISPORIC ACIDS .....................................151

Review of post cyclisation oxidations by cytochrome $\mathbf{P} 450$ oxygenases in

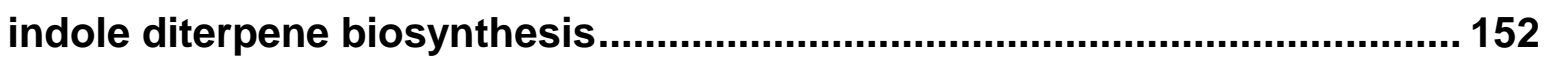

The NOD cluster lacks highly homologous cytochrome P450 oxygenases 152 P450 analysis: An approach to functionally confirm non-homologous genes

Transformation of $P$. paxilli with P450-encoded vectors ............................... 156

Identification of nodulisporic acid F (82) ................................................ 158

Confirmation that five genes are required for nodulisporic acid biosynthesis 159

Enhancing nodulisporic acid F (82) biosynthesis ..................................... 163

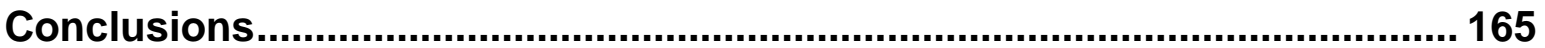

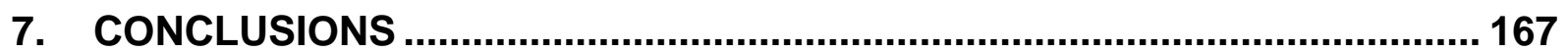

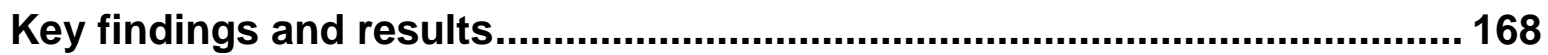

Future work: Functional analysis of the remaining NOD genes ................... 171

What is the benefit of this work and what will be done with all that has been

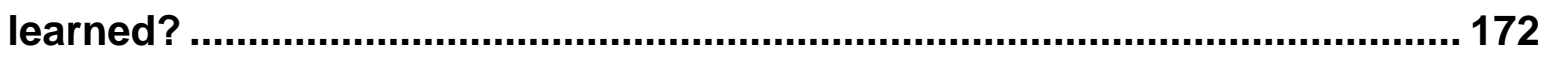

The opportunity for a metabolic engineering business................................ 173

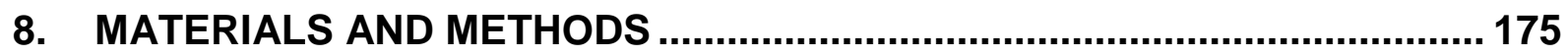


gDNA isolation for genome sequencing and transcription unit module amplification.

Genome sequencing, identification and annotation of the $\boldsymbol{H}$. pulicicidum (ATCC ${ }^{\circledR} 74245^{\mathrm{TM}}$ ) NOD gene cluster and GenBank accession numbers 177

Molecular biology reagents 178

Bacterial strains .179

PCR 179

Construction of MIDAS vectors 179

Construction of the pML1 source vector. 180

Construction of pML2 shuttle vectors. 182

Construction of the pML3 destination vector. 188

Golden Gate assembly reactions 191

Preparation of MIDAS constructs. 191

Primer design. 191

Protocols for MIDAS Level-1 module cloning ….........................................193

Protocols for MIDAS Level-2 transcription unit assembly ............................193

Protocols for MIDAS Level-3 multigene assembly ......................................194

Penicillium paxilli PAX gene deletion mutants ..........................................195

Media and reagents used for fungal work ....................................................197

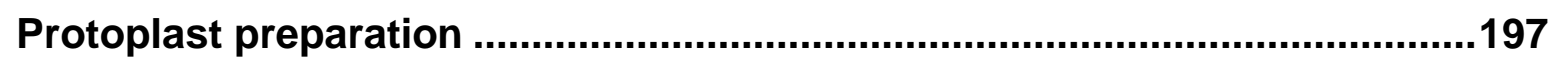

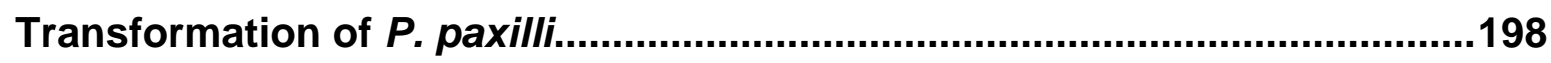

Fungal transformations and analysis of indole diterpene chemotypes........199

Indole diterpene production and extraction ...............................................199

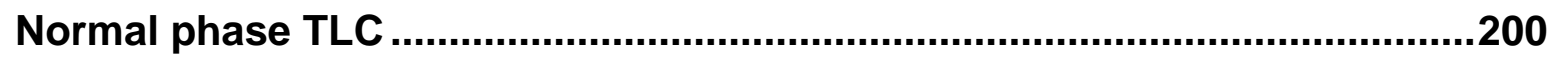

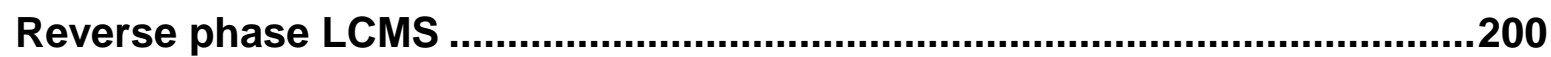

Large scale indole diterpene purification for NMR spectroscopy analysis..201

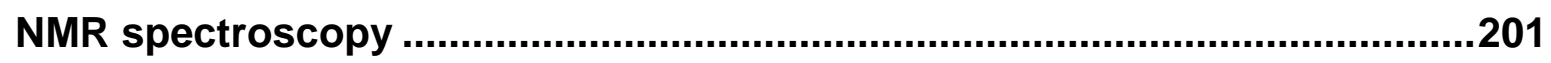

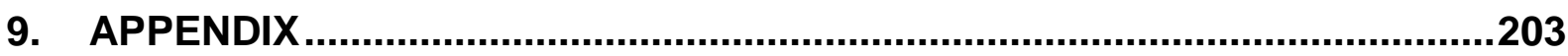

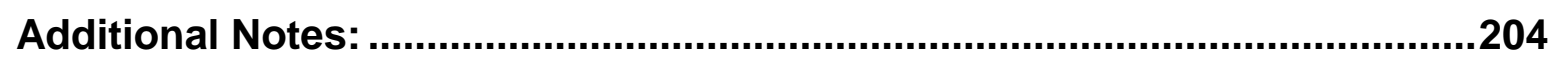

Introduction: Additional radiography feeding studies:....................................204

Chapter 3: Testing of the paxC promoter region (paxCProUTR-2) ........................204

Bioinformatics analyses to identify the potential genetic elements involved in the biosynthesis of nodulisporic acids. .205 
Assembling of genetic elements (5'UTRs, CDS, 3'UTRs, etc.) into MIDAS vectors

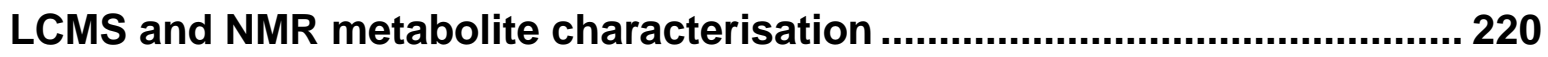

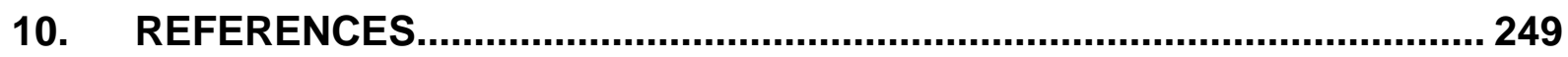


1. INTRODUCTION 


\section{Fungal secondary metabolites}

Secondary metabolites are natural products that are not required for the growth of the producing species. Although the systematic study of fungal secondary metabolites began in $1922,{ }^{4}$ it was not until the discovery and development of the most well known fungal secondary metabolite, penicillin - the first broad spectrum antibiotic - that pharmaceutical companies invested in screening programs directed toward fungal secondary metabolites. ${ }^{5}$ Since then, thousands of fungal secondary metabolites have been isolated, characterised, and bioactively profiled. An impressive review of 1,500 fungal metabolites that were identified between 1993 and 2001 showed that over half of the compounds had antimicrobial or antitumor activities. ${ }^{6}$

One method used to classify the array of fungal secondary metabolites differentiates them based on the enzyme classes used in their biosynthesis. ${ }^{5}$ According to this classification method, four main classes of fungal secondary metabolites exist: polyketides, non-ribosomal peptides, terpenes, and indole alkaloids. This introduction focuses on one subclass of the terpene secondary metabolites known as indole diterpenes.

\section{The indole diterpenes}

The indole diterpenes are of particular interest due to their wide range of chemical diversity and concomitant bioactivities, which include insecticidal, ${ }^{7-11}$ anti-cancer, ${ }^{12-13}$ anti-MRSA,${ }^{14}$ anti-H1N1,${ }^{15}$ and tremorgenic ${ }^{16}$ activities. The differences in the bioactive properties of indole diterpenes arise from the variety of ways the indole diterpene core structure is cyclised and the functional groups that decorate the cyclised cores (Figure 1.1, Note: Table 9.10, located at the end of the APPENDIX, contains a list of numbered compounds and corresponding structures.). Genetic variation of the native indole diterpene producers gives rise to the different cyclisation mechanisms and functional groups of indole diterpenes. Understanding how these compounds are biosynthesised and subsequently identifying enzymes from biosynthetic pathways can enable enhanced production of compounds that are difficult or impossible to access from natural sources, and provides the opportunity to engineer efficiently novel compounds with enhanced bioactive properties. 


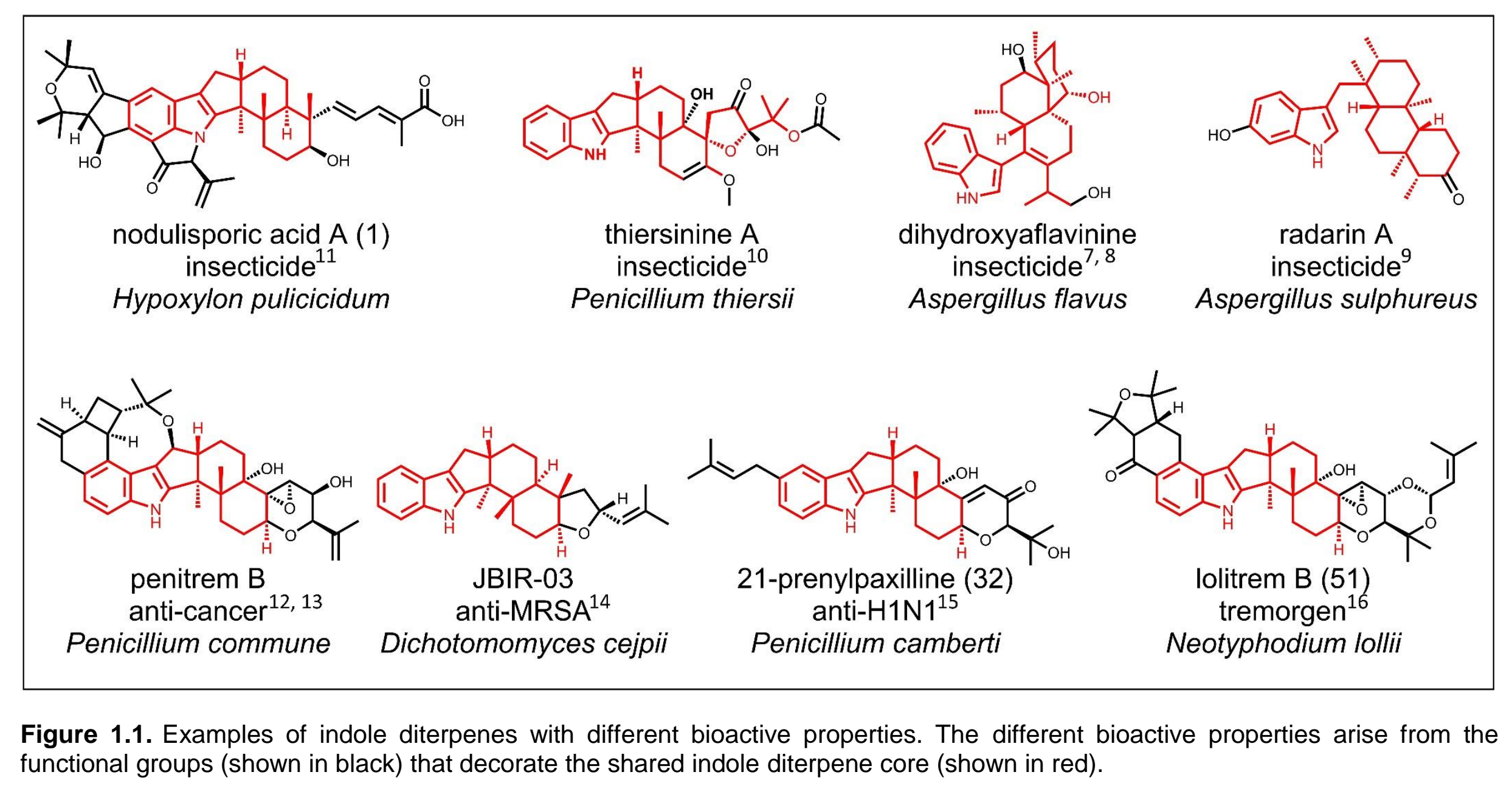


The main goal of this thesis is to identify the genes involved in the biosynthesis of the indole diterpene nodulisporic acid A (1) - structure shown in Figure 1.1. Therefore, to start, what is currently known about the biosynthesis of indole diterpenes will be described to provide a foundation of knowledge that will be exploited throughout the rest of this thesis and used to examine how nodulisporic acids are produced by their native host, Hypoxylon pulicicidum (Nodulisporium sp.). ${ }^{17}$

\section{A review of indole diterpene biosynthesis: From early labelled substrate feeding studies to recent pathway reconstitution studies}

The biosynthesis of indole diterpenes was initially explored throughout the 1970s90s using labelled substrate feeding studies; ${ }^{18-22}$ where labelled precursors shed insight on how the indole diterpene core scaffolds were formed by their native fungal producers. In the late 1990s the first potential indole diterpene gene cluster, the PAX cluster, responsible for paxilline (2) production in the filamentous fungi Penicillium paxilli, was identified. ${ }^{23-24}$ Throughout the 2000s to the early 2010 s, the PAX cluster served as the pivotal reference for the identification of seven other indole diterpene clusters. $^{25-33}$ During this time, the individual functions of the secondary-metabolic genes involved in indole diterpene biosynthesis have been elucidated, ${ }^{25-28,}{ }^{31-43}$ which, when combined together, establish a database of information that can be used to probe for novel indole diterpene gene clusters. This section will review how the current understanding of indole diterpene biosynthesis has been established.

The biosynthesis of indole diterpenes has been established from the functional analysis of a number of indole diterpene genes through a combination of feeding studies with labelled ${ }^{19-20,22,44-46}$ or unlabelled precursors, ${ }^{29-30,36,39-40,42}$ the analysis of indole diterpene products produced by knockout strains, ${ }^{24}, 26,29-32,38$ and the reconstruction of biosynthetic pathways. ${ }^{27-29,} 31,33,38,40-41,43$ Studies have found that the structural variability of indole diterpenes, which corresponds to their various bioactive properties, arises from genetic variation of specific indole diterpene genes within the genomes of the indole diterpene producing hosts (Figure 1.2). Typically, the complexity of the final indole diterpene product correlates with the number of genes required for its biosynthesis, where the more complex products like the lolitrems ${ }^{26-27,29}$ and penitrems ${ }^{31-32}$ require more genes for their biosynthesis (10 genes and 17 genes 
respectively), than the simpler indole diterpene products like paxillines ${ }^{24,34-40,42}$ and aflatrems $^{25,28,39,41}$ (7 genes each). To date, the functionality of genes in eight different indole diterpene biosynthetic pathways, which give rise to the paxillines, ${ }^{24,} 34-40,42$ aflatrems, ${ }^{25}, 28,39,41$ lolitrems, ${ }^{26-27,} 29$ shearinines, ${ }^{32,} 43$ penitrems, ${ }^{31-32}$ terpendoles, ${ }^{30}$ aflavinines, ${ }^{33}$ and emindoles, ${ }^{33,40}$ have been confirmed. Notably, the function of all the secondary-metabolic genes required for the biosynthesis of the major products in four of these indole diterpene classes, the paxillines, ${ }^{35-36,38,40,42}$ aflatrems, 39,41 shearinines, ${ }^{43}$ and penitrems, ${ }^{31}$ have been confirmed and thus provide an excellent database to understand the complex indole diterpene biosynthetic mechanisms.

For example, the genes required to produce these four classes of indole diterpenes, six including the lolitrems and terpendoles, have all been found clustered together in the genomes of their native fungal producers (Figure 1.2), where each gene product in the cluster is linked to the catalysis of a specific biochemical reaction in the indole diterpene biosynthetic pathway. Naming of genes in indole diterpene clusters has followed the Aspergillus nidulans naming convention, where genes are given a name with a three-letter prefix in lower case that designates species, followed by a single letter suffix in upper case that designates gene function written in italic font (e.g. paxG). Naming of the corresponding protein product follows the same rules except that the initial letter of the prefix is upper case and the entire name is written in normal (nonitalic) font (e.g. PaxG is the protein product of paxG). Therefore, homologous genes from different species (with the exception of the ATM clusters in Aspergillus flavus and Aspergillus oryzae $)^{28}$ contain a different three letter prefix but have been given the same single letter suffix (e.g. paxG is from P. paxilli and ItmG is from Epicloë festucae).

This introduction begins by highlighting the first four secondary-metabolic steps in indole diterpene biosynthesis that give rise to the library of indole diterpene cyclised cores that are often further decorated in distinct ways to install specific bioactive properties. From there, attention will turn to how these cyclised cores are decorated and aspects of the different indole diterpene gene clusters will be examined; including how the clusters were identified and how the genes have been functionally analysed. After elaborating on what is known about indole diterpene biosynthesis, the history of the main indole diterpenes of this thesis, the nodulisporic acids, will be presented. 


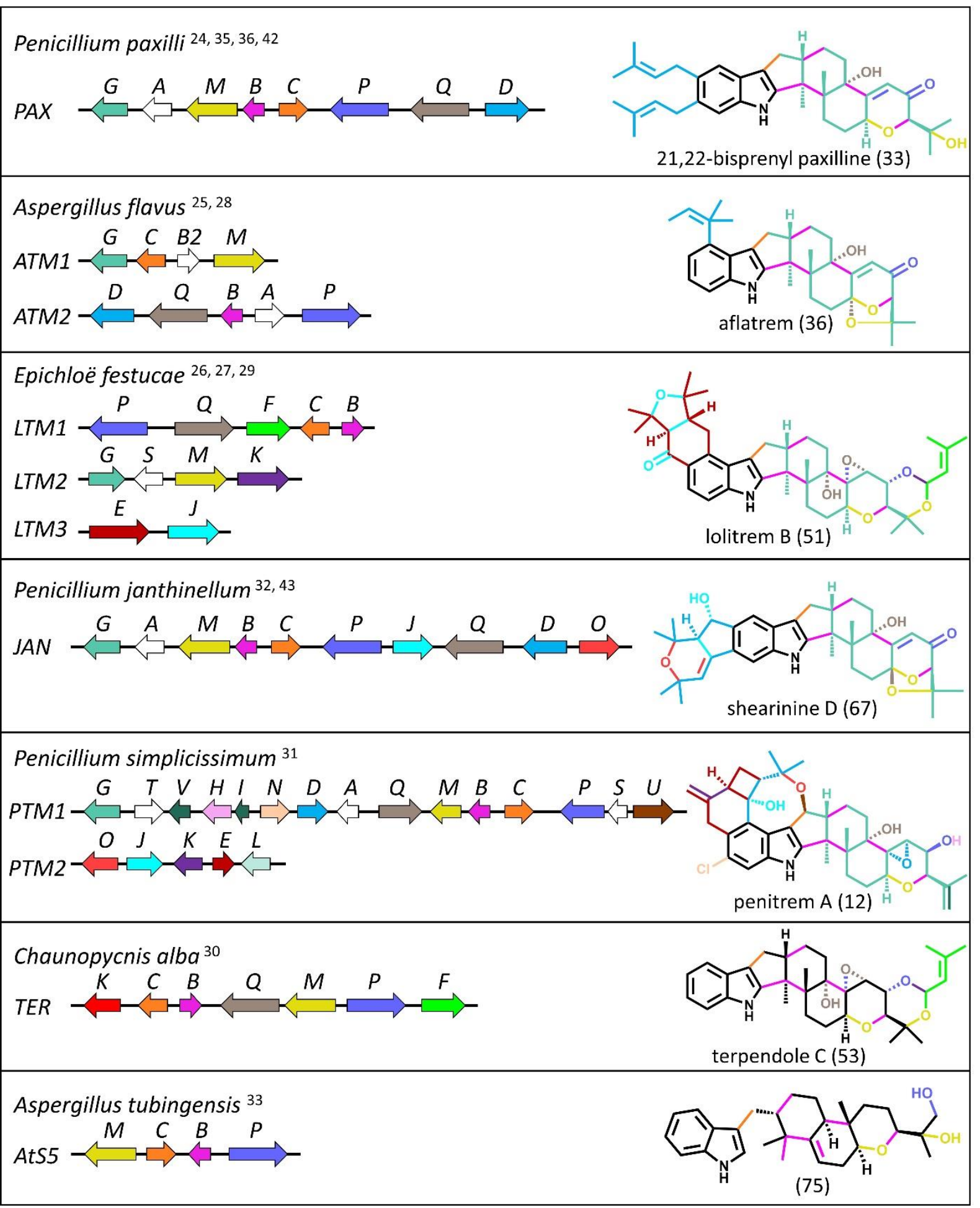

Figure 1.2. Compilation of known indole diterpene gene clusters. The name of the organism that each gene cluster was isolated from is listed above the cartoon of its corresponding gene cluster. Genes are represented by coloured arrows and named with a letter. The colour and letter of each gene corresponds to the function of the gene in indole diterpene biosynthesis. Genes that are homologous to one another have the same gene letter and colour. One of the indole diterpene products that each gene cluster codes for is listed to the right of the cluster, where the colour in the indole diterpene product indicates the specific moiety that is installed by the protein product of the corresponding coloured gene. 


\section{Primary metabolites involved in indole diterpene biosynthesis}

The results from various indole diterpene biosynthesis studies have revealed that indole diterpenes are biosynthesised from the condensation of an indole-containing precursor and a twenty-carbon chain (diterpene) of geranylgeranyl pyrophosphate (3). ${ }^{20-21,40,44}$ The indole-containing precursor is preferentially indole-3-glycerol phosphate $(4)^{40,44}$ but alternate indole cores like indole $(5)^{40}$ and tryptophan $(6)^{19,}, 22$ can also serve as a substrate for some geranylgeranyl transferase enzymes. These three indole-containing precursors are synthesised via the tryptophan (6) biosynthetic pathway, in which the key substrate, indole-3-glycerol phosphate (4), is specifically derived from anthranilate and phosphoribosyl pyrophosphate. ${ }^{44}$

Geranylgeranyl pyrophosphate (3) is synthesised from the two common terpene building blocks of activated isoprene units known as isopentyl pyrophosphate (7) and dimethylallyl pyrophosphate (8) shown in Figure 1.3. In fungi, animals, plants (cytosol), and archaea isopentyl pyrophosphate (7) is synthesised from 3 units of acetate (9) as an intermediate of the mevalonate pathway and dimethylallyl pyrophosphate (8) is synthesised from isopentyl pyrophosphate (7) by isopentyl pyrophosphate (7)isomerase. In eubacteria, green algae, and higher plants isopentyl pyrophosphate and dimethylallyl pyrophosphate (8) are synthesised from D-glyceraldehyde 3-phosphate and pyruvate via the 2-C-methyl-D-erythritol 4-phosphate (MEP) pathway (syn. 1deoxy-D-xylulose 5-phosphate or non-mevalonate pathway). ${ }^{47}$ To form the fifteencarbon chain of farnesyl pyrophosphate (10), one unit of dimethylallyl pyrophosphate (8) sequentially condenses with two units of isopentyl pyrophosphate (7). Farnesyl pyrophosphate (10) then condenses with isopentyl pyrophosphate (7) in a geranylgeranyl pyrophosphate (3) synthase-mediated condensation to form geranylgeranyl pyrophosphate (3) (Figure 1.3). 


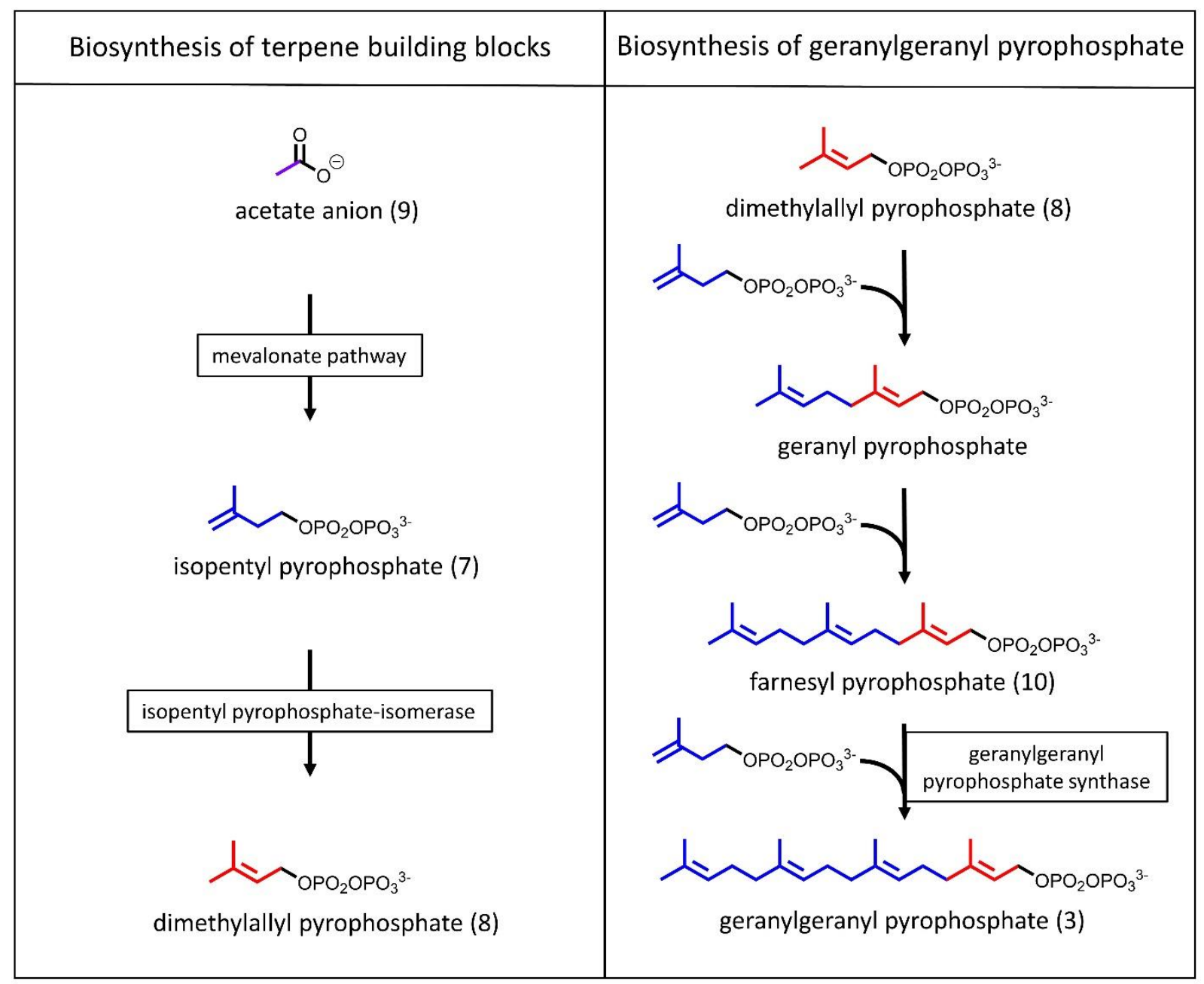

Figure 1.3. Depiction of the biosynthesis of the diterpene component [geranylgeranyl pyrophosphate (3)] of indole diterpenes.

\section{Secondary metabolism step 1: Formation of geranylgeranyl pyrophosphate (3)}

A combination of feeding studies with labelled substrates and gene functionality studies have established that the synthesis of is the first secondary-metabolic step in indole diterpene biosynthesis (Figure 1.4). Genomes of the known indole diterpene producers often contain two geranylgeranyl pyrophosphate (3) synthases - one dedicated to primary metabolism and one dedicated to secondary metabolism. ${ }^{37} \mathrm{~A}$ key difference between the two is that the secondary- geranylgeranyl pyrophosphate (3) synthases contain a DDXDD motif in their $\mathrm{N}$-terminus that is not present in any of the primary geranylgeranyl pyrophosphate (3) synthases. ${ }^{24}$ 


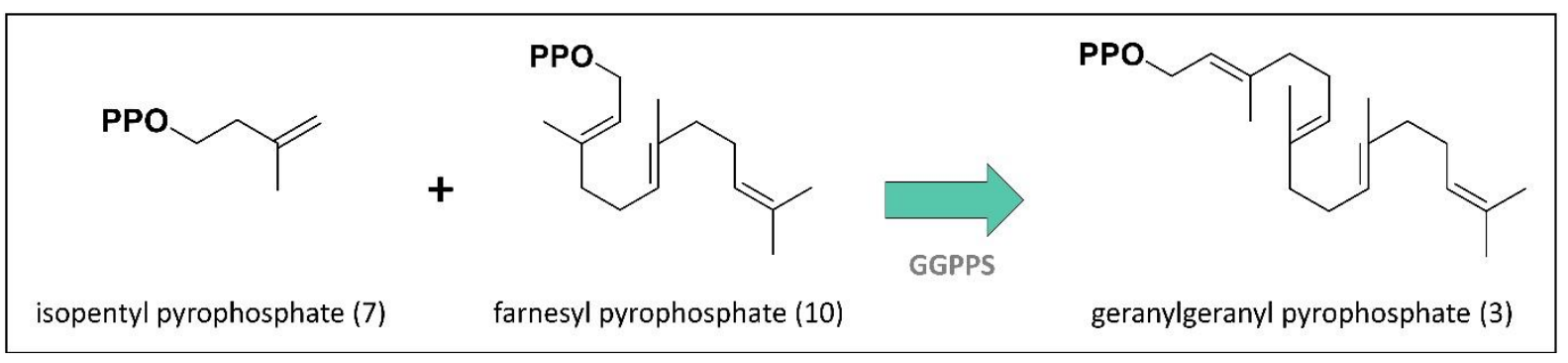

Figure 1.4. First secondary-metabolic step in indole diterpene biosynthesis catalysed by a geranylgeranyl pyrophosphate (3) synthase (GGPPS) enzyme (turquoise arrow). By convention, indole diterpene geranylgeranyl pyrophosphate (3) synthases are identified by the suffix $\mathrm{G}$. For illustrative purposes block arrows have been used to link genes to chemistry. The name of the enzyme that the gene encodes is depicted below the arrow and the direction of the arrow indicates reaction direction. The likely cofactor for GGPPS enzymes is $\mathrm{Mg}^{2+}$ but not all enzymatic mechanisms have been thoroughly examined.

Feeding studies with labelled substrates have clearly shown that the diterpene moiety of the indole diterpene core is biosynthesised from twelve units of acetate (9), which make up the four isoprene units of geranylgeranyl pyrophosphate (3). Acklin et al. (1977) were the first to show that twelve units of $\left[{ }^{13} \mathrm{C}\right]$-acetate (9) were an essential component of the diterpene moiety in paspaline (11) biosynthesis in Claviceps paspali. ${ }^{18}$ Further feeding studies on Penicillium crustosum by de Jesus et al. (1983) using ${ }^{13} \mathrm{C}$ and ${ }^{2} \mathrm{H}$-labelled acetate (9), mevalonate, and mevalonolacetone concluded that six mevalonate units, four for the indole diterpene core and two for the northern region, were involved in the biosynthesis of penitrem $A(12) \cdot{ }^{21}$ Similarly, feeding studies by Byrne et al. (2002) on Hypoxylon pulicicidum (formerly Nodulisporium sp.) using ${ }^{13} \mathrm{C}$ - labelled acetate (9) and mevalonolacetone showed that nodulisporic acids were biosynthesised from seven isoprene units, four for the indole diterpene core, two in the western region, and one in the southern region; ${ }^{44}$ thereby confirming that twelve acetates (9) were involved in geranylgeranyl pyrophosphate (3) biosynthesis and nine involved in three isopentyl pyrophosphates (7) that form western and southern regions.

The identification of the first indole diterpene gene cluster, linked to paxilline (2) production in $P$. paxilli, further supported the involvement of a geranylgeranyl pyrophosphate (3) synthase due to the findings that a geranylgeranyl pyrophosphate (3) synthase (paxG), located within the PAX gene cluster, was essential for paxilline (2) biosynthesis. ${ }^{24}$ Since then, five other secondary-metabolic geranylgeranyl pyrophosphate (3) synthase-encoding gene sequences [atmG (A. flavus), ${ }^{28}$ ItmG (E. 
festucae), ${ }^{29}$ janG ( $P$. janthinellum), ${ }^{32}$ ptmG ( $P$. simplicissimum),${ }^{31}$ and penG $(P$. crustosum $)^{32}$ ] have been found clustered with their downstream indole diterpene genes (Figure 1.2).

Interestingly, there has been one reported indole diterpene producing organism, Chaunopycnis alba, that does not seem to have a secondary-metabolic geranylgeranyl pyrophosphate (3) synthase gene that is homologous to the known geranylgeranyl pyrophosphate (3) synthase genes (i.e. paxG, atmG, ItmG). ${ }^{30} \mathrm{C}$. alba produces a group of indole diterpenes known as terpendoles, for which the TER gene cluster has been identified. Since the TER cluster clearly lacks a secondary-metabolic geranylgeranyl pyrophosphate (3) synthase gene, the C. alba, genome was screened with degenerate PCR primers designed to functionally-confirmed secondary-metabolic geranylgeranyl pyrophosphate (3) synthase genes but no homologues were identified. The high titres of indole diterpenes that $C$. alba produces suggest high flux of geranylgeranyl pyrophosphate into the pathway. Therefore, it has been speculated that $C$. alba does have a secondary-metabolic geranylgeranyl pyrophosphate (3) synthase gene but that no homolgoues were identified due to low sequence similarity. ${ }^{30}$

\section{Secondary metabolism step 2: Geranylgeranylation of indole-containing substrate}

The second biosynthetic step in indole diterpene biosynthesis is catalysed by a geranylgeranyl transferase (GGT) enzyme, which facilitates the indole condensation of geranylgeranyl pyrophosphate (3) and an indole-containing donor to make 3geranylgeranylindole (13) as shown in Figure 1.5. Results from feeding experiments with labelled ${ }^{19}, 22,44$ and unlabelled ${ }^{40}$ indole-core precursors have shown that geranylgeranyl transferases from different organisms have variability in their indole donor substrate preferences. 


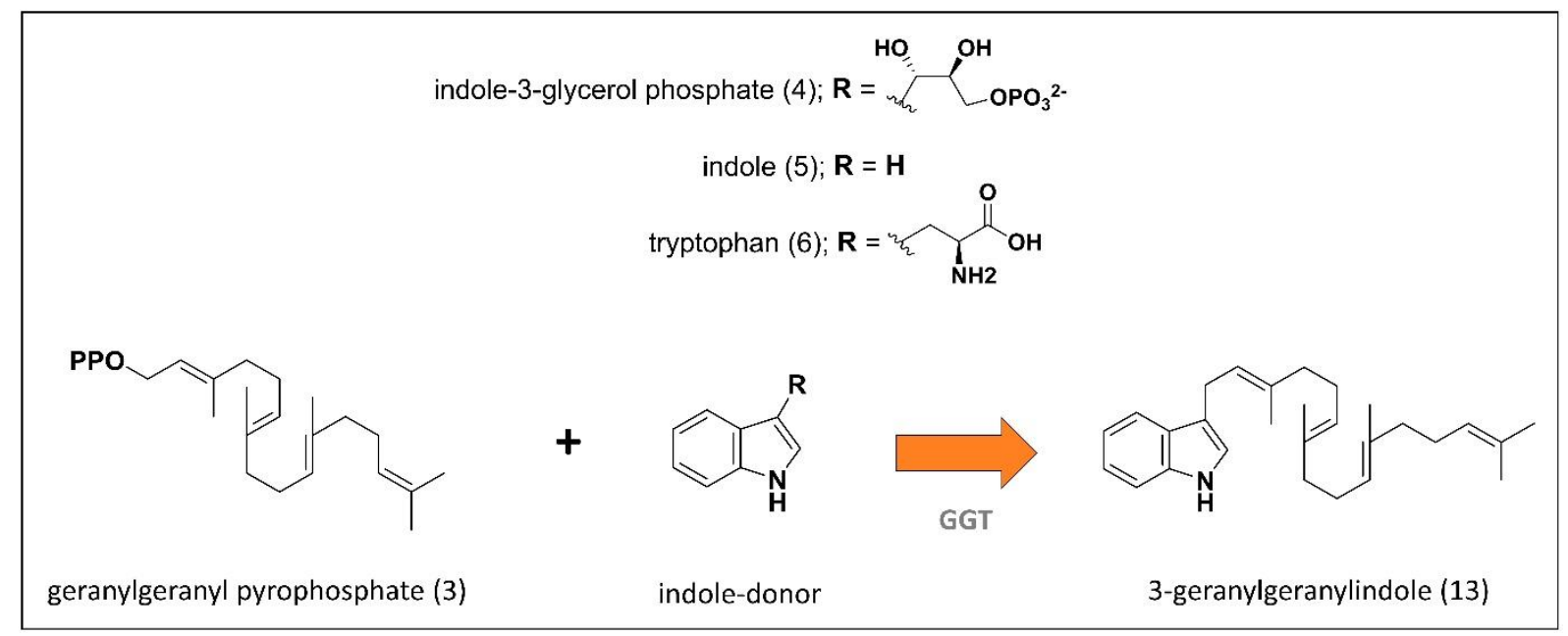

Figure 1.5. Second secondary-metabolic step in indole diterpene biosynthesis catalysed by a geranylgeranyl transferase (GGT) enzyme (orange arrow). By convention, indole diterpene geranylgeranyl transferases are identified by the suffix $C$. The likely cofactor for GGT enzymes is $\mathrm{Mg}^{2+}$ but not all enzymatic mechanisms have been thoroughly examined.

The first reported labelled indole-core precursor feeding studies on indole diterpene biosynthesis examined the effects of feeding two ${ }^{14} \mathrm{C}$-labelled tryptophan (6) precursors, (2S)-[3- ${ }^{14} \mathrm{C}$-tryptophan (6) and (2R/S)-[benzene-ring-U- $\left.{ }^{14} \mathrm{C}\right]$-tryptophan (6), to Penicillium crustosum, a known producer of penitrems [see Figure 1.2 for srtructure of penitrem $A(12)] .{ }^{19}$ Results from this study revealed that the indole portion of tryptophan (6) was incorporated into penitrem $A(12)$, but further examination into alternate indole-containing donors was never conducted.

For around 20 years it was generally assumed that tryptophan (6) was the preferred indole-donor in indole diterpene biosynthetic pathways - as the specific substrate for the geranylgeranyl transferase step of indole diterpene biosynthesis had not been identified. In further support of this, Mantle et al. published results in 1994, where they found that $\left[{ }^{14} \mathrm{C}\right]$-tryptophan (6) fed to Penicillium paxilli was incorporated into its indole diterpenes, the paxillines, but again, no other indole-containing precursors were tested. ${ }^{22}$

It was not until Byrne et al. (2002) published results of their indole-core precursor feeding studies with the nodulisporic acid indole diterpene producer $H$. pulicicidum (formerly Nodulisporium sp. ${ }^{17}$ ) that researchers in the field began to really question the substrate preference of the geranylgeranyl transferases. Byrne et al. (2002) were the first to propose the geranylgeranyl transferase substrate preference of indole-3- 
glycerol phosphate (4), the direct precursor of tryptophan (6), on the basis that they were unable to incorporate indole- $-{ }^{14} \mathrm{C}$-tryptophan (6), $2-{ }^{13} \mathrm{C}$-indole-tryptophan (6), or $2-{ }^{13} \mathrm{C}$-indole (5) into any of the nodulisporic acids but were successfully able to incorporate (ring- ${ }^{14} \mathrm{C} 6$ )-anthranilic acid and $1-{ }^{13} \mathrm{C}$-D-ribose. ${ }^{44}$

To explain the conflicting results presented for the substrate specificity of the geranylgeranyl transferases, Tagami et al. (2013) carried out in vitro substrate conversion experiments using recombinant PaxC. ${ }^{40}$ They individually subjected three indole-containing donors, indole-3-glycerol phosphate (4), indole (5), and tryptophan (6), to a PaxC-mediated reaction with geranylgeranyl pyrophosphate (3) and $\mathrm{Mg}^{2+}$ and conducted subsequent kinetic experiments. High-performance liquid chromatography (HPLC) analysis of their reaction products revealed high quantities of 3geranylgeranylindole (13) in the reaction with indole-3-glycerol phosphate (4), moderate quantities of 3-geranylgeranylindole (13) in the reaction with indole (5), and trace quantities of 3-geranylgeranylindole (13) in the reaction with tryptophan (6). In accordance with the HPLC results, their subsequent kinetic experiments further exemplified that indole-3-glycerol phosphate (4) was the preferred indole-core substrate for PaxC ( $k_{c a t} / K_{M}$ for indole-3-glycerol phosphate (4) was $28.2 \mathrm{mM}^{-1} \mathrm{~s}^{-1}$ and for indole (5) was $3.6 \mathrm{mM}^{-1} \mathrm{~s}^{-1}$ ). Notably, the in vitro analysis of PaxC avoids interference from other enzymes in the fungi, which could convert the fed substrate [e.g. $\left[{ }^{14} \mathrm{C}\right.$-tryptophan (6)] into alternate compounds [e.g. $\left[{ }^{14} \mathrm{C}\right]$-indole-3-glycerol phosphate (4)] that in turn could be used as the geranylgeranyl transferase substrate. Therefore, it can be concluded that indole-3-glycerol phosphate (4) is the preferred indole-containing donor in the paxilline (2) biosynthetic pathway, but to ensure that this result can be extended to all indole diterpene biosynthetic pathways additional substrate conversion experiments using recombinant geranylgeranyl transferases and subsequent kinetic experiments are required.

In addition to PaxC's promiscuity around accepting various indole-containing donors, Liu et al. (2013) revealed PaxC's promiscuity around the prenyl donor. ${ }^{39}$ PaxC kinetic experiments involving farnesyl pyrophosphate (10) as the prenyl donor revealed catalytic conversion to farnesylindole (14), albeit with a lower $k_{c a t} / K_{M}$ value $\left(16.6 \mathrm{~s}^{-1}\right.$ $\mathrm{mM}^{-1}$ ) than Tagami et al. (2013) noted for geranylgeranyl pyrophosphate (3) $\left(278.1 \mathrm{~s}^{-}\right.$ $\left.{ }^{1} \mathrm{mM}^{-1}\right) .{ }^{40}$ These results indicate that geranylgeranyl pyrophosphate (3) is the preferred prenyl donor in comparison to farnesyl pyrophosphate (10). Unfortunately, no other 
prenyl donors were tested, but this single observation of farnesyl pyrophosphate (10) conversion suggests that $\mathrm{PaxC}$ may readily accept a range of synthetic prenyl donor derivatives that contain a carbon tail composed of 15 to 25 carbons. One could presumably determine the extent of the substrate specificity through additional PaxC kinetic experiments using a wide range of prenyl donors.

Although $\mathrm{PaxC}$ is the only geranylgeranyl transferase that has been functionally analysed in vitro, functional analysis of two other geranylgeranyl transferases, AtmC from A. flavus ${ }^{33}$ and LtmC from E. festucae,${ }^{27}$ have been completed in vivo. AtmC was functionally characterised using pathway reconstitution of atm $G$ and atm $C$ in Saccharomyces cerevisiae. Unexpectedly, the extract of the atmG-atmC transformant did not contain 3-geranylgeranylindole (13) [as seen in the paxG-paxC transformant from Tagami et al. $(2013)^{40}$ ] but instead contained the 17,18-epoxy-3geranylgeranylindole (15), which Tang et al. (2015) attributed to a putative endogenous yeast epoxidase. ${ }^{33}$ Even though Tang et al. (2015) did not get 3-geranylgeranylindole (13) from the atmG-atmC transformant, they concluded that AtmC was a functional orthologue of PaxC. In contrast, LtmC was functionally characterised by Young et al. (2006) using complementation of ItmC with a P. paxilli $\triangle$ paxC deletion mutant. While the $\triangle$ paxC deletion mutant was unable to synthesise paxilline, the $\operatorname{ItmC}$ transformant restored paxilline (2) biosynthesis demonstrating that $I t m C$ is a functional orthologue of $p a x C$.

In addition to $\operatorname{pax} C$ and $\operatorname{atm} C$, four other homologous geranylgeranyl transferase encoding genes, clustered with known indole diterpene genes, have been identified through sequence similarity [ $p t m C$ ( $P$. simplicissimum),${ }^{31}$ penC ( $P$. crustosum) ${ }^{32}$ janC $\left(P\right.$. janthinellum) ${ }^{32}$ and terC (Chaunopycnis alba) ${ }^{30}$. The high sequence similarity and their location within an indole diterpene cluster, suggests that these geranylgeranyl transferase encoding genes are functional orthologues of paxC. It may prove to be the case that the level of promiscuity of each gene product differs and thus further kinetic studies on recombinant geranylgeranyl transferases are required to identify subtle differences between these proteins, and to ascertain if sequence differences correspond to any differences in function. 


\section{Secondary metabolism step 3: Epoxidation of 3-geranylgeranylindole}

At the third step in indole diterpene biosynthesis, the geranylgeranyl tail of 3geranylgeranylindole (13) is activated for cyclisation by epoxidation. Fueki et al. (2004) first established that 3-geranylgeranylindole (13) was a common essential intermediate in indole diterpene biosynthesis through feeding studies with deuterium labelled $\left[{ }^{2} \mathrm{H}_{3}\right]-$ 3-geranylgeranylindole on P. paxilli and Emericella desertorum. ${ }^{45}$ Results showed that $\left[{ }^{2} \mathrm{H}_{3}\right]$-3-geranylgeranylindole was readily incorporated into the respective indole diterpene products, paxilline (2) and emindole DA indicating that different epoxidised indole diterpene products utilised the same 3-geranylgeranylindole (13) substrate.

To date, three different types of epoxidations of the geranylgeranyl tail of 3geranylgeranylindole (13) have been proposed (Figure 1.6) that result in three unique epoxidised products: (1) a single epoxide product, 13,14-epoxy-3geranylgeranylindole (16), where the third alkene from the indole core is epoxidised, (2) a double epoxide product, bisepoxy-3-geranylgeranylindole (17), where the third and fourth alkene from the indole core are epoxidised, and (3) a single epoxide product, 17,18-epoxy-3-geranylgeranylindole (15), where the fourth alkene from the indole ring is epoxidised. 


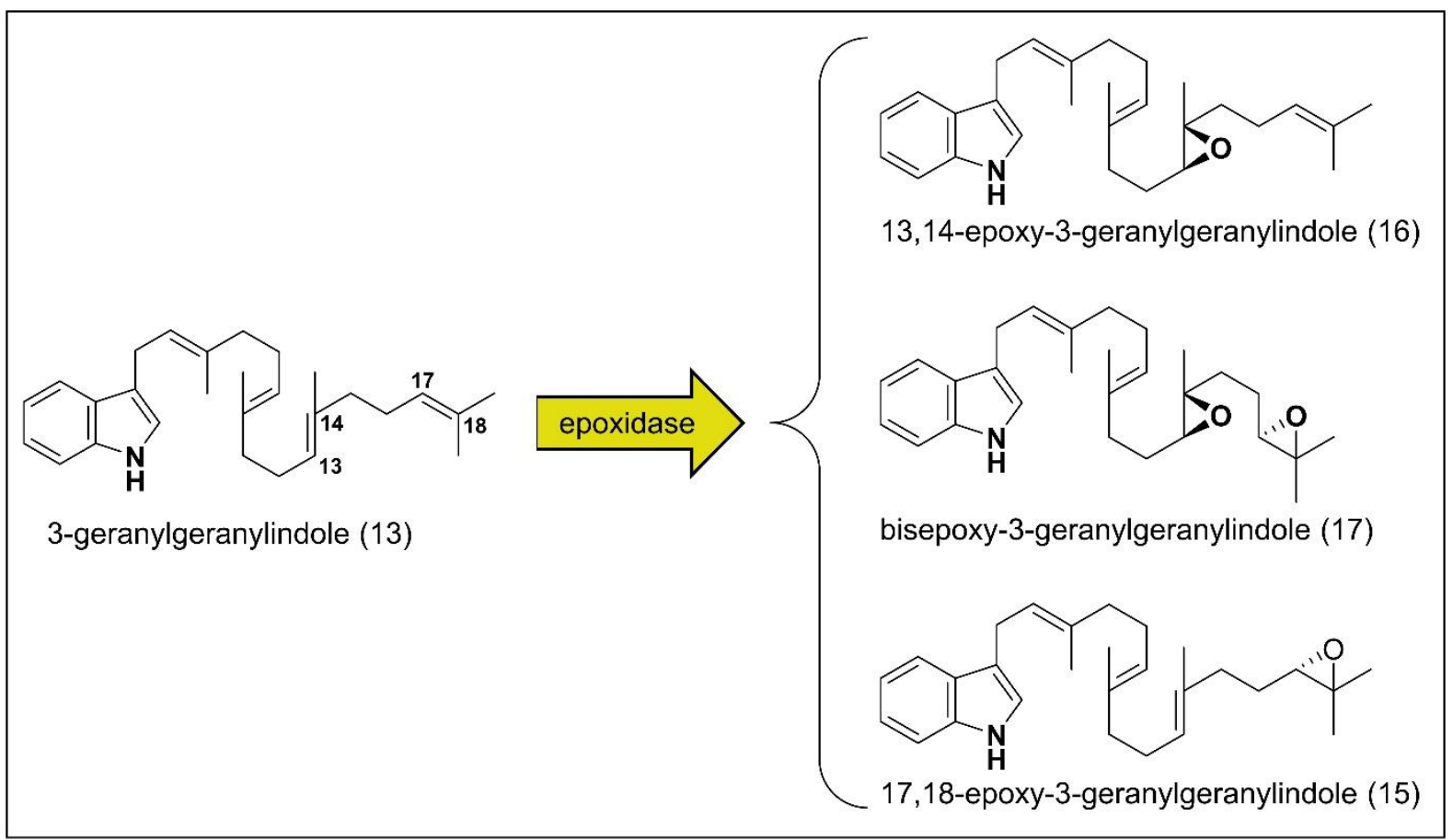

Figure 1.6. Third secondary-metabolic step in indole diterpene biosynthesis catalysed by an indole diterpene epoxidase enzyme (yellow arrow). Indole diterpene epoxidases from different species have the capacity to produce different epoxidised products [13,14-epoxy-3-geranylgeranylindole (16), bisepoxy-3-geranylgeranylindole (17) and 17,18-epoxy-3-geranylgeranylindole (15)]. By convention, indole diterpene 3geranylgeranylindole (13)-epoxidases are identified by the suffix $M$. The likely cofactors for indole diterpene expoxidase enzymes are $\mathrm{NADPH}$ and $\mathrm{O}_{2}$ but enzymatic mechanisms have not been thoroughly examined.

Full in vitro functional analysis of any of the indole diterpene epoxidases that give rise to 13,14-epoxy-3-geranylgeranylindole (16), bisepoxy-3-geranylgeranylindole (17) and/or 17,18-epoxy-3-geranylgeranylindole (15) have not been completed, but putative functions have been deduced from the results of pathway reconstitution experiments. $^{33,40}$ In 2013, Tagami et al. published results from their stepwise reconstruction of the $P A X$ cluster from $P$. paxilli into in Aspergillus oryzae. Interestingly, HPLC analysis of the extract from the paxG, paxC, and paxM transformant produced two compounds not present in the wild type strain, 13,14-epoxy-3-geranylgeranylindole (16) and its respective diol, but no bisepoxy-3-geranylgeranylindole (17) was observed. Subsequent studies on the indole diterpene cyclase, $\operatorname{pax} B$, revealed that $\operatorname{pax} B$ readily cyclised 13,14-epoxy-3-geranylgeranylindole (16) to emindole SB (18) and bisepoxy3-geranylgeranylindole (17) to paspaline (11) leading Tagami et al. (2013) to propose the first two-path stepwise epoxidation scheme of 3-geranylgeranylindole (13) shown in Figure $1.7 ; ;^{40}$ where in path 1 the epoxidase (paxM) carries out a single epoxidation 
to produce 13,14-epoxy-3-geranylgeranylindole (16) followed by a second epoxidation to produce bisepoxy-3-geranylgeranylindole (17) that gets passed onto paxB for cyclisation to paspaline (11), and in path 213,14 -epoxy-3-geranylgeranylindole (16) is cyclised (by paxB) to form emindole SB (18) and then subjected to a second epoxidation (by paxM) and second cyclisation (by paxB) to form paspaline (11). Path 1 of their two path scheme seems the most reasonable due to likely substrate specificity problems of the paxM gene product, but the fact that no bisepoxy-3geranylgeranylindole (17) was identified in the extract from the paxG, $\operatorname{pax} C$, paxM transformant makes any conclusion on the preferred route from 3-geranylgeranylindole (13) to paspaline (11) unclear. 


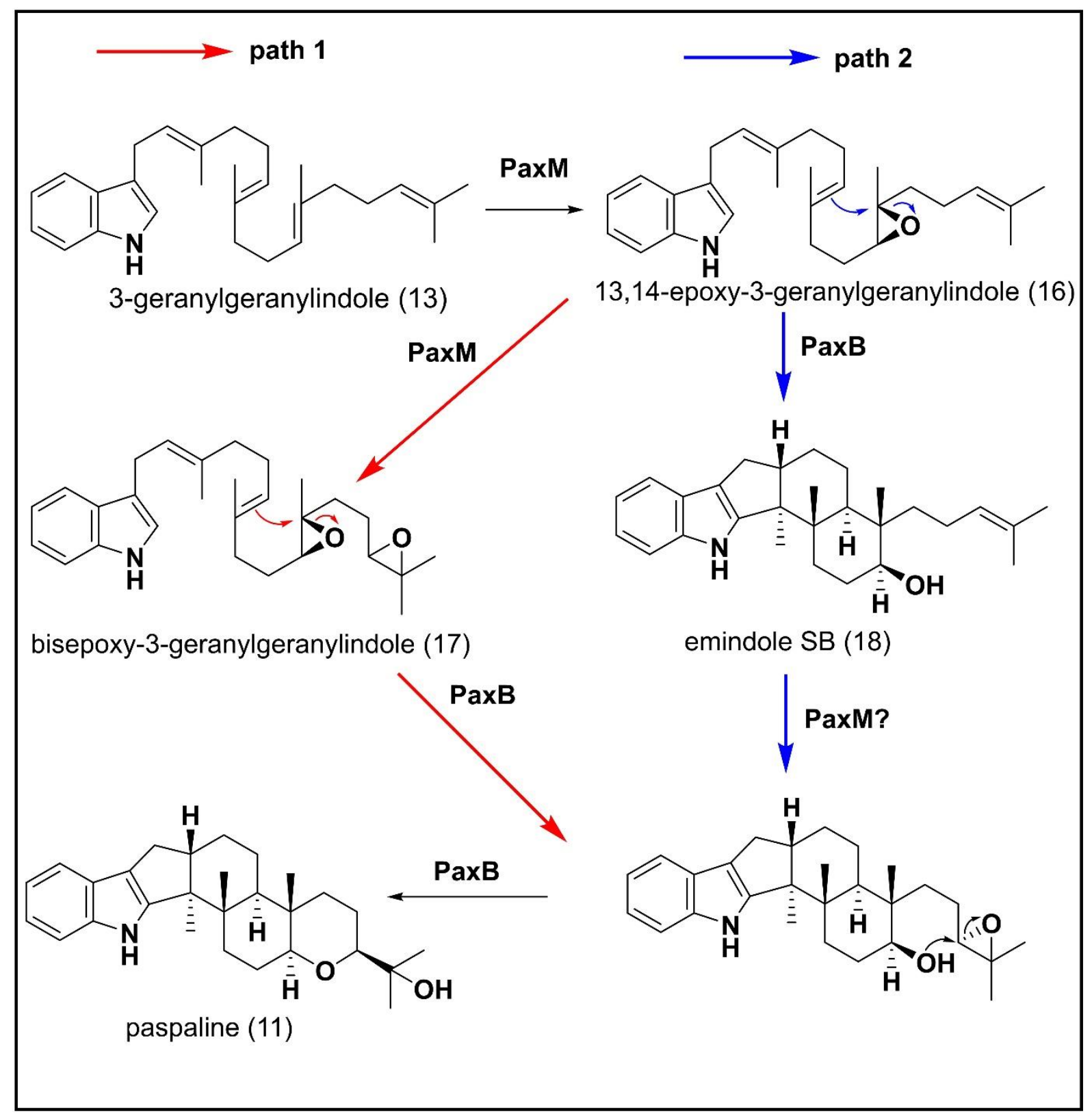

Figure 1.7. Two-path stepwise epoxidation scheme of 3-geranylgeranylindole (13) involving the epoxidase (PaxM) and the cyclase (PaxB) proposed by Tagami et al. (2013). ${ }^{40}$

A more recent study by Tang et al. (2015) published results from the reconstruction of part of the ATM gene cluster from Aspergillus flavus in Saccharomyces cerevisiae. ${ }^{33}$ Notably, extract from the atmG, atmC transformant unexpectedly contained 17,18epoxy-3-geranylgeranylindole (15), which they proposed was a product of an endogenous yeast epoxidase. Therefore, the bisepoxy-3-geranylgeranylindole (17) that they observed in the extract of the $\operatorname{atmG}$, atm $C$, atmM transformant cannot conclusively be linked to activity of the atmM gene product alone. Overall, the 
mechanism by which 3-geranylgeranylindole (13) gets epoxidised remains inconclusive and further in vitro substrate studies with recombinant epoxidases are required to reveal the preferred epoxidation mechanism.

In addition to the two epoxidases that were functionally analysed through pathway reconstitution [PaxM (P. paxilli) ${ }^{40}$ and AtmM (A. flavus $)^{33}$ ] and predicted to give rise to both the mono- and di-epoxide products 13,14-epoxy-3-geranylgeranylindole (16) and bisepoxy-3-geranylgeranylindole (17), one other epoxidase, LtmM (E. festucae), ${ }^{29}$ has been functionally characterised through complementation of ItmM into a $P$. paxilli $\triangle$ pax $M$ deletion mutant. ${ }^{26}$ While the $\Delta$ pax $M$ deletion mutant was unable to synthesise paxilline, the ItmM transformant restored paxilline (2) biosynthesis demonstrating that ItmM is a functional orthologue of paxM and thereby encodes the epoxidase capable of producing the necessary epoxide product for paxilline (2) biosynthesis; however discrepancies around whether that is the mono- or di-epoxide product [13,14-epoxy-3geranylgeranylindole (16) or bisepoxy-3-geranylgeranylindole (17)] remains unclear.

In addition to these three functionally characterised epoxidases (PaxM, AtmM, and LtmM), four others [(PtmM ( $P$. simplicissimum), ${ }^{31}$ PenM ( $P$. crustosum) ${ }^{32}$ JanM $(P$. janthinellum), ${ }^{32}$ TerM (Chaunopycnis alba) ${ }^{30}$ ], have been identified through sequence similarity and are proposed to be functional orthologues of PaxM, AtmM, and LtmM based on their hosts' abilities to produce paspaline (11)-derived indole diterpene products (i.e. penitrems, shearinines, and terpendoles; examples shown in Figure 1.2). Unlike the epoxidases that give rise to 13,14-epoxy-3-geranylgeranylindole (16) and bisepoxy-3-geranylgeranylindole (17), epoxidases that only give rise to the single epoxide product, 17,18-epoxy-3-geranylgeranylindole (15), have not been identified making the biosynthesis of compounds derived from 17,18-epoxy-3geranylgeranylindole (15) relatively underexplored.

Together, the three epoxidised products [13,14-epoxy-3-geranylgeranylindole (16), bisepoxy-3-geranylgeranylindole (17) and 17,18-epoxy-3-geranylgeranylindole (15)] establish the potential biosynthetic pathways by which the indole diterpenes can be cyclised (Figure 1.8). The first epoxidised product, 13,14-epoxy-3geranylgeranylindole (16), where only the third alkene from the indole core is epoxidised, serves as a substrate in the biosynthesis of emindole SB (18) derived indole diterpenes (like the nodulisporic acids) and non-emindole SB derived compounds (like the aflavinines, anominines, tubingensins, eujindoles, emindole 
SA/DA, and emeniveol-derivatives). The second epoxidised product, bisepoxy-3geranylgeranylindole (17), where both the third and fourth alkenes are epoxidised, is used as a substrate in the biosynthesis of paspaline (11)-derived indole diterpenes (like the paxillines, lolitrems, aflatrems, penitrems, terpendoles, shearinines, janthitrems, and sulpinines) and some non-paspaline-derived indole diterpenes (like the thiersinines, anthcolorins, AtS5 compounds, emindole DB (19), and emindoles PCPA). The third epoxidised product, 17,18-epoxy-3-geranylgeranylindole (15), where the fourth alkene from the indole core is epoxidised, acts as the substrate for the relatively unexplored biosynthesis of radarins and petromindole (20). The cyclisation mechanisms of the three epoxides [13,14-epoxy-3-geranylgeranylindole (16), bisepoxy-3-geranylgeranylindole (17) and 17,18-epoxy-3-geranylgeranylindole (15)] will be explored in detail in the next section (Indole diterpene biosynthesis: Step 4). 


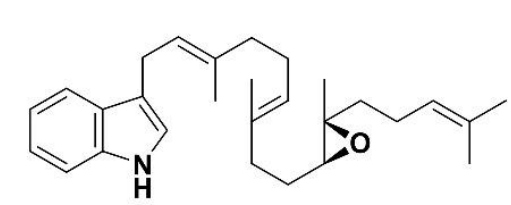

13,14-epoxy-3-geranylgeranylindole (16)

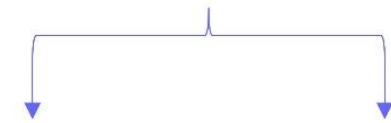

mindole SB

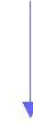

nodulisporic acids

\section{aflavinines}

anominines

tubingensins

eujindoles

emindole SA/DA

emeniveol-derivatives

etc.

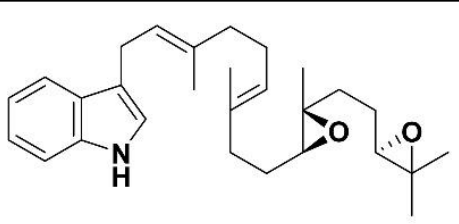

bisepoxy-3-geranylgeranylindole (17)

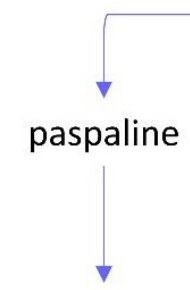

paxillines

aflatrems

lolitrems

terpendoles

penitrems

shearinines

janthitrems

sulpinines

etc.

thiersinines

anthcolorins

emindole $\mathrm{DB}$

etc.

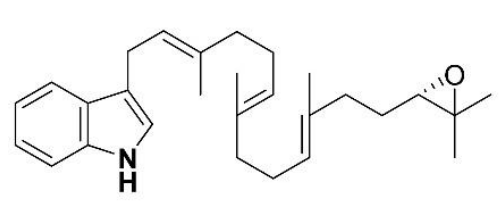

17,18-epoxy-3-geranylgeranylindole (15)

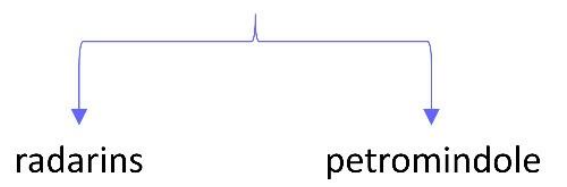

AtS5 compounds

emindoles PC-PA

Figure 1.8. Depiction of the different biosynthetic pathways that arise from the three epoxidised products 13,14-epoxy-3geranylgeranylindole (16), bisepoxy-3-geranylgeranylindole (17) and 17,18-epoxy-3-geranylgeranylindole (15). 


\section{Secondary metabolism step 4: Formation of cyclic indole diterpene scaffolds}

In the fourth indole diterpene biosynthesis step the activated 3-geranylgeranylindole

$$
\text { epoxides [13,14-epoxy-3-geranylgeranylindole }
$$

(16),

bisepoxy-3geranylgeranylindole (17) and 17,18-epoxy-3-geranylgeranylindole (15)] are cyclised to form the skeleton library of tetra-, penta-, and hexa-cyclic indole diterpene scaffolds as shown in Figure 1.9. Each skeleton is derived from a cyclisation mechanism that follows either a Markovnikov reaction mechanism or an anti-Markovnikov reaction mechanism often coupled with selected sigmatropic shifts (e.g. $\mathrm{H}$ shifts, $\mathrm{CH}_{3}$ shifts, and bond shifts). The cyclisation mechanisms can be divided into four key paths (Figure 1.9, blue arrows) from which all known indole diterpenes are biosynthesised. Three of these paths involve Markovnikov cyclisation mechanisms (Figure 1.9, path 1, 2 and 4) and one of them involves an anti-Markovnikov cyclisation mechanism (Figure 1.9, path 3). Each cyclisation path has been proposed based on results from the metabolic profiles from native fungi, ${ }^{48}$ or the metabolic profiles from precursor feeding studies $^{18,21,44}$ and/or pathway reconstruction studies. ${ }^{33}$ 


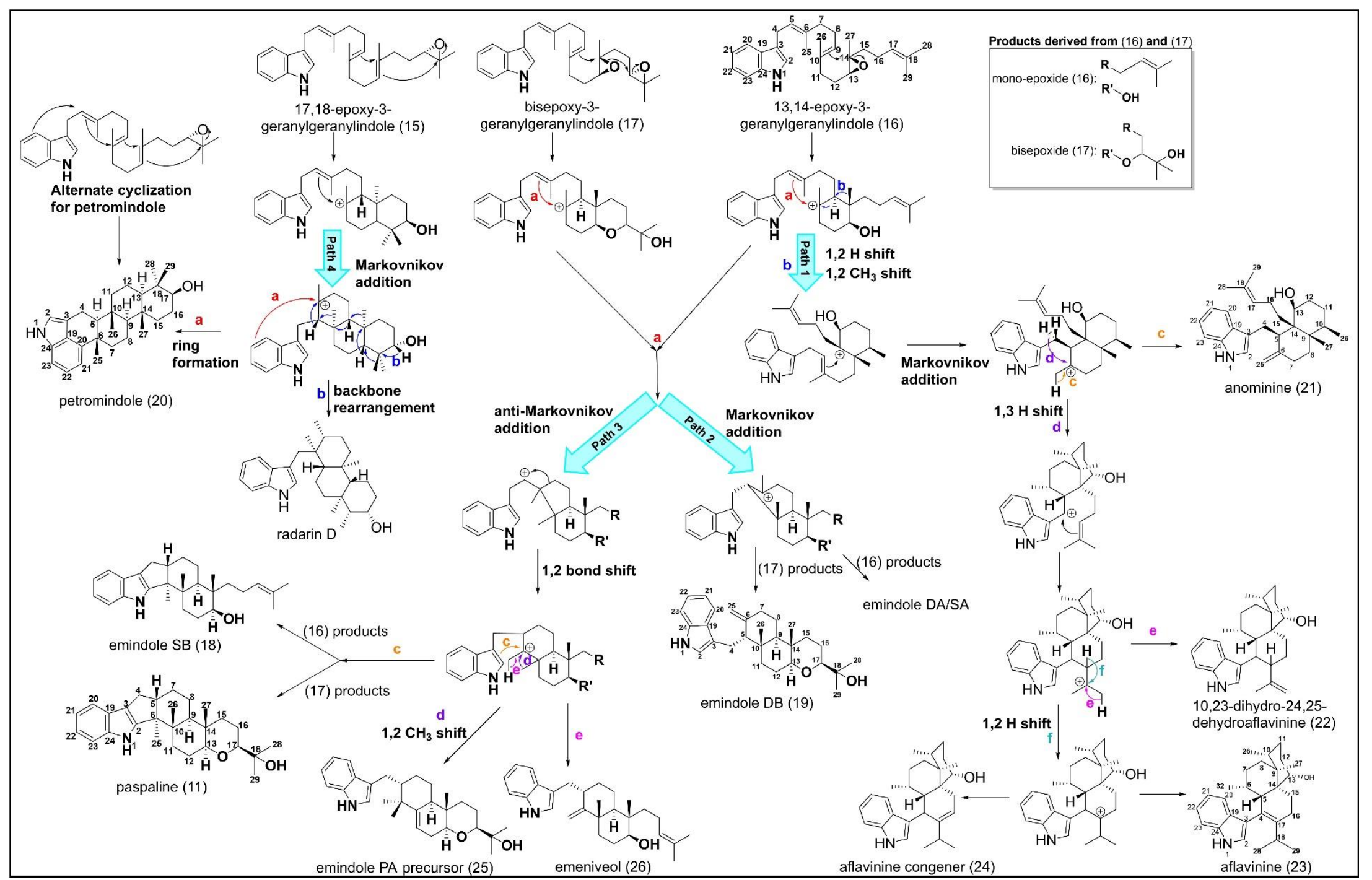

Figure 1.9. Fourth secondary-metabolic step in indole diterpene biosynthesis catalysed by an indole diterpene cyclase enzyme. Indole diterpene cyclases from different species have the capacity to cyclise the epoxidised products [13,14-epoxy-3-geranylgeranylindole (16), bisepoxy-3-geranylgeranylindole (17) and 17,18-epoxy-3-geranylgeranylindole (15)] in different ways. 


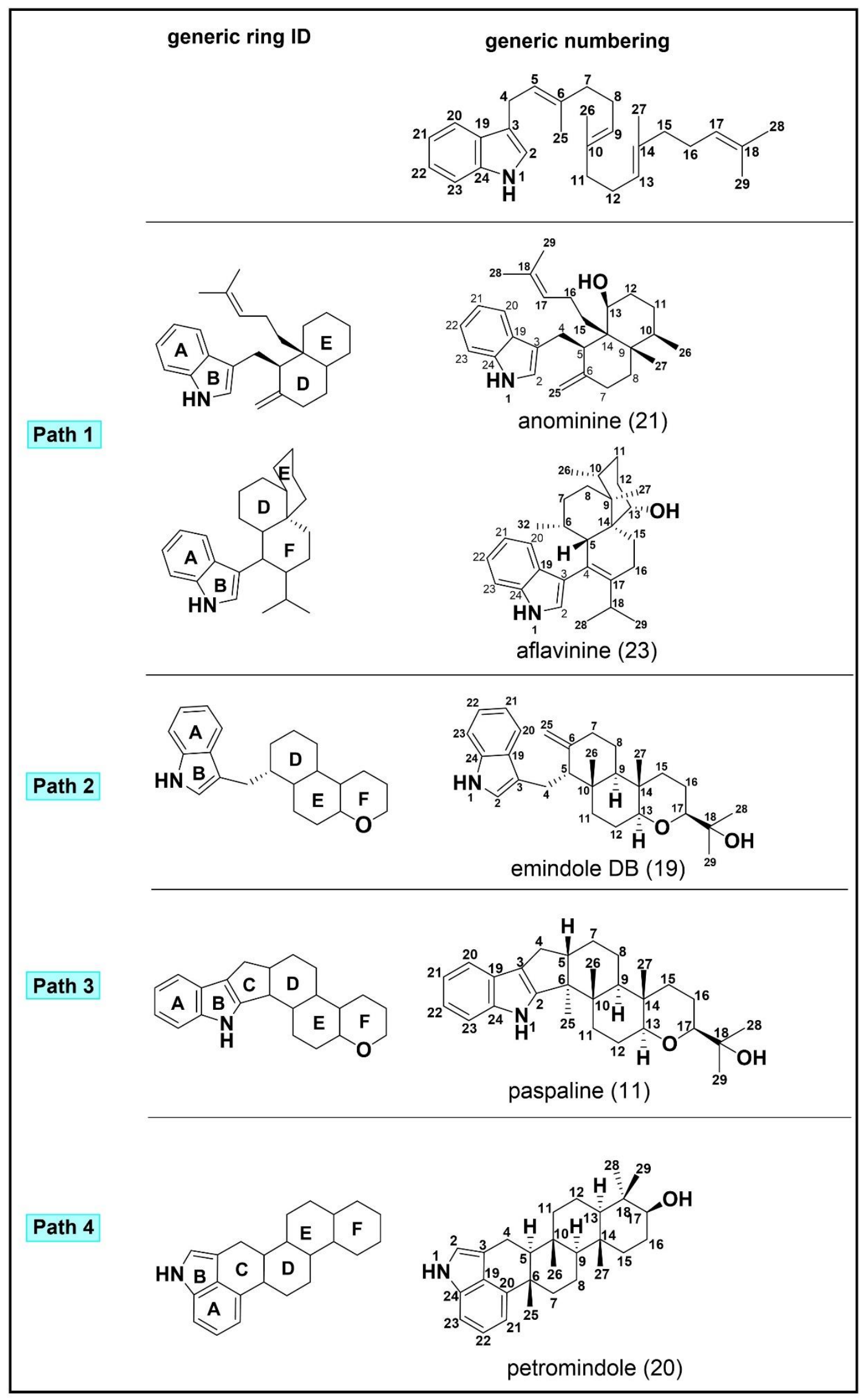

Figure 1.10. Summary of generic numbering scheme used in this thesis to classify the indole diterpene scaffolds. 
Path 1 of Figure 1.9, first proposed by Tang et al. (2015), was established on the basis of phylogeny guided pathway reassembly of two unclustered indole diterpene cyclases (AtS2B, and AfB from Aspergillus tubingensis and Aspergillus flavus respectively) in Saccharomyces cerevisiae. ${ }^{33}$ Results from their reconstitution studies revealed that AtS2B and AfB readily cyclised 13,14-epoxy-3-geranylgeranylindole (16) into anominine (21) and 10,23-dihydro-24,25-dehydroaflavinine (22) and that AfB could additionally cyclise 13,14-epoxy-3-geranylgeranylindole (16) into aflavinine (23) and its congener (24). They were then able to propose the most likely cyclisation mechanism that the two enzymes catalysed in which 13,14-epoxy-3-geranylgeranylindole (16) undergoes a 1,2- $\mathrm{H}$ shift and 1,2- $\mathrm{CH}_{3}$ shift followed by subsequent Markovnikov driven cyclisation before splitting into two subpaths ( $c$ and $d$ of Path 1 ) to produce anominine (21) as shown in path $c$ and the aflavinines as shown in path $d$. Where subpath $c$ of Path 1 involves a simple deprotonation, path d involves a 1,3- $\mathrm{H}$ shift and ring closure before branching into two additional subpaths e and f to deliver 10,23-dihydro-24,25dehydroaflavinine (22) via deprotonation (path e) and aflavinine (23) and its congener (24) via a 1,2-H shift and subsequent deprotonation (path $\mathrm{f}$ ). Since these cyclisation mechanisms are simply proposals, future work with labelled substrates is required to confirm the exact chemical mechanisms of Path 1 indole diterpene cyclases.

In contrast to Path1 of Figure 1.9, no sigmatropic shifts of the indole diterpene carbon skeleton are required to produce the various emindole indole diterpene products derived from Path 2 [e.g. emindole DA,SA, and DB (19)]. Instead, products from Path 2 are derived from the single or double epoxide products 13,14-epoxy-3geranylgeranylindole (16) and bisepoxy-3-geranylgeranylindole (17) via a simple Markovnikov driven ring closure and subsequent deprotonation. Notably, Path 2 was first proposed by Nozawa et al. (1988) and the indole diterpene cyclases that catalyse this pathway have yet to be identified.

Similar to Path 2 of Figure 1.9, Path 3 involves cyclisation of both the single and double epoxide products 13,14-epoxy-3-geranylgeranylindole (16) and bisepoxy-3geranylgeranylindole (17) but follows an anti-Markovnikov driven ring closure. The ring closure is the common precursor for a variety of subpaths (c,d, and e) to produce emindole SB (18), paspaline (11), the emindole PA precursor (25), and emeniveol (26). The involvement of a 1,2-bond shift has been proposed on the basis of coupling observed between C5 and C7 (See paspaline (11) in Figure 1.10) in the $\left[2{ }^{13} \mathrm{C}\right]$-acetate 
(9) derived indole diterpene products (paspalines, penitrems, and nodulisporic acids) from feeding studies on C. paspali, ${ }^{18}$ P. crustosum, ${ }^{21}$ and $\mathrm{H}$. pulicicidum ${ }^{44}$ respectively. Notably all of the indole diterpene products involved in the acetate (9)-labelled feeding studies are derived from subpath $\mathrm{c}$ of Path 3 and the functional examination of three indole diterpene cyclases $\left[\right.$ AtmB (A. flavus), ${ }^{33}$ AtS5B1( A. tubingensis), ${ }^{33}$ PaxB ( $P$. paxilli ${ }^{40}$ ], has revealed that all three cyclases have the ability to produce both subpath c products, emindole SB (18) and paspaline (11). In addition to the three cyclases that have been functionally confirmed, five others $\left[\mathrm{PtmB}\left(P\right.\right.$. simplicissimum), ${ }^{31} \mathrm{LtmB}(E$. festucae), ${ }^{29}$ PenB (P. crustosum), ${ }^{32}$ JanB (P. janthinellum), ${ }^{32}$ TerB (Chaunopycnis alba $)^{30}$ ], have been proposed to have the same functionality as AtmB, AtS5B1, and PaxB based on sequence identity and location within indole diterpene gene clusters. As for the other two subpaths ( $d$ and $e$ ) of Path 3, one indole diterpene cyclase [AtS5B1 (A. tubingensis $)^{33}$ ] has been identified for subpath d involving a $1,2-\mathrm{CH}_{3}$ shift, and none have been identified for subpath $\mathrm{e}$.

In comparison to Paths 1-3 of Figure 1.9, Path 4 is unique in that it does not involve a cyclisation scheme activated by an internal epoxide moiety. Rather, a cyclisation scheme involving the formation of two carbocyclic rings ( $E$ and $F$, See figure 1.10, path 4) from 17,18-epoxy-3-geranylgeranylindole (15) is proposed to give rise to petromindole (20) and the radarins via subpath $a$ and $b$ of Path 4 respectively. The lack of the internal epoxide is linked to re-face addition as opposed to the si-face addition seen in Paths 1-3 giving rise to the reversal in stereochemistry of the petromindole (20) and radarin stereocenters. To date, only one Path 4 cyclase has been identified and surprisingly it was not from a fungus known to produce petromindole (20) or radarins. Rather, the cyclase identified was a lupeole synthase (LUP1) from the plant species Arabidopsis thaliana, leading Xiong et al. (2003) to hypothesise that the cyclase in the petromindole (20) and radarin producing endophytes is a modified oxidosqualene cyclase distant from other indole diterpene cyclases. ${ }^{49}$ They further proposed that the putative indole diterpene cyclase likely evolved from a lanosterol synthase or a pentacyclic triterpene synthase. Overall, more work is required to identify the petromindole (20) and radarin indole diterpene cyclases and confirm their mechanisms of action.

In conclusion, the indole diterpene cyclases encompass a class of enzymes that catalyse an extremely wide range of reactions to establish the core library of cyclised 
indole diterpene compounds. These core compounds are often decorated further to install the potent bioactive properties seen across the known indole diterpenes. In the next section, genes encoding enzymes for these decorative steps will be reviewed.

\section{Decoration of the cyclic scaffolds: Gene identification and elucidation of the decoration steps}

Genes encoding decorative enzymes have been identified through sequence analysis followed by functional studies on gene knockout strains, ${ }^{24}, 29-30,32,34-35$ pathway complementation ${ }^{25-29}$ or reconstitution studies, ${ }^{31}, 33,35,40-41,43$ precursor feeding studies, ${ }^{29,} 31,36$ and in vitro recombinant enzyme analyses. ${ }^{31,} 39,42-43$ These decorative enzymes fall into five categories [cytochrome P450 oxygenases (P450s), FAD/FMN dependent oxygenases (FOs), NAD(P)+ dependent oxidoreductases (NOs), prenyl transferases (PTs), acetyl transferases (ATs), and unknowns (UKs)] where each category represents a type of reaction. To date, indole diterpene decorative enzymes from seven fungal species within two fungal classes [Sordariomycetes (Aspergillus flavus, Aspergillus terreus, Penicillium janthinellum, Penicillium paxilli, Penicillium simplicissimum) and Eurotiomycetes (Chaunopycnis alba, Epichloë festucae)] have been functionally identified creating a library of 36 known decorative genes, which include $21 \mathrm{P} 450 \mathrm{~s}$ [PaxPQ (P. paxilli), ${ }^{36,} 40 \mathrm{AtmPQ}$ (A. flavus), ${ }^{28,41}$ LtmPQJK (Epichloë festucae), ${ }^{29}$ TerPQK (Chaunopycnis alba), ${ }^{30}$ PtmPQJKLU (P. simplicissimum), ${ }^{31}$ JanPQJ (Penicillium janthinellum), ${ }^{43}$ and AtS5-P450 (A. terreus) $\left.)^{33}\right], 4$ FOs [PaxO ( $P$. paxillI), ${ }^{38} \mathrm{JanO}$ (Penicillium janthinellum), ${ }^{43} \mathrm{PtmNO}$ (P. simplicissimum) $\left.{ }^{31}\right], 1 \mathrm{NO}[\mathrm{PtmH}$ (P. simplicissimum) ${ }^{31}$ ], 8 PTs [PaxD ( $P$. paxillI) ${ }^{42}$ AtmD (A. flavus), ${ }^{39}$ LtmFE (Epichloë festucae), ${ }^{29}$ TerF (Chaunopycnis alba), ${ }^{30}$ PtmDE (P. simplicissimum), ${ }^{31}$ JanD (Penicillium janthinellum)], ${ }^{43} 1$ AT [PtmV (P. simplicissimum $\left.)^{31}\right]$, and 1 unknown [Ptml (P. simplicissimum $)^{31}$. This gene library establishes a suite of information that essentially provides a 'recipe' book to install selectively various functional groups onto the indole diterpene skeletons formed in Step 4 of indole diterpene biosynthesis. The various classes of indole diterpene decorative genes will be discussed in detail below. 


\section{Identification of gene clusters enables rapid confirmation of individual gene function}

The various gene clusters shown in Figure 1.2 were discovered over the course of 2001-2015 and their discovery enabled subsequent gene functionality studies. During this time the technological advancements around genetic sequencing and molecular biology rapidly expedited the amount of time required to identify novel genes and functionally confirm their roles in indole diterpene biosynthesis. Although the feeding studies on native indole diterpene producers with labelled substrates gave insight into how the various indole diterpene cores were biosynthesised, it was the identification of the indole diterpene gene clusters themselves that was paramount in understanding how the various indole diterpene cores were decorated. Below, methods of how the known indole diterpene genes have been identified and functionally characterised will be examined.

\section{Identification of the $P A X$ gene clusters and functional analysis of paxP, paxQ and paxD}

The PAX cluster (Figure 1.11) was the first indole diterpene cluster identified. Using plasmid mutagenesis Young et al. (1998) isolated three paxilline-negative mutants, the first by random integration of the pAN7-1 plasmid into the $P$. paxilli genome and the other two by homologous integrations of a replacement construct at the locus identified by the first random integration. ${ }^{23}$ Their results indicated that genes required for paxilline (2) biosynthesis were located between a 100-200 kb sequence on chromosome $\mathrm{Va}$ of the $P$. paxilli genome.

To determine where the PAX genes were located within the $100-200 \mathrm{~kb}$ sequence, Young et al. (2001) carried out a second round of plasmid mutagenesis with pAN7-1 using restriction enzyme-mediated integration. ${ }^{24}$ From the thin-layer chromatography (TLC) analysis of the extracts of 750 transformants they isolated one transformant (LM662) that did not produce paxilline. Molecular analysis of the plasmid insertion site revealed that the locus tagged with pAN7-1 was unlikely to be involved in paxilline (2) biosynthesis. Subsequent southern hybridisation of the LM-662 genome revealed a second site mutation corresponding to a $22.3 \mathrm{~kb}$ deletion that contained a portion of 
the 17 genes (paxGMCPQDNRSHTOUVWXY) they hypothesised may be involved in paxilline (2) biosynthesis.

\begin{tabular}{|l|l|c|}
\hline \multicolumn{3}{|c|}{ PAX cluster from Penicillium paxilli (strain PN2013) } \\
\hline PaxG & Geranylgeranyl pyrophosphate (3) synthase & 1 \\
\hline PaxA & Unknown & -3 \\
\hline PaxM & FAD-dependent oxygenase (epoxidase) & 4 \\
\hline PaxB & Cyclase & 2 \\
\hline PaxC & Geranylgeranyl transferase & 6 \\
\hline PaxP & Cytochrome P450 oxygenase & 7 \\
\hline PaxQ & Cytochrome P450 oxygenase & 8 \\
\hline PaxD & Prenyl transferase & \\
\hline PaxO & FAD-dependent oxygenase & \\
\hline
\end{tabular}

Figure 1.11. Depiction of the PAX cluster from $P$. paxilli strain PN2013. ${ }^{38}$

As this was the first indole diterpene cluster identified, and the whole $P$. paxilli genome was not known, Young et al. (2001) were surprised to find that $P$. paxilli paxilline-negative mutants were able to survive without a geranylgeranyl pyrophosphate (3) synthase (paxG). This led them to search for another geranylgeranyl pyrophosphate (3) synthase, which they found on chromosome IV, using degenerate primers designed to conserved domains of fungal geranylgeranyl pyrophosphate (3) synthases, and termed ggs1. Molecular analysis of each geranylgeranyl pyrophosphate (3) synthase revealed that paxG was unique from all other geranylgeranyl pyrophosphate (3) synthases characterised at the time as it 
contained a DDXDD motif in the $N$-terminus that was not present in any other geranylgeranyl pyrophosphate (3) synthase. To confirm the involvement of paxG in paxilline (2) biosynthesis, they disrupted paxG in the wild-type strain and observed the loss of paxilline (2) production. Thus, they confirmed that paxG was a geranylgeranyl pyrophosphate (3) synthase dedicated to secondary metabolic synthesis and specifically the synthesis of paxilline.

Finally, Young et al. (2001) examined the expression levels of mRNA for paxG, paxM, and paxP to see if they were upregulated during paxilline (2) biosynthesis. Semiquantitative RT-PCR monitoring revealed that all three genes were upregulated with the onset of paxilline (2) production ( $60 \mathrm{~h}$; Figure 1.12, A) further suggesting that they had identified the gene locus responsible for paxilline (2) biosynthesis and highlighting that there is a coregulation of the PAX genes with the onset of paxilline (2) production.

From there, attention was shifted to two of the P450 oxygenases in the PAX cluster, PaxP and PaxQ. McMillan et al. (2003) showed that PaxP and PaxQ deletion mutants were unable to produce paxilline (2) and instead accumulated paspaline (11) and 9desoxypaxilline (27), respectively as shown in Figure 1.12, $C^{34}$ (notably, the ' 9 ' in 9desoxypaxilline (27) is numbered based on the numbering scheme shown in Figure 1.10 and will be so numbered throughout this thesis). In reports published by Barry Scott's lab they have used a different numbering scheme where this compound was named 13-desoxypaxilline). $.44,36,38$ These results provided the first evidence that PaxP was responsible for the $\mathrm{C} 14$ demethylation and subsequent $\mathrm{C} 16$ oxidation to form 9desoxypaxilline (27) and that PaxQ catalysed the C9 oxidation of 9-desoxypaxilline (27) to form paxilline.

Working from the insights of Young et al. (2001) ${ }^{24}$ and McMillan et al. (2003), ${ }^{34}$ Saikia et al. (2006) ${ }^{35}$ planned to define the minimum number of genes required for paspaline (11) biosynthesis. Results from $P A X$ gene deletion experiments revealed that paxG [Young et al. (2001)], paxC (unpublished results from Young et al.), ${ }^{35}$ paxM (unpublished McMillan et al.), ${ }^{35}$ paxA, and paxB [Saikia et al. (2006)] deletion mutants lacked the ability to produce identifiable indole diterpenes. Thus, they predicted that a maximum of five genes (paxGCMBA) may be involved in paspaline (11) biosynthesis. They prepared plasmids containing various combinations of these five genes and transformed them into a $P$. paxilli knockout mutant that lacked the entire $P A X$ cluster (strain CY2). Their results indicated that only four genes, paxGCMB, were necessary 
for paspaline (11) production (Figure 1.12, B) thereby leaving discrepancies around the absence of indole diterpenes produced by the previously generated paxA deletion mutant. One possibility is that a portion of the paxA-flanking genes, paxG or paxM, may have been deleted as well in the construction of the mutant, which prevented paspaline (11) biosynthesis; however, re-introduction of wild-type paxA restored paxilline (2) production in the pax $A$ knockout strain indicating that pax $A$ may have some significant role. Notably, paxA homologues are conserved throughout many other known indole diterpene clusters (i.e. atmA, janA, ptmA) indicating they may have a role in indole diterpene biosynthesis; however, they are not required for heterologous biosynthesis of indole diterpenes. For example, Tagami et al. (2013) initiated paxilline (2) production in $A$. oryzae by heterologously expressing six $P A X$ genes $(\text { paxGCMBPQ })^{40}$ and van Dolleweerd et al. (2018) restored paxilline (2) production in a full $P A X$ cluster $P$. paxilli knockout strain (CY2) by reconstituting the same six $P A X$ genes (paxGCMBPQ), ${ }^{3}$ clearly demonstrating that paxA is not required for paxilline (2) biosynthesis. 


\section{A. Gene expression and deletion mutants [Young et al. (2001), McMillan et al. (2003), Saikia et al. (2006)]}

\begin{tabular}{|c|l|l|}
\hline PAX & Gene expression & Products of gene deletion mutants \\
\hline G & Upregulated with onset of paxilline production $(\sim 60 \mathrm{~h})$ [Young et al. (2001)] & no identifiable IDTs [Young et al. (2001)] \\
\hline M & Upregulated with onset of paxilline production $(\sim 60 \mathrm{~h})$ [Young et al. (2001)] & no identifiable IDTs (McMillan et al., unpublished) \\
\hline C & & no identifiable IDTs (Young et al., unpublished) \\
\hline P & Upregulated with onset of paxilline production $(\sim 60 \mathrm{~h})$ [Young et al. (2001)] & paspaline [McMillan et al. (2003)] \\
\hline Q & & 9-desoxypaxilline [McMillan et al. (2003)] \\
\hline A & & no identifiable IDTs [Saikia et al. (2006)] \\
\hline B & & no identifiable IDTs [Saikia et al. (2006)] \\
\hline
\end{tabular}

\section{B. Pathway reconstitution [Saikia et al. (2006)]}

Assembled in Penicillium paxilli $P A X$-cluster deletion mutant strain
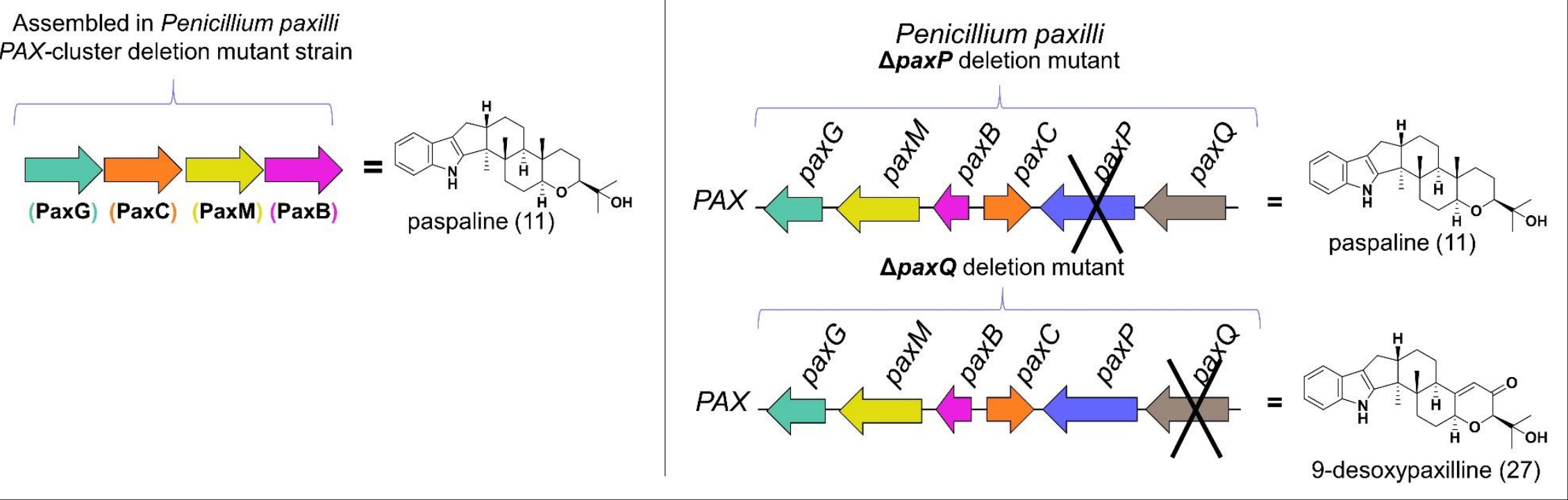

Figure 1.12. Summary of $P A X$ gene expression, deletion, and pathway reconstitution results from Young et al. (2001), ${ }^{24}$ McMillan et al. (2003), ${ }^{34}$ and Saikia et al. (2006). ${ }^{35}$ 
Identification of the minimum number of genes required for paspaline (11) biosynthesis enabled Tagami et al. (2013) to evaluate the individual biosynthetic steps involved in paspaline (11) biosynthesis by reconstituting paspaline (11) biosynthesis in the heterologous host Aspergillus oryzae. ${ }^{40}$ Stepwise introduction of paxGCMB combined with in vitro reconstituted protein studies revealed that $\mathrm{PaxC}$ is a geranylgeranyl transferase responsible for condensation of the indole-containing core and geranylgeranyl pyrophosphate (3) to form 3-geranylgeranylindole (13) and that PaxM and PaxB catalyse the stepwise epoxidation and cyclisation mechanism to form emindole SB (18) and paspaline (11) as shown in Figure 1.13.

Following the functional analysis of paxC, paxM and paxB, Tagami et al. (2013) tandemly transformed paxP and paxPQ into their four gene (paxGCMB) paspaline (11)-producing $A$. oryzae mutant, which initiated 9-desoxypaxilline (27) and paxilline (2) production from the respective paxGCMBP and paxGCMBPQ strains. Taken together, all of these results indicate that paxilline (2) production requires six genes (paxGCMBPQ; Figure 1.13). 


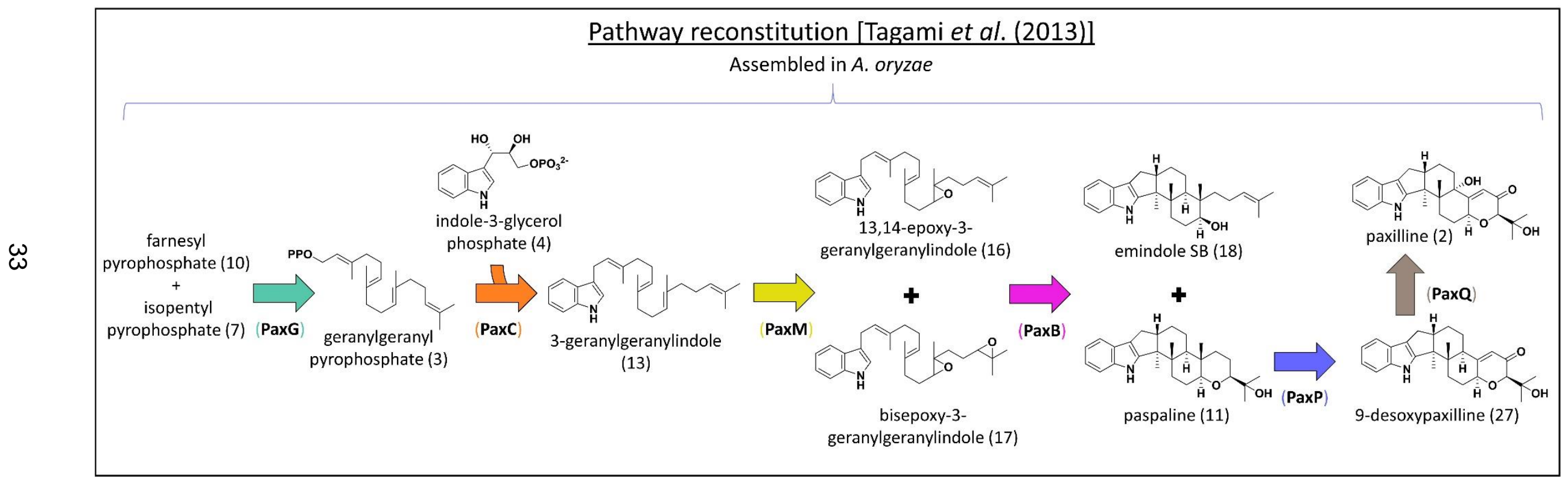

Figure 1.13. Summary of results pathway reconstitution experiments by Tagami et al. (2013). ${ }^{40}$ Arrows represent enzymatic steps encoded by the genes in the PAX cluster. 
Prior to the reconstitution study by Tagami et al. (2013), Saikia et al. (2007) completed the first functional analysis of the two P450 oxygenase-encoding genes in the $P A X$ cluster, $\operatorname{pax} P$ and $\operatorname{pax} Q .{ }^{36}$ As suggested by the $\operatorname{pax} P Q$ gene knockout results from McMillan et al. (2003), the conversion of paspaline (11) to paxilline (2) is catalysed by paxP and paxQ. Thus, Saikia et al. (2007) conducted substrate feeding experiments on $P$. paxilli mutants containing either paxP or pax $Q$ to confirm the catalytic abilities of each gene product. Upon feeding paspaline (11) to the paxP mutant strain, they observed conversion to two new metabolites, one major and one minor, identified as 9-desoxypaxilline (27) and $\beta$-PC-M6 (28) respectively (Figure 1.14, i.). They predicted that PaxP was capable of stepwise oxidation of paspaline (11) to first form $\beta-P C-M 6$ (28) and then 9-desoxypaxilline (27). Subsequent feeding studies of $\beta$-PC-M6 (28) and $\alpha-P C-M 6$ (29) on the paxP mutant showed that PaxP readily converted $\beta$-PC-M6 (28) to 9-desoxypaxilline (27) as shown in Figure 1.14, ii. Notably, they saw no conversion of a-PC-M6 (29) to 9-desoxypaxilline (27) as shown in Figure 1.14, iv., demonstrating that the stereochemistry around the $\mathrm{C} 16$ hydroxyl group is a determining factor in the substrate specificity of PaxP. In their final PaxP feeding experiment, they fed $\alpha$-paxitriol (30) and $\beta$-paxitriol (31) to the paxP mutant strain and found that $\beta$-paxitriol (31)-fed strains produced paxilline (2) whereas no conversion of $\alpha$-paxitriol (30) was observed (Figure 1.14, iii. and iv.). Together these results showed that PaxP catalyses the demethylation of $\mathrm{C} 14$ and the complete oxidation of $\mathrm{C} 16$.

Saikia et al. (2007) completed similar feeding experiments on a paxQ mutant strain and found that PaxQ readily metabolises 9-desoxypaxilline (27) and a-PC-M6 (29) to form respectively paxilline (2) and a-paxitrio (30) as shown in Figure $1.14 \mathrm{v}$. and vi. Intriguingly, they did not see any PaxQ-mediated metabolism of $\beta$-PC-M6 (28) to form $\beta$-paxitriol (31) as shown in Figure 1.14 vii. Together these results indicate that $P a x Q$ catalyses the hydroxylation at the C9 position and that the stereochemistry around the oxidised $\mathrm{C} 16$ must be in the $\alpha$-conformation (in contrast to the $\beta$-conformation for PaxP) or have the carbonyl moiety in order for $P a x Q$ to metabolise the substrate. This functional analysis of $\operatorname{pax} P$ and $\operatorname{pax} Q$ by Saikia et al. (2007) was the first functional analysis of indole diterpene "decoration" genes and established a foundation for further analysis of other decoration genes like the prenyl transferase, paxD, which flanks paxQ (Figure 1.11) and is potentially poorly expressed. ${ }^{43}$ 


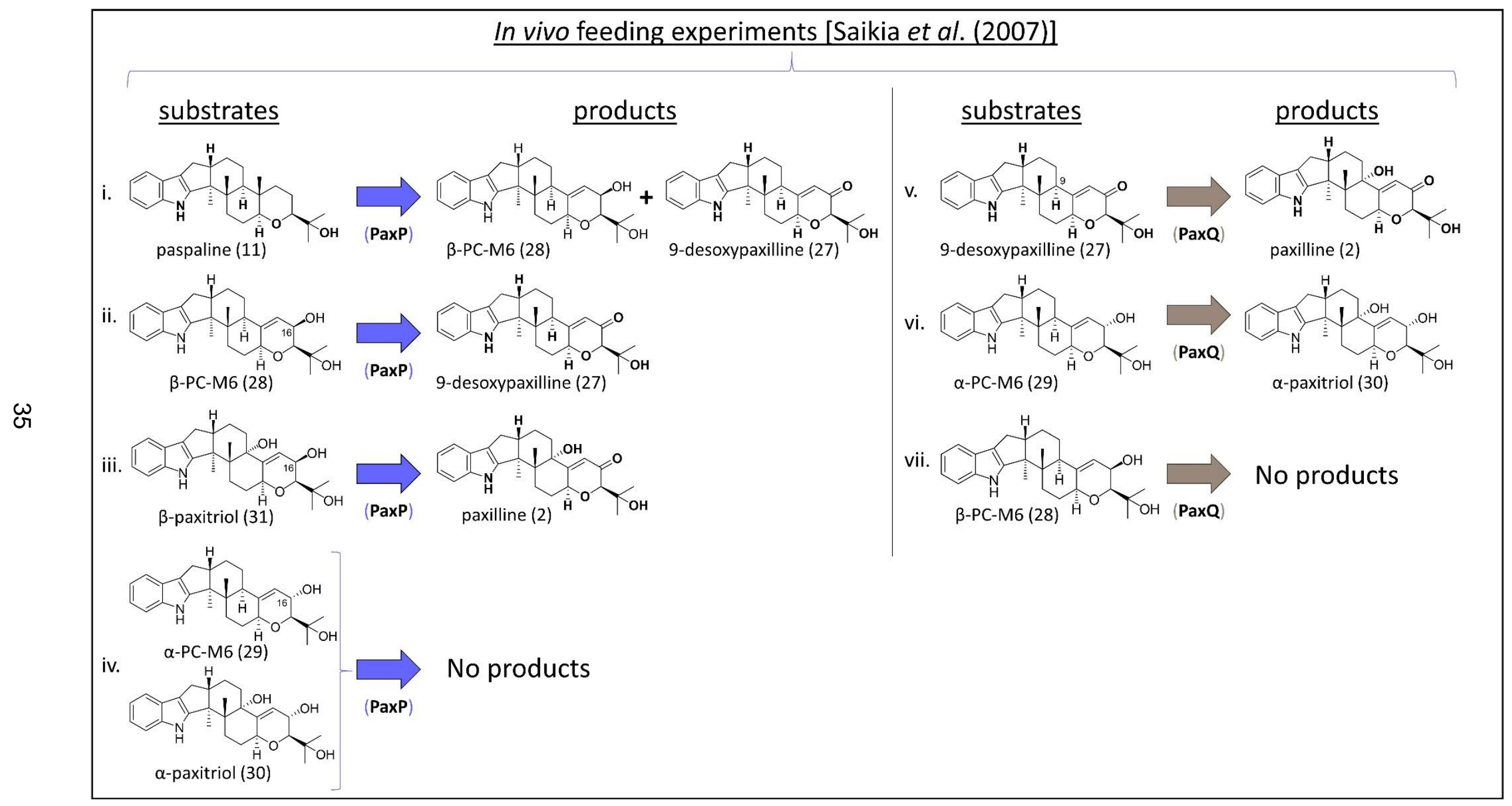

Figure 1.14. Summary of results from the feeding experiments by Saikia et al. (2007). ${ }^{36}$ 
To functionally analyse paxD, Liu et al. (2014) expressed paxD in E. coli and purified PaxD for in vitro analysis. ${ }^{42}$ Recombinant PaxD was incubated with dimethylallyl pyrophosphate (8) and paxilline (2) and yielded two products, determined to be 21prenylpaxilline (32) and 21,22-bisprenylpaxilline (33) as the minor and major products respectively (Figure 1.15, i.). Since these prenylated paxillines had not been observed in $P$. paxilli extracts, they explored the natural production of prenylated paxillines and found that they were present in production medium in quantities $\sim 0.5 \%$ of paxilline. Therefore, they concluded that PaxD is naturally expressed at low levels or not partitioned correctly within the cell. Further analysis of PaxD by Liu et al. (2013) showed that it catalysed the monoprenylation of paspaline (11) at C21 to form 21prenylpaspaline (34) and at C22 to form 22-prenylpaspaline (35) as shown in Figure 1.15 , ii., indicating that indole diterpene $D$-enzymes can prenylate a variety of indole diterpene substrates and that the type of prenylation is substrate specific. ${ }^{39}$ Intriguingly, Liu et al. (2013) also experimented with the substrate specificity of PaxC and found that it readily converted farnesyl pyrophosphate (10) and indole-3-glycerol phosphate (4) to farnesylindole (14) as shown in Figure 1.15, iii., demonstrating that many of these indole diterpene enzymes are rather promiscuous in their choice of substrate. 


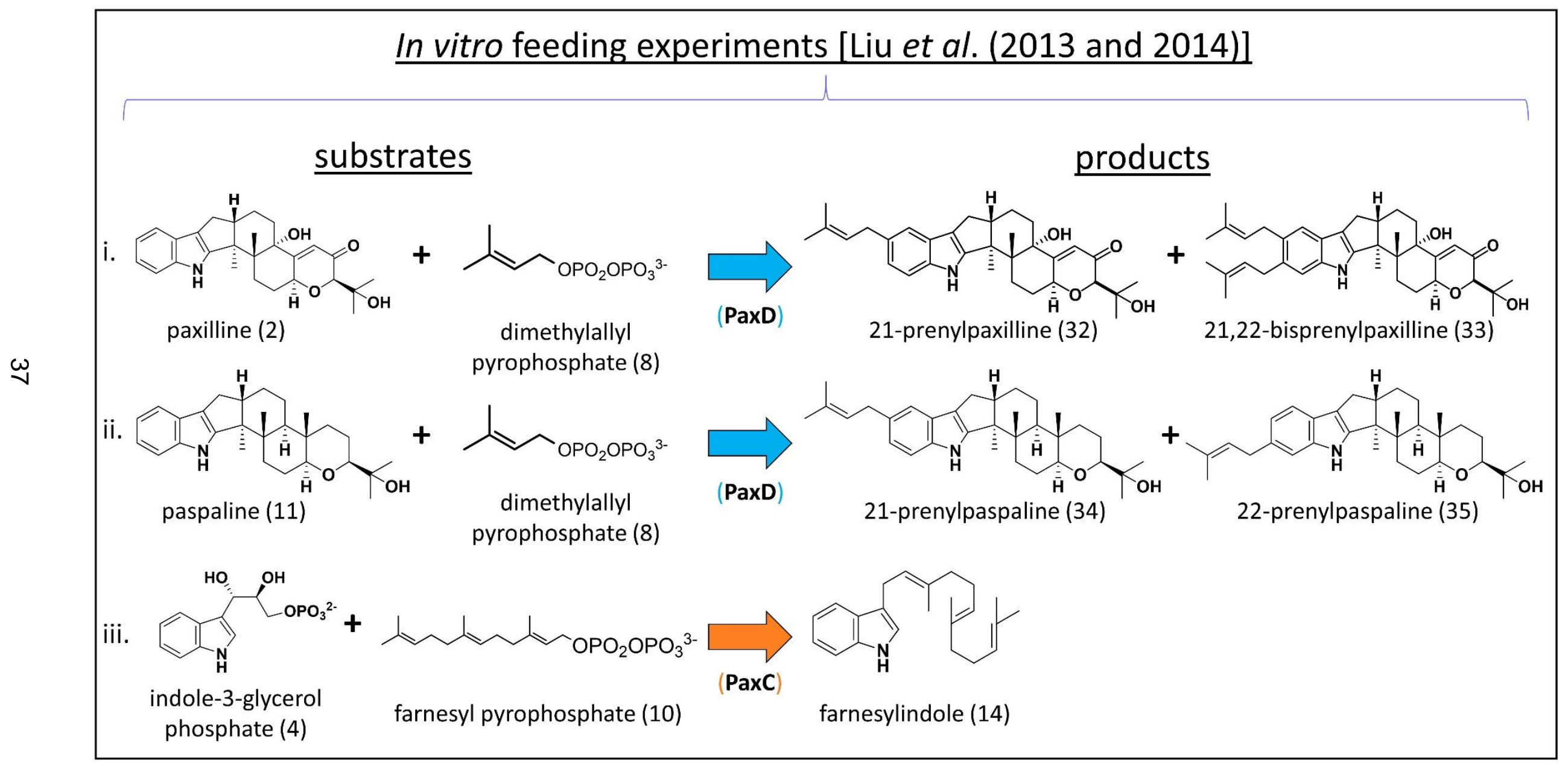

Figure 1.15. Summary of results from feeding experiments by Liu et al. (2013/2014). 39, 42 
Flanking $\operatorname{pax} D$ is a "decorative" FAD-dependent oxygenase-encoding gene, paxO. To date, paxO has not been functionally analysed but its close homologue janO has been shown to encode a protein product that catalyses the oxidative ring closure in janthitremane biosynthesis. ${ }^{43}$ Additional evidence that PaxD and PaxO may have at one point induced janthitremane synthesis in $P$. paxilli is supported by the fact that they are upregulated during paxilline (2) biosynthesis. ${ }^{38}$ It seems that alterations in gene expression/regulation or mutations of $\operatorname{pax} D$ and paxO over time may have somehow altered the expression or effectiveness of their protein products and thus the main indole diterpene produced by $P$. paxilli is paxilline.

Overall, the sequence identities of genes within the PAX cluster established a foundation for further sequence analysis guided identification of homologous indole diterpene genes in other indole diterpene producing fungi; thereby enabling more rapid identification of additional indole diterpene producing gene clusters and especially facilitating the localization of clusters of indole diterpene genes found in more than one specific locus as seen with the ATM, LTM and PTM clusters from A. flavus, E. festucae and $P$. simplicissimum respectively (Figure 1.2). 


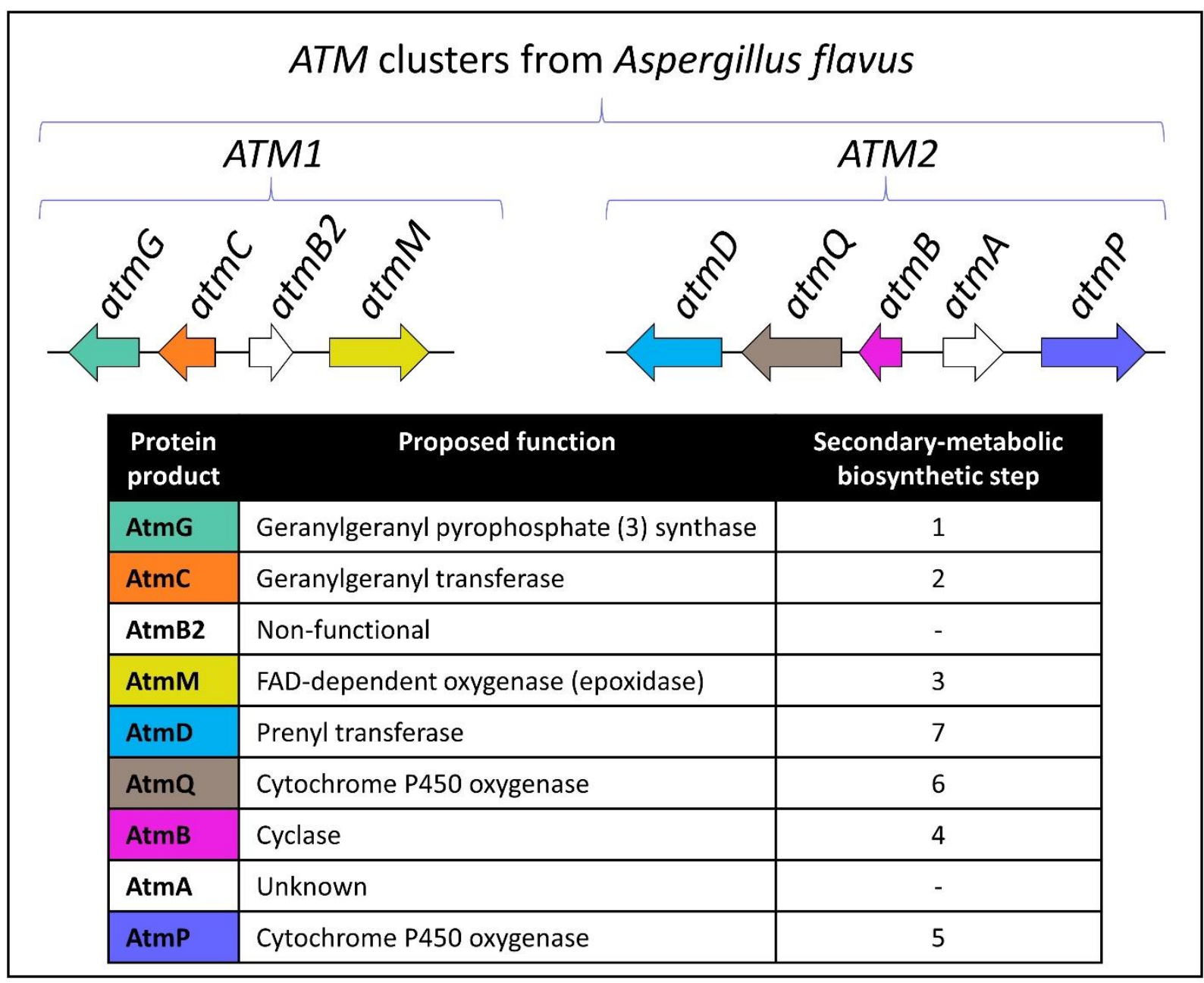

Figure 1.16. Depiction of $A T M$ gene clusters from Aspergillus flavus. ${ }^{25,} 28$

Zhang et al. (2004) provided the first genetic evidence for the biosynthetic pathway of aflatrem (36) in A. flavus by locating the first cluster of ATM genes (atmG, atmC, and atmM; Figure 1.16) that were highly homologous to respective gene products in the PAX cluster (49\% to PaxG, 55\% to PaxC, and $52 \%$ to PaxM respectively). ${ }^{25}$ Degenerate primers for conserved domains of geranylgeranyl pyrophosphate (3) synthases were used to identify atm $G$ and subsequent chromosome walking revealed the closely clustered $\operatorname{atm} C$ and atmM. They showed that gene expression for these three ATM genes increased during the onset of aflatrem (36) biosynthesis ( 108 hr; Figure 1.17, A) and that atmM was orthologous to paxM through complementation of atmM into the $P$. paxilli $\triangle$ paxM knockout strain (Figure 1.17, B). 
With the availability of complete genome sequences, Nicholson et al. (2009) were able to tBLASTn the genomes of $A$. flavus and $A$. oryzae to locate the second ATM cluster containing five homologous indole diterpene genes $(\operatorname{atm} D, \operatorname{atm} Q, \operatorname{atm} B, \operatorname{atm} A$, and atmP; Figure 1.16) to those found in the PAX cluster of $P$. paxilli (29\% to PaxD, $55 \%$ to $\mathrm{PaxQ}, 61 \%$ to $\mathrm{PaxB}, 29 \%$ to $\mathrm{PaxA}$, and $61 \%$ to $\mathrm{PaxP}) .{ }^{28}$ In contrast to the $A T M$ genes in cluster 1 , they found that these five decorative genes were expressed prior to the onset of aflatrem (36) biosynthesis at $108 \mathrm{~h}(48 \mathrm{~h}$ for $\operatorname{atm} Q, \operatorname{atm} B$, and $\operatorname{atm} A$ and $60 \mathrm{~h}$ for atmD and atmP; Figure 1.17, A). Complementation experiments of atmP and atm $Q$ into respective $\triangle p a x P$ and $\triangle p a x Q P$. paxilli mutant strains revealed that atm $P$ is a functional orthologue of $\operatorname{paxP}$ [i.e. atmP readily demethylates $\mathrm{C} 14$ and oxidises $\mathrm{C} 16$ of paspaline (11)] and that atmQ is not a functional orthologue of paxQ (Figure 1.17, B). Instead, the atmQ transformant was shown to produce two new indole diterpene products, paspalicine (37) and paspalinine (38); thereby indicating that AtmQ is able to catalyse the same oxidation as $\operatorname{PaxQ}$ (i.e. the hydroxylation at $C 9$ ) in addition to the acetal ring formation at $\mathrm{C} 13$. 


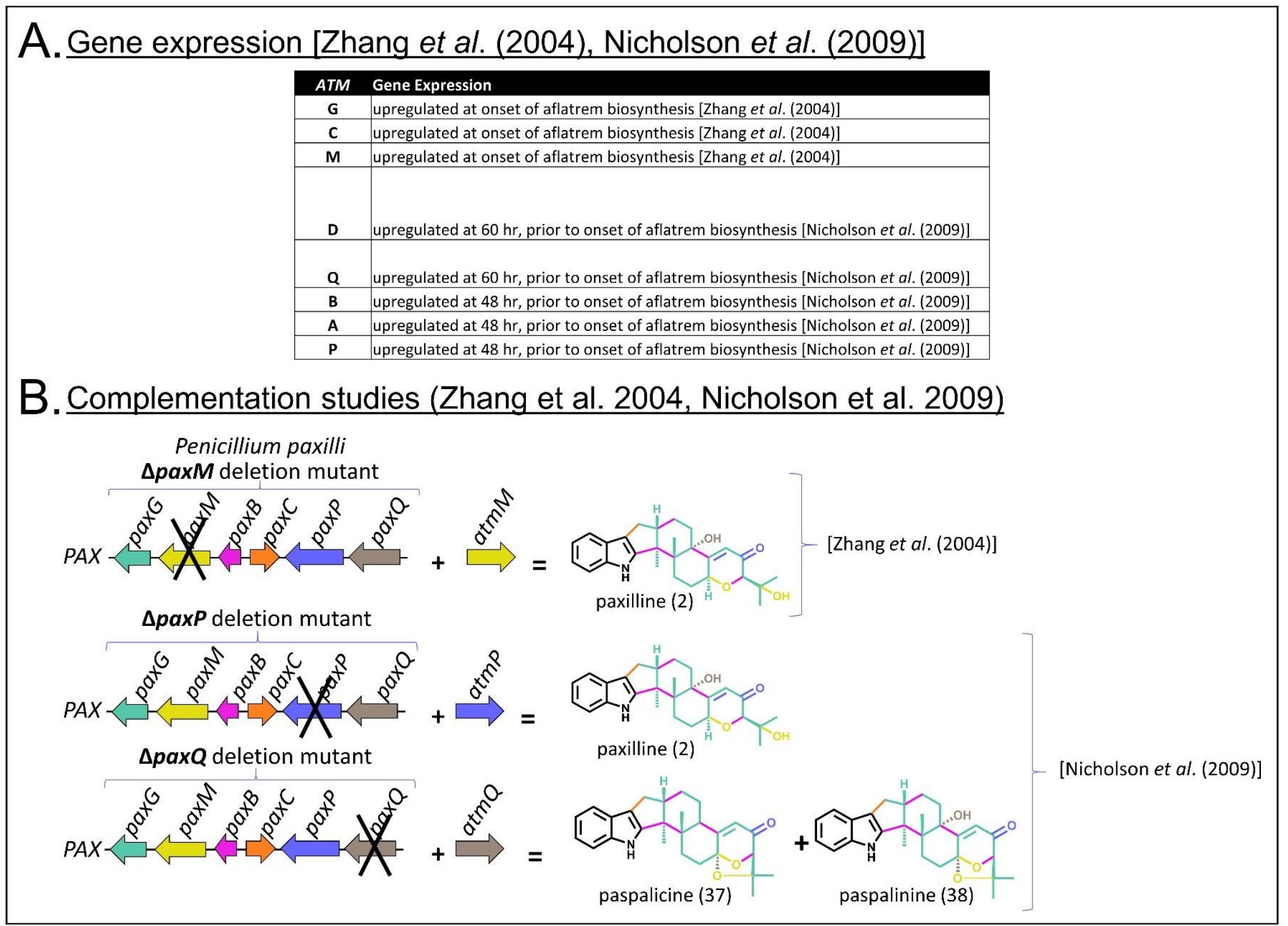

Figure 1.17. Summary of results from gene expression and complementation experiments done by Zhang et al. (2004) and Nicholson et al. (2009). ${ }^{25,28}$ 
Subsequent in vitro analysis of recombinant AtmD by Liu et al. (2013) showed that AtmD was highly promiscuous. ${ }^{39}$ AtmD was incubated with dimethylallyl pyrophosphate (8) and one of four different substrates: farnesylindole (14), 3geranylgeranylindole (13), paspaline (11), or paxilline. Firstly, the incubation with paxilline (2) led to the formation of three monoprenylated products, two major and one minor (Figure 1.18). The two major products were determined to be reversely 20 prenylated paxilline (39) and reversely 21-prenylated paxilline (40) (Liu et al. (2013) used 'regularly' and 'reversely' to describe the prenylations they observed, which correspond respectively to a 'head' or 'tail' isoprenoid condensation). As for the minor product, the most likely candidate is reversely 22-prenylated paxilline (41) but not enough compound was produced to complete the structural identification. These results suggested that $A t m D$ catalyses the same reverse prenylations of paspalinine (38) to form aflatrem (36) and $\beta$-aflatrem (42).

Liu et al. (2013) then investigated incubation of AtmD with farnesylindole (14), 3geranylgeranylindole (13), and paspaline (11). Liquid chromatography-mass spectrometry (LCMS) analysis of the completed reactions revealed that all three substrates were monoprenylated and that the yield of the 3-geranylgeranylindole (13)reaction was too low for clear product identification (Figure 1.18). They identified the products of the farnesylindole (14)-reaction to be regularly 5-prenylated farnesylindole (43) and regularly 6-prenylated farnesylindole (44). The products from the paspaline (11) reaction were determined to be regularly 21-prenylated paspaline (34) and regularly 22-prenylated paspaline (35). Finally, the nuclear magnetic resonance (NMR) spectroscopy results of the product mixture from the 3-geranylgeranylindole (13)reaction revealed typical signals for regular dimethylallyl moiety indicating that 3geranylgeranylindole (13) was monoprenylated in the same regular prenylation fashion as farnesylindole (14) and paspaline (11) to produce regularly 5-prenylated 3geranylgeranylindole (45) and regularly 6-prenylated 3-geranylgeranylindole (46). 


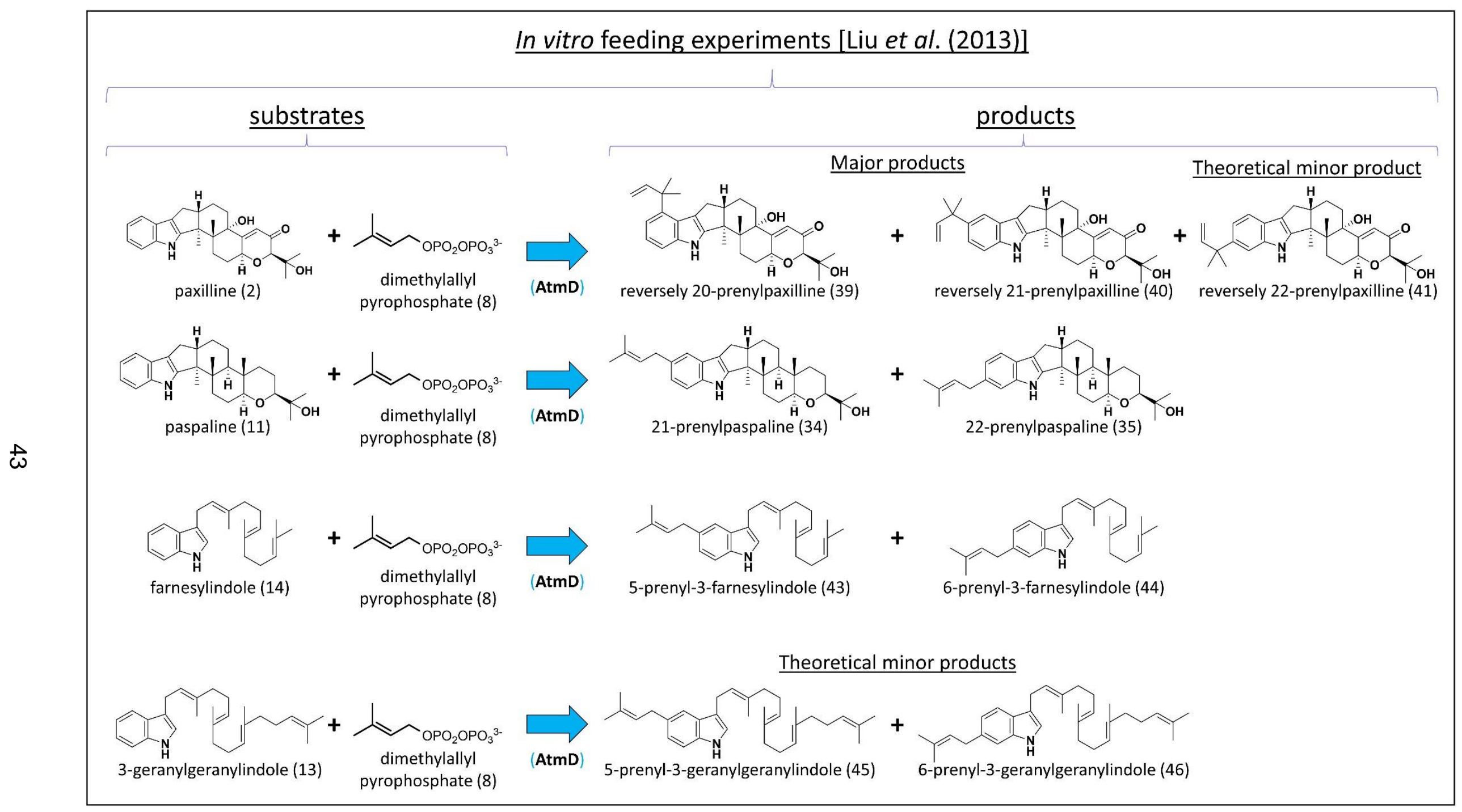

Figure 1.18. Summary of results from in vitro feeding experiments of AtmD by Liu et al. (2013). ${ }^{39}$ 
Tagami et al. (2014) concluded that seven genes (atmGCMBPQD) are involved in aflatrem (36) biosynthesis, and confirmed that AtmD reversely prenylated paspalinine (38) at the $C 20$ and $C 21$ positions to form aflatrem (36) and $\beta$-aflatrem (42) respectively, by rapidly reconstituting four $P A X$ genes (paxGCMB) and three ATM genes (atmPQD) in a heterologous expression host, $A$. oryzae (Figure 1.19). ${ }^{41}$ Amazingly, only two rounds of tandem transformations, involving four different plasmids (containing paxGC, paxMB, atmPQ, or atmD), were used to fully reconstruct the aflatrem (36) biosynthetic machinery in $A$. oryzae.

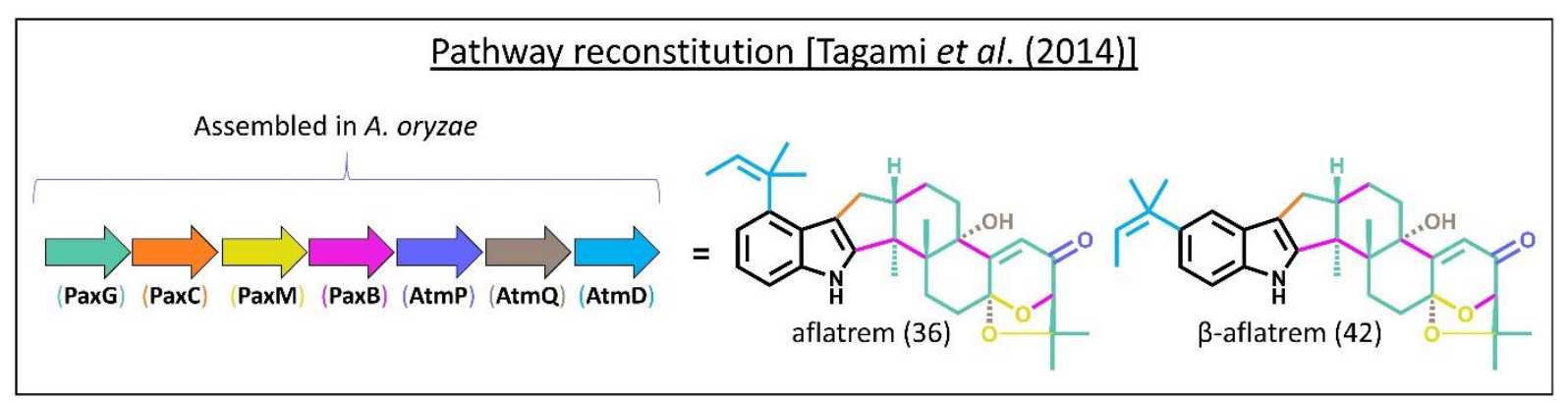

Figure 1.19. Summary of results from pathway reconstitution studies by Tagami et al. (2014) ${ }^{41}$

\section{Identification of the LTM gene clusters and functional analysis of $L T M$ genes}

During the discovery of the ATM biosynthetic genes from Aspergillus spp., similar studies were being conducted on Neotyphodium lolii and E. festucae to discover the LTM gene clusters responsible for producing the potent rye-grass stagger-inducing lolitrems. The first genes that were proposed to be essential for lolitrem biosynthesis in Neotyphodium Iolii and E. festucae were discovered by Young et al. (2005). ${ }^{26}$ Just as with the discovery of the first ATM gene cluster, degenerate primers designed to conserved domains of fungal geranylgeranyl pyrophosphate (3) synthases were used to discover ItmG and subsequent chromosome walking was used to locate the closely clustered ItmM and ItmK (Figure 1.20). 


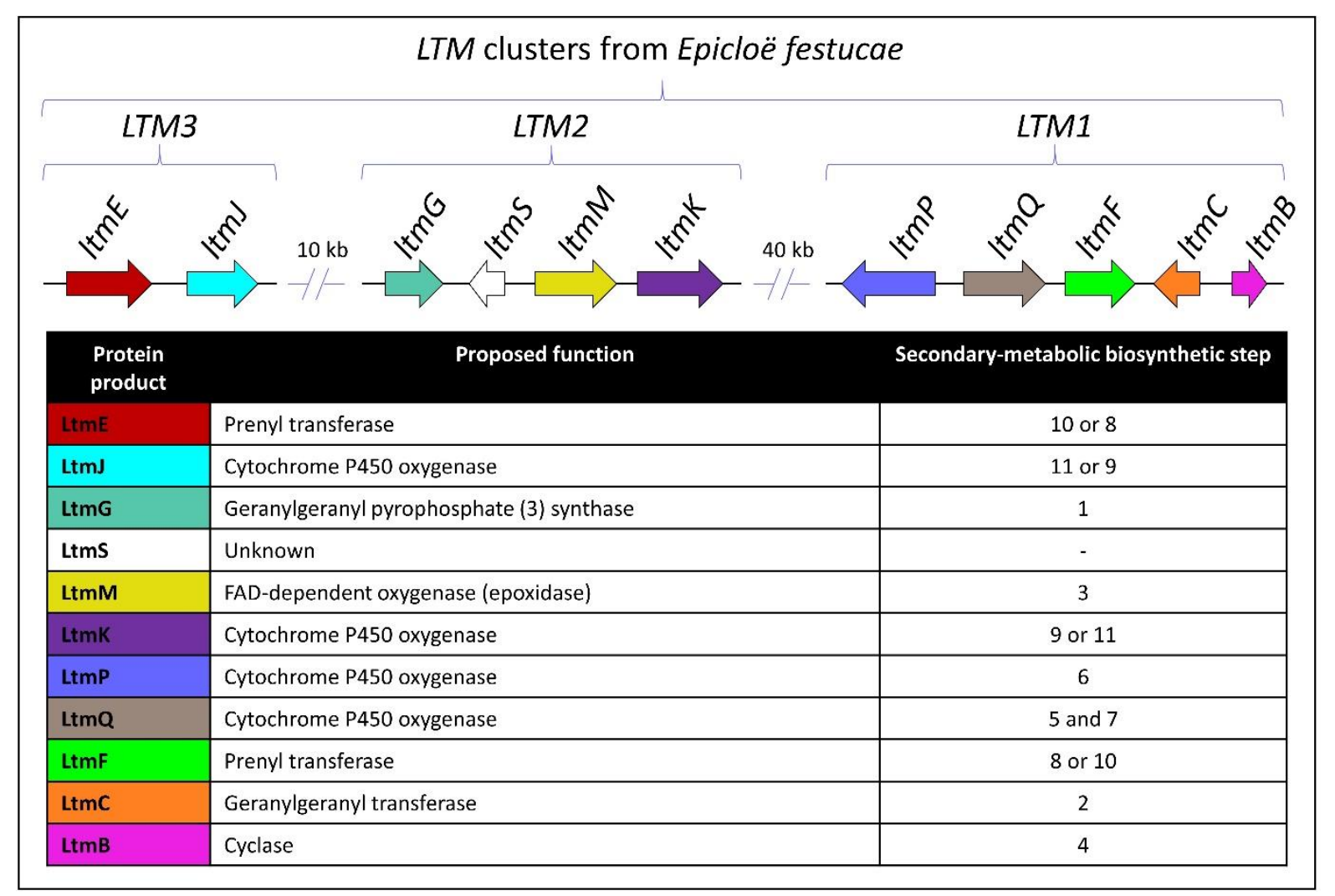

Figure 1.20. Schematic of LTM clusters identified in E. festucae. ${ }^{26-27,29}$

Young et al. (2005) showed that ItmM was a functional orthologue of paxM through complementation of paxM into the E. festucae $\Delta /$ tmM mutant strain (Figure 1.21). Intriguingly, this three gene $L T M$ cluster was flanked by large blocks of retrotransposon sequence preventing them from using chromosome walking to identify any other potential LTM genes. Therefore, they changed tactics to suppression subtractive hybridisation, and generated subtracted Expressed Sequence Tag libraries. Young et al. (2006) compared these Expressed Sequence Tag libraries to GenBank databases, SwissProt databases, and $P$. paxilli biosynthesis genes using BLAST algorithms. ${ }^{27}$ Results from these BLAST sequence comparisons revealed three new LTM genes: ItmC, ItmP, and ItmJ. Subsequent contig generation revealed that these three genes were part of two additional separate clusters, named $L T M$ cluster 2 and $L T M$ cluster 3. Five genes were found in $L T M$ cluster 2 (ItmP, ItmQ, ItmF, ItmC, and ItmB) and two genes were found in LTM cluster 3 (ItmE and ItmJ) (Figure 1.20). Of these seven new LTM genes, Young et al. 2006 showed that ItmC is a functional orthologue of paxC through complementation of ItmC into the $P$. paxilli pax $C$ mutant strain (Figure 1.21). 


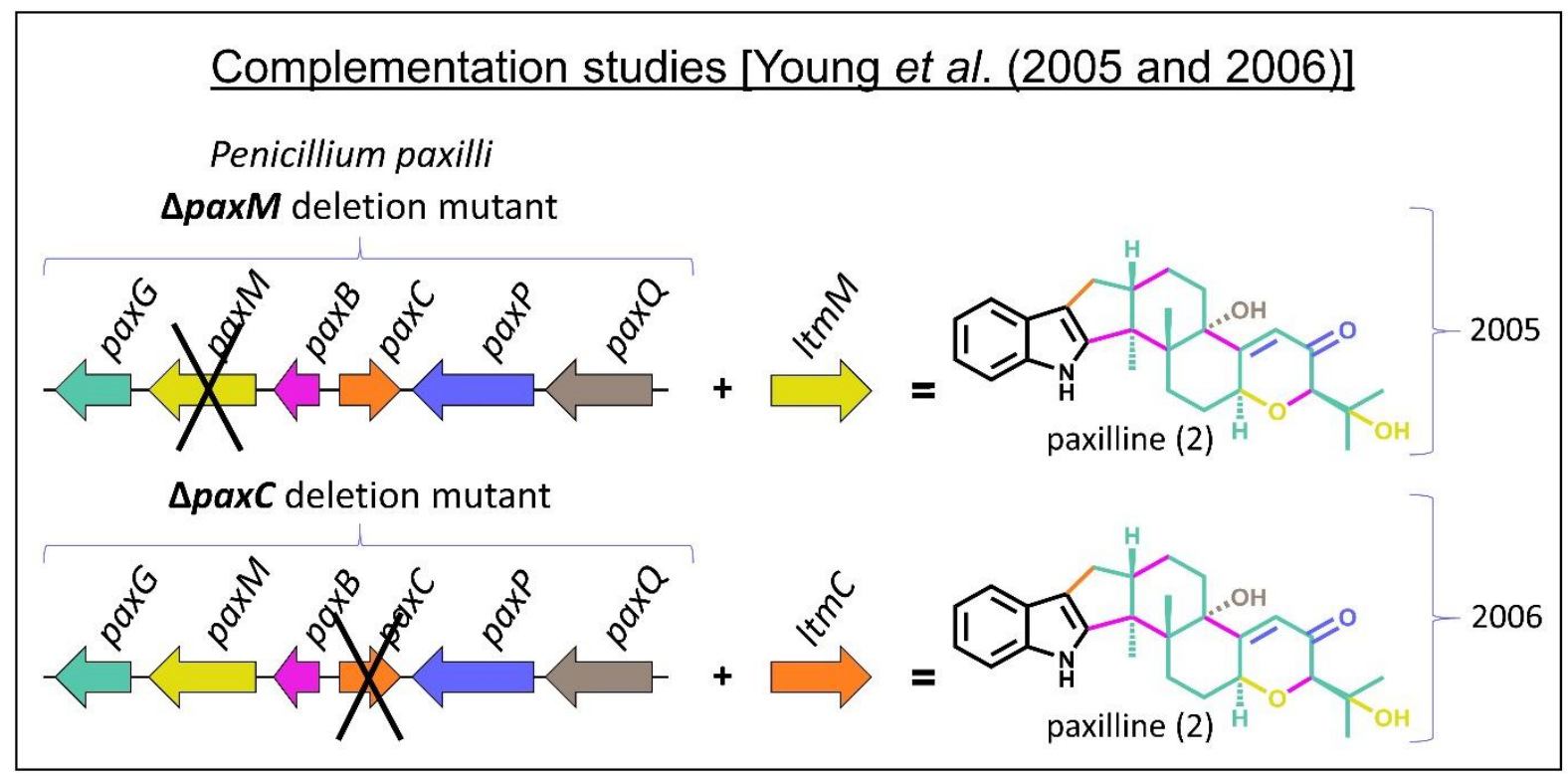

Figure 1.21. Summary of results from complementation experiments by Young et al. (2005 and 2006). ${ }^{26-27}$

For the next six years, the functionality of the rest of the LTM genes remained a mystery; then Saikia et al. (2012) published results from their studies on the exploration of the functionality of E. festucae genes in these three LTM clusters. ${ }^{29}$ They used a mixture of gene knockout studies of LTM genes in E. festucae, complementation experiments of $L T M$ genes into $P$. paxilliknockout strains, and feeding studies on $L T M$ gene-containing $P$. paxilli mutants to propose a potential metabolic biosynthetic grid for lolitrem biosynthesis. Unfortunately, results from their studies did not resolve the functionality of the first two cytochrome P450 oxygenases, LtmP and LtmQ, because the novel compounds produced from feeding experiments were never structurally confirmed by NMR spectroscopy analysis. That said, they were still able to provide a great deal of valuable information about these two cytochrome P450 oxygenases. 


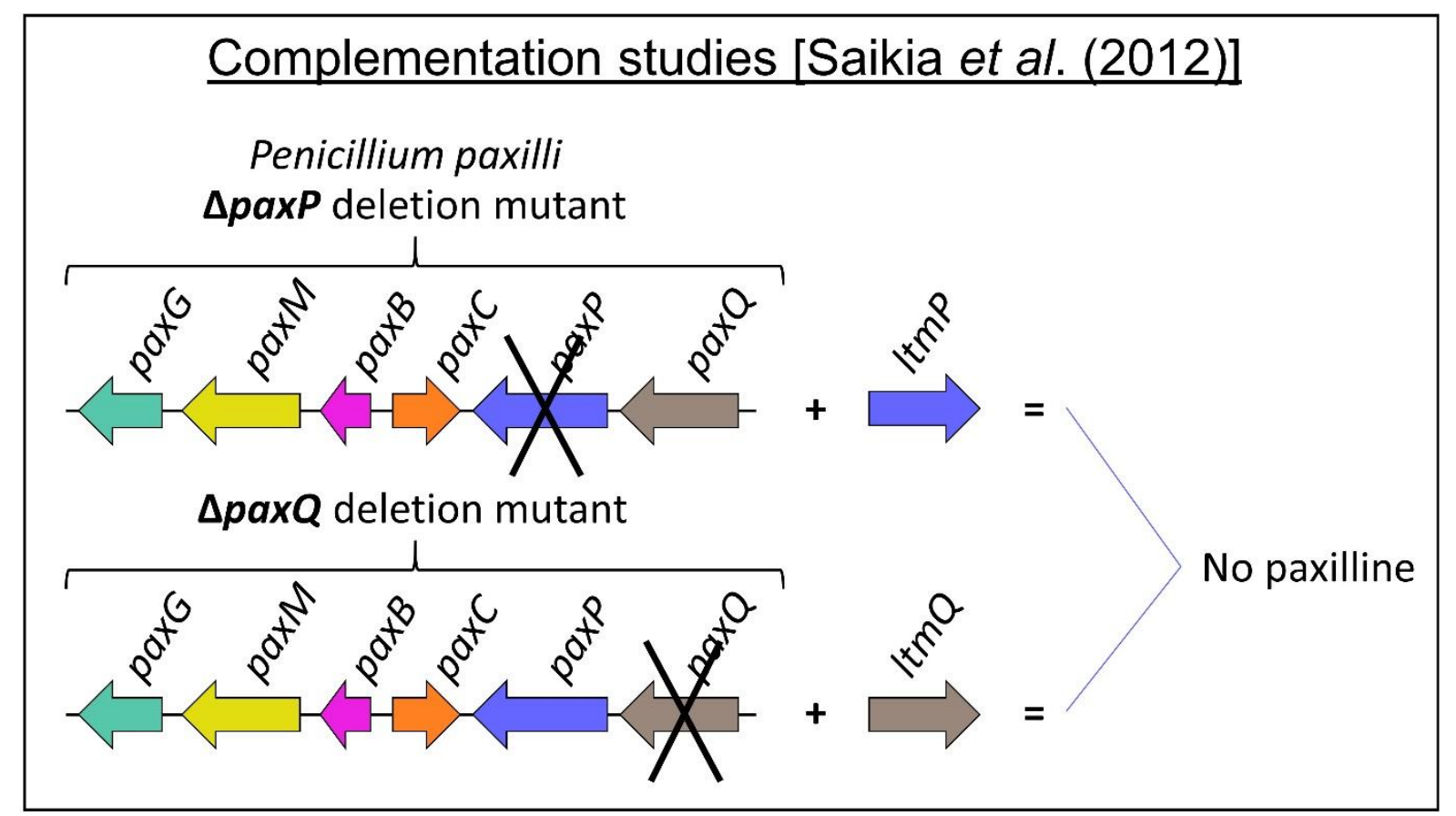

Figure 1.22. Summary of LTM gene complementation attempts by Saikia et al. (2012). ${ }^{29}$

To start, ItmP and ItmQ were unable to complement respective $\triangle$ paxP and $\triangle$ paxQ $P$. paxilli mutant strains (Figure 1.22) indicating that $\operatorname{Itm} P$ and $\operatorname{Itm} Q$ were not orthologous to paxP and paxQ. Therefore, three $P$. paxilli mutant strains containing only ItmP, ItmQ, or both $\operatorname{Itm} P$ and $\operatorname{Itm} Q$ were prepared for substrate feeding experiments to analyse the LtmP and LtmQ functionality in vivo. Paspaline (11) fed to the ItmP containing mutant was readily converted to PC-M6 [they identified this as $\beta$ PC-M6 (28), but based on the lack of NMR spectroscopy analysis and the $\alpha$ stereochemistry of the C16 hydroxyl group of lolitrems, this product was most likely $\alpha$ PC-M6 (29)] and two unknown indole diterpenes with $[\mathrm{M}+\mathrm{H}]^{+} 438 \mathrm{~m} / \mathrm{z}$ that were paspaline (11) derivatives based on tandem mass spectrometry fragmentation patterns (Figure 1.23, i). Intriguingly, no 9-desoxypaxilline was produced from this experiment highlighting why ItmP was unable to complement the $P$. paxilli $\triangle$ paxP mutant strain. In the LtmQ analysis, they found that extract from the $\triangle / t m Q$ gene knockout $E$. festucae strain revealed wild-type levels of paspaline (11) production and a large accumulation of 9-desoxypaxilline (27) - see Figure 1.23, iv - supporting the potential involvement of 9-desoxypaxilline (27) as an intermediate in lolitrem biosynthesis; however, feeding of 9-desoxypaxilline (27) to the ItmQ-containing and ItmP/ItmQ-containing P. paxilli mutant strains showed only minor conversion to paxilline (2) and did not yield any other 
novel indole diterpene products (Figure 1.23, ii) indicating that 9-desoxypaxilline (27) is unlikely a direct intermediate in lolitrem biosynthesis. Thus, they fed paspaline (11) to the $\operatorname{tm} P Q$ double mutant and found conversion to seven indole diterpene products (Figure 1.23, iii); the same three metabolites observed when paspaline (11) was fed to the single ItmP-mutant (PC-M6, and the two unknown indole diterpenes), and four additional indole diterpene products: terpendole $E$ (47), terpendole $F(48)$, and two additional unknown compounds with $[\mathrm{M}+\mathrm{H}]^{+} 422 \mathrm{~m} / \mathrm{z}$ and $452 \mathrm{~m} / \mathrm{z}$. Notably, none of these compounds, including terpendole $E(47)$ and $F(48)$, were structurally confirmed by NMR spectroscopy nor compared to standards and thus, further structural confirmation is required to make definitive conclusions. 


\section{In vivo feeding studies and deletion mutant experiments [Saikia et al. (2012)]}

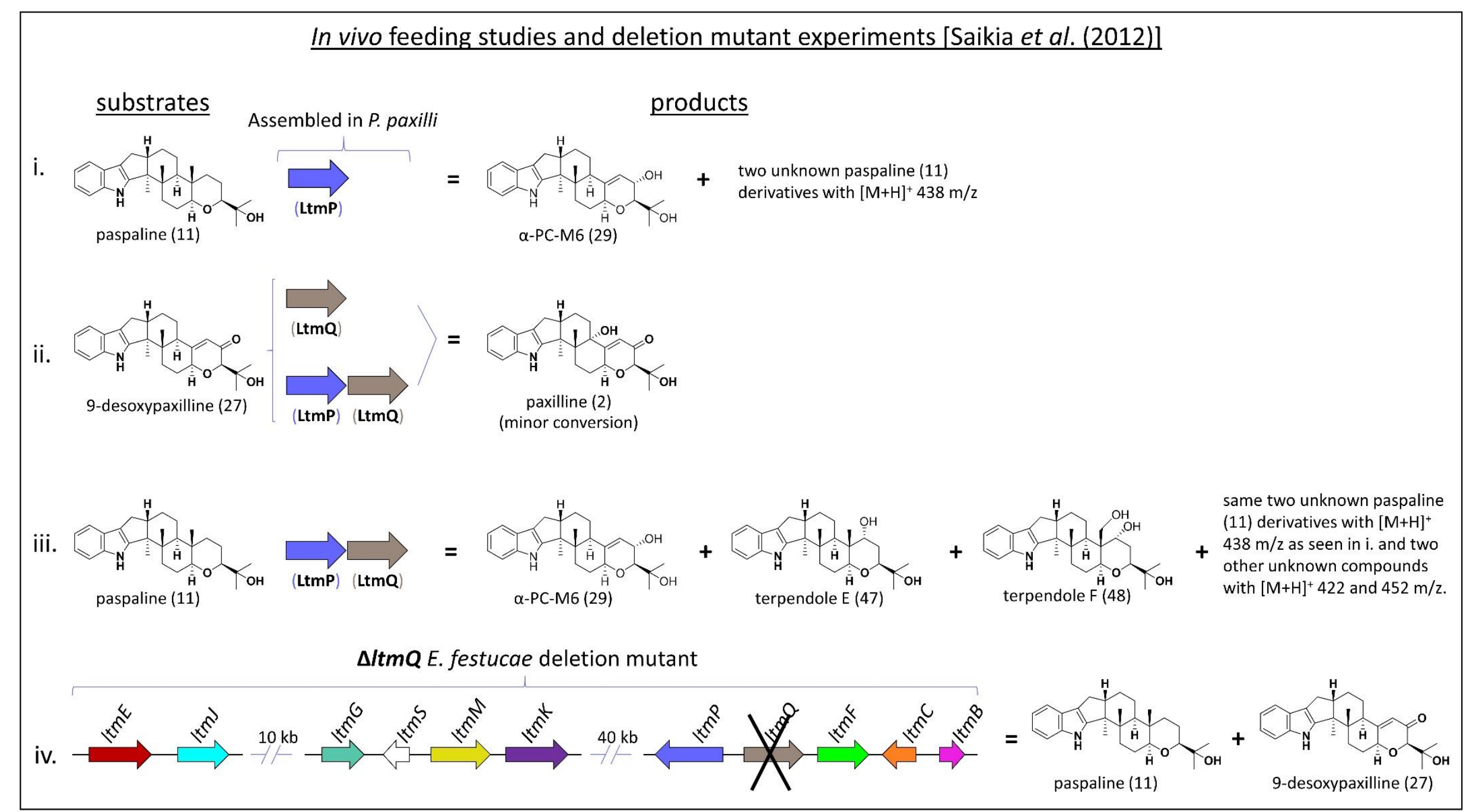

Figure 1.23. Summary of results from experiments used to elucidate the function of LtmP and LtmQ (Saikia et al. 2012). ${ }^{29}$ Saikia et al. 2012 identified the PC-M6 as $\beta$-PC-M6 (28), but based on the lack of NMR spectroscopy analysis and the $\alpha$-stereochemistry of the C16 hydroxyl group of lolitrems, this product was most likely a-PC-M6 (29). Therefore, $\alpha$-PC-M6 (29) is shown in the figure. 
Overall, Saikia et al. (2012) showed that LtmP was able to oxidise C16 (with unconfirmed stereochemistry) and LtmQ was able to oxidise C9 but more studies must be completed to clearly functionally characterise LtmP and LtmQ. ${ }^{29} \mathrm{~A}$ good starting point would be to analyse the novel compounds produced in feeding studies by NMR spectroscopy and feed the LtmQ strain paspaline (11) to see if terpendole $E(47)$ is produced.

In addition to LtmP and LtmQ, Saikia et al. (2012) also analysed two more cytochrome P450 oxygenases ( $\mathrm{LtmK}$ and LtmJ) and two prenyl transferases ( $\mathrm{LtmF}$ and LtmE) by studying the chemical compounds of the respective gene knockout strains. To start, the ItmF knockout mutant produced lolitriol (49) and lolitrem J (50) but no Iolitrem B (51) - structure shown in Figure 1.2, lolitrem E (52), or O-prenylated paspalanes (i.e. terpendoles $C(8)$ and J) (Figure 1.24, i). Thus they concluded that LtmF catalysed the prenylation of the C18-hydroxyl group. In contrast, the ItmK knockout strains lacked lolitrem B (51) and terpendole C (53) but contained lolitrem E (52) as shown in Figure 1.24, ii, showing that LtmK is responsible for oxidative acetyl ring formation. Finally, based on absence of lolitremanes in the ItmE and ItmJ knockout strains but the presence of simple and O-prenylated paspalanes like terpendole C (53) and terpendole $M(54)$ shown in Figure 1.24, iii-iv, they proposed that LtmE catalyses the prenylations at position $\mathrm{C} 20$ and $\mathrm{C} 21$ of the lolitremane core and that LtmJ catalyses the oxidative cyclisation of the two prenyl groups to form the upper left portion of lolitremanes. With these results, they were able to propose a biosynthetic metabolic grid of lolitremane biosynthesis, but as noted above, there is still a great deal of work to be done to elucidate the precise biosynthetic pathway of the lolitremanes. 


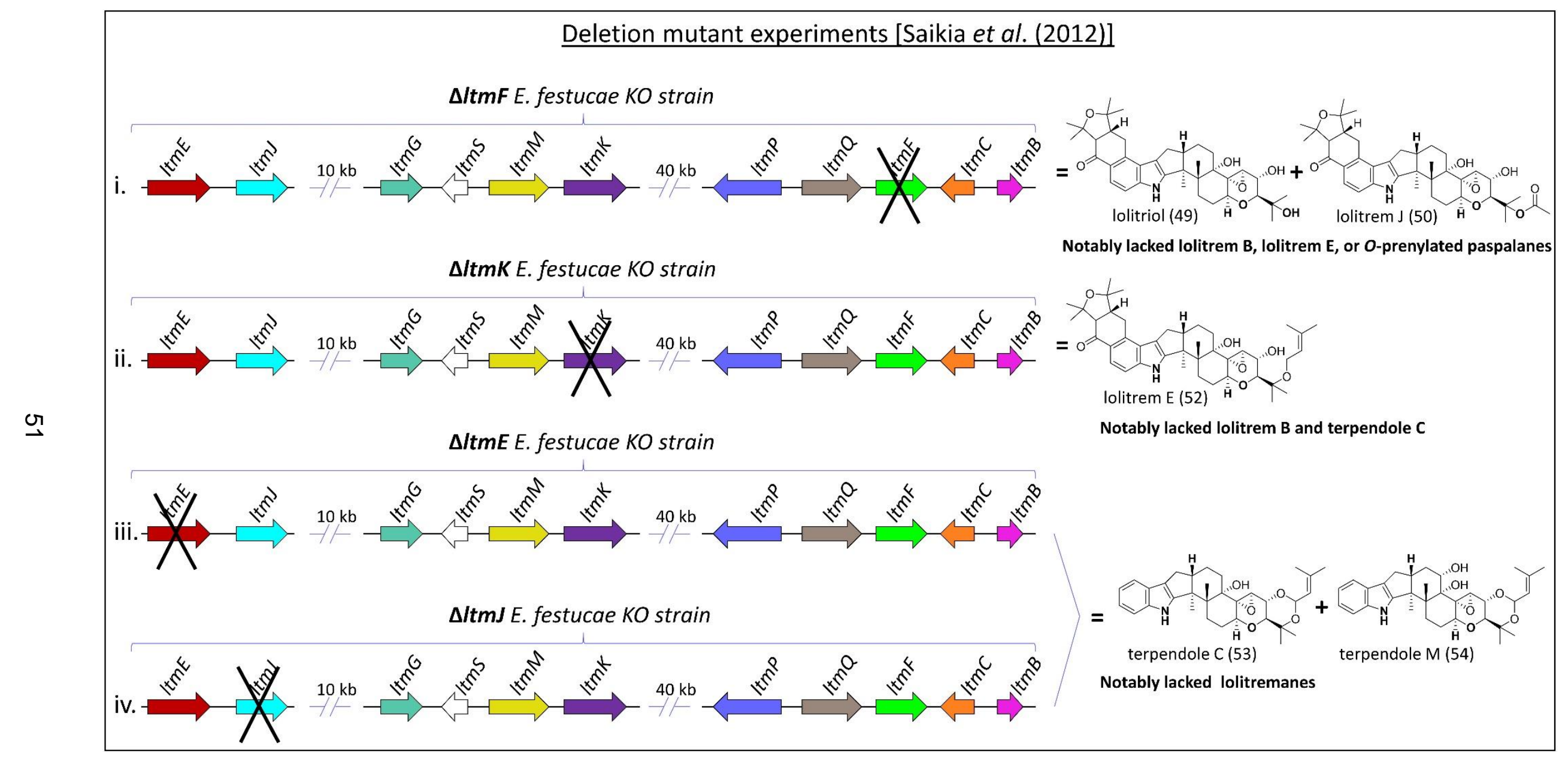

Figure 1.24. Summary of results from E. festucae gene knockout studies (Saikia et al. 2012). ${ }^{29}$ 


\section{Identification of the TER gene cluster and functional analysis of terP and terQ}

The TER gene cluster is responsible for terpendole production in Chaunopycnis alba and was identified in hope of generating a way to produce efficiently terpendole $E(47)$, a known kinesin Eg5 inhibitor for use as an anti-cancer agent. Using a similar degenerate primer method that Zhang et al. (2004) and Young et al. (2005) used to identify the ATM and LTM clusters, Motoyama et al. (2012) designed degenerate primers to locate a paxP homologue within the $C$. alba genome. ${ }^{30}$ Upon location of the pax $P$ homologue, named ter $P$, a $25 \mathrm{~kb}$ region of DNA around ter $P$ was cloned by genome walking and sequenced. This $25 \mathrm{~kb}$ region (Figure 1.25) contained seven genes (terK, terC, terB, terQ, terM, terP, and terF) that were highly similar to known indole diterpene genes and thus predicted to encode the gene products required for terpendole biosynthesis. Notably, the amino acid sequences of these seven gene products were most similar to those found in the lolitrem B (51) producer N. lolii (LtmK, 54\%; LtmC, 55\%; LtmB, 70\%; LtmQ, 61\%; LtmM, 49\%; LtmP, 61\%; and LtmF, 54\%).

\begin{tabular}{|l|l|c|}
\hline \multicolumn{2}{|c|}{ PER cluster from Chaunopycnis alba } \\
\hline $\begin{array}{l}|c| \\
\text { Protein } \\
\text { product }\end{array}$ & Proposed function & 8 \\
\hline TerK & Cytochrome P450 oxygenase & 2 \\
\hline TerC & Geranylgeranyl transferase & 4 \\
\hline TerB & Cyclase & 5 \\
\hline TerQ & Cytochrome P450 oxygenase & 3 \\
\hline TerM & FAD-dependent oxygenase (epoxidase) & 6 \\
\hline TerP & Cytochrome P450 oxygenase & 7 \\
\hline TerF & Prenyl transferase & Stabolic \\
\hline
\end{tabular}

Figure 1.25. Depiction of TER gene cluster identified in C. alba by Motoyama et al. (2012). ${ }^{30}$ 
As the main goal of the study conducted by Motoyama et al. (2012) was to create more terpendole $E(47)$, their experiments focused on confirming the function of two key terpendole genes, terP and terQ, which they predicted could be involved in terpendole E (47) biosynthesis. Generation of three $C$. alba gene knockout strains (terP-knockout, terQ-knockout, and terP/terQ-knockout) showed that the terP knockout strain accumulated terpendole E (47) in reasonably high quantities $(36 \mathrm{mg} / \mathrm{L}$ after 2 days growth), the terQ knockout strain initially accumulated paspaline (11) that was readily converted to 9-desoxypaxilline (27), a-PC-M6 (29), and a putative 9desoxypaxilline (27) analogue with +16 mass of 9-desoxypaxilline (27), and that the terQ/terP-double knockout strain only accumulated paspaline (11) (Figure 1.26). 


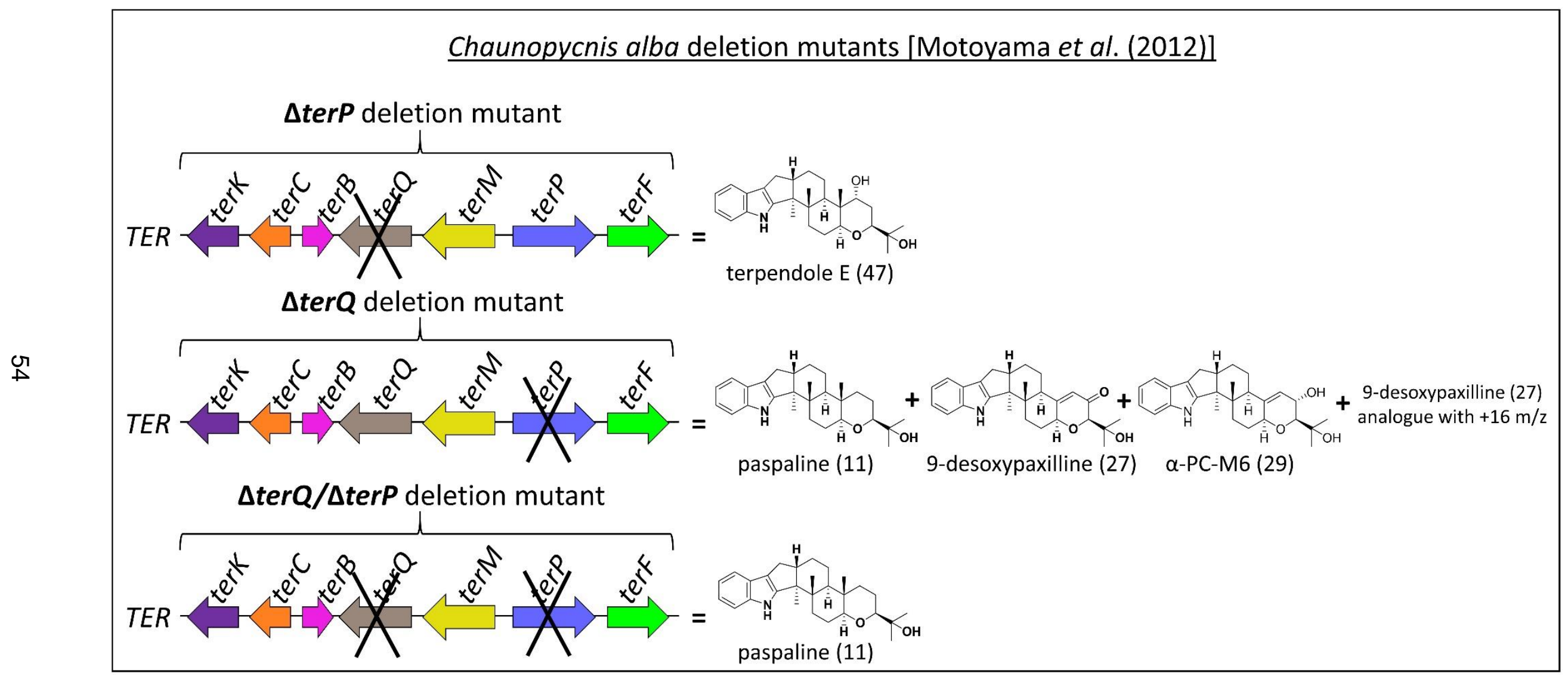

Figure 1.26. Summary of results from gene knockout studies completed by Motoyama et al. (2012). ${ }^{30}$ 
Subsequent feeding experiments on $C$. alba strains containing only ter $P$ or only ter $Q$ revealed the specific oxidative properties of TerP and TerQ. The terP-only strain converted terpendole E (47) to 9-desoxyterpendole I (55), paspaline (11) to 9desoxypaxlline, a-PC-M6 (29), and the putative 9-desoxypaxilline (27) analogue, and a-PC-M6 (29) to 9-desoxypaxilline (27) as shown in Figure 1.27; and the terQ-only strain metabolised a-PC-M6 (29) to some unidentified compounds, 9-desoxypaxilline (27) to paxilline (2) and an unidentified paxilline (2) derivative with +14 mass of 9 desoxy paxilline, paspaline (11) to terpendole $E$ (47), and 9-desoxyterpendole I (55) to terpendole I (56) as shown in Figure 1.27. Together these results indicate that TerP catalyses the $C 14$ demethylation and $C 16$ oxidation of terpendole $E(47)$ and paspaline (11) and that TerQ catalyses the C9 hydroxylation of 9-desoxypaxilline (27) and 9desoxyterpendole I (55) and the C15 hydroxylation of paspaline (11). Notably, the TerP catalysed $\mathrm{C} 14$ demethylation of terpendole $\mathrm{E}$ (47) triggers the C14-C15 epoxide formation to form 9-desoxyterpendole I (55). Additionally, since a-PC-M6 (29) and 9desoxypaxilline (27) were not converted to any terpendoles by the terP or terQ-only strains, this suggests that 9-desoxypaxilline (27) is a shunt metabolite in terpendole biosynthesis. As a final note, they demonstrated that TerQ and PaxQ have different substrate specificities by feeding paspaline (11) and 9-deoxypaxilline to a paxQ-only strain. As expected, the paxQ-only strain readily converted 9-desoxypaxilline (27) to paxilline (2) but did not convert paspaline (11) to terpendole $E$ (47). 


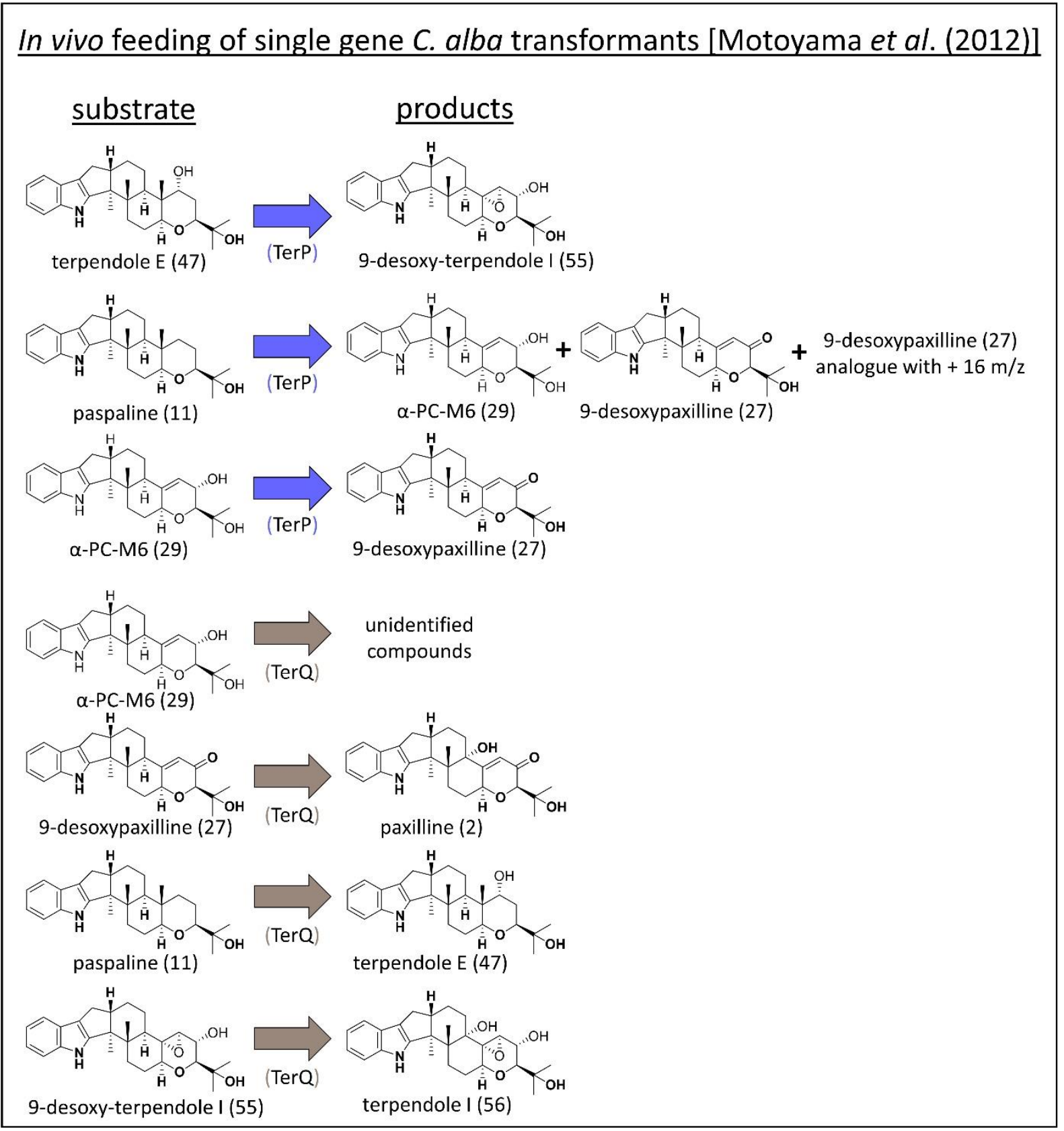

Figure 1.273. Summary of results from feeding experiments completed by Motoyama et al. (2012). ${ }^{30}$

To date, the terP and terQ genes are the only TER genes that have been functionally analysed, but based on sequence similarity to LTM gene products, Motoyama et al. 2012 proposed that the encoded products of terCMB deliver paspaline (11) and terFK convert terpendole I (56) to terpendole C (53). 


\section{Identification of the PTM and PEN gene clusters and functional analysis of the genes}

The PTM and PEN gene clusters encode the genes responsible for allowing penitrem production in Penicillium simplicissimum and $P$. crustosum respectively. Liu et al. (2015) were the first to publish their results on the identification of the PTM cluster from P. simplicissimum (Figure 1.28, A) and functional characterisation of 13 of the 17 PTM genes through heterologous reconstitution in $A$. oryzae. ${ }^{31}$ Shortly after, Nicholson et al. 2015 published their findings of the $P E N$ cluster from $P$. crustosum and confirmation that pen $P$ was essential for penitrem production through generation of a $\triangle$ penP $P$. crustosum knockout strain (Figure 1.28, B). ${ }^{32}$ Below, the methods each group used to identify the PTM and PEN clusters and how Liu et al. (2015) functionally confirmed the genes responsible for penitrem biosynthesis will be explored.

To start, Liu et al. (2015) employed gene cluster searching methods (i.e. Blast searching and 2ndFind program) to identify the first penitrem gene cluster spanning 44 $\mathrm{kb}$ and containing 15 putative genes (ptmGTVHINDAQMBCPSU) in Penicillium simplicissimum. Subsequent RNA sequencing analysis on another penitrem producer, $P$. crustosum, revealed a second $11 \mathrm{~kb}$ cluster containing 5 additional genes (ptmOJKEL) that were expressed with the onset of penitrem production (Figure 1.28, A and D). In contrast, Nicholson et al. (2015) used degenerate primers designed to conserved regions of indole diterpene biosynthetic genes homologous to paxC and paxP to create a fragment of the putative PEN gene cluster that was used to generate radioactively labelled hybridisation probes for cosmid library screening. Results from the cosmid screening were used to generate a contiguous sequence of $66 \mathrm{~kb}$ that contained 23 putative genes (PC-01 to PC-23, which included penPCBMQADGO) that were potentially involved in penitrem biosynthesis. 


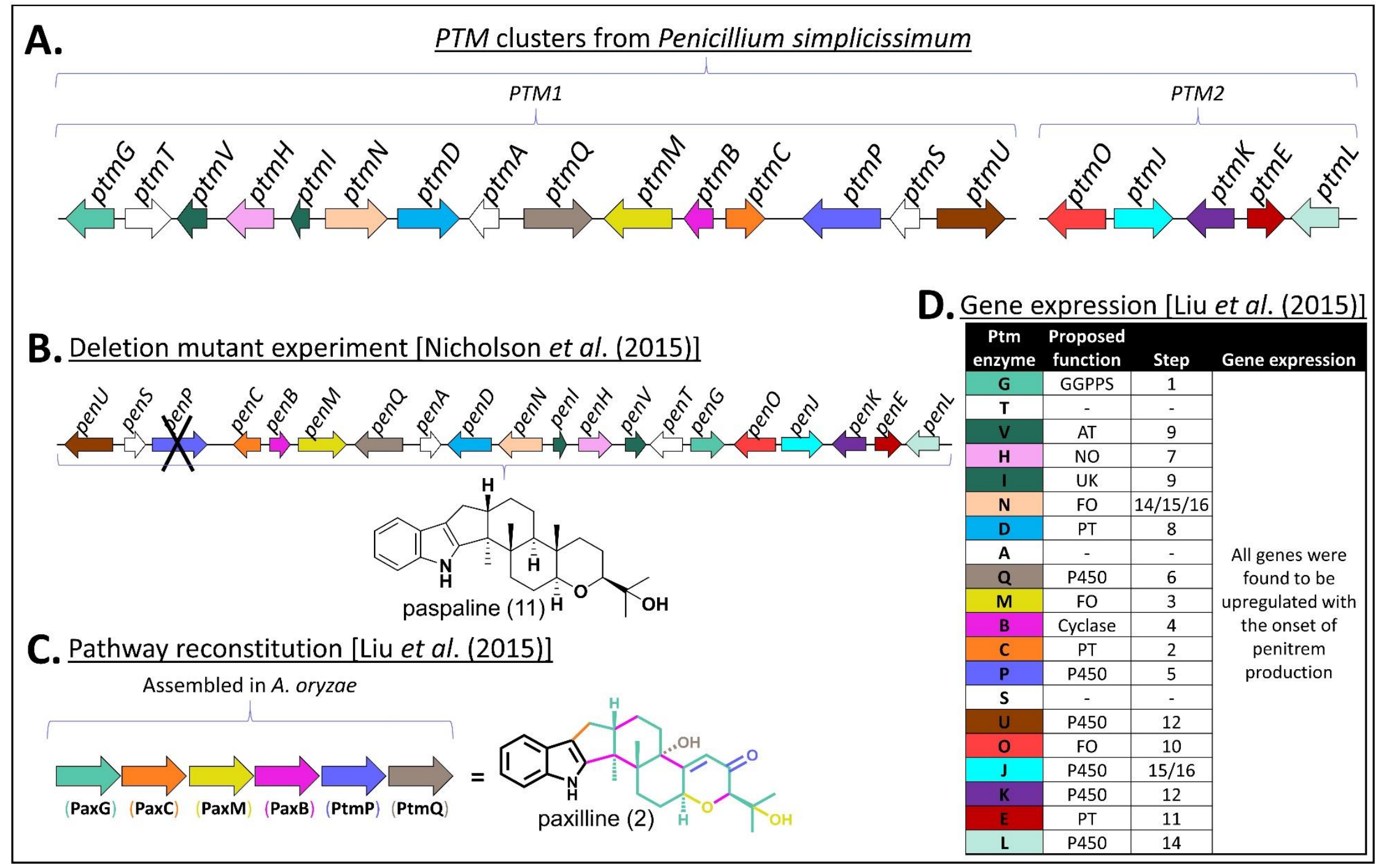

Figure 1.28. Summary of initial results from Liu et al. (2015) (A, C, D) ${ }^{31}$ and Nicholson et al. (2015) (B). ${ }^{32}$ Abbreviations: GGPPS (geranylgeranyl pyrophosphate synthase), AT (acetyl transferase), NO [NAD(P)+ dependent oxygenase], UK (unknown), FO (FAD/FMN dependent oxygenase), PT (prenyl transferase) and P450 (cytochrome P450 oxygenase). 
After identifying the two PTM gene clusters, Liu et al. (2015) first examined the functionality of $p t m P$ and $p t m Q$. They introduced $p t m P$ and $p t m Q$ into an $A$. oryzae paspaline (11)-producing strain containing paxGCMB and found that $p t m P Q$ initiated production of paxilline, but not $\beta$-paxitriol (31) as shown in Figure 1.28, C. From there, they introduced $p t m H$ and $p t m D$ into an $A$. oryzae strain without any other indole diterpene genes. Paxilline (2) fed to the $p t m H D$ strain was metabolised to $\beta$-paxitriol (31) and 20-prenyl- $\beta$-paxitriol (57) indicating that $\mathrm{ptmH}$ reduced the $\mathrm{C} 16$ ketone of paxilline (2) and ptmD prenylated C20 of B-paxitriol (Figure 1.29, i). Ambiguities over the genes required for the next step in penitrem biosynthesis led them to design four additional strains containing ptmHDV, ptmHDIE, ptmHDVI, ptmHDVIE, or ptmHDVIOE (Figure1.29, ii-vi). Whereas incubation of paxilline (2) with the ptmHDV, and ptmHDIE strains did not yield any new products, the ptmHDVI and ptmHDVIE strain showed metabolism of paxilline (2) to 20-prenylpenijanthine A (58), the dehydration product of 20-prenyl- $\beta$-paxitriol (57), showing that $p t m V$ and $p t m /$ are likely both essential for the dehydration. Incubation of paxilline (2) with the ptmHDVIOE strain showed that this strain readily converted paxilline (2) to PC-M4 (59) indicating that PtmE was capable of catalysing the construction of the highly unusual bicyclo[4.2.0]heptane skeleton of PC-M4 (59). 


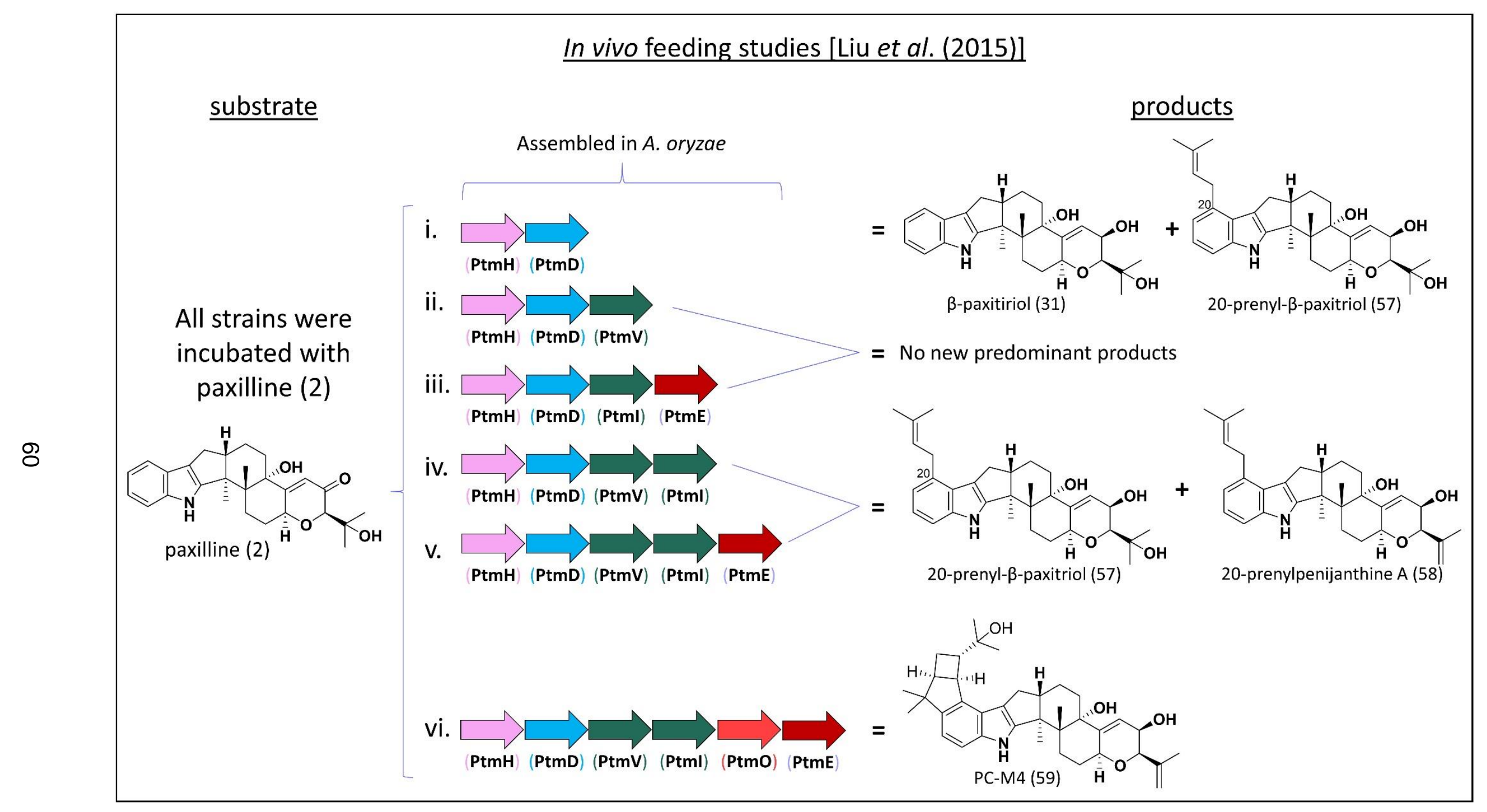

Figure 1.294. Summary of Liu et al. (2015) feeding studies used to delineate the biosynthetic steps from paxilline (2) to PC-M4 (59). ${ }^{31}$ 
Liu et al. (2015) then analysed whether PC-M5 (60) and PC-M4 (59) were shunt metabolites (i.e. metabolites not directly involved in the production of penitrems) by conducting feeding experiments on two additional strains that contained ptmKULNJ or ptmKULNO. ${ }^{31}$ PC-M4 (59) was metabolised by the ptmKULNJ-strain to penitrem A (12) (Figure 1.30, vi) and the ptmKULNO-strain to penitrem $F(61)$ as shown in Figure 1.30, iii, indicating that five genes ( $p t m K U L N J$ ) are involved in the transformation of PC-M4 (59) to penitrem A (12) and that ptmJ is essential for the final benzylic hydroxylation. To elucidate the biosynthetic pathway from PC-M4 (59) to penitrem F (61), four more strains were assembled containing only ptmKUN, ptmK, ptmN, or ptmJ. Incubation of PC-M4 (59) with the ptmKUN strain resulted in penitrem $C$ (62) as shown in Figure 1.30, ii, production showing that $p t m L$ was essential for the $\mathrm{C} 14-\mathrm{C} 15$ epoxide formation. Incubation of PC-M4 (59) with the ptmK-strain showed conversion to secopenitrem D (63) as shown in Figure 1.30, i, indicating that PtmK catalyses the ring expansion and olefin formation of PC-M4 (59) to form secopenitrem D (63). Subsequent feeding of penitrem B (64) to the ptmN-strain showed conversion to penitrem $F(61)$ as shown in Figure 1.30, v, indicating that $\operatorname{PtmN}$ catalyses the C22chlorination in penitrem biosynthesis and that ptmU is essential for the 8-membered ether ring formation of penitrem $D$ from secopenitrem $D(63)$. Finally, penitrem $F(61)$ fed to the ptmJ-strain was converted to penitrem A (12) (Figure 1.30, vi), confirming that $p t m J$ is essential for the final benzylic hydroxylation. 


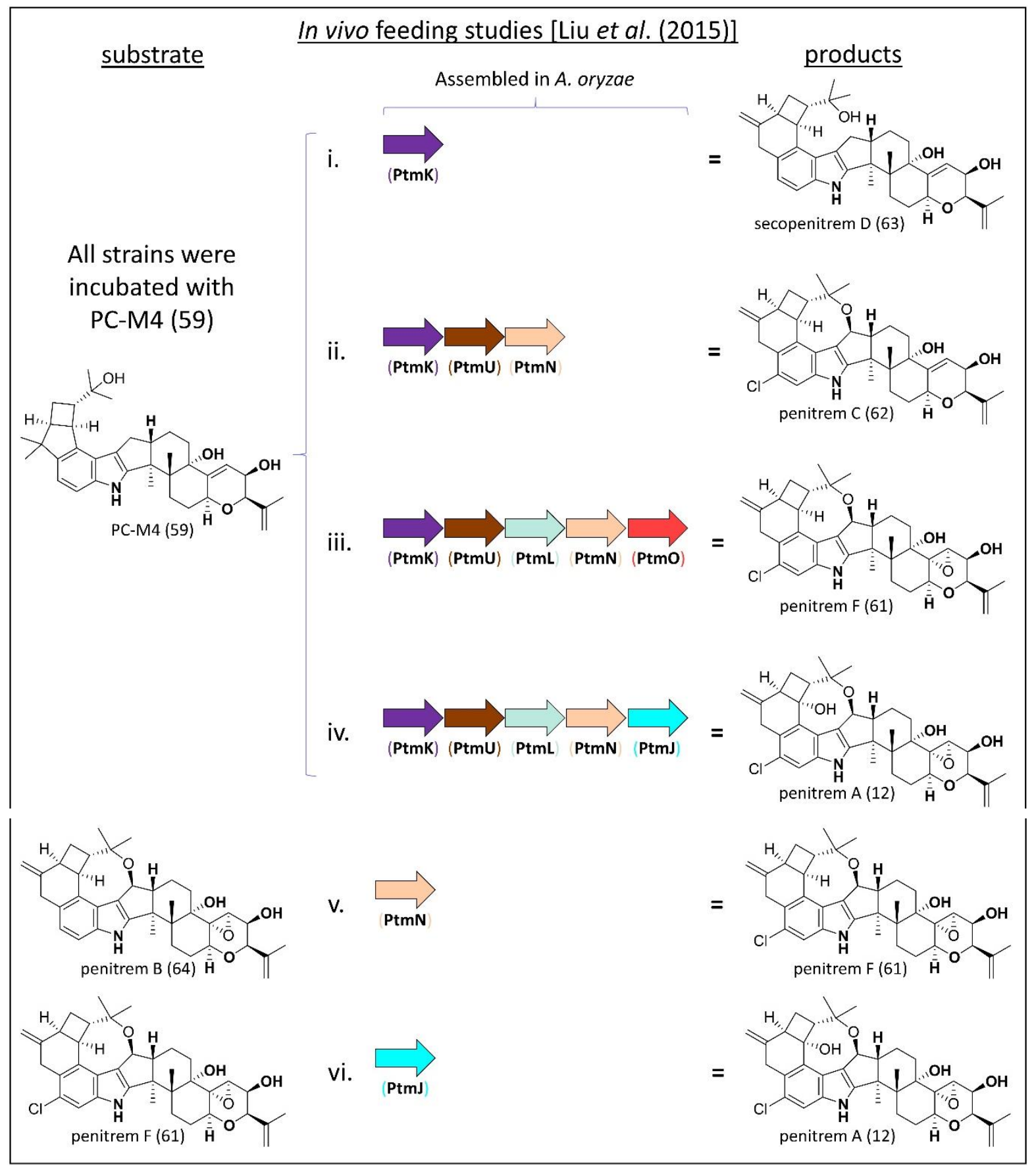

Figure 1.30. Summary of Liu et al. (2015) feeding studies used to delineate the biosynthetic steps from PC-M4 (59) to penitrem A (12). ${ }^{31}$

With the final five cytochrome P450 oxidation steps confirmed, Liu et al. (2015) turned their attention back to the intricacies of the biosynthetic steps involved in converting 20-prenylpenijanthine A (58) to PC-M4 (59). In vitro analysis of recombinant PtmO incubated with 20-prenylpenijanthine A (58) yielded PC-M5 (60) as shown in Figure 1.31, demonstrating that PtmO terminally hydroxylated the $\mathrm{C} 20$ prenyl group. To confirm the unusual action of PtmE, they analysed PtmE in vitro. Incubation of PtmE 
with PC-M5 (60), $\mathrm{Mg}^{2+}$, and dimethylallyl pyrophosphate (8) yielded PC-M4 (59) as shown in Figure 1.31, confirming that just a single enzyme was capable of catalysing the prenylation construction of the highly unusual bicyclo[4.2.0]heptane skeleton of PC-M4 (59).

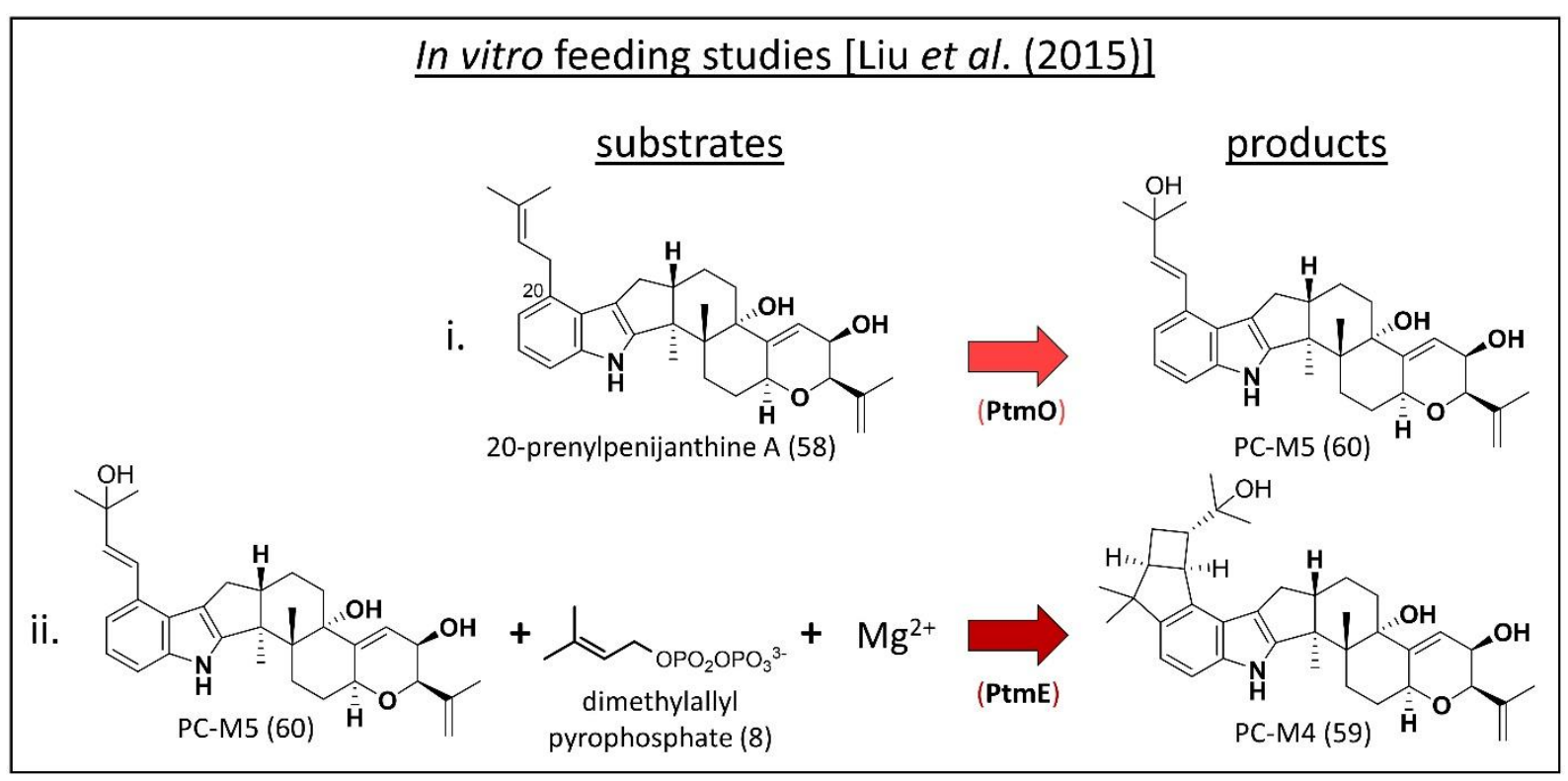

Figure 1.31. Summary of Liu et al. (2015) recombinant enzyme analyses used to functionally examine PtmO and PtmE; the biosynthetic steps from 20prenylpenijanthine $\mathrm{A}(58)$ to PC-M4 (59). ${ }^{31}$

Overall, Liu et al. (2015) clearly confirmed the function of each of the thirteen genes involved in the biosynthetic pathway required to convert paspaline (11) to penitrem A (12). ${ }^{31}$ This work is easily the most comprehensive and impressive analysis of the genes involved in the biosynthesis of an indole diterpene product, as they first-off identified the novel PTM gene clusters and then functionally confirmed the identity of every unique gene involved in penitrem biosynthesis.

\section{Identification of the JAN gene cluster and functional analysis of the genes}

In the same report where Nicholson et al. (2015) announced the identification of the $P E N$ cluster in $P$. crustosum, they also announced the identification of the JAN cluster for janthitremane (i.e. shearinine) biosynthesis in P. janthinellum. ${ }^{32}$ As with the PEN cluster, they used degenerate primers designed to conserved regions of indole 
diterpene biosynthetic genes homologous to $\operatorname{pax} C$ and $\operatorname{pax} P$ to create a fragment of the putative JAN gene cluster that was used to generate radioactively labelled hybridisation probes for cosmid library screening. Results from the cosmid screening were used to generate a contiguous sequence of $68 \mathrm{~kb}$ that contained 10 putative genes (janGAMBCPJQDO) that were potentially involved in janthitremane biosynthesis (Figure 1.32, A). Notably, the nine JAN genes share high amino acid identity compared with their respective $P A X$ homologues: JanG $(42 \%)$, JanC $(71 \%)$, JanM (67\%), JanA (50\%), JanB (77\%), JanP (71\%), JanQ (65\%), JanD (66\%), JanO (72\%). Interestingly, the orientations of all of the genes and the positions of introns are the same in the PAX and JAN clusters except for the presence of janJ. Therefore, Liu et al. (2016) speculated that the PAX cluster could produce janthitremanes with a paxilline (2) core, but this has not been observed because paxD and paxO are naturally expressed at low levels in $P$. paxilli. ${ }^{43}$ 


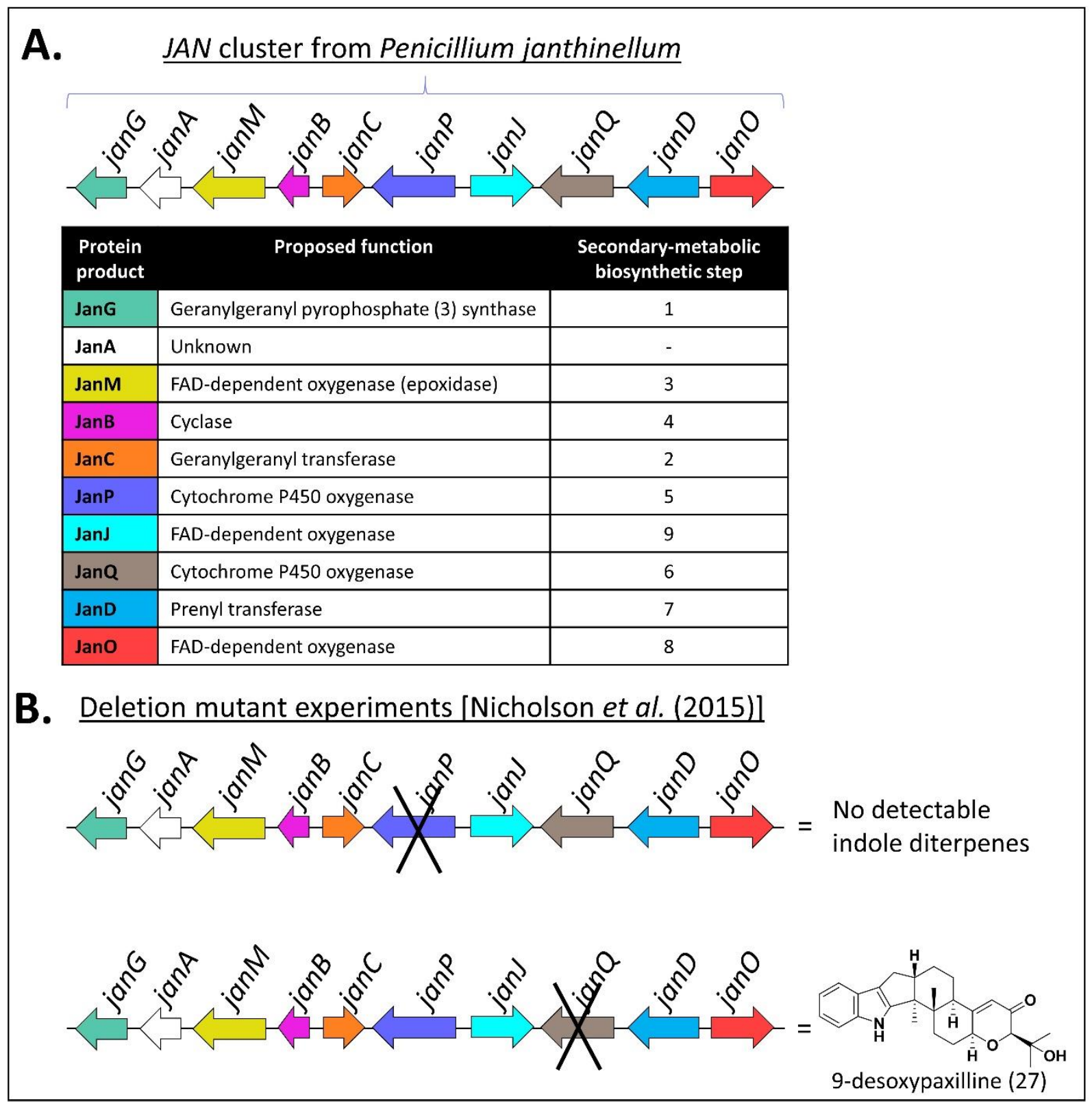

Figure 1.32. Summary of results from Nicholson et al. (2015). The JAN cluster is depicted in $\mathbf{A}$ and the knockout strains with their corresponding indole diterpene porducts are shown in B. ${ }^{32}$

To confirm the involvement of these janP and janD in janthitremane biosynthesis, Nicholson et al. (2015) prepared two $P$. janthinellum knockout mutants where janP or jan $D$ was removed. ${ }^{32}$ They noted the absence of all indole diterpene compounds in the extract of the $\triangle j a n P$-mutant and increased accumulation of 9-desoxypaxilliine with lack of janthitremane production in the $\Delta j a n D$-mutant (Figure 1.32, B), thereby confirming that both genes were involved in janthitremane biosynthesis. Based on the genes in the JAN cluster, Nicholson et al. (2015) proposed the most likely biosynthetic pathway 
for shearninine A biosynthesis, but full elucidation of individual gene functionality remained unclear until Liu et al. (2016) published their findings from their analysis of janQDOJ using their $A$. oryzae heterologous expression system. ${ }^{43}$

Liu et al. (2016) prepared three A. oryzae transformants that contained janQ, janQDO, or janQDOJ and conducted feeding experiments on each strain using 9desoxypaxilline (27). ${ }^{43}$ They found that the janQ-transformant readily metabolised 9desoxypaxilline (27) to paspalinine (38) and that no paxilline (2) was produced indicating that janQ catalysed the $C 9$ hydroxylation and the acetal ring formation of the terminal hydroxide of paspaline (11) to form paspalinine (38) as shown in Figure 1.33, i. Subsequent incubation of 9-desoxypaxilline (27) with the janQDO-transformant yielded shearinine $\mathrm{K}(65)$ and shearinine A (66) showing that JanD and JanO were responsible for the prenylation of paspalinine (38) to form shearinine $K(65)$ and subsequent oxidative ring closure of shearinine $K(65)$ to form shearinine $A(66)$ as shown in Figure 1.33, ii. Finally, incubation of 9-desoxypaxilline (27) with the janQDOJtransformant yielded sheadinine $D$ showing that JanJ catalysed the hydroxylation of shearinine A (66) to form shearinine $D(67)$ as shown in Figure 1.33, iii.

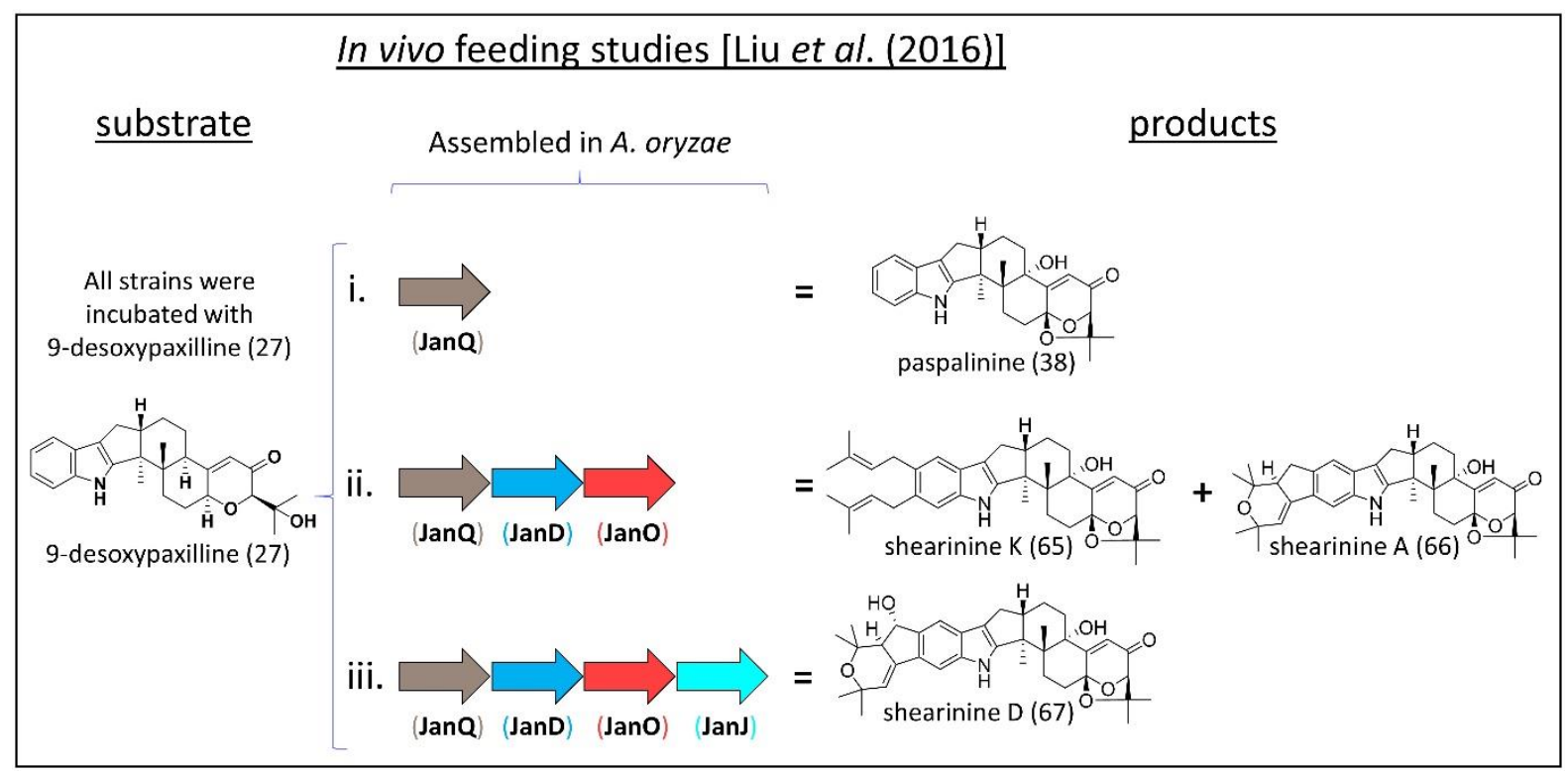

Figure 1.33. Summary of results from heterologous feeding study of JAN genes in $A$. oryzae by Liu et al. (2016). ${ }^{43}$

To further investigate the detailed mechanism of the janDO prenylation and oxidative cyclisation, they analysed the JanD and JanO recombinant enzymes in vitro. They 
incubated recombinant JanD with paspalinine (38) and paxilline (2) and found that paspalinine (38) was specifically bisprenylated to shearinine $K(65)$ whereas paxilline (2) was unspecifically bisprenylated to form both 21,22-bisprenylpaxilline (33) and 20,21-bisprenylpaxilline (68) as shown in Figure 1.34, i and ii. Additionally, they analysed recombinant PaxD and found that it readily converted paxilline (2) to 21,22bisprenylpaxilline (33) as shown in Figure 1.15, i) and also converted paspalinine (38) to shearinine $K(65)$ as shown in Figure 1.34, ii. Next, they incubated recombinant JanO with shearinine $K(65)$ and saw rapid complete conversion to shearinine $A(66)$ as a shown in Figure 1.34, iii, indicating that JanO was solely responsible for the oxidative cyclisation to form shearinine A (66). Finally, they conducted a large-scale JanO mediated oxidation of 21,22-bisprenylpaxilline (33) and a coupled JanD-JanO reaction with paxilline (2) as shown respectively in Figure 1.34, v and vi. Interestingly JanO poorly oxidised 21,22-bisprenylpaxilline (33) to 22-protoshearinine (69) and shearinine B (70) but was unable to oxidise or cyclise the 20,21-prenylpaxilline. In accordance with this, incubation of paxilline (2) with JanD-JanO readily yielded 20,21bisprenylpaxilline (68) and 21,22-bisprenylpaxilline (33) but only produced very small quantities of shearinine B (70) and 22-protoshearinine (69). 


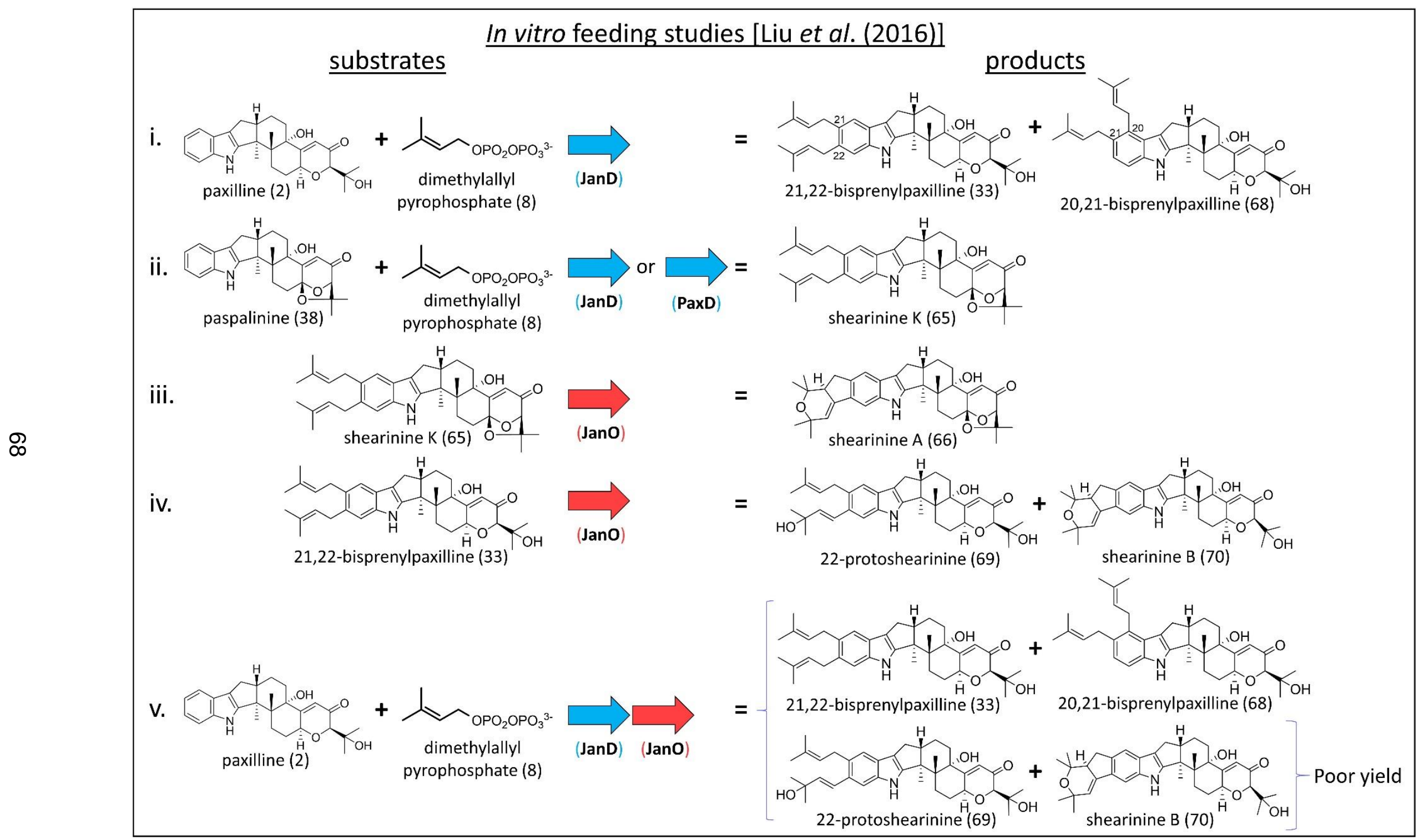

Figure 1.34. Summary of recombinant enzyme substrate studies of JanD and JanO by Liu et al. (2016).43 
The diprenylation-flavoprotein oxidative cyclisation mechanism of JanD and JanO in shearinine biosynthesis provides a third pathway by which the indole diterpene cores are prenylated and oxidised. In contrast to this mechanism, the bicylco[3.2.0]heptane in penitrem biosynthesis is catalysed by a prenyl transferase (PtmE) acting on the hydroxylated-prenyl group of PC-M5 (60), and the bicylic core of lolitrems is formed by a diprenylation-P450-catalysed oxidative cyclisation. Thus, there are three different known mechanisms that fungi use during construction of the bisprenylation-oxidation sequence (Figure 1.35). 


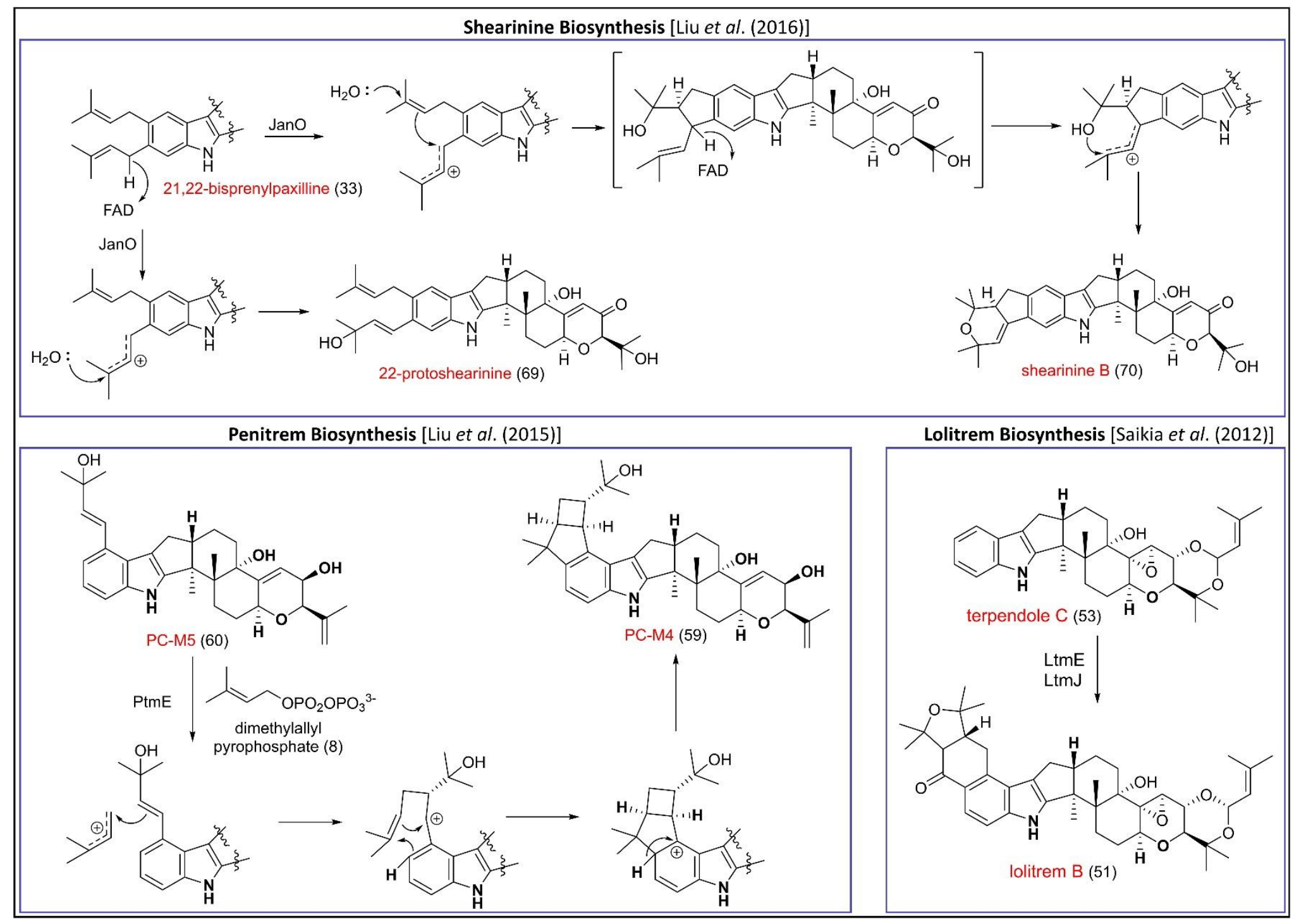

Figure 1.35. Summary of the three different kinds of oxidative cyclisation mechanisms proposed by Saikia et al. (2012) for the lolitrems, ${ }^{29}$ Liu et al. (2015) for the penitrems, ${ }^{31}$ and Liu et al. (2016) for the shearinines. ${ }^{43}$ 
Identification and functional analysis of unclustered indole diterpene genes, which led to the identification of another indole diterpene cluster, ATS5

The identification and functional confirmation of the various clustered indole diterpene genes established a sequence library that Tang et al. (2015) used to selectively probe for novel, unclustered indole diterpene genes. ${ }^{33}$ Their studies specifically focused around the identification and functional analysis of three novel indole diterpene cyclases (AtS2B, AtS5B1, and AfB) but further exploration around one of the novel cyclases (Ats5B1) revealed a new indole diterpene cluster, the ATS5 locus, in Aspergillus tubingenesis (Figure 1.36). Rather than use the typical filamentous fungi expression system for their heterologous pathway reconstitution studies, they showed, for the first time, that yeast (Saccharomyces cerevisiae) readily accepted indole diterpene genes for production of indole diterpene products.

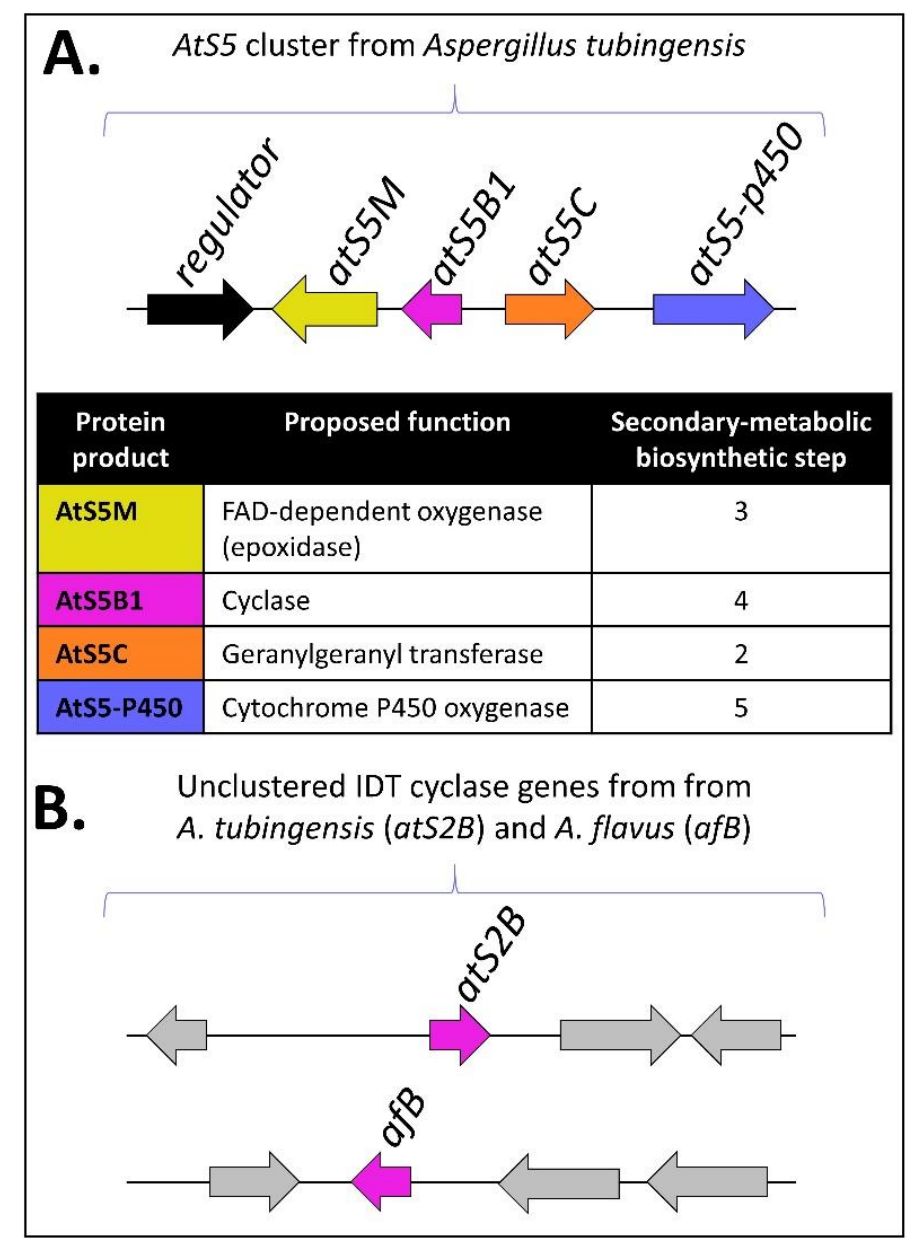

Figure 1.36. Schematic of AtS5 locus in $\mathbf{A}$ and the location of the two unclustered indole diterpene cyclases AtS2B and AfB in B (Tang et al. 2015). ${ }^{33}$ 
To demonstrate that their yeast expression system worked, Tang et al. (2015) first reassembled the core indole diterpene genes (atmGCM) from the aflatrem (36) biosynthetic pathway into S. cerevisiae. ${ }^{33}$ When AtmG and AtmC were co-expressed they unexpectedly saw generation of 17,18-epoxy-3-geranylgeranylindole (15), which they attributed to an endogenous yeast oxygenase (Figure 1.37, i). Upon addition of AtmM to the atmGC-mutant, they saw formation of the two expected products shown in Figure 1.37, ii [13,14-epoxy-3-geranylgeranylindole (16) and bisepoxy-3geranylgeranylindole (17)]. The triple gene transformant provided the epoxysubstrates required for the analysis of various indole diterpene cyclases. Thus, as a proof of concept, the first indole diterpene cyclase they tested was AtmB. When atmB was introduced into the atmGCM-strain, paspaline (11) was detected as the major product and emindole SB (18) was detected as the minor product (Figure 1.37, iii) confirming that their expression system was suitable for the analysis of other indole diterpene cyclases. 


\section{Pathway reconstitution [Tang et al. (2015)]}

Assembled in Saccharomyces cerevisiae

i.

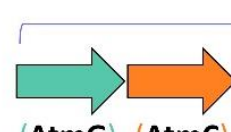

AtmG) (AtmC

ii.

(AtmG) (AtmC) AtmM
$=\mathbb{N}_{\mathrm{N}}$

17,18-epoxy-3-

geranylgeranylindole (15)

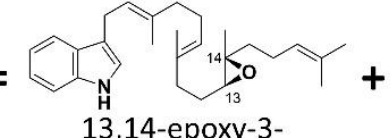

geranylgeranylindole (16) iv.

iii.

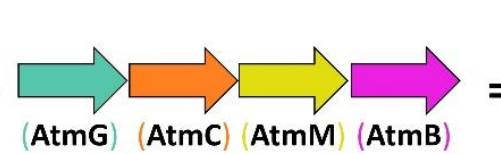

AtmG (AtmC) AtmM (AtS5B1)<smiles>CC(C)=CCCC1CCC2CCC3Cc4c([nH]c5ccccc45)C3CCC2C1O</smiles>
emindole SB (18) (minor)

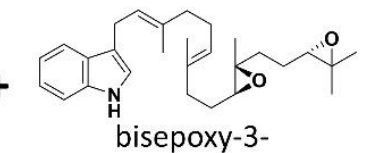

geranylgeranylindole (17)

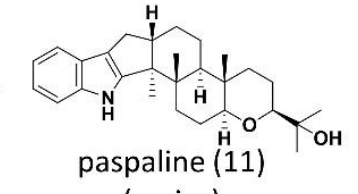

paspaline (11)

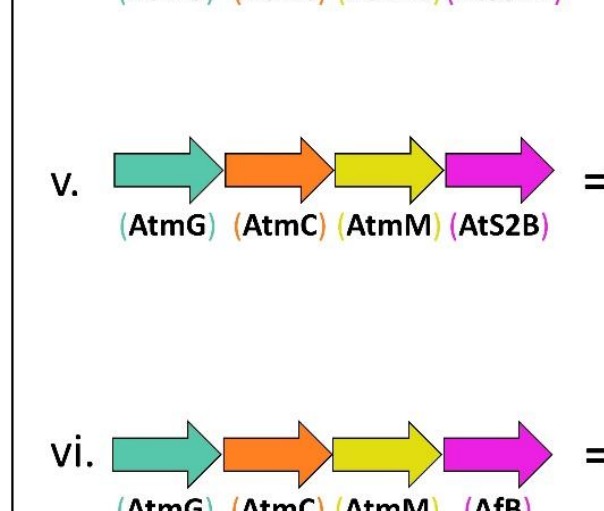

AtmG (AtmC) AtmM (AfB)

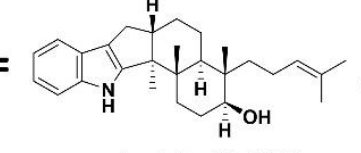

emindole SB (18) (minor)

\section{$=$}

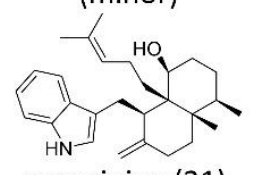

anominine (21)

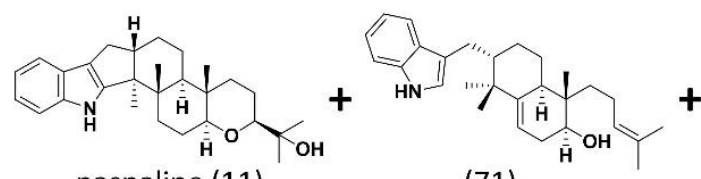

(71) paspaline (11) (major)
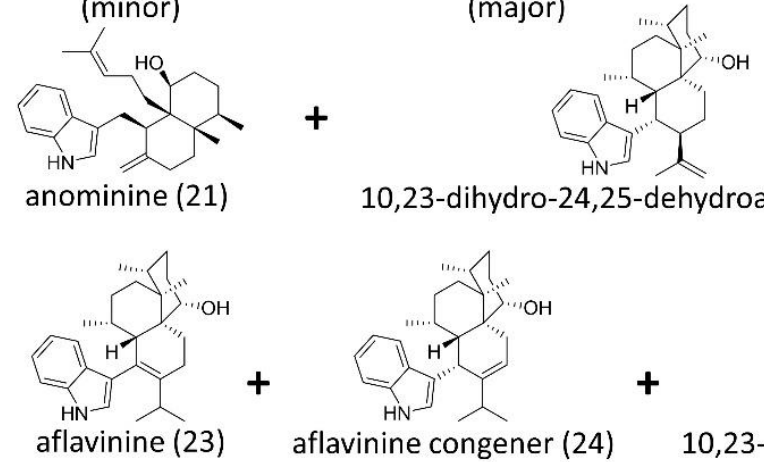

10,23-dihydro-24,25-dehydroaflavinine (22) (major) (minor)

10,23-dihydro-24,25-dehydroaflavinine (22) (minor)

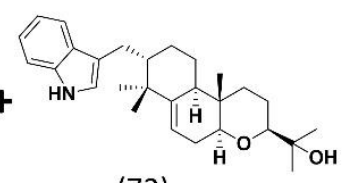

(72)
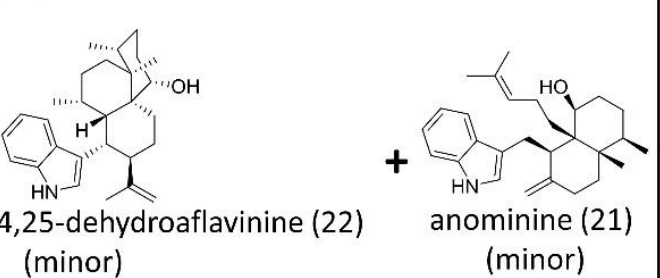

Figure 1.375. Summary of heterologous expression studies used to analyse indole diterpene cyclases [Tang et al. (2015)]. ${ }^{33}$ 
Tang et al. (2015) chose to focus on identifying indole diterpene cyclases that were responsible for the formation of the Markovnikov-derived indole diterpenes anominine (21) and aflavinine (23) because no indole diterpene cyclases had been identified that gave rise to any Markovnikov-derived indole diterpene products. ${ }^{33}$ From Protein BLAST searches on AtmB homologues they generated a phylogenetic tree containing 140 homologous enzymes grouped into five clades. From the phylogenetic tree they systematically chose three indole diterpene cyclases to study; two from Aspergillus tubingenesis (AtS2B and AtS5B1) and one from A. flavus (AfB). AtS2B and AfB were both from Clade 4 of their tree, which notably contained standalone indole diterpene cyclases, and AtS5B1 was from Clade 5, the same clade as the clustered indole diterpene cyclases that give rise to paspaline (11)-derived indole diterpenes (i.e. AtmB, $\mathrm{PaxB}$, etc.). To examine whether phylogenetically-grouped indole diterpene cyclases had similar cyclisation regioselectivity they first tested AtS5B1. Upon cloning atS5B1 into the atmGCM-strain, they saw formation of the expected paspaline (11) and emindole SB (18) as well as two new products (71) and (72) shown in Figure 1.37, iv. Intriguingly, compound (71) and (72) are derived from a different cyclisation pattern than paspaline (11) and emindole SB (18), which involves a 1,2-methyl migration and concomitant ring expansion to yield the 6-6 bicyclic terpene moiety. Although the cyclisation patterns differ, both involve anti-Markovnikov generated ring closures thereby demonstrating that these phylogenetically-grouped indole diterpene cyclases share similar cyclisation mechanisms.

Tang et al. (2015) then turned their attention to the standalone indole diterpene cyclases, AtS2B and AfB. When AtS2B was introduced into the atmGCM-strain two new compounds, confirmed by NMR spectroscopy as anominine (21) and 10,23dihydro-24,25-dehydroaflavinine (22), were produced (Figure 1.37, v). In contrast, when AfB was introduced into the atmGCM-strain, aflavinine (23) was produced as the major product and anominine (21), 10,23-dihydro-24,25-dehydroaflavinine (22), and aflavinine cogener (24) were produced as minor products (Figure 1.37, vi). Thus the two standalone indole diterpene cyclases are solely responsible for the cyclisation mechanism that gives rise to anominine (21) and aflavinines.

Finally, Tang et al. (2015) further demonstrated the power of the phylogeneticallyguided yeast platform by identifying and confirming the function of a P450 (AtS5-P450) that was clustered with AtS5B1. Co-expression of AtS5-P450 in a mutant containing 
atmGC, atS5M (the ATS5 cluster homologue of atmM), and atS5B1 yielded three novel terminally-hydroxylated compounds (73), (74) and (75) shown in Figure 1.38, i. Surprisingly, when AtS5-P450 was combinatorially co-expressed with atmGCM and atS2B, two terminally-hydroxylated anominine (21) two new derivatives were produced, compound (76) and (77) as shown in Figure 1.38, ii. Overall, these results showed that phylogeny-guided pathway reassembly is a promising method for the characterisation of novel genes, which in this case were indole diterpene cyclases and an oxygenase.

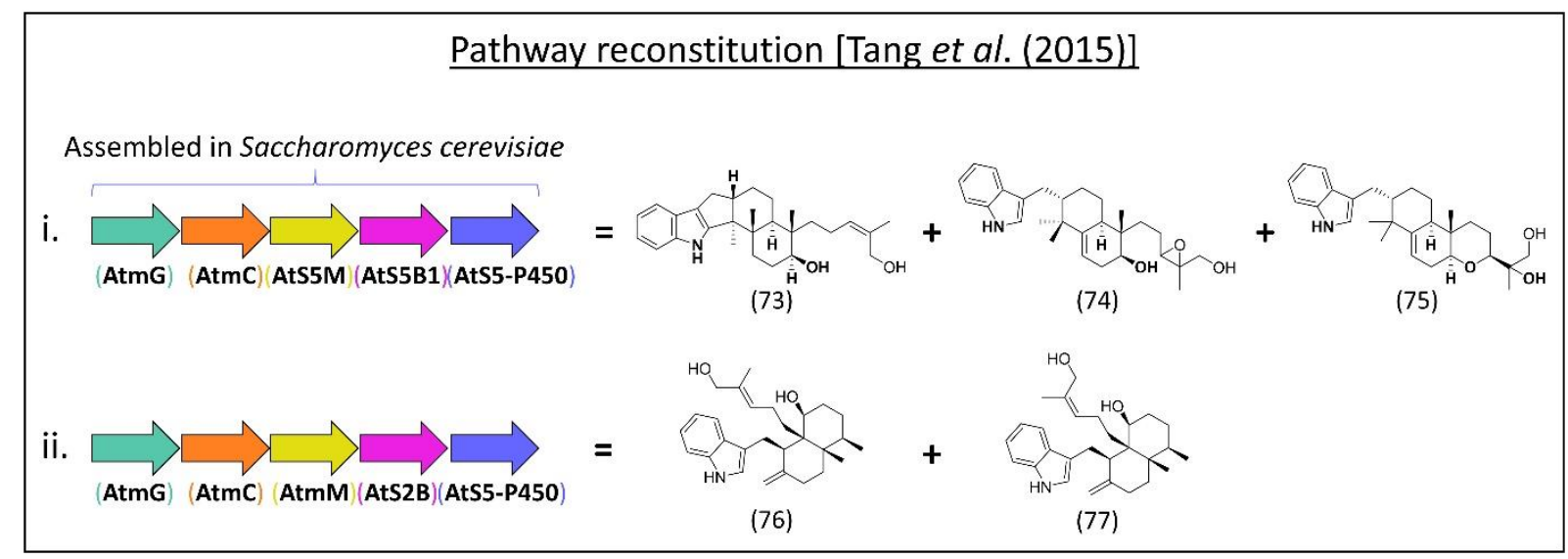

Figure 1.38. Summary of Tang et al. (2015) results from heterologous expression studies with AtS5-P450. ${ }^{33}$

\section{Conclusions}

This section has explored how the indole diterpenes are biosynthesised and the methods that have been used to identify and functionally analyse the indole diterpene genes. In summary, it is clear that the functional groups around the indole diterpene core structures are derived from the differences in the genes from their host organism. Some of the differences are obvious, as in the specific biosynthetic pathway divergence seen between the hosts that produce paspaline (11)-derived indole diterpenes; where the Eurotiomycetes (Penicilli and Aspergilli) use 9-desoxypaxilline (27) as substrate and have $\beta$-stereochemistry at $\mathrm{C} 10$, and the Sordariomycetes ( $C$. alba, N. Iolii, E. festucae) use terpendole $E(47)$ as substrate and have $\alpha$ stereochemistry at $\mathrm{C} 10$. But other differences, like the under-utilization of $\mathrm{PaxD}$ and $\mathrm{PaxO}$ in $P$. paxilli in comparison to JanD and JanO in $P$. janthinellum remain a bit of a 
mystery. Mysteries like this one will be exposed as more information about the genes and gene products is amassed. With the identification of each new indole diterpene gene and functional characterisation of the gene product, comes insight into the key differences in gene sequence that introduce substrate specificity and gene function.

As the databases grow, it becomes easier to envision a future where made-to-order chemical products can be easily engineered by altering genetic information. Components of a chemical's pharmacophore will be alterable to remove toxic moieties and deliver more potent therapeutic properties (i.e. mixing and matching the decorative genes from different organisms may enable creation of made-to-order pseudo-natural products with prescribed bioactivities).

In parallel, approaches will be developed to enhance compound production to enable access to useful compounds that are produced in very small quantities or potentially not at all (e.g. turning on silent genes). This thesis serves as a proof of concept to address this parallel approach and explore how to gain access to the valuable nodulisporic acids that have remained just beyond reach for the past three decades. The following section reviews what is known about the nodulisporic acids to provide a convincing argument for the purpose of pursuing this research project. 


\section{Nodulisporic acids: the target for this research}

Nodulisporic acid A (1) (Figure 1.39) was first discovered in 1992 by Merck Research Laboratories during an ongoing study to identify unique bioactive compounds. ${ }^{11}$ Merck scientists isolated nodulisporic acid A (1), using bioassay-guided isolation, from the fermentation extracts of the endophytic filamentous fungus, Hypoxylon pulicicidum (formerly classified as Nodulisporium sp. ${ }^{17}$ MF 5954, ATCC 74245), which they acquired from the tropical woody plant, Bontia daphnoides, in Hawaii. ${ }^{11}$ The discovery of the potent bioactive profile associated with nodulisporic acid led to an extensive screening project aimed at identifying additional nodulisporic acid $A$ (1) producers. Twelve new strains of Nodulisporium spp. (presumably all Hypoxylon spp.) were identified across seven tropical regions on four continents; and in a parallel approach to identify congeners and/or natural analogues of nodulisporic acid A (1) that were inaccessible by chemical derivitization, Merck scientists created a library of chemically mutated Hypoxylon strains that overproduced key nodulisporic acid intermediates (i.e. A1, A2, A4, $\Delta 23-A 4, B, B 1, B 2, C, C 1, C 2, \Delta 23-C 4, D, D 1, D 2, D 3, E$, and F; Figure 1.39). ${ }^{11,50-53}$ They screened the bioactive profiles of this nodulisporic acid compound library and found that nodulisporic acid A (1) had the most potent insecticidal properties of all of the naturally occurring nodulisporic acid derivatives. Although nodulisporic acid A (1) had very good in vitro and in vivo activity against fleas, its potency and pharmacokinetic properties did not justify its development as a drug, leading Merck to launch a medicinal chemistry study to optimise the nodulisporic acid A (1) profile. Merck scientists generated and screened a library of more than 1000 nodulisporic acid A (1) analogues leading to a comprehensive understanding of the nodulisporic acid A (1) pharmacophore and the identification of an ideal drug target, $N$-tert-butyl nodulisporamide (78)..$^{54-55}$ Details of Merck's studies are discussed below. 


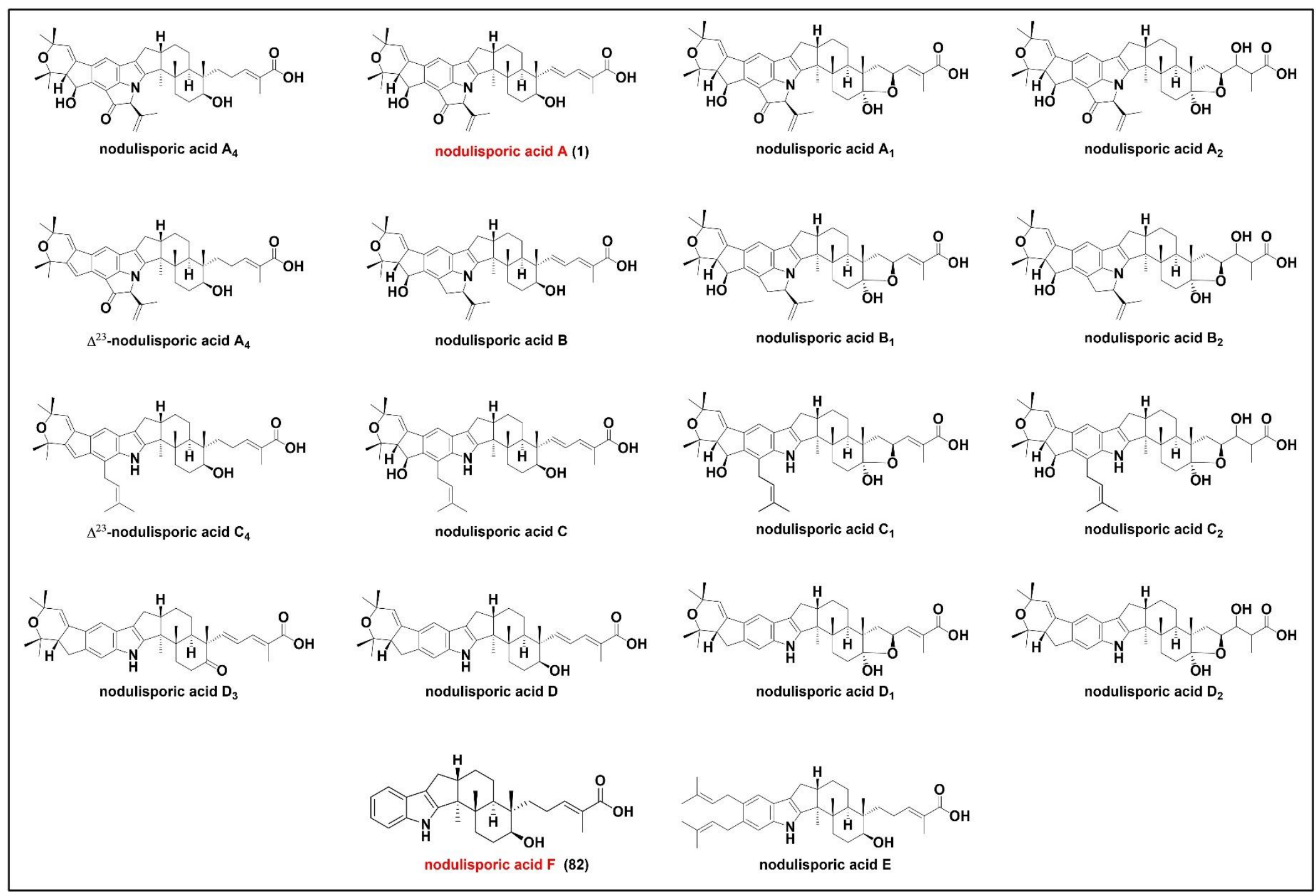

Figure 1.39. Collection of known nodulisporic acids. ${ }^{11,50}$ 


\section{The discovery of nodulisporic acid A's (1) insecticidal activity}

The insecticidal bioactivity of nodulisporic acid A (1) was first detected by Merck in 1992 during crude natural product extract screening with an in vitro Lucilia sericata (blowfly) assay. ${ }^{56}$ In parallel to the $L$. sericata assay, Merck employed an in vivo bioassay using Cimex lectularius (bedbug) infected mice to isolate nodulisporic acid A (1) through evaluations of crude broth fractionations (i.e. infected mice were dosed with fractions of the fungal extract). ${ }^{57-58}$ Once nodulisporic acid A (1) was isolated, pure compound was found to have a lethal concentration necessary to kill $50 \%$ (LC50) against $L$. sericata of 300-1000 ppb. ${ }^{56,58}$ These results demonstrated that nodulisporic acid $A$ (1) had potential for use as an insecticide because it was more potent than the widely used pyrethroids ( 22,000-28,000 ppb). ${ }^{11}$ Thus, additional bioassays were completed to analyse nodulisporic acid A's (1) potency to a range of other ectoparasites.

Merck first screened pure nodulisporic acid A (1) in an in vivo C. lectularius bioassay, and observed complete parasite paralysis at $0.4 \mathrm{ppm}$, which, when compared to 5.0 ppm of ivermectin, indicated that nodulisporic acid A (1) could be developed into a useful therapeutic to fight blood-feeding arthropods. To test this theory further, nodulisporic acid A (1) was assayed against the cat flea (Ctenocephalides felis) in an artificial membrane flea-feeding apparatus, and again found to be roughly ten-times more potent than ivermectin (i.e. $1 \mathrm{ppm}$ vs 10 ppm). ${ }^{59}$ Nodulisporic acid A (1) was further tested against the hard tick (Dermacentor variabilis) in a similar in vivo bioassay to that used with $C$. lectularius, but no observable activity was observed when mice were treated with a single oral dose of $2.5 \mathrm{mg} / \mathrm{kg} .{ }^{60}$ Notably, anthelmintic activity was also tested using in vitro and in vivo assays but no desirable activity was observed. ${ }^{11}$

\section{Nodulisporic acid A (1) selectively targets glutamate gated chloride ion channels}

The potency that nodulisporic acid A (1) exhibited toward fleas prompted Merck scientists to examine nodulisporic acid A's (1) mechanism of action. To this end, they developed a series of nodulisporic acid $A(1)$ and avermectin derived radioligands and used them to establish that nodulisporic acid A (1) selectively activates glutamategated chloride ion channels that are unique to invertebrates. ${ }^{61-62}$ Activation of these channels silences neuronal activity and leads to paralysis, starvation and death of the 
insect. Importantly, they found that nodulisporic acid A (1) does not activate glycineand GABA-gated chloride ion channels, which are found across both invertebrates and vertebrates and are notably activated by ivermectin. Therefore, the ion channel selectivity of nodulisporic acid A (1) imparts it with a unique vertebrate safety profile that confers the ability to orally dose cats and dogs at higher levels than ivermectin, allowing for greater anitparasitic activity and enhanced therapeutic potential.

\section{Nodulisporic acid A (1) is an orally safe, systemically active, ectoparasiticide}

In the case of animal therapeutics, oral treatments are preferred over topical as they ensure uniform drug coverage and are not subject to variation in drug effectiveness caused by environmental factors. That said, systemically active oral therapeutics are much harder to come by as they must be nontoxic (i.e. cannot interfere detrimentally with the inner workings of the animal undergoing treatment). Therefore, nodulisporic acid $A(1)$ is an ideal oral therapeutic because it is a systemically active ectoparasiticide that shows no acute mammalian toxicity ${ }^{59}$ and does not interfere with ligand gated channels (GABA, kainite, glycine) or sodium and potassium voltage gated channels, ${ }^{62}$ which is an essential property of long-lasting therapeutics (i.e. therapeutics dosed on a monthly basis) as they will be exposed to plasma for extended periods of time. Additionally, the unique targeting of nodulisporic acid $A(1)$ to the glutamate-gated chloride ion channels imparts nodulisporic acid A (1) with a novel mechanism of action compared to the widely used pyrethroids, which alter the kinetics of sodium gated ion channels. ${ }^{63}$ Therefore, by targeting a different ion channel, nodulisporic acid A (1) circumvents the problem of insecticidal resistance that has developed toward the common insecticides like the pyrethroids, ${ }^{64}$ carbamates, and organophosphates. ${ }^{65-67}$

To directly demonstrate nodulisporic acid A's (1) unique vertebrate safety profile, Merck completed in vivo bioassays using C. felis (flea) infected dogs. ${ }^{59}$ Dogs were experimentally infected with $C$. felis and administered nodulisporic acid A (1) orally, one time, at $15 \mathrm{mg} / \mathrm{kg}$ in two studies. Upon a 48 hour post-dose examination the nodulisporic acid A (1)-treated dogs harboured $80 \%$ and $99.7 \%$ fewer fleas than the control dogs. Additional new fleas were placed on dogs four and five days after initial administration of nodulisporic acid $A(1)$ and examined 48 hours later, day six and seven post-dose (study dependent), at which point $50 \%$ residual efficacy was 
observed. These results led Merck to examine the efficacy of oral administration and the pharmacodynamics of nodulisporic acid A (1) in vivo.

Merck scientists examined the efficacy of oral administration by comparing the maximum blood plasma concentration and plasma half-life of nodulisporic acid A (1) when administered orally versus intravenously. ${ }^{59}$ They found that the results were similar upon an intravenous administration of $1.5 \mathrm{mg} / \mathrm{kg}$ nodulisporic acid A (1) and an oral administration of $15 \mathrm{mg} / \mathrm{kg}$ nodulisporic acid $A(1)$, where the maximum blood plasma concentration was respectively $2.2 \mathrm{mg} / \mathrm{ml} 1$ hour post-dose compared to 1.0 $\mathrm{mg} / \mathrm{ml} 8$ hours post-dose and the plasma half-life was 23 hours compared to 19.3 hour, indicating that oral administration was viable. Importantly, the pharmacodynamic data from this dog study showed that blood concentrations of less than $0.1 \mathrm{mg} / \mathrm{ml}$ nodulisporic acid A (1) were effective at killing $~ 50 \%$ of fleas.

Together these results demonstrated that nodulisporic acid A (1) had potent in vitro and in vivo activity toward fleas but that its pharmacodynamics profile was not ideal for drug development. Thus, Merck launched a medicinal chemistry effort to design a nodulisporic acid A (1) analogue that had a longer plasma half-life and more potent insecticidal properties.

\section{Medicinal chemistry studies: elucidation of the nodulisporic acid $A$ (1) pharmacophore and development of nodulisporic acid A (1) analogues with more desirable properties}

When Merck scientists launched their medicinal chemistry efforts they were unaware of how modifications to different parts of the nodulisporic acid A (1) structure would affect its inherent bioactive properties. As an initial approach they made a mixture of simple modifications to each reactive centre on the nodulisporic acid A (1) scaffold to elucidate the key components of the nodulisporic acid A (1) pharmacophore that were essential for its potent ectoparasiticidal activity. In particular, they altered the oxidation state of the various functional groups (Figure 1.40) and evaluated a variety of dienoic chain modifications by inserting esters, thioesters, and amides. ${ }^{54}$ 


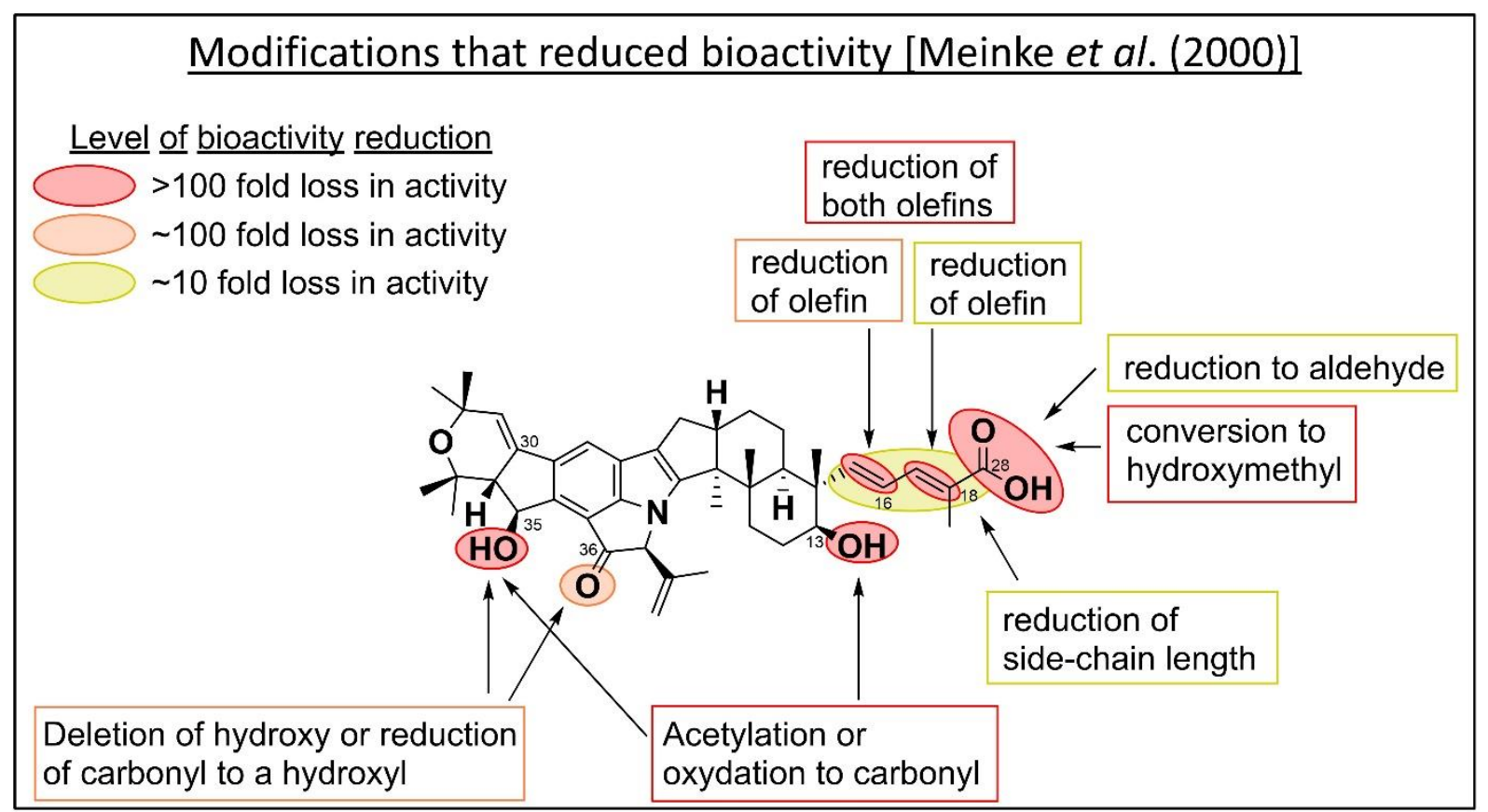

Figure 1.40. Summary of modifications cited in by Meinke et al. (2000) that diminished the inherent insecticidal properties of nodulisporic acid A (1). ${ }^{54}$

Results from these initial modification studies revealed that alterations to the core nodulisporic acid A (1) structure were deleterious to its inherent insecticidal properties whereas many modifications to the dienoic acid sidechain enhanced desirable bioactive properties. This led them to characterise the nodulisporic acid A (1) core as "non-permissive" and the dienoic acid side chain as "permissive" to chemical modifications (Figure 1.41). ${ }^{11}$

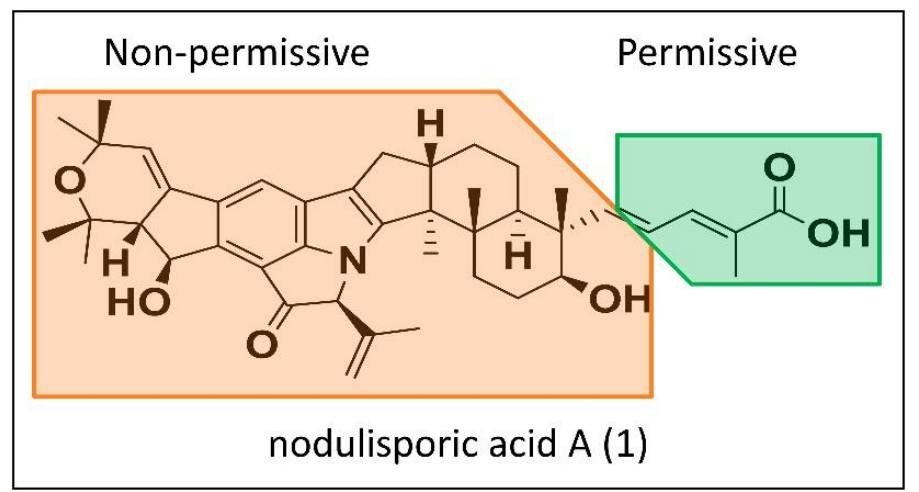

Figure 1.41. Depiction of the non-permissive and permissive regions of nodulisporic acid $A$ (1). ${ }^{11}$ All chemical modifications to the non-permissive region were deleterious to the inherent insecticidal activity of nodulisporic acid A (1) whereas some key modifications to the permissive region greatly enhance the insecticidal profile of nodulisporic acid A (1). 
Although many of their amide side chain modifications resulted in more potent antiflea activity (i.e. $>1$ order of magnitude), there were two key amide modifications to the acidic tail of nodulisporic acid $\mathrm{A}(1)$, the mono-substituted $\mathrm{NHC}(\mathrm{Me})_{2} \mathrm{C}(\mathrm{O}) \mathrm{NMe}_{2}$ compound (79) and the disubstituted $N-1-\left[\left(4-\mathrm{SO}_{2} \mathrm{Me}\right)\right.$ Piperazinyl] compound (80) shown in Figure 1.42, that enhanced the flea efficacy by two orders of magnitude (i.e. flea $L C \geq 80$ of $0.01 \mathrm{ppm}$ vs $1 \mathrm{ppm}$ ). ${ }^{54}$ With this in mind, Merck scientists directed their focus to further enhancing the anti-tick and pharmacokinetic properties of nodulisporic acid A (1) by developing more intricate side chain modifications.

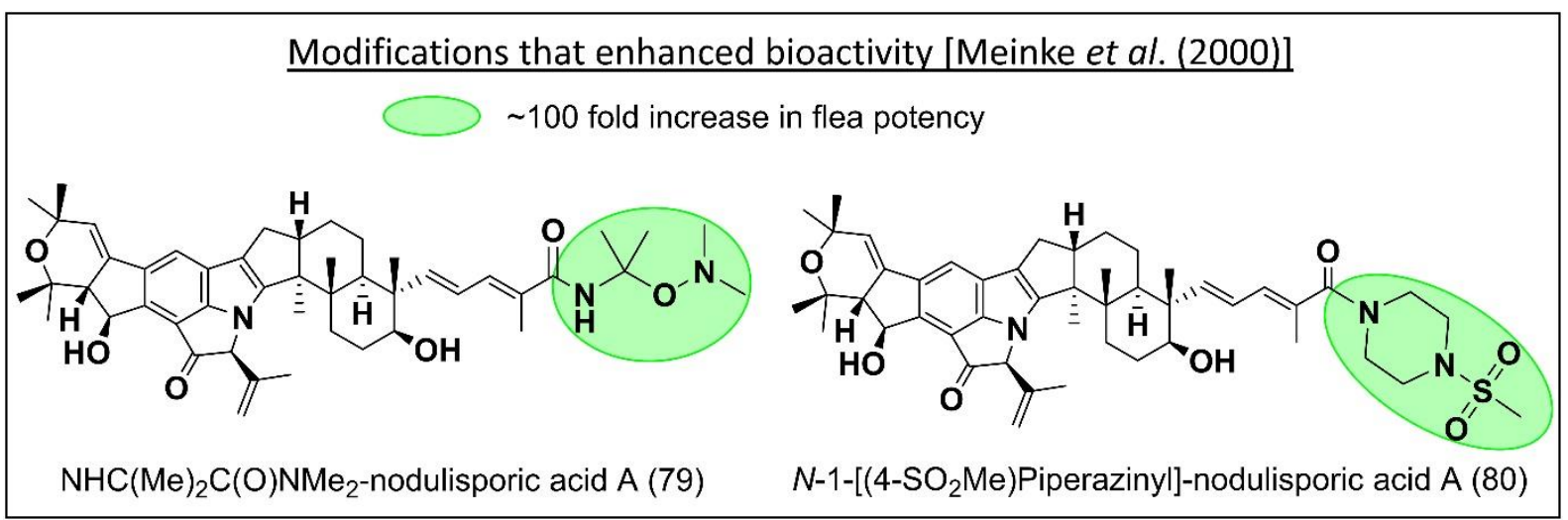

Figure 1.42. Depiction of the two key nodulisporamides that were nearly two orders of magnitude more potent to fleas than nodulisporic acid A (1). ${ }^{54}$

One approach led by Berger et al. (2001) was to install heterocylic oxazoles and thiazoles into the 17,18-olefin of the dienoic acid chain of nodulisporic acid $A(1) .{ }^{60}$ Of the three key heterocycles tested, the dimethylamino oxazole compound (81) shown in Figure 1.43 was the most well balanced as it demonstrated superior in vivo flea and tick efficacy to nodulisporic acid A (1) - 1.5 weeks longer protection from fleas and 4 weeks protection from ticks - and had an improved plasma half-life - 2.8 days vs 0.8 days for nodulisporic acid A (1). Unfortunately, the synthesis of the dimethylamino oxazole compound (81) was uneconomical preventing further development of bioactive properties of the heterocylic derivatives. ${ }^{55}$ 


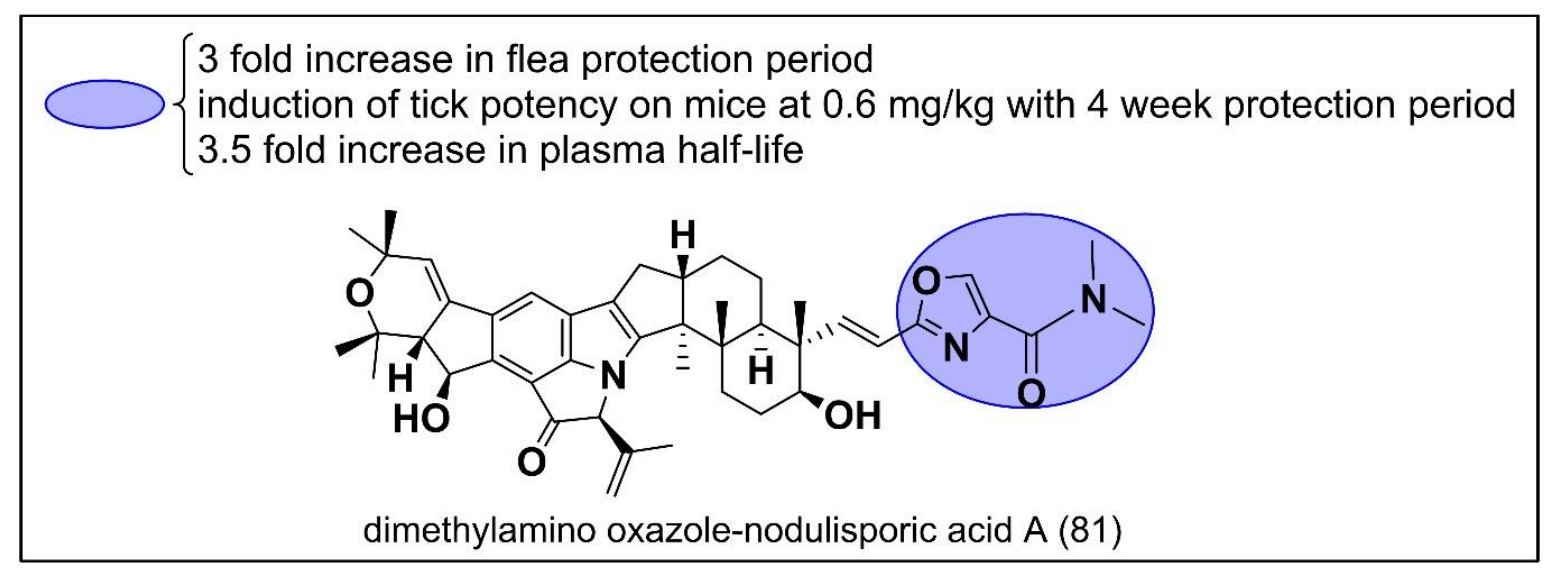

Figure 1.43. Depiction of the key dimethylamino oxazole that installed potent anti-tick activity onto the nodulisporic acid A (1) core [Berger et al. (2001)]. ${ }^{60}$

With a general understanding of the nodulisporic acid A (1) pharmacophore and the chemical modifications that would likely install desirable properties, Merck scientists pushed forward with their efforts to create a safe, economically viable nodulisporamide derivative that could be dosed monthly for the prevention of fleas and ticks on companion animals. They prepared a library of 335 nodulisporamides and screened them in vitro using their artificial flea-feeding assay and in vivo using a mouse/bedbug assay. ${ }^{55}$ Of the 335 nodulisporamides tested, 66 that had promising properties (i.e. flea $\mathrm{LC}_{50} \leq 1.0 \mathrm{ppm}$ and bedbug ED $50 \leq 1.0 \mathrm{mg} / \mathrm{kg}$ ) were analysed in an in vitro dog/flea assay, of which twelve were screened more thoroughly in an eight-week dog/flea assay, four-week dog/tick assay, and four-week cat/flea assay. Of the twelve nodulisporamides tested, all of which were synthesised in a single chemical reaction from nodulisporic acid A (1), one, $\mathrm{N}$-tert-butyl nodulisporamide (78) shown in Figure 1.44 , displayed the most desirable ectoparasitic activity and was selected for drug development.

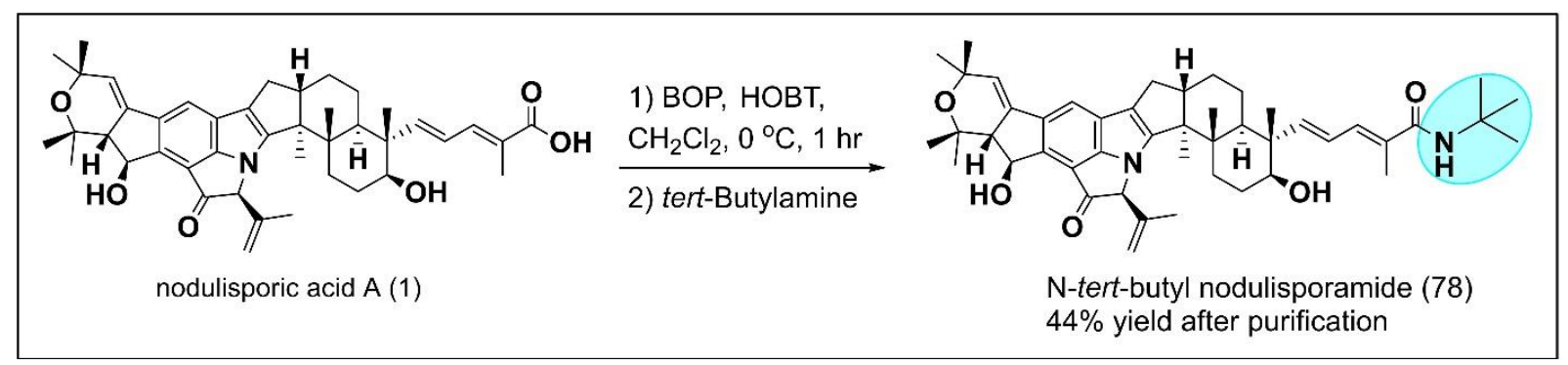

Figure 1.44. $N$-tert-butyl nodulisporamide (78) can be synthesised from nodulisporic acid A (1) using a single chemical reaction [Meinke et al. (2009)]. ${ }^{55}$ 


\section{Analysis of the ideal nodulisporic acid A (1) analogue drug target: $N$-tert-butyl nodulisporamide (78)}

\section{Flea efficacy of $\boldsymbol{N}$-tert-butyl nodulisporamide (78) in dogs}

To validate the development of $N$-tert-butyl nodulisporamide (78) into a drug, Merck scientists conducted additional comparison studies against two widely used topical agents, fipronil and imidacloprid. ${ }^{5}$ They conducted a nine-week dog/flea efficacy study where $N$-tert-butyl nodulisporamide (78) was orally dosed at $10 \mathrm{mg} / \mathrm{kg}$, and fipronil and imidacloprid were applied as described in their packaging. Following the single oral dose of $N$-tert-butyl nodulisporamide (78) complete (100\%) flea control was observed for six weeks and marginal loss of efficacy was observed in week seven and eight until nearly $50 \%$ loss in activity was reached in week nine. Corresponding pharmacokinetic data showed that the flea control diminished when the plasma drug levels decreased to $\leq 0.20 \mu \mathrm{g} / \mathrm{mL}$. In comparison, fipronil controlled $100 \%$ fleas until week five and had $>90 \%$ efficacy throughout week six to nine and imidacloprid exhibited $100 \%$ flea control for four weeks and diminished activity in week five and six. A subsequent test of $\mathrm{N}$-tertbutyl nodulisporamide (78) flea efficacy was completed where dogs were dosed with $30 \mathrm{mg} / \mathrm{kg}$ and checked for fleas at 61 and 90 days post-dose. At day $61,100 \%$ flea control was observed but by day 90 there was an exceptional loss in efficacy (23\% control). These results demonstrated that orally dosed $\mathrm{N}$-tert-butyl nodulisporamide (78) competes well against the most widely used topical flea therapeutics.

\section{Flea efficacy of $\mathbf{N}$-tert-butyl nodulisporamide (78) in cats}

From their initial study of fleas on cats, Merck found that cats eliminated $\mathrm{N}$-tert-butyl nodulisporamide (78) from their system more quickly than dogs resulting in a shorter half-life of 6.6 days vs 8.7 days and lower maximum concentration of $1.52 \pm 1.65$ vs $9.8 \pm 0.28 \mu \mathrm{M}$, and thus a greatly reduced anti-flea therapeutic affect when dosed with $10 \mathrm{mg} / \mathrm{kg} .{ }^{55}$ Therefore, Merck conducted a second four-week flea challenge on cats given a single oral dose of either $15 \mathrm{mg} / \mathrm{kg}$ or $20 \mathrm{mg} / \mathrm{kg}$ or a topical dose of fipronil or imidacloprid. Although the cats given $15 \mathrm{mg} / \mathrm{kg} \mathrm{N}$-tert-butyl nodulisporamide (78) experienced flea breakthrough at three weeks, those dosed with $20 \mathrm{mg} / \mathrm{kg}$ were completely protected (100\%) from fleas for three weeks with $97 \%$ and $94 \%$ protection 
on week four and five, respectively. By week six the efficacy dropped to $76 \%$ where the systemic plasma concentration was $0.2-0.3 \mu \mathrm{g} / \mathrm{mL}$. In contrast, fipronil and imidacloprid provided nearly complete flea protection for four weeks and showed only moderate loss of efficacy in week five, $94 \%$ and $97 \%$ respectively. In week six, flea breakthrough was observed for fipronil whereas imidacloprid showed $>97 \%$ efficacy.

\section{Tick efficacy of $\mathbf{N}$-tert-butyl nodulisporamide (78) in dogs}

Merck scientists examined the tick ectoparasitic efficacy of $\mathrm{N}$-tert-butyl nodulisporamide (78) compared to fipronil over a four-week tick/dog challenge (imidacloprid was not assessed because it does not exhibit anti-tick activity). ${ }^{55}$ They found that a $10 \mathrm{mg} / \mathrm{kg}$ oral dose of $\mathrm{N}$-tert-butyl nodulisporamide (78) was $80 \%, 78 \%$, and $41 \%$ effective against brown dog ticks (Riphicephalus sanguineus) on dogs at days 12,19 , and 26 respectively. In comparison, fipronil was $91 \%$ and $77 \%$ effective at days 19 and 26 showing that a higher oral dose of $\mathrm{N}$-tert-butyl nodulisporamide (78) would be required to have competitive advantage over fipronil. Therefore, Merck launched a second four-week tick challenge where dogs were challenged with $R$. sanguineus or Lone Star ticks (Amblyoma americanum) and given a single oral dose of $30 \mathrm{mg} / \mathrm{kg}$. Although $100 \%$ protection was observed at day 14 for both tick species, the drug efficacy dropped to $85 \%$ and $87 \%$ at day 21 and $84 \%$ and $92 \%$ at day 28 for $R$. sanguineus and $A$. americanum respectively. The corresponding pharmacokinetic data showed that tick control diminished when the plasma drug levels decreased to $\leq$ $1 \mu \mathrm{g} / \mathrm{mL}$. Notably, these results demonstrated that a single $30 \mathrm{mg} / \mathrm{kg}$ oral dose of $N$ tert-butyl nodulisporamide (78) had a greater flea and tick efficacy than fipronil. 


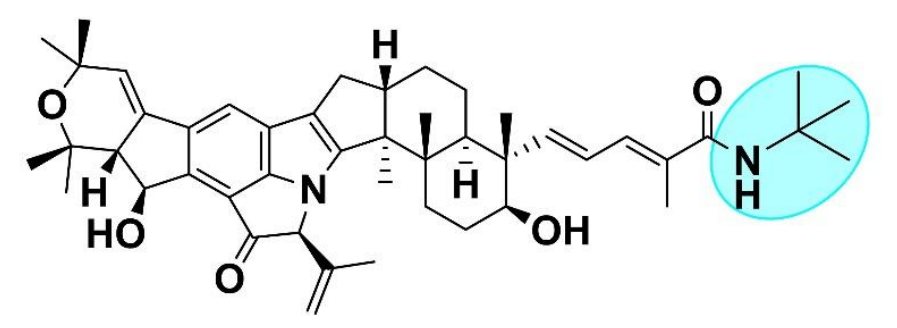

N-tert-butyl nodulisporamide (78)

\section{Summary of bioactivity testing [Meinke et al. (2009)]}

- 7 fold increase in flea potency $\left(0.1 \mathrm{ppm} \mathrm{LC} \mathrm{C}_{50}\right)$ compared to NAA

- 8 fold increase in bedbug potency $\left(0.125 \mathrm{mg} / \mathrm{kg} \mathrm{ED}_{50}\right.$ on mice) compared to NAA

- 8.5 fold increase in plasma half life of dogs dosed with $10 \mathrm{mg} / \mathrm{kg}$ compared to NAA

- 7 week flea prevention and induction of tick prevention for 3 weeks at $10 \mathrm{mg} / \mathrm{kg}$ dose on dogs

- 9 week flea prevention (100\%) and 4 week tick prevention (84-92\%) of dogs dosed with $30 \mathrm{mg} / \mathrm{kg}$

- 3-4 week flea prevention on cats dosed with $10 \mathrm{mg} / \mathrm{kg}$

- 5 week flea prevention (94\%) of cats dosed with $20 \mathrm{mg} / \mathrm{kg}$

Figure 1.45. Summary of bioactive properties of $\mathrm{N}$-tert-butyl nodulisporamide (78). ${ }^{55}$

\section{Further examination of the $\mathrm{N}$-tert-butyl nodulisporamide (78) pharmacokinetic safety profile}

To further analyse the safety profile of $\mathrm{N}$-tert-butyl nodulisporamide (78), Merck conducted a series of 102 in vitro assays against $\mathrm{N}$-tert-butyl nodulisporamide (78) at $10 \mu \mathrm{M} .{ }^{55}$ Of the 102 assays, monoamine uptake was the only one that showed meaningful activity where $90 \%$ and $52 \%$ inhibition were observed at $10 \mu \mathrm{M}$ and $1 \mu \mathrm{M}$ respectively. A subsequent covalent binding study using radiolabelled $\mathrm{N}$-tert-butyl nodulisporamide (78) in rat and dog liver microsomes showed $<50 \mathrm{pM}$ equiv/mg protein labelling. Importantly, $\mathrm{N}$-tert-butyl nodulisporamide (78) was found to bind very tightly to plasma proteins (99.3\%), an attribute that likely enhances its drug delivery and mammalian safety profile. A further study looking into $\left[{ }^{14} \mathrm{C}\right] \mathrm{N}$-tert-butyl nodulisporamide (78) drug distribution over time found that at 8 hours postdose the majority of the drug is present in the stomach and gastrointestinal tract whereas at 21 and 28 days postdose the majority of the drug is in the abdominal and altilateral adipose tissue. This distribution study suggests that $\mathrm{N}$-tert-butyl nodulisporamide (78) is initially absorbed into the adipose tissue and gradually leeches out, which confers it with a prolonged half-life and enhanced efficacy toward ectoparasites. 


\section{Difficulties with nodulisporic acid A (1) production prevent drug development}

Although Merck scientists were able to identify an ideal nodulisporic acid A (1) analogue for drug development, they were hampered along the way by severe limitations in the availability of nodulisporic acid A (1); a limitation that has also prevented commercialization of $\mathrm{N}$-tert-butyl nodulisporamide (78). ${ }^{55}$ Firstly, the structural complexity of nodulisporic acid A (1) has precluded total synthesis of the compound for decades - the mechanisms for the total synthesis of nodulisporic acid $\mathrm{F}$ $(82)^{68}$ and nodulisporic acid $D^{69}$ have been elucidated but total synthesis of nodulisporic acid $A$ (1) has not been achieved. ${ }^{70}$ Secondly, the fermentation of nodulisporic acid A (1) from its natural producer, H. pulicicidum, is exceptionally difficult where the reported nodulisporic acid A (1) biosynthesis methods require that $H$. pulicicidum be grown for 21 days in complete darkness in highly nutrient rich medium. ${ }^{44}$ Notably, personal attempts to isolate nodulisporic acid A (1) from $\mathrm{H}$. pulicicidum using reported fermentation methods ${ }^{44,50}$ have been unsuccessful and more than 8 months of attempts at eliciting nodulisporic acid A (1) biosynthesis yielded nothing.

Thus the questions at the foundation of this thesis presents itself: What secondarymetabolic genes and corresponding enzymatic steps are involved in nodulisporic acid biosynthesis? In order to answer this question, the $H$. pulicicidum genome was sequenced, a gene cluster responsible for nodulisporcic acid biosynthesis was predicted, and four genes in the cluster were functionally characterised through gene complementation and pathway reconstitution studies using a more robust fungal host, Penicillium paxilli. 


\section{THE GENE CHARACTERISATION TOOLBOX}

In this chapter the four main tools used to functionally characterise genes involved in nodulisporiac acid biosynthesis are described. The four tools that make up the gene characterisation toolbox include bioinformatics, DNA assembly, heterologous expression, and compound analysis (Figure 2.1). First, bioinformatics are used to predict the genes that are likely involved in a biosynthetic pathway of interest. Second, the predicted genes are assembled into plasmids for amplification. Third, amplified plasmids are inserted into a new host for heterologous expression in a process called transformation. Fourth, transformants are grown in production cucltures, compounds are extracted and extraction profiles are analysed for compounds of interest.

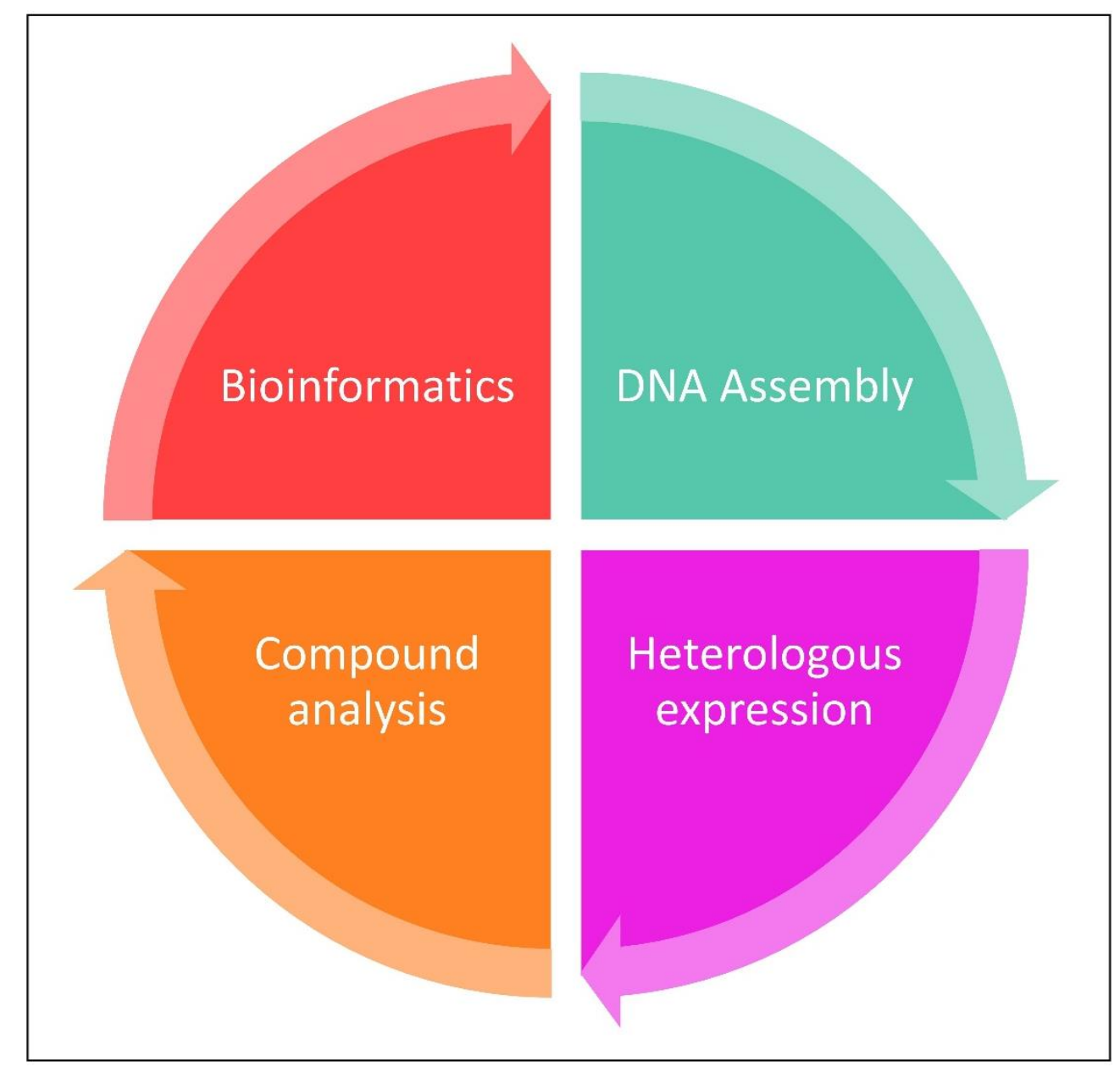

Figure 2.1. Depiction of the main tools in the gene characterisation toolbox used in this thesis. 
In order to establish the gene characterisation toolbox used for this thesis, four main components were required:

1. Genes encoding information for the biosynthesis of the compound of interest (i.e. H. pulicicidum genes encoding information for nodulisporic acid biosynthesis)

2. A DNA assembly system to combine genetic components [i.e. the Modular Idempotent DNA Assembly System (MIDAS)]

3. A library of expression hosts for confirming gene function (i.e.the library of $P$. paxilli deletion mutants)

4. A means to evaluate compound production (i.e. extraction solvents, purification materials, TLC, LCMS and NMR spectroscopy techniques)

Each of these components are described in detail below.

\section{Bioinformatics: Identification of the NOD cluster}

In order to understand the molecular mechanisms of the biosynthesis of nodulisporic acids, the genomic DNA sequence of the natural nodulisporic acid producer, Hypoxylon pulicicidum, was required. To this end, H. pulicicidum (ATCC ${ }^{\circledR} 74245^{\mathrm{TM}}$ ) was obtained from the American Type Culture Collective, DNA was extracted from $\mathrm{H}$. pulicicidum mycelia, and full genome sequencing was commissioned (New Zealand Genomics Limited). ${ }^{2}$ By comparing the genome sequence to that of genes that have been confirmed to be involved in indole diterpene biosynthesis, a cluster of thirteen potential genes involved in nodulisporic acid biosynthesis was identified (Figure 2.2). Designation of the cluster boundaries was supported by identification of flanking genes (described in table 2.1) that have highly conserved homologues in the genome of a closely related species of Hypoxylon (CO27-5), ${ }^{71}$ most of which were found at a single locus in the related genome. Notably, the closely related species (CO27-5) lacks the $N O D$ gene cluster and does not produce nodulisporic acids. Details ragrding the genome assembly, annotation and cluster identification can be found in Chapter 8. MATERIALS AND METHODS: gDNA isolation for genome sequencing and transcription unit module amplification and Genome sequencing, identification and annotation of the $H$. pulicicidum $\left(\mathrm{ATCC}^{\circledR} 74245^{\mathrm{TM}}\right.$ ) NOD gene cluster and GenBank accession numbers. 


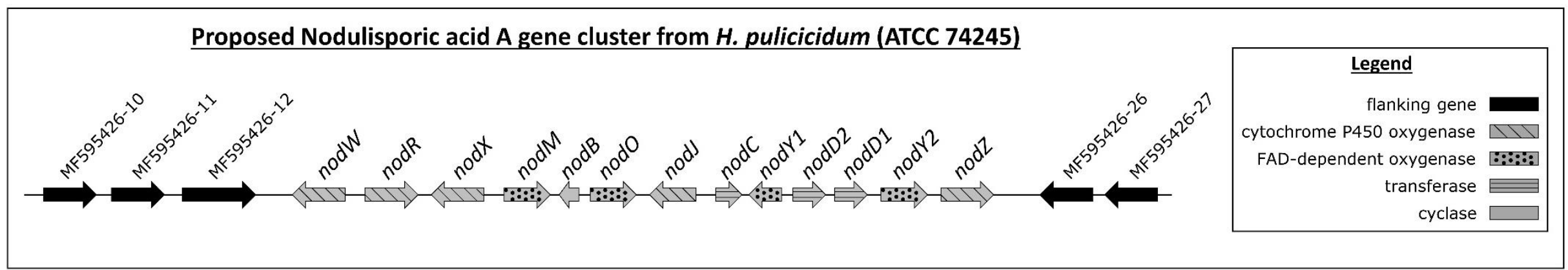

Figure 2.2. Proposed nodulisporic acid gene cluster (NOD) discovered in the genome of $H$. pulicicidum (ATCC 74245). Figure has been modified from Van de Bittner et al. 2018. ${ }^{1}$ 
Table 2.1. Functional assignment of predicted genes in and around the nodulisporic acid gene cluster. A nod name was assigned to each $H$. pulicicidum gene in the nodulisporic acid $A(1)$ gene cluster [shaded green $(\square)$ ]. H. pulicicidum genes that share homology $(>$ $35 \%$ amino acid identity of predicted translational products) with genes found in known indole diterpene pathways are followed by an asterisk $\left(^{*}\right)$ and, with the exception of nodR, were given letters corresponding to known confirmed genes (e.g. the protein encoded by nodC shares $52.8 \%$ amino acid identity with the protein product of pax $C$ ). The genes that do not share homology with known indole diterpene genes were assigned letters that are not shared with any of the confirmed indole diterpene genes. Notably, the cluster contains two sets of paralogous genes (share $>40 \%$ amino acid identity with each other), which are distinguished by numbers (i.e. nodD1/nodD2 and nodY1/nodY2). Genes flanking the cluster have been included in the table and are positioned relative to how they flank the nod cluster. Closest matches were identified using BLASTp (protein-protein BLAST) against the non-redundant protein sequence database with 'expect threshold' set at 10 and 'word size' set at 6 . The BLOSUM62 scoring matrix was applied with a gap opening penalty of 11 and a gap extension penalty of 1 with conditional compositional score matrix adjustment. Table has been modified from Van de Bittner et al. 2018. ${ }^{1}$

\begin{tabular}{|c|c|c|c|c|c|}
\hline \multirow{3}{*}{ Gene } & \multirow{3}{*}{$\begin{array}{l}\text { Size of } \\
\text { encoded } \\
\text { protein } \\
\text { (aa) }\end{array}$} & \multirow{3}{*}{$\begin{array}{l}\text { Predicted function } \\
\text { [Specific Function] }\end{array}$} & \multicolumn{3}{|c|}{ Most notable BLASTp match } \\
\hline & & & \multirow[b]{2}{*}{ Organism } & E-value & \multirow{2}{*}{$\begin{array}{c}\text { Protein name } \\
\text { and accession } \\
\text { number }\end{array}$} \\
\hline & & & & $\begin{array}{l}\text { \% identity/ } \\
\% \text { coverage }\end{array}$ & \\
\hline \multirow{2}{*}{ MF595426-10 } & \multirow{2}{*}{500} & \multirow{2}{*}{ MFS transporter } & \multirow{2}{*}{$\begin{array}{c}\text { Hypoxylon sp. } \\
\text { CO27-5 }\end{array}$} & 0 & \multirow{2}{*}{ OTA93954 } \\
\hline & & & & $88 \%$ ID/99\% & \\
\hline \multirow{2}{*}{ MF595426-11 } & \multirow{2}{*}{496} & \multirow{2}{*}{ FAD-dependent oxygenase } & \multirow{2}{*}{$\begin{array}{c}\text { Hypoxylon sp. } \\
\text { CO27-5 }\end{array}$} & 0 & \multirow{2}{*}{ OTA93953 } \\
\hline & & & & $84 \%$ ID/96\% & \\
\hline \multirow{2}{*}{ MF595426-12 } & \multirow{2}{*}{1664} & \multirow{2}{*}{ WD40 domain protein } & \multirow{2}{*}{$\begin{array}{l}\text { Hypoxylon sp. } \\
\text { CO27-5 }\end{array}$} & $\begin{array}{c}0 \\
\end{array}$ & \multirow{2}{*}{ OTA80149 } \\
\hline & & & & $36 \%$ ID $/ 80 \%$ & \\
\hline \multirow{2}{*}{ nodW } & \multirow{2}{*}{608} & \multirow{2}{*}{$\begin{array}{c}\text { Cytochrome P450 oxygenase } \\
\text { [terminal-C dioxygenase] }\end{array}$} & \multirow{2}{*}{$\begin{array}{l}\text { Aspergillus } \\
\text { aculeatus }\end{array}$} & $9.00 \mathrm{E}-153$ & \multirow{2}{*}{ XP_020058732 } \\
\hline & & & & $44 \%$ ID/ $97 \%$ & \\
\hline
\end{tabular}




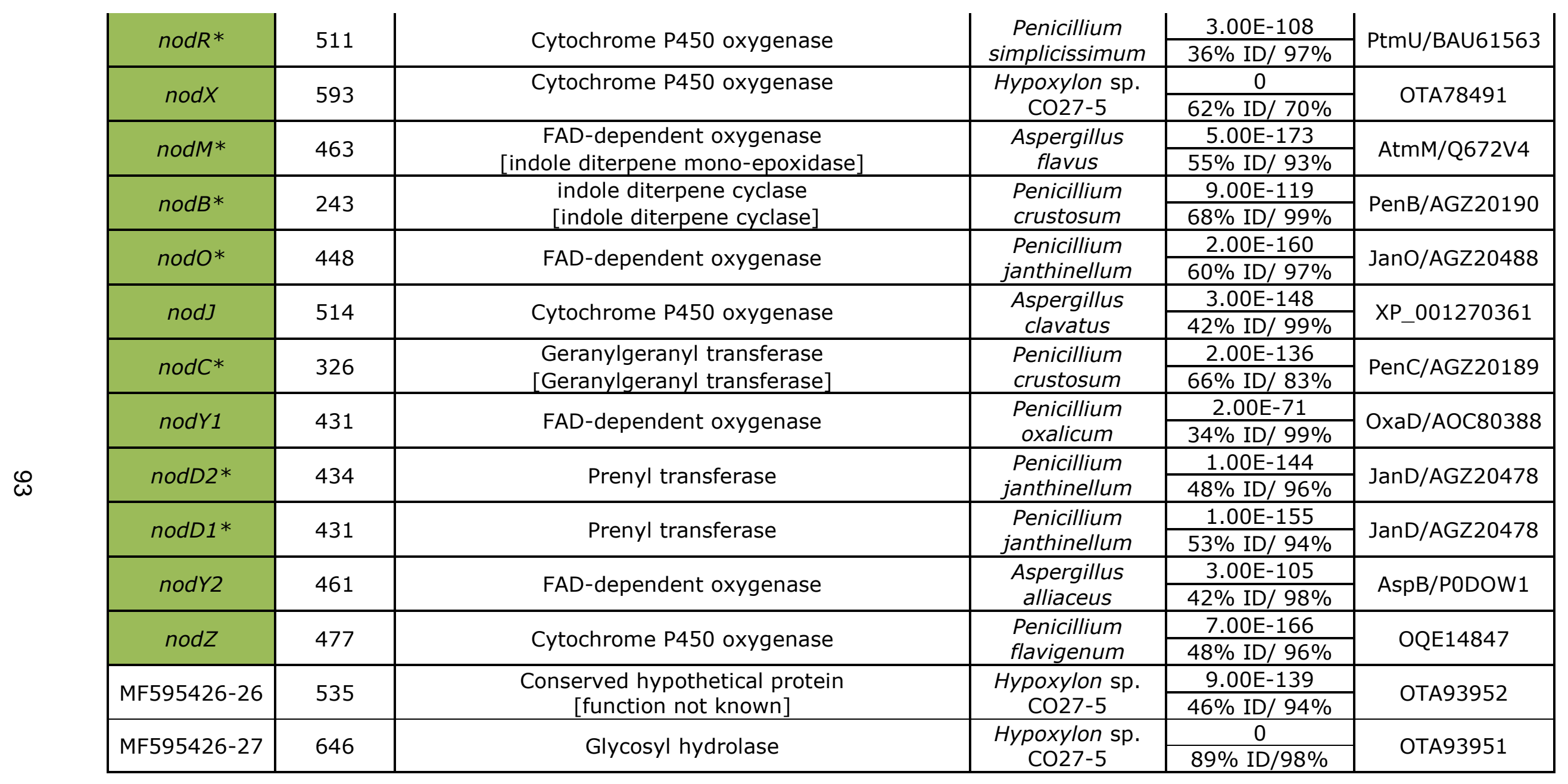


Details of the predicted genes and their proposed function are shown in Table 2.1. Importantly, six of the genes in the cluster $(\operatorname{nod} M, \operatorname{nod} B, \operatorname{nod} O, \operatorname{nod} C, \operatorname{nod} D 2$, and nodD1) encode protein products with notably high amino acid sequence identities to those of known indole diterpene biosynthetic genes (e.g. $>40 \%$ to homologues in the $P A X$ cluster of $P$. paxilli; see Appendix, Figures 9.2 to 9.4, 9.6, 9.7) indicating the likelihood that this gene cluster was involved in indole diterpene biosynthesis. The functionalities of the protein products of these six homologous genes were predicted to include a geranylgeranyl transferase (NodC), a regioselective flavin adenine dinucleotide-dependent epoxidase (NodM), an indole diterpene cyclase (NodB), two paralogous prenyl transferases (NodD2 and NodD1), and one flavin adenine dinucleotide-dependent cyclo-oxygenase (NodO). The other seven genes encode five cytochrome P450 oxygenases (NodW, NodR, NodX, NodJ and NodZ), and a pair of paralogous flavin adenine dinucleotide-dependent oxygenases (NodY1 and NodY2; see Appendix, Table 9.1). By combining the information about predicted gene functionality with the previously hypothesised biosynthetic pathway of nodulisporic acid biosynthesis ${ }^{50}$ twelve of the genes in the cluster were tentatively assigned to their most likely role in the proposed pathway (Figure 2.3). 


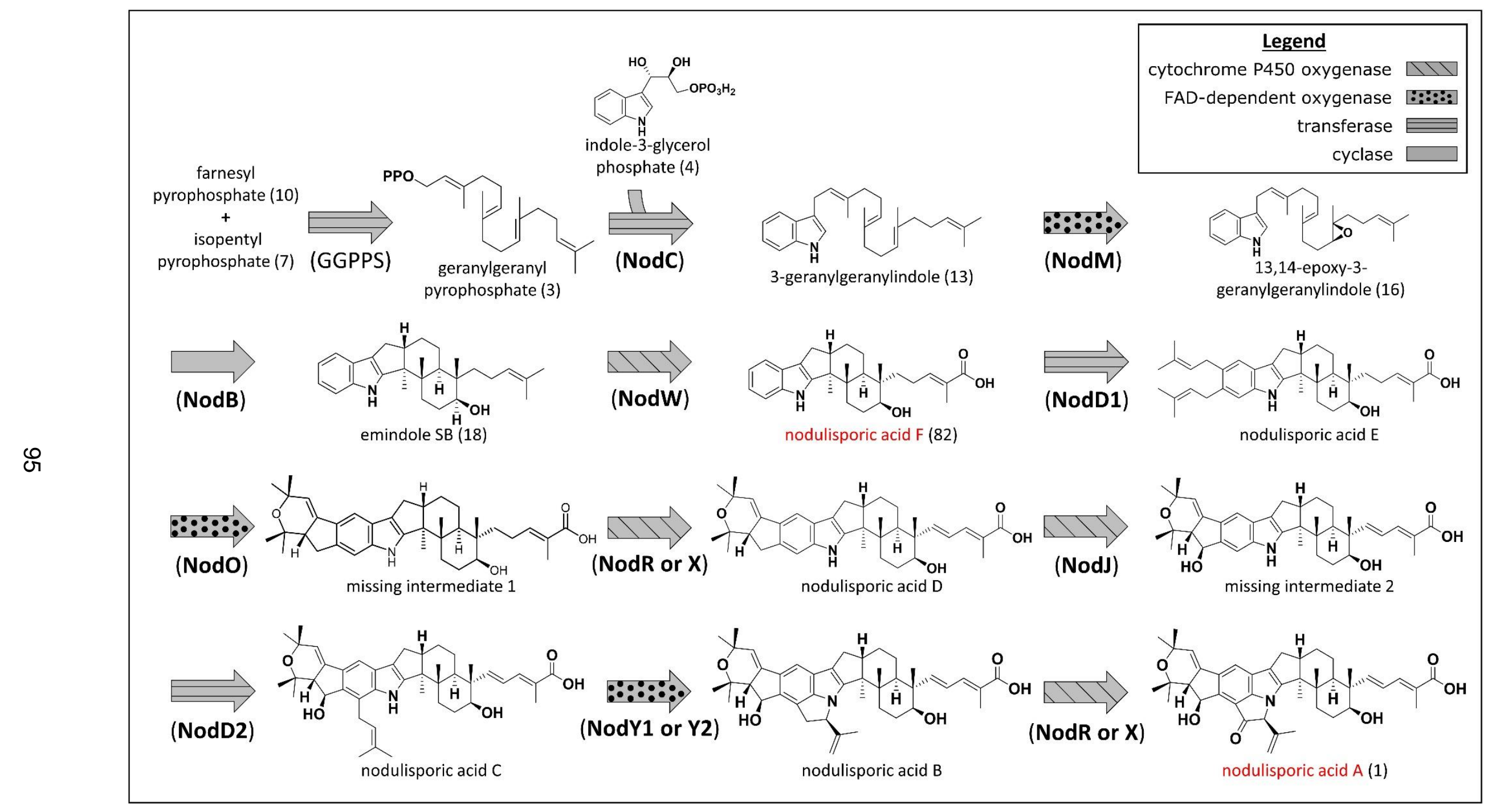

Figure 2.3. Proposed pathway for the biosynthesis of nodulisporic acids. Each arrow represents an enzymatic step and the arrow decoration correspond to enzymatic function. The protein products of gene(s) in the NOD cluster have been assigned to their most likely enzymatic step in the proposed pathway. Figure has been modified from Van de Bittner et al. 2018.1 
Intriguingly, the NOD cluster lacks a secondary metabolic geranylgeranyl pyrophosphate (3) synthase-encoding gene, which has previously been assigned as the first secondary-metabolic step in indole diterpene biosynthesis. ${ }^{33,}$, 37-38, 40-41 That said, other indole diterpene gene clusters have been identified that lack a secondary metabolic geranylgeranyl pyrophosphate (3) synthase-encoding gene, like the TER gene cluster from Chaunopycnis alba (Tolypocladium album) ${ }^{30}$ and the AtS5 cluster from Aspergillus tubingensis, ${ }^{33}$ respectively responsible for terpendole and emindoleanalogue biosynthesis. To explore whether the $H$. pulicicidum genome contained a secondary metabolic geranylgeranyl pyrophosphate (3) synthase-encoding gene outside of the cluster, amino acid sequence comparisons of the full $H$. pulicicidum genome against the sequences of functionally confirmed primary and secondary metabolic geranylgeranyl pyrophosphate (3) synthases were completed to generate a phylogenetic tree (Figure 2.4 A) and corresponding similarity matrix (Figure $2.4 \mathrm{~B}$ ). Searches revealed that only one other geranylgeranyl pyrophosphate (3)-encoding gene (Nod ggs1) was present and that based on its location outside of the NOD-cluster and the amino acid sequence homology of its protein product to primary geranylgeranyl pyrophosphate (3) synthases, Nod ggs1 was most likely a primary geranylgeranyl pyrophosphate (3) synthase. 


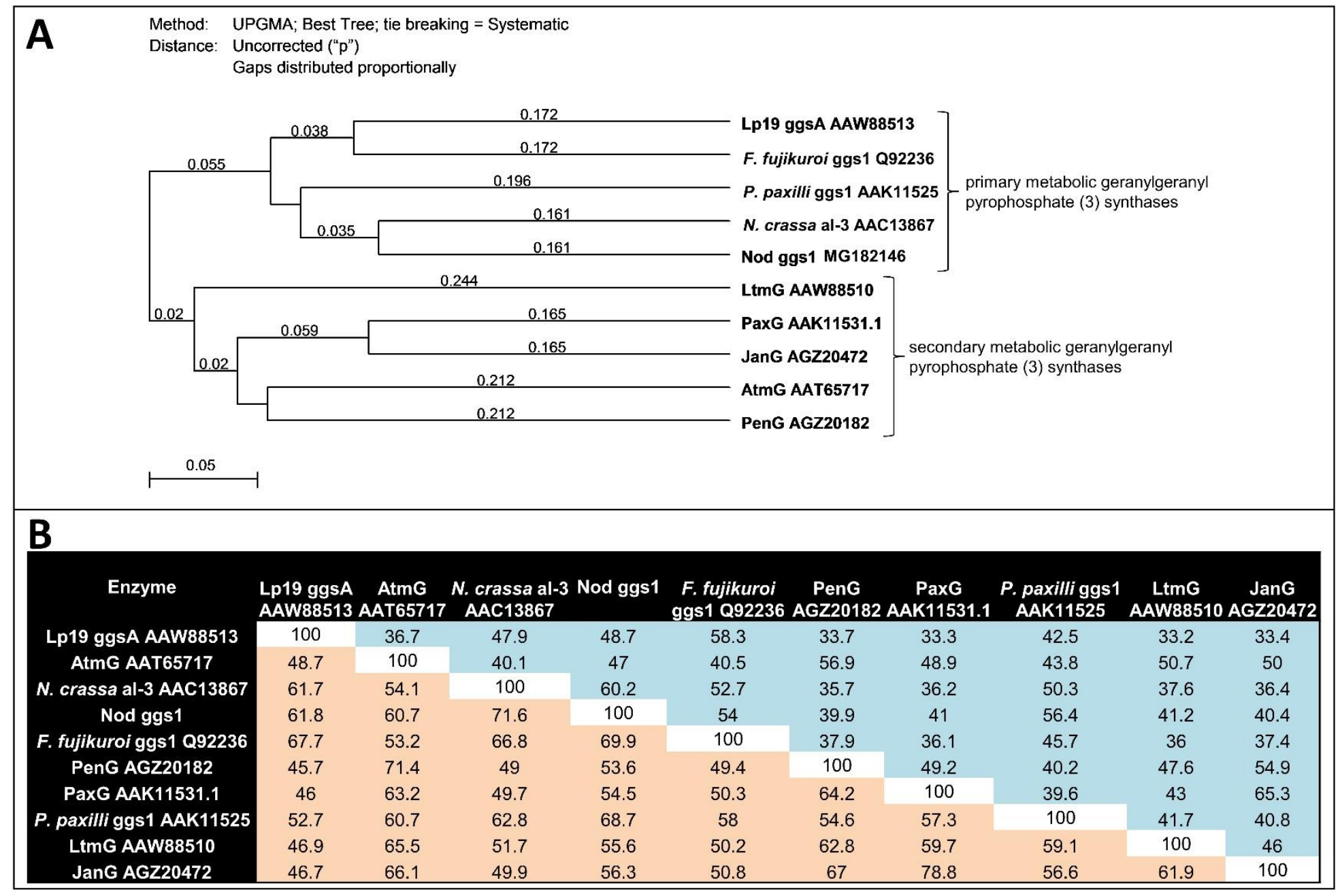

Figure 2.4. Phylogenetic tree $(\mathbf{A})$ and similarity matrix $(\mathbf{B})$ of geranylgeranyl synthases ('ggs enzymes' are involved in primary metabolism and ' $G$ ' enzymes are involved in secondary metabolism). The phylogenetic tree depicts the difference in \% identity scores for the amino acid residues of the enzymes. The similarity matrix depicts the \% identity scores (blue shaded regions $(D)$ ) and the \% similarity scores (orange shaded regions $($ )) for the amino acid residues of the enzymes. Figure has been modified from Van de Bittner et al. 2018. ${ }^{1}$ 


\section{DNA assembly: The Modular Idempotent DNA Assembly System (MIDAS)}

In order to test the gene functionality of the NOD genes through heterologous expression, a method to isolate the predicted NOD gene coding sequences and combine them with promoters that would work efficiently in an expression host was required. To this end, a DNA assembly system, termed MIDAS for Modular Idempotent DNA Assembly System, was developed in a collaborative effort with Callaghan Innovation. ${ }^{3}$

MIDAS is a powerful synthetic biology platform with higher efficiency, fidelity and modularity than similar assembling systems. It allows for the seamless, efficient and flexible multigene assembly of tailored combinations of transcription unit modules (e.g. promoters, coding sequences, and terminators) from one or more biosynthetic pathways. MIDAS is composed of three Levels (Figure 2.5) that enable full user-control over the components contained within individual transcription units as well as the direction, orientation and position of transcription units within multigene constructs. MIDAS separates itself from other MoClo systems, in that at the third level of the system, components can be added iteravely into a single construct indefinitely (in theory), rather than having to move into additional levels to make longer, more complex constructs. $^{3}$

In Level-1, transcription unit modules (e.g. promoters, coding sequences and terminators) are cloned into the Level-1 destination vector (pML1) to build defined, fully reusable, sequence-verified module libraries. In Level-2, compatible sets of Level-1 modules are released from pML1 and assembled into full length transcription units in a Level-2 destination vector (pML2) to create a transcription unit library. In Level-3, Level-2 transcription units are sequentially assembled into a Level-3 vector (pML3) to form functional multigene constructs. The complete Level-3 multigene constructs are then transformed into an expression host to test gene functionality. 




Figure 2.5. Overview of MIDAS. First (A), modules such as promoters (ProUTRs), coding sequences (CDSs) and terminators (UTRterms) are amplified or synthesized. Second (B), modules are cloned, via BsmBI-mediated restriction-ligation reactions, into the pML1 source vector. Third (C), cloned modules are assembled, via Bsalmediated restriction-ligation reactions, into one of eight pML2 vectors to form full length transcription units (TUs). Fourth (D), full length TUs are iteratively assembled, via alternating Aarl- and BsmBI-mediated restriction-ligation reactions, into Level-3 vectors to form multigene constructs. Additional pML2 vectors are shown in Figure 8.4. 
Assembly at each Level of the MIDAS platform is performed in a one-pot GoldenGate-type assembly reaction that is mediated by a type IIS restriction enzyme ( $B s m \mathrm{BI}$, Bsal or Aarl). Each of these enzymes has a unique binding sequence and creates a defined 4-nucleotide overhang (see Figure 2.5) that is used to ensure correct assembly of the transcription unit modules into $\mathrm{pML1}$, transcription unit assembly into $\mathrm{pML2}$, and multigene assemble in pML3. As part of the MIDAS platform, all type IIS restriction sites within original transcription unit module sequences are removed during primer design in a process called domestication. In Chapter 3 , a full explanation of each level of the MIDAS platform is provided. For additional details of the MIDAS platform including the success rate of each step of MIDAS cloning, how this was affected by the number of fragments being assembled in a single step, etc. please refer to the van Dolleweerd et al. 2018 MIDAS manuscript and its supporting information. ${ }^{3}$

\section{Heterologous expression}

Heterologous expression is a powerful bioengineering tool to, among other things, identify the function of genes and reconstruct biosynthetic pathways. It is often used to work towards industrially relevant titres of target compound and especially useful when working with genes that are poorly expressed or highly regulated in their native host, because it imparts the ability to alter the gene expression levels (e.g. by promoter swapping or increasing copy number) and to bypass natural regulatory mechanisms (e.g. specific light, temperature or nutrient source).

At the basis of a heterologous expression system, genes from one organism (e.g. the natural producer of nodulisporic acids, Hypoxylon pulicicidum) are transferred to another organism (e.g. Penicillium paxilli) that serves as the heterologous expression host and often has some sort of beneficial role (e.g. grows faster and produces more compound).

In the case of this project, heterologous expression was used to confirm gene function of genes involved in nodulisporic acid biosynthesis because the native nodulisporic acid producer, $\mathrm{H}$. pulicicidum, produces very low titres of nodulisporic acids and the biosynthetic pathway appears to be highly regulated (i.e. to elicit nodulisporic acid production $H$. pulicicidum must be grown in complete darkness in nutrient rich medium for three weeks). ${ }^{44}, 50$ Therefore, performing gene knockout studies on $\mathrm{H}$. pulicicidum was not an efficient method to confirm gene function because it would involve long, complicated growth procedures and, due to low compound 
production, likely lead to discrepancies around assigning functionality (i.e. a gene knockout may produce compounds at levels below the limit of detection that could lead to false conclusions).

\section{A library of expression hosts for confirming gene function}

There are a number of things to consider when making a choice about an expression host for confirming gene function through heterologous expression, and for each type of expression system the ideal host will likely change. In most cases though, an ideal expression host will grow quickly on relatively nutrient poor medium (to reduce time and supply costs), lack undesirable regulatory elements for the target pathway, encode the metabolic machinery required to produce the target compound(s) of interest, produce high titres of target compound(s), and be safe and easy to work with (e.g. does not produce deadly toxins, is susceptible to antibiotics for transformant screening, has morphology that enables easy separation from fermentation broths, etc.).

With these selection criteria in mind, it was determined that the filamentous fungi, Penicillium paxilli, which has been extensively used to confirm the function of the genes involved in the biosynthesis of various indole diterpenes, ${ }^{25-29}, 35-36,38$ would be an excellent expression host for nodulisporic acids. P. paxilli naturally produces a class of indole diterpenes known as paxillines, the biosynthesis of which has been fully elucidated (Figure 2.6, A) ${ }^{38}$ In total, the gene cluster for the biosynthesis of paxillines contains nine genes, of which seven have been functionally analysed and are involved in the biosynthesis of prenylated paxillines (Figure 2.6, B). ${ }^{35-38,40,42}$ These seven genes encode a geranylgeranyl pyrophosphate (3) synthase (PaxG), a geranylgeranyl transferase (PaxC), a regioselective flavin adenine dinucleotide-dependent epoxidase (PaxM), an indole diterpene cyclase (PaxB), two cytochrome P450 oxygenases (PaxP and $P a x Q)$, and a prenyl transferases (PaxD). The two other genes encode a gene with unknown function (PaxA) and one flavin adenine dinucleotide-dependent cyclooxygenase (PaxO). 


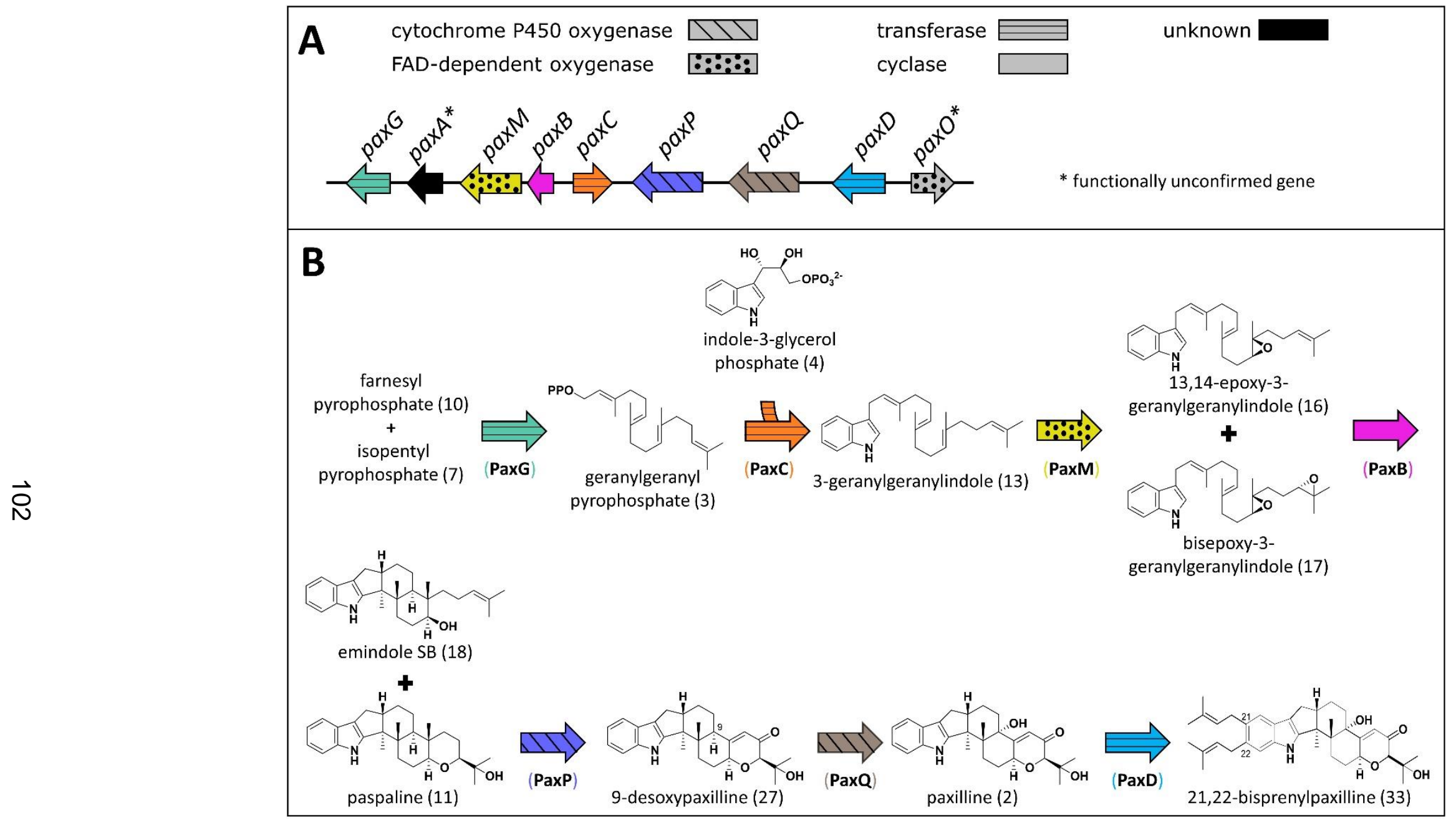

Figure 2.6. Depiction of the identified paxilline (2) gene cluster from Penicillium paxilli (A) and corresponding paxilline (2) biosynthetic pathway (B). The gene cluster contains nine genes, of which $\operatorname{six}(\operatorname{pax} G, M, B, C, P, Q)$ are required for paxilline (2) biosynthesis. ${ }^{40}$ Of the other three genes, (paxA, paxD and paxO) only paxD has been functionally analysed for its role in converting paxilline (2) to 21prenylpaxilline (32) and 21,22-bisprenylpaxilline. ${ }^{42}$ Arrows represent individual genes and arrow decorations represent gene function. Figure has been modified from Van de Bittner et al. 2018. ${ }^{1}$ 
During the identification and functional analysis of the genes in the PAX cluster a series of single and multigene-deletion mutants ${ }^{24}, 35,37-38$ were generated that are unable to synthesise the indole diterpenes normally produced by wild-type $P$. paxilli (strain PN201372). The selective deletion of a gene within the paxilline (2) biosynthetic pathways prevents paxilline (2) biosynthesis at a specific biosynthetic step. For example, a $\triangle p a x C$ deletion mutant is not able to produce any of the metabolites downstream of the PaxC-catalysed step (i.e. condensation of indole-3glycerolphosphate and geranylgeranyl pyrophosphate (3) to form gernaylgeranylindole). These single gene deletion mutants are excellent platforms for testing gene orthology - i.e. the ability to see if a homologous gene from a different indole diterpene producer has the same function as that of the gene that has been deleted in the $P$. paxilli deletion mutant. For instance, if a pax $C$ homologue (e.g. nodC) were heterologously expressed in the $\triangle$ pax $C P$. paxilli deletion mutant and paxilline (2) were observed in the transformant extract, then that would indirectly indicate that the paxC homologue was orthologous to paxC because it restored paxilline (2) biosynthesis. In contrast to the single-gene deletion mutants, multigene-deletion mutants are ideal for ensuring that the paxilline (2) biosynthesis genes do not have an influence on heterologously expressed genes and thereby serve as preferred hosts to examine how multiple genes from a single multigene construct interact with one another. ${ }^{1}$ Overall, the $P$. paxilli deletion mutant library provides a useful resource for confirming gene function and individually testing whether MIDAS-assembled transcription units contain all the necessary cis-acting elements to successfully transcribe and express their coding sequences.

\section{Compound analysis}

In order to link genes to biosynthetic steps, a method had to be developed to produce, extract and analyse the compounds of interest. In the case of the heterologous expression system used in this thesis, after genes are introduced into $P$. paxilli, the transformants are grown in production medium for a week and indole diterpenes are extracted with an organic solvent. The extracts are initially screened using TLC to identify transformants that produce compounds not present in the respective $P$. paxilli deletion mutant. Extracts containing novel compounds are then subjected to LCMS analysis to identify compounds by their mass/charge ratio and 
fragmentation patterns. If a novel compound is produced for which no standard is available, then the transformant that produced the unknown product is grown on larger scales and the compound of interest is extracted and purified by semi-preparative HPLC for analysis by NMR spectroscopy.

\section{Conclusion}

As a whole, these four components: the NOD cluster, MIDAS, the P. paxilli expression host library and the techniques to analyse indole diterpenes give rise to the gene characterisation toolbox used in this thesis that provided a means to confirm gene functionality and partially reconstruct the nodulisporic acid biosynthetic pathway. The next four chapters exemplify how each of these techniques has been used to confirm the function of individual genes within the NOD cluster - thereby demonstrating that this toolbox provides a powerful platform to reconstruct biosynthetic pathways of secondary metabolites. 


\section{FUNCTIONAL CHARACTERISATION OF NodC}

\section{Proposed Nodulisporic acid A gene cluster from H. pulicicidum (ATCC 74245)}

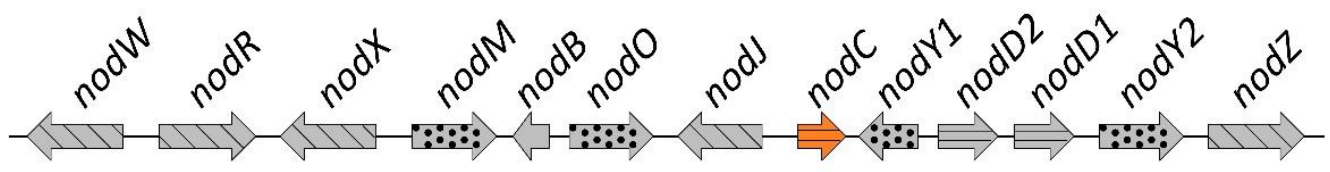

cytochrome P450 oxygenase FAD-dependent oxygenase

::::::

transferase cyclase

Figure 3.1. Depiction of the predicted nodulisporic acid gene cluster (NOD) from $H$. pulicicidum (ATCC $\left.{ }^{\circledR} 74245^{\mathrm{TM}}\right)$. The nodC gene is highlighted in orange. 
As shown in Figure 3.1, the NOD gene cluster does not contain a secondarymetabolic geranylgeranyl pyrophosphate (3) synthase-encoding gene, which has been shown to encode the enzyme responsible for catalysing the first secondary-metabolic step in indole diterpene biosynthesis, the condensation of farnesyl pyrophosphate (10) and isopentyl pyrophosphate (7) to form geranylgeranyl pyrophosphate (3) shown in Figure 3.2. Therefore, attention was turned to the second secondary-metabolic step in indole diterpene biosynthesis, the production of geranylgeranylindole from the condensation of geranylgeranyl pyrophosphate (3) and indole-3-glycerol phosphate (4) shown in Figure 3.2 .

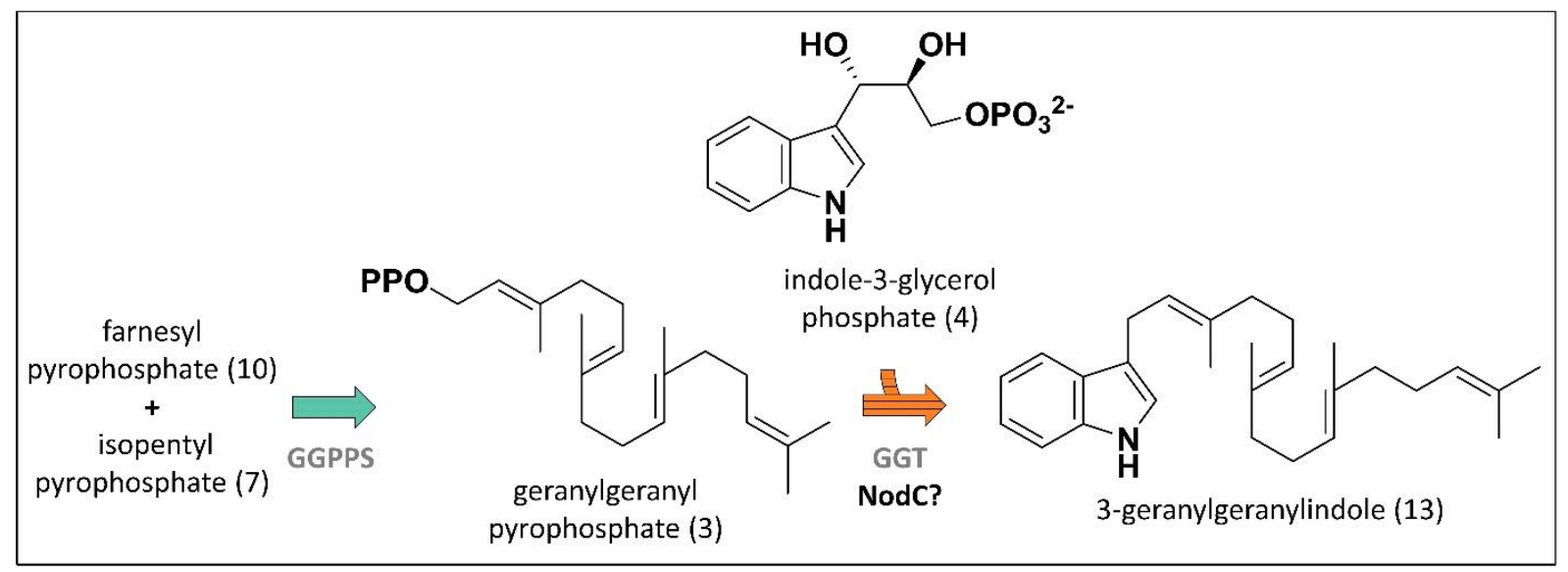

Figure 3.2. Schematic of the first two secondary-metabolic steps in indole diterpene biosynthesis. In step 1 a geranylgeranyl pyrophosphate (3) synthase (GGPPS, turquoise arrow) catalyses the condenation of farnesyl pyrophosphate (10) and isopentyl pyrophosphate to form geranylgeranyl pyrophosphate (3). In step 2, a geranylgeranyl transferase (GGT, orange arrow) catalyses the condensation of geranylgeranyl pyrophosphate (3) and indole-3-glycerol phosphate (4) to produce 3geranylgeranylindole (13). Notably the NOD cluster isolated from $H$. pulicicidum does not contain a GGPPS-encoding gene but it does contain a gene candidate (nodC) that is predicted to encode the geranylgeranyl transferase necessary for the biosynthesis of 3-geranylgeranylindole (13). The likely cofactor for GGT enzymes is $\mathrm{Mg}^{2+}$ but not all enzymatic mechanisms have been thoroughly examined.

\section{Prediction of nodC}

It has been well established that production of geranylgeranyl indole is catalysed by a class of indole diterpene geranylgeranyl transferase enzymes that conventionally go by the name " $\mathrm{C}$ " (e.g. the PaxC in paxilline (2) biosynthesis). ${ }^{33,40}$ Using sequence homology comparisons of the amino acid sequences from functionally confirmed indole 
diterpene geranylgeranyl transferases to the predicted gene products within the $N O D$ cluster, a likely geranylgeranyl transferase encoding-gene named "nodC" was identified (Figure 3.3, A and B). NodC shares greater than $50 \%$ amino acid identity to four other known geranylgeranyl transferases (PaxC, PenC, AtmC, and JanC), the highest being JanC with $55.4 \%$ amino acid identity.

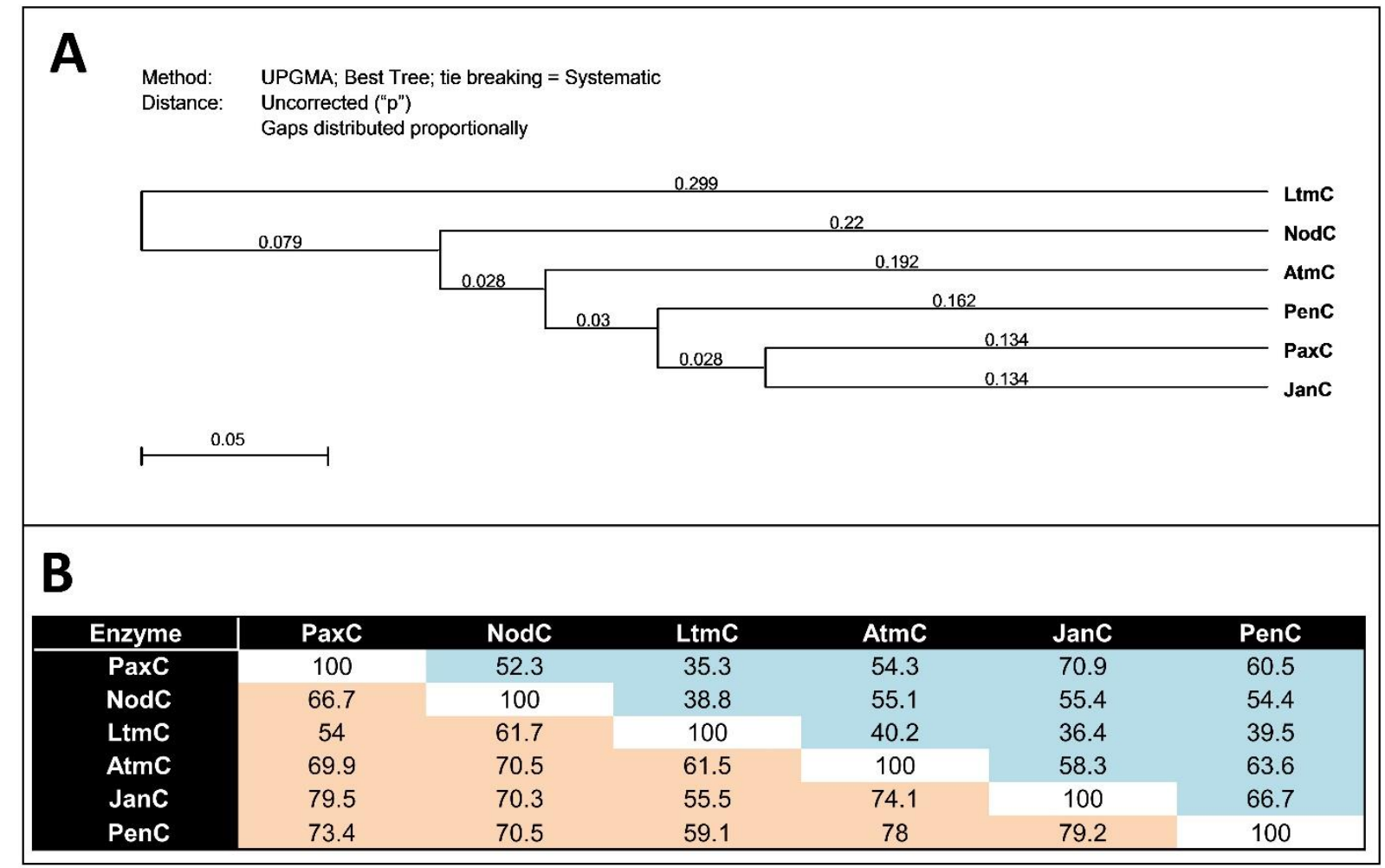

Figure 3.3. Phylogenetic tree (A) and similarity matrix (B) of geranylgeranyl transferases ('C' enzymes). The phylogenetic tree depicts the difference in $\%$ identity scores for the amino acid residues of the ' $C$ ' enzymes. The similarity matrix depicts the $\%$ identity scores (blue shaded regions ( ()$)$ and the \% similarity scores (orange shaded regions ( )) for the amino acid residues of the ' $C$ ' enzymes.

\section{Functional analysis of NodC}

To assess whether NodC was a functional orthologue of the indole diterpene geranylgeranyl transferases, and establish directly the role of nodC in nodulisporic acid A (1) biosynthesis, a series of MIDAS Level-3 plasmids was constructed, which were then introduced into the P. paxilli $\triangle$ paxC deletion mutant strain PN2290. As mentioned in Chapter 2, MIDAS is composed of a three-tiered system (Figure 3.4), termed MIDAS Level-1, Level-2, and Level-3, which employs the use of unique MIDAS destination 
vectors for the assembly of transcription unit modules (promoters, coding sequences, and terminators) in Level-1, full length transcription units in Level-2, and multigene assemblies in Level-3. ${ }^{3}$

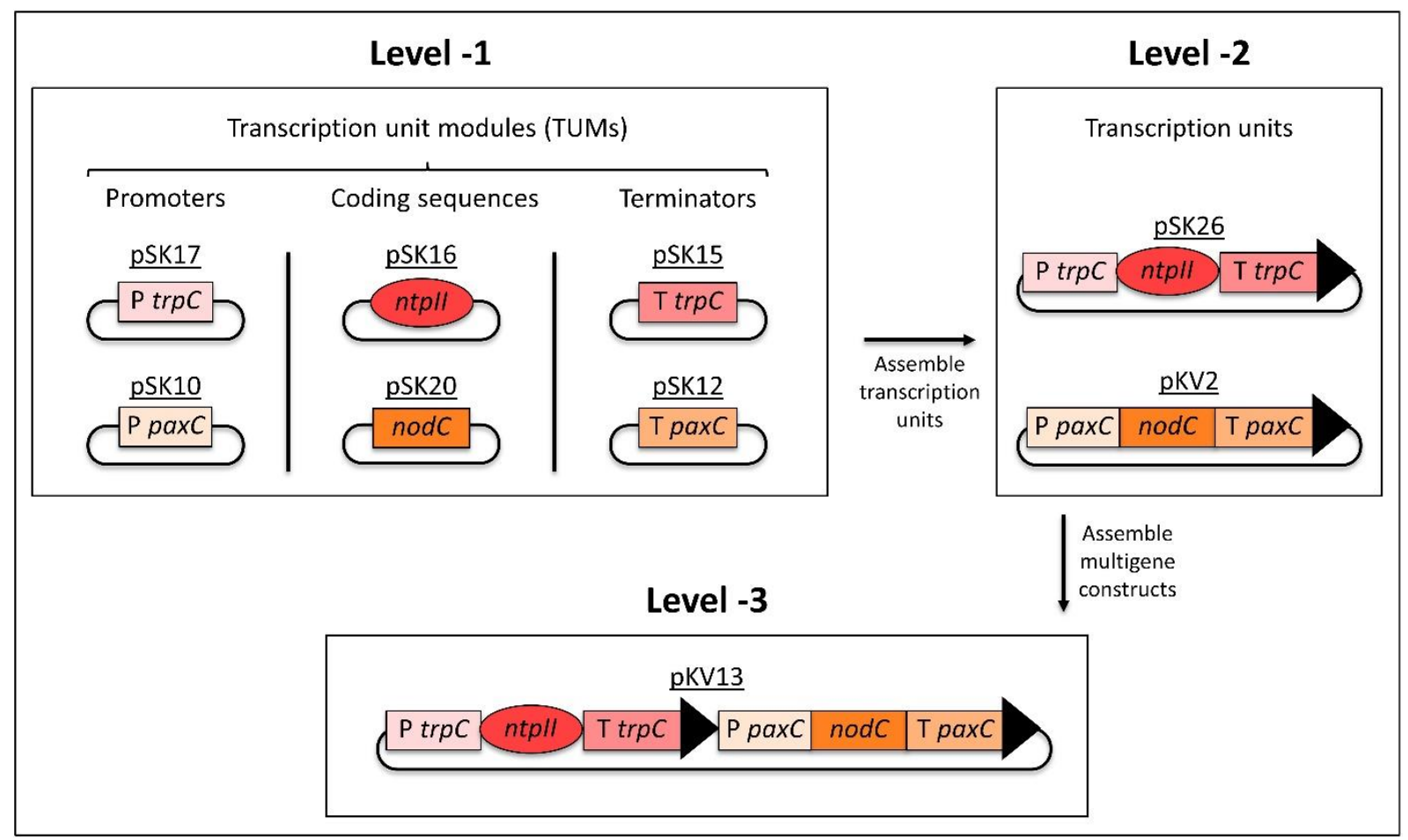

Figure 3.4. Overview of the MIDAS platform for plasmid assembly of pKV13

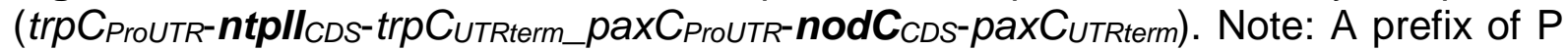
or $\mathrm{T}$ respectively represent a promoter or terminator region.

Assembly of the first MIDAS Level-3 nodC $C_{C D S}$-containing plasmid [pKV13, (trpC ProUTR-ntpll $_{C D S}$-trpCUTRterm_pax $C_{\text {ProUTR-nod }} \boldsymbol{C}_{C D S}$-paxCUTRterm)] is described in detail to provide a thorough introduction to the MIDAS platform that was used throughout this project to assemble plasmids for heterologous expression studies of genes within the $N O D$ cluster. The nod $C_{C D S}$-containing plasmid (pKV13) was designed to harbour a geneticin resistance cassette, used to screen transformed colonies, and a nod $C$ transcription unit, used to assess the role of nodC in nodulisporic acid $A$ (1) biosynthesis. In accordance with the MIDAS vector assembly platform, six Level-1 MIDAS constructs, three for the geneticin resistance cassette [pSK17 (trp $C_{\text {ProUTR), }}$ pSK16 (ntp/l $C D S$ ), pSK15 (trpCUTRterm)] and three for the nodC transcription unit [pSK10

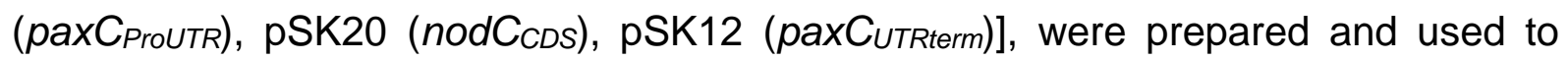
assemble the two MIDAS Level-2 constructs, pSK26 (trpC $C_{\text {ProUTR- }}$ ntpll ${ }_{C D S}$-trpCUTRterm) 
and pKV2 (pax ProUtR-nod $_{\text {CDS-pax }}$ - $C_{U T R t e r m)}$ respectively. An in-depth description of each Level of the MIDAS platform is presented throughout this chapter.

\section{Preparation of MIDAS Level-1 plasmids}

Two key steps are involved in the preparation of MIDAS Level-1 plasmids: (1) polymerase chain reaction (PCR) primers are designed and used to generate PCR products, and (2) PCR products are assembled into a Level-1 destination vector and propagated in bacteria. In this project, all PCR products were generated from the genomic DNA (gDNA) of specific fungal species in a fungal library. They were then inserted into the MIDAS Level 1 destination vector (pML1) via a one-pot BsmBImediated reaction and cloned in E. coli (Stellar). Alternatively, domesticated synthetic modules could be ordered from a DNA synthesis company and directly assembled into the Level-1 destination vector (pML1).

\section{Generation of PCR products}

The foundation of the MIDAS Level-1 plasmids relies on incorporation of MIDASspecific sequences that flank each end of the transcription unit modules (i.e. promoters, coding sequences, and terminators), which can be achieved by appropriately designing PCR primers. Flanking primers are designed to amplify all of the transcription unit modules with two notable characteristics; the first is the $\mathrm{BsmBI}$ enzyme-specific recognition sites and corresponding sticky ends that are essential to the correct assembly of PCR fragments into the MIDAS Level-1 destination vector, and the second is the Bsal-mediated sticky ends that encode the "4-nucleotide address system' essential for proper transcription unit module assembly into MIDAS Level-2 destination vectors to form complete transcription units. In addition to the generic transcription unit module flanking primers, additional primers, known as domestication primers, must be designed to remove any transcription unit module internal recognition sites for Aarl, Bsal and BsmBI. Transcription unit modules that require domestication are therefore amplified in separate fragments that are reassembled into full transcription unit modules in MIDAS Level-1. For example, the nod $C$ coding sequence contains an internal $B s m B I$ restriction site and thus had to be amplified as two fragments (Figure 3.6, B) in order to eliminate the BsmBI site. Upon PCR amplification 
of each transcription unit module or fragment thereof, PCR products of expected size were spin-column purified and assessed by agarose gel electrophoresis as shown in Figure 3.5, A.

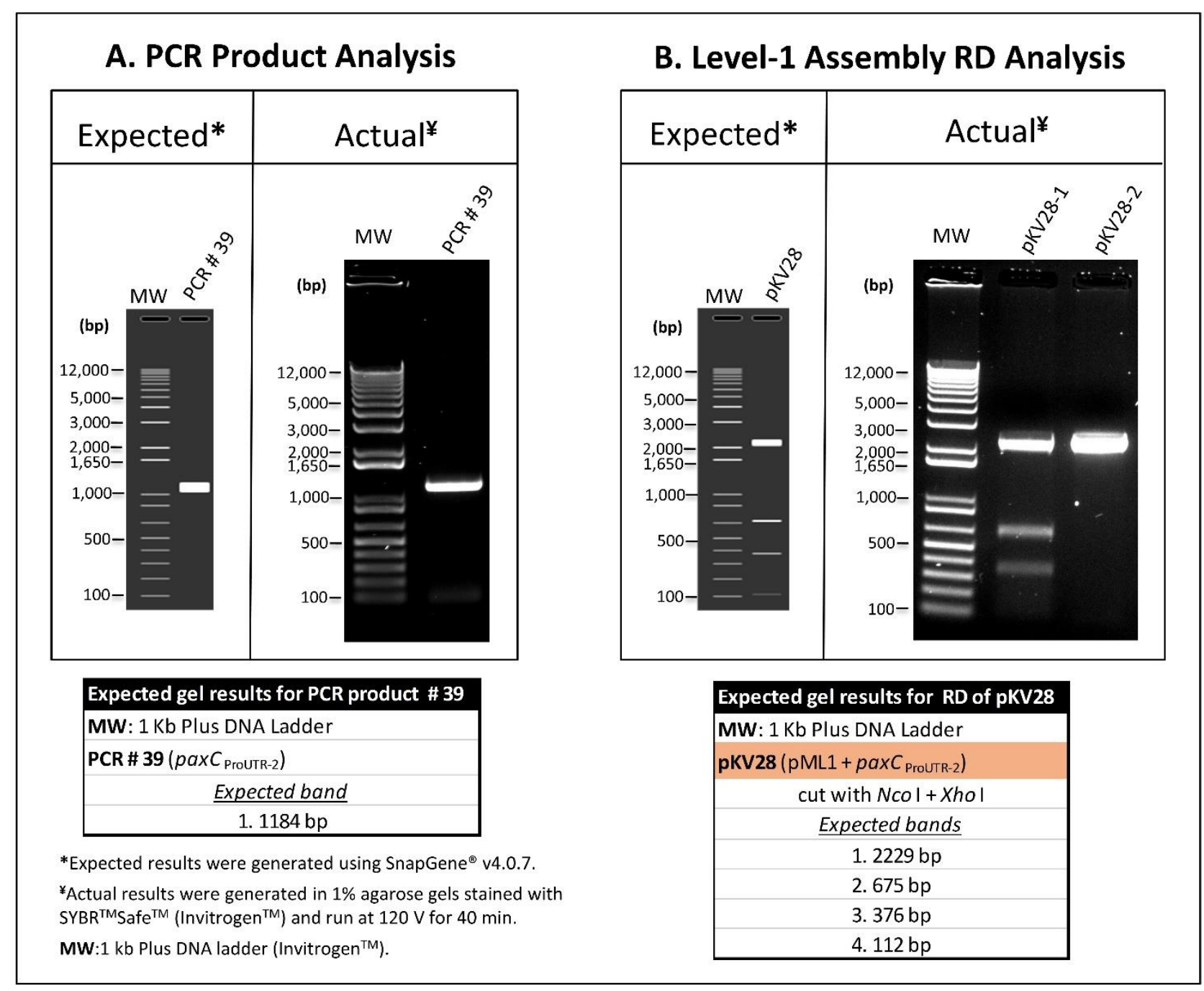

Figure 3.5. An example of the expected and actual gel results of a PCR product (PCR \# 39) used to assemble a promoter transcription unit module (paxCProUTR-2) in the pML1 MIDAS Level-1 destination vector (A) and an example of the expected and actual gel results of the restriction digest of two MIDAS Level-1 plasmids, pKV28-1 and pKV282, assembled from PCR \#39 (B).

\section{Assembly of Level-1 plasmids}

PCR products that matched their expected length were assembled into transcription unit modules (i.e. promoters, coding sequences and terminators) in the MIDAS Level1 plasmid, pML1, by BsmBI-mediated Golden Gate assembly (as illustrated in Figure 3.6). Plasmids were then cloned in E. coli. Successful cloning via BsmBI-mediated 
Golden Gate assembly resulted in disruption of the lacZ gene (note absence of lacZ in Figure 3.6 plasmid products) so only white colonies were selected for analysis by restriction digest. As an example, results from the cloning of pKV28 are shown in Figure 3.5, B, where only one of the three clones picked (pKV28-1) was positive by restriction analysis. To ensure that the Level-1 transcription unit modules were free of errors introduced by $\mathrm{PCR}$ and that the $\mathrm{BsmBI}$ assembly junctions were correct, typically one plasmid with the correct restriction profile (in this case pKV28-1) was sequenced. Once Level-1 transcription unit modules were sequence-verified they were used to assemble the transcription units in MIDAS Level-2. 


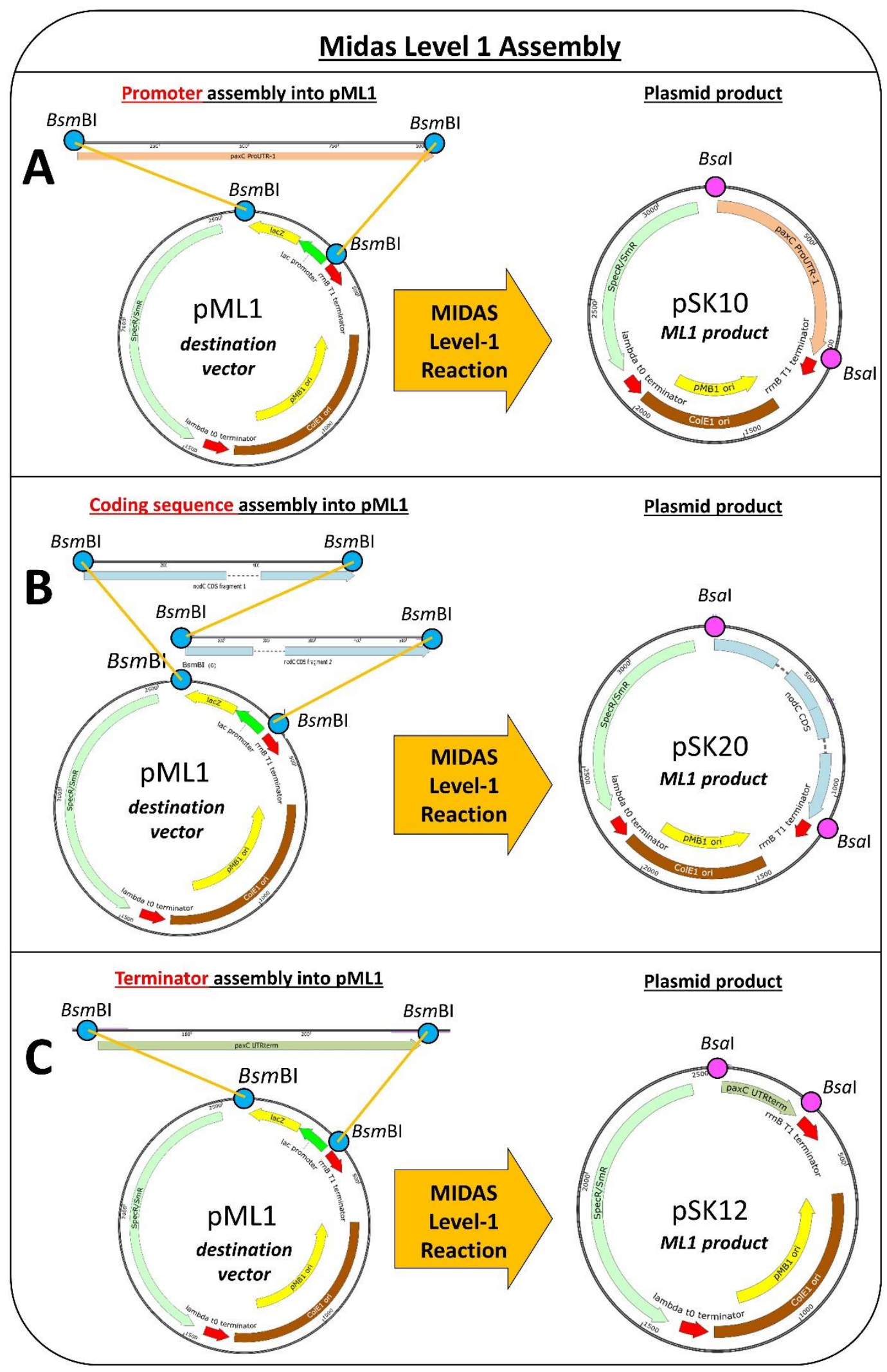

Figure 3.6. Depiction of MIDAS Level-1 assembly of the paxC ProUTR transcription unit module, pSK10 (A), the nodCCDS transcription unit module, pSK20 (B), and the paxCUTRterm transcription unit module, pSK12 (C). All plasmids were assembled using a BsmBI-mediated Golden Gate reaction into the pML1 destination vector to form the plasmid products. 


\section{Preparation of MIDAS Level-2 plasmids}

MIDAS Level-2 assembly provides the backbone of MIDAS, which implements the unique modularity of transcription unit direction and position within the final MIDAS Level-3 vector. Eight MIDAS Level-2 destination vectors [pML2(+)BF, pML2(-)BF, pML2(+)WF, pML2(-)WF, pML2(+)BR, pML2(-)BR, pML2(+)WR and pML2(-)WR); Figure 8.4] are available for assembly of the MIDAS Level-1 transcription unit modules, but due to the simplicity of testing the function of a single gene, nod $C$ as discussed in this chapter, only two of the Level-2 destination vectors (pML2(+)WF and pML2(+)BF) were used to assemble the two Level-2 transcription units [pSK26 (trp ProUTR-ntpll $_{C D S-}$

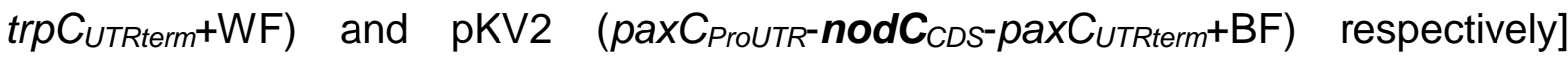
required for the stepwise MIDAS Level-3 assembly of pKV22 (trpC ProUTR-ntpll $_{C D S}$

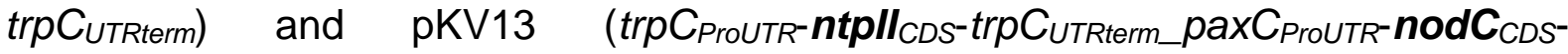
paxCUTRterm).

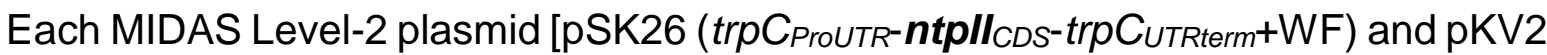
(pax $C_{\text {ProUtR-nod }} \boldsymbol{C}_{\text {CDS-pax }}$-putRterm+BF)] was assembled in a 1-pot Bsal-mediated Golden Gate-type assembly reaction containing three MIDAS Level-1 plasmids and one Level-2 destination vector. For example, Figure 3.7 shows the MIDAS Level-2 assembly of pKV2 (pax $C_{\text {ProUtR-nod }} \boldsymbol{C}_{C D S}$-pax $C_{U T R t e r m+B F}$ ) from three MIDAS Level-1 plasmids, pSK10 (pax $C_{\text {ProUtR) }}$ ), pSK20 (nod $C_{C D S)}$ ) and pSK12 (paxCUtRterm), and one MIDAS Level-2 destination vector, pML2+BF. Correct assembly of the Level-1 transcription unit modules into the full length Level-2 transcription unit is dependent upon the sticky ends accounted for during primer design known as the MIDAS Level2 '4-nucleotide address system' (Figure 3.7, red and blue sequences). Upon assembly, Level-2 plasmids were transformed into competent E. coli cells and typically three colonies were picked for propagation and plasmid purification. Purified plasmids were subjected to restriction analysis and, for these initial plasmids, sequence analysis. It became apparent that the sequence verification of Level-2 plasmids was unnecessary and this step was redundant as the Level-1 sequence verification already ensured that no errors were present within sequences of the transcription unit modules. Therefore, restriction analysis became the preferred method to verify proper assembly of the Level-2 transcription units prior to MIDAS Level-3 assembly. 


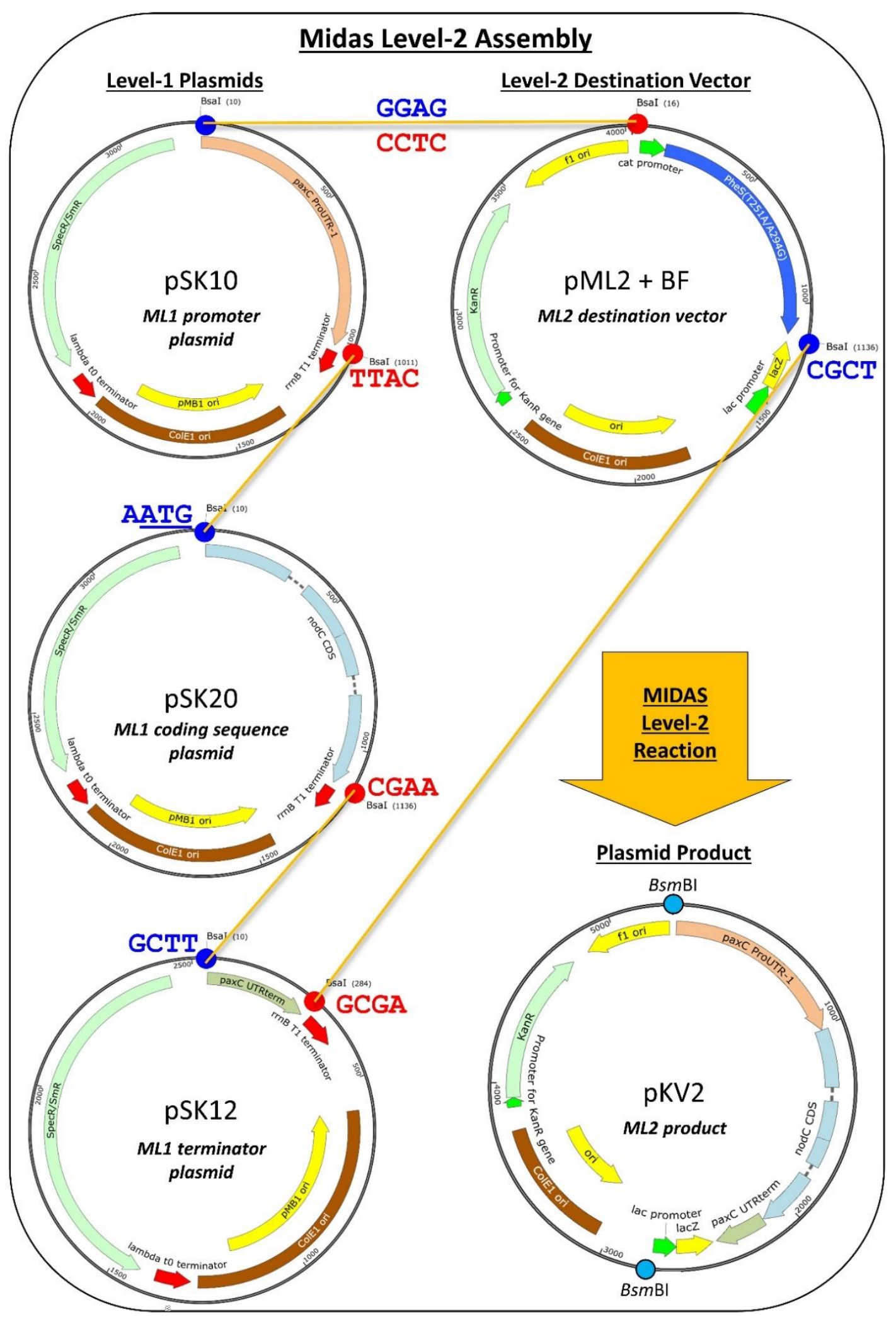

Figure 3.7. Depiction of MIDAS Level 2 Assembly using three MIDAS Level-1 plasmids

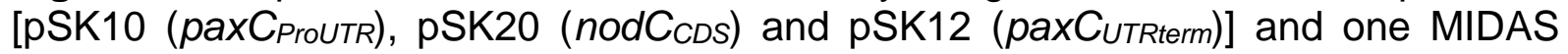
Level-2 destination vector (pML2+BF) to create a MIDAS Level-2 plasmid product [pKV2 (pax ProUTR-nod $_{\text {CDS-pax }}$ CUTRterm $_{\text {-BF) }}$ ]. The reaction was carried out in a single tube and the sticky ends created upon Bsal digestion are depicted in blue (leading DNA strand, 5' to $3^{\prime}$ ) and red (complementary DNA strand, 3' to 5'). The nod $C_{C D S}$ ATG start site is underlined in the leading strand overhang of pSK20 (nod $C_{C D S}$ ). 
The white Level-2 destination vectors impart Aarl recognition sites that flank the

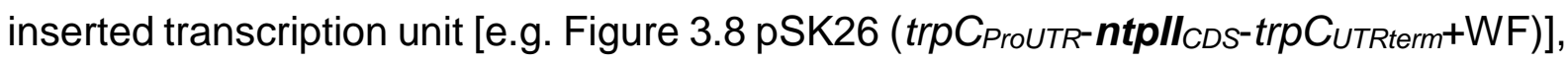
whereas the blue Level-2 destination vectors impart $B s m B I$ recognition sites that flank the inserted transcription unit [e.g. Figure 3.7 and Figure 3.8, pKV2 (pax $C_{\text {ProUtR- }}$ nod $C_{\text {CDs }}$-pax $\left.\left.C_{\text {UtRterm }}+\mathrm{BF}\right)\right]$. The Aarl and $B s m B I$ recognition sites are used in the stepwise assembly of the Level-3 plasmids, where the first Level-3 assembly reaction into pML3.1 is always mediated by Aarl and the second is always mediated by $B s m B I$ as shown respectively in A and B of Figure 3.8 (Note: an additional Level-3 destination vector could be synthesised to be mediated first by a $B s m B I$ reaction, but this was not required for this work). Therefore, the first transcription unit that is placed into the Level3 destination vector (pML3.1) must be inserted into a white (W) Level-2 destination vector. In the case of this project, introducing geneticin resistance (encoded by the $\left.n p t I_{C D S}\right)$ into all of the Penicillium paxilli knockout strains intended for use in heterologous expression experiments, with the exception of strain PN2458 ( $\triangle$ paxB) (see Table 8.5 in Materials and Methods), would allow easy screening of transformed colonies that contained desired gene(s) of interest. To this end, the first Level-3 assembly reaction was designed to create a Level-3 destination plasmid [pKV22 (trpC ProUTR-ntpll $_{C D S}$-trpC $C_{U T R t e r m}$ ) shown in Figure $\left.3.8, \mathrm{~A}\right]$ that contained a ntpll ${ }_{C D S}$ transcription unit for geneticin resistance; and thus the ntpll $C D S$ cassette was assembled into a white Level-2 destination vector (pML2(+) $\underline{\mathrm{W} F})$ to create the Level-2 plasmid product pSK26 (trpC ProUTR-ntpll $_{\text {CDS-trpC }}$ UTRterm $_{\text {WF }}$ ). 


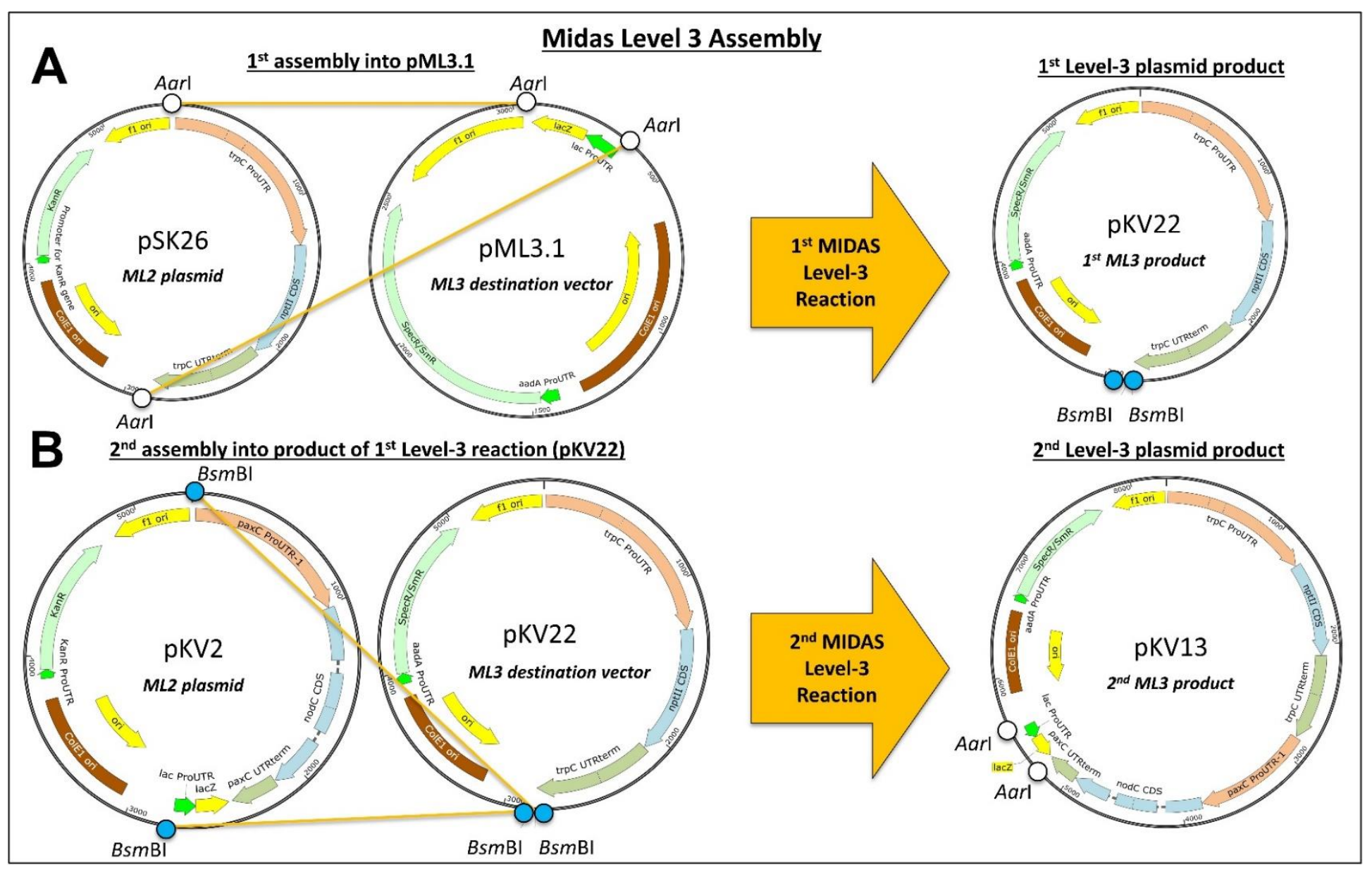

Figure 3.8. Depiction of the 2-step MIDAS Level-3 Assembly used to insert two transcription units, the npt/l $I_{C D S}$ cassette from pSK26 and the nod $C_{C D S}$ cassette from pKV2, into a single MIDAS Level-3 plasmid [pKV13 (trpC $C_{\text {ProUTR-ntpll }}$ CDS-trpC $_{\text {UTRterm_pax }}$ ProUTR- $_{\text {- }}$

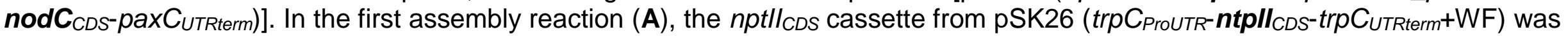
inserted into the pML3.1 destination vector using an Aarl-mediated Golden Gate assembly reaction to create the $1^{\text {st }}$ Level-3 plasmid product, pKV22 (trp $C_{\text {ProUTR-ntpll }}$ CDS-trp $_{\text {UTRterm). In the second assembly reaction (B), the nodC }}$ CDS cassette from pKV2 was inserted into the $1^{\text {st }}$ Level-3 plasmid product, pKV22 (trp ProUTR-ntpll $_{C D S}$-trpCUTRterm), using a BsmBI-mediated Golden Gate assembly reaction

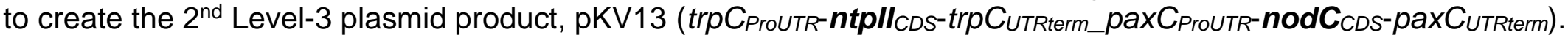


Assembly of a white Level-2 plasmid product [e.g. pSK26 (trpC ProUTR-ntpll $_{C D S^{-}}$ trp $\left.C_{U T R t e r m}+\mathrm{WF}\right)$ ] into a Level-3 destination vector imparts $B s m B I$ recognition sites into

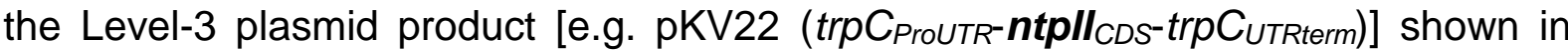
Figure 3.8, A]. Therefore, the next transcription unit that is assembled into the Level-3 plasmid product [e.g. pKV22 (trpC ProUTR-ntpll $_{\text {CDS-trpC }}$ UTRterm)] must be assembled in a blue Level-2 destination vector (e.g. pML2(+) $\underline{B} F)$. Accordingly, the nod $C_{C D S}$ transcription unit was assembled into a blue Level-2 destination vector (pML2(+) $\underline{B} F)$ to create the Level-2 plasmid product pKV2 (pax $C_{\text {ProUtR-nod }} \boldsymbol{C}_{\text {CDS-pax }}$ - UTRterm $\mathrm{BF}$ ) shown in Figure 3.8. Since direction (forward or reverse as indicated respectively by the $\mathrm{F}$ and $\mathrm{R}$ in the Level-2 destination vectors) or orientation (positive orientation of ProUTR-CDS-UTRterm versus negative orientation of 4 UTRterm-CDS-ProUTR as indicated respectively by the (+) and (-) of the Level-2 destination vectors) of the $n p t I_{C D S}$ transcription unit and the nod $C_{C D S}$ transcription unit were not seemingly paramount to successful expression of either gene, both transcription units were assembled into (+)F Level-2 destination vectors.

\section{Preparation of MIDAS Level-3 plasmids}

MIDAS Level-3 assembly reactions are used to create the plasmids necessary for fungal transformation. A combination of Aarl-mediated Golden Gate assembly reactions followed by BsmBI-mediated Golden Gate assembly reactions are used to assemble transcription units from Level-2 plasmids into Level-3 plasmids in a stepwise fashion until the final plasmid product, used for fungal transformation, is assembled. For example, the Level-3 plasmid pKV13 (trpC ProUTR-ntpll $_{\text {CDS-trpC }}$ UTRterm_pax $C_{\text {ProUTR- }}$ nod $\boldsymbol{C}_{C D S}$-pax $\left.C_{U T R t e r m}\right)$ was assembled stepwise using two MIDAS Level-3 assembly reactions (Figure 3.8, A and B); the first being the assembly of pKV22 (trpC $C_{\text {ProUTR- }}$ ntpll ${ }_{C D S}$-trpC $C_{U T R t e r m)}$ from the Level-2 plasmid pSK26 (trpC $C_{\text {ProUTR-ntpll }}$ CDStrpCutRterm+WF) into the Level-3 destination vector pML3.1 (Figure 3.8, A), and the second being the assembly of pKV13 (trpC $C_{\text {ProUTR-ntpll }}$ CDS-trpC UTRterm_pax $C_{\text {ProUTR- }}$ nod $C_{C D S}$-pax $\left.C_{U T R t e r m}\right)$ from the Level-2 plasmid pKV2 (pax $C_{\text {ProUTR-nod }} \boldsymbol{C}_{C D S}$

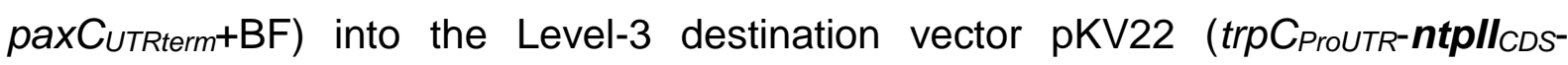
trpC UTRterm) (Figure 3.8, B).

Following transformation of the assembled products into E. coli, three transformants analysed by restriction analysis all contained the correctly assembled insert. One of the properly assembled Level-3 plasmid products was used as either a destination 
vector for the next MIDAS Level-3 assembly if more genes needed to be added [as done with pKV22 (trpC ProUTR-ntpll $_{C D S-\text { trp }} C_{U T R \text { term }}$ )] or for fungal transformation if it contained all of the desired genes [as done with pKV13 (trpC $C_{\text {ProUTR-ntpll }}$ CDS- $^{-}$ trpC UTRterm_pax ProUTR-nod $_{\text {CDS-pax }}$ - UTRTterm)].

\section{Control plasmids}

To control for any problems that may have arisen from the MIDAS plasmid assembly

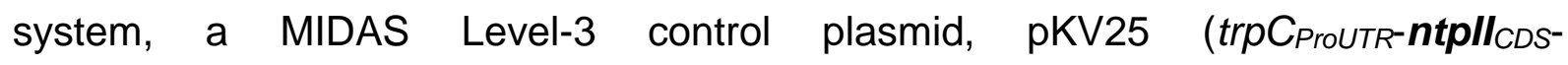
trpC UTRterm_pax $C_{\text {ProUtR-pax }} \boldsymbol{C}_{\text {CDS-pax }}$ - $C_{\text {UTRterm) }}$ shown in Figure 3.9, was assembled

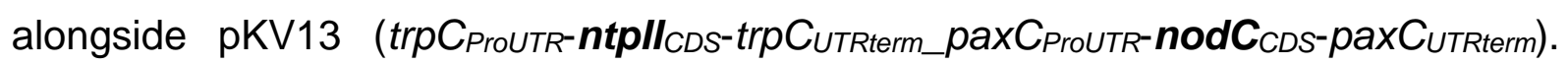

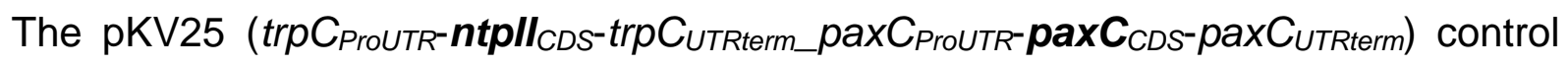
plasmid was designed exactly as pKV13 (trpC $C_{\text {ProUTR-ntpll }}$ CDS-trpC $C_{U T R t e r m}$ ppax $C_{\text {ProUTR- }}$ nod $\boldsymbol{C}_{C D S}$-pax $\left.\boldsymbol{C}_{U T R t e r m}\right)$ with the exception of the coding sequence in the second transcription unit being pax $C_{C D S}$ in pKV25 (trp $C_{\text {ProUTR-ntpll }}$-nDS-trp $C_{U T R t e r m \_p a x} C_{\text {ProUTR- }}$ pax $\boldsymbol{C}_{C D S}$-pax $C_{U T R t e r m}$ ) instead of nod $C_{C D S}$ as described in pKV13 (trp $C_{\text {ProUTR-ntpll }}$-nDS-

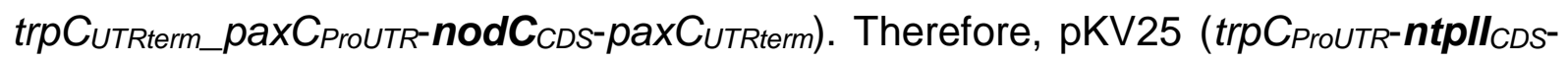
trpC UTRterm_pax ProUTR-pax $_{\text {CDS-pax }}$ CUTRterm) was assembled from the same Level-3

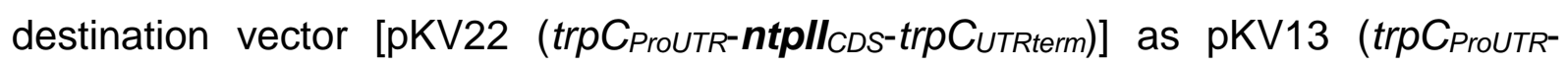
ntpll CDS-trpC $_{\text {UTRterm_pax }}$ ProUTR-nod $_{\text {CDS-pax }} \boldsymbol{C}_{U T R \text { term) }}$ (shown in Figure 3.8, B), but with a different Level-2 plasmid, pKV24 (pax $C_{\text {ProUtR-pax }} \boldsymbol{C}_{C D S}$-pax $C_{U T R t e r m+B F)}$ ), which contained the pax $C_{C D s}$ transcription unit. In addition to this first control plasmid [pKV25

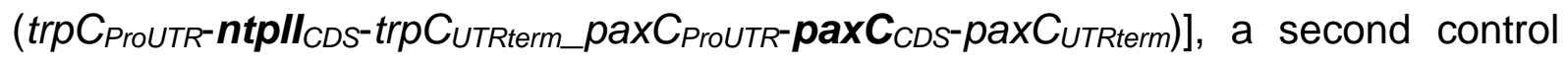

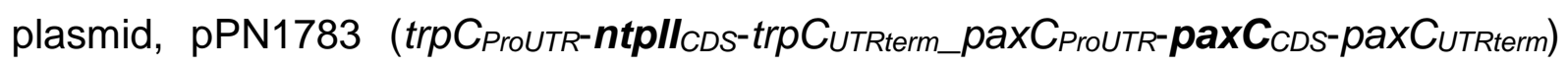
(plasmid map unavailable), which harbours the native pax $C_{C D S}$ transcription unit and was used to restore paxilline (2) production in the P. paxilli $\triangle$ paxC deletion mutant strain (PN2290) during the initial paxilline (2) biosynthetic pathway confirmation experiments, ${ }^{38}$ was obtained from Prof. Barry Scott's lab. 


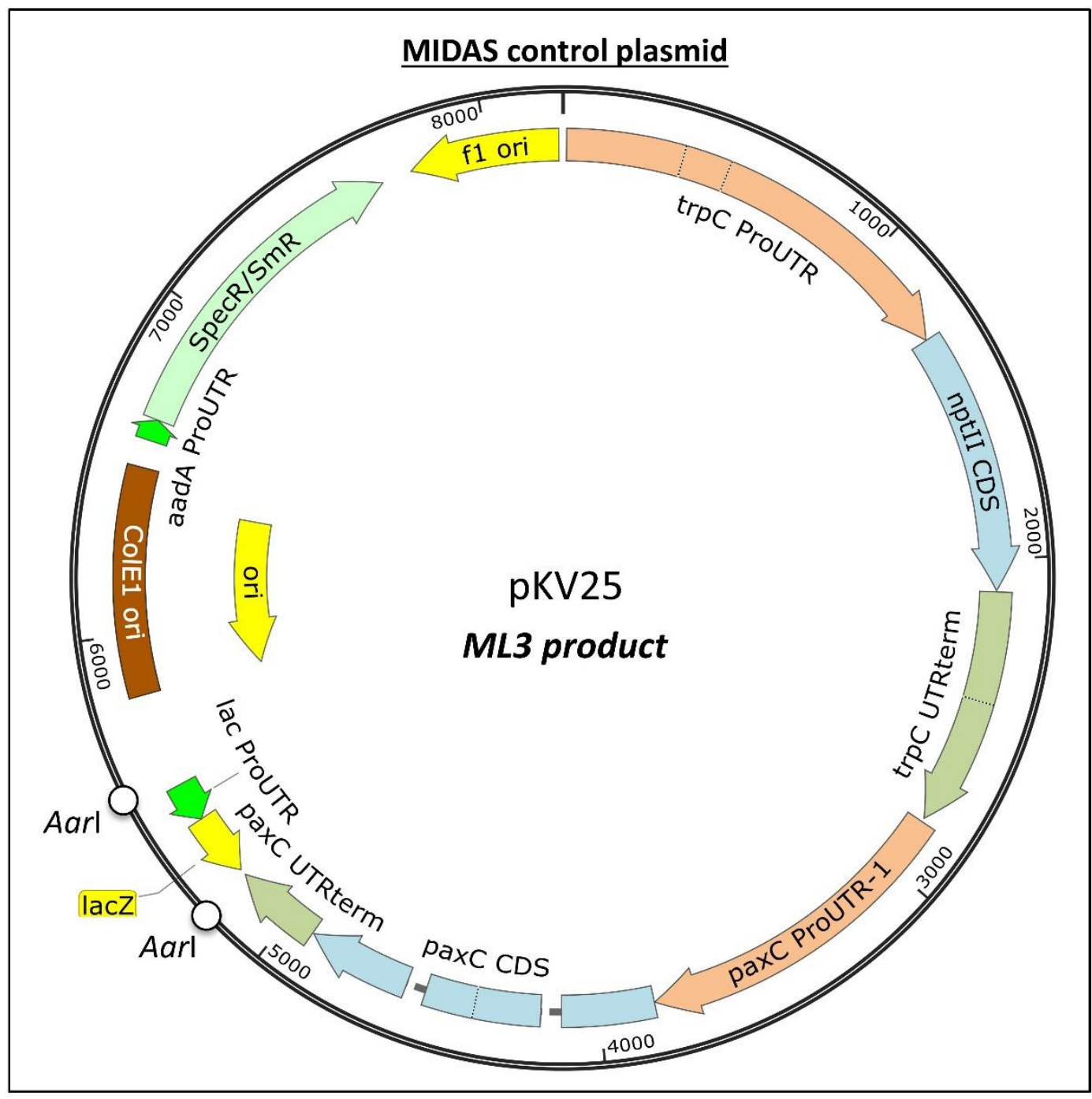

Figure 3.9. Map of the pKV25 (trpC $C_{\text {ProUTR- }}$ ntpll ${ }_{C D S}$-trpC $C_{U T R t e r m}$ pax $C_{\text {ProUTR-pax }} \boldsymbol{C}_{C D S^{-}}$ pax $\left.C_{U T R t e r m}\right)$ MIDAS control plasmid used during the pKV13 (trpC $C_{\text {ProUTR- }}$ ntpll CDS $^{-}$ trpCUTRterm_pax ProUTR-nod $_{\text {CDS-pax }}$ CUTRterm):PN2290(_paxC) fungal transformation experiment. Notes: SpecR/SmR imparts spectinomycin resistance, lacZ is used for blue/white selective screening, ColE1 ori is the bacterial origin of replication and F1 ori is the fungal origin of replication.

\section{Fungal transformations}

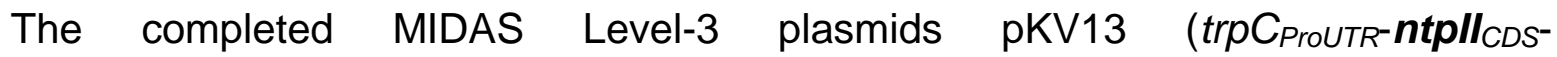

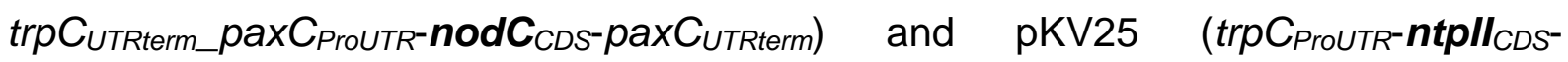
trp $C_{U T R t e r m \_p a x} C_{\text {ProUTR-pax }} \boldsymbol{C}_{C D S}$-pax $C_{U T R t e r m)}$ provided the DNA required for the first fungal transformation experiment. Protoplasts of the $P$. paxilli $\triangle p a x C$ deletion mutant strain (PN2290), which does not produce paxilline (2) as shown in lane 2 of Figure 3.10, were transformed with the three plasmids [pKV13 (trp $C_{\text {ProUTR- }}$ ntpll $_{C^{\prime} S^{-}}$

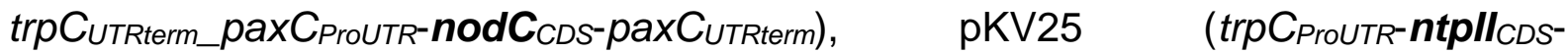
trpCUTRterm_pax $C_{\text {ProUTR-pax }} \boldsymbol{C}_{C D S}$-pax $\left.C_{U T R t e r m}\right)$, and pPN1783 (trp $C_{\text {ProUTR-ntpll }}$ CDS $^{-}$ 
trpC UtRterm_pax ProUtR-pax $_{\text {CDS-pax }}$ UUTRterm)] individually to test for the ability to restore paxilline (2) production. Contrary to expectation, neither of the MIDAS Level-3

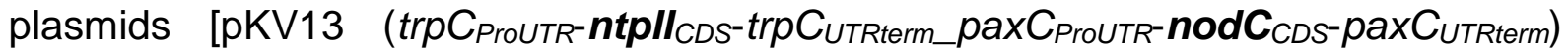

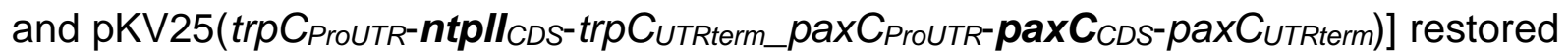
paxilline (2) production (Figure 3.10, lanes $4-11$ and 12-17 respectively) but the positive control plasmid, pPN1783 (trpC ProUTR-ntpll $_{\text {CDS-trpC }}$ UTTRterm_pax $_{\text {ProUTR- }}$ pax $C_{C D S-p a x} C_{U T R t e r m}$ ), did (Figure 3.10, lane 3 ), thereby indicating that the lack of paxilline (2) production seen in the MIDAS transformants was likely not due to the transformation procedure, but rather a problem within the MIDAS constructs.

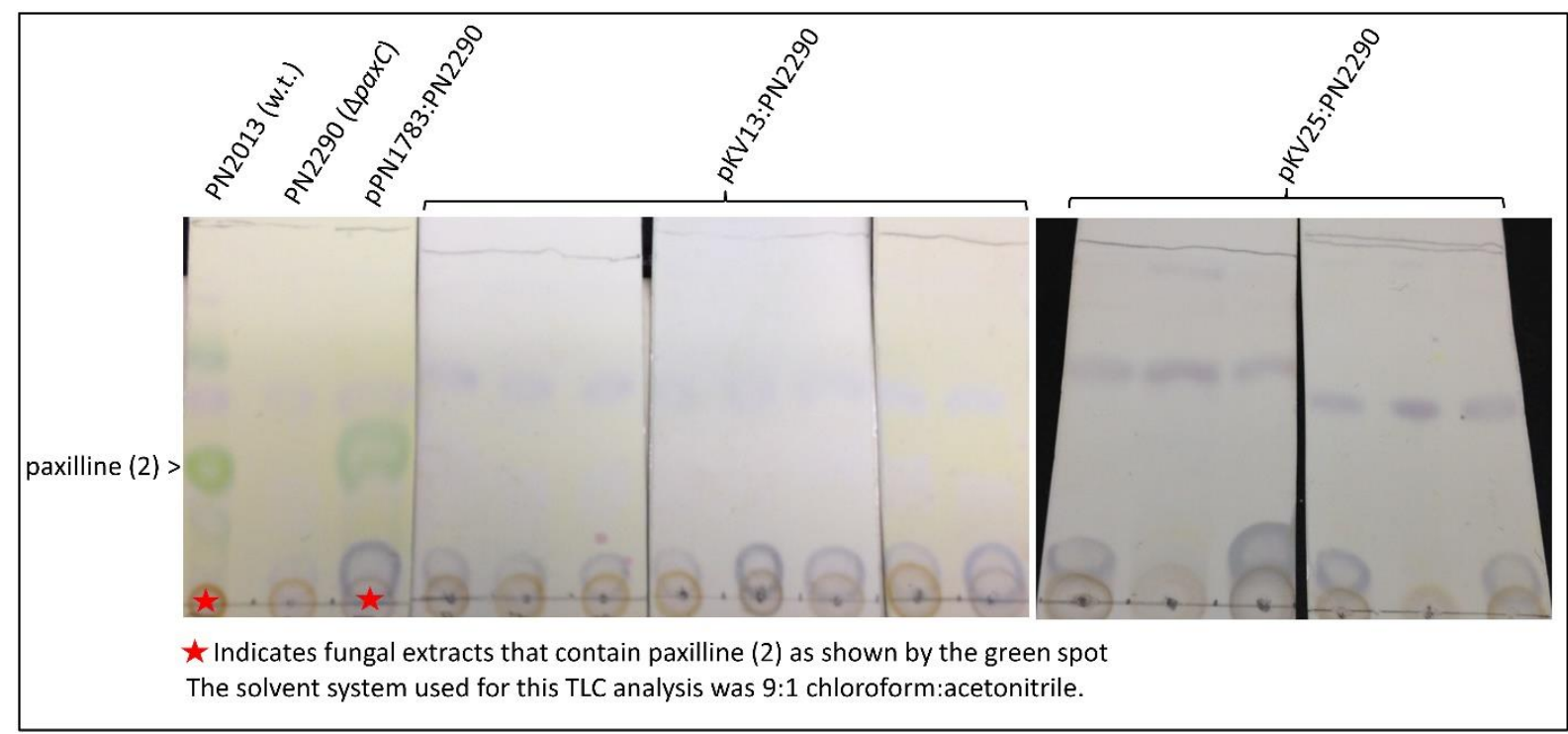

Figure 3.10. TLC results of fungal extracts from the individual transformation of $P$. paxilli $\triangle$ paxC deletion mutant PN2290 with three plasmids [pKV13 (trpC ProUTR- $^{-n t p}$ II $_{C D S^{-}}$

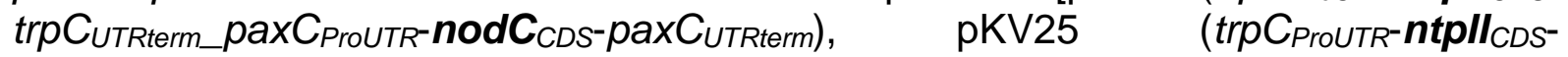

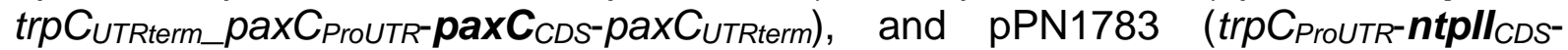
trpC UTRterm_pax ProUTR-pax $_{\text {CDS-pax }}$ ( $\left.C_{\text {UTRterm) }}\right]$. Results from the fungal extracts of the $P$. paxilli wild-type (w.t.) strain $\mathrm{PN} 2013$ and the $P$. paxilli $\triangle$ paxC deletion mutant PN2290 have been included as positive and negative controls respectively.

\section{Resolution of MIDAS construct problem}

Direct comparison of the sequences of the MIDAS pax $C_{C D S}$ construct [pKV25

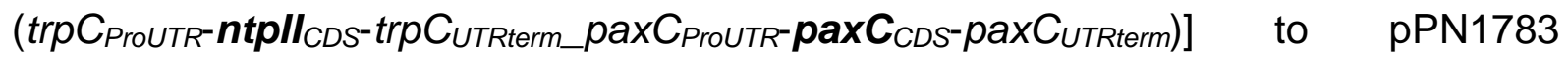
(trp $C_{\text {ProUTR-ntpll }}$ CDS-trpC $C_{U T R t e r m \_p a x} C_{\text {ProUTR-pax }} C_{C D S}$-pax $C_{U T R t e r m}$ ) showed that the

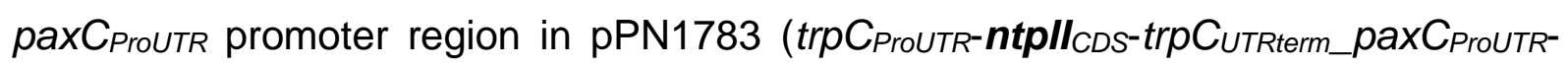

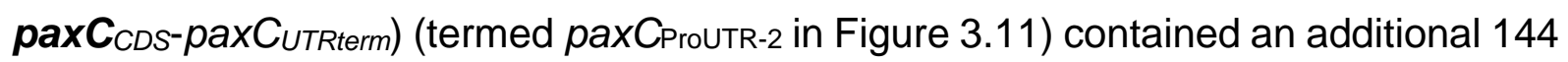
bp upstream of the pax $C_{\text {ProUTR }}$ promoter region used in pKV25 (trpC ProUTR-ntpll $_{C D S}$ trp $C_{U T R t e r m \_p a x} C_{\text {ProUtR-pax }} \boldsymbol{C}_{\text {CDS-pax }} C_{\text {UTRterm) }}$ (termed pax $C_{\text {ProUtR-1 }}$ in Figure 3.11). 
These extra 144 bp were not accounted for in the MIDAS constructs because the pax $C_{\text {ProUTR }}$ promoter region was designed based on annotation of the paxilline (2) biosynthetic pathway located on GenBank, which, as demonstrated, contains designated promoter regions that have not been functionally confirmed.

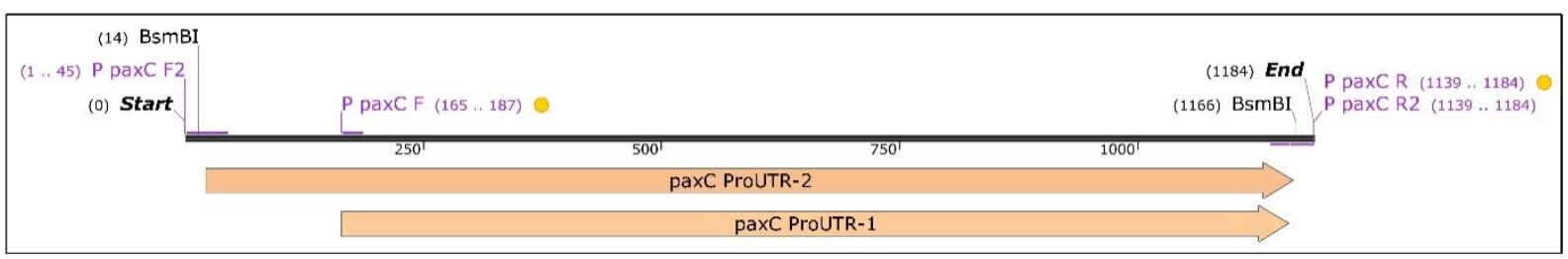

Figure 3.11. Direct comparison of the paxCProUTR-1 promoter region used in pKV13 (trpC ProUTR-ntpll $_{\text {CDS }- \text { trpC }}$ UTRterm_pax ProUTR-nod $_{\text {CDS-paxCUTRterm) }}$ and pKV25

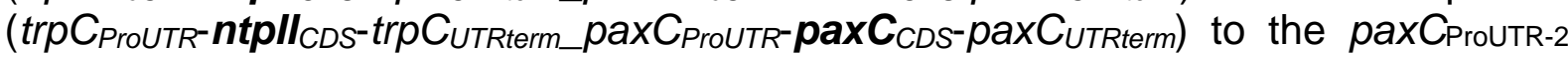
promoter region used in pPN1783 (trpC $C_{\text {ProUTR-ntpll }}$ CDS-trp $C_{\text {UTRterm_pax }}$ ProUTR- $_{\text {- }}$ pax $C_{C D S}$-pax $C_{\text {UTRterm) }}$. Notably paxCProUTR-1, which is 144 bp shorter than paxCProUTR2, did not promote the expression of nod $C_{C D S}$ or pax $C_{C D S}$ whereas the pax $C_{\text {ProUtR-2 }}$ did promote the expression of pax $C_{C D S}$.

Since these results suggested that there was a problem with the promoter region used in pKV13 (trpC $C_{\text {ProUtR-ntpll }}$ CDS-trpC $C_{\text {UTRterm_pax }} C_{\text {ProUTR-nod }}$-nods-paxCUTRterm) and

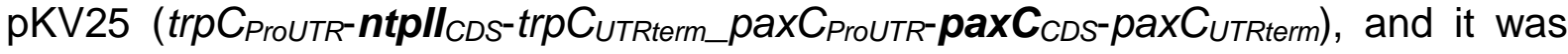
apparent that the trpC $C_{\text {Proutr }}$ promoter region worked from the colony growth of the transformed MIDAS constructs in the presence of geneticin, the modularity of the MIDAS system was used to create a MIDAS Level-3 plasmid, pKV27 (trpC $C_{\text {ProUTR- }}$

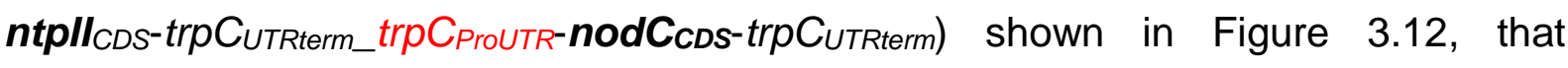
harboured nod $C_{C D S}$ driven by trpCProutR promoter region. 


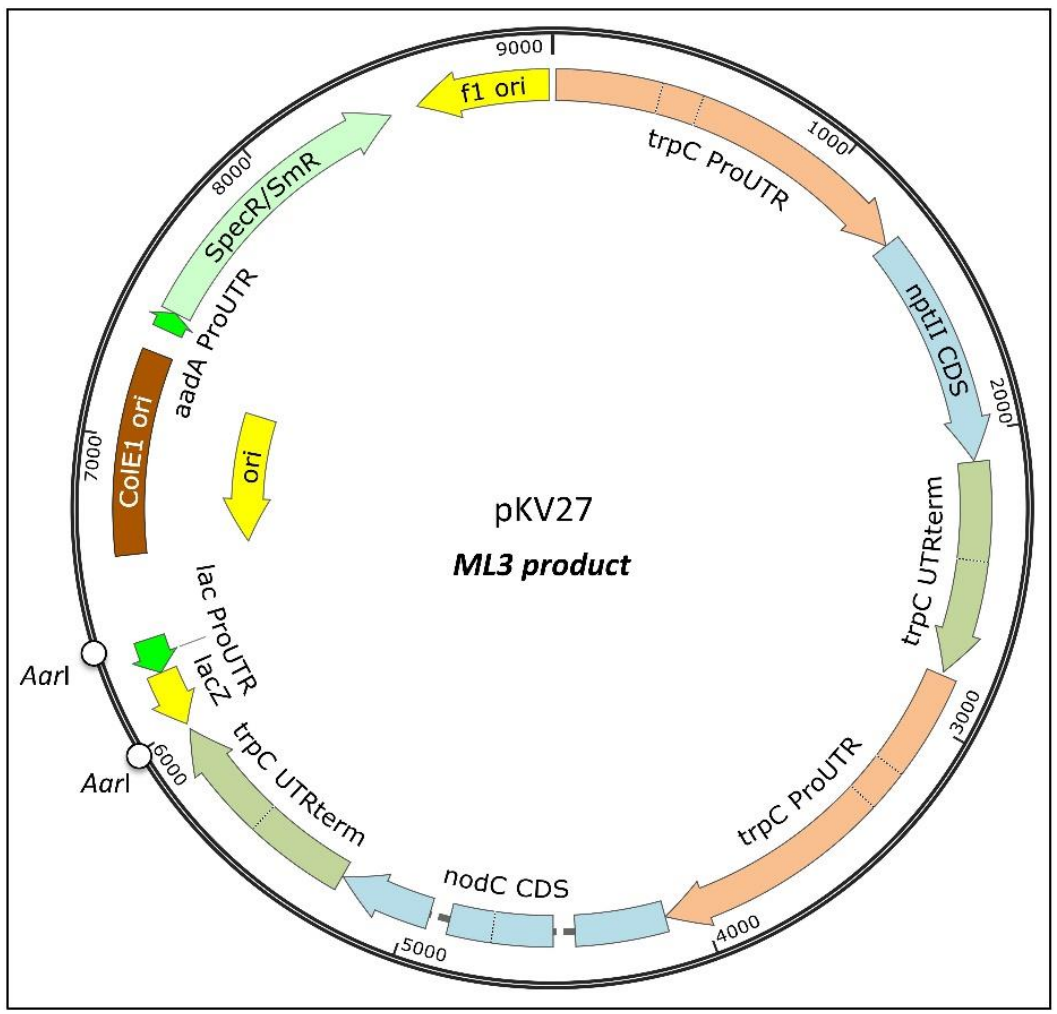

Figure 3.12. Plasmid map of pKV27 (trp $C_{\text {ProUTR-ntpll }}$ CDS $_{\text {-trp }} C_{U T R t e r m}$ trp $C_{\text {ProUTR- }}$ nod $C_{C D S}$-trp $C_{U T R t e r m)}$ showing the use of the trpC $C_{\text {ProUTR }}$ promoter region to drive expression of the nod $C_{C D S}$ coding sequence. Notes: SpecR/SmR imparts spectinomycin resistance, lacZ is used for blue/white selective screening, ColE1 ori is the bacterial origin of replication and $\mathrm{F} 1$ ori is the fungal origin of replication.

\section{Successful complementation of nodC into the $\triangle$ paxC $P$. paxilli deletion mutant}

Transformation of $P$. paxilli $\triangle$ paxC deletion mutant strain (PN2290) with pKV27 (trp $C_{\text {ProUTR-ntpll }}$ CDS-trpC $C_{\text {UTRterm_trp }} C_{\text {ProUTR-nod }} \boldsymbol{C}_{\text {CDS-trpC }} C_{\text {UTRterm) }}$ ) restored paxilline (2) production in at least six of ten transformants as determined by TLC analysis (Figure 3.13, lanes 1-5 and 7). Some of the pKV27 (trpC ProUTR-ntpll $_{\text {CDS }}$-trp $C_{U T R t e r m}$ trp $C_{\text {ProUTR- }}$ nod $\boldsymbol{C}_{C D S}$-trp $C_{U T R t e r m)}$ transformants produce paxilline (2) and some do not because of variations in integration site (i.e. some plasmid DNA may insert into a particular portion of the genome that prevents compound production) and partial integration (i.e. only a portion of the plasmid has integrated). Notably, the production of paxilline (2) was much lower in the nod $C_{C D S}$-containing (pKV27) transformants (lanes 1-5 and 7) than in the pax $C_{C D S}$-containing (pPN1783) control transformants (lanes 8 and 9). One possible explanation for this was promoter choice because the nod $C_{C D S}$ coding sequence was driven by the trp $C_{\text {ProUTR }}$ promoter region and the pax $C_{C D S}$ coding sequence was driven by the paxCProUtR promoter region. In the next chapter, "NodM, The key epoxidase," 
a study was designed to test how promoter choice affected compound production and conclusions can be found in the chapter's subsection: "A note about promoter choice".

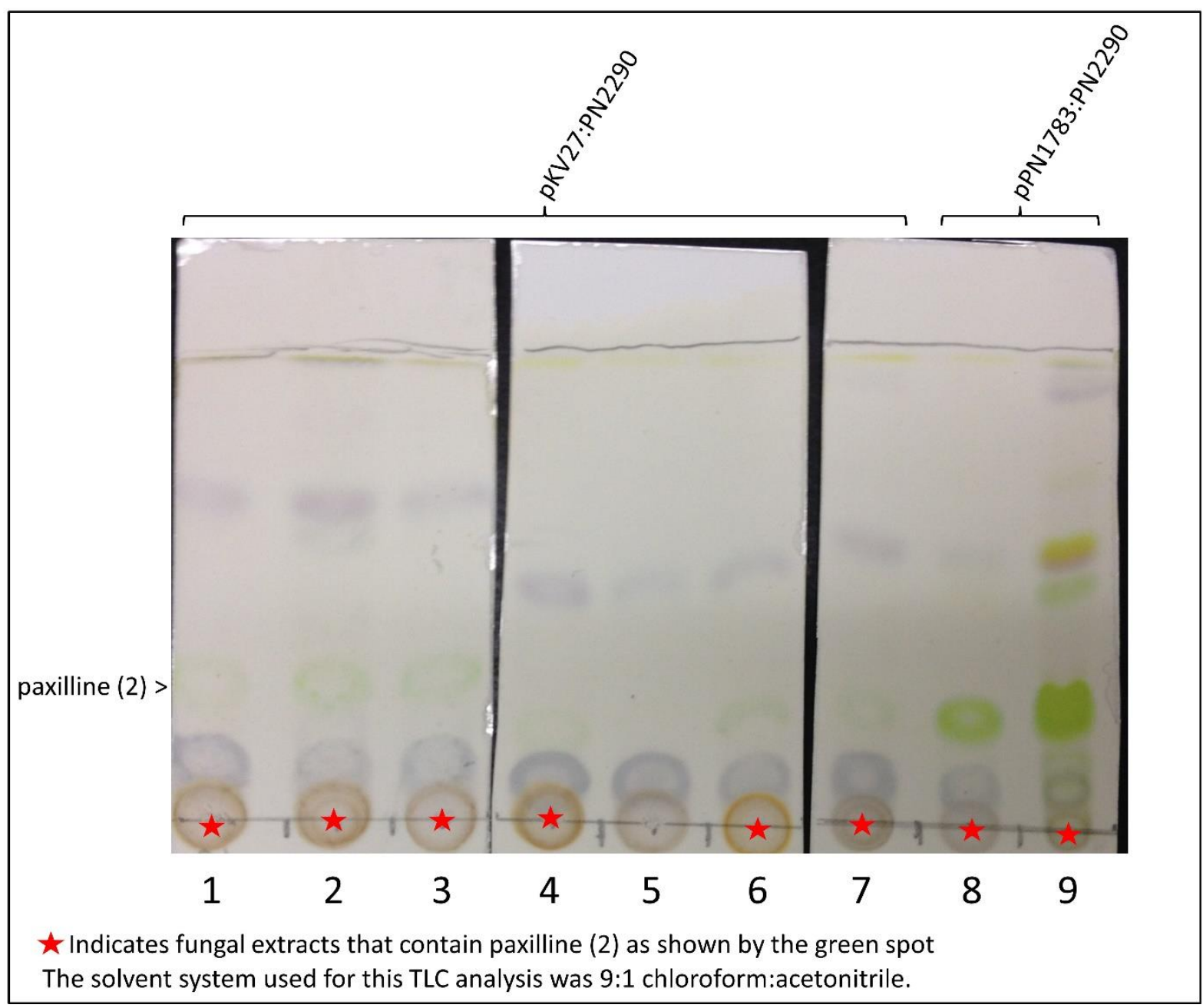

Figure 3.13. TLC results from seven of ten fungal extracts from the transformation of

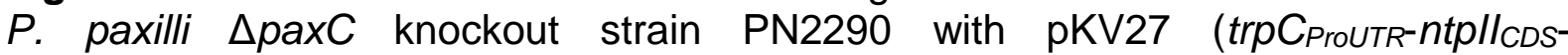

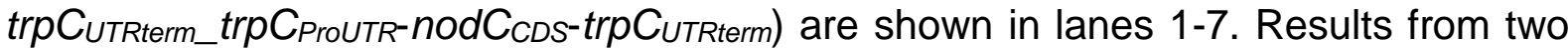
of three extracts of the transformation of $P$. paxilli $\triangle$ paxC knockout strain PN2290 with the positive control plasmid pPN1783 (trpC $C_{\text {ProUTR-ntpll }}$ CDS-trpC $C_{\text {UTRterm_pax }}$ ProUTRpax $C_{C D S}$-pax $C_{\text {UTRterm) }}$ are shown in lanes 8 and 9. Note that the TLC results shown are from a second round of TLC analysis and that extracts that are not shown (e.g. three of ten nod $C_{C D S}$-containing pKV27 transformants) did not contain visible indole diterpenes in the first analysis.

\section{LCMS confirmation of paxilline (2) production}

The production of paxilline (2) by the nod $C_{C D S}$-containing transformants was confirmed by LCMS. In particular, a new compound was present, that was not present in the extract of the $\triangle p a x C$ deletion mutant strain PN2290 (Figure 3.14, trace i), whose 
retention time $(5.2 \mathrm{~min})$ and high resolution-mass spectra $\left([\mathrm{M}+\mathrm{H}]^{+}=436.2485 \mathrm{~m} / \mathrm{z}\right)$ corresponded to that of the paxilline (2) standard (Figure 3.14 trace ii).

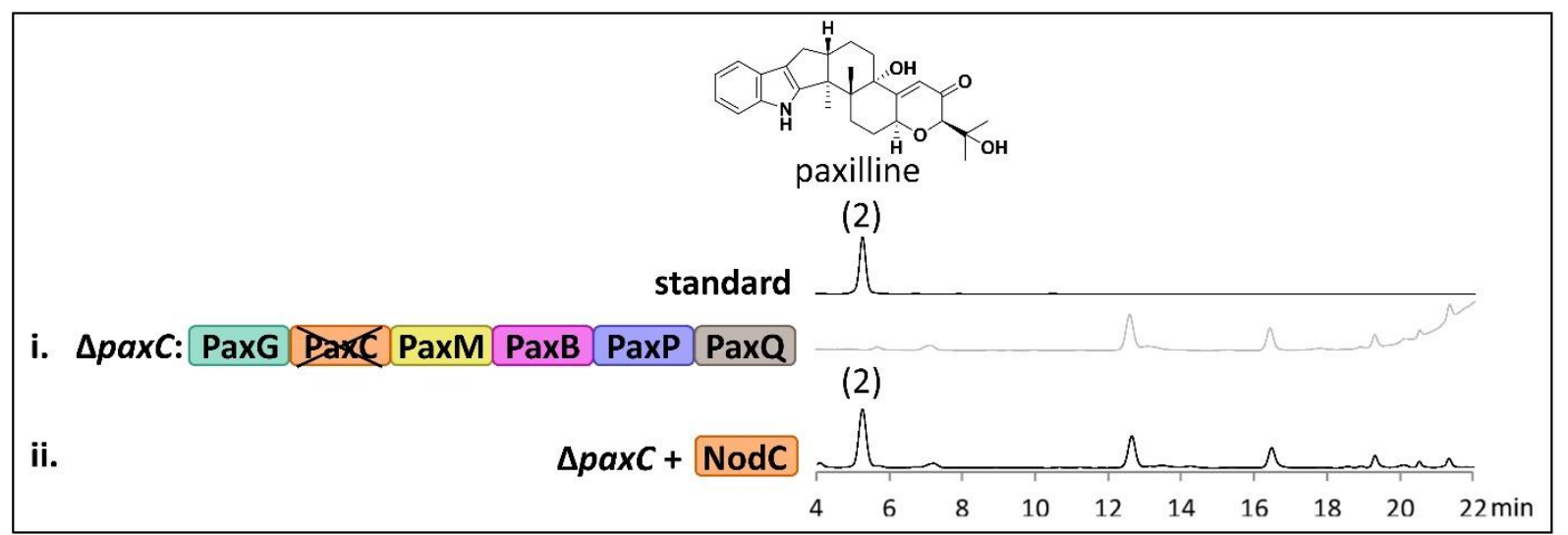

Figure 3.14. HPLC analysis $(271 \mathrm{~nm})$ of fungal extracts from the $P$. paxilli $\triangle$ paxC deletion mutant strain, PN2290 (trace i), and the nod $C_{C D S}$-containing pKV27 transformed $P$. paxilli $\triangle$ paxC deletion mutant strain (pKV27:PN2290) expressing the NodC enzyme from $H$. pulicicidum (trace ii). Extracted ion chromatograms are located in the Appendix (Figure 9.8).

\section{Conclusions}

Restoration of paxilline (2) biosynthesis by the introduction of the nod $C_{C D S}$ coding sequence into the $\triangle p a x C$ deletion mutant strain (PN2290) indirectly demonstrated that NodC is a functional orthologue of $\mathrm{PaxC}$, giving the first indication that the MIDAS system was a viable method for gene cluster reconstitution and corroborating the hypothesis that the NOD cluster located in the $H$. pulicicidum genome is responsible for nodulisporic acid A (1) biosynthesis. With this, attention was turned to the gene likely responsible for the expression of the protein product that catalysed the next step in the proposed nodulisporic acid biosynthetic pathway, the epoxidation of 3geranylgeranylindole (13). 


\section{NodM, THE KEY EPOXIDASE}

\section{Proposed Nodulisporic acid A gene cluster from H. pulicicidum (ATCC 74245)}

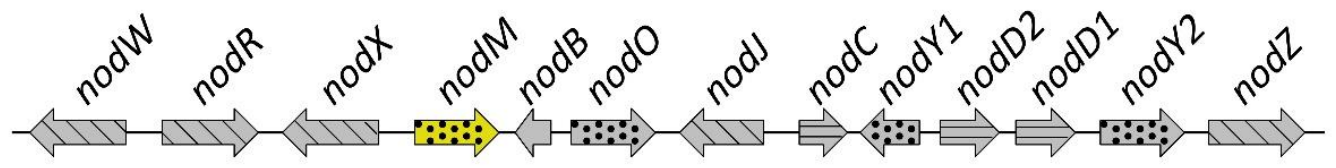

cytochrome P450 oxygenase $\square \backslash$

transferase

FAD-dependent oxygenase

[:::::

cyclase

Figure 4.1. Depiction of the predicted nodulisporic acid gene cluster (NOD) from $\mathrm{H}$. pulicicidum (ATCC ${ }^{\circledR} 74245^{\mathrm{TM}}$ ). The nodM gene is highlighted in yellow. 


\section{The indole diterpene epoxidases}

In step three of indole diterpene biosynthesis the geranylgeranyl tail of geranylgeranylindole, the product of the previous NodC-catalysed reaction, gets primed for cyclisation by epoxidation. Three different types of epoxidation of the geranylgeranyl tail of 3-geranylgeranylindole (13) have been described in indole diterpene biosynthetic pathways and are shown in Figure 4.2. In the first type, the C13C14 alkene is epoxidised to form 13,14-epoxy-3-geranylgeranylindole (16); in the second, both the C13-C14 and C17-C18 alkenes are epoxidised to form bisepoxy-3geranylgeranylindole (17); and in the third, the $\mathrm{C} 17-\mathrm{C} 18$ alkene is epoxidised to form 17,18-epoxy-3-geranylgeranylindole (15). Each epoxidation primes the indole diterpene scaffold for cyclisation in a variety of unique indole diterpene cores. In other words, this epoxidation serves as the key step that gives rise to the first branches of the complex indole diterpene biosynthetic tree.

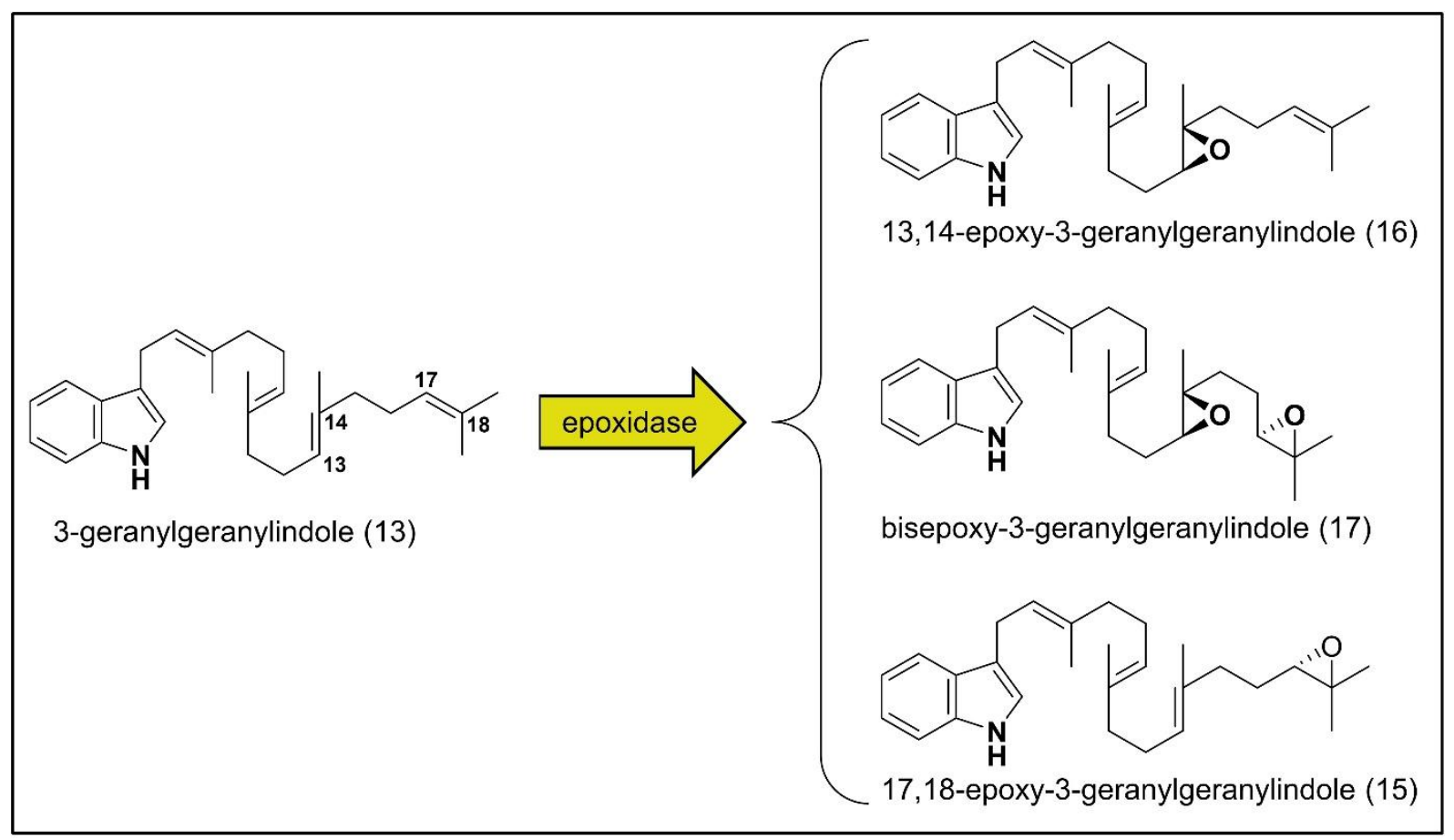

Figure 4.2. Depiction of the third secondary-metabolic step of indole diterpene biosynthesis. In this step, 3-geranylgeranylindole (13) is epoxidised into one or more epoxidised products: 13,14-epoxy-3-geranylgeranylindole (16), bisepoxy-3geranylgeranylindole (17) and 17,18-epoxy-3-geranylgeranylindole (15). The type of epoxidase (yellow arrow) is species dependant and determines what epoxidised product(s) is/are formed. The predicted cofactors for these epoxidases are NADPH and $\mathrm{O}_{2}$ but the majority of the in vitro enzyme characterisations have not been completed. 


\section{Identifying the NOD epoxidase}

For the biosynthesis of nodulisporic acid A (1) it was predicted that 13,14-epoxy-3geranylgeranylindole (16) is the dedicated geranylgeranylindole-epoxide intermediate (Figure 2.3) because all of the nodulisporic acids have a pentacyclic skeleton containing the key $\mathrm{C} 17-\mathrm{C} 18$ alkene moiety. By comparing the amino acid sequences of the functionally confirmed indole diterpene epoxidases to the gene products in the NOD cluster, one gene product, NodM, was identified that shares $>57 \%$ amino acid identity with five other indole diterpene epoxidases (Figure 4.3; 57.2\% to LtmM, 63.4\% AtmM, 65.8\% PaxM, 66\% PenM, and 67\% JanM). As seen in the NodC similarity screening, the enzyme from the JAN cluster, JanM, had the highest sequence similarity $(67 \%)$ to NodM. Intriguingly, all of the functionally characterised NodM homologues catalyse both the single epoxidation reaction of geranylgeranylindole to form 13,14 epoxy-3-geranylgeranylindole (16) as well as the double epoxidation to form bisepoxy3-geranylgeranylindole (17).26, 33, 40 Therefore, if NodM only catalysed the single epoxidation of geranylgeranyl indole to form 13,14-epoxy-3-geranylgeranylindole (16), as hypothesised, then it would be the first report of a functionally confirmed enzyme of this kind. 


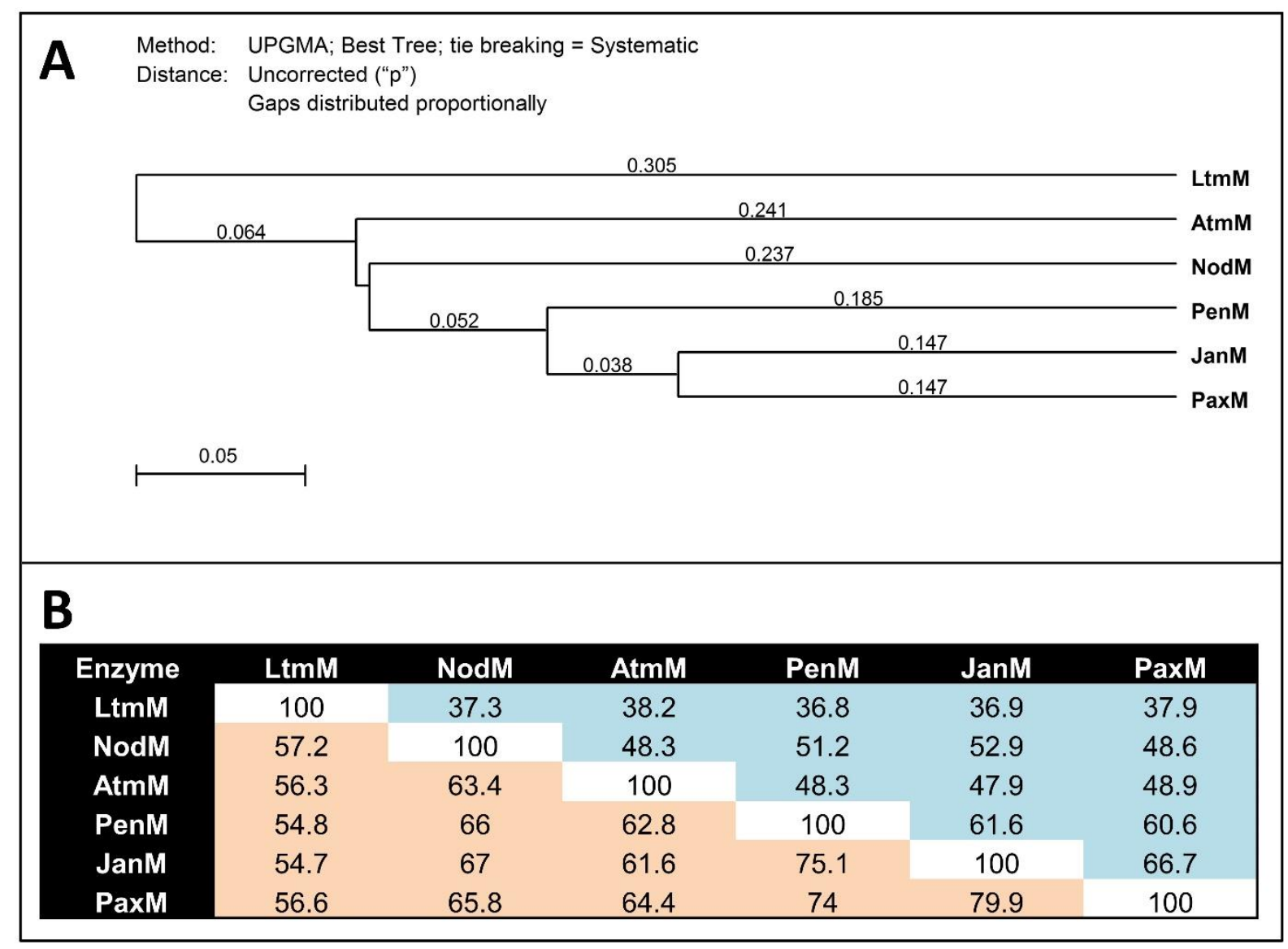

Figure 4.3. Phylogenetic tree $(\mathbf{A})$ and similarity matrix (B) of indole diterpene epoxidases (' $M$ ' enzymes). The phylogenetic tree depicts the difference in $\%$ identity scores for the amino acid residues of the 'M' enzymes. The similarity matrix depicts the \% identity scores (blue shaded regions ( $)$ ) and the \% similarity scores (orange shaded regions ( )) for the amino acid residues of the ' $M$ ' enzymes.

\section{Functional analysis of nodM}

To assess the epoxidative capacity of NodM (i.e. how many and what type of epoxidations it can catalyse), to establish directly its role in nodulisporic acid A (1) biosynthesis, and to explore how promoter choice affected compound biosynthesis, two MIDAS Level 3 vectors shown in Figure 4.4 [pKV34 (trpC $C_{\text {ProUtR-ntpll }}$ CDStrpC $C_{\text {UTRterm_trp }} C_{\text {ProUTR-nodM }} M_{C D S}$-trp $\left.C_{\text {UTRterm }}\right)$ and pKV63 (trpC $C_{\text {ProUTR-ntpll }}$ CDS $^{-}$ trpCUTRterm_paxMProUTR-nodMCDS-paxMUTRterm)] were designed that respectively contained the nodM $M_{C D S}$ driven by the trp $C_{\text {ProUTR }}$ promoter region from Aspergillus nidulans or driven by the paxM ProUTR promoter region from P. paxilli and inserted them into the $\triangle p a x M$ P. paxilli deletion mutant strain (PN2257). It was hypothesised that 
rather than restore paxilline (2) production, a $P$. paxilli strain that specifically produced emindole SB (18) would be generated.

\section{MIDAS assembly}

The MIDAS assembly of two plasmid constructs [pKV34 (trpC ProUTR-ntp/l $_{C D S}$ trpC $C_{\text {UTRterm_trp }} C_{\text {ProUTR-nodM }}$ CDS-trpC $\left._{\text {UTRterm }}\right)$ and pKV63 (trpC $C_{\text {ProUTR-ntpll }}$ CDStrpCUtRterm_paxMProUTR-nodM the nodMCDS are depicted in Figure 4.4. A total of six Level-1 MIDAS vectors encoding transcription unit modules for the trpCProUTR promoter region (pSK17), paxMProUTR promoter region (pSK4), nodM $M_{C D S}$ coding sequence (pSK18), ntp/l ${ }_{C D S}$ coding sequence (pSK16), trpCUTRterm terminator region (pSK15), and paxMUTRterm terminator region (pSK6) were used in the assembly of these two nodM $M_{C D S}$-containing Level-3 vectors. While Level-1 MIDAS vectors containing the trpC $C_{\text {ProUTR }}$ promoter region (pSK17), $n p t I_{C D S}$ coding sequence (pSK16) and trpCUTRterm terminator region (pSK15) were assembled during the functional analysis of nodC, the other three Level 1 MIDAS vectors encoding the paxMProUTR promoter region (pSK4), nodMCDS coding sequence (pSK18), and paxMUTRterm terminator region (pSK6) were newly assembled into individual Level-1 destination vectors. The six Level-1 vectors were used to assemble three unique transcription units in individual Level-2 MIDAS vectors pSK26 (trpC ProUTR-

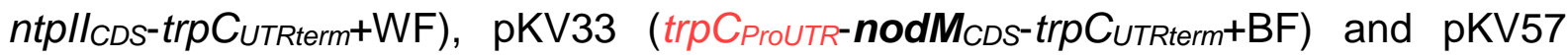
(paxM $M_{\text {ProUtR-nodM }}$ CDS-paxMUtRterm+BF). The final two Level-3 MIDAS vectors for use in fungal transformation pKV34 (trpC $C_{\text {ProUtR-ntpll }}$ CDS-trpC $C_{U T R t e r m}$ trp $C_{\text {ProUTR-nodM }}$ CDStrpC UTRterm) and pKV63 (trpC ProUTR-ntpll $_{\text {CDS-trpC }}$ UTRterm_paxMProUTR-nodM CDS- $_{\text {- }}$ paxMUTRterm) were respectively generated from the individual assembly of either pKV33

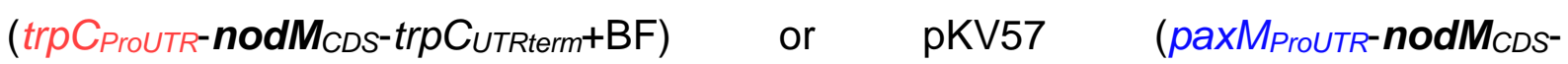

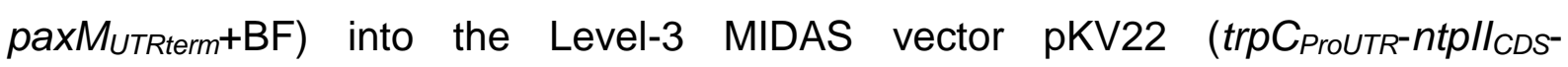
trpCutRterm), which was generated during nodC functionality testing and contains the geneticin resistance (npt/l) cassette. 


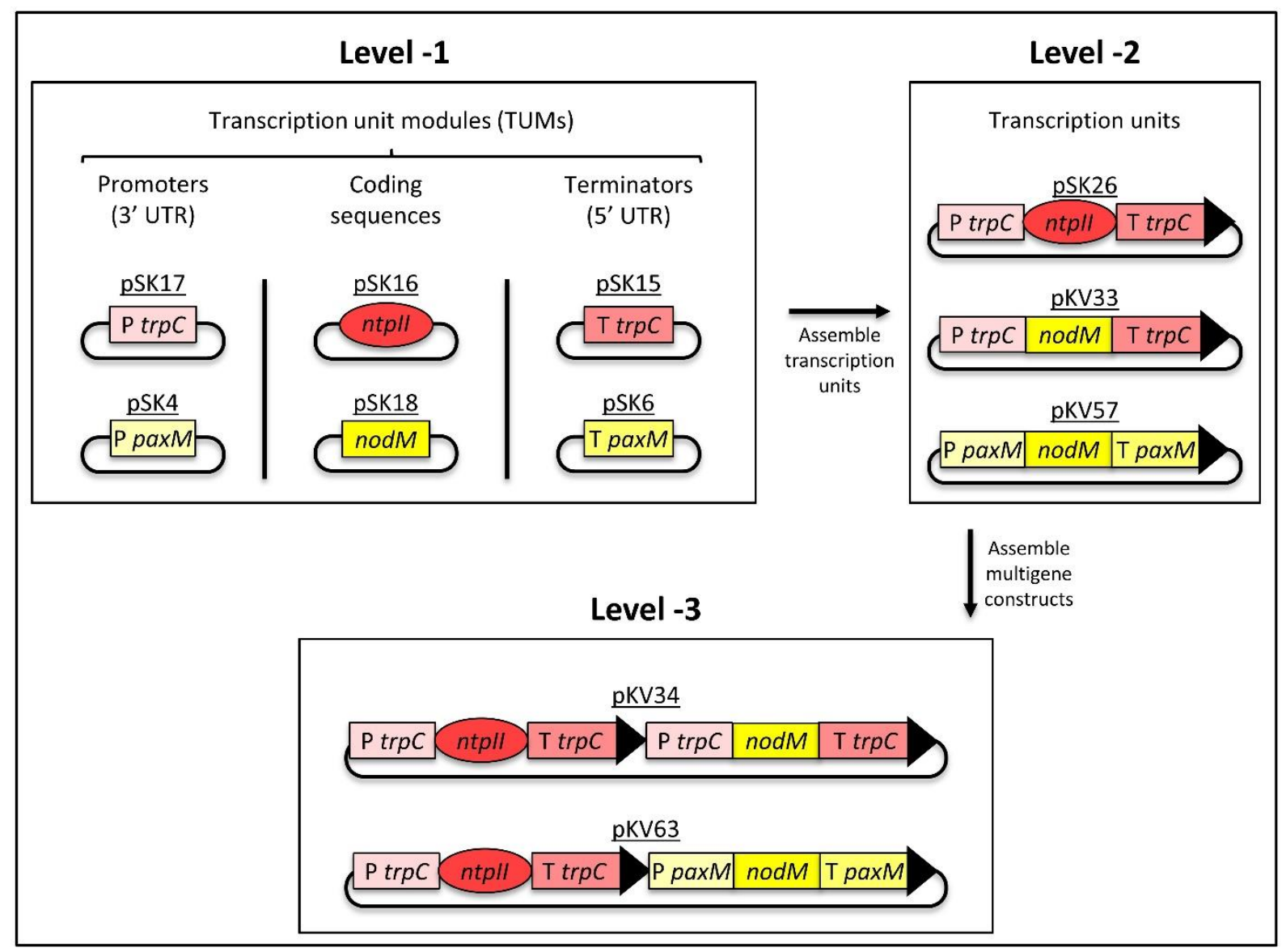

Figure 4.4. Depiction of the assembly of MIDAS vectors pKV34 (trp $C_{\text {ProUTR-ntpll }}$ CDS- $^{-}$

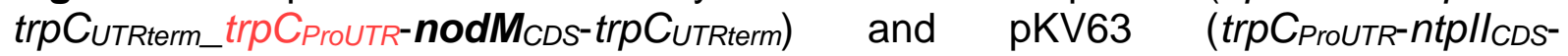
trpC UTRterm_paxM ProUTR-nodM MDS-paxMUTRterm) used to confirm the function of the nodMCDS coding sequence.

\section{Fungal transformations}

The two Level-3 nodM-containing vectors, pKV34 (trpC $C_{\text {ProUTR- }} n t p / I_{C D S}-$

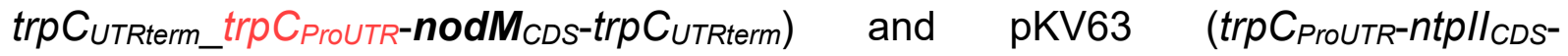
trpCUTRterm_paxMProUTR-nodMCDS-paxMUTRterm), were each individually inserted into protoplasts of the $\triangle p a x M$ deletion mutant strain PN2257, and a parallel transformation with vector pPN1857 (trpC $C_{\text {ProUTR-ntpll }}$ CDS-trpC UTRterm_paxMProUTR-paxMCDSpaxMUTRterm) was used as a positive control. The pPN1857 vector harbours the native paxM transcription unit and was used to restore paxilline (2) production during initial paxilline (2) biosynthetic pathway confirmation experiments. ${ }^{38}$ Six transformants were selected from the pKV34 (trpC $C_{\text {ProUTR-ntpll }}{\text { CDS-trp } C_{U T R t e r m} \text { trp } C_{\text {ProUTR-nodM }} \text { CDS- }}$ trp $C_{\text {UTRterm }}$ ) transformation, ten were selected from the pKV63 (trp $C_{\text {ProUTR-ntpll }}$ CDS- $^{-}$ trpCUTRterm_paxMProUTR-nodM MDS-paxMUTRterm) transformation, and a total of eight 
transformants were selected from two pPN1857 (trpC ProUTR-ntp/l $_{C D S^{-}}$ trpC UTRterm_paxM ProUTR-paxM $M_{C D S}$-paxMUTRterm) control transformations. The 24 transformants were grown in production medium and analysed by TLC and LCMS as shown in Figures 4.5 and 4.6. As expected, the initial TLC screening suggested that paxilline (2) was produced by seven of eight paxMCDS-containing control transformants (Figure 4.5, lanes: 7-10 and 21-23) and none of the nodMCDS-containing transformants (Figure 4.5, lanes: 1-6 and 11-20), and that at least eight of sixteen nodM $M_{C D S}$-containing transformants [two of six pKV34 (trpC ProUTR-ntp/ICDS-trpCUTRterm_trpC $C_{\text {ProUTR-nodM }}$ CDS- $_{\text {- }}$ trpCUTRterm) and five of ten pKV63 (trpC $C_{\text {ProUTR-ntpll }}$ CDS-trpCUTRterm_paxMProUTR-

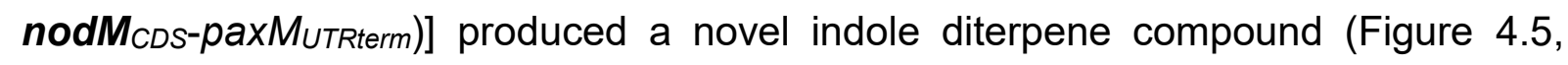
lanes: $4,5,11,13,15,16,20)$. 


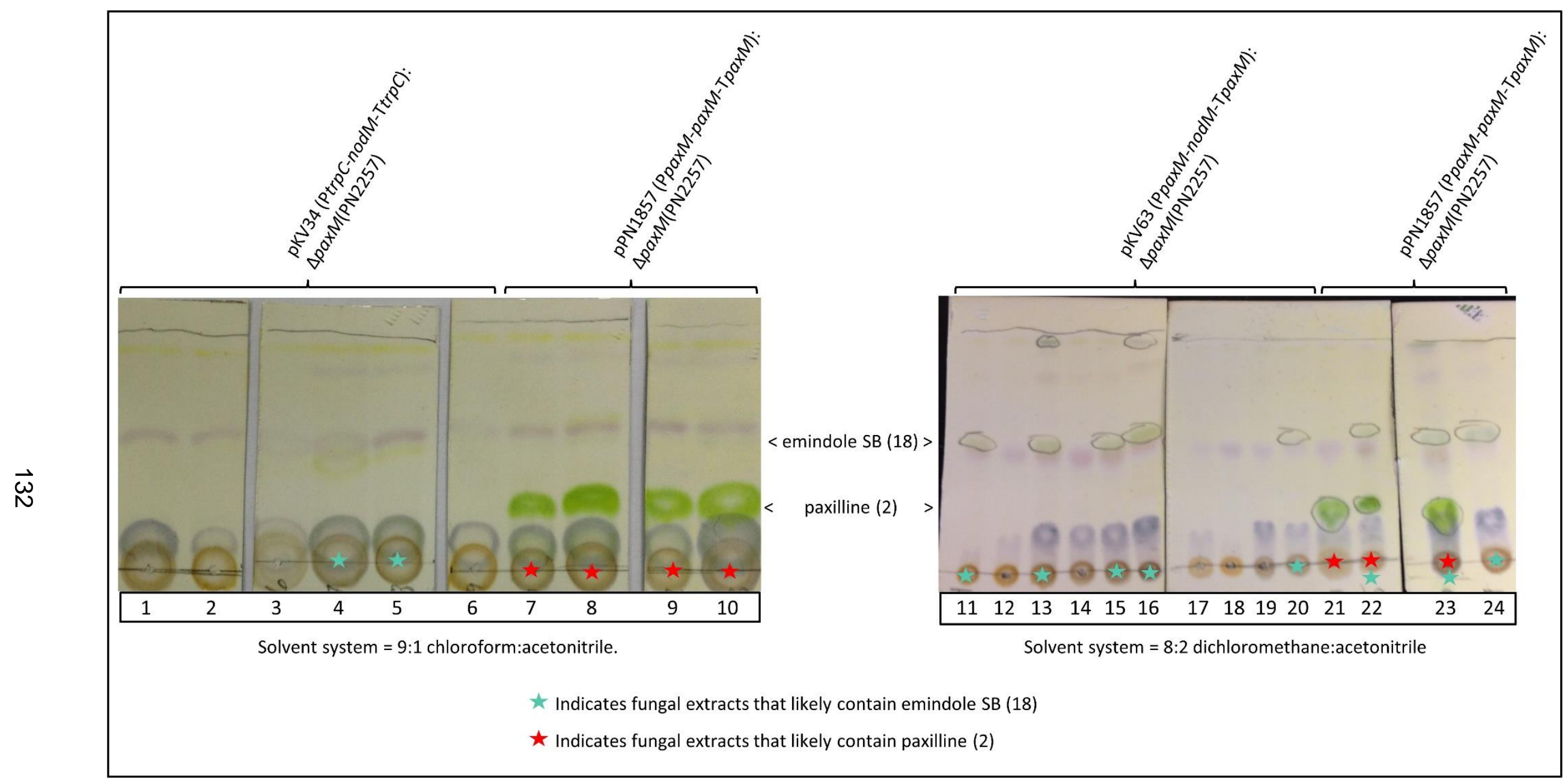

Figure 4.5. TLC results of fungal extracts from the individual transformation of $P$. paxilli $\triangle$ paxM deletion mutant PN2257 with three

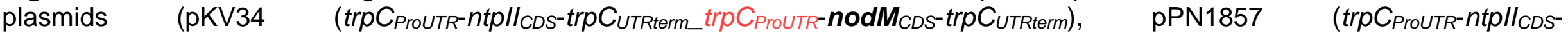
trpCUTRterm_paxM ProUTR-paxM $M_{C D S}$-paxMUTRterm), and pKV63 (trpC $C_{\text {ProUTR-ntpll }}$ CDS-trpC UTRterm_paxMProUTR-nodMCDS-paxMUTRterm)). 


\section{A note about promoter choice}

Importantly, in these experiments it was observed that there was greater compound production when the nodM $M_{C D S}$ coding sequence was driven by the paxM $M_{\text {proUTR }}$ promoter region (pKV63) than when driven by the trpC $C_{\text {proUTR }}$ promoter region (pKV34) (Figure 4.5 , lane 4 and 5 vs. lanes $11,13,14,15)$. Although this difference was not quantified by LCMS, the visual inspection by TLC clearly implied that promoter choice greatly influenced compound production. It is especially important to consider promoter choice during host optimisation because there are multiple hurdles to overcome viz. negative feedback loops and overexpression, which could lead to a decrease in cell performance and potentially result in cell death. ${ }^{73}$

\section{Novel compound identification}

To identify the novel compound that was produced by the nodMCDS-containing transformants, a high producing strain (as determined by visual inspection of TLCs)

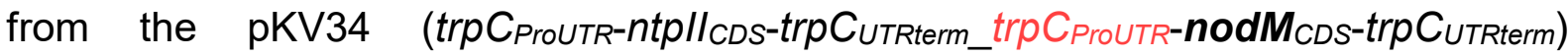
transformants (Figure 4.5, lane 5) and the pKV63 (trpC ProUTR-ntpll $_{\text {CDS- }}$ trpC UTRterm_paxMProUTR-nodMCDS-paxMUTRterm) transformants (Figure 4.5, lane 13) were analysed by LCMS. Evaluation of the spectra revealed that, based on the retention time $(19.3 \mathrm{~min})$ and mass (high resolution-mass spectra $[\mathrm{M}+\mathrm{H}]^{+}=406.3109$ $\mathrm{m} / \mathrm{z}$, corresponding to $\mathrm{C}_{28} \mathrm{H}_{39} \mathrm{NO}$ ), the novel compound was emindole $\mathrm{SB}$ (18). Notably, no paxilline (2) or paspaline (11) were present in the either of the transformant extracts 
[results only shown for the the pKV63 (trpC ProUTR-ntpll $_{\text {CDS-trpC UTRterm_paxMProUTR- }}$

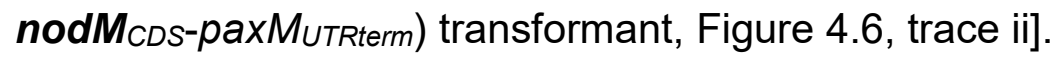

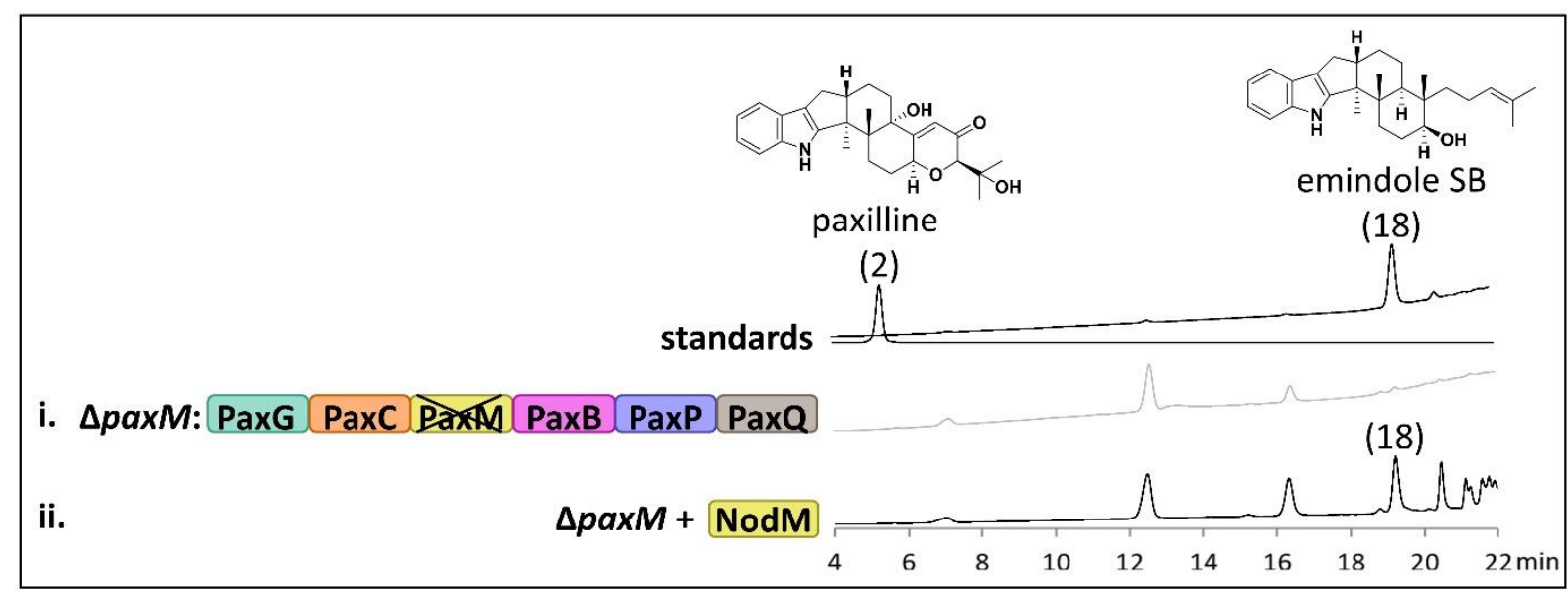

Figure 4.6. HPLC analysis $(271 \mathrm{~nm})$ of fungal extracts from the $P$. paxilli $\triangle$ paxM deletion mutant strain, PN2257 (trace i), and the P. paxilli $\triangle$ paxM deletion mutant strain

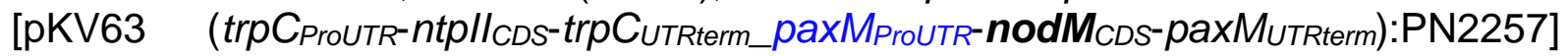
expressing the NodM enzyme from $H$. pulicicidum (trace ii). Extracted ion chromatograms are located in the Appendix (Figure 9.9).

\section{Conclusions}

\section{Explanation for emindole SB (18) production}

It has previously been shown that paxB, a gene present in the $\triangle$ paxM P. paxilli mutant strain, is capable of cyclising both 13,14-epoxy-3-geranylgeranylindole (16) and bisepoxy-3-geranylgeranylindole (17) to produce emindole SB (18) and paspaline (11) respectively (see Figure 4.7). ${ }^{40}$ Thus, these results indirectly confirmed the hypothesis that NodM was only capable of a single epoxidation at the C13-C14 position of 3geranylgeranylindole (13) to produce 13,14-epoxy-3-geranylgeranylindole (16) because the absence of paspaline (11) or paxilline (2) production demonstrate that this enzyme was not capable of producing the double epoxidised product bisepoxy-3geranylgeranylindole (17). NodM is the first functionally confirmed epoxidase that performs only one epoxidation of 3-geranylgeranylindole (13) to form 13,14-epoxy-3geranylgeranylindole (16). By solely epoxidising the C13-C14 alkene of geranylgeranylindole, NodM ensures that the only available substrate for cyclisation is 13,14-epoxy-3-geranylgeranylindole (16); thereby establishing the foundation of nodulisporic acid biosynthesis. 


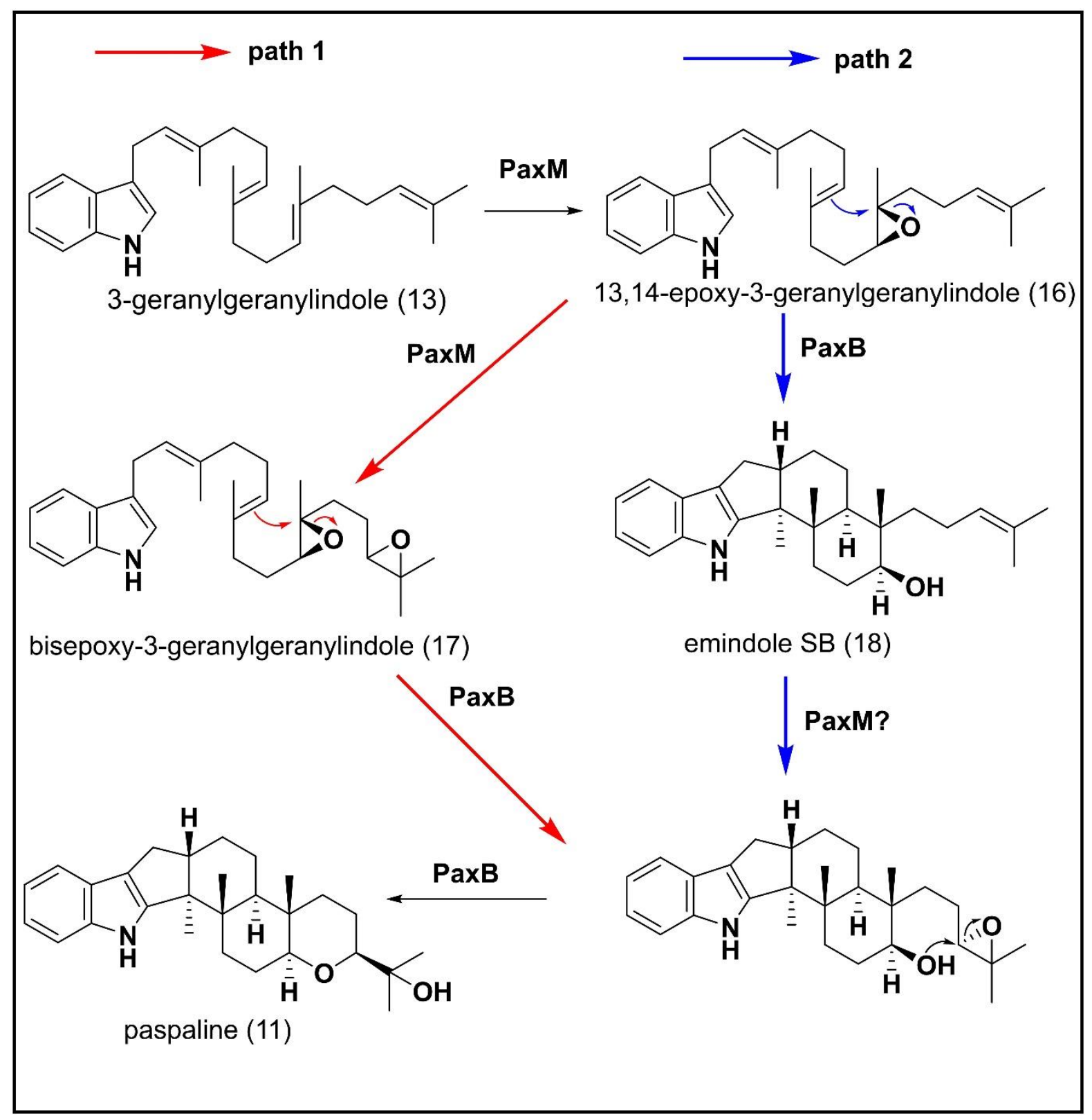

Figure 4.7. Two-path stepwise epoxidation scheme of 3-geranylgeranylindole (13) involving the epoxidase (PaxM) and the cyclase (PaxB) proposed by Tagami et al. (2013). .40 


\section{NodB, A PROMISCUOUS INDOLE DITERPENE CYCLASE}

Proposed Nodulisporic acid A gene cluster from H. pulicicidum (ATCC 74245)

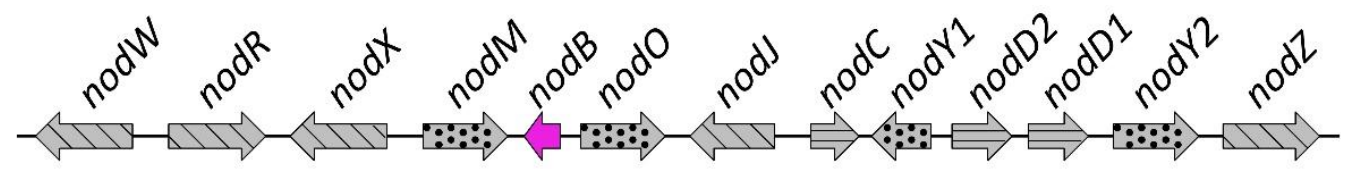

cytochrome P450 oxygenase

Figure 5.1. Depiction of the predicted nodulisporic acid gene cluster $(N O D)$ from $H$. pulicicidum $\left(\mathrm{ATCC}^{\circledR} 74245^{\mathrm{TM}}\right)$. The nodB gene is highlighted in pink. 


\section{Indole diterpene cyclases are notoriously promiscuous}

The indole diterpene cyclases are the most elaborate group of enzymes in indole diterpene biosynthesis as they have an extremely diverse capacity to cyclise the three epoxide products [13,14-epoxy-3-geranylgeranylindole (16), bisepoxy-3geranylgeranylindole (17) and 17,18-epoxy-3-geranylgeranylindole (15)] into a variety of distinct core compounds (Figure 5.2). The cyclases produce a range of ring closures, often coupled to sigmatropic shifts, which result in the addition of 2-4 rings to the epoxidised-geranylgeranyl tail of the epoxide substrate. To date, every functionally confirmed indole diterpene cyclase has shown promiscuity around its ability to cyclise more than one of the three epoxide substrates [13,14-epoxy-3-geranylgeranylindole (16), bisepoxy-3-geranylgeranylindole (17) and 17,18-epoxy-3-geranylgeranylindole (15)]. ${ }^{33,40}$ While their promiscuity leads to the delivery of a wide array of compounds, the cyclases usually deliver only one major cyclised core that is used in one specific biosynthetic pathway of the native indole diterpene producer. For example, the cyclase PaxB, used in the paxilline (2) biosynthetic pathway of $P$. paxilli, shows substrate promiscuity around its ability to cyclise 13,14-epoxy-3-geranylgeranylindole (16) into emindole SB (18) and bisepoxy-3-geranylgeranylindole (17) into paspaline (11); of which paspaline (11) is the major product and dedicated substrate for paxilline (2) biosynthesis. 


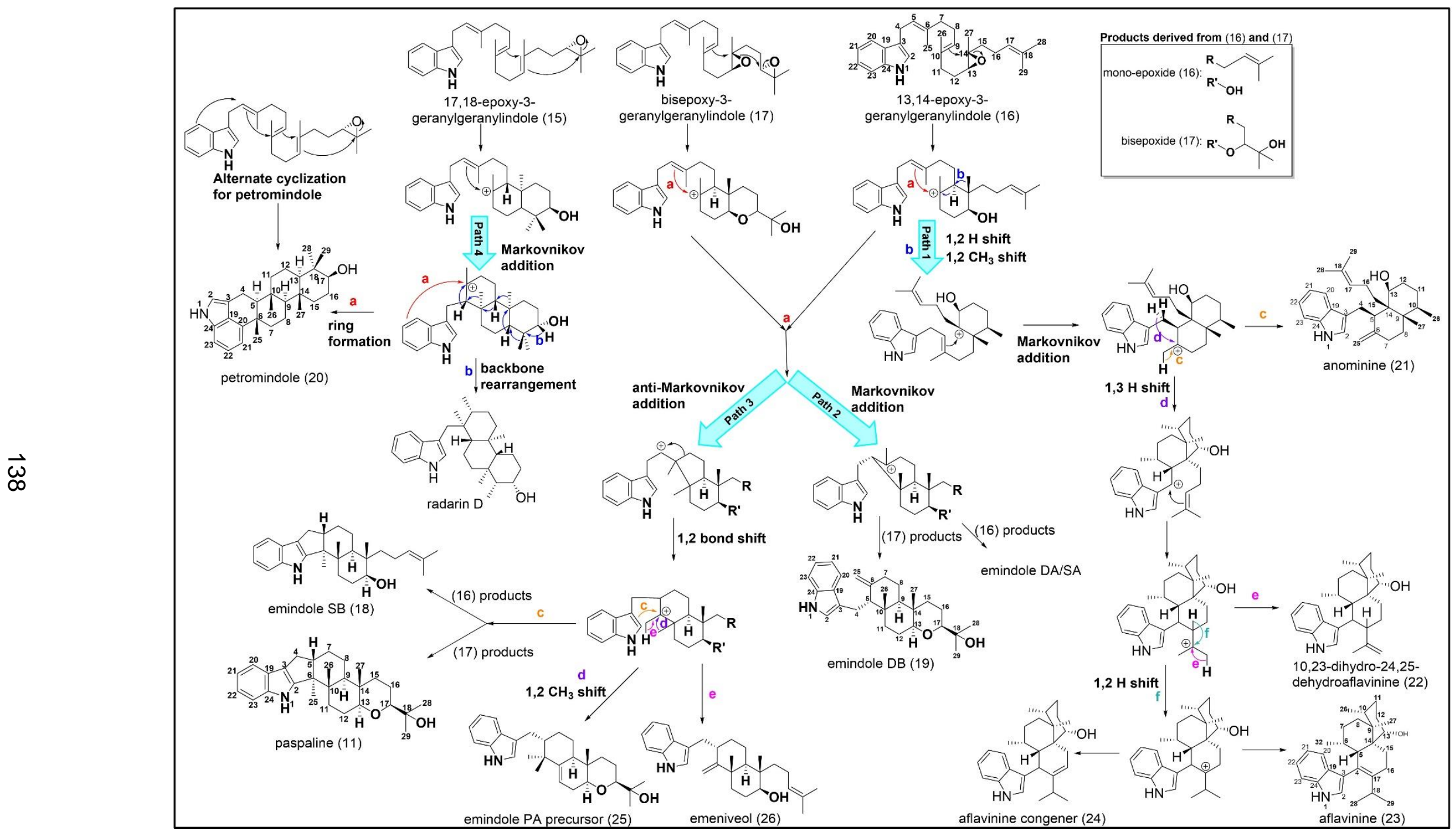

Figure 5.2. Depiction of the pathway diversity generated by indole diterpene cyclases. Indole diterpene cyclases from different species have the capacity to cyclise the epoxidised substrates [(15), (16), and (17)] in different ways using either a Markovnikov reaction mechanism or an anti-Markovnikov reaction mechanism often coupled with select sigmatropic shifts. 


\section{Identifying the NOD cyclase}

In terms of nodulisporic acid biosynthesis, emindole SB (18) was predicted to be the dedicated pentacyclic core intermediate (Figure 2.3). Using amino acid sequence comparisons of the functionally confirmed indole diterpene cyclases to the gene products in the NOD cluster a likely indole diterpene cyclase, NodB, that shares $>48 \%$ amino acid identity with five other indole diterpene cyclases (Figure 5.3; 48.8\% LtmB, 63\% PaxB, 64.2\% AtmB, 65.4\% JanB, 67.9\% PenB) was identified. As with the previous steps, the MIDAS platform was used to generate plasmids for expression and functional characterisation of nodB.

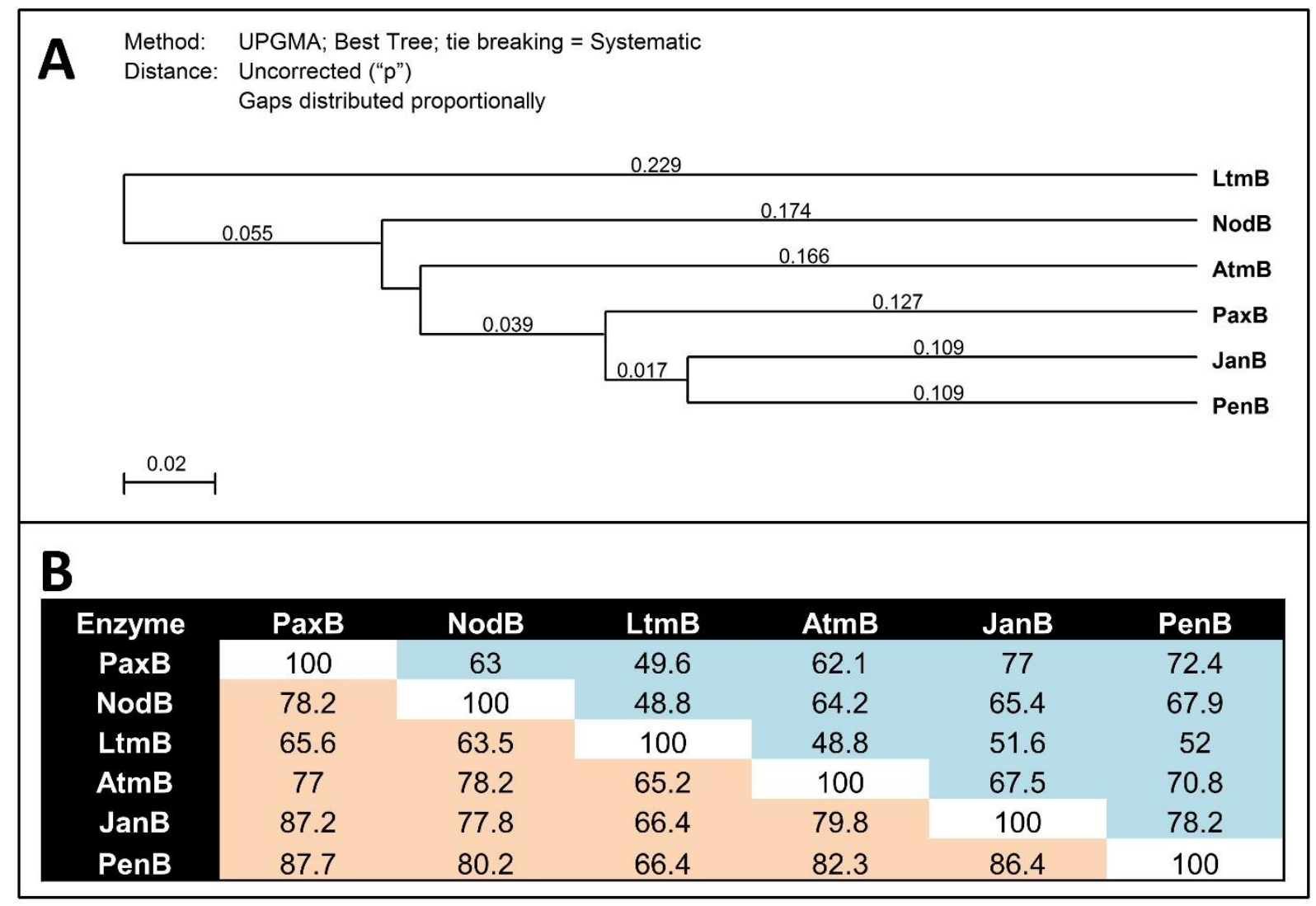

Figure 5.3. Phylogenetic tree (A) and similarity matrix (B) of indole diterpene epoxidases (' $B$ ' enzymes). The phylogenetic tree depicts the difference in $\%$ identity scores for the amino acid residues of the 'B' enzymes. The similarity matrix depicts the $\%$ identity scores (blue shaded regions ( $)$ ) and the \% similarity scores (orange shaded regions ( )) for the amino acid residues of the 'B' enzymes. 


\section{Functional analysis of nodB}

\section{Initial approach}

Initially, the functionally characterisation of $\operatorname{nod} B$ was attempted using a single gene heterologous expression experiment of a $\operatorname{nod} B_{C D S}$-containing transcription unit in a $P$. paxilli $\triangle$ paxB deletion mutant (PN2458). To this end, the nodBCDs coding sequence, driven by the trp $C_{\text {ProUTR }}$ promoter region from $A$. nidulans, was assembled, along with the hygromycin resistance cassette encoded by the $h p h$ transcription unit, into a Level 3 MIDAS vector pKV36 ( $h p h_{\text {ProUTR-hph }}$ CDs-hphUTRterm_trpC $C_{\text {ProUTR-nodB }}$ CDs-trpC UTRterm) and introduced into the P. paxilli $\triangle$ paxB deletion mutant strain PN2458. Contrary to expectations, all of the extracts from ten transformants did not contain any detectable indole diterpenes as shown in Figure 5.4, lane 1-10.

Notably, no control plasmid with the native paxB transcription unit was available to confirm that its complementation of the P. paxilli $\triangle$ paxB deletion mutant strain PN2458 would restore paxilline (2) biosynthesis. In part, this was due to the fact that $P$. paxilli $\triangle$ paxB deletion mutant strain PN2458 was never transformed with the native paxB transcription unit, as was done with the $P$. paxilli $\triangle$ pax $C$ and $\triangle$ paxM deletion mutants using pPN1783 and pPN1857 respectively. ${ }^{38}$ Careful examination of the PAX cluster revealed that a portion of the paxMProutR promoter region ( $750 \mathrm{bp}$ ) overlaps with the pax $B_{C D S}$ coding sequence. Therefore, when the $P$. paxilli $\triangle$ pax $B$ deletion mutant was created, it may have also incurred a paxM deletion. Rather than explore the intricacies of the potential problems within the genome of the $P$. paxilli $\triangle$ paxB deletion mutant (PN2458), a different approach involving the generation of larger multigene constructs was used to analyse the function of nodB. 


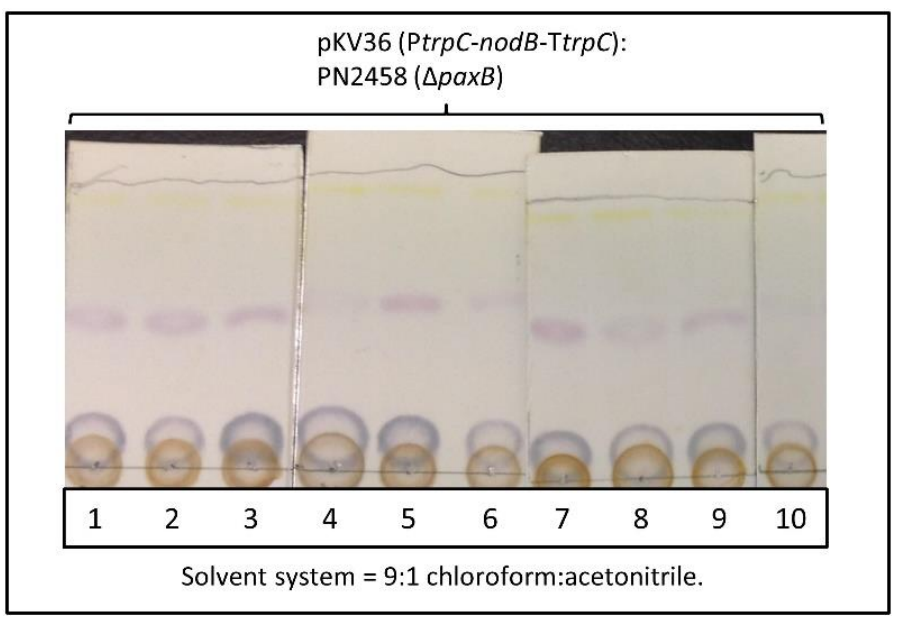

Figure 5.4. TLC results of fungal extracts from ten transformants of plasmid pKV36

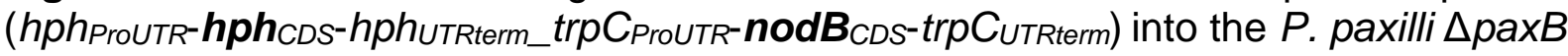
deletion mutant PN2458.

\section{Assembly of multigene constructs}

As opposed to the single indole diterpene transcription unit that was used in the initial attempt to elucidate the function of nodB, the multigene approach required the assembly of four indole diterpene transcription units that encoded the pax $G_{C D S}$, nod $C_{C D S}$, nod $M_{C D S}$ and nodB $B_{C D S}$. Rather than use the MIDAS Level 2 vectors that were assembled during the functional analyses of nod $C$, nod $M$ and the initial nod $B$ approach for assembly of the multigene construct, new MIDAS Level 2 vectors were designed so that the position and direction of each transcription unit in the final multigene construct resembled that found in the native $P A X$ and $N O D$ gene clusters as shown in Figure 5.5. The final multigene construct contained five transcription units encoding

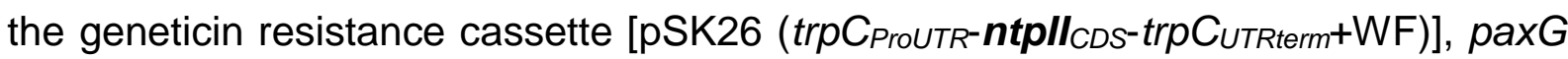

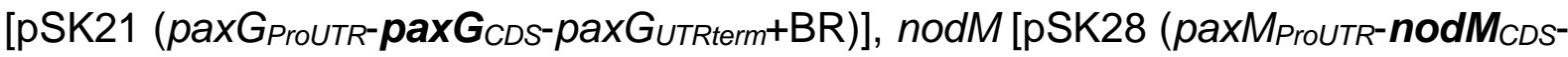
paxMutRterm+WF)], nodB [pSK29 (paxB ProutR-nodB $_{\text {CDs-paxBUtRterm }}+\mathrm{BR}$ )] and nodC [pSK60 (pax ProUtR-nod $_{\text {CDS }}$-pax $C_{\text {UTRterm }}+\mathrm{WF}$ )] that were respectively assembled into

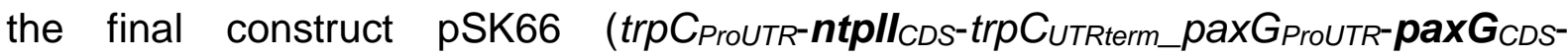

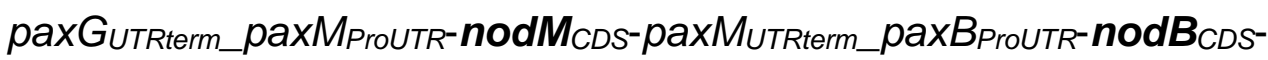
paxB UTRterm_pax $C_{\text {ProUTR-nod }} \boldsymbol{C}_{\text {CDS }}$ pax $C_{\text {UTRterm) }}$ in an iterative approach (Figure 5.6). 


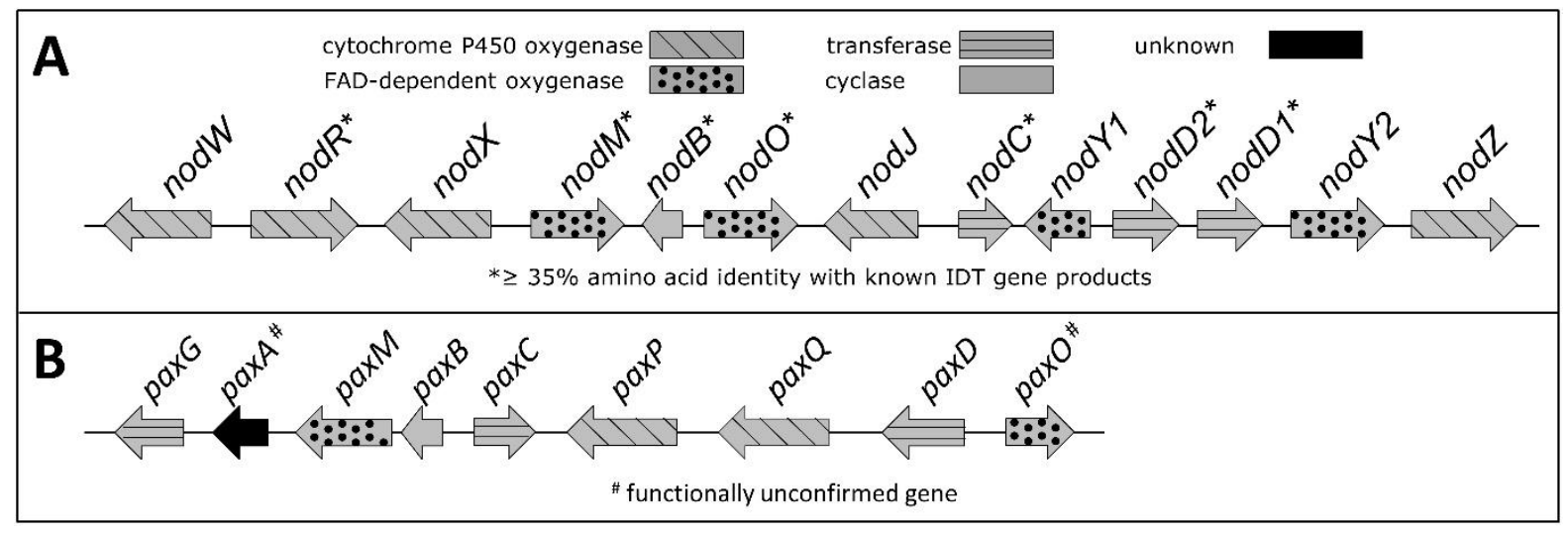

Figure 5.5. Figure of the $N O D$ cluster $(\mathbf{A})$ and the $P A X$ cluster (B) showing the order and direction of each gene (arrows). 


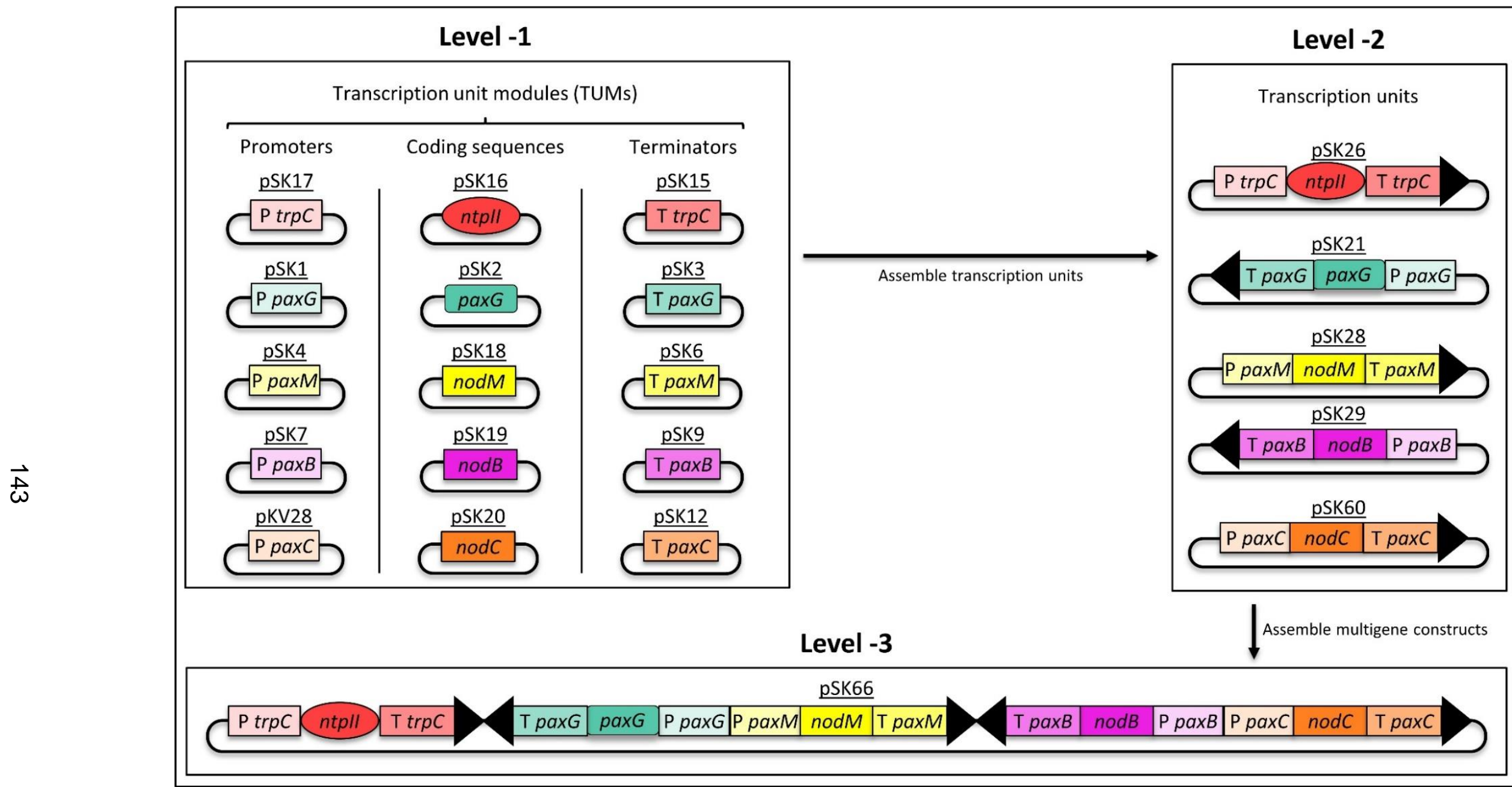

Figure 5.6. Depiction of the MIDAS approach used to assemble the multigene construct pSK66 (trpC ProUTR-ntpll $_{C D S^{-}}$

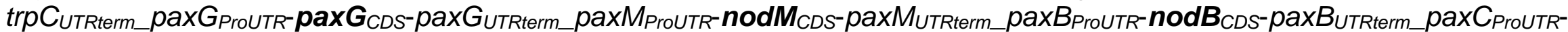
nod $C_{C D S}$-pax $C_{U T R t e r m)}$. In Level-1, the plasmids encoding transcription unit modules (promoters, coding sequences, terminators) are shown. In Level-2 the plasmids that encode the five full length transcription units are shown -where the arrow represents the direction of transcription. In Level-3, the final multigene plasmid (pSK66) that was used for fungal transformation is depicted. 
The high percentage amino acid identity that NodB shared with the functionally confirmed indole diterpene cyclases suggested that NodB was capable of similar catalytic function and was therefore also likely to be capable of performing two cyclisations; however, only one type of cyclised indole diterpene core, that corresponding to the core of the nodulisporic acids, has ever been isolated from the native nodulisporic acid producer, $H$. pulicicidum. One possible explanation is that only one epoxidised intermediate, 13,14-epoxy-3-geranylgeranylindole (16), is provided by NodM, as was demonstrated during the functional confirmation of NodM, and thus only one cyclised product is formed. Alternatively, NodB may only be able to cyclise one epoxide intermediate and thus NodM and NodB would work in conjunction to establish the foundation of nodulisporic acid biosynthesis. To test whether NodM solely established the foundation of nodulisporic acid production or whether NodM and NodB worked in conjunction NodB would have to be presented with an alternative epoxide product, bisepoxy-3-geranylgeranylindole (17) and/or 17,18-epoxy-3geranylgeranylindole (15), and extracts would have to be examined for alternative cyclic core compounds [e.g. paspaline (11)]. Hence, a second multigene construct was assembled to do just that.

The second multigene construct, pKV74 (trpC $C_{\text {ProUTR-ntpll }}$ CDS-trpC $_{U T R t e r m}$ pax ProUTR- $_{\text {- }}$ paxGCDS-paxGUTRterm_paxMProUTR-paxM $M_{C D S}$-paxMUTRterm_paxB ProUTR-nodB $_{C D S}-$

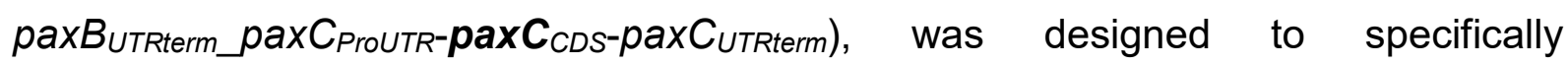
incorporate a paxM transcription unit and a nodB transcription unit into the same Level3 vector (Figure 5.7, Level-3). The paxM transcription unit was used in this second multigene construct because it catalyses the production of bisepoxy-3geranylgeranylindole (17) (Figure 5.8), the ideal substrate to test the promiscuity of NodB because all of the functionally confirmed cyclases that NodB is highly similar to are capable of cyclising bisepoxy-3-geranylgeranylindole (17) to paspaline (11). ${ }^{33,40}$ Therefore, it was hypothesised that paspaline (11) would be produced if NodB were provided with bisepoxy-3-geranylgeranylindole (17) (Figure 5.8). 


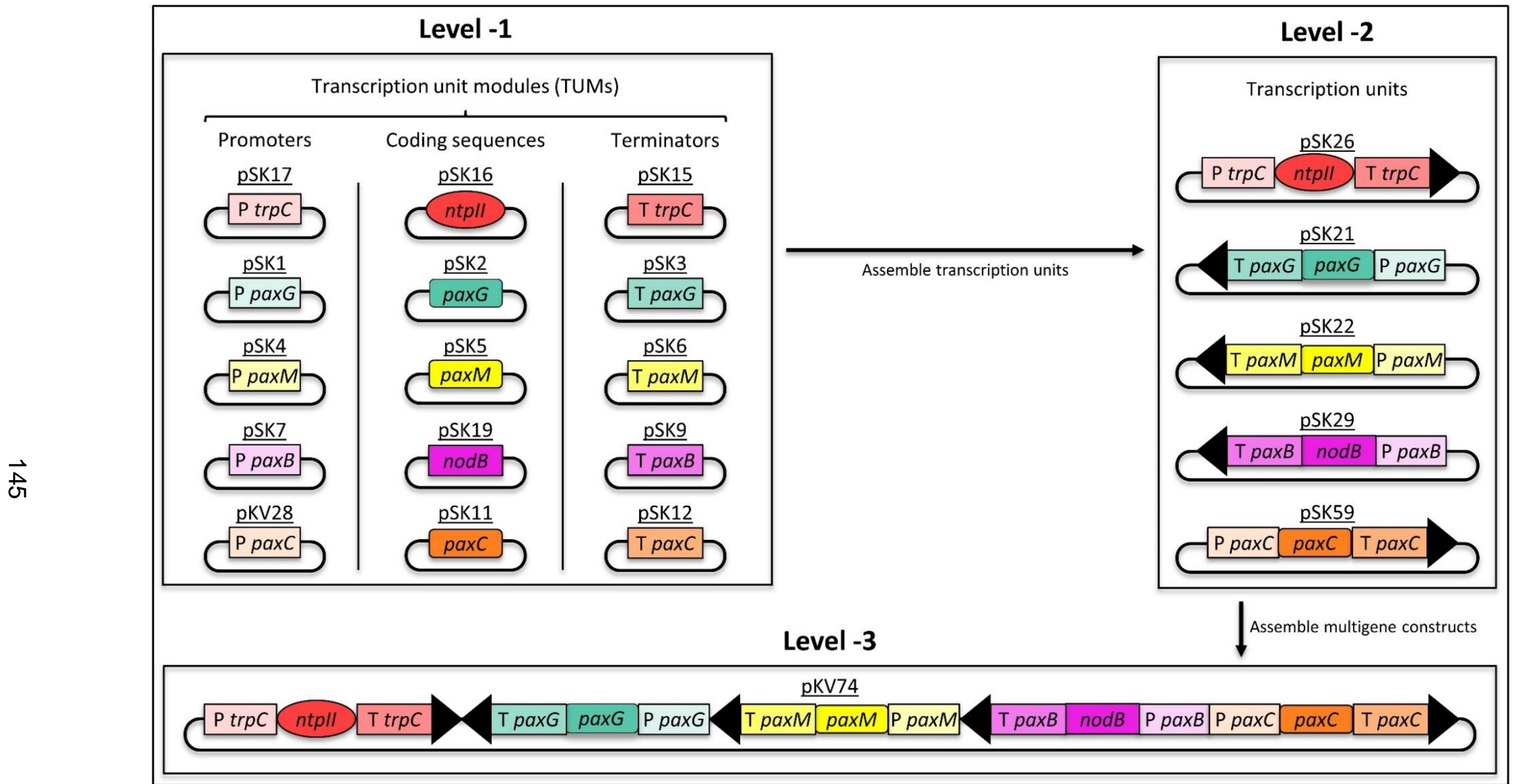

Figure 5.7. Depiction of the MIDAS approach used to assemble the multigene construct pKV74 (trpC ProUTR-ntpll $_{C D S^{-}}$

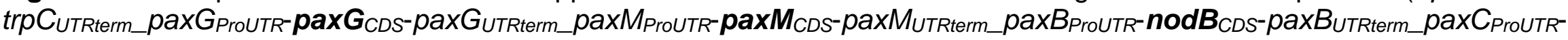
pax $C_{C D S}$-pax $C_{U T R t e r m)}$. In Level-1, the plasmids encoding transcription unit modules (promoters, coding sequences, terminators) are shown. In Level-2 the plasmids that encode the five full length transcription units are shown -where the arrow represents the direction of transcription. In Level-3, the final multigene plasmid (pKV74) that was used for fungal transformation is depicted. 


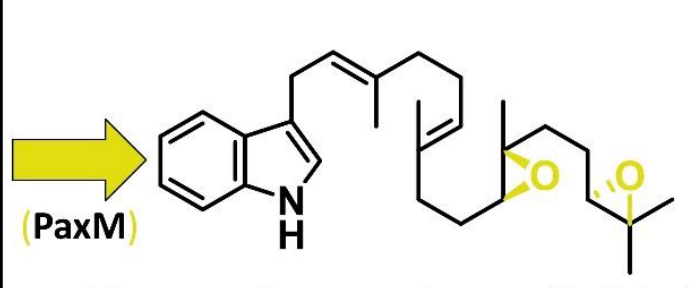

bisepoxy-3-geranylgeranylindole (17)

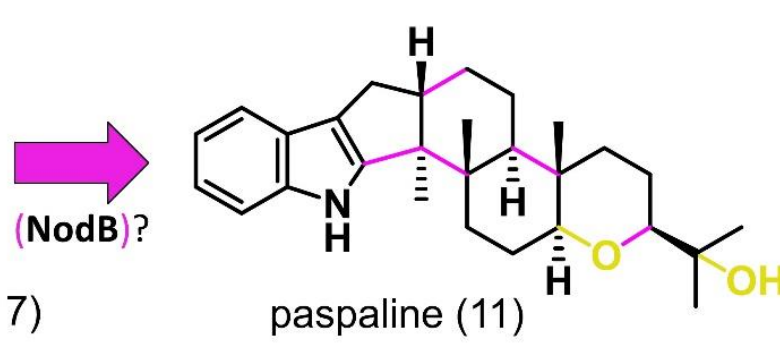

paspaline (11)

Figure 5.8. Is NodB capable of cyclising bisepoxy-3-geranylgeranylindole (17) to paspaline (11)?

In addition to the paxM and nodB transcription units, the second multigene construct,

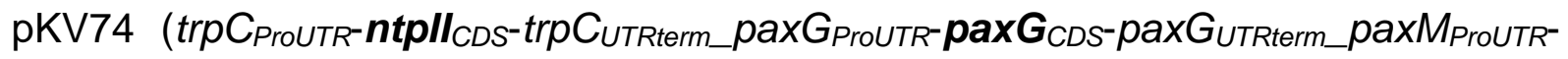
paxM $M_{C D S}$-paxMUTRterm_paxB ProUTR-nodB $_{C D S}$-paxBUTRterm_pax $C_{\text {ProUTR-pax }}$ CDS $_{\text {- }}$ pax $($ UtRterm), also contained the geneticin resistance cassette and the pax $G$ and paxC transcription units (Figure 5.4, Level-3). Thus, as with the first multigene construct

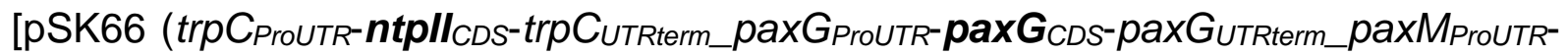
nodM $M_{C D S}$-paxMUTRterm_paxB ProUTR-nodB $B_{C D S}$-paxBUTRterm_pax $C_{\text {ProUTR-nod }}$ CDS $_{\text {- }}$ pax $\left.\left.C_{\text {UTRterm }}\right)\right]$, the second [pKV74 (trpC ProUTR-ntpll $_{\text {CDS-trpC }}$ UTRterm_paxG $G_{\text {ProUTR- }}$ paxG $G_{C D S}$-paxGUTRterm_paxMProUtR-paxM MDS-paxMUTRterm_paxBProUTR-nodB $B_{C D S}$ paxBUTRterm_pax $C_{\text {ProUTR-pax }} \boldsymbol{C}_{C D S}$-pax $\left.C_{U T R t e r m)}\right)$ also contained a total of five transcription units, three that were from the same Level 2 MIDAS vectors as used in the first multigene construct (pSK66): the geneticin resistance cassette [pSK26 (trp $C_{\text {ProUTR-ntpll }}$ CDS-trpC $\left.\left._{\text {UTRterm }}+\mathrm{WF}\right)\right], \quad$ paxG $\quad\left[\mathrm{pSK} 21 \quad\right.$ (paxG ProUTR-paxG $_{C D S^{-}}$

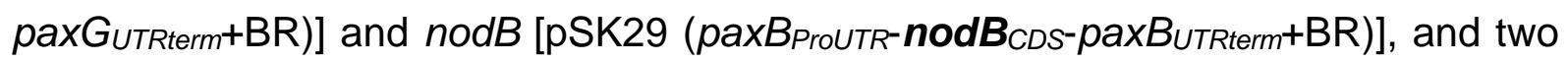

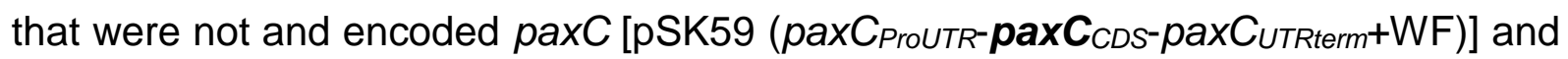

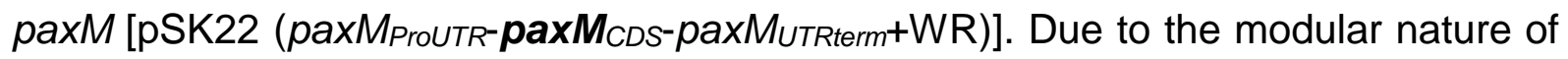
MIDAS and the iterative addition of transcription units to the final Level-3 construct, the Level-3 MIDAS construct that contained the geneticin resistance cassette and the

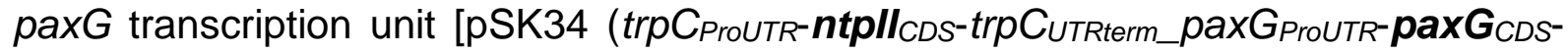
paxGUTRterm)], assembled during construction of the first multigene construct (pSK66), were used as the first destination vector for this second multigene construct (pKV74). 


\section{Transformations of the multigene constructs}

Upon completion of the multigene assembly of pSK66 (trp $C_{\text {ProUTR-ntpll }}$ CDStrpC UTRterm_paxG ProUTR-pax CDS-paxG UTRterm_paxMProUTR-nodM $_{\text {CDS- }}$

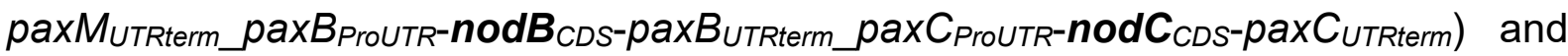

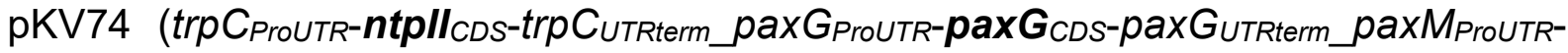

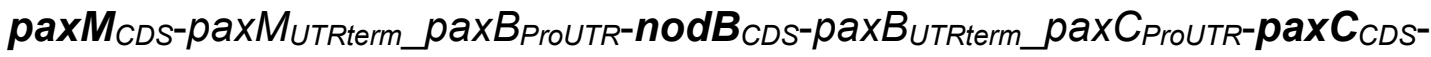

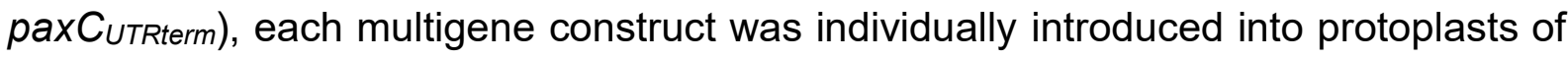
the P. paxilli strain PN2250 (CY2) that contains a deletion of the entire PAX locus. Fifteen transformants were picked from the pSK66 transformation and ten transformants were picked from the pKV74 transformation. TLC analysis of the pSK66 transformant extracts indicated that eleven of fifteen produced an indole diterpene compound with the same retention time as that of emindole SB (18) as shown in Figure 5.9 , lanes 2,4 to $8,10,13-16$. In contrast, no obvious indole diterpene compounds were visible from the TLC analysis of the nine pKV74 transformants (Figure 5.9, lanes 17 to 26). To confirm the production of emindole SB (18) by the pSK66 transformants and to assess if any indole diterpenes were present in the pKV74 transformants that may have been below the limit of detection of the TLC analysis, three LCMS samples were prepared from the extracts of each group of transformants and analysed. 


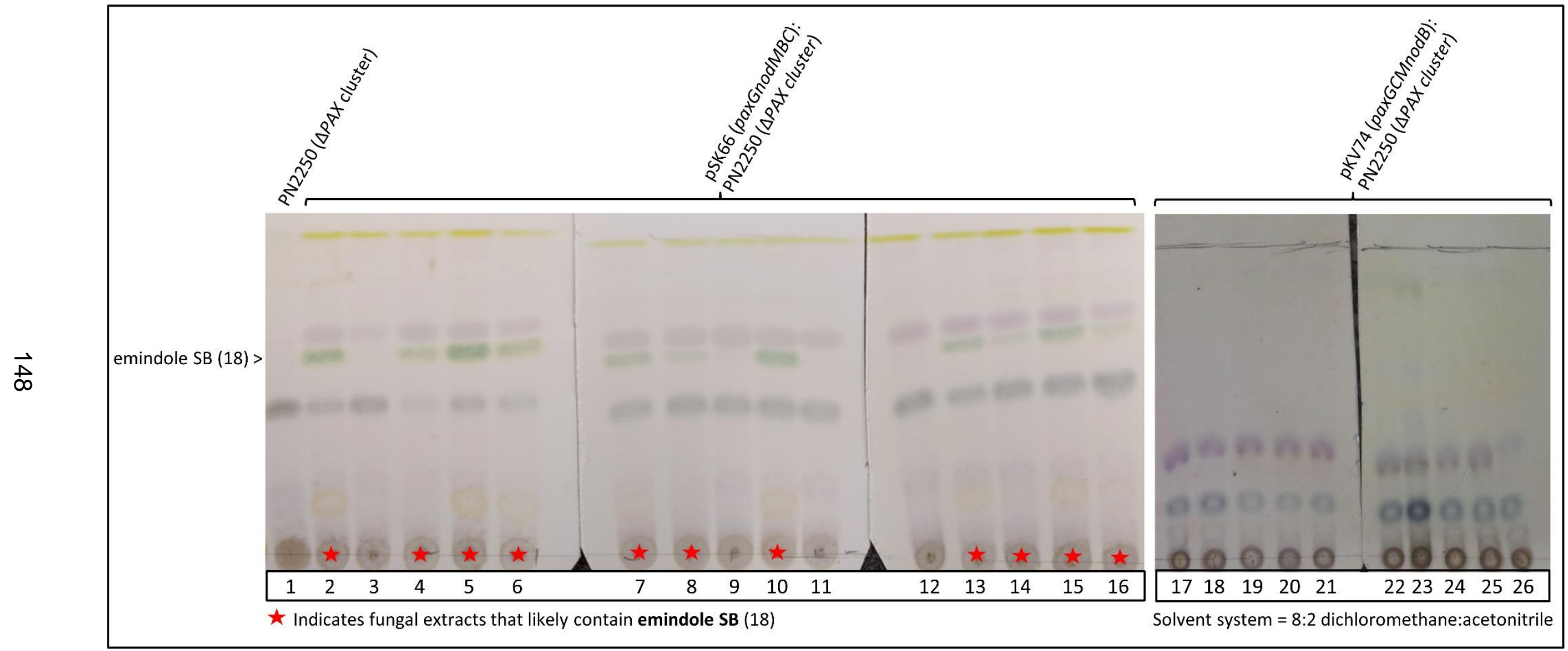

Figure 5.9. TLC results of fungal extracts from the individual transformation of $P$. paxilli $\triangle P A X$-cluster deletion mutant PN2250 with

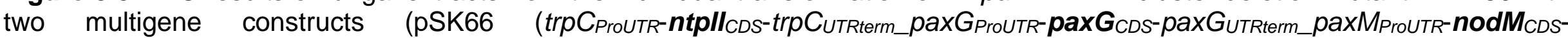

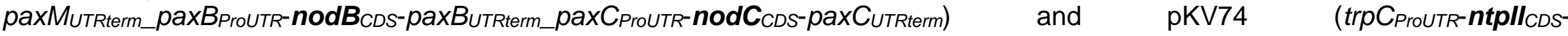

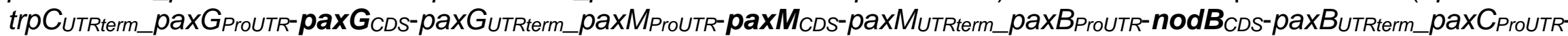
pax $C_{C D S}$-pax $\left.C_{U T R t e r m)}\right)$. As a negative control, lane 1 contains extract from the $P$. paxilli $P A X$-cluster deletion mutant $P N 2250$. 


\section{Analysis of LCMS results}

As expected, LCMS analysis revealed that the pSK66 (trp $C_{\text {ProUTR-ntplI }}$ CDS- $^{-}$ trpCUTRterm_paxGProUTR-paxG CDS-paxGUTRterm_paxM ProUTR-nodM $_{\text {CDS- }}$

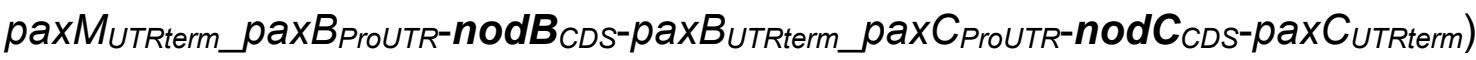
transformants were indeed producing emindole SB (18) (high resolution-mass spectra $406.3109 \mathrm{~m} / \mathrm{z}$; Figure 5.10, trace ii) - indicating that NodB was capable of cyclising 13,14-epoxy-3-geranylgeranylindole (16) to form emindole SB (18). Additionally,

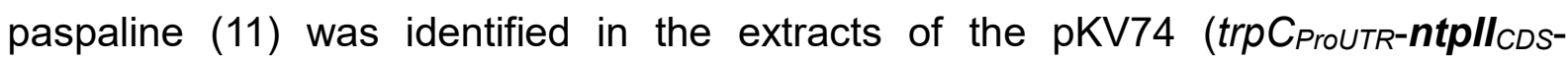

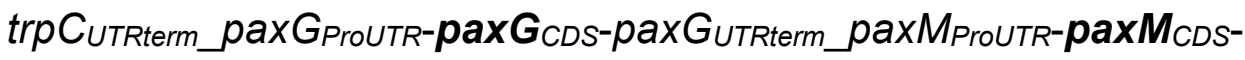

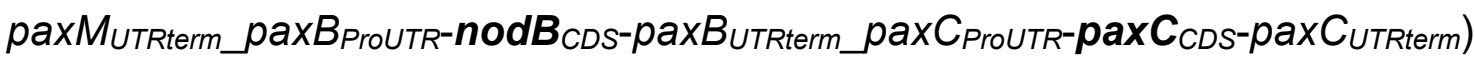
transformants (high resolution-mass spectra $[\mathrm{M}+\mathrm{H}]^{+}=422.3055 \mathrm{~m} / \mathrm{z}$; Figure 5.10 , trace iii.)- showing that NodB was also capable of cyclising bisepoxy-3-geranylgeranylindole (17) to form paspaline (11).

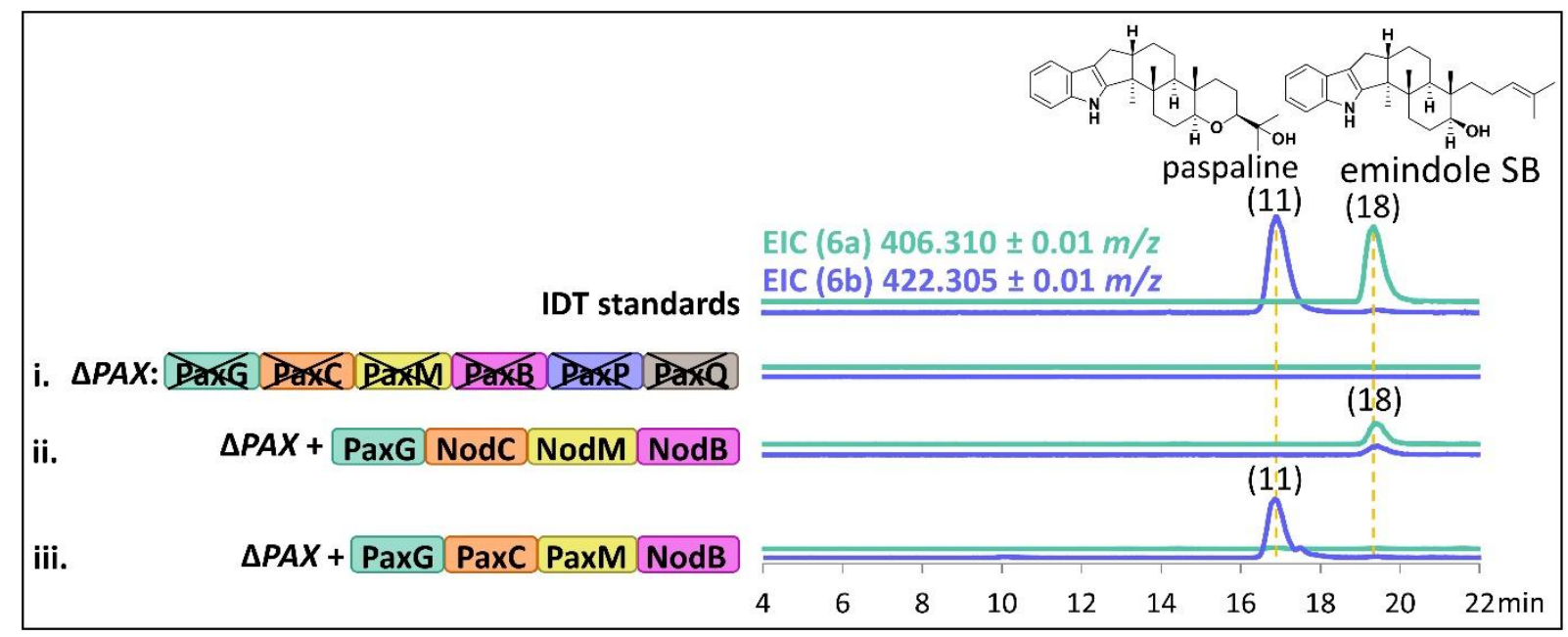

Figure 5.10. Mass spectrometry extracted ion chromatogram traces corresponding to the $[\mathrm{M}+\mathrm{H}]^{+}$ion of emindole SB (18) $(406.310 \pm 0.01 \mathrm{~m} / \mathrm{z})$ in turquoise and paspaline (11) $(422.305 \pm 0.01 \mathrm{~m} / \mathrm{z})$ in purple. Indole diterpene (IDT) standards [emindole SB (18) and paspaline (11)] are depicted at the top, traces from the full $\triangle P A X$-cluster deletion mutant (strain PN2250) are depicted in i., traces from the paxG, nodCMB (pSK66) transformant are depicted in ii., and traces from the paxGCM, nodB (pKV74) transformant are depicted in iii. Notably, there is a peak associated to $[\mathrm{M}+\mathrm{H}]^{+}$of 422.305 in trace ii. That has same retention time as emindole SB (18) but no compounds with these properties have been characterized. 


\section{Conclusions}

As suggested by NodB's high level of similarity compared with functionally characterised indole diterpene cyclases, it was confirmed that NodB possesses the same promiscuous capability in that it is able to catalyse the cyclisation of both 13,14 epoxy-3-geranylgeranylindole (16) and bisepoxy-3-geranylgeranylindole (17) to respectively form emindole SB (18) and paspaline (11). Therefore, NodB is a functional orthologue of PaxB and the other functionally confirmed indole diterpene cyclases that share high amino acid sequence identity. This shows that NodB and NodM do not work in conjunction to streamline nodulisporic acid biosynthesis; rather, by only epoxidising geranylgeranylindole at the $\mathrm{C} 14-\mathrm{C} 15$ alkene, NodM prevents the formation of shunt products [e.g. paspaline (11)] by NodB and is thereby solely responsible for streamlining nodulisporic acid biosynthesis. 


\section{NodW, THE FUNNEL TO NODULISPORIC ACIDS}

\section{Proposed Nodulisporic acid A gene cluster from H. pulicicidum (ATCC 74245)}

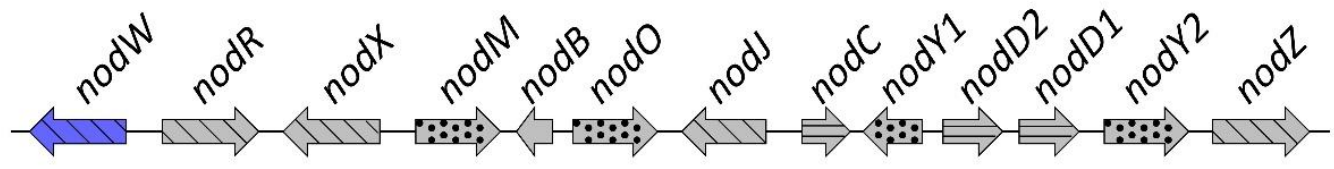

cytochrome P450 oxygenase

Figure 6.1. Depiction of the predicted nodulisporic acid gene cluster (NOD) from $H$. pulicicidum $\left(\mathrm{ATCC}^{\circledR} 74245^{\mathrm{TM}}\right.$ ). The nodW gene is highlighted in purple. 


\section{Review of post cyclisation oxidations by cytochrome P450 oxygenases in indole diterpene biosynthesis}

After cyclisation (i.e. step four of indole diterpene biosynthesis), structurally complex indole diterpenes are typically oxidised in a two-step manner by two separate cytochrome P450 oxygenases (Figure 6.2). For example, in the case of paxilline (2) biosynthesis by $P$. paxilli, the cyclised core, paspaline (11), is oxidised first by PaxP to form 9-desoxypaxilline (27) and then by $P a x Q$ to form paxilline. ${ }^{36,40}$ This same pattern is not only seen in the biosynthesis of paxilline-derived indole diterpenes like penitrems ( $P$. simplicissimum), ${ }^{31}$ but also in the biosynthesis of non-paxilline-derived indole diterpenes like the aflatrems ( $A$. flavus), ${ }^{28,41}$ shearinines (Penicillium janthinellum), ${ }^{32}$, 43 terpendoles (Chaunopycnis alba) ${ }^{30}$ and lolitrems (Epichloë festucae) ${ }^{29}$

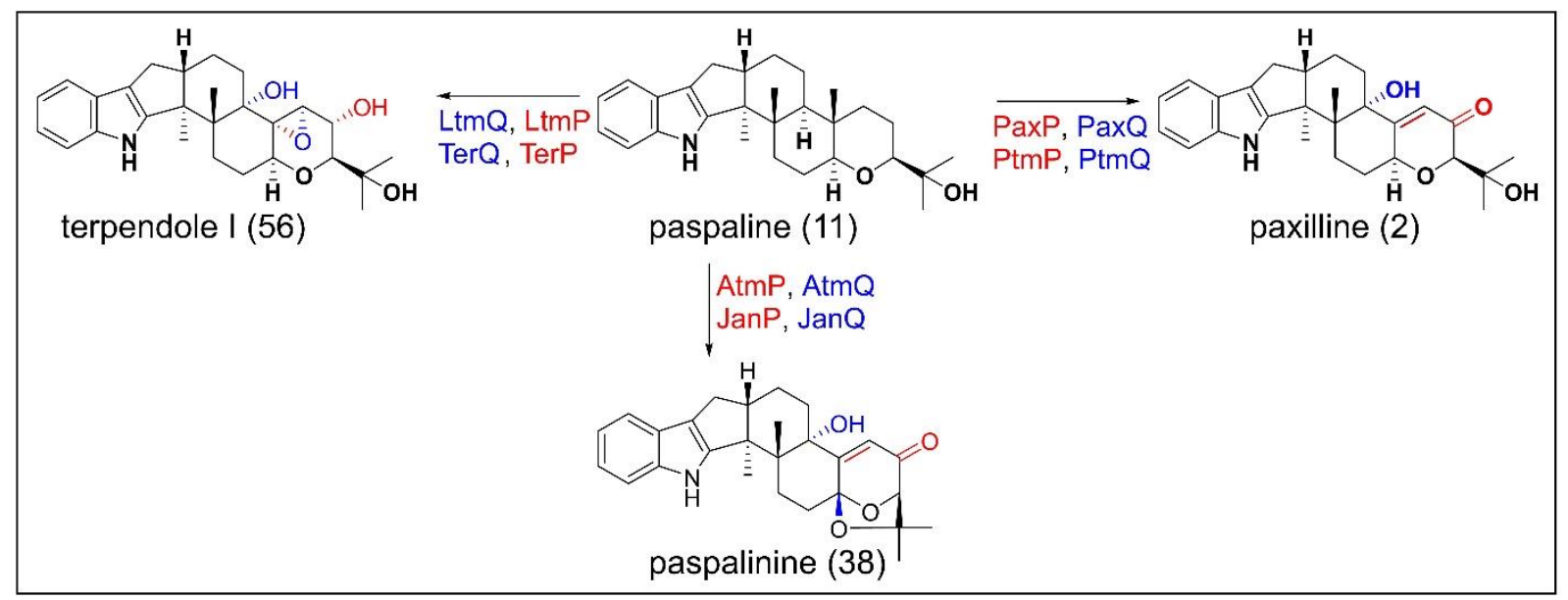

Figure 6.2. Depiction of the typical two-step oxidation that occurs in indole diterpene biosynthesis post-cyclisation. ${ }^{28-29,} 31-32,36,40-41,43$

\section{The NOD cluster lacks highly homologous cytochrome P450 oxygenases}

When considering the biosynthesis of nodulisporic acids it was predicted that the cyclised core compound, emindole SB (18), was oxidised at one of its two terminal methyl carbons to form nodulisporic acid $F(82)$ (Figure 2.3). The oxidation of a nonfunctional terminal carbon to form the carboxylic acid moiety of nodulisporic acid $F(82)$ is consistent with the involvement of a cytochrome P450 oxygenase, but no indole diterpene cytochrome P450 oxygenases have ever been functionally confirmed that 
perform this type of oxidation and thus it was unclear whether only one cytochrome P450 oxygenase was responsible for installing the carboxylic acid moiety or whether multiple cytochrome P450 oxygenases were involved. Additionally, because no other indole diterpene cytochrome P450 oxygenases had been identified with this type of functionality, sequence homology screening could not be used to delineate what cytochrome P450 oxygenase(s) within the five cytochrome P450 oxygenases in the $N O D$ gene cluster (nodW, nodR, nodX, nodJ, and nodZ) were responsible for this oxidation (Figure 6.3). Therefore, an experiment was designed to test all five of the cytochrome P450 oxygenases for their ability to catalyse the oxidation of emindole SB (18) to form nodulisporic acid F (82).

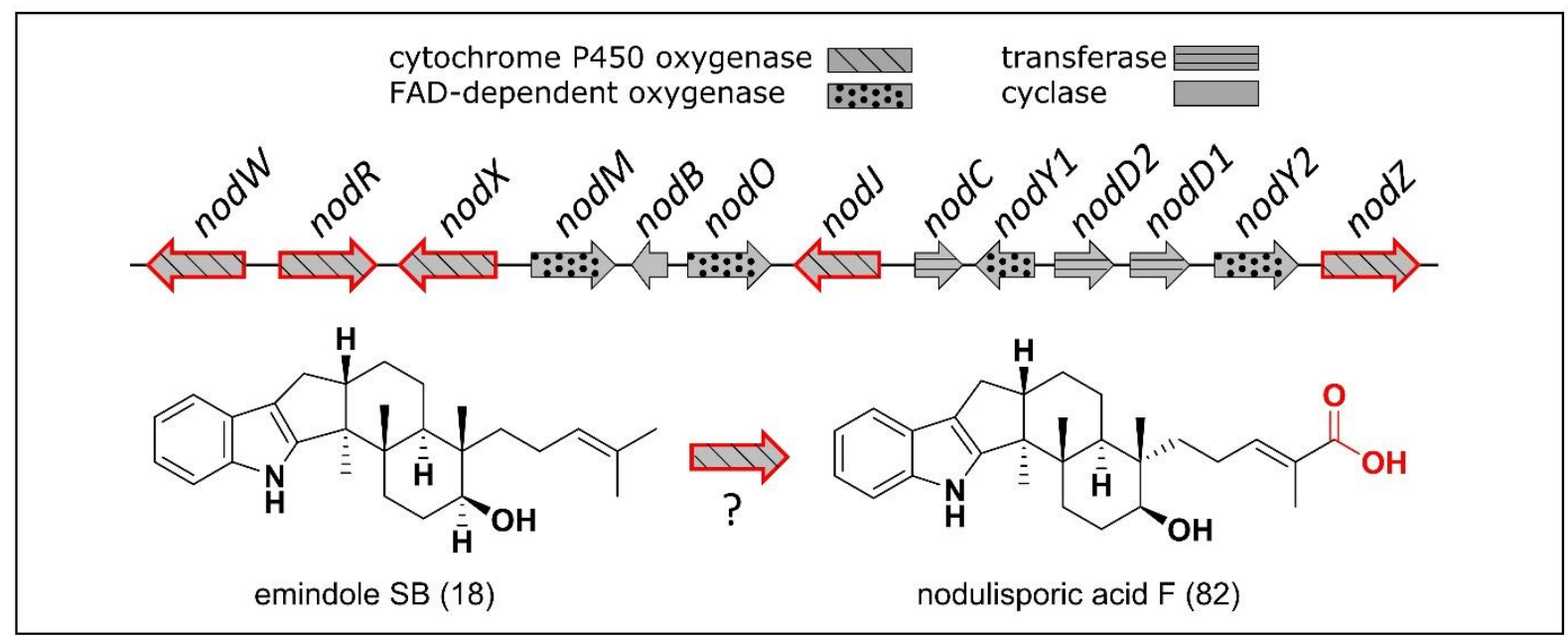

Figure 6.3. Depiction of the $N O D$ cluster with the five-predicted cytochrome $P 450$ oxygenases outlined in red and a schematic of the oxidation of emindole SB (18) to form nodulisporic acid $F$ (82).

\section{P450 analysis: An approach to functionally confirm non-homologous genes}

During the gene functionality analysis of nodM it was confirmed that introduction of nod $M$ into the $P$. paxilli $\triangle$ paxM deletion mutant resulted in production of emindole $S B$ (18), the substrate required for functional analysis of the NOD cluster cytochrome P450 oxygenases. In addition, it was demonstrated that when nodM was driven by the paxMProUTR promoter region it produced more emindole SB (18) than when it was driven by the trp $C_{\text {ProUTR }}$ promoter region. Therefore, in order to test the five NOD 
cytochrome P450 oxygenases, each of the genes predicted to encode a cytochrome P450 oxygenase was assembled into a separate multigene construct [pKV64 (trpC $C_{\text {ProUTR-ntpll }}$ CDS-trpC CUTRterm_paxMProUTR-nodMCDS-paxMUTRterm_trpC $C_{\text {ProUTR- }}$

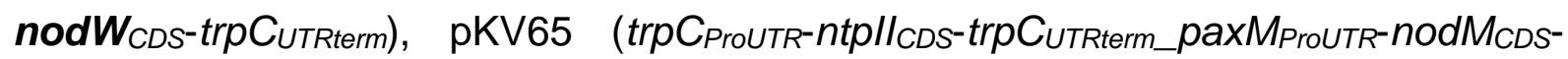
paxMUtRterm_trp $C_{\text {ProUTR-nodR }}$ CDS-trpC $\left.C_{U T R t e r m}\right), \quad$ pKV66 (trpC $C_{\text {ProUTR-ntpll }}$ CDS-

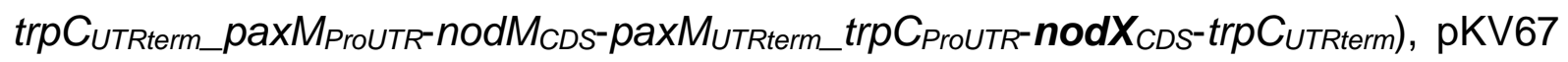

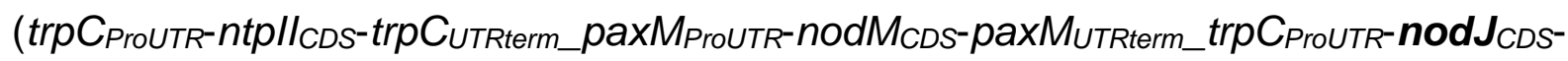
trpC UTRterm), and pKV68 (trpC $C_{\text {ProUTR-ntpll }}$ CDS-trpC UTRterm_paxMProUTR-nodMCDSpaxMUTRterm_trp $C_{\text {ProUTR-nod }} Z_{C D S}$-trp $\left.\left.C_{U T R t e r m}\right)\right]$ that contained the nodMCDS coding sequence driven by the paxMProUTR promoter region. Due to the versatility of the MIDAS system, the same Level-3 geneticin resistance cassette-containing/nodM-containing destination vector [pKV63 (trpC $C_{\text {ProUTR-ntpll }}$ CDS-trpCUTRterm_paxMProUTR-nodM CDS- $_{\text {- }}$ paxMutRterm)] was used for the assembly of each cytochrome P450 oxygenase fungaltransformation vector. Since the destination vector (pKV63) required insertion of a white level 2 vector, five white MIDAS Level-2 vectors, each containing a separate NOD cytochrome P450 oxygenase coding sequence were assembled with the trp $C_{\text {ProUTR }}$ promoter and trpC UTRterm terminator regions. The Level-2 vectors were designed to incorporate each cytochrome P450 oxygenase coding sequence into the final destination vector in the same orientation that it has in the NOD cluster. Therefore, nod $W$, nod $R$ and nod $J$ were assembled into white forward Level-2 destination vectors and nod $X$ and nod $Z$ were assembled into white reverse Level-2 destination vectors (Figure 6.4). 


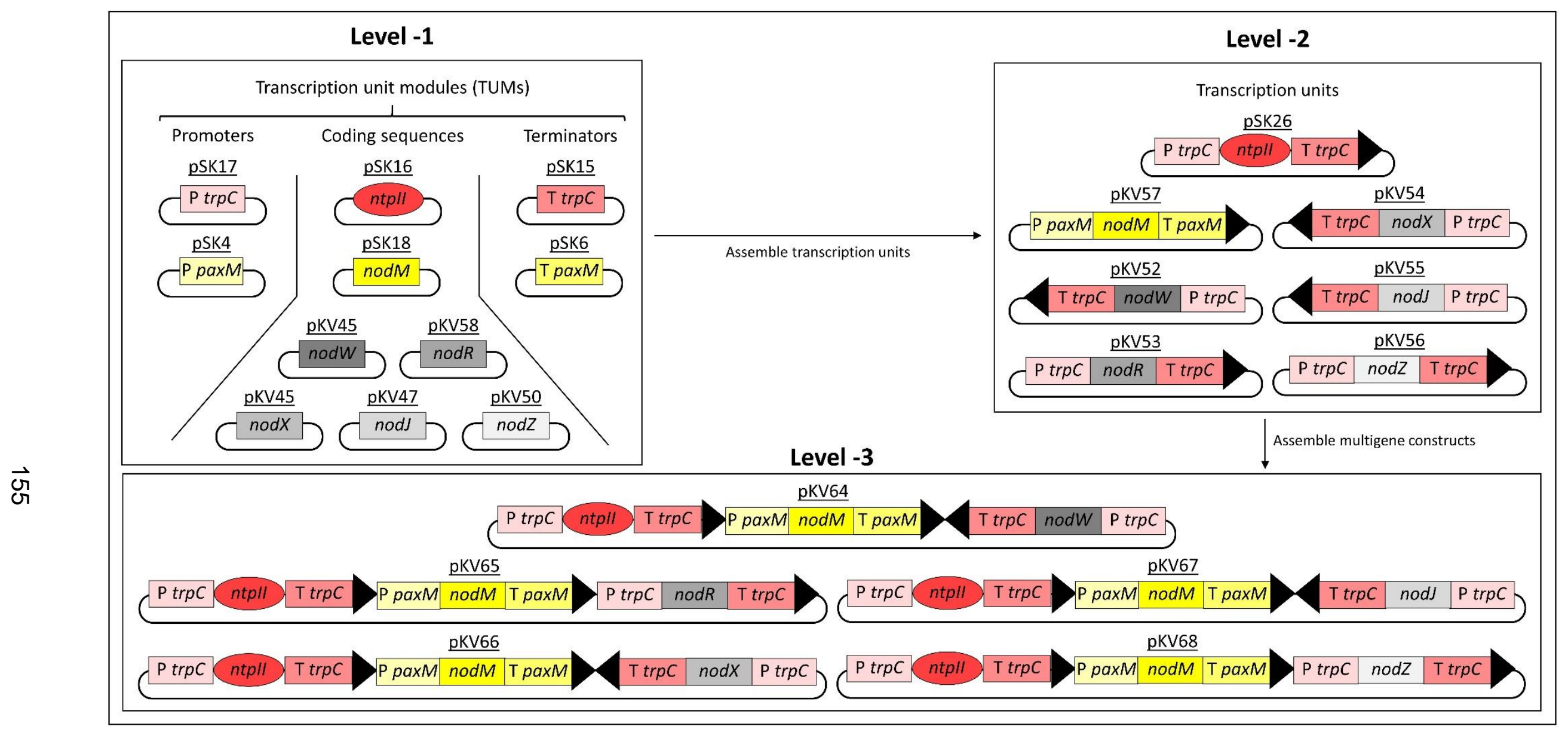

Figure 6.4. Depiction of the MIDAS approach used to assemble the five multigene constructs (pKV64, pKV65, pKV66, pKV67, and pKV68) used to identify the cytochrome P450 involved in the oxidation of emindole SB (18) to form nodulisporic acid F (82). In Level1 , the plasmids encoding transcription unit modules (promoters, coding sequences, terminators) are shown. In Level-2 the plasmids that encode the five full length transcription units are shown -where the arrow represents the direction of transcription. In Level-3, the final multigene plasmids that was used for fungal transformation are depicted. 


\section{Transformation of $\boldsymbol{P}$. paxilli with P450-encoded vectors}

Protoplasts of the $P$. paxilli $\triangle$ paxM deletion mutant strain PN2257 were transformed individually with each of the five multigene P450-encoding Level-3 vectors. Ten transformants from each transformation were picked and fermented in production medium. Initial TLC analysis of all of the transformant extracts showed that emindole SB (18) was present in in a selection of extracts from every transformation (Figure 6.5) - showing that nodM had been successfully incorporated and expressed thereby suggesting that the cytochrome P450 oxygenases were also successfully incorporated into the genomes of these transformants. Since the TLC analysis showed that there was a wide range of variation in compound production of the transformants, a selection of three extracts from each transformation was chosen for further LCMS analysis. 


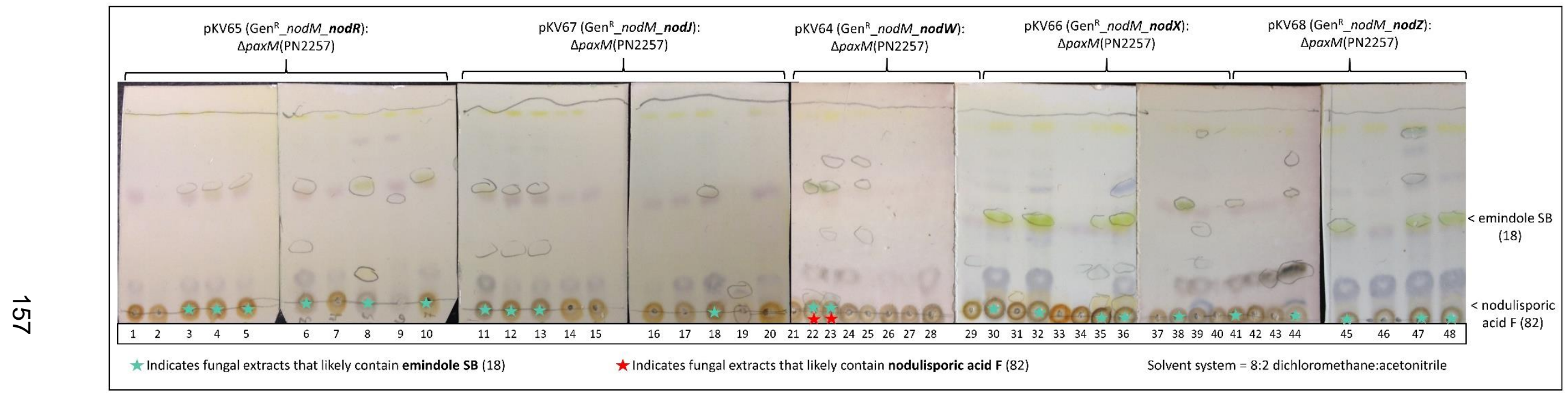

Figure 6.5. TLC results of fungal extracts from the individual transformations of $P$. paxilli $\triangle$ paxM deletion mutant PN2257 with five

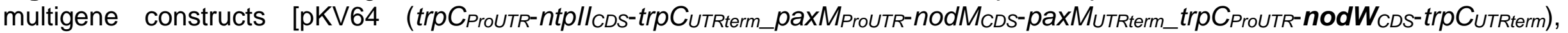

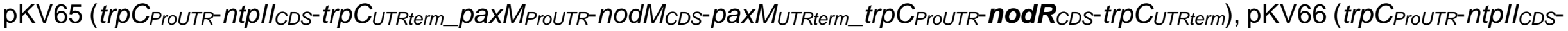

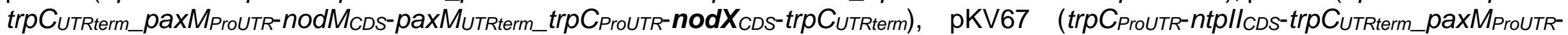

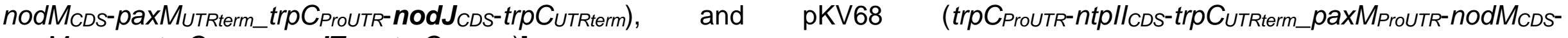
paxMUTRterm_trp $C_{\text {ProUTR-nod }}$ CDS-trpC $\left.C_{U T R t e r m)}\right]$. 


\section{Identification of nodulisporic acid $\mathrm{F}$ (82)}

The LCMS analysis of the selection of extracts tested from each transformation revealed that emindole SB (18) was present in transformants from every nodM/P450 transformation (Figure 6.6, traces iii.-vii.). Importantly, a novel indole diterpene compound with a mass corresponding to that of nodulisporic acid $\mathrm{F}$ (82) (high resolution-mass spectra $[\mathrm{M}+\mathrm{H}]^{+}=$ $436.2870 \mathrm{~m} / \mathrm{z}$, r.t. $5.6 \mathrm{~min}$ ) was present in the extracts of only the nod $W$-harbouring transformant (Figure 6.6, trace iii.). The identity of the novel compound was confirmed by NMR spectrometry as nodulisporic acid $F$ (82). A table of the ${ }^{1} \mathrm{H}$ and ${ }^{13} \mathrm{C}$ NMR assignments are shown in the Appendix in Table 9.9 and the corresponding 1-dimensional and 2-dimensional NMR spectra are shown in Figures 9.22 to 9.25. The production of nodulisporic acid $F(82)$ by the the nod $W$-harbouring transformant indicates that a single cytochrome P450 oxygenase (NodW) catalyses the oxidation of the terminal carbon on emindole SB (18) to form the reactive carboxylicic acid handle of nodulisporic acid $F(82)$. 


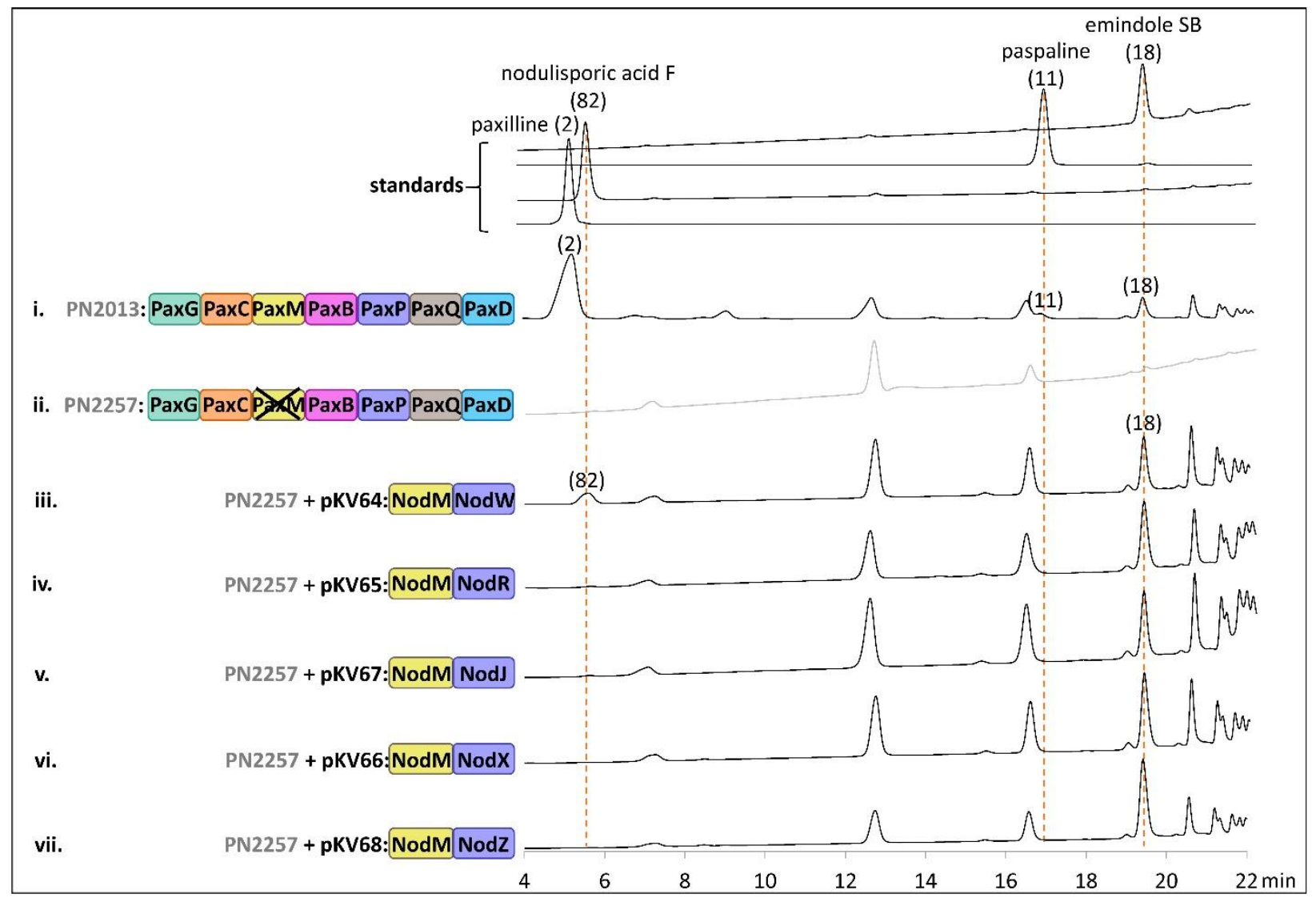

Figure 6.6. HPLC analysis $(271 \mathrm{~nm})$ of fungal extracts from the $P$. paxilli wild-type strain (PN2013) (trace i.), P. paxilli $\triangle$ paxM deletion mutant strain, PN2257 (trace ii.), pKV64 transformed $P$. paxilli $\triangle$ paxM deletion mutant strain (pKV64:PN2257) expressing the NodM and NodW enzymes from H. pulicicidum (trace iii.), pKV65 transformed $P$. paxilli $\triangle$ paxM deletion mutant strain (pKV65:PN2257) expressing the NodM and NodW enzymes from H. pulicicidum (trace iv.), pKV67 transformed $P$. paxilli $\triangle$ paxM deletion mutant strain (pKV67:PN2257) expressing the NodM and NodJ enzymes from H. pulicicidum (trace v.), pKV66 transformed $P$. paxilli $\triangle$ paxM deletion mutant strain (pKV66:PN2257) expressing the NodM and NodX enzymes from $H$. pulicicidum (trace vi.), and pKV68 transformed $P$. paxilli $\triangle$ paxM deletion mutant strain (pKV68:PN2257) expressing the NodM and NodZ enzymes from $H$. pulicicidum (trace vii.). Extracted ion chromatograms are located in the Appendix (Figures 9.8 to 9.11).

\section{Confirmation that five genes are required for nodulisporic acid biosynthesis}

We were aware that the $\triangle p a x M$ deletion mutant strain PN2257 contained decoration genes (i.e. $\operatorname{pax} P, \operatorname{pax} Q, \operatorname{pax} D$, and $\operatorname{pax} O$ ) that could have influenced to the production of nodulisporic acid $F(82)$ and wanted to ensure that none of these genes were involved in 
its biosynthesis. Therefore, to establish that only five genes were involved in nodulisporic acid $F(82)$ biosynthesis a six-gene construct encoding $n t p l l, \operatorname{pax} G, \operatorname{nod} C, \operatorname{nod} M, \operatorname{nod} B$ and nodW [pSK68 (trpC $C_{\text {ProUTR-ntpll }}$ CDS-trpCUTRterm_paxG $G_{\text {ProUTR-paxG }}$-pDS-

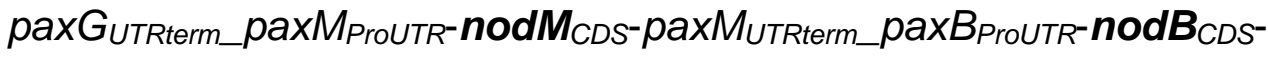

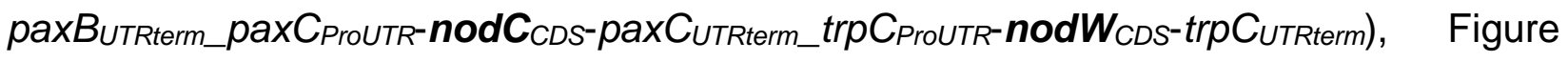
6.7] was assembled and inserted it into the entire $\triangle P A X$-cluster deletion mutant strain PN2250. With the exception of the promoter region for nodW, native PAX promoter regions were used to drive their respective genes or gene homologues for paxG, nodC, nod $M$ and nodB. As the trp $C_{\text {ProUTR }}$ promoter region was used in the initial testing of nodW, and the strength of the promoter regions in the promoter library had not yet been assessed, the trpC promoter region was selected to drive nodW in pSK68. Transformation of the P. paxilli PAX deletion mutant strain PN2250 with this six-gene construct (pSK68) potentially established nodulisporic acid $F(82)$ production in at least two of six transformants, as confirmed by TLC analysis (Figure 6.8). Subsequent LCMS analysis revealed that nodulisporic acid $F(82)$ was only present in one of the transformant extracts and that it was only present in very low amounts (Figure 6.9). Therefore, an attempt was made to enhance nodulisporic acid $F(82)$ biosynthesis by changing the promoter region used to drive the nod $W_{C D S}$ coding sequence. 


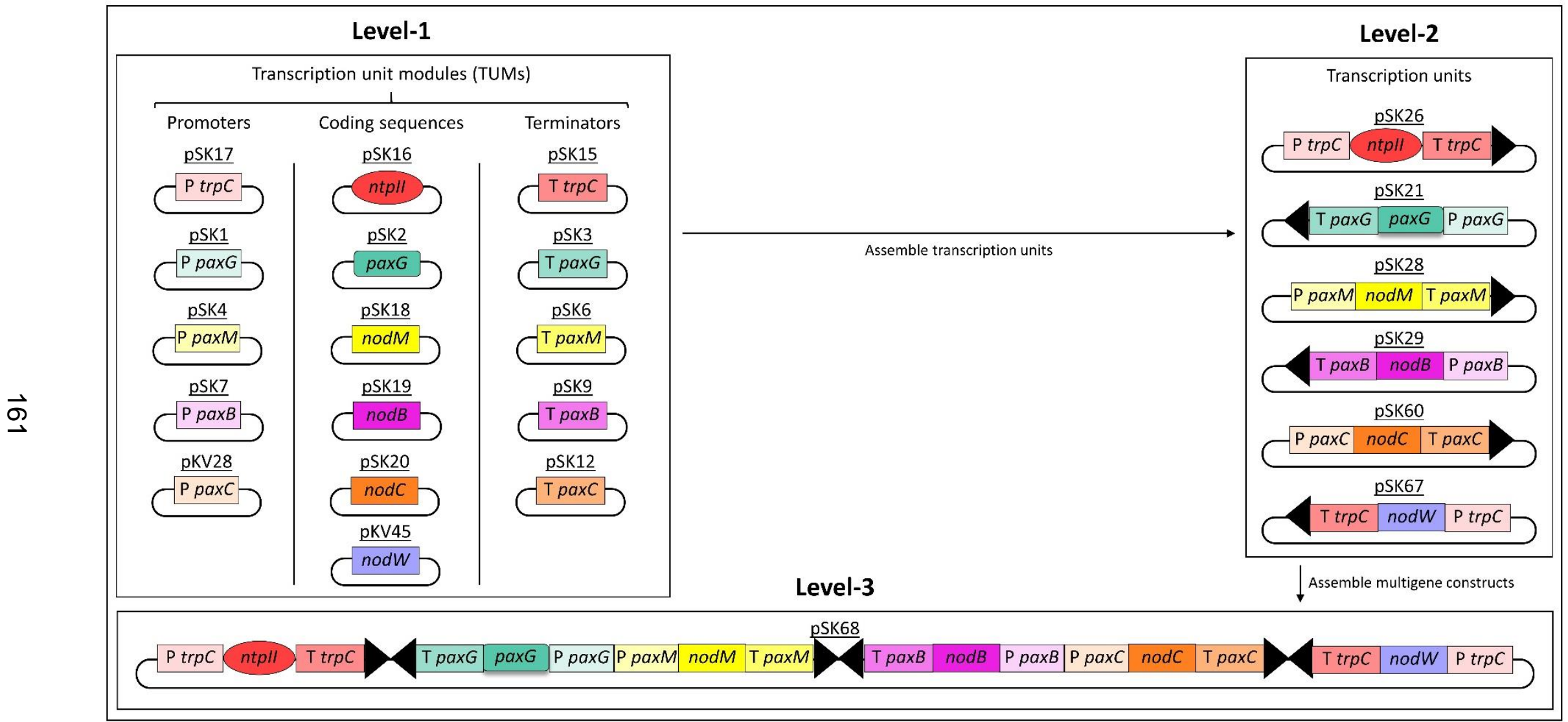

Figure 6.7. Depiction of the MIDAS approach used to assemble the multigene construct pSK68 (trpC ProUTR-ntpll $_{C D S-}$

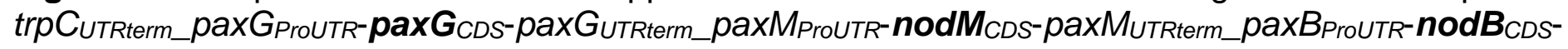

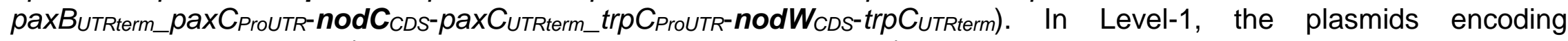
transcription unit modules (promoters, coding sequences, terminators) are shown. In Level-2 the plasmids that encode the five full length transcription units are shown -where the arrow represents the direction of transcription. In Level-3, the final multigene plasmid (pSK68) that was used for fungal transformation is depicted. 


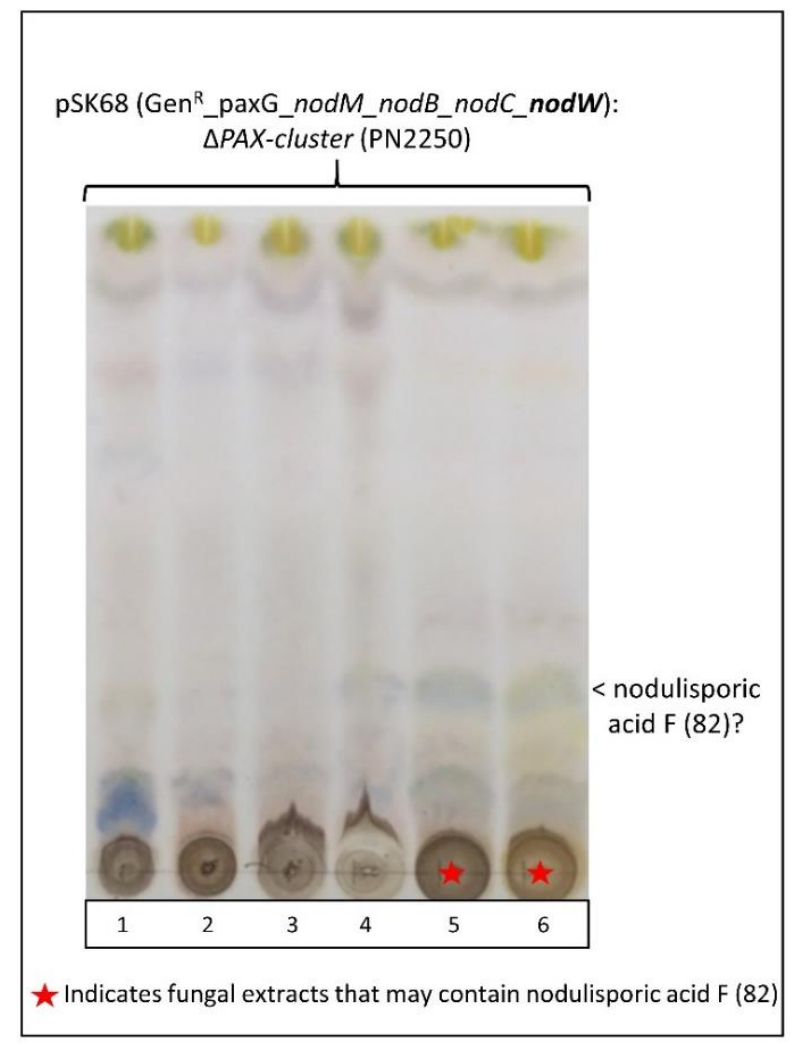

Figure 6.8. TLC results of fungal extracts from the transformation of $P$. paxilli $\triangle P A X-$ cluster deletion mutant PN2250 with the multigene construct pSK68 (trpC ProUTR-ntpll $_{C D S}$

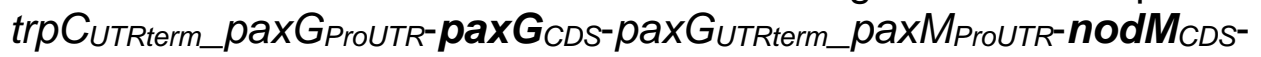
paxMUTRterm_paxB ProUTR-nodB $_{C D S}-$ paxB $B_{U T R t e r m \_p a x}$ ProUTR-nod $_{C D S^{-}}$ paxCUTRterm_trp $C_{\text {ProUTR-nod }}$ WDS-trp $C_{U T R \text { Tterm) }}$.

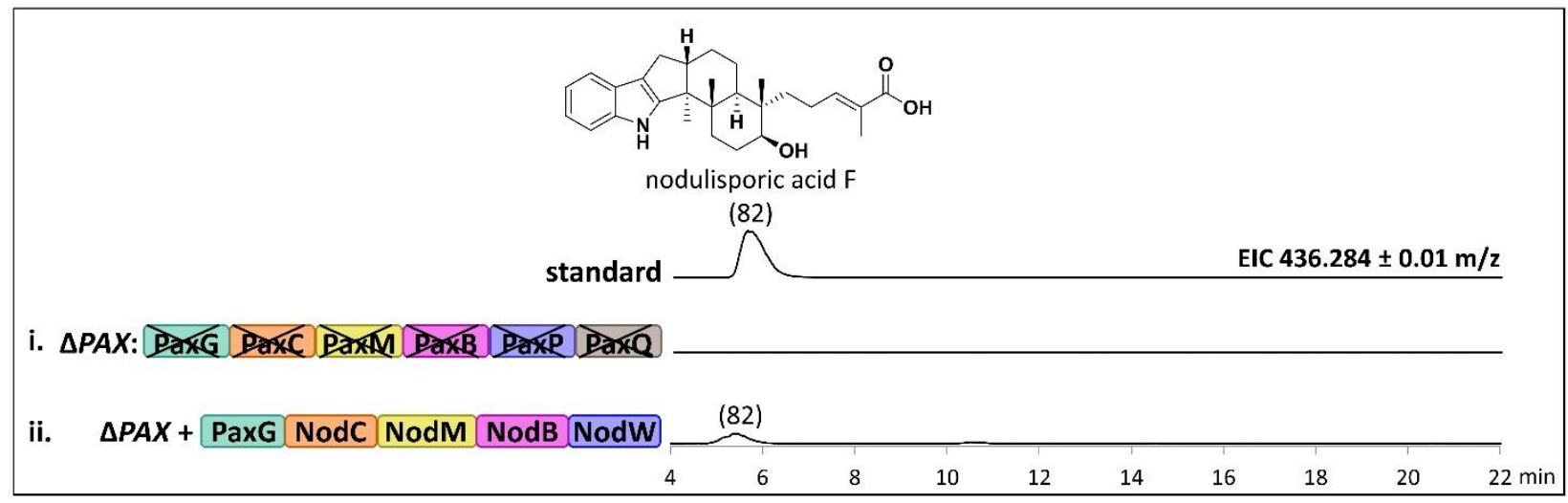

Figure 6.9. Mass spectrometry extracted ion chromatogram (EIC) traces corresponding to the $[\mathrm{M}+\mathrm{H}]^{+}$ion of nodulisporic acid $\mathrm{F}(82)(436.284 \pm 0.01 \mathrm{~m} / \mathrm{z})$. The EIC trace of the nodulisporic acid $F(82)$ standard is depicted at the top, the EIC of extract from the full $\triangle P A X$-cluster deletion mutant (strain PN2250) is depicted in trace $\mathbf{i}$., and the EIC of extract from the paxG, nodCMBW (pSK68) transformant is depicted in ii. 


\section{Enhancing nodulisporic acid $F(82)$ biosynthesis}

To try to enhance the biosynthesis of nodulisporic acid $F(82)$ a new six-gene construct was assembled that directly resembled pSK68 (trpC ProUTR-ntpll $_{C D S^{-}}$

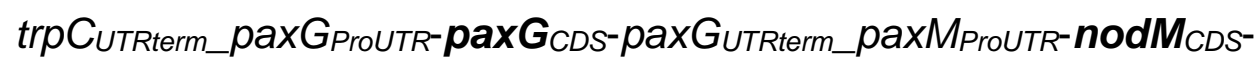

paxMUTRterm_paxB $B_{\text {ProUTR-nodB }}$ CDS-paxBUTRterm_pax $C_{\text {ProUTR-nod }} \boldsymbol{C}_{C D S^{-}}$

paxCUTRterm_trpC $C_{\text {ProUTR-nod }}$-nods-trpCUTRterm) with the exception of the promoter region used to drive the nod $W_{C D S}$ coding sequence (Figure 6.10). In this new six-gene construct,

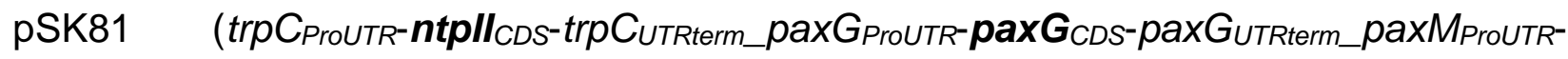
nodM $M_{C D S}$-paxMUTRterm_paxB $B_{\text {ProUTR-nod }} \boldsymbol{B}_{C D S}$-paxB $B_{U T R t e r m \_p a x} C_{\text {ProUTR-nod }} \boldsymbol{C}_{C D S}$

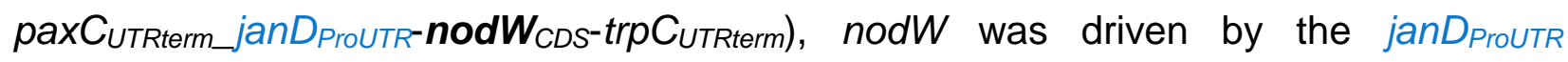
promoter region. Upon assembly, pSK81 was inserted into protoplasts of the entire PAX deletion mutant strain PN2250. The TLC analysis of fifteen transformants (Figure 6.11) showed that at least six (lanes 1, 3, 4, 8, 10, and 12) produced nodulisporic acid $F(82$ ) and that nodulisporic acid $F(82)$ was produced in higher concentrations than that of the pSK68 transformants (See Figure 6.8 for analysis of pSK68). Subsequent LCMS analysis of the pSK81 transformants (Figure 6.12) also showed much higher concentrations of nodulisporic acid $F(82)$ than were found in the pSK68 transformants (the nodulisporic acid $F(82)$ in the extracts of the pSK68 transformants was not observed in the UV trace and only detectable by the mass spectrometer) - confirming that promoter swapping of a single gene in a multi-gene construct could greatly enhance compound biosynthesis. 


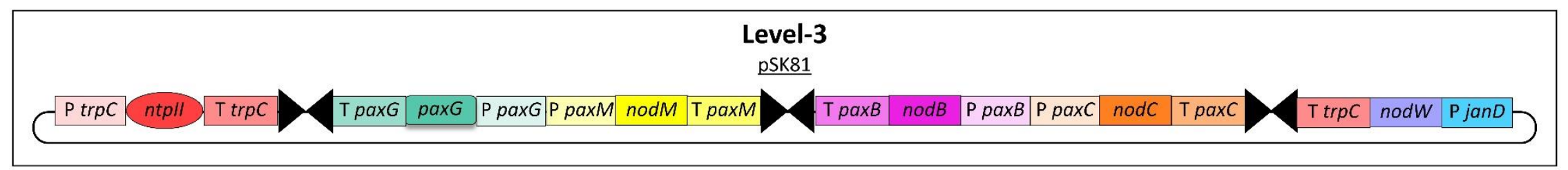

Figure 6.10. Representation of the six-gene construct pSK81 used to test how promoter region swapping (the trp $C_{\text {ProUTR }}$ promoter region in pSK68 to the janD ProUTR promoter region in pSK81) of a single gene (nodW) in this construct would affect biosynthesis of nodulisporic acid $\mathrm{F}(82)$.

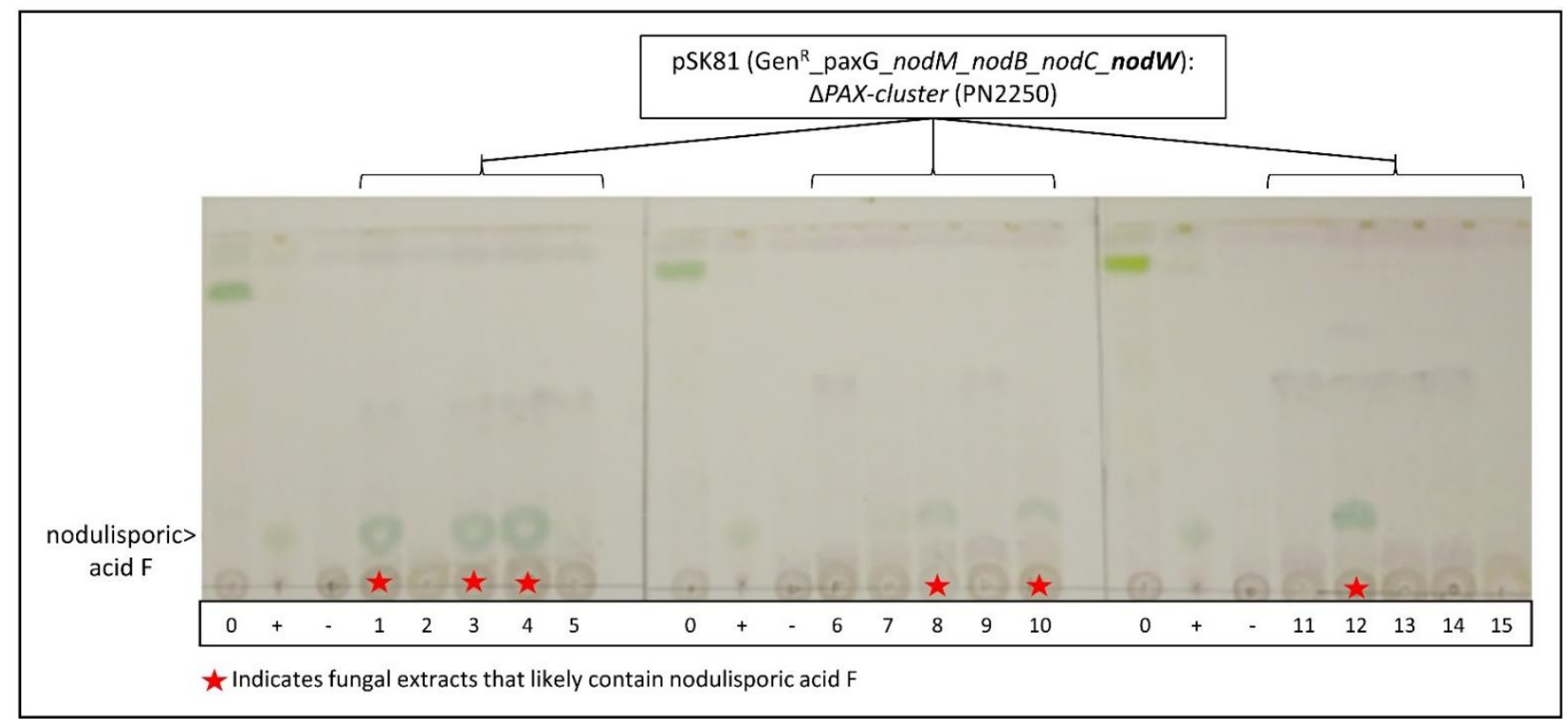

Figure 6.11. TLC results of fungal extracts from the transformation of $P$. paxilli $\triangle P A X$-cluster deletion mutant $P N 2250$ with the multigene construct pSK81 (trpC $C_{\text {ProUTR-ntpll }}$ CDS-trpCUTRterm_paxGProUTR-paxG CDS-paxGUTRterm_paxMProUTR-nodM $_{C D S}$

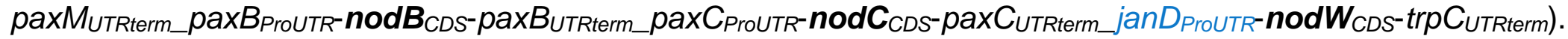
controls, extract from the $P$. paxilli wildtype strain is shown in lanes with a (0), the nodulisporic acid $F(82)$ standard is shown in lanes with a $(+)$, and extract from the $P$. paxilli $\triangle P A X$-cluster deletion mutant PN2250 is shown in lanes with a (-). 


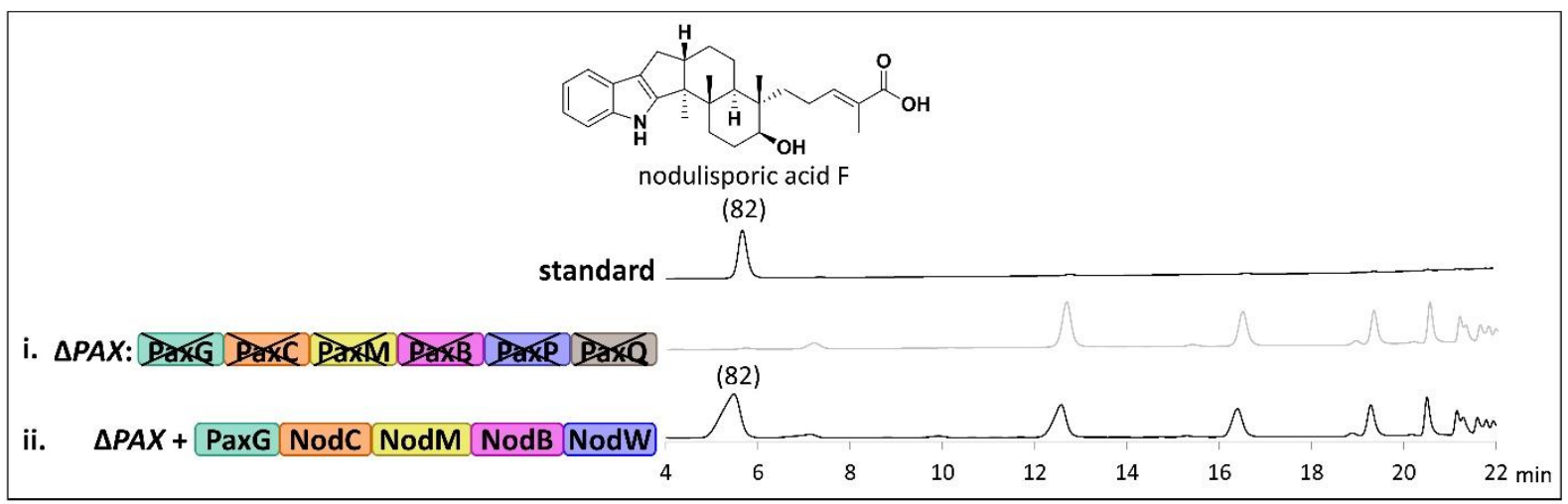

Figure 6.12. HPLC analysis $(271 \mathrm{~nm})$ of fungal extracts from the $P$. paxilli $\triangle P A X-$ cluster deletion mutant strain, PN2250 (trace i), and one of the pSK81 (paxG, nodCMBW) transformants (trace ii.). The top trace corresponds to the nodulisporic acid $F(82)$ standard. Extracted ion chromatograms are located in the Appendix (Figure 9.11).

\section{Conclusions}

Overall, this work confirms that five secondary metabolic genes (paxG, nodC, nodM, nod $B$ and nod $W$ ) are involved in the heterologous biosynthesis of nodulisporic acid $F$ (82) by P. paxilli (Figure 6.13). Importantly, previous studies of the nodulisporic acid pharmacophore established that the carboxylic acid handle of nodulisporic acids is readily modified using chemical synthesis to enhance the bioactivity and insecticidal specificity of the natural compounds. ${ }^{11,54-55,60}$ Thus, the identification of nodW provides access to a key modifiable core structure, nodulisporic acid $F(82)$, for exploration of novel indole diterpenes. Additionally, the functional confirmation of the nod $W_{C D S}$ coding sequence establishes a new subclass of indole diterpene cytochrome P450 oxygenases and will facilitate the identification of other oxygenases that may modify the indole diterpene cores in a similar but discrete manner. Furthermore, it was demonstrated that simple promoter region exchange of a single coding sequence within a multi-gene construct can dramatically enhance the biosynthesis of nodulisporic acid $F(82)$. Most important of all though is that nodulisporic acid $F(82)$ was produced in one week, in the light and in relatively nutrient poor medium showing that heterologous expression is a powerful method to bypass the regulatory mechanisms found in the native nodulisporic acid producer, H. pulicicidum. 


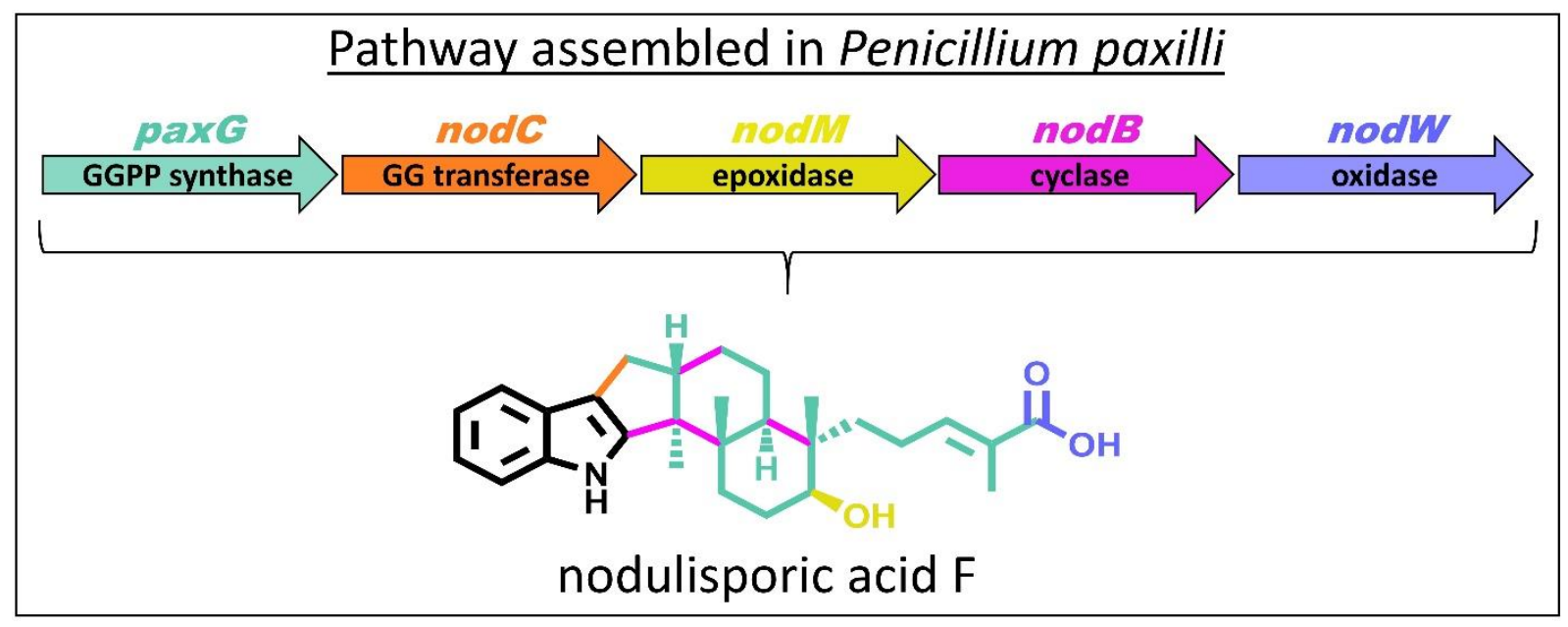

Figure 6.13. Summary of the biosynthetic genes involved in the heterologous biosynthesis of nodulisporic acid $\mathrm{F}$ (82) by P. paxilli (Van de Bittner et al. 2018). ${ }^{1}$ 
7. CONCLUSIONS 


\section{Key findings and results}

In summary, the genome of Hypoxylon pulicicidum (ATCC $\left.{ }^{\circledR} 74245^{\mathrm{TM}}\right)^{2}$ has been sequenced and sequence homology comparisons have been used to locate a cluster of thirteen genes, named the NOD cluster, which as described in this thesis, is responsible for nodulisporic acid biosynthesis. Using the efficient gene reassembly of the Modular Idempotent DNA Assembly System (MIDAS) ${ }^{3}$ in combination with heterologous expression in a high producing indole diterpene species, Penicillium paxilli, the functions of four of the thirteen genes (nodC, nodM, nodB and nod $W$ ) within this cluster have been confirmed. These four genes encode the enzymes that catalyse the second through fifth secondary-metabolic steps of nodulisporic acid biosynthesis shown in Figure 7.1: nodC encodes the geranylgeranyl transferase (NodC) that catalyses the condensation of geranylgeranyl pyrophosphate (3) and indole-3-glycerol phosphate (4) to form 3-geranylgeranylindole (13), nodM encodes a flavin adenine dinucleotide monooxygenase (NodM) that catalyses the mono-epoxidation of 3geranylgeranylindole (13) at the C13-C14 alkene to form 13,14-epoxy-3geranylgeranylindole (16), nodB encodes an indole diterpene cyclase (NodB) that catalyses the cyclisation of 13,14-epoxy-3-geranylgeranylindole (16) into emindole SB (18), and nodW encodes a cytochrome P450 oxygenase (NodW) that catalyses the oxidation of emindole SB (18) to form nodulisporic acid $F(82)$. Of these four genes, two - nodM and nodW - had functions that were previously unreported and are specifically involved in streamlining nodulisporic acid biosynthesis. 
A

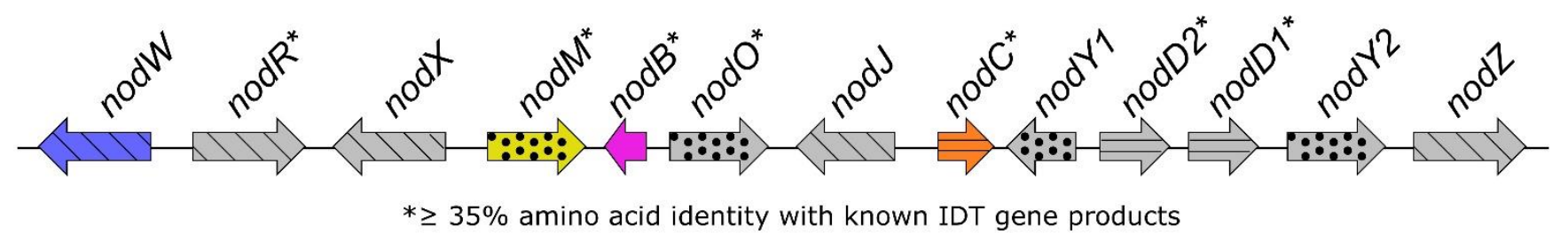

$* \geq 35 \%$ amino acid identity with known IDT gene products

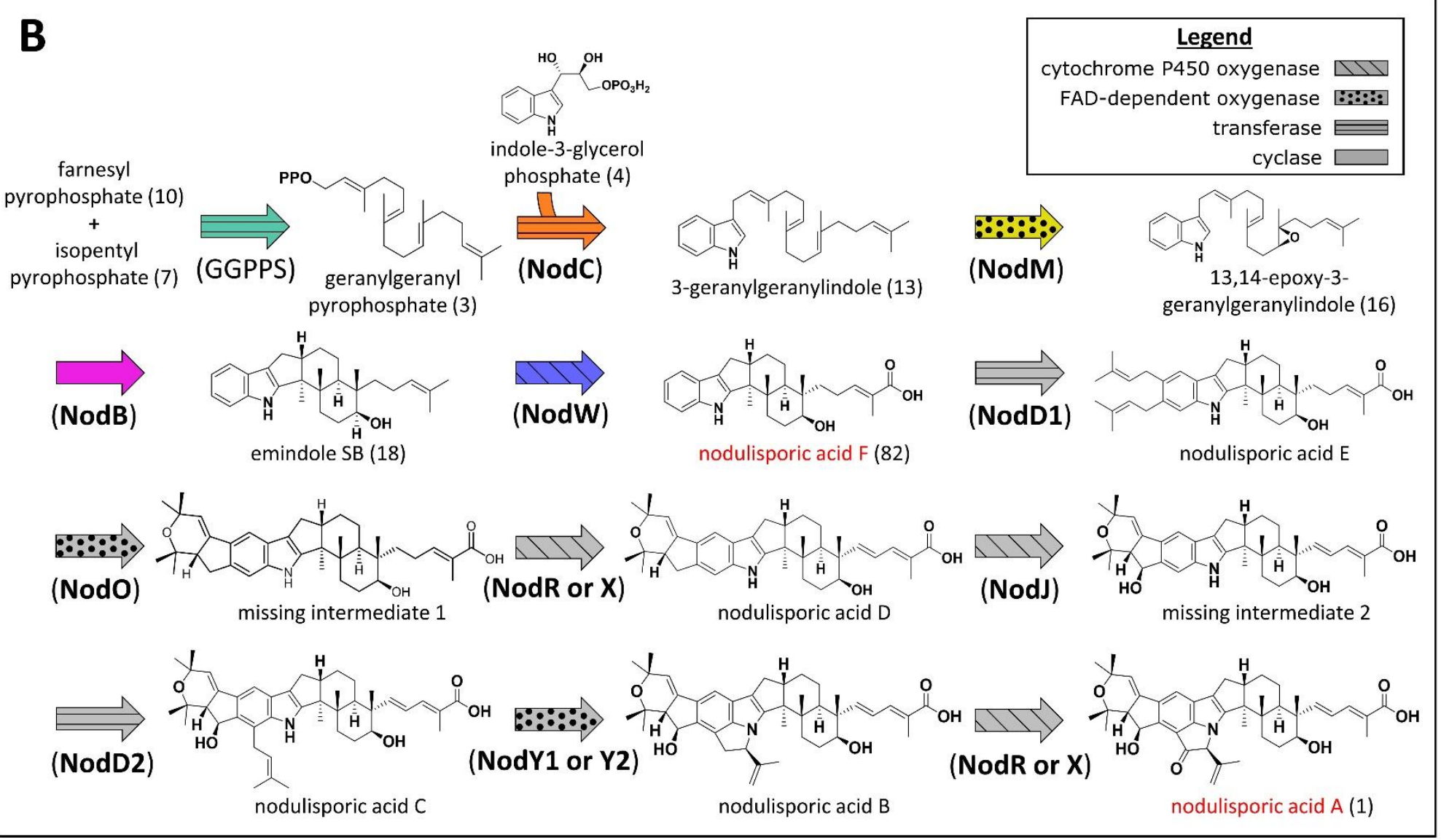

Figure 7.1. The nodulisporic acid gene cluster from Hypoxylon pulicicidum $(\mathbf{A})$ and the predicted nodulisporic acid $\mathbf{A}(1)$ biosynthetic pathway (B). The coloured genes (arrows) have been functionally characterised. 
By introducing nod $M$ into the $P$. paxilli $\triangle$ paxM deletion mutant it was demonstrated that NodM specifically epoxidised 3-geranylgeranylindole (13) at the C13-C14 alkene and not at the $\mathrm{C} 17-\mathrm{C} 18$ alkene, a characteristic that is not shared with any of its functionally characterised homologues [paxM (P. paxilli) ${ }^{40}$, atmM (A. flavus) ${ }^{33}$ and ItmM (E. festucae $\left.)^{26}\right]$. In turn, by epoxidizing 3-geranylgeranylindole (13) at the one specific location essential for nodulisporic aicd biosynthesis, nodM prevents the diversion of resources into shunt products (e.g. paspaline) that are not essential to the nodulisporic acid pathway. In a similar manner, it was demonstrated that nodW installs emindole SB (18) with the distinctive carboxylic acid moiety of nodulisporic acids to form nodulisporic acid $F(82)$. As nodulisporic acid $F(82)$ is the first nodulisporic acid in the pathway, this oxidation step acts as a 'funnel' for the biosynthesis of nodulisporic acids. An oxidation of this kind on an indole diterpene core has, until now, never been functionally characterised, and thus this discovery establishes a new subclass of indole diterpene cytochrome P450 oxygenases that will facilitate the identification of other oxygenases that may modify indole diterpene cores in a similar but discrete manner.

Notably, the cluster lacks a gene that encodes a secondary-metabolic geranylgeranyl pyrophosphate (3) synthase to catalyse the first secondary metabolic step of nodulisporic acids, the production geranylgeranyl pyrophosphate (3). The absence of a secondary-metabolic geranylgeranyl pyrophosphate (3) synthase provides a possible explanation as to why $\mathrm{H}$. pulicicidum produces nodulisporic acids in such low yield. The low production is a problem for both resolving gene function and useful generation of the compounds. Heterologous expression has provided a way to overcome both of these problems as it was demonstrated that nodulisporic acid $F$ (82) can be produced in a third of the time (one week in $P$. paxilli vs. 3 weeks in $H$. pulicicidum $)^{50}$ and without using the strict growth protocols required to elicit nodulisporic acid production in H. pulicicidum (i.e. H. pulicicidum cultures cannot be exposed to the light and required relatively nutrient-rich medium, while $P$. paxilli cultures can be exposed to light and grown in relatively nutrient-poor medium). Additionally, by exchanging promoter regions of the NOD gene coding sequences it was demonstrated that nodulisporic acid biosynthesis can be enhanced, further providing evidence that heterologous expression is a promising means to gain access to valuable compounds. 


\section{Future work: Functional analysis of the remaining NOD genes}

From here, efforts will be dedicated to confirming the function of the remaining genes within the NOD cluster involved in the biosynthesis of nodulisporic acid $A$ (1). The biosynthetic pathway of nodulisporic acid $F(82)$ that was elucidated as part of this thesis provides a foundation for the relatively straightforward identification of the "decoration" steps to make nodulisporic acid A (1). Already, using gene product homology comparisons (see Appendix Figures 9.1 to 9.7, Table 9.1), each gene has been ascribed to its most likely step within the biosynthesis of nodulisporic acid A (1) as shown in Figure 7.1, and functionality testing will be carried out using a bottom-up and top-down approach. The bottom-up approach will follow the previous gene functional analysis studies in this thesis, where multigene constructs will be assembled and introduced into either the $\triangle$ paxM P. paxilli deletion mutant strain PN2257 or the $\triangle P A X$-cluster $P$. paxilli deletion mutant strain PN2250. For the top-down approach, a MIDAS-modified bacterial artificial chromosome has been developed that will be used to assemble and introduce the entire $N O D$ cluster into the $\triangle P A X$-cluster $P$. paxilli mutant strain PN2250.

Along side the functional characterisation of genes involved in the nodulisporic acid A (1) biosynthetic pathway, efforts will be directed toward quantifying titres and optimising the expression host system to produce the highest possible yields of nodulisporic acid A (1). Many steps will be involved in yield-optimisation and some promising places to explore include the exchange of promoter regions, combinatorial anaylsis of different promoters, controlling integration sites, varying the copy number of specific genes, experimenting with different host species, trialling different fermentation conditions, development of quantitative assays for all intermediates in the pathway, and work to understand flux bottlenecks and engineer improved titres using classical metabolic engineering approaches.

Overall, work in this thesis demonstrates the power of using a gene characterisation platform to functionally characterise genes and identify how valuable compounds are produced. 


\section{What is the benefit of this work and what will be done with all that has been learned?}

As indole diterpenes are well known to have potent insecticidal7-10, 74-78 and anticancer properties, ${ }^{13}, 30,79-82$ they are ideal for application in both the agri-technology and pharmaceutical industries. Fundamentally, this thesis has focused around the development of a powerful biosynthetic platform for the functional analysis of genes to enable commercialization of one valuable indole diterpene, nodulisporic acid $A(1)$, for use as an animal health therapeutic; but that is only the tip of the iceberg. Over 200 indole-diterpene compounds have been identified from biological samples so far, representing different natural combinations of genes with overlapping and distinct gene-encoded chemistry. Approximately 40 secondary metabolic indole diterpene genes have been characterised, which naturally deliver around 80 unique indole diterpene products. The chemical diversity within the indole diterpenes arises from the role of different gene products that modify one general starting block into multiple bioactive products.

The functional confirmation of genes within biosynthetic pathways provides access to natural products not only for derivatisation by synthetic chemical approaches but also through derivatisation by gene recombination approaches. Therefore, upon completion of the functional characterisation of each gene in the nodulisporic acid $A$ (1) biosynthetic pathway, analogues will be generated by mixing-and-matching functionally confirmed genes from different indole diterpene pathways. MIDAS will be used to combine genes from different biosynthetic pathways into Level-3 vectors that will be heterologously expressed in appropriate $P$. paxilli deletion mutants (Figure 7.2). As an example, a theoretical non-natural product is depicted in Figure 7.2 that could be biosynthesised by mixing two decoration genes (ItmE and ItmJ) from the lolitrem biosynthetic pathway of Epicloë festucae with the genes from the nodulisporic acid pathway. 


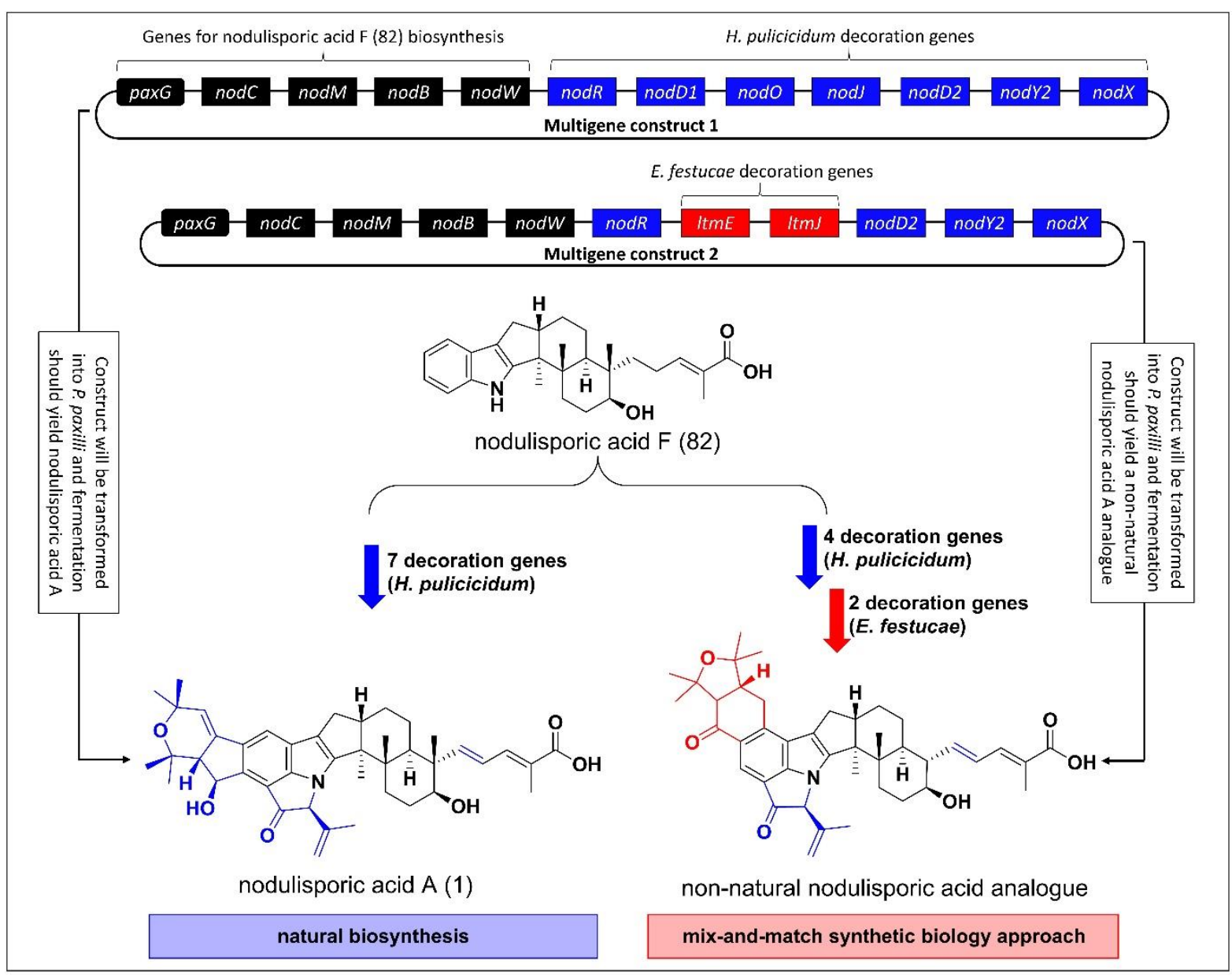

Figure 7.2. Depiction of two theoretical MIDAS-generated multigene constructs for the biosynthesis of a natural product or a non-natural analogue. Multigene construct 1 would be used for the biosynthesis of the natural product nodulisporic acid A (1) and Multigene construct 2 would be used to create a non-natural nodulisporic acid A (1) analogue through a mix-and-match synthetic biology approach.

\section{The opportunity for a metabolic engineering business}

As mentioned in a recent review, major efforts are being put forth toward metabolic engineering of microbes for the sustainable production of biofuels, novel chemicals, pharmaceuticals and nutraceuticals. ${ }^{83}$ Taken as a whole, the work presented in this thesis provides an opportunity create a metabolic engineering business based around providing natural products and their non-natural derivatives for use across many industries. Accordingly, the general business model would follow a pipeline similar to that presented in Figure 7.3, where biosynthetic gene clusters will be identified, genes will be annotated, MIDAS will be used to assemble the genes into expression vectors, genes will be heterologously expressed, transformants will be chemotyped for novel compounds, novel compounds will be characterised and microbial factories will be 
optimised for market. As with any business model, there are risks associated along every step of the pipeline from not being able to locate essential biosynthetic genes to producing chemicals that have no commercial value. Many of these risks can be mitigated by targeting compounds that are clearly valuable (i.e. have useful chemical properties) and belong to a family of compounds with well characterised biosynthetic pathways (i.e. homologous genes sequences are available to identify new genes using sequence comparisons). The prospects are exciting as the tools are now available to exploit efficiently the nearly infinite pool of natural products that nature provides.

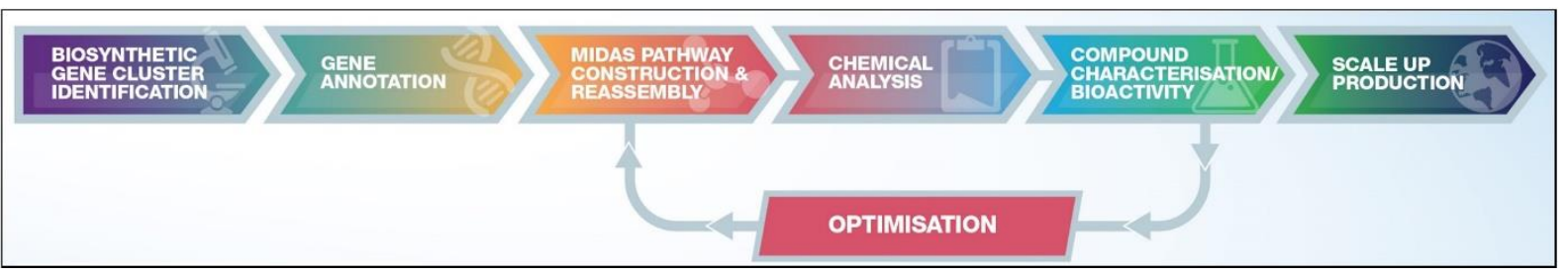

Figure 7.3. Depiction of business model for microbial factories. 


\section{MATERIALS AND METHODS}

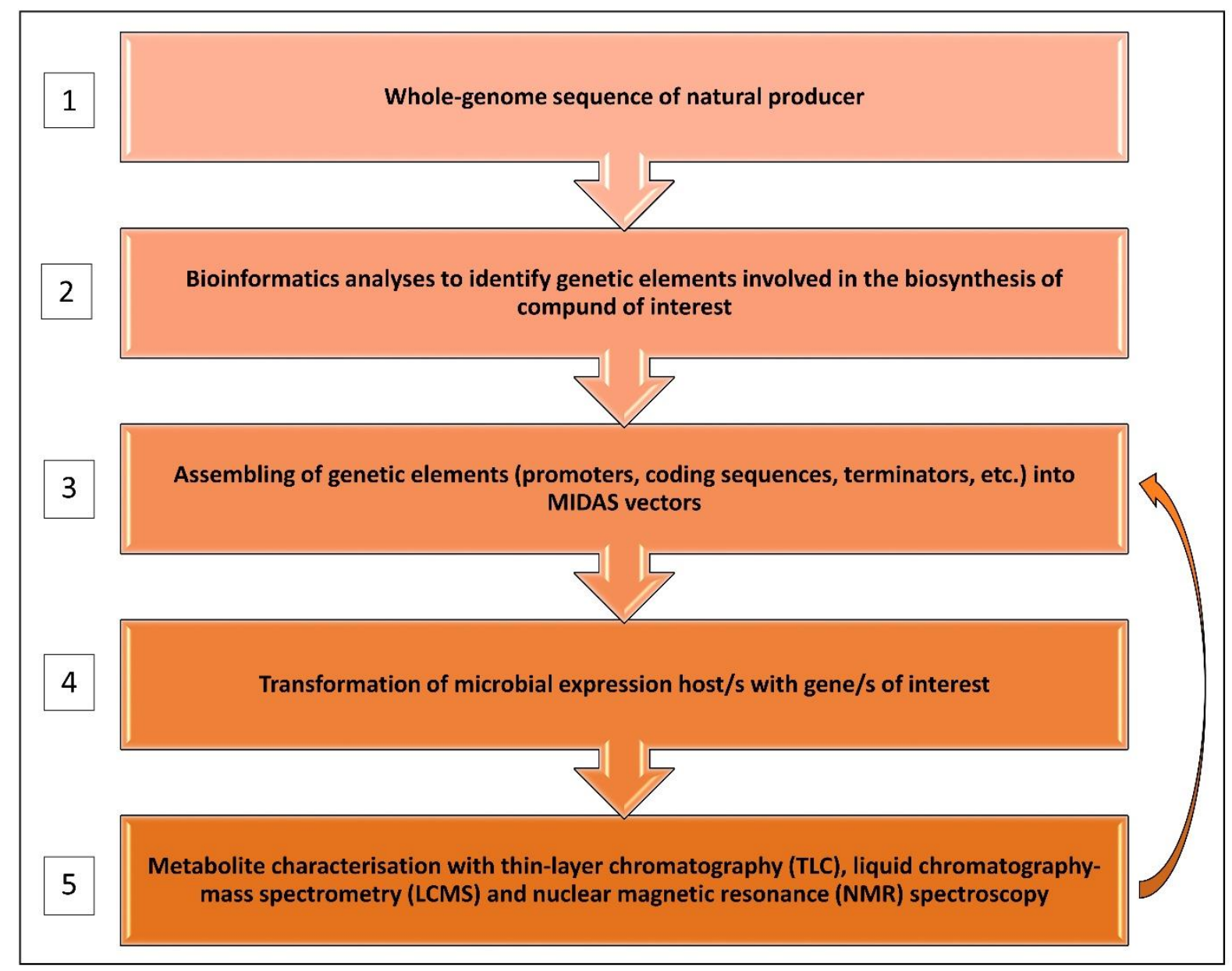

Figure 8.1. Overview of methods used in this thesis.

All of the below methods were described in Van de Bittner et al. (2018) ${ }^{1}$ or van Dolleweerd et al. (2018) ${ }^{3}$ and have been listed here for convenience. Some of the methods have been slightly modified from their published versions. 


\section{gDNA isolation for genome sequencing and transcription unit module amplification}

Initially, genomic DNA for genome sequencing and transcription unit module amplification by PCR was isolated from Penicillium paxilli strain ATCC ${ }^{\circledR} 26601^{\mathrm{TM}}$ (PN2013) ${ }^{72}$ and Hypoxylon pulicicidum strain ATCC $^{\circledR} 74245^{\text {TM }} 84$ according to Byrd et al. ${ }^{85}$ with modifications. Sterile $2.4 \%(\mathrm{w} / \mathrm{v})$ Difco ${ }^{\mathrm{TM}}$ potato dextrose broth (Becton, Dickinson and Company, Maryland, U.S.A.) in Milli- ${ }^{\circledR}$ water was prepared in $25 \mathrm{~mL}$ aliquots in $125 \mathrm{~mL}$ Erlenmeyer flasks and inoculated with $5 \times 10^{6}$ spores or $\sim 1 \mathrm{~cm}^{2}$ freshly ground mycelia (for non-sporulating strains). Cultures were incubated for 2-4 days at $22^{\circ} \mathrm{C}$ with shaking (200 rpm). The fermentation broth was filtered through a sterile nappy liner and the mycelia were rinsed three times with sterile water. Mycelia were transferred to a sterile $15 \mathrm{~mL}$ centrifuge tube and flash frozen in liquid nitrogen for lyophilization for 24-48 hours. $15-20 \mathrm{mg}$ freeze dried mycelia was placed in a mortar with liquid nitrogen and ground into a powder. The ground mycelia were transferred into a $2 \mathrm{~mL}$ tube and resuspended in $1 \mathrm{~mL}$ extraction buffer (150 mM EDTA, $50 \mathrm{mM}$ Tris- $\mathrm{HCl}$, and $1 \%(\mathrm{w} / \mathrm{v})$ sodium lauroyl sarcosine). $1.6 \mathrm{mg}$ proteinase $\mathrm{K}$ was added to the tube and contents were incubated at $37^{\circ} \mathrm{C}$ for $30 \mathrm{~min}$. The tube was centrifuged at $13,000 \mathrm{rpm}$ for $10 \mathrm{~min}$ and the supernatant was transferred to a fresh $2 \mathrm{~mL}$ tube. 500 $\mu \mathrm{L}$ phenol and $500 \mu \mathrm{L}$ chloroform were added to the tube and the contents were mixed by vortex before centrifugation for $10 \mathrm{~min}$ at 13,000 rpm. The aqueous phase was transferred to a fresh $2 \mathrm{~mL}$ tube and washed two more times with $500 \mu \mathrm{L}$ phenol and $500 \mu \mathrm{L}$ chloroform as previously described. The aqueous phase was then transferred to a fresh $2 \mathrm{~mL}$ tube and washed (vortexed and centrifuged at 13,000 rpm for $10 \mathrm{~min}$ ) with $1 \mathrm{~mL}$ chloroform. The aqueous phase was transferred to a fresh $2 \mathrm{~mL}$ tube and mixed with $1 \mathrm{~mL}$ chilled isopropanol. The DNA was precipitated overnight at $20^{\circ} \mathrm{C}$ and pelleted at 13,000 rpm for $10 \mathrm{~min}$. The supernatant was discarded and the DNA was resuspended in $1 \mathrm{~mL} 1 \mathrm{M} \mathrm{NaCl}$. The tube was incubated for $10 \mathrm{~min}$ at room temperature and then centrifuged at $13,000 \mathrm{rpm}$ for $10 \mathrm{~min}$ to pellet polysaccharides. The supernatant was transferred to a fresh tube and mixed with $1 \mathrm{~mL}$ isopropanol. The tube was incubated at room temperature for $10 \mathrm{~min}$ and DNA was pelleted by centrifugation at 13,000 rpm for $10 \mathrm{~min}$. The supernatant was discarded and $1 \mathrm{~mL}$ chilled $70 \%$ ethanol was added to the pellet without resuspension. The tube was centrifuged for $2 \mathrm{~min}$ at 13,000 rpm and the supernatant was discarded. The tube was 
centrifuged for $1 \mathrm{~min}$ at 13,000 rpm and residual 70\% ethanol was pipetted off. The pellet was air dried at room temperature, resuspended in $50 \mu \mathrm{L}$ Milli-Q ${ }^{\circledR}$ water and stored at $-20^{\circ} \mathrm{C}$.

Alternatively, genomic DNA was isolated using the ZR Fungal/Bacterial DNA MicroPrep ${ }^{\text {TM }}$ Kit from Zymo Research. First, sterile 2.4\% (w/v) DifcoTM potato dextrose broth (Becton, Dickinson and Company, Maryland, U.S.A.) in Milli-Q® water was prepared in $25 \mathrm{~mL}$ aliquots in $125 \mathrm{~mL}$ Erlenmeyer flasks and inoculated with $5 \times 106$ spores or $\sim 1 \mathrm{~cm} 2$ freshly ground mycelia (for non-sporulating strains). Cultures were incubated for $2-4$ days at $22^{\circ} \mathrm{C}$ with shaking (200 rpm). The fermentation broth was filtered through a sterile nappy liner and the mycelia were rinsed three times with sterile water. Mycelia were transferred to a $1.5 \mathrm{~mL}$ Safe-Lock tube containing $750 \mu \mathrm{L}$ Lysis Solution (ZR Fungal/Bacterial DNA MicroPrepTM, Zymo Research) and $\sim 100 \mu \mathrm{L} 1.0$ mm zirconium oxide beads (Next Advance). Samples were placed in a Storm 24 Bullet Blender (Next Advance) and homogenised at speed 8 for 5 min. Tubes were centrifuged at $10,000 \times \mathrm{g}$ for $1 \mathrm{~min}$ and $400 \mu \mathrm{L}$ supernatant was transferred to a ZymoSpinTM IV Spin Filter in a Collection Tube and ultra-pure DNA was purified as detailed in the ZR Fungal/Bacterial DNA MicroPrepTM kit (Zymo Research). DNA was eluted in Milli-Q尺 water rather than the elution buffer provided with the ZR Fungal/Bacterial DNA MicroPrepTM kit (Zymo Research) and stored at $-20^{\circ} \mathrm{C}$.

\section{Genome sequencing, identification and annotation of the $H$. pulicicidum (ATCC ${ }^{\circledR} 74245^{\mathrm{TM}}$ ) NOD gene cluster and GenBank accession numbers}

Genomic DNA was sequenced on an Illumina MiSeq instrument by New Zealand Genomics Limited (NZGL) obtaining approximately 49-fold genome coverage. Reads were dynamically trimmed using the SolexaQA++ package to their longest fragment such that base call error rates did not exceed $P=0.01$ and the minimum length of paired end reads was $25 \mathrm{bp}$. De novo assembly was performed using SPAdes Genome Assembler (version 3.5.0) with the default parameters using a kmer range of 39-127, and scaffolding was performed using SSPACE version 1.10 and GapFiller version 3.0.

Bioinformatic analyses were performed using BLAST and Fgenesh gene finding software $^{86}$ to facilitate identification and manual annotation of the gene cluster that is 
responsible for nodulisporic acid biosynthesis (Chapter 2, Table 2.1). The annotated nucleotide sequence of the $H$. pulicicidum (ATCC74245) NOD gene cluster has been deposited with GenBank ${ }^{2}$ with accession number MG182145. The accession number for the $H$. pulicicidum (ATCC74245) primary geranylgeranyl pyrophosphate (3) synthase is MG182146. Preparation of similarity matrices and phylogenetic trees for cluster identification.

All similarity matrices and phylogenetic trees (See Appendix, Figures 9.1 to 9.7 and Table 9.1) were constructed in MacVector 10.0.2 and based on pairwise and multiple sequence alignments generated using ClustalW (v1.83). Alignments were performed with the following parameters: open gap penalty $=10$, extend gap penalty $=0.1$, and using the Gonnet similarity matrix. Additional parameters for multiple alignments were: delay divergent $=40 \%$ and gap distance $=8$. Phylogenetic trees were calculated using UPGMA best tree with systematic tie breaking. Distance was uncorrected and gaps were distributed proportionally.

\section{Molecular biology reagents}

Restriction endonucleases were purchased from New England Biolabs, except Aarl, which was purchased from Thermo Fisher Scientific. T4 DNA Ligase, 10× T4 DNA Ligase buffer and $10 \mathrm{mM}$ ATP were from New England Biolabs. Primers and gBlocks were synthesised by Integrated DNA Technologies. Other synthetic polynucleotides were synthesised by Epoch Life Science, Inc. Plasmids pBB528 and pBB535 were a gift from Bernd Bukau (Addgene plasmids \#27390 and \#27392, respectively). Kits for purification of plasmid DNA and PCR products using spin-column protocols were purchased from Macherey-Nagel. All PCRs for the construction of the MIDAS source, shuttle and destination vectors, and for amplification of MIDAS modules were performed using Phusion High-Fidelity PCR Master Mix with HF Buffer (New England Biolabs). Construction of the MIDAS vectors is described below. Isopropyl $\beta-D-1-$ thiogalactopyranoside (IPTG) was purchased from Calbiochem, 5-bromo-4-chloro-3indolyl- $\beta$-D-galactopyranoside (X-Gal) from PanReac AppliChem, and 4-chloro-DLphenylalanine (4CP) from Sigma. Antibiotics used in this work were; Geneticin (G418, 
from Sigma), kanamycin (PanReac AppliChem) and spectinomycin (Gold Biotechnology).

\section{Bacterial strains}

Routine growth of Escherichia coli was performed at $37^{\circ} \mathrm{C}$ in LB broth. Chemically competent E. coli HST08 Stellar cells (Clontech Laboratories, Inc.) were used for routine transformations and maintenance of all plasmids (including source, shuttle and destination vectors, and all plasmids assembled at Level-1, Level-2, and Level-3).

\section{PCR}

All PCRs for the construction of the MIDAS source, shuttle and destination vectors, and for amplification of MIDAS modules were performed using Phusion High-Fidelity PCR Master Mix with HF Buffer (New England Biolabs). Reactions were completed in $200 \mu \mathrm{L}$ tubes typically containing $10 \mu \mathrm{L}$ of $5 X$ Phusion Buffer, $1 \mu \mathrm{L} 10 \mathrm{mM}$ dNTPs, $2 \mu \mathrm{L}$ template (e.g. $20 \mathrm{ng}$ genomic DNA), $2.5 \mu \mathrm{L} 10 \mu \mathrm{M}$ forward primer, $2.5 \mu \mathrm{L} 10 \mu \mathrm{M}$ reverse primer, $31.5 \mu \mathrm{L}$ Milli-Q ${ }^{\circledR}$ water, and $0.5 \mu \mathrm{L}$ Phusion polymerase for a total volume of 50 $\mu \mathrm{L}$. Reactions were carried out in a DNA Engine PTC-200 Peltier Thermal Cycler (BioRad) using the following parameters: 2 minutes at $98^{\circ} \mathrm{C}, 35$ cycles of (30 seconds at $98^{\circ} \mathrm{C}, 30$ seconds at $\mathrm{n}-2^{\circ} \mathrm{C}$ (where $\mathrm{n}$ is the lowest primer melting temperature) and 90 seconds at $72^{\circ} \mathrm{C}$ ), and 5 minutes at $72^{\circ} \mathrm{C}$ followed by an infinite hold at $4^{\circ} \mathrm{C}$.

\section{Construction of MIDAS vectors}

The initial plasmids produced during construction of the MIDAS vectors were constructed in a modular fashion by Bsal-mediated Golden Gate assembly of PCRgenerated DNA fragments containing terminal, convergent Bsal recognition sites (Tables 8.1, 8.2 and 8.3 below). Since all MIDAS source, shuttle and destination vectors were required to be domesticated (i.e. made free of recognition sites for Aarl, Bsal and $B s m \mathrm{BI})$, the PCR fragments were chosen to correspond to specific, functional plasmid structures (e.g., origin of replication, resistance markers, etc.), and by taking into account the number of internal Type IIS sites to be removed. Typically, 1-2 $\mu \mathrm{L}$ of

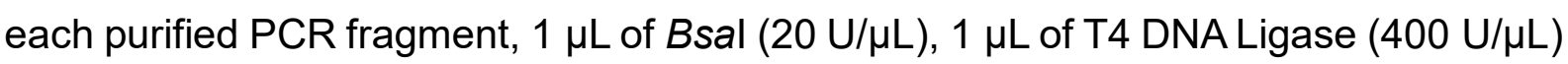


and $2 \mu \mathrm{L}$ of $10 \times$ T4 DNA Ligase buffer in a total reaction volume of $20 \mu \mathrm{L}$ were incubated overnight at $37^{\circ} \mathrm{C}$. Construction of the plasmids is described in detail below.

\section{Construction of the pML1 source vector.}

A precursor to source vector PML1 was generated using a four-way Bsal-mediated Golden Gate reaction between PCR fragments CV-161, CV-162, CV-74 and CV-152 (Table 8.1). Following Golden Gate assembly, an aliquot of each reaction was transformed into E. coli HST08 Stellar competent cells by heat shock and spread onto LB plates supplemented with $50 \mu \mathrm{g} / \mathrm{mL}$ spectinomycin, $1 \mathrm{mM}$ IPTG and $50 \mu \mathrm{g} / \mathrm{mL}$ XGal. Blue colonies were screened by restriction enzyme digestion of purified plasmid DNA, and confirmed by sequencing. 
Table 8.1. PCR primers used in the construction of the pML1 source vector. Type IIS restriction enzyme recognition sites are colour-coded for Bsal (ggtctc) and BsmBI (cgtctc), with overhangs shaded grey. Ncol (ccatgg) and Xhol (ctcgag) cloning sites are shown underlined. Spec ${ }^{\mathrm{R}}$ denotes spectinomycin resistance.

\begin{tabular}{|c|c|c|c|c|}
\hline $\begin{array}{l}\text { PCR name } \\
\text { (Size, bp) }\end{array}$ & Plasmid fragment & Fragment description & $\begin{array}{l}\text { Template } \\
\text { (source/ } \\
\text { reference) }\end{array}$ & $\begin{array}{l}\text { Primer name (length): } \\
\left.\text { Primer sequence (5' to } 3^{\prime}\right)\end{array}$ \\
\hline \multirow{2}{*}{$\begin{array}{l}\text { CV-161 } \\
(1125)\end{array}$} & \multirow{2}{*}{$\begin{array}{l}\text { Bsal[ACCG]-P aadA-Spec }{ }^{R_{-}} \\
\text {[GAAT]Bsal }\end{array}$} & \multirow{2}{*}{$\begin{array}{l}\text { Bacterial spectinomycin } \\
\text { resistance gene driven by the } \\
\text { aadA promoter }\end{array}$} & \multirow{2}{*}{ pBB535 ${ }^{87}$} & $\begin{array}{l}\text { cvd2016-01-13a (41-mer): } \\
\text { cgctcacggtctcaACCGgacgtcgatatccggatgaaggc }\end{array}$ \\
\hline & & & & $\begin{array}{l}\text { cvd2016-01-13b (45-mer): } \\
\text { tgaacgaggtctcaATTCttatttgccgactaccttggtgatctc }\end{array}$ \\
\hline \multirow{2}{*}{$\begin{array}{l}\text { CV-162 } \\
(591)\end{array}$} & \multirow{2}{*}{$\begin{array}{l}\text { Bsal[GAAT]-f1 ori- } \\
\text { [GTGT]Bsal }\end{array}$} & \multirow{2}{*}{$\begin{array}{l}\text { Bacteriophage } \mathrm{f} 1 \text { origin of } \\
\text { replication }\end{array}$} & \multirow{2}{*}{$\begin{array}{l}\text { pET-28a }(+) \\
\text { (Novagen) }\end{array}$} & $\begin{array}{l}\text { cvd2016-01-13c (55-mer): } \\
\text { gatgagttggtctcaGAATtaattcatgagcggatacatatttgaatgtatt } \\
\text { tag }\end{array}$ \\
\hline & & & & $\begin{array}{l}\text { cvd2015-11-18d (35-mer): } \\
\text { gaggaacggtctcCACACtggcgaatgggacgcgc }\end{array}$ \\
\hline \multirow{2}{*}{$\begin{array}{l}\text { CV-74 } \\
(372)\end{array}$} & \multirow{2}{*}{$\begin{array}{l}\text { Bsal[GTGT]-lacZa- } \\
\text { [GGCA]Bsal }\end{array}$} & \multirow{2}{*}{$\begin{array}{l}\text { Wild type lacZa fragment, } \\
\text { driven by the lac promoter. }\end{array}$} & \multirow{2}{*}{$\begin{array}{l}\text { E. coli K12 } \\
\text { ER2925 (New } \\
\text { England Biolabs) }\end{array}$} & $\begin{array}{l}\text { cvd2015-05-28b (47-mer): } \\
\text { ctttccggtctcaGTGTccatggttattaccaggcaaagcgccattc }\end{array}$ \\
\hline & & & & $\begin{array}{l}\text { cvd2015-05-28k (54-mer): } \\
\text { gacaggtttggtctcgTGCCctcgagcagctggcgcaacgcaattaatgtga } \\
\text { gt }\end{array}$ \\
\hline \multirow{2}{*}{$\begin{array}{l}\text { CV-152 } \\
(1070)\end{array}$} & \multirow{2}{*}{$\begin{array}{l}\text { Bsal[GGCA]-pMB1 ori- } \\
\text { [ACCG]Bsal }\end{array}$} & \multirow{2}{*}{$\begin{array}{l}\text { Bacterial pMB1 plasmid origin } \\
\text { of replication. }\end{array}$} & \multirow{2}{*}{$\begin{array}{l}\text { pUC19 } \\
\text { (Clontech } \\
\text { Laboratories, Inc.) }\end{array}$} & $\begin{array}{l}\text { cvd2015-11-18a }(39-\text {-mer }): \\
\text { cacattaaggtctctGGCAtcactgccegctttccagtc }\end{array}$ \\
\hline & & & & $\begin{array}{l}\text { cvd2015-11-18b }(50-\text { mer }): \\
\text { tgattaggtctcgCGGTctgtcagaccaagtttactcatatatactttag }\end{array}$ \\
\hline \multirow{2}{*}{$\begin{array}{l}\text { CV-81 } \\
(367)\end{array}$} & \multirow{2}{*}{$\begin{array}{l}\text { Ncol-[CTCG]BsmBI-lacZa- } \\
\text { BsmBI[AGAC]-Xhol }\end{array}$} & \multirow{2}{*}{$\begin{array}{l}\text { Fragment containing the pML1 } \\
\text { Golden Gate cloning cassette, } \\
\text { i.e., lacZa (driven by the lac } \\
\text { promoter) flanked by divergent } \\
\text { BsmBI recognition sites. }\end{array}$} & \multirow{2}{*}{$\begin{array}{l}\text { E. coli K12 } \\
\text { ER2925 } \\
\text { (New England } \\
\text { Biolabs) }\end{array}$} & $\begin{array}{l}\text { cvd2015-05-28d (50-mer): } \\
\text { cagctttcccatggtCTCGtgagacgttattaccaggcaaagcgccattc }\end{array}$ \\
\hline & & & & $\begin{array}{l}\text { cvd2015-05-281 (46-mer): } \\
\text { tcccgacctcgagGTCTagagacggcgcaacgcaattaatgtgagt }\end{array}$ \\
\hline
\end{tabular}


The $329 \mathrm{bp} \mathrm{Ncol-Xhol} \mathrm{fragment} \mathrm{of} \mathrm{the} \mathrm{pML1} \mathrm{precursor} \mathrm{was} \mathrm{replaced,} \mathrm{by} \mathrm{restriction}$ enzyme digestion and ligation, with the 346 bp Ncol-Xhol fragment of PCR CV-81, which contains a lacZa-based Golden Gate cloning cassette ([CTCG]BsmBI-lacZ $\alpha$ BsmBI[AGAC]). The resultant plasmid is the Level-1 source vector, pML1 (Figure 8.2).

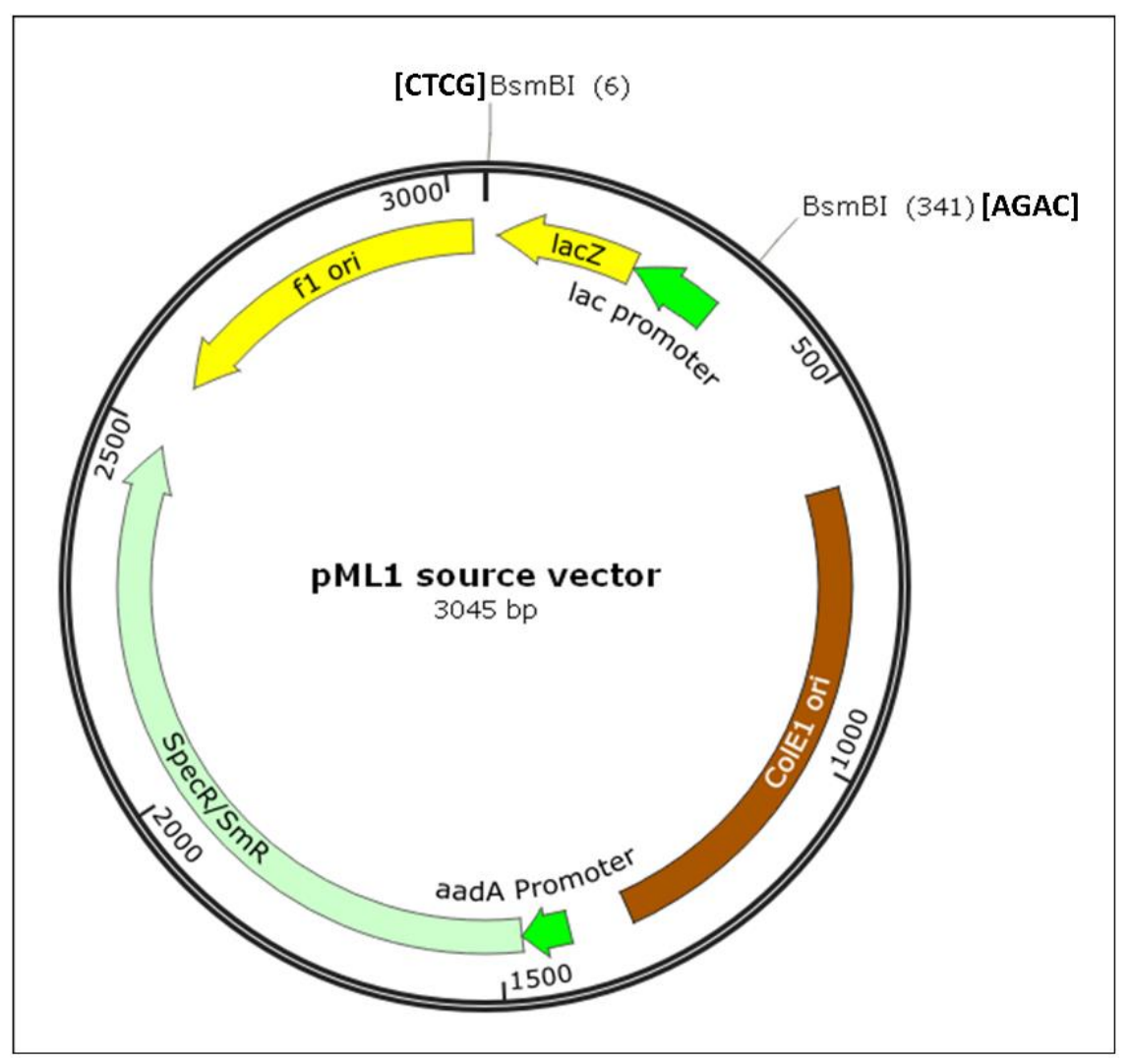

Figure 8.2. pML1 source vector.

\section{Construction of pML2 shuttle vectors.}

A precursor to the pML2 shuttle vectors was generated using a four-way Bsalmediated Golden Gate reaction between PCR fragments CV-153, CV-154, CV-77 and CV-152 (Table 8.2). Following Golden Gate assembly, an aliquot of each reaction was transformed into E. coli HST08 Stellar competent cells by heat shock and spread onto LB plates supplemented with $75 \mu \mathrm{g} / \mathrm{mL}$ kanamycin, $1 \mathrm{mM} \mathrm{IPTG}$ and $50 \mu \mathrm{g} / \mathrm{mL}$ X-Gal. Blue colonies were screened by restriction enzyme digestion of purified plasmid DNA, and confirmed by sequencing. 
Table 8.2. PCR primers used in the construction of the pML2 shuttle vectors. The Bsal restriction enzyme recognition sites are colour-coded (ggtctc), with overhangs shaded grey. Ncol (ccatgg) and Xhol (ctcgag) cloning sites are shown underlined. Kan ${ }^{\mathrm{R}}$ denotes kanamycin resistance.

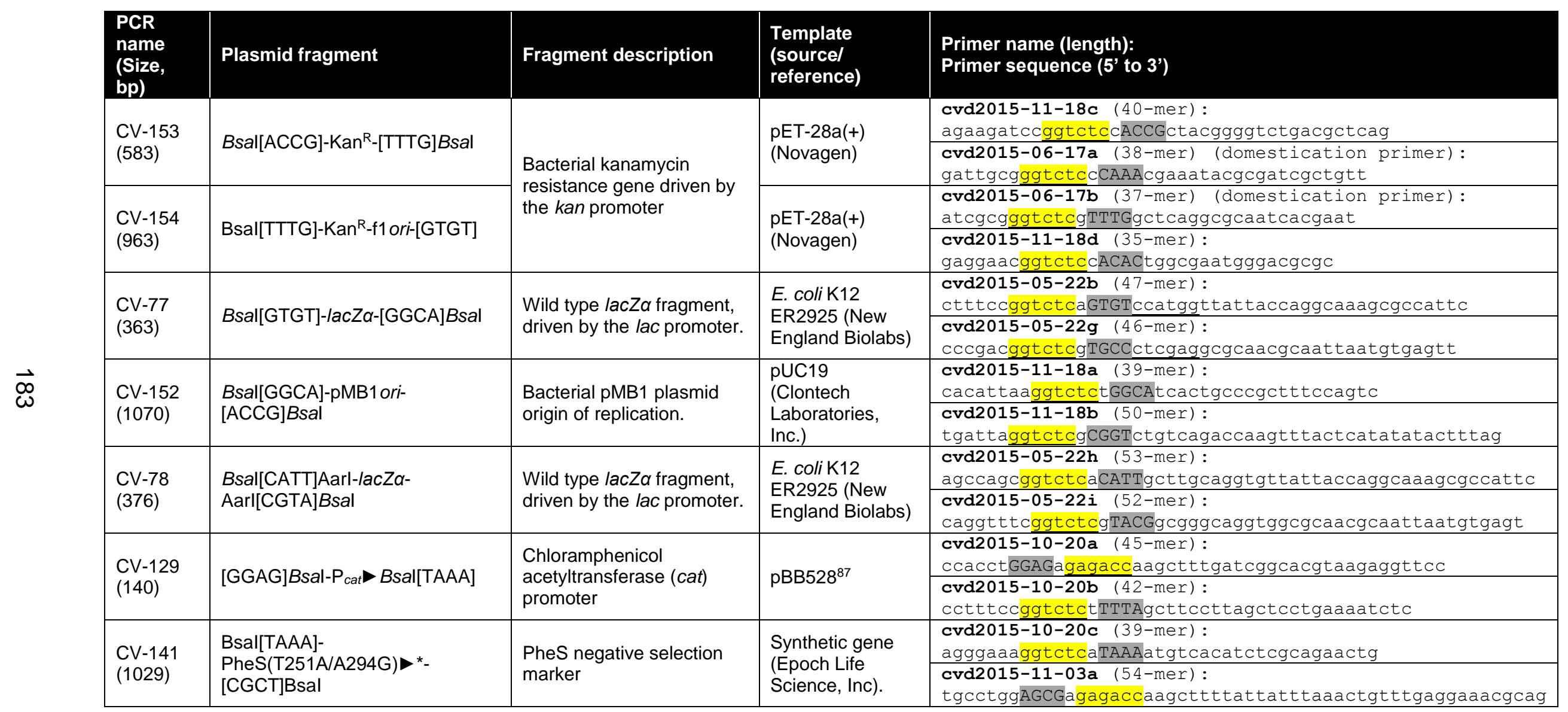


The pML2 precursor (Figure 8.3) was digested with $\mathrm{Ncol}+$ Xhol and ligated to gBlocks digested with the same enzymes. The eight gBlocks (Table 8.3) are classified into two groups - one group comprising four "White" gBlocks (ML2(+)WF, ML2(+)WR, ML2(-)WF and ML2(-)WR) and a second group composed of four "Blue" gBlocks (ML2(+)BF, ML2(+)BR, ML2(-)BF or ML2(-)BR). Ligations were transformed into $E$. coli Stellar and spread onto LB plates supplemented with $75 \mu \mathrm{g} / \mathrm{mL}$ kanamycin, $1 \mathrm{mM}$ IPTG and $50 \mu \mathrm{g} / \mathrm{mL}$ X-Gal. White colonies were analysed by restriction enzyme digestion of purified plasmid DNA, and confirmed by sequencing. The resultant eight plasmids were designated "gBlock precursor plasmids", comprising four "White" gBlock precursor plasmids and four "Blue" gBlock precursor plasmids.

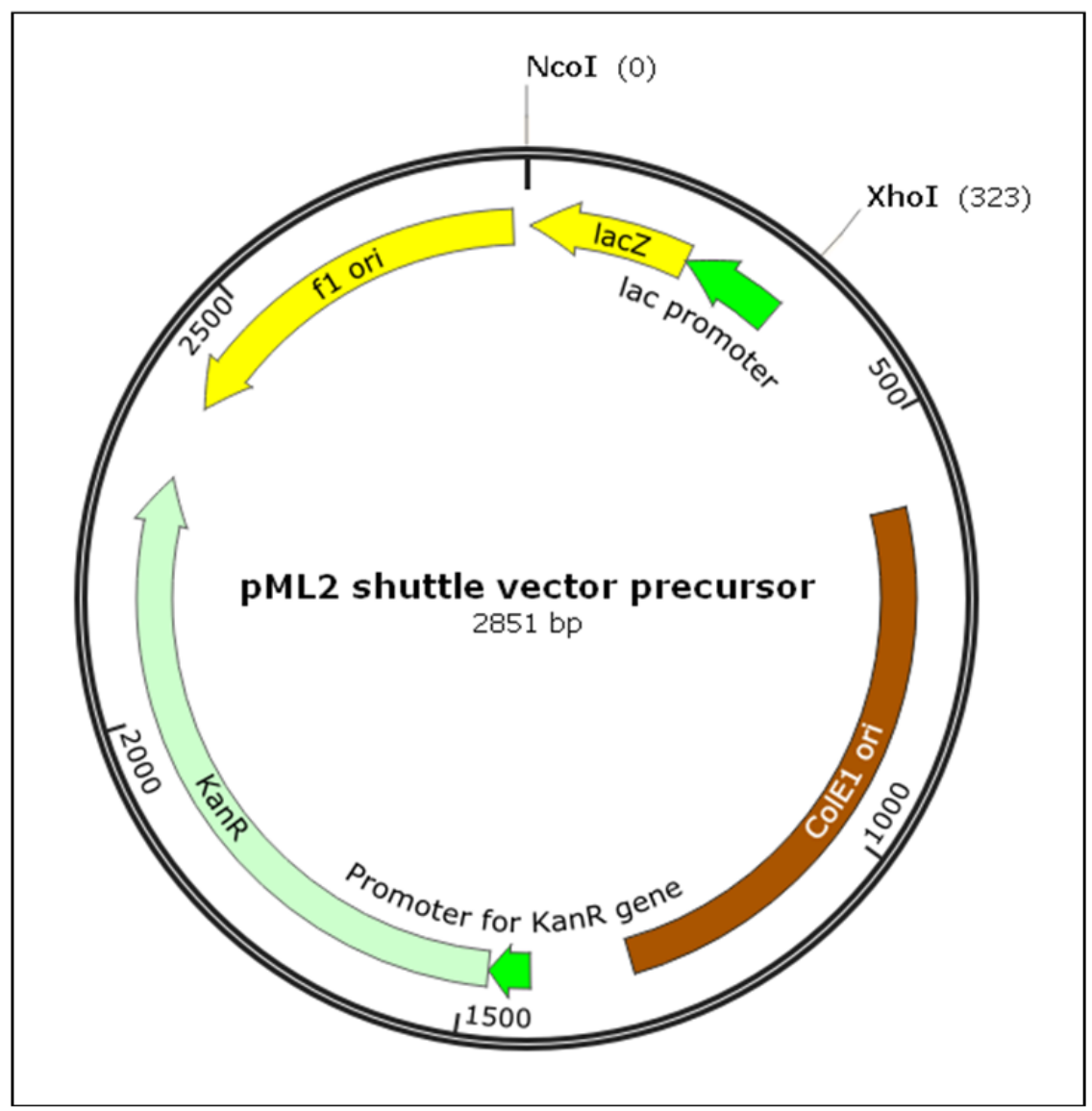

Figure 8.3. pML2 shuttle vector precursor. 
Table 8.3. gBlock sequences used for the construction of the pML2 shuttle vectors. gBlocks are synthesised as double stranded DNA; only the top strand (5' to 3') is shown in the table. Ncol (ccatgg) and Xhol (ctcgag) cloning sites are shown underlined. Type IIS restriction enzyme recognition sites are colour-coded for Aarl (cacctgc), Bsal (ggtctc) and BsmBI (cgtctc), with overhangs shaded grey.

\begin{tabular}{|c|c|c|}
\hline & $\begin{array}{l}\text { gBlock } \\
\text { name }\end{array}$ & gBlock sequence ( $5^{\prime}$ to $\left.3^{\prime}\right)$ \\
\hline \multirow{4}{*}{$\begin{array}{l}\text { "White" } \\
\text { gBlocks }\end{array}$} & ML2(+)WF & $\begin{array}{l}\text { gaccccgtaggtgtccatggcacctgcagctcattggagagagaccttaagcttgcagcagcggtctctcgc } \\
\text { tcattagagacggccggattgcggccgctaaccggccgtctcacgtaatatgcaggtgctcgagggcatcaa } \\
\text { at }\end{array}$ \\
\hline & ML2(+)WR & $\begin{array}{l}\text { gaccccgtaggtgtccatggcacctgcagctcattagcgagagaccttaagcttgcagcagcggtctctctc } \\
\text { ccattagagacggccggattgcggccgctaaccggccgtctcacgtaatatgcaggtgctcgagggcatcaa } \\
\text { at }\end{array}$ \\
\hline & ML2(-)WF & $\begin{array}{l}\text { gaccccgtaggtgtccatggcacctgcagctcattagagacggccggattgcggccgctaaccggccgtctc } \\
\text { acgtaggagagagaccttaagcttgcagcagcggtctctcgctcgtaatatgcaggtgctcgagggcatcaa } \\
\text { at }\end{array}$ \\
\hline & ML2(-)WR & $\begin{array}{l}\text { gaccccgtaggtgtccatggcacctgcagctcattagagacggccggattgcggccgctaaccggccgtctc } \\
\text { acgtaagcgagagaccttaagcttgcagcagcggtctctctcccgtaatatgcaggtgctcgagggcatcaa } \\
\text { at }\end{array}$ \\
\hline \multirow{4}{*}{$\begin{array}{l}\text { "Blue" } \\
\text { gBlocks }\end{array}$} & ML2(+)BF & $\begin{array}{l}\text { gaccccgtaggtgtccatggcgtctcacattggagagagaccttaagcttgcagcagcggtctctcgctcat } \\
\text { tatatgcaggtggccggattgcggccgctaaccggccacctgcagctcgtaagagacgctcgagggcatcaa } \\
\text { at }\end{array}$ \\
\hline & ML2(+)BR & $\begin{array}{l}\text { gaccccgtaggtgtccatggcgtctcacattagcgagagaccttaagcttgcagcagcggtctctctcccat } \\
\text { tatatgcaggtggccggattgcggccgctaaccggccacctgcagctcgtaagagacgctcgagggcatcaa } \\
\text { at }\end{array}$ \\
\hline & ML2(-)BF & $\begin{array}{l}\text { gaccccgtaggtgtccatggcgtctcacattatatgcaggtggccggattgcggccgctaaccggccacctg } \\
\text { cagctcgtaggagagagaccttaagcttgcagcagcggtctctcgctcgtaagagacgctcgagggcatcaa } \\
\text { at }\end{array}$ \\
\hline & ML2(-)BR & $\begin{array}{l}\text { gaccccgtaggtgtccatggcgtctcacattatatgcaggtggccggattgcggccgctaaccggccacctg } \\
\text { cagctcgtaagcgagagaccttaagcttgcagcagcggtctctctcccgtaagagacgctcgagggcatcaa } \\
\text { at }\end{array}$ \\
\hline
\end{tabular}


A synthetic polynucleotide sequence encoding the $\mathrm{Thr}^{251} \mathrm{Ala} / \mathrm{Ala}{ }^{294} \mathrm{Gly}$ double mutant of the E. coli pheS, as described by Miyazaki, ${ }^{88}$ was supplied by Epoch Life Science, Inc. The coding sequence of the pheS gene was amplified (PCR CV-141) and placed under the control of the chloramphenicol acetyltransferase (cat) promoter by Bsalmediated Golden Gate assembly with PCR CV-129. The Bsal was heat-inactivated and the assembled pheS gene was then ligated into each of the eight gBlock precursor plasmids generated in the previous step that had also been treated with Bsal. Ligations were transformed by heat shock into E. coli HST08 Stellar competent cells, spread onto LB plates supplemented with $75 \mu \mathrm{g} / \mathrm{mL}$ kanamycin, and colonies analysed by restriction enzyme analysis and sequencing. For the four "White" gBlock precursor plasmids, ligation of the assembled pheS gene resulted in the four "White" Level-2 shuttle vectors: pML2(+)WF, pML2(+)WR, pML2(-)WF and pML2(-)WR (Figure 8.4). 


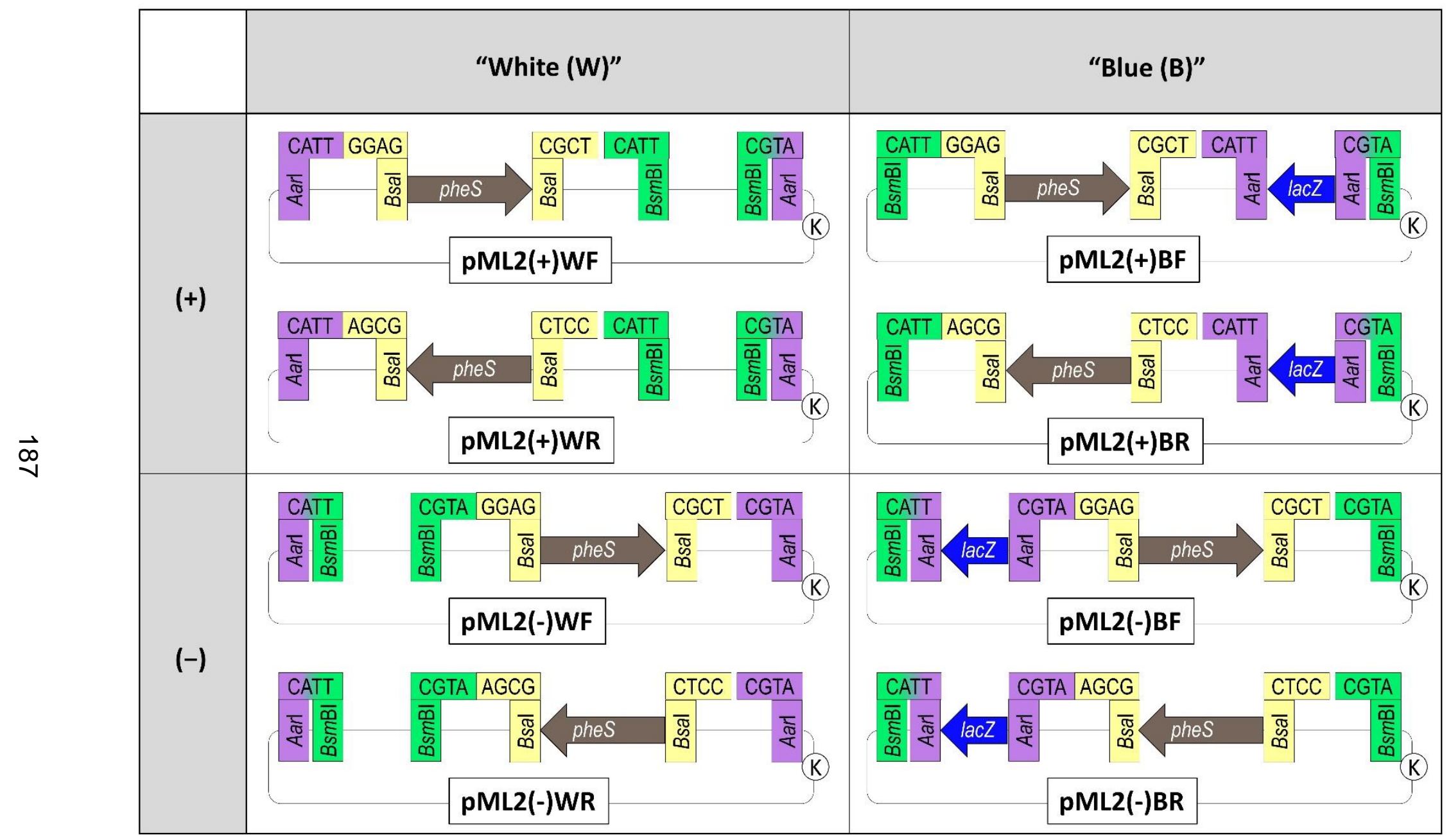

Figure 8.4. Overview of MIDAS cassettes. The structure of the MIDAS cassette in each of the eight pML2 shuttle vectors is depicted schematically. Within each MIDAS cassette, the dashed box shows the Golden Gate cloning cassette (comprised of divergent Bsal sites flanking a pheS negative selection marker) used for Level-2 assembly of TUs. 
To produce the four "Blue" Level-2 shuttle vectors, the four "Blue" gBlock precursor plasmids harbouring the assembled pheS gene from the previous step, were digested with Aarl and ligated to Bsal-digested PCR CV-78, resulting in the four "Blue" Level-2 shuttle vectors: pML2(+)BF, pML2(+)BR, pML2(-)BF and pML2(-)BR (Figure 8.4). Ligations were transformed into E. coli HST08 Stellar, spread onto LB plates supplemented with $75 \mu \mathrm{g} / \mathrm{mL}$ kanamycin, $1 \mathrm{mM}$ IPTG and $50 \mu \mathrm{g} / \mathrm{mL}$ X-Gal. Blue colonies were analysed by restriction analysis and sequencing.

Functionality of the mutant pheS gene in each of the eight pML2 shuttle vectors was confirmed by transforming $0.1 \mu \mathrm{g}$ of each plasmid into $E$. coli HST08 Stellar competent cells and spreading transformation mixes onto LB plates supplemented with $1.25 \mathrm{mM}$ $4 \mathrm{CP}$ and $75 \mu \mathrm{g} / \mathrm{mL}$ kanamycin, and onto LB plates supplemented with $75 \mu \mathrm{g} / \mathrm{mL}$ kanamycin only (no 4CP). In each case, no colonies were observed when 4CP. containing plates were spread with undiluted transformation mixes (giving a calculated efficiency of $<3.25 \times 10^{4} \mathrm{Kan}^{\mathrm{R}} \mathrm{CFU} / \mu \mathrm{g}$ ), while $>1.3 \times 10^{6} \mathrm{Kan}^{\mathrm{R}} \mathrm{CFU} / \mu \mathrm{\mu g}$ plasmid DNA was calculated for each vector following growth on plates devoid of $4 \mathrm{CP}$, indicating high counter-selection efficiency.

\section{Construction of the pML3 destination vector.}

Plasmid pML3 (Figure 8.5) was generated using a four-way Bsal-mediated Golden Gate reaction between PCR fragments CV-161, CV-162, CV-79 and CV-152 (Table 8.4). Following Golden Gate assembly, an aliquot of each reaction was transformed into E. coli HST08 Stellar competent cells by heat shock and spread onto LB plates supplemented with $50 \mu \mathrm{g} / \mathrm{mL}$ spectinomycin, $1 \mathrm{mM}$ IPTG and $50 \mu \mathrm{g} / \mathrm{mL}$ X-Gal. Blue colonies were screened by restriction enzyme digestion of purified plasmid DNA, and confirmed by sequencing. 


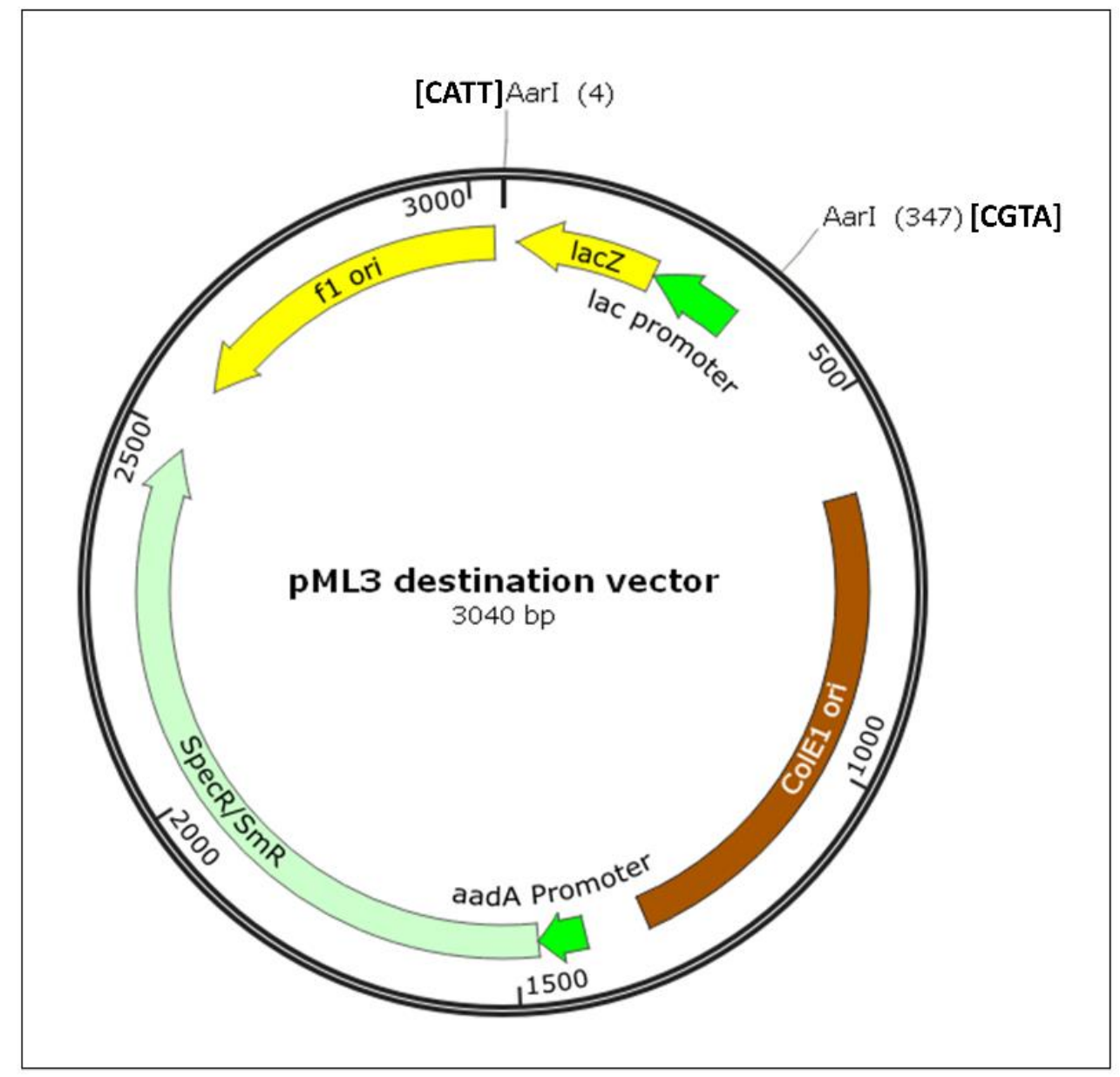

Figure 8.5. pML3 destination vector. 
Table 8.4. PCR primers used in the construction of the pML3 destination vector. Type IIS restriction enzyme recognition sites are colour-coded for Bsal (ggtctc) and Aarl (cacctgc), with overhangs shaded grey. Spec ${ }^{\mathrm{R}}$ denotes spectinomycin resistance.

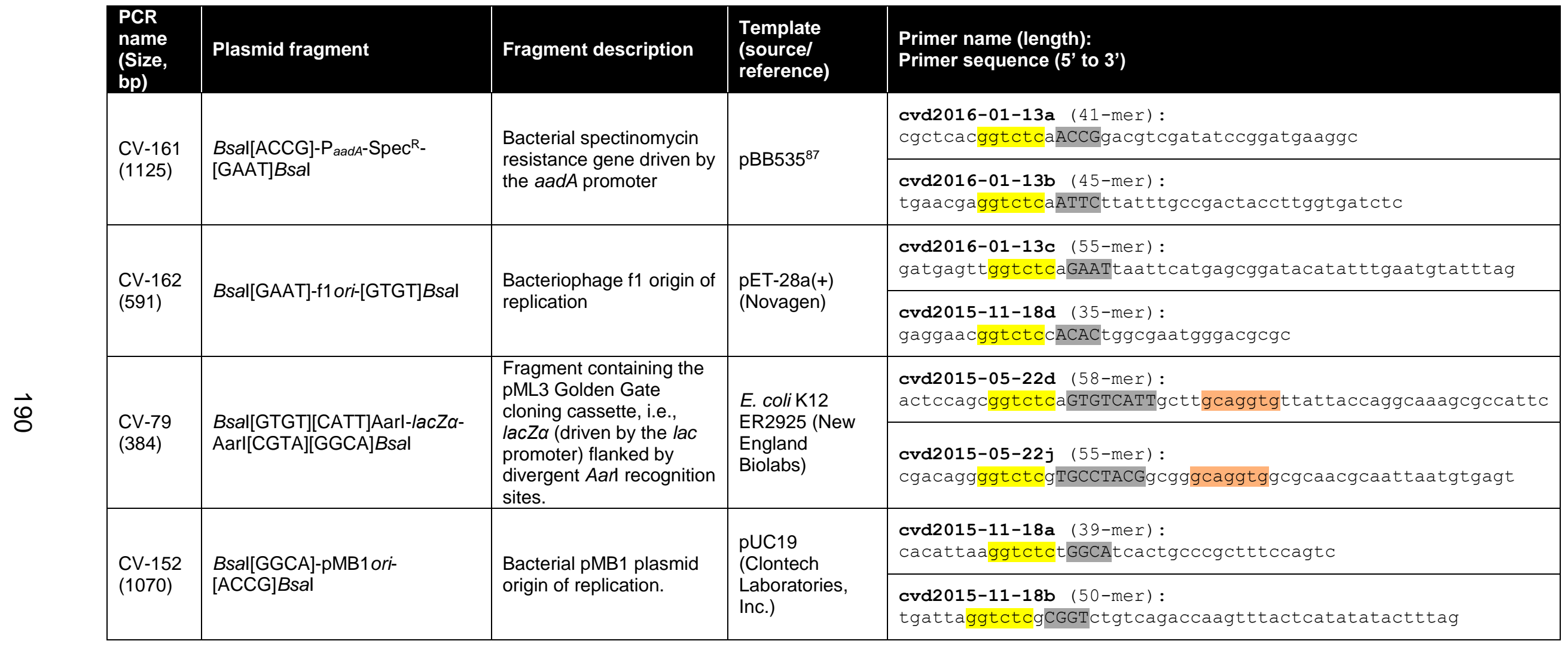




\section{Golden Gate assembly reactions}

One-pot Golden Gate (restriction-ligation) reactions were used to assemble transcription unit modules at Level-1, transcription units at Level-2 and multigene plasmids at Level-3. Detailed methods for each of these reactions are given below.

\section{Preparation of MIDAS constructs}

MIDAS constructs for use in heterologous expression studies were prepared as described below. Accordingly, coding sequences of the $H$. pulicicidum genes of interest were amplified (see Appendix, Table 9.2 for primers) and cloned into the MIDAS Level1 destination vector, pML1 (Table 9.3). At MIDAS Level-2, the cloned coding sequences were placed under the control of heterologous promoter and transcriptional terminator modules to generate full-length transcription units, transcription units (Table 9.4), which were then used to generate the multigene plasmids (Table 9.5).

\section{Primer design.}

As each transcription unit module is defined by its flanking prefix and suffix nucleotides, these module-specific bases effectively form an address system for each transcription unit module and they determine its position and orientation within the assembled transcription unit. The developers of MoClo and GoldenBraid2.0 have already worked in concert to develop a common syntax or set of standard addresses (referred to as 'fusion sites' in the MoClo system and 'barcodes' in GoldenBraid2.0) for a wide variety of transcription unit modules to facilitate part exchangeability for plant expression, ${ }^{89}$ and this standard is also adopted here for MIDAS-based assembly of transcription units for expression in filamentous fungi (Figure 8.6). 


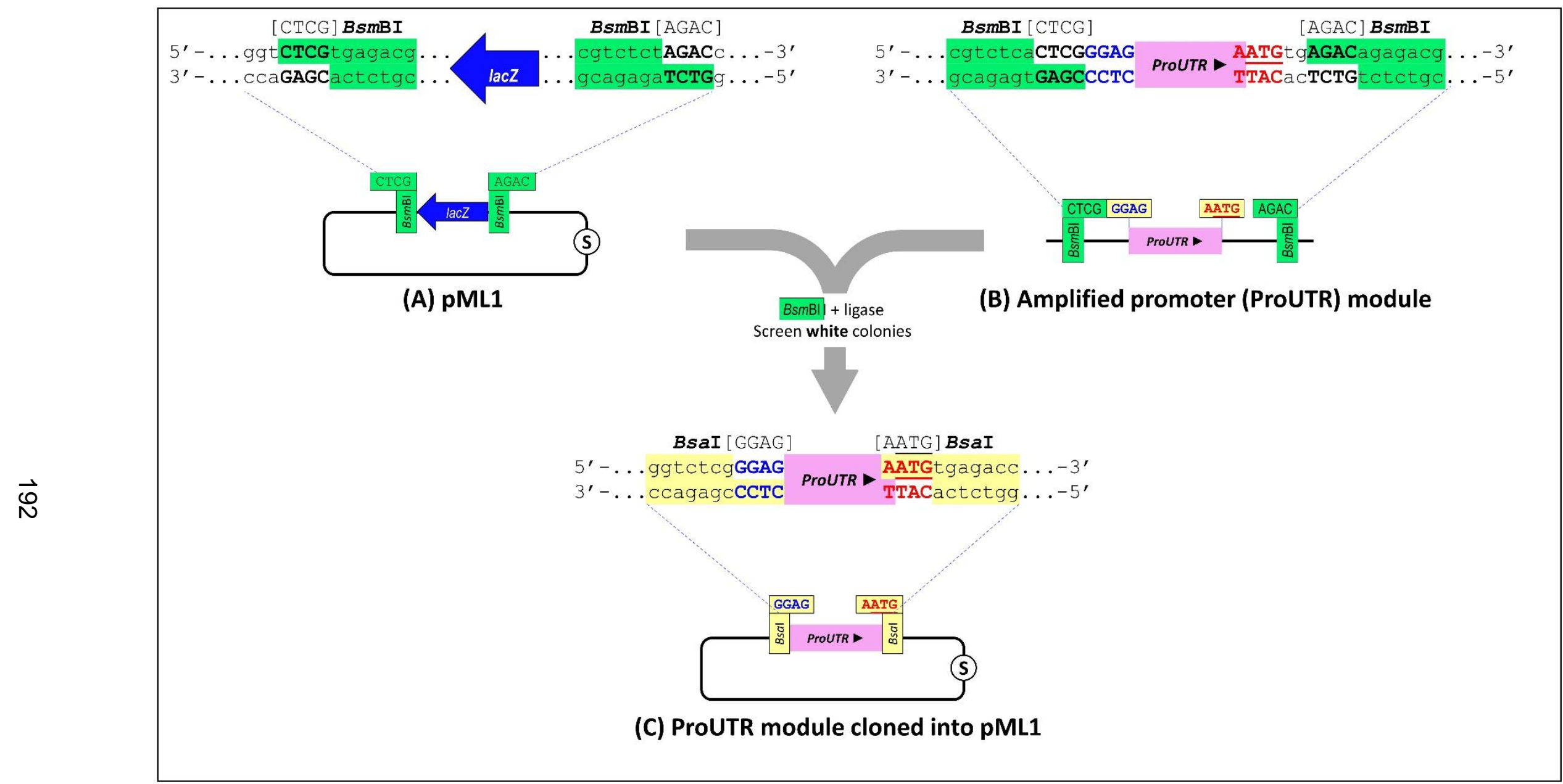

Figure 8.6. Principle of MIDAS Level-1 cloning. (A) The Golden Gate cloning cassette (lacZa flanked by divergent BsmBI sites) of the pML1 source vector. (B) A PCR product containing a promoter (ProUTR) module flanked by convergent BsmBI sites. The 4 bp sequences GGAG and AATG represent, respectively, the prefix and suffix signature unique to ProUTR modules (as mentioned, prefix and suffix signatures differ for coding sequences (AATG and GCTT) and terminators [UTRterm <GCTT and CGCT>]). Following a $B s m B I-m e d i a t e d$ Golden Gate reaction between pML1 and the amplified ProUTR module, a plasmid consisting of the ProUTR module cloned into pML1 is obtained (C), and the cloned module is now flanked by convergent Bsal recognition sites. 
Considerations for the design of PCR primers for amplifying promoters (ProUTR), coding sequence and terminator (UTRterm) transcription unit modules are shown in the Appendix, Table 9.2. Since the module-specific bases are incorporated as part of the PCR primer sequences (and not as part of the pML1 source vector), MIDAS is completely adaptable to the addition of new parts (i.e., new fusion site combinations) and part types. For example, using the module address standard described by Patron et al., ${ }^{89}$ ProUTR parts can be broken down into smaller parts, fusion proteins can be constructed, and $\mathrm{N}$ - or $\mathrm{C}$-terminal tags can be added to coding sequences.

Furthermore, to enable subsequent (i.e., Level-2) assembly of full-length transcription units, each transcription unit module is designed to be flanked by four module-specific, user-defined nucleotides at the 5' end (prefix), and four, different, module-specific, user-defined nucleotides at the 3 ' end (suffix), which are included as part of the PCR primer sequences.

\section{Protocols for MIDAS Level-1 module cloning}

PCR-amplified modules were purified using spin-column protocols and cloned into the MIDAS Level-1 plasmid, pML1, by BsmBI-mediated Golden Gate assembly. Typically, 1-2 $\mu \mathrm{L}$ (approximately 50-200 ng) of pML1 plasmid DNA from a miniprep was mixed with 1-2 $\mu \mathrm{L}$ of each purified PCR fragment, $1 \mu \mathrm{L}$ of $B s m B I(10 \mathrm{U} / \mu \mathrm{L}), 1 \mu \mathrm{L}$

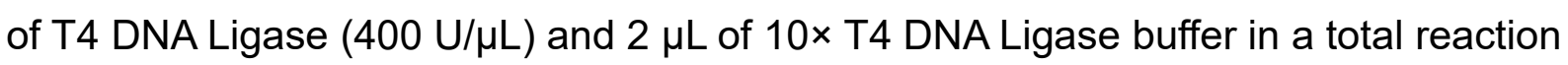
volume of $20 \mu \mathrm{L}$. Reactions were incubated at $37^{\circ} \mathrm{C}$ for $>4$ hours and an aliquot (typically 2-3 $\mu \mathrm{L}$ ) was transformed into $50 \mu \mathrm{L}$ of E. coli HST08 Stellar competent cells by heat shock at $42^{\circ} \mathrm{C}$. Following a recovery period (addition of $100 \mu \mathrm{L} \mathrm{SOC}$ medium and incubation at $37^{\circ} \mathrm{C}$ and $200-250 \mathrm{rpm}$ for 1 hour), aliquots of the transformation mix were spread onto LB agar plates supplemented with $50 \mu \mathrm{g} / \mathrm{mL}$ spectinomycin, $1 \mathrm{mM} \mathrm{IPTG}$ and $50 \mu \mathrm{g} / \mathrm{mL}$ X-Gal. Plates were incubated overnight at $37^{\circ} \mathrm{C}$, and white colonies were chosen for analysis.

\section{Protocols for MIDAS Level-2 transcription unit assembly}

Using the modules cloned at Level-1, full-length transcription units were assembled into MIDAS Level-2 plasmids by Bsal-mediated Golden Gate assembly. Typically, 40 fmol of pML2 plasmid DNA was mixed with $40 \mathrm{fmol}$ of plasmid DNA of each Level-1 


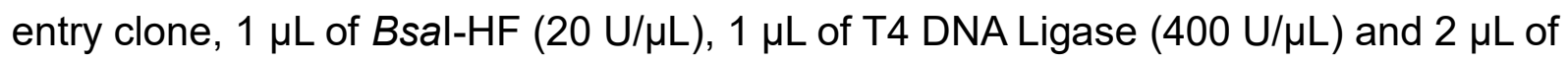
10x T4 DNA Ligase buffer in a total reaction volume of $20 \mu \mathrm{L}$. Reactions were incubated at $37^{\circ} \mathrm{C}$ for $>4$ hours and an aliquot and transformed into E. coli HST08 Stellar competent cells as described under Protocols for MIDAS Level-1 module cloning and spread onto LB agar plates containing $75 \mu \mathrm{g} / \mathrm{mL}$ kanamycin and $1.25 \mathrm{mM}$ $4 \mathrm{CP}$. Following overnight incubation at $37^{\circ} \mathrm{C}$, colonies were picked for analysis.

\section{Protocols for MIDAS Level-3 multigene assembly}

Full-length transcription units assembled at Level-2, were used to create multigene assemblies in the Level-3 destination vector by alternating Golden Gate assembly using either Aanl (for transcription units cloned into pML2 "White" shuttle vectors) or $B s m B I$ (for transcription units cloned into pML2 "Blue" shuttle vectors). Typically, 40 fmol of Level-3 destination vector plasmid DNA was mixed with $40 \mathrm{fmol}$ of Level-2 entry clone plasmid DNA. For BsmBI-mediated assemblies, $1 \mu \mathrm{L}$ of $B s m B I(10 \mathrm{U} / \mu \mathrm{L}), 1 \mu \mathrm{L}$ of T4 DNA Ligase (400 U/ $\mu \mathrm{L}$ ) and $2 \mu \mathrm{L}$ of $10 \times$ T4 DNA Ligase buffer, was added to the plasmid DNA in a final reaction volume of $20 \mu \mathrm{L}$. Two different reaction conditions were tested for Aarl-mediated assemblies; (i) conventional Golden Gate reactions performed in T4 DNA Ligase buffer and (ii) reactions performed in Aarl restriction enzyme buffer supplemented with ATP. For Aarl-mediated reactions performed in T4 DNA Ligase buffer, $1 \mu \mathrm{L}$ of $A$ arl $(2 \mathrm{U} / \mu \mathrm{L}), 0.4 \mu \mathrm{L}$ of $50 \times$ oligonucleotide $(25 \mu \mathrm{M}$, supplied with the enzyme), $1 \mu \mathrm{L}$ of T4 DNA Ligase (400 U/ $\mu \mathrm{L})$ and $2 \mu \mathrm{L}$ of T4 DNA Ligase buffer was added to the plasmid DNA, in a final reaction volume of $20 \mu \mathrm{L}$. For Aarl-mediated reactions carried out in restriction enzyme buffer, $1 \mu \mathrm{L}$ of $\operatorname{Aarl}(2 \mathrm{U} / \mu \mathrm{L})$, $0.4 \mu \mathrm{L}$ of $50 \times$ oligonucleotide ( $25 \mu \mathrm{M}$, supplied with the enzyme), $2 \mu \mathrm{L}$ of $10 \times$ Buffer Aarl (supplied with the enzyme), $1 \mu \mathrm{L}$ of T4 DNA Ligase (400 U/ $\mu \mathrm{L}$ ) and $2 \mu \mathrm{L}$ of $10 \mathrm{mM}$ ATP was added to the plasmid DNA, in a final reaction volume of $20 \mu \mathrm{L}$. All Level-3 reactions ( $B s m B I-$ and $A$ arl-mediated) were incubated $37^{\circ} \mathrm{C}$ for $>4$ hours and reactions were transformed into E. coli HST08 Stellar competent cells as described under Protocols for MIDAS Level-1 module cloning and spread onto LB agar plates supplemented with $50 \mu \mathrm{g} / \mathrm{mL}$ spectinomycin, $1 \mathrm{mM}$ IPTG and $50 \mu \mathrm{g} / \mathrm{mL}$ X-Gal. Plates were incubated overnight at $37^{\circ} \mathrm{C}$. For Aarl-mediated assembly reactions, white colonies were chosen for analysis while, for BsmBI-mediated assembly reactions, blue colonies were screened. 


\section{Penicillium paxilli PAX gene deletion mutants}

Penicillium paxilli and Hypoxylon pulicicidum strains used in this study are shown in Table 8.5. Gene deletion mutants of the PAX cluster have been described in the literature; the $\triangle p a x G,{ }^{37} \Delta p a x M^{38}$ and $\Delta p a x C^{38}$ single gene deletion mutants and the CY2 full PAX cluster deletion mutant ${ }^{35}$ of $P$. paxilli (strains PN2662, PN2257, PN2290, and PN2250 respectively) are unable to synthesise the indole diterpenes emindole SB (18), paspaline (11) and paxilline (2) normally produced by wild type $P$. paxilli (strain PN2013). These mutants provide a useful resource for confirming gene function and individually testing whether MIDAS-assembled transcription units contain all the necessary cis-acting elements to successfully transcribe and express their coding sequences. Thus, production of novel compounds (e.g. nodulisporic acids) or restoration of emindole SB (18), paspaline (11) and/or paxilline (2) production would indicate successful reconstitution of the deletion mutant and show, in turn, the function(s) of the transformed gene(s). 
Table 8.5. Table of fungal species used in this thesis.

\begin{tabular}{|c|c|c|c|c|}
\hline \multirow{2}{*}{$\begin{array}{l}\text { Hypoxylon pulicicidum } \\
\text { (Nodulisporium sp.) strain }\end{array}$} & \multirow[b]{2}{*}{ Description } & \multicolumn{2}{|c|}{$\begin{array}{c}\text { Indole diterpene } \\
\text { phenotype }\end{array}$} & \multirow[b]{2}{*}{ Source reference } \\
\hline & & \multicolumn{2}{|c|}{ Nodulisporic acid A (1) } & \\
\hline ATCC $^{\circledR} 74245^{\mathrm{TM}}$ & Wild type & \multicolumn{2}{|c|}{+} & ATCC ${ }^{\otimes 84}$ \\
\hline \multirow[b]{2}{*}{ Penicillium paxilli strain } & \multirow[b]{2}{*}{ Description } & \multicolumn{2}{|c|}{$\begin{array}{c}\text { Indole diterpene } \\
\text { phenotype }\end{array}$} & \multirow[b]{2}{*}{ Source reference } \\
\hline & & Paspaline (11) & Paxilline (2) & \\
\hline PN2013 (ATCC $\left.{ }^{\circledR} 26601^{\mathrm{TM}}\right)$ & Wild type & + & + & $\begin{array}{l}\text { Barry Scott, } \\
\text { Massey University }{ }^{72}\end{array}$ \\
\hline PN2250 (CY2) & PN2013/Deletion of entire $P A X$ locus $(\triangle P A X) ; \mathrm{Hyg}^{\mathrm{R}}$ & - & - & $\begin{array}{l}\text { Barry Scott, } \\
\text { Massey University } 35\end{array}$ \\
\hline PN2257 & 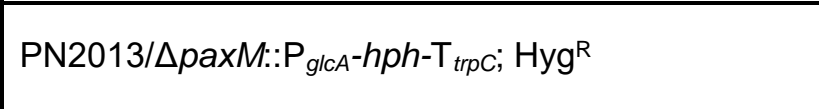 & - & - & $\begin{array}{l}\text { Barry Scott, } \\
\text { Massey University } 38\end{array}$ \\
\hline PN2290 & $\mathrm{PN} 2013 / \Delta p a x C:: \mathrm{P}_{t r p c}-h p h ; \mathrm{Hyg}^{\mathrm{R}}$ & - & - & $\begin{array}{l}\text { Barry Scott, } \\
\text { Massey University } 38\end{array}$ \\
\hline PN2458 & PN2013/ApaxB:: $P_{t r p c}-n p t I-t r p C ; G^{R}{ }^{R}$ & - & - & $\begin{array}{l}\text { Barry Scott, } \\
\text { Massey University } 38\end{array}$ \\
\hline
\end{tabular}




\section{Media and reagents used for fungal work}

CDYE (Czapex-Dox/Yeast extract) medium with trace elements was made with deionised water and contained 3.34\% (w/v) Czapex-Dox (Oxoid Ltd., Hampshire, England), $0.5 \%(\mathrm{w} / \mathrm{v})$ yeast extract (Oxoid Ltd., Hampshire, England), and 0.5\% (v/v) trace element solution. For agar plates, Select agar (Invitrogen, California, USA) was added to $1.5 \%(\mathrm{w} / \mathrm{v})$.

Trace element solution was made in deionised water and contained $0.004 \%(\mathrm{w} / \mathrm{v})$ cobalt(II) chloride hexahydrate (Ajax Finechem, Auckland, New Zealand), 0.005\% (w/v) copper(II) sulfate pentahydrate (Scharlau, Barcelona, Spain), $0.05 \%$ (w/v) iron(II) sulfate heptahydrate (Merck, Darmstadt, Germany), 0.014\% (w/v) manganese(II) sulfate tetrahydrate, and $0.05 \%(\mathrm{w} / \mathrm{v})$ zinc sulfate heptahydrate (Merck, Darmstadt, Germany). The solution was preserved with 1 drop of $12 \mathrm{M}$ hydrochloric acid.

Regeneration $(R G)$ medium was made with deionised water and contained $2 \%(\mathrm{w} / \mathrm{v})$ malt extract (Oxoid Ltd., Hampshire, England), 2\% (w/v) D(+)-glucose anhydrous (VWR International BVBA, Leuven, Belgium), 1\% (w/v) mycological peptone (Oxoid Ltd., Hampshire, England), and 27.6\% sucrose (ECP Ltd. Birkenhead, Auckland, New Zealand). Depending on whether the medium was to be used for plates (1.5\% RGA) or overlays ( $0.8 \%$ RGA), Select agar (Invitrogen, California, USA) was added to $1.5 \%$ or $0.8 \%(\mathrm{w} / \mathrm{v})$, respectively.

\section{Protoplast preparation}

The preparation of fungal protoplasts for transformation was according to Yelton et al. $1984^{90}$ with modifications. Five $25 \mathrm{~mL}$ aliquots of CDYE medium with trace elements, in $100 \mathrm{~mL}$ Erlenmeyer flasks, were inoculated with $5 \times 10^{6}$ spores and incubated for 28 hours at $28^{\circ} \mathrm{C}$ with shaking (200 rpm). The fermentation broth from all five flasks was filtered through a sterile nappy liner and the combined mycelia were rinsed three times with sterile water and once with OM buffer (10 $\mathrm{mM} \mathrm{Na}_{2} \mathrm{HPO}_{4}$ and $1.2 \mathrm{M} \mathrm{MgSO}_{4} .7 \mathrm{H}_{2} \mathrm{O}$, brought to $\mathrm{pH} 5.8$ with $100 \mathrm{mM} \mathrm{NaH} 2 \mathrm{PO}_{4} .2 \mathrm{H}_{2} \mathrm{O}$ ). Mycelia were weighed, resuspended in $10 \mathrm{~mL}$ of filter-sterilised Lysing Enzymes solution (prepared by resuspending Lysing Enzymes from Trichoderma harzianum (Sigma) at $10 \mathrm{mg} / \mathrm{mL}$ in OM buffer) per gram of mycelia, and incubated for 16 hours at $30^{\circ} \mathrm{C}$ with shaking at $80 \mathrm{rpm}$. Protoplasts were filtered through a sterile nappy liner into a $250 \mathrm{~mL}$ 
Erlenmeyer flask. Aliquots $(5 \mathrm{~mL})$ of filtered protoplasts were transferred into sterile 15 $\mathrm{mL}$ centrifuge tubes and overlaid with $2 \mathrm{~mL}$ of ST buffer $(0.6 \mathrm{M}$ sorbitol and $0.1 \mathrm{M}$ Tris$\mathrm{HCl}$ at $\mathrm{pH}$ 8.0). Tubes were centrifuged at $2600 \times g$ for 15 minutes at $4^{\circ} \mathrm{C}$. The white layer of protoplasts that formed between the OM and ST buffers in each tube was transferred (in $2 \mathrm{~mL}$ aliquots) into sterile $15 \mathrm{~mL}$ centrifuge tubes, gently washed by pipette resuspension in $5 \mathrm{~mL}$ of STC buffer $(1 \mathrm{M}$ sorbitol, $50 \mathrm{mM}$ Tris- $\mathrm{HCl}$ at pH 8.0, and $50 \mathrm{mM} \mathrm{CaCl}_{2}$ ) and centrifuged at $2600 \times \mathrm{g}$ for 5 minutes at $4^{\circ} \mathrm{C}$. The supernatant was decanted off and pelleted protoplasts from multiple tubes were combined by resuspension in $5 \mathrm{~mL}$ aliquots of STC buffer. The STC buffer wash was repeated three times until protoplasts were pooled into a single $15 \mathrm{~mL}$ centrifuge tube. The final protoplast pellet was resuspended in $500 \mu \mathrm{L}$ of STC buffer and protoplast concentration was estimated with a hemocytometer. The protoplast stock was diluted to give a final concentration of $1.25 \times 10^{8}$ protoplasts per $\mathrm{mL}$ of STC buffer. Aliquots of protoplasts $(100 \mu \mathrm{L})$ were used immediately for fungal transformations and excess protoplasts were preserved in $8 \%$ PEG solution ( $80 \mu \mathrm{L}$ of protoplasts were added to $20 \mu \mathrm{L}$ of $40 \%$ (w/v) PEG 4000 in STC buffer) in $1.7 \mathrm{~mL}$ micro-centrifuge tubes and stored at $-80^{\circ} \mathrm{C}$.

\section{Transformation of $P$. paxilli}

Fungal transformations - modified from Vollmer and Yanosfsky $1986^{91}$ and Oliver et al. $1987^{92}$ - were carried out in $1.7 \mathrm{~mL}$ micro-centrifuge tubes containing $100 \mu \mathrm{L}$ $\left(1.25 \times 10^{7}\right)$ protoplasts, either freshly prepared in STC buffer, or stored in $8 \%$ PEG solution (as described above). A solution containing $2 \mu \mathrm{L}$ of spermidine (50 mM in $\left.\mathrm{H}_{2} \mathrm{O}\right), 5 \mu \mathrm{L}$ heparin ( $5 \mathrm{mg} / \mathrm{mL}$ in STC buffer), and $5 \mu \mathrm{g}$ of plasmid DNA $(250 \mu \mathrm{g} / \mathrm{mL})$ was added to the protoplasts and, following incubation on ice for 30 minutes, $900 \mu \mathrm{L}$ of $40 \%$ PEG solution (40\% (w/v) PEG 4000 in STC buffer) was added. The transformation mixture was incubated on ice for a further $15-20$ minutes, transferred to $17.5 \mathrm{~mL}$ of $0.8 \%$ RGA medium (prewarmed to $50^{\circ} \mathrm{C}$ ) in sterile $50 \mathrm{~mL}$ tubes, mixed by inversion, and $3.5 \mathrm{~mL}$ aliquots were dispensed onto $1.5 \%$ RGA plates. Following overnight incubation at $25^{\circ} \mathrm{C}, 5 \mathrm{~mL}$ of $0.8 \% \mathrm{RGA}$ (containing sufficient $\mathrm{G} 418$ to achieve a final concentration of $150 \mu \mathrm{g}$ per $\mathrm{mL}$ of solid medium) was overlaid onto each plate. Plates were incubated for a further 4 days at $25^{\circ} \mathrm{C}$ and spores were picked from individual colonies and streaked onto CDYE agar plates supplemented with $150 \mu \mathrm{g} / \mathrm{mL}$ G418. 
Streaked plates were incubated at $25^{\circ} \mathrm{C}$ for a further 4 days. Spores from individual colonies were suspended in $50 \mu \mathrm{L}$ of $0.01 \%(\mathrm{v} / \mathrm{v})$ triton $\mathrm{X}-100$ and $5 \times 5 \mu \mathrm{L}$ aliquots of the spore suspension was transferred onto new CDYE agar plates supplemented with $150 \mu \mathrm{g} / \mathrm{mL}$ G418. Sporulation plates were incubated at $25^{\circ} \mathrm{C}$ for 4 days and spore stocks were prepared as follows. Colony plugs from the sporulation plates were suspended in $2 \mathrm{~mL}$ of $0.01 \%(\mathrm{v} / \mathrm{v})$ triton $\mathrm{X}-100$, and $800 \mu \mathrm{L}$ of suspended spores were mixed with $200 \mu \mathrm{L}$ of $50 \%(\mathrm{w} / \mathrm{v})$ glycerol in a $1.7 \mathrm{~mL}$ micro-centrifuge tube. Spore stocks were used to inoculate $50 \mathrm{~mL}$ of CDYE medium, flash frozen in liquid nitrogen and stored at $-80^{\circ} \mathrm{C}$.

\section{Fungal transformations and analysis of indole diterpene chemotypes}

In a series of complementation or pathway reconstitution experiments, a selection of the Level-3 plasmids produced in this work were transformed into $P$. paxilli strains harbouring appropriate genetic backgrounds (Table 8.5). Indole diterpene chemotypes [emindole SB (18), paspaline (11), paxilline (2), and/or nodulisporic acid F (82)] of G418-resistant fungal transformants were determined by an initial TLC screen of mycelial extracts and confirmed by LCMS and NMR. For these purposes, paspaline (11) and paxilline (2) reference standards were prepared from extracts of wild type $P$. paxilli (strain PN2013) and a nodulisporic acid F (82) reference standard was prepared from a pKV64(nodM/nodW): $\Delta$ paxM(PN2257) P. paxilli transformant (sKV170) by semipreparative HPLC. The HPLC peaks were analysed by high-resolution mass spectrometry, which identified $[\mathrm{M}+\mathrm{H}]^{+}$masses of $406.3109 \mathrm{~m} / \mathrm{z}$ at 19.3 minutes, $422.3055 \mathrm{~m} / \mathrm{z}$ at 17.1 minutes, $436.2485 \mathrm{~m} / \mathrm{z}$ at 5.3 minutes and $436.2870 \mathrm{~m} / \mathrm{z}$ at 5.6 minutes corresponding to the masses of emindole SB (18) (calc. $[\mathrm{M}+\mathrm{H}]^{+} 406.310 \mathrm{~m} / \mathrm{z}$ ), paspaline (11) (calc. $[\mathrm{M}+\mathrm{H}]^{+} 422.305 \mathrm{~m} / \mathrm{z}$ ), paxilline (2) (calc. $[\mathrm{M}+\mathrm{H}]^{+} 436.248 \mathrm{~m} / \mathrm{z}$ ) and nodulisporic acid $\mathrm{F}(82)$ (calc. $[\mathrm{M}+\mathrm{H}]^{+} 436.284 \mathrm{~m} / \mathrm{z}$ ) respectively (see Appendix Figures 9.8 to 9.11). NMR spectroscopy was used to confirm the structures of the purified reference standards (see Appendix Tables 9.7 to 9.9 and Figures 9.12 to 9.25 ).

\section{Indole diterpene production and extraction}

Fungal transformants were grown in $25 \mathrm{~mL}$ of CDYE medium with trace elements for 7 days at $28^{\circ} \mathrm{C}$ in shaker cultures ( $\left.\geq 200 \mathrm{rpm}\right)$, in $125 \mathrm{~mL}$ Erlenmeyer flasks capped 
with cotton wool. Mycelia were isolated from fermentation broths by filtration through nappy liners, transferred to $50 \mathrm{~mL}$ centrifuge tubes (Lab Serv ${ }^{\circledR}$, Thermo Fisher Scientific) and indole diterpenes were extracted by vigorously shaking the mycelia ( $\geq 200 \mathrm{rpm}$ ) in 2-butanone for $\geq 45$ minutes.

\section{Normal phase TLC}

The 2-butanone supernatant (containing extracted indole diterpenes) was used for TLC analysis on solid phase silica gel 60 aluminum plates (Merck). Indole diterpenes were chromatographed with 9:1 chloroform:acetonitrile or 8:2 dichloromethane:acetonitrile and visualised with Ehrlich's reagent $(1 \%(w / v) p$ dimethylaminobenzaldehyde in $24 \%(\mathrm{v} / \mathrm{v}) \mathrm{HCl}$ and $50 \%$ ethanol).

\section{Reverse phase LCMS}

Samples were prepared for LCMS from selected transformants that were identified by TLC. Accordingly, a $1 \mathrm{~mL}$ sample of the 2-butanone supernatant (containing extracted indole diterpenes) was transferred to a $1.7 \mathrm{~mL}$ micro-centrifuge tube and the 2-butanone was evaporated overnight. Contents were resuspended in $100 \%$ acetonitrile and filtered through a $0.2 \mu \mathrm{m}$ membrane into a LCMS vial. LCMS samples were chromatographed on a reverse phase Thermo Scientific Accucore $2.6 \mu \mathrm{m}$ C18 $\left(50 \times 2.1 \mathrm{~mm}\right.$ ) column attached to an UltiMate ${ }^{\circledR} 3000$ Standard LC system (Dionex, Thermo Fisher Scientific) run at a flow rate of $0.200 \mathrm{~mL} /$ minute and eluted with aqueous solutions of acetonitrile containing $0.01 \%$ formic acid using a multistep gradient method (Table 8.6). Mass spectra were captured through in-line analysis on

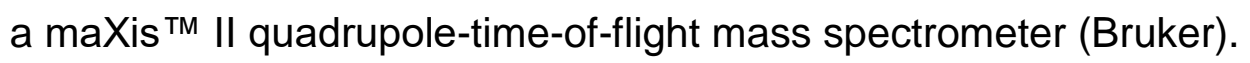


Table 8.6. Multistep acetonitrile gradient used for LCMS analysis of fungal extracts.

\begin{tabular}{|c|c|}
\hline $\begin{array}{c}\text { Time } \\
\text { (minutes) }\end{array}$ & $\begin{array}{c}\%(\mathbf{v} / \mathbf{v}) \text { of acetonitrile + } \\
\mathbf{0 . 0 1} \%(\mathbf{v} / \mathbf{v}) \text { formic acid }\end{array}$ \\
\hline 0 & 50 \\
\hline 1 & 50 \\
\hline 15 & 70 \\
\hline 20 & 95 \\
\hline 25 & 95 \\
\hline 28 & 50 \\
\hline 38 & 50 \\
\hline
\end{tabular}

\section{Large scale indole diterpene purification for NMR spectroscopy analysis}

Fungal transformants that produced high levels of novel indole diterpenes were grown in $\geq 1 \mathrm{~L}$ of CDYE medium with trace elements, as described above under "Indole diterpene production and extraction". Mycelia were pooled into $1 \mathrm{~L}$ Schott bottles containing stir bars. 2-butanone was added and indole diterpenes were extracted overnight with stirring ( $\geq 700 \mathrm{rpm})$. Extracts were filtered through Celite $\AA^{8} 545$ (J. T. Baker $\left.{ }^{\circledR}\right)$ and dry loaded onto silica with rotary evaporation for crude purification by silica column prior to a final purification by semi-preparative HPLC. A $1 \mathrm{~mL}$ aliquot of crude extract was injected onto a semi-preparative reversed phase Phenomenex $5 \mu \mathrm{m}$ C18(2) $100 \AA(250 \times 15 \mathrm{~mm})$ column attached to an UltiMate $\AA 3000$ Standard LC system (Dionex, Thermo Fisher Scientific) run at a flow rate of $8.00 \mathrm{~mL} / \mathrm{minute}$. Multistep gradient methods were optimised for the purification of different sets of indole diterpenes. The purity of each indole diterpene was assessed by LCMS and the structure was identified by NMR spectroscopy.

\section{NMR spectroscopy}

Paxilline and paspaline (11) samples were prepared in deuterated chloroform and the nodulisporic acid F (82) sample was prepared in deuterated methanol. Proton $\left({ }^{1} \mathrm{H}\right)$ and carbon $\left({ }^{13} \mathrm{C}\right)$ NMR spectra were recorded on an Agilent 400-MR instrument operating for ${ }^{1} \mathrm{H}$ NMR at $400 \mathrm{MHz}$ and $100 \mathrm{MHz}$ for ${ }^{13} \mathrm{C}$ NMR. All chemical shifts are quoted on the $\delta$-scale in ppm using residual solvent as an internal standard. ${ }^{1} \mathrm{H}$ and ${ }^{13} \mathrm{C}$ spectra were assigned using correlation spectroscopy (COSY), heteronuclear single quantum correlation (HSQC) spectroscopy, and heteronuclear multiple bond 
correlation (HMBC) spectroscopy. The nodulisporic acid F (82) HMBC spectra was captured on a Varian Unity Inova 600-MR instrument operating for ${ }^{1} \mathrm{H}$ NMR spectroscopy at $600 \mathrm{MHz}$ and $150 \mathrm{MHz}$ for ${ }^{13} \mathrm{C}$ NMR spectroscopy. Spectra were acquired with VNMRJ 3.2 or VNMRJ 4.2 acquisition software and processed with VNMRJ 3.2, VNMRJ 4.2, or MestReNova processing software. All NMR spectra can be found in the Appendix (Tables 9.7 to 9.9 and Figures 9.12 to 9.25 ). 
9. APPENDIX 


\section{Additional Notes:}

\section{Introduction: Additional radiography feeding studies:}

[14C]-paxilline was efficiently incorporated into the major indole diterpenes produced by $P$. janczewskii (penitrem $\mathrm{A}, \mathrm{E}$, and pennigritrem) and $P$. janthinellum (janthitrems $\mathrm{B}$ and C). ${ }^{93}$

$\alpha$-[14C]-paxitriol and $\beta$-[14C]-paxitriol feeding on $P$. janczewskii and $P$. janthinellum showed incorporation of $\beta$-[14C]-paxitriol into indole diterpene end products. A small amount of janthitrem $B$ became radiolabelled from $\alpha-[14 \mathrm{C}]$-paxitriol indicating some isomerase activity in $P$. janthinellum but no penitrems were radiolabelled from $\alpha-[14 \mathrm{C}]-$ paxitriol in $P$. janczewskii. There was clear incorporation of $\beta$-[14C]-paxitriol and [14C]paxilline into janthitrem $D$ but none into janthitrem $A .{ }^{94} \beta-10-O$-acetyl[14C]paxilline was incorporated into janthitrem $B$ in small amounts and janthitrem $C$ in large amounts but most of the substrate remained unutilised in contrast to the $\beta$-[14C]-paxitriol and [14C]paxilline, which were fully utilised - indicating that $\beta-10-0$-acetyl[14C]paxilline is unlikely a direct intermediate. From results, suggest that paxilline (2) is likely reduced to $\beta$-[14C]-paxitriol during janthitrem and penitrem biosynthesis. ${ }^{93}$

\section{Chapter 3: Testing of the paxC promoter region (pax $C_{\text {ProUTR-2) }}$}

To confirm that the pax $C$ promoter region was functional with the additional $144 \mathrm{bp}$, MIDAS primers were eventually redesigned to include the missing base pairs in a new

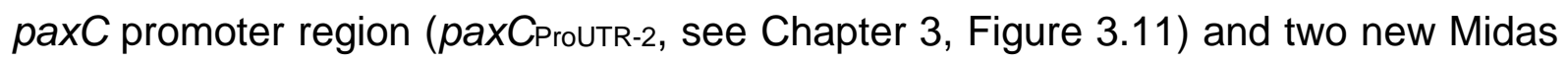
Level-3 plasmids were created harbouring pax $C$ driven by pax $C_{\text {ProUTR-2 }}(\mathrm{pKV} 30)$ and nodC driven by paxCProUtR-2 (pKV32). These two plasmids (pKV30 and pKV32) were individually transformed into the $P$. paxilli $\triangle$ paxC deletion mutant strain (PN2290) and chemical analysis of the transformant extracts revealed that paxilline (2) biosynthesis had been restored (Results not shown) thereby confirming that the paxCProUTR-2 was functional. 
Bioinformatics analyses to identify the potential genetic elements involved in the biosynthesis of nodulisporic acids 


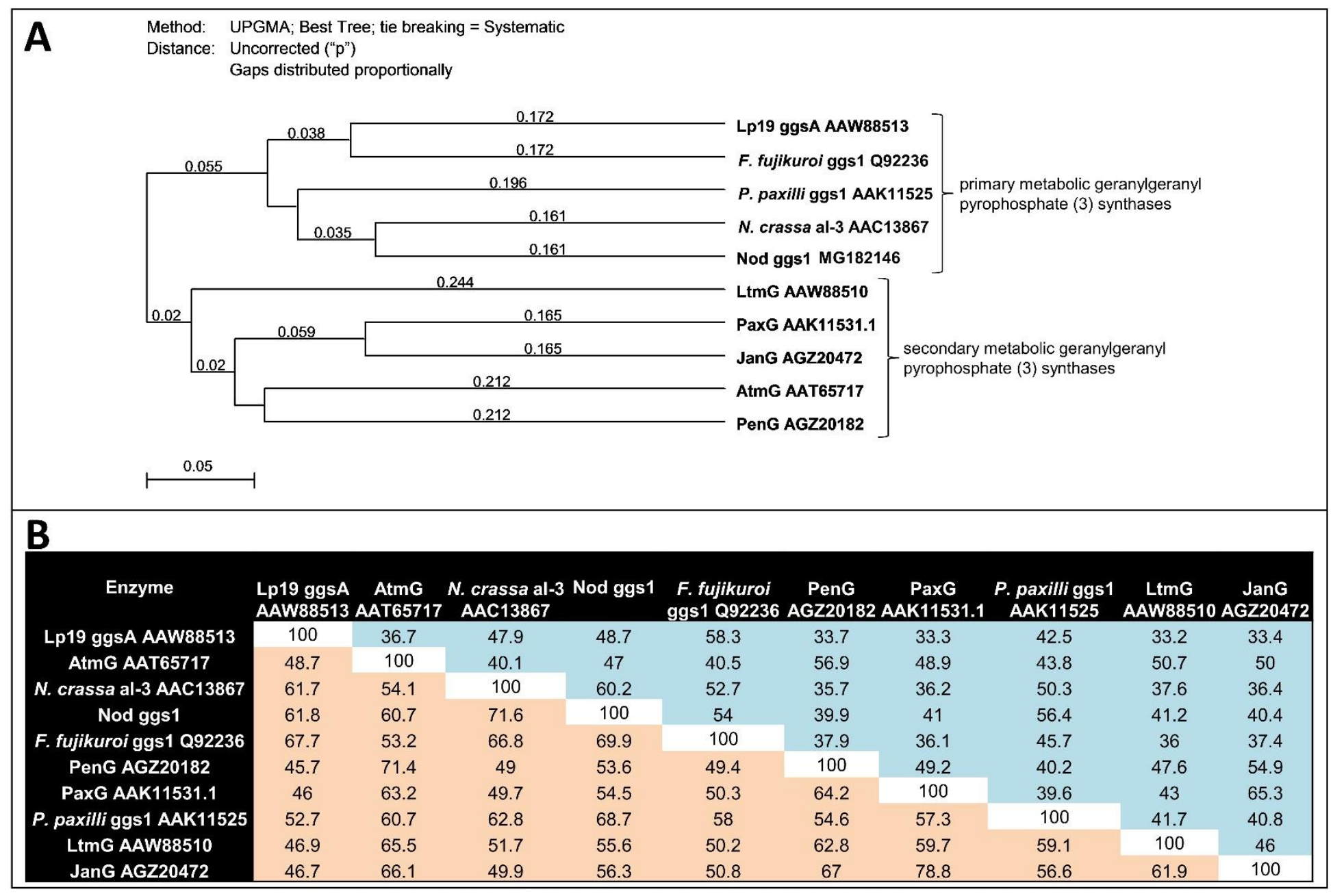

Figure 9.1. Phylogenetic tree (A) and similarity matrix (B) of geranylgeranyl synthases ('ggs enzymes' are involved inprimary metabolism and ' $G$ ' enzymes are involved in secondary metabolism). The phylogenetic tree depicts the difference in \% identity scores for the amino acid residues of the enzymes. The similarity matrix depicts the \% identity scores (blue shaded regions $(D)$ ) and the \% similarity scores (orange shaded regions ( )) for the amino acid residues of the enzymes. Figure has been modified from Van de Bittner et al. 2018. ${ }^{1}$ 


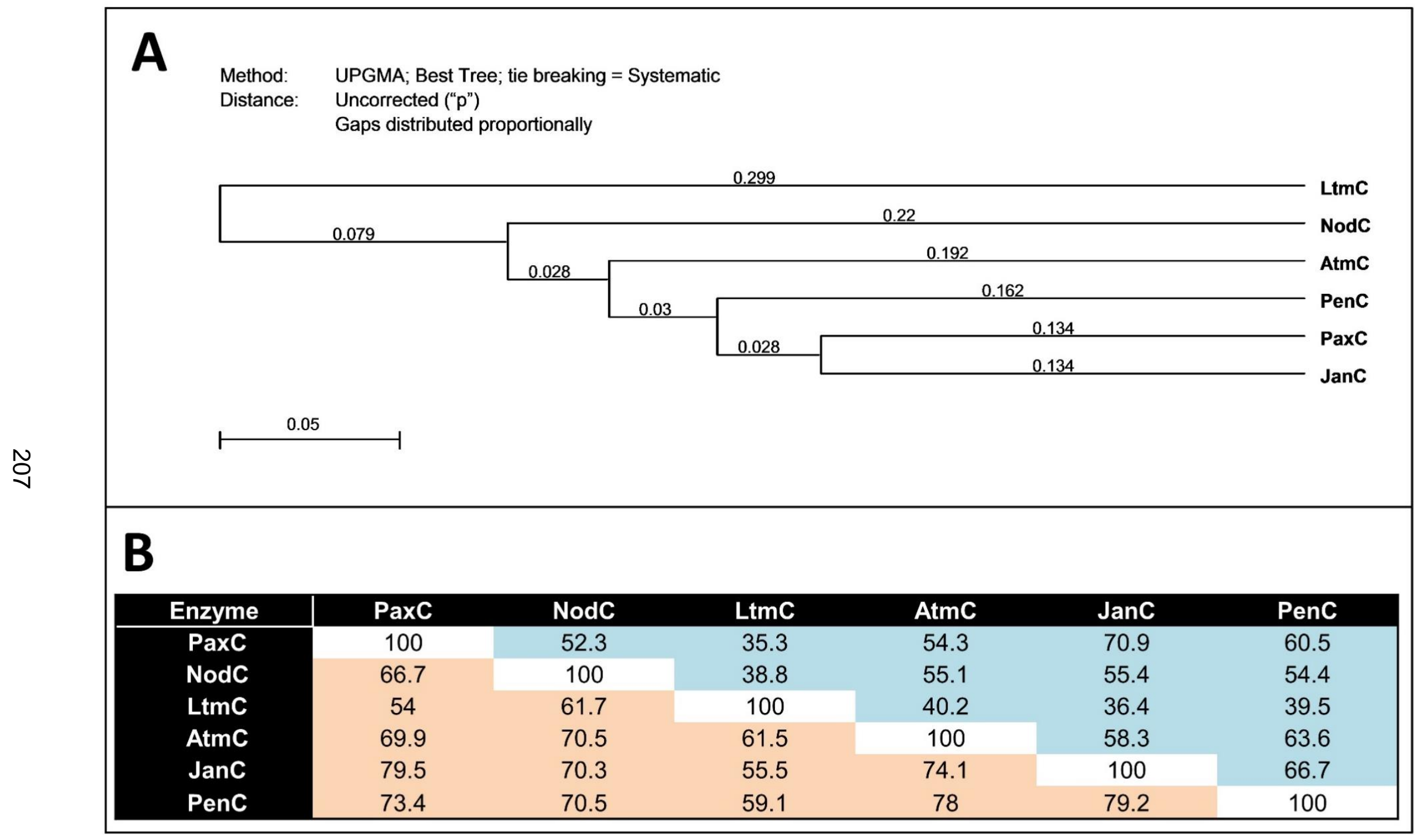

Figure 9.2. Phylogenetic tree $(\mathbf{A})$ and similarity matrix $(\mathbf{B})$ of geranylgeranyl transferases ('C' enzymes). The phylogenetic tree depicts the difference in \% identity scores for the amino acid residues of the ' $\mathrm{C}$ ' enzymes. The similarity matrix depicts the $\%$ identity scores (blue shaded regions () ) and the \% similarity scores (orange shaded regions () ) for the amino acid residues of the ' $\mathrm{C}$ ' enzymes. 


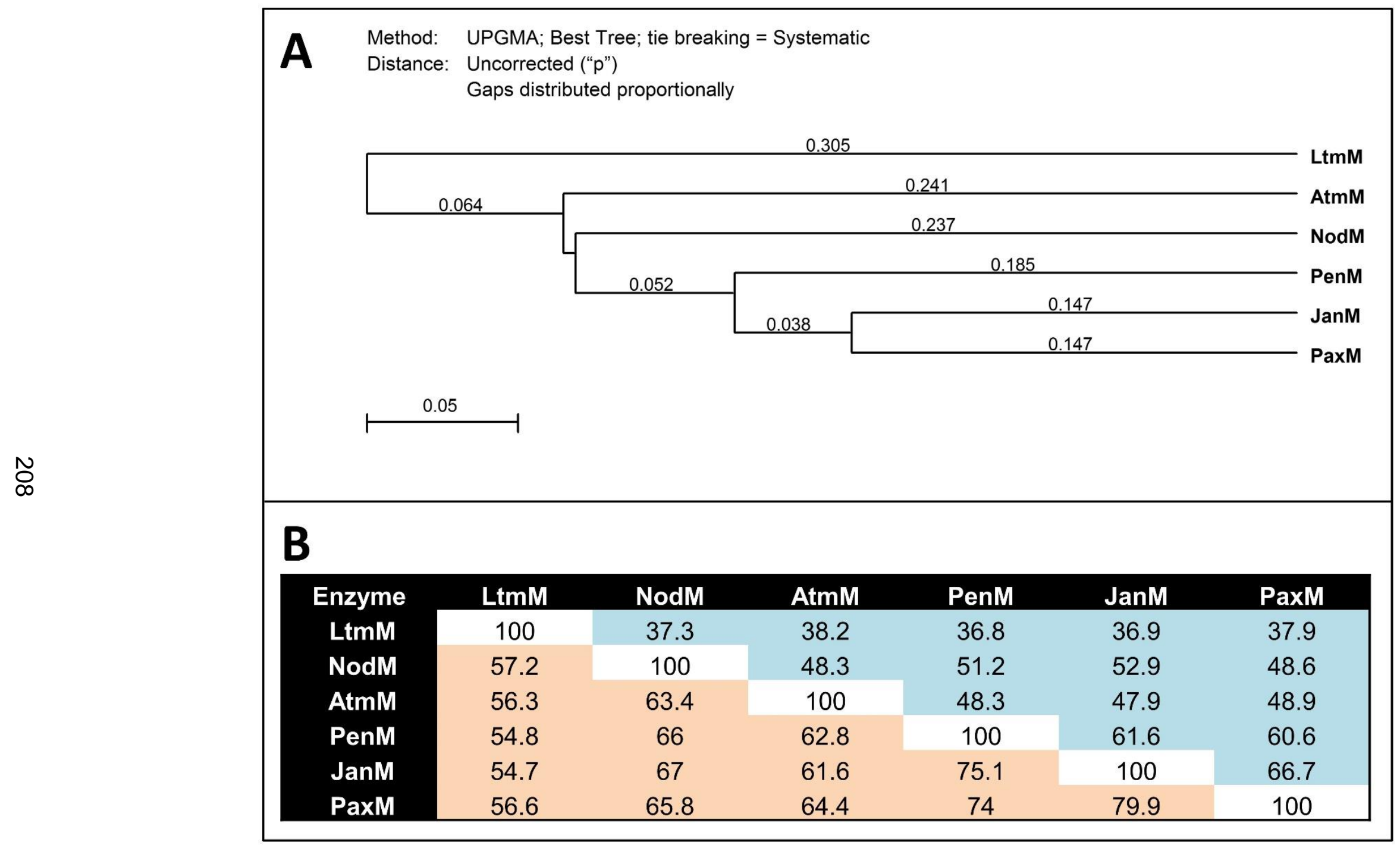

Figure 9.3. Phylogenetic tree $(\mathbf{A})$ and similarity matrix $(\mathbf{B})$ of indole diterpene epoxidases ('M' enzymes). The phylogenetic tree depicts the difference in \% identity scores for the amino acid residues of the ' $M$ ' enzymes. The similarity matrix depicts the \% identity scores (blue shaded regions ( )) and the \% similarity scores (orange shaded regions ( )) for the amino acid residues of the 'M' enzymes. 


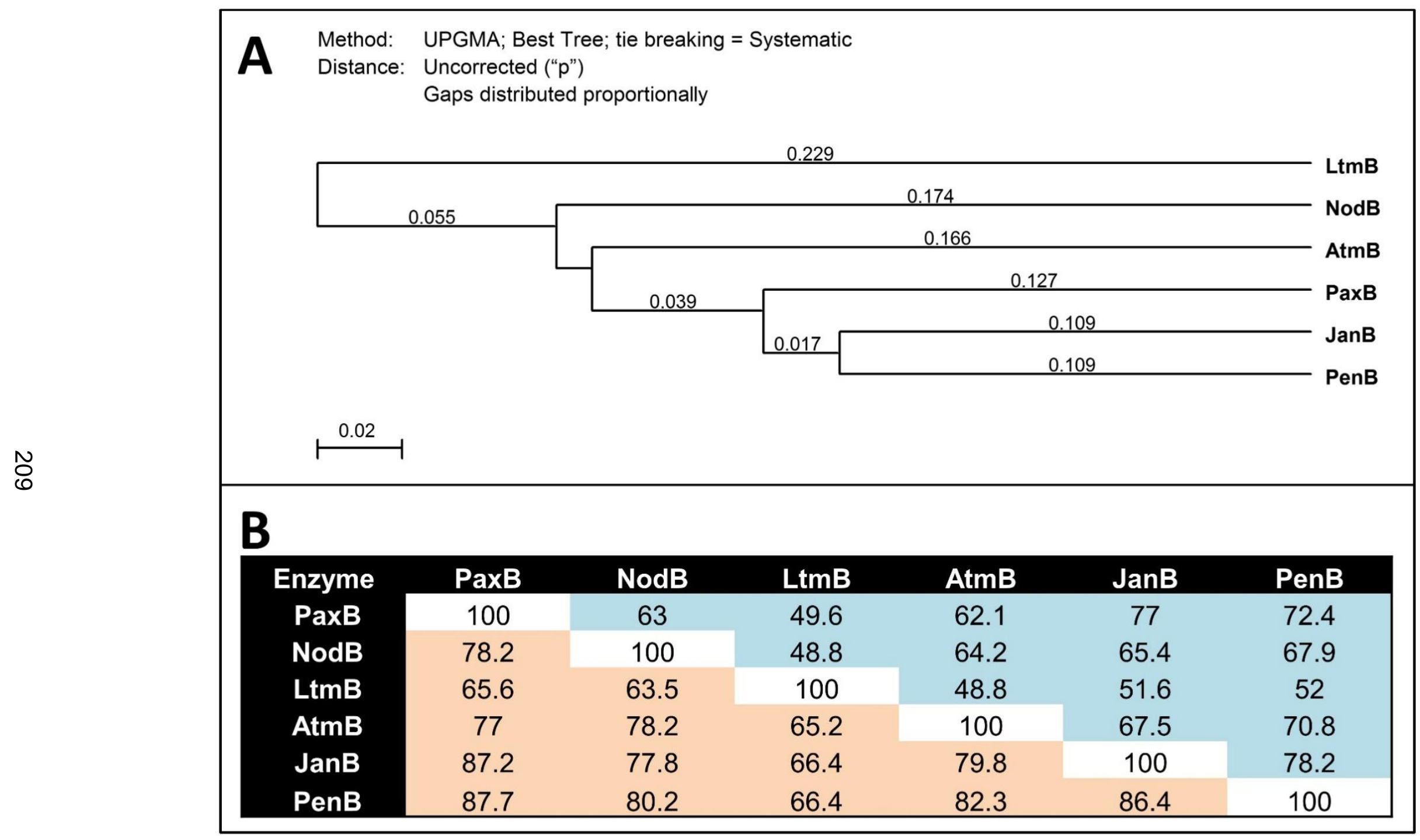

Figure 9.4. Phylogenetic tree $(\mathbf{A})$ and similarity matrix $(\mathbf{B})$ of indole diterpene epoxidases ('B' enzymes). The phylogenetic tree depicts the difference in \% identity scores for the amino acid residues of the ' $\mathrm{B}$ ' enzymes. The similarity matrix depicts the \% identity scores (blue shaded regions ( )) and the \% similarity scores (orange shaded regions ( )) for the amino acid residues of the 'B' enzymes. 


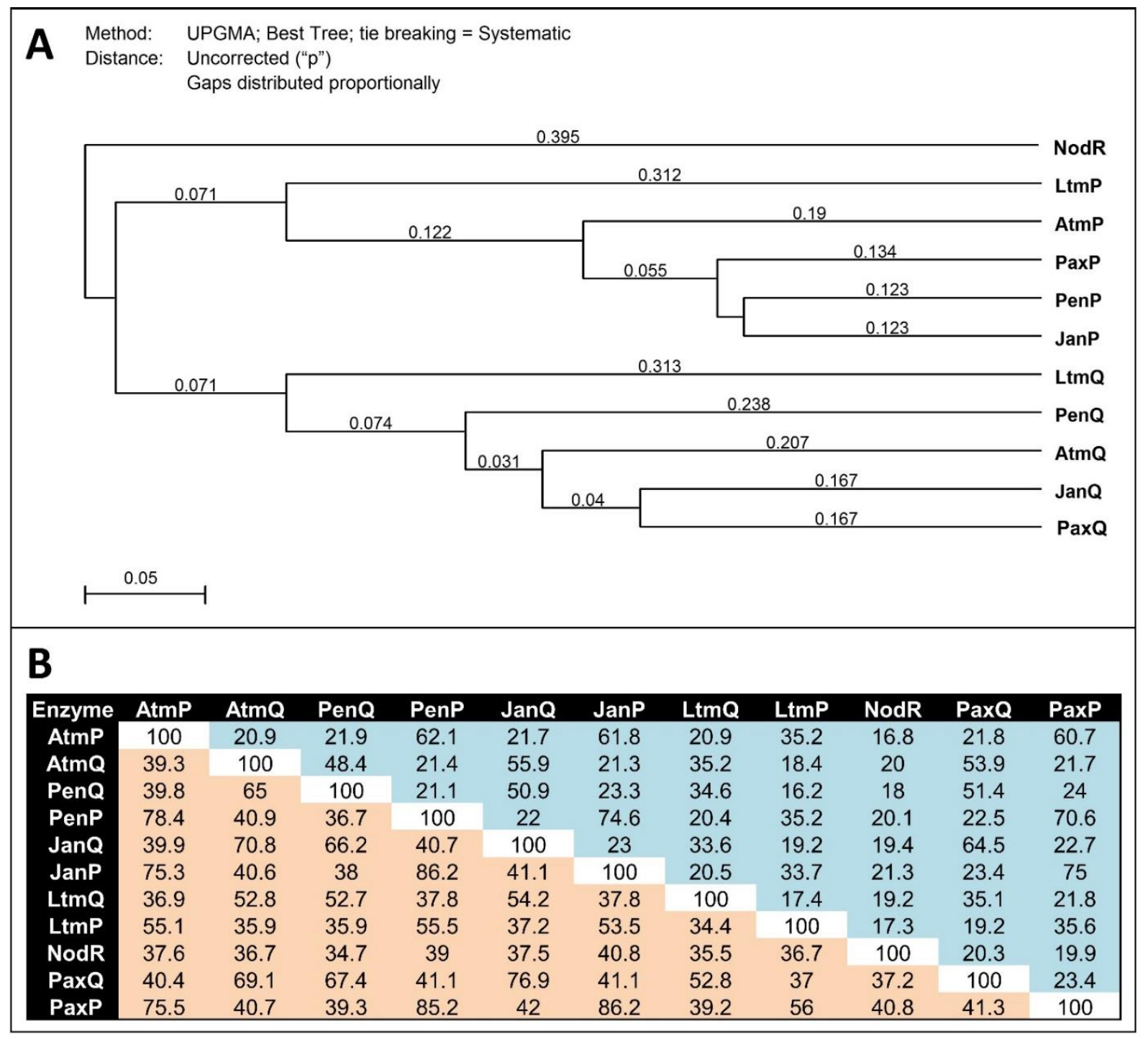

Figure 9.5. Phylogenetic tree $(\mathbf{A})$ and similarity matrix $(\mathbf{B})$ of indole diterpene cytochrome P450 oxygenases (' $P$ ' and ' $Q$ ' enzymes compared to NodR). The phylogenetic tree depicts the difference in \% identity scores for the amino acid residues of the enzymes. The similarity matrix depicts the \% identity scores (blue shaded regions ( )) and the \% similarity scores (orange shaded regions ( )) for the amino acid residues of the enzymes. 


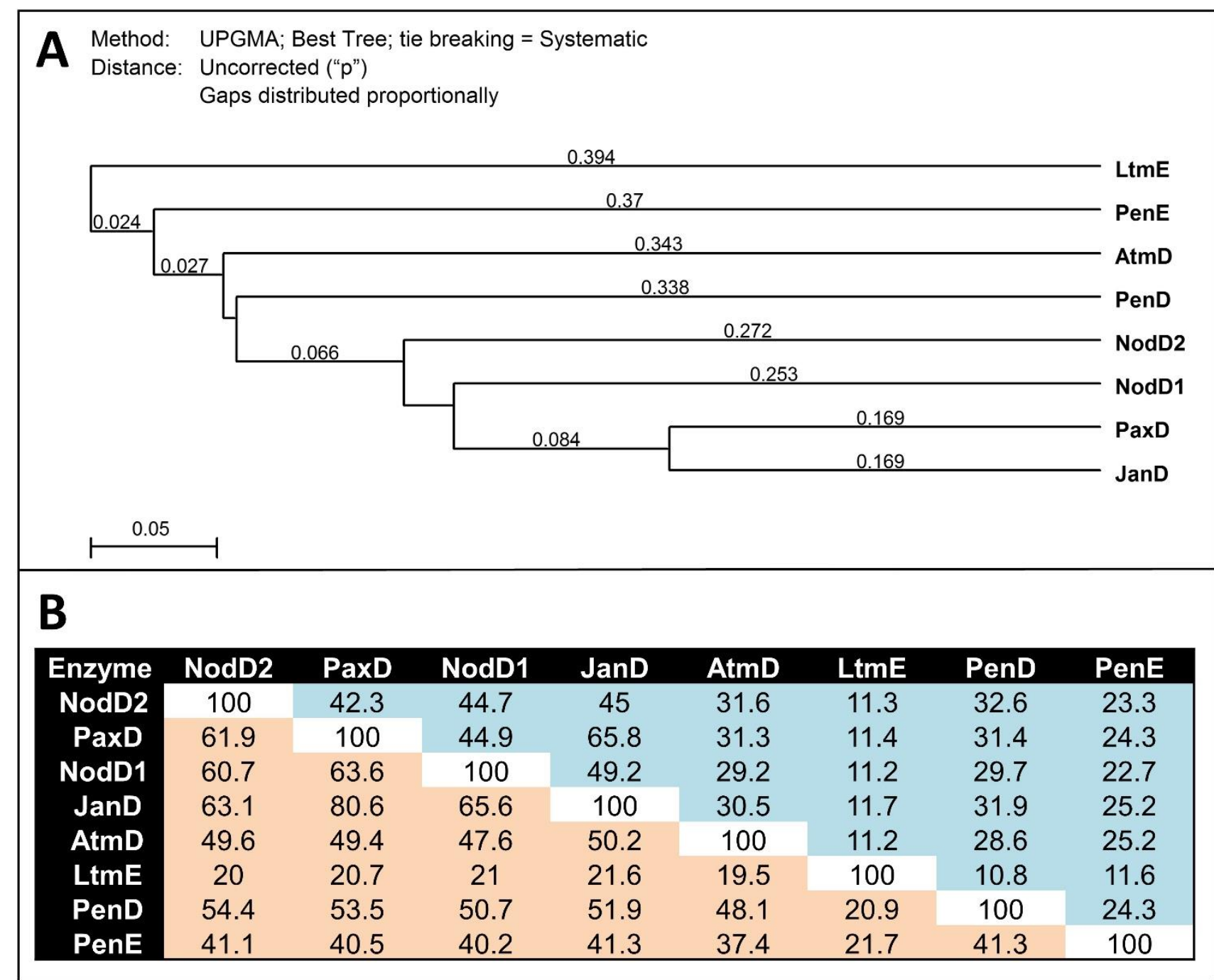

Figure 9.6. Phylogenetic tree $(\mathbf{A})$ and similarity matrix (B) of indole diterpene prenyl transferases ('D' and 'E' enzymes). The phylogenetic tree depicts the difference in \% identity scores for the amino acid residues of the enzymes. The similarity matrix depicts the \% identity scores (blue shaded regions () ) and the \% similarity scores (orange shaded regions () ) for the amino acid residues of the enzymes. 


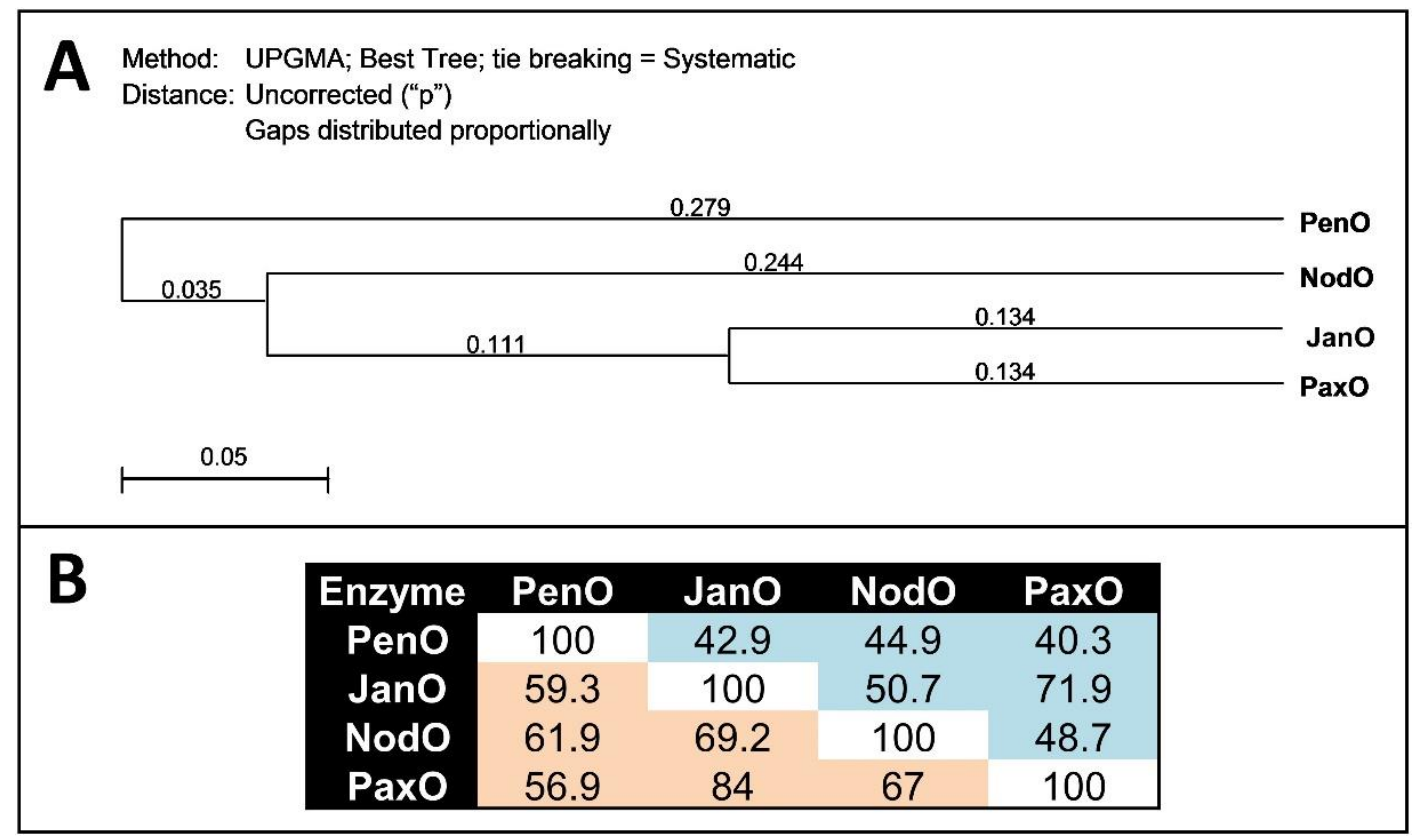

Figure 9.7. Phylogenetic tree $(\mathbf{A})$ and similarity matrix $(\mathbf{B})$ of indole diterpene flavin adenine dinucleotide-dependent oxidative cyclases ('O' enzymes). The phylogenetic tree depicts the difference in \% identity scores for the amino acid residues of the enzymes. The similarity matrix depicts the \% identity scores (blue shaded regions () ) and the \% similarity scores (orange shaded regions () ) for the amino acid residues of the enzymes.

Table 9.1. Similarity matrix of NodY enzymes. Blue shaded regions ( ) represent \% identity scores and orange shaded regions ( ) represent \% similarity scores for amino acid residues.

\begin{tabular}{|c|c|c|}
\hline Enzyme & NodY2 & NodY1 \\
\hline NodY2 & 100 & 41.1 \\
NodY1 & 60 & 100 \\
\hline
\end{tabular}




\section{Assembling of genetic elements (5'UTRs, CDS, 3'UTRs, etc.) into MIDAS vectors}

Table 9.2. PCR primers for amplification of transcription unit modules (TUMs). The forward and reverse PCR primers used for amplification of TUMs (i.e. promoters [ProUTR], coding sequences [CDSs], and terminators [UTRterm]) are listed. Primers used to amplify TUM fragments for domestication purposes (i.e. removal of internal sites for Aarl, Bsal or BsmBI) are shaded in orange ( ). The template for amplification of nod CDSs was genomic DNA from Hypoxylon pulicicidum strain ATCC ${ }^{\circledR} 74245^{\mathrm{TM}} .84$ The template for amplification of pax gene TUMs was genomic DNA from Penicillium paxilli strain ATCC ${ }^{\circledR} 26601^{\mathrm{TM}}$ (PN2013) [Accession HM171111]. ${ }^{72}$ The PCR products used to produce the trpC ProUTR module, nptII CDS module (conferring resistance to geneticin (G418)), and trpCutRterm module were all amplified from plasmid pll99. ${ }^{95}$ The $B s m B I$ recognition sites are colour coded (cgtctc), with the overhangs generated following BsmBI cleavage shown by the grey shading ( $)$. The 5' (prefix) and 3' (suffix) nucleotide bases, which flank each TUM and form the basis of the address system for each of the MIDAS modules, are shown in blue and red respectively.

\begin{tabular}{|c|c|c|}
\hline TUM & Primer name & Primer sequence (5' to $\left.3^{\prime}\right)$ \\
\hline \multicolumn{3}{|c|}{ Hypoxylon pulicicidum primers } \\
\hline \multicolumn{3}{|r|}{ nodw } \\
\hline \multirow{4}{*}{ nodW $\mathrm{CDS}$} & P4502 frag $1 \mathrm{~F}$ & cgatgtacgtctcaCTCGAATGactttagctattttaggcatcagttgcC \\
\hline & P4502 frag $1 \mathrm{R}$ & actgctcgtctcaACTCCcgctgcgagccgct \\
\hline & P4502 frag $2 \mathrm{~F}$ & acgtaccgtctccGAGTccggtcctggtggagtgatc \\
\hline & P4502 frag $2 \mathrm{R}$ & gacctttcgtctctGTCTCaAAGCctaagttatgcccagatatttccag \\
\hline \multicolumn{3}{|r|}{ nodR } \\
\hline \multirow{4}{*}{ nodRcDs } & nodP frag $1 \mathrm{~F}$ & cgatgtacgtctcaCTCGAATGtttgatattgattttggcattctatttcC \\
\hline & nodP frag $1 R$ & gtacagcgtctcaTTGAgtctccttcaggacgctgtc \\
\hline & nodP frag $2 \mathrm{~F}$ & gtcactcgtctcaTCAAcgcctaaaacctatgcaaatc \\
\hline & nodP frag $2 \mathrm{R}$ & gacctttcgtctctGTCTCaAAGCttacagttgaatctcttctttccgc \\
\hline \multicolumn{3}{|r|}{ nodX } \\
\hline \multirow{10}{*}{ nod $X_{\mathrm{CDS}}$} & $\begin{array}{l}\text { NA- } \\
\text { 03_frag1_F }\end{array}$ & cgatgtacgtctcaCTCGAATGgagtcaagtgcggataatacag \\
\hline & $\begin{array}{l}\text { NA- } \\
\text { 03_frag1_R }\end{array}$ & actgaccgtctcgGAACaaggttgcgtggtcgctatc \\
\hline & $\begin{array}{l}\text { NA- } \\
03 \text { frag2_F }\end{array}$ & gtatcgegtctctGTTCetcatgtcccccatgc \\
\hline & $\begin{array}{l}\text { NA-03_frag2- } \\
\text { R }\end{array}$ & actgaccgtctcgTCCCaccttgccatttttctgg \\
\hline & NA-03_frag3-F & gtatcgcgtctctGGGActgtatgaggtcattagggaccC \\
\hline & $\begin{array}{l}\text { NA-03_frag3- } \\
\text { R }\end{array}$ & actgaccgtctcgGCCAgcggtcgcttggg \\
\hline & NA-03_frag4-F & gtatcgcgtctccTGGCcgggctgaaggtg \\
\hline & $\begin{array}{l}\text { NA-03_frag4- } \\
\text { R }\end{array}$ & actgaccgtctcgGCGTagatctgccggcc \\
\hline & NA-03_frag5-F & gtatcgcgtctctACGCcgtgagattgtgagagatgagg \\
\hline & $\begin{array}{l}\text { NA- } \\
\text { 03_frag5_R }\end{array}$ & gacctttcgtctctGTCTCaAAGCctaatctgcgatggaccctgac \\
\hline
\end{tabular}




\begin{tabular}{|c|c|c|}
\hline \multicolumn{3}{|r|}{ nodM } \\
\hline \multirow{4}{*}{ nodMcDs } & nodM frag1 $\mathrm{F}$ & cgatgtacgtctcaCTCGAATGtctacccctgagttcaagg \\
\hline & nodM frag1 R & cagtcacgtctcaACGCctctcaagaacgatgtgggaaattc \\
\hline & nodM frag2 F & gtgcatcgtctcaGCGTagtgtaatcgcaccagag \\
\hline & nodM frag2 R & $\begin{array}{l}\text { gacctttcgtctctGTCTcaAAGCctatgaagcgatgtctctaatatggagtaa } \\
\text { c }\end{array}$ \\
\hline \multicolumn{3}{|r|}{ nodB } \\
\hline \multirow{2}{*}{ nodBcDs } & nodB F & cgatgtacgtctcaCTCGAATGgatggattcgatcgttccaatg \\
\hline & nodB R & gacctttcgtctctGTCTcaAAGCttattgagccttccgcgcattg \\
\hline \multicolumn{3}{|r|}{ nodJ } \\
\hline \multirow{4}{*}{ nodJCDS } & P4501 frag $1 \mathrm{~F}$ & cgatgtacgtctcaCTCGAATGgaacttatcgttattatcataactctcg \\
\hline & P4501 frag $1 \mathrm{R}$ & actgaccgtctcaCGGTgtctgtatggaacttgcaag \\
\hline & P4501 frag 2 F & gtatcgcgtctctACCGgcaggggagttcatggtga \\
\hline & P4501 frag 2 R & gacctttcgtctctGTCTCaAAGCttacccaactagcagtggaaacttc \\
\hline \multicolumn{3}{|r|}{ nodC } \\
\hline \multirow{4}{*}{ nodCCDs } & nodC frag1 F & cgatgtacgtctcaCTCGAATGtccttaggtttacagtgcttgg \\
\hline & nodC frag1 R & cattgacgtctcgGTCAcgtcgccaaaccagcga \\
\hline & nodC frag2 F & gtcacgcgtctctTGACggcctcactagctttcc \\
\hline & nodC frag2 R & gacctttcgtctctGTCTCaAAGCtcaatgcgtaagatcgagtttctcctttct \\
\hline \multicolumn{3}{|r|}{ nodz } \\
\hline \multirow{6}{*}{ nodZCDs } & P4503 frag $1 \mathrm{~F}$ & cgatgtacgtctcaCTCGAATGataacagcatccacttccg \\
\hline & P4503 frag $1 \mathrm{R}$ & actgtccgtctcaGAGTccaatcaggacgcaactaatcatc \\
\hline & P4503 frag $2 \mathrm{~F}$ & actgcacgtctctACTCccatggtgtcgagatgag \\
\hline & P4503 frag 2 R & ctacgtcgtctctCGCTtctctggccacaaaacggaaag \\
\hline & P4503 frag $3 \mathrm{~F}$ & ctgactcgtctctAGCGtttcacacagaggggaaag \\
\hline & P4503 frag $3 \mathrm{R}$ & gacctttcgtctctGTCTCaAAGCtaggtatccaccacacggttc \\
\hline \multicolumn{3}{|r|}{ Penicillium paxilli primers } \\
\hline \multicolumn{3}{|r|}{ paxG } \\
\hline \multirow{2}{*}{ paxGProutR } & PpaxG F & cgatgtacgtctcaCTCGGGAGattcacgacctgtgactagtcaa \\
\hline & PpaxG R & gacctttcgtctctGTCTCaCATTggcgtcgaacttgatgaagttttc \\
\hline \multirow{8}{*}{ paxGcos } & paxG frag1 F & cgatgtacgtctcaCTCGAATGtcctacatccttgcagaag \\
\hline & paxG frag1 R & cttctacgtctcgTACTgttctaatcgtgcttggtg \\
\hline & paxG frag2 F & gcacgacgtctccAGTAcaggtgctagaagatgacgttgac \\
\hline & paxG frag2 R & aggcgccgtctccACCAatctctttcaatcttgcttgttgga \\
\hline & paxG frag3 F & gattgacgtctctTGGTgacccccgcgectt \\
\hline & paxG frag3 R & gtcgaccgtctctTTCCctagtatattggaagctccccg \\
\hline & paxG frag4 F & tccaatcgtctcgGGAAaccctaagtcgacttagtgcg \\
\hline & paxG frag4 R & gacctttcgtctctGTCTcaAAGCttaaactcttcctttctcattagtaggg \\
\hline \multirow{2}{*}{ paxGUTRterm } & TpaxG F & cgatgtacgtctcaCTCGGCTTtcaatcgtgctgcatttctctt \\
\hline & TpaxG R & gacctttcgtctctGTCTcaAGCGtcactcccgagcaatattgct \\
\hline \multicolumn{3}{|r|}{ paxc } \\
\hline \multirow{2}{*}{$\begin{array}{l}\text { paxCProUTR- } \\
1\end{array}$} & $\mathrm{P} \operatorname{paxCF}$ & cgatgtacgtctcaCTCGGGAGtgaccatgatgcgtattaacggc \\
\hline & $\mathrm{P} \operatorname{paxCR}$ & gacctttcgtctctGTCTCaCATTCaaaatgggacctacaccctgaa \\
\hline \multirow{2}{*}{$\begin{array}{l}\text { paxCProutR- } \\
2\end{array}$} & PpaxC F2 & cgatgtacgtctcaCTCGGGAGacaacaaaaagatcagccaatgg \\
\hline & PpaxC R2 & gacctttcgtctctGTCTCaCATTaaaatgggacctacaccctgaa \\
\hline paxCcDs & paxC frag1 F & cgatgtacgtctcaCTCGAATGggcgtagcaggga \\
\hline
\end{tabular}




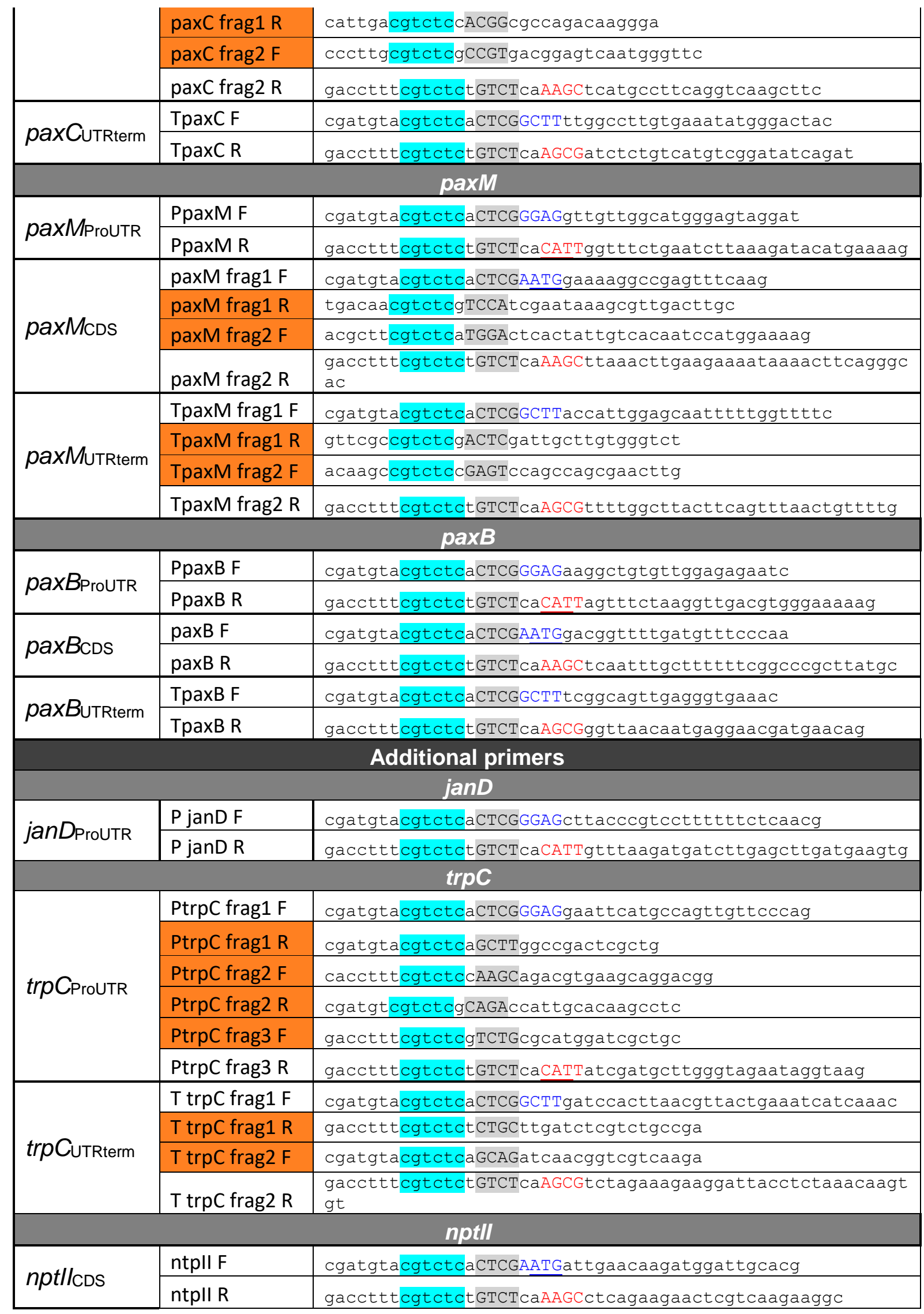


Table 9.3. MIDAS Level-1 plasmid library: Assembly of transcription unit modules (i.e. promoters [ProUTR], coding sequences [CDSs], and terminators [UTRterm]) in pML1. This table represents the MIDAS level-1 transcription unit modules that were used to assemble MIDAS level-2 transcription units (Table 9.4). The 4 base prefixes and suffixes ( $5^{\prime}$ to $3^{\prime}$ ) that flank each transcription unit module are shown at the top of the table to highlight the sequences used to bind the transcription unit modules together to make MIDAS level-2 transcription units. These 4 base flanking regions are depicted in the primer table (Table 9.2) in blue (forward addresses) and red (reverse addresses).

\begin{tabular}{|c|c|c|c|c|c|}
\hline [GGAG] & \multicolumn{2}{|c|}{ [AATG] } & \multicolumn{2}{|c|}{ [GCTT] } & \\
\hline \multicolumn{2}{|c|}{ ProUTR modules } & \multicolumn{2}{|c|}{ CDS modules } & \multicolumn{2}{|c|}{ UTRterm modules } \\
\hline Plasmid name & Description & Plasmid name & Description & Plasmid name & Description \\
\hline $\mathrm{pSK} 1$ & paxGProUTR & pKV45 & nodWcDs & pSK3 & paxGUTRterm \\
\hline pSK10 & pax ProUTR-1 $_{1}$ & pKV58 & nodR $R_{\mathrm{CDS}}$ & pSK12 & paxCUTRterm \\
\hline pKV28 & pax ProUTR-2 $_{\text {P }}$ & pKV46 & nod $X_{\mathrm{CDS}}$ & pSK6 & paxMutRterm \\
\hline pSK4 & paxMProUtR & pKV59 & \multirow{2}{*}{ nodMcDs } & pSK9 & paxButRterm \\
\hline pSK7 & paxB ProUTR & pSK18 & & pSK15 & trpCuTRterm \\
\hline pSK17 & $\operatorname{trp} C_{\text {ProUTR }}$ & pSK19 & nodBCDs & & \\
\hline pLB9 & janDProUTR & pKV47 & nodJ $J_{\mathrm{CDS}}$ & & \\
\hline & & pSK20 & nodC $\mathrm{CDS}$ & & \\
\hline & & pKV50 & nodZCDs & & \\
\hline & & pSK2 & paxGCDS & & \\
\hline & & pSK11 & paxCcos & & \\
\hline & & pSK5 & paxMcDs & & \\
\hline & & pSK16 & $n p t / l_{\text {CDS }}$ & & \\
\hline
\end{tabular}


Table 9.4. MIDAS Level-2 plasmid library: Assembly of transcription units (TUs) in pML2 destination vectors. This table represents the construction of the MIDAS level-2 TUs that were used to assemble MIDAS level-3 multi-gene plasmids (Table 9.5) for heterologous expression studies. TUs are described by the coding sequence (CDS) they contain (in the TU column) and the Level-1 entry clones used for TU assembly are depicted in their corresponding promoter (ProUTR), CDS, or terminator (UTRterm) columns. The column labelled pML2 destination vector describes the type of MIDAS Level-2 plasmid that was used to assemble each TU. The names of the Level-2 entry plasmids produced are shown in the blue shaded ( ) column and the TU orientation, determined by the pML2

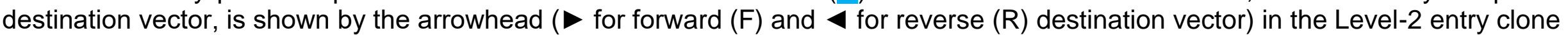
description.

\begin{tabular}{|c|c|c|c|c|c|c|}
\hline \multirow[b]{3}{*}{ TU } & \multirow{2}{*}{\multicolumn{3}{|c|}{ Level-1 entry clones used for TU assembly }} & \multirow{3}{*}{$\begin{array}{l}\text { PML2 } \\
\text { destination } \\
\text { vector }\end{array}$} & \multicolumn{2}{|r|}{ Level-2 entry clones } \\
\hline & & & & & & \\
\hline & ProUTR & CDS & UTRterm & & Name & Description \\
\hline \multirow{3}{*}{ nodW } & & \multirow{3}{*}{ pKV45 } & \multirow{3}{*}{ pSK15 } & pML2(+)WR & pKV52 & $\boldsymbol{4}\left(T_{\text {trpc }}-n o d W-P_{t r p c}\right): p M L 2(+) W R$ \\
\hline & pSK17 & & & \multirow{2}{*}{ pML2(+)BR } & pSK67 & $\left(T_{\text {trpc }}-n o d W-P_{t r p c}\right): p M L 2(+) B R$ \\
\hline & pLB9 & & & & pSK80 & $\varangle\left(\mathrm{T}_{\text {trpc }}-n o d W-\mathrm{P}_{j a n D}\right): \mathrm{pML} 2(+) \mathrm{BR}$ \\
\hline nodR & pSK17 & pKV58 & pSK15 & pML2(+)WF & pKV53 & $\left(P_{t r p c}-n o d R-T_{t r p c}\right): p M L 2(+) \mathrm{WF}$ \\
\hline $\operatorname{nod} X$ & pSK17 & pKV46 & pSK15 & pML2(+)WR & pKV54 & $4\left(T_{t r p c}-n o d X-P_{t r p c}\right): p M L 2(+)$ WR \\
\hline \multirow{3}{*}{ nodM } & pSK17 & pSK18 & pSK15 & \multirow{2}{*}{$\mathrm{pML2}(+) \mathrm{BF}$} & pKV33 & $\left(P_{t r p c}-n o d M-T_{t r p c}\right): p M L 2(+) B F$ \\
\hline & \multirow{2}{*}{ pSK4 } & pKV59 & \multirow{2}{*}{ pSK6 } & & pKV57 & $\left(P_{\text {paxM- }}\right.$-nodM-T $\left.T_{\text {paxM }}\right):$ pML2(+)BF \\
\hline & & pSK18 & & pML2(+)WF & pSK28 & $\left(P_{\text {paxM-nodM- }} T_{\text {paxM }}\right):$ :pML2(+)WF \\
\hline \multirow{2}{*}{ nodB } & pSK17 & \multirow{2}{*}{ pSK19 } & pSK15 & $\mathrm{pML2(+)BF}$ & pKV35 & $\left(\mathrm{P}_{t r p c}-n o d B-T_{t r p c}\right): \mathrm{pML2}(+) \mathrm{BF}$ \\
\hline & pSK7 & & pSK9 & pML2(+)BR & pSK29 & $4\left(T_{p a \times B}-n o d B-P_{p a \times B}\right): p M L 2(+) B R$ \\
\hline nodJ & pSK17 & pKV47 & pSK15 & pML2(+)WR & pKV55 & $4\left(T_{t r p c}-n o d J-P_{t r p c}\right): p M L 2(+) \mathrm{WR}$ \\
\hline \multirow{3}{*}{ nodC } & pSK17 & \multirow{3}{*}{ pSK20 } & pSK15 & $\mathrm{pML} 2(+) \mathrm{BF}$ & pKV26 & $\left(P_{t r p c}-n o d C-T_{t r p c}\right): p M L 2(+) B F$ \\
\hline & pKV28 & & \multirow{2}{*}{ pSK12 } & pML2(+)WF & pSK60 & $\left(P_{\text {paxc-2- }}\right.$-nodC- $\left.T_{\text {pax }}\right) \vee: \mathrm{pML} 2(+) \mathrm{WF}$ \\
\hline & pSK10 & & & $\mathrm{pML2}(+) \mathrm{BF}$ & pKV2 & $\left(P_{p a x C-1}-n o d C-T_{p a x}\right) \vee: p M L 2(+) \mathrm{BF}$ \\
\hline nodZ & pSK17 & pKV50 & pSK15 & pML2(+)WF & pKV56 & $\left(P_{t r p c}-n o d Z-T_{t r p c}\right): \mathrm{pML2}(+) \mathrm{WF}$ \\
\hline paxG & pSK1 & pSK2 & pSK3 & pML2(+)BR & pSK21 & $4\left(T_{\text {paxG }}-\right.$ paxG- $\left.P_{\text {paxG }}\right):$ pML2(+)BR \\
\hline \multirow{2}{*}{$\operatorname{pax} C$} & pSK10 & \multirow{2}{*}{ pSK11 } & \multirow{2}{*}{ pSK12 } & $\mathrm{pML2}(+) \mathrm{BF}$ & pKV24 & $\left(P_{\text {paxc-1 }}-\right.$ paxC- $\left.T_{\text {paxc }}\right)$ :pML2(+)BF \\
\hline & pKV28 & & & pML2(+)WF & pSK59 & $\left(P_{p a x c-2}-p a x C-T_{p a x c}\right): p M L 2(+) \mathrm{WF}$ \\
\hline paxM & pSK4 & pSK5 & pSK6 & pML2(+)WR & pSK22 & $4\left(T_{\text {pax }}-\right.$ paxM- $\left.P_{\text {paxM }}\right): \mathrm{pML2}(+) \mathrm{WR}$ \\
\hline nptII & pSK17 & pSK16 & pSK15 & pML2(+)WF & pSK26 & $\left(P_{t r p c}-n p t I I-T_{t r p c}\right):$ :pML2(+)WF \\
\hline
\end{tabular}


Table 9.5. MIDAS Level-3 plasmid library: Multigene assemblies in pML3. This table shows the Level-2 entry clone and Level-3 destination vectors used to construct the Level-3 product plasmids. The number of level 3 assembly reactions used to create the level-3 plasmid is indicated by number in the step column. The name and description of each Level-2 entry clone and the Level-3 destination vector that the Level-2 entry clones were assembled into are shown in their respective columns. The enzyme (Aarl or $B s m \mathrm{BI}$ ) used to mediate the Level-3 reaction is listed in the Golden Gate reaction column. The name (blue shaded ( ) column), description and size of plasmids produced during each cycle of Level-3 assembly are shown in their respective Level-3 product plasmid columns. In the Level-3 product plasmid descriptions, transcription units are annotated with the name of the coding sequence they contain and transcription unit orientation is shown by the arrowhead.

\begin{tabular}{|c|c|c|c|c|c|c|c|}
\hline \multirow[b]{2}{*}{ Step } & \multicolumn{2}{|r|}{ Level-2 entry clone } & \multirow[b]{2}{*}{$\begin{array}{l}\text { Destination } \\
\text { vector }\end{array}$} & \multirow{2}{*}{$\begin{array}{l}\text { Golden } \\
\text { Gate } \\
\text { reaction }\end{array}$} & \multicolumn{3}{|c|}{ Product Level-3 plasmid } \\
\hline & Name & Description & & & Name & Description & $\begin{array}{l}\text { Plasmid } \\
\text { size (kb) }\end{array}$ \\
\hline 1 & pSK26 & $\left(\mathrm{P}_{t r p c}-n p t I I-\mathrm{T}_{t r p c}\right): \mathrm{pML} 2(+) \mathrm{WF}$ & pML3 & Aarl & pKV22 & pML3:nptII & 5.6 \\
\hline 2 & pKV2 & $\left(P_{p a x C-1}-n o d C-T_{p a x C}\right): p M L 2(+) B F$ & pKV22 & BsmBI & pKV13 & pML3:nptII :nodC & 8.3 \\
\hline 2 & pKV24 & $\left(P_{\left.p a x c-1-p a x C-T_{p a x c}\right)}\right)$ pML2(+)BF & pKV22 & BsmBI & pKV25 & pML3:nptII :paxC & 8.2 \\
\hline 2 & pKV26 & $\left(\mathrm{P}_{t r p c}-n o d C-T_{t r p c}\right): \mathrm{pML2}(+) \mathrm{BF}$ & pKV22 & BsmBI & pKV27 & pML3:nptII>:nodC & 9.0 \\
\hline 2 & pKV33 & $\left(\mathrm{P}_{t r p c}-n o d M-T_{t r p c}\right): \mathrm{pML} 2(+) \mathrm{BF}$ & pKV22 & $B s m \mathrm{BI}$ & pKV34 & pML3:nptII :nodM & 9.5 \\
\hline 2 & pKV35 & $\left(\mathrm{P}_{t r p c}-n o d B-T_{t r p c}\right): \mathrm{pML} 2(+) \mathrm{BF}$ & pKV22 & $B s m \mathrm{BI}$ & pKV36 & pML3:nptII :nodB & 8.0 \\
\hline 2 & pKV57 & $\left(P_{\text {paxM-nodM- }} T_{\text {paxM }}\right):$ :pML2(+)BF & pKV22 & $B s m B I$ & pKV63 & pML3:nptII :nodM & 9.4 \\
\hline 3 & pKV52 & $\boldsymbol{\Psi}\left(T_{t r p C}-n o d W-P_{t r p C}\right): p M L 2(+) W R$ & pKV63 & Aarl & pKV64 & pML3:nptII :nodM : 4 nodW & 13.0 \\
\hline 3 & pKV53 & $\left(P_{t r p C}-n o d R-T_{t r p c}\right)$ :pML2(+)WF & pKV63 & Aarl & pKV65 & pML3:nptII :nodM :nodR & 13.0 \\
\hline 3 & pKV54 & $\boldsymbol{\triangleleft}\left(T_{t r p c}-n o d X-P_{t r p c}\right): p M L 2(+)$ WR & pKV63 & Aarl & pKV66 & pML3:nptII :nodM : 4 nodX & 13.2 \\
\hline 3 & pKV55 & $\boldsymbol{4}\left(T_{\text {trpc }}-n o d J-P_{t r p c}\right): p M L 2(+) W R$ & pKV63 & Aarl & pKV67 & pML3:nptII :nodM : $\triangleleft$ nodJ & 12.9 \\
\hline 3 & pKV56 & $\left(P_{t r p c}-n o d Z-T_{t r p c}\right): p M L 2(+) W F$ & pKV63 & Aarl & pKV68 & pML3:nptII :nodM :nodZ & 13.0 \\
\hline 1 & pSK26 & $\left(\mathrm{P}_{t r p c}-n p t I l-\mathrm{T}_{t r p c}\right) \mathrm{:pML2(+) \textrm {WF }}$ & pML3 & Aarl & pSK33 & pML3:nptII & 5.6 \\
\hline 2 & pSK21 & $\checkmark\left(T_{\left.p a x G-p a x G-P_{p a x G}\right): p M L 2(+) B R}\right.$ & pSK33 & BsmBI & pSK34 & pML3:nptII>: 4 paxG & 8.2 \\
\hline 3 & pSK22 & $\downarrow\left(T_{\text {paxм }}-\right.$ paxM-P $\left.P_{\text {paxм }}\right): \mathrm{pML} 2(+) \mathrm{WR}$ & pSK34 & Aarl & pSK36 & pML3:nptII : $\triangleleft$ paxG: $\triangleleft$ paxM & 11.5 \\
\hline 4 & pSK29 & $\downarrow\left(T_{p a x B}-n o d B-P_{p a x B}\right): p M L 2(+) B R$ & pSK36 & BsmBI & pKV73 & pML3:nptII : 4 paxG: 4 paxM: 4 nodB & 14.1 \\
\hline 5 & pSK59 & $\left(P_{p a x c-2}-p a x C-T_{p a x c}\right): p M L 2(+) W F$ & pSK73 & Aarl & pKV74 & pML3:nptII : \paxG: \paxM: \nodB:paxC & 16.3 \\
\hline 3 & pSK28 & $\left(P_{\text {paxM-nodM-T }}-T_{\text {paxM }}\right): \mathrm{pML2}(+) \mathrm{WF}$ & pSK34 & Aarl & pSK35 & pML3:nptII : 4 paxG:nodM & 11.5 \\
\hline 4 & pSK29 & $\checkmark\left(T_{p a x B}-n o d B-P_{p a x B}\right): p M L 2(+) B R$ & pSK35 & BsmBI & pSK38 & 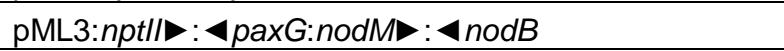 & 14.1 \\
\hline 5 & pSK60 & $\left(P_{\text {paxC-2-nodC- }} T_{\text {paxC }}\right):$ :pML2 $(+) \mathrm{WF}$ & pSK38 & Aarl & pSK66 & pML3:nptII : ४ paxG:nodM〉: ४nodB:nodC & 16.3 \\
\hline 6 & pSK67 & $\boldsymbol{\Psi}\left(T_{t r p c}-n o d W-P_{t r p c}\right): p M L 2(+) B R$ & pSK66 & BsmBI & pSK68 & pML3:nptII : $४$ paxG:nodM : $\triangleleft$ nodB:nodC $: \triangleleft$ nodW & 20.5 \\
\hline 6 & pSK80 & $\boldsymbol{4}\left(T_{t r p C}-n o d W-P_{j a n D}\right): \mathrm{pML} 2(+) \mathrm{BR}$ & pSK66 & BsmBI & pSK81 & pML3:nptII : $\triangleleft$ paxG:nodM : $\triangleleft$ nodB:nodC $: \triangleleft$ nodW & 20.3 \\
\hline
\end{tabular}


Table 9.6. Table of notable fungal strains generated in this thesis.

\begin{tabular}{|c|c|c|}
\hline $\begin{array}{c}\text { Newly generated } P \text {. paxilli } \\
\text { strains }\end{array}$ & Description (plasmid/destination strain) & Notable indole diterpene phenotype \\
\hline sKV50 & pKV13/PN2290 & none \\
\hline sKV60 & pKV25/PN2290 & none \\
\hline sKV44 & pPN1783/PN2290 & paxilline \\
\hline sKV66 & pKV27/PN2290 & paxilline \\
\hline sKV98 & pKV34/PN2257 & emindole SB (18) \\
\hline sKV101 & pPN1857/PN2257 & paxilline \\
\hline sKV136 & pKV63/PN2257 & emindole SB (18) \\
\hline sKV88 & pKV36/PN2458 & none \\
\hline sSK66-14 & pSK66/PN2250 & emindole SB (18) \\
\hline sKV254 & pKV74/PN2250 & paspaline (11) \\
\hline sKV170 & pKV64/PN2257 & nodulisporic acid $F(82)$ \\
\hline sKV157 & pKV65/PN2257 & emindole SB (18) \\
\hline sKV160 & pKV67/PN2257 & emindole SB (18) \\
\hline sKV185 & pKV66/PN2257 & emindole SB (18) \\
\hline sKV193 & pKV68/PN2257 & emindole SB (18) \\
\hline sSK68-11 & pSK68/PN2250 & nodulisporic acid $F(82)$ \\
\hline sSK81-4 & pSK81/PN2250 & nodulisporic acid $F(82)$ \\
\hline
\end{tabular}




\section{LCMS and NMR metabolite characterisation}

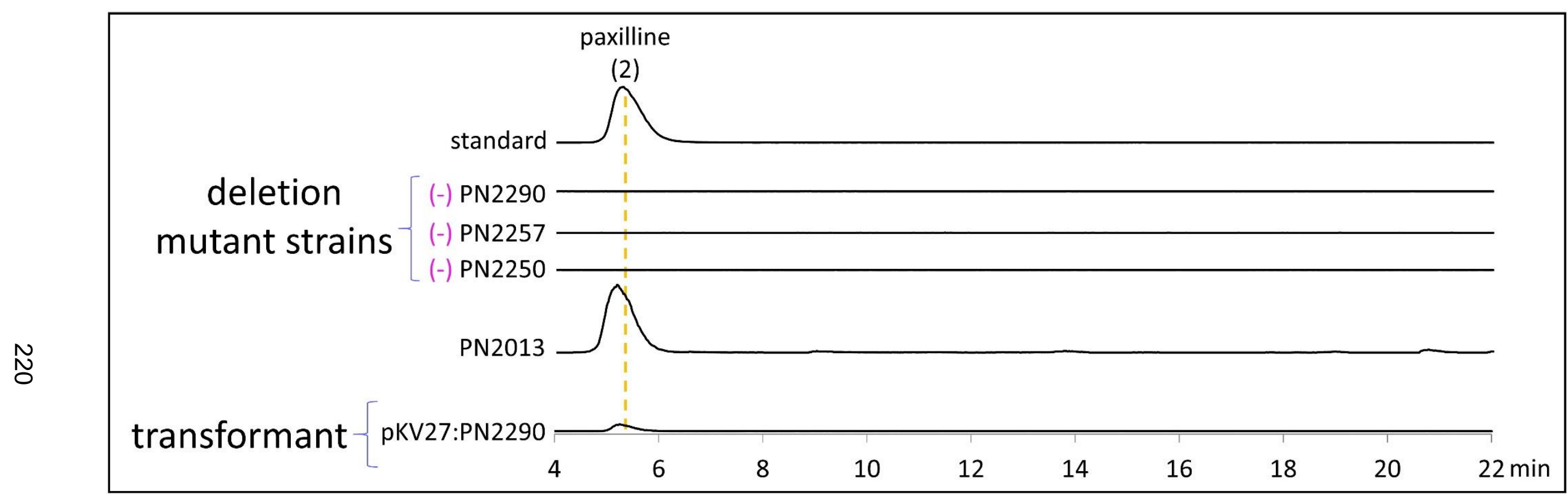

Figure 9.8. Extracted ion chromatograms (EICs) used to detect paxilline (2) (5.3 minutes, $436.248 \pm 0.01 \mathrm{~m} / \mathrm{z})$. A pink negative sign (-) indicates traces where paxilline (2) was not detected. All EICs have been scaled to 300,000 AU. 


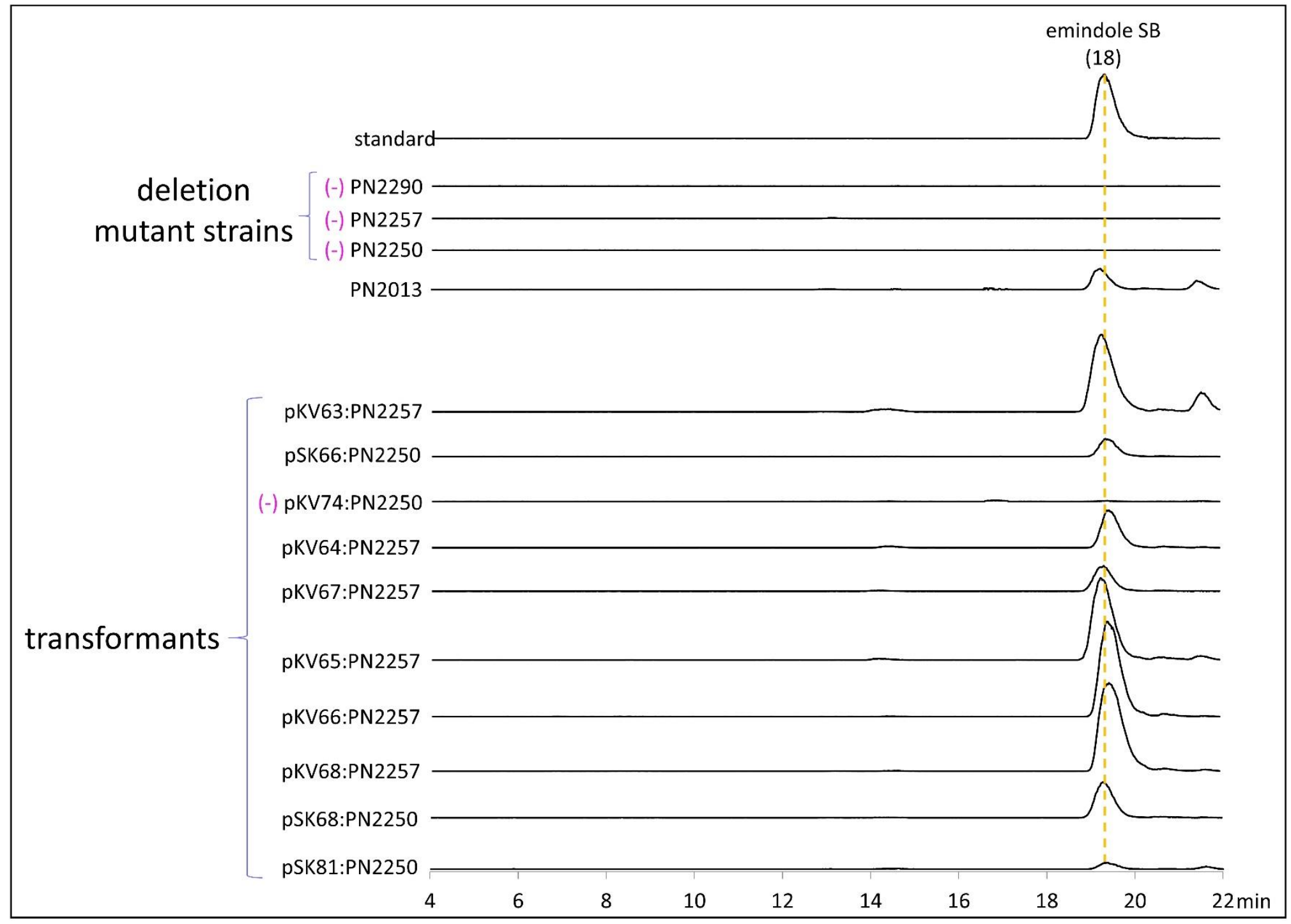

Figure 9.9. Extracted ion chromatograms (EICs) used to detect emindole SB (18) (19.3 min, $406.31 \pm 0.01 \mathrm{~m} / \mathrm{z})$. A pink negative sign (-) indicates traces where emindole SB (18) was not detected. All EICs have been scaled to 300,000 AU. 


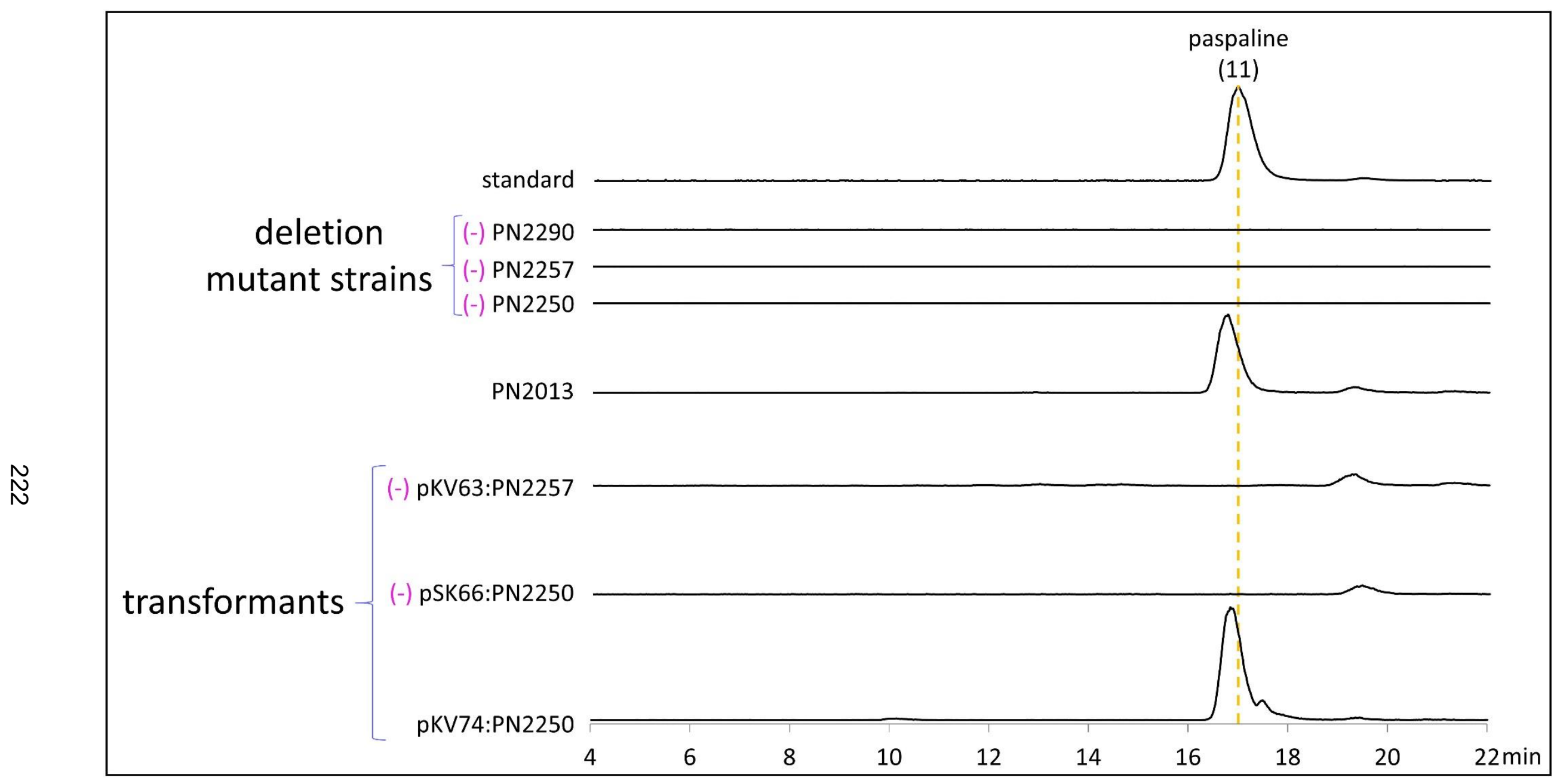

Figure 9.10. Extracted ion chromatograms (EICs) used to detect paspaline (11) (17.6 minutes, $422.305 \pm 0.01 \mathrm{~m} / \mathrm{z}$ ). A pink negative sign (-) indicates traces where paspaline (11) was not detected. All EICs have been scaled to 300,000 AU. 


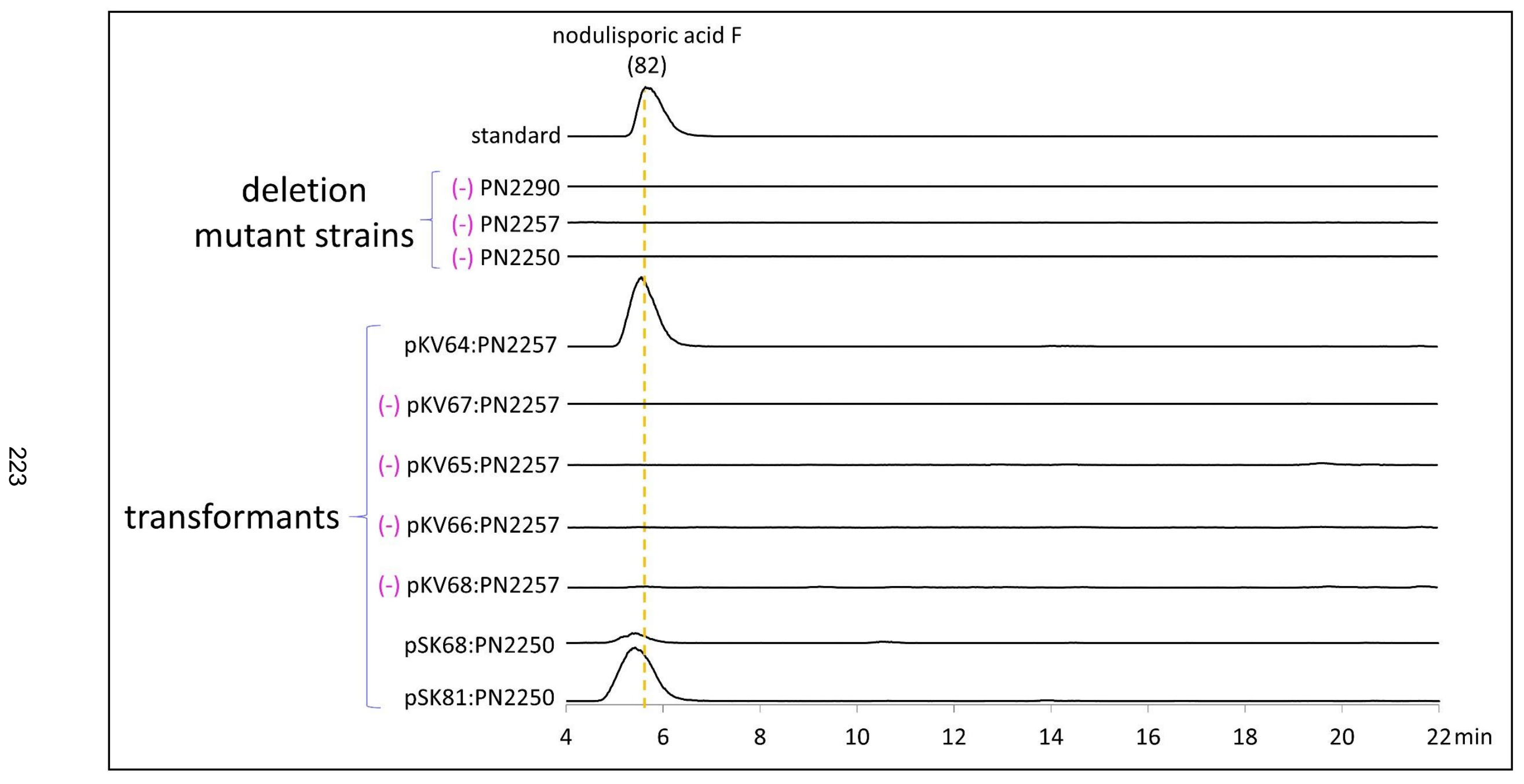

Figure 9.11. Extracted ion chromatograms (EICs) used to detect nodulisporic acid $F(82)$ (6.2 minutes, $436.284 \pm 0.01 \mathrm{~m} / \mathrm{z})$. A pink negative sign (-) indicates traces where nodulisporic acid $F(82)$ was not detected. All EICs have been scaled to $300,000 \mathrm{AU}$. 
Table 9.7. ${ }^{1} \mathrm{H}$ and ${ }^{13} \mathrm{C}$ NMR assignment of paspaline (11) in $\mathrm{CDCl}_{3}$. Paspaline (11) spectra are depicted in Figure 9.12 to 9.16.

\begin{tabular}{|c|c|c|c|}
\hline \multirow{2}{*}{ Position } & \multicolumn{2}{|c|}{ Paspaline (11) } & \multirow[b]{2}{*}{ COSY } \\
\hline & ${ }^{1} \mathrm{H}$ & ${ }^{13} \mathrm{C}$ & \\
\hline 1 & - & - & - \\
\hline 2 & $3.21, \mathrm{dd}$ & $84.64, \mathrm{CH}$ & $\mathrm{H} 3$ \\
\hline 3 & $1.35, \mathrm{~m} \mathrm{1.67, \textrm {m }}$ & $21.93, \mathrm{CH}_{2}$ & \\
\hline 4 & 1.12, m 1.83, m & $37.62, \mathrm{CH}_{2}$ & \\
\hline $4 a$ & & $36.52, \mathrm{C}$ & \\
\hline $4 b$ & $1.45, \mathrm{~m}$ & $46.38, \mathrm{CH}$ & $\mathrm{H} 5$ \\
\hline 5 & $1.45, \mathrm{~m} \mathrm{1.66,m}$ & $21.90, \mathrm{CH}_{2}$ & \\
\hline 6 & $1.59, \mathrm{~m} \mathrm{1.77, \textrm {m }}$ & $25.24, \mathrm{CH}_{2}$ & $\mathrm{H} 5$ \\
\hline $6 a$ & $2.76, \mathrm{~m}$ & 48.72, $\mathrm{CH}$ & $\begin{array}{l}\mathrm{H} 6, \\
\mathrm{H} 7\end{array}$ \\
\hline 7 & $\begin{array}{l}\text { 2.32, dd 2.66, } \\
\text { dd }\end{array}$ & $27.48, \mathrm{CH}_{2}$ & \\
\hline $7 a$ & & 118.22, C & \\
\hline $7 b$ & & $125.09, \mathrm{C}$ & \\
\hline 8 & $7.41, \mathrm{~m}$ & 118.35, $\mathrm{CH}$ & $\mathrm{H} 9$ \\
\hline 9 & $7.06, \mathrm{~m}$ & $119.52, \mathrm{CH}$ & \\
\hline 10 & $7.06, \mathrm{~m}$ & $120.40, \mathrm{CH}$ & \\
\hline 11 & $7.28, \mathrm{~m}$ & $111.40, \mathrm{CH}$ & $\mathrm{H} 10$ \\
\hline $11 \mathrm{a}$ & & 139.94, C & \\
\hline 12 & 7.72, brs & & \\
\hline $12 a$ & & 150.82, C & \\
\hline $12 b$ & & $52.97, \mathrm{C}$ & \\
\hline $12 c$ & & $39.98, \mathrm{C}$ & \\
\hline 13 & $1.62, \mathrm{~m} \mathrm{1.94,m}$ & $33.88, \mathrm{CH}_{2}$ & \\
\hline 14 & $1.69, \mathrm{~m}$ & $24.57, \mathrm{CH}_{2}$ & \\
\hline $14 a$ & $3.01, \mathrm{dd}$ & $85.68, \mathrm{CH}$ & \\
\hline 15 & $1.01, \mathrm{~s}$ & $14.50, \mathrm{CH}_{3}$ & \\
\hline 16 & $1.12, \mathrm{~s}$ & $19.93, \mathrm{CH}_{3}$ & \\
\hline 17 & $0.87, \mathrm{~s}$ & $12.61, \mathrm{CH}_{3}$ & \\
\hline 18 & & $71.98, \mathrm{C}$ & \\
\hline 19 & $1.18, \mathrm{~s}$ & $26.02, \mathrm{CH}_{3}$ & \\
\hline 20 & $1.16, \mathrm{~s}$ & $23.64, \mathrm{CH}_{3}$ & \\
\hline
\end{tabular}

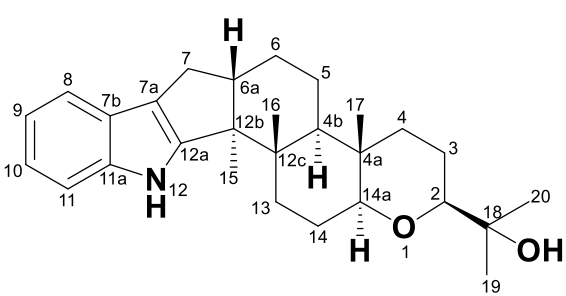

paspaline (11)

$\mathrm{C}_{28} \mathrm{H}_{39} \mathrm{NO}_{2}$

Exact Mass: 421.30

calc. $[\mathrm{M}+\mathrm{H}]+422.3054 \mathrm{~m} / \mathrm{z}$

obs. $[\mathrm{M}+\mathrm{H}]+422.3055 \mathrm{~m} / \mathrm{z}$

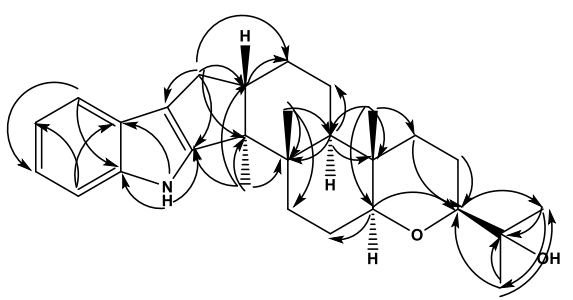

Key HMBC correlations of paspaline, (11) 


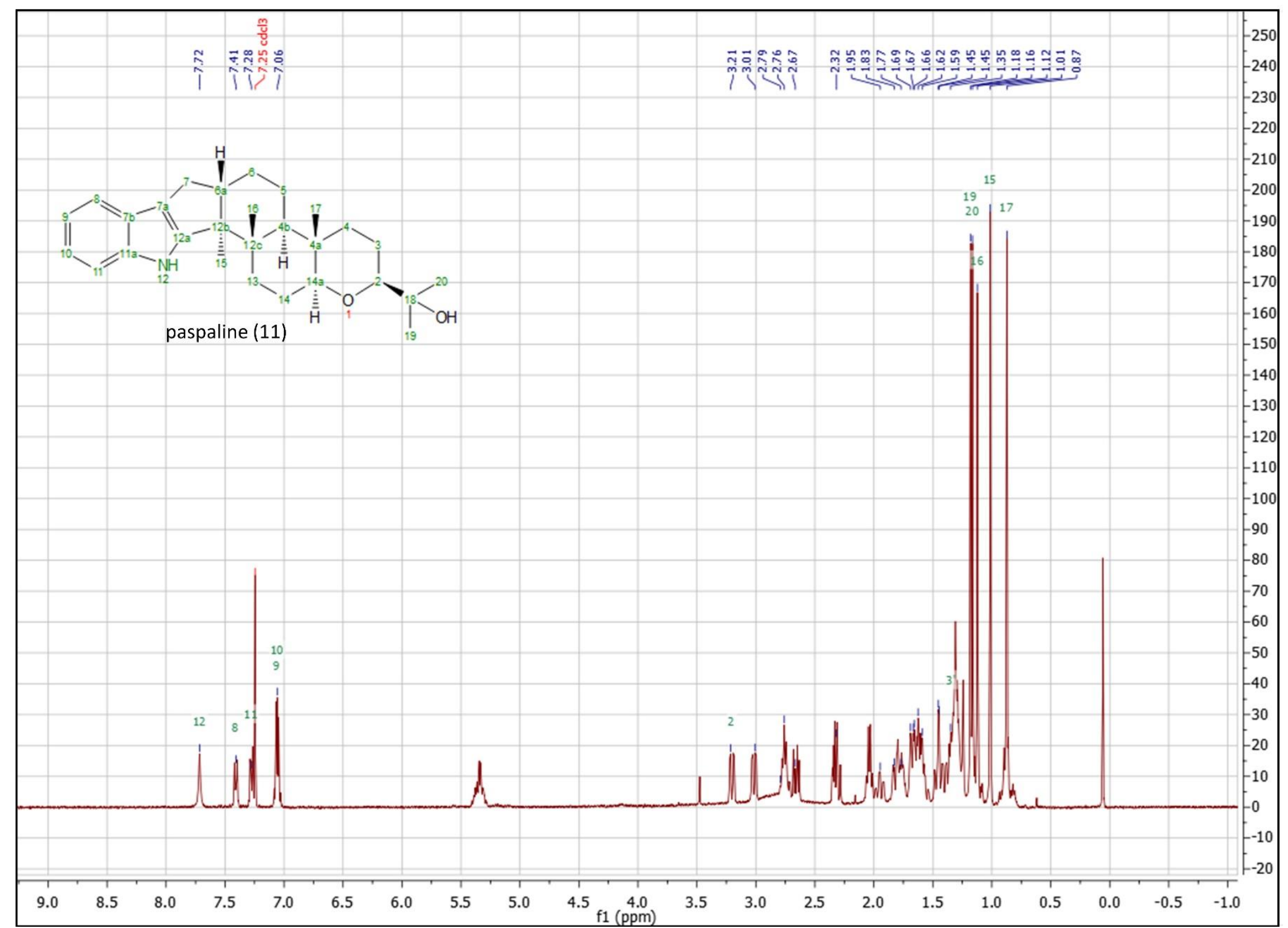

Figure 9.12. ${ }^{1} \mathrm{H}-\mathrm{NMR}$ spectra for paspaline (11) standard in $\mathrm{CDCl}_{3}$ at $400 \mathrm{MHz}$. 


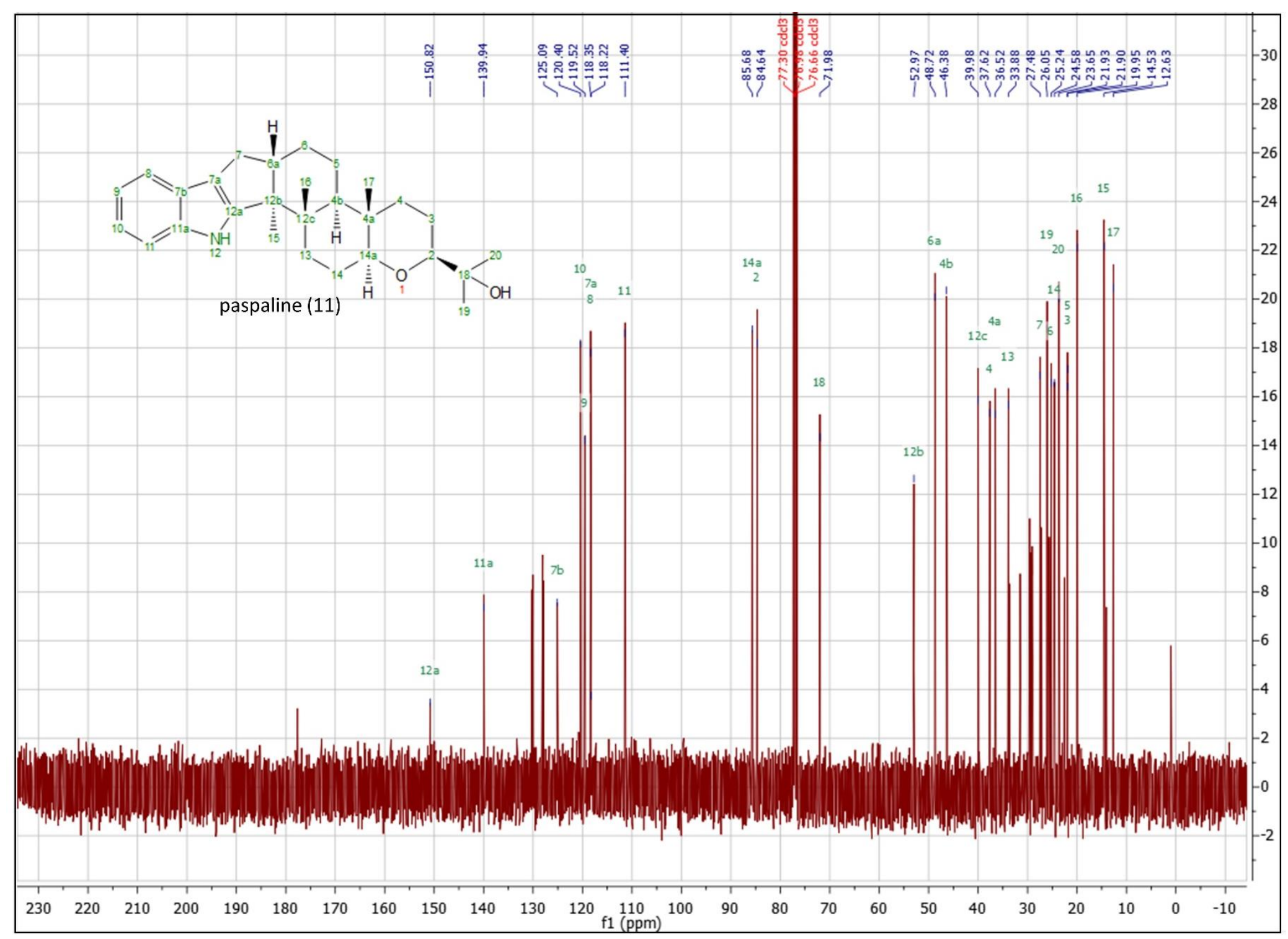

Figure 9.13. ${ }^{13} \mathrm{C}-\mathrm{NMR}$ spectra for paspaline (11) standard in $\mathrm{CDCl}_{3}$ at $100 \mathrm{MHz}$. 


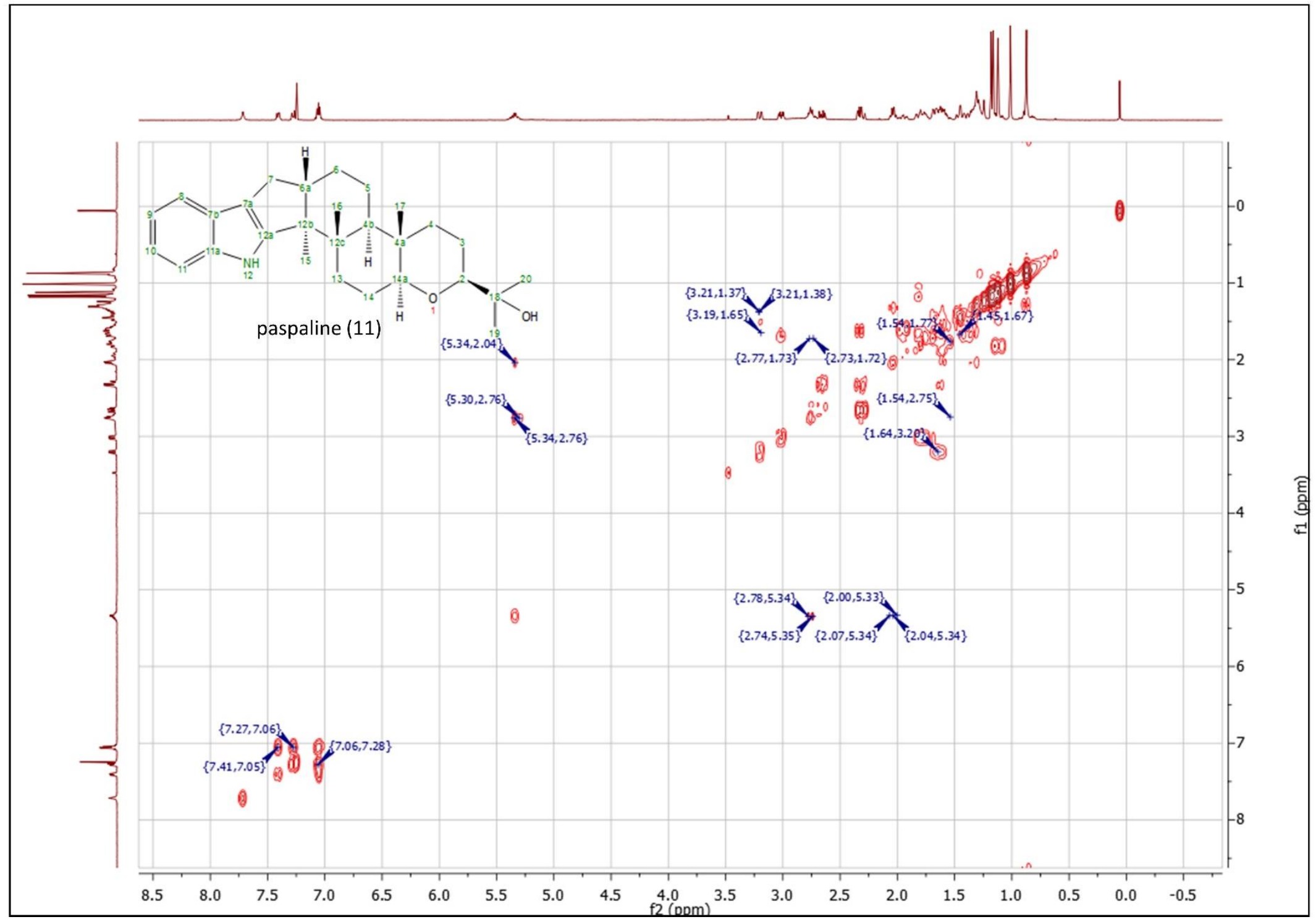

Figure 9.14. ${ }^{1} \mathrm{H}-{ }^{1} \mathrm{H}$ COSY-NMR spectra for paspaline (11) standard in $\mathrm{CDCl}_{3}$ at $400 \mathrm{MHz}$. 


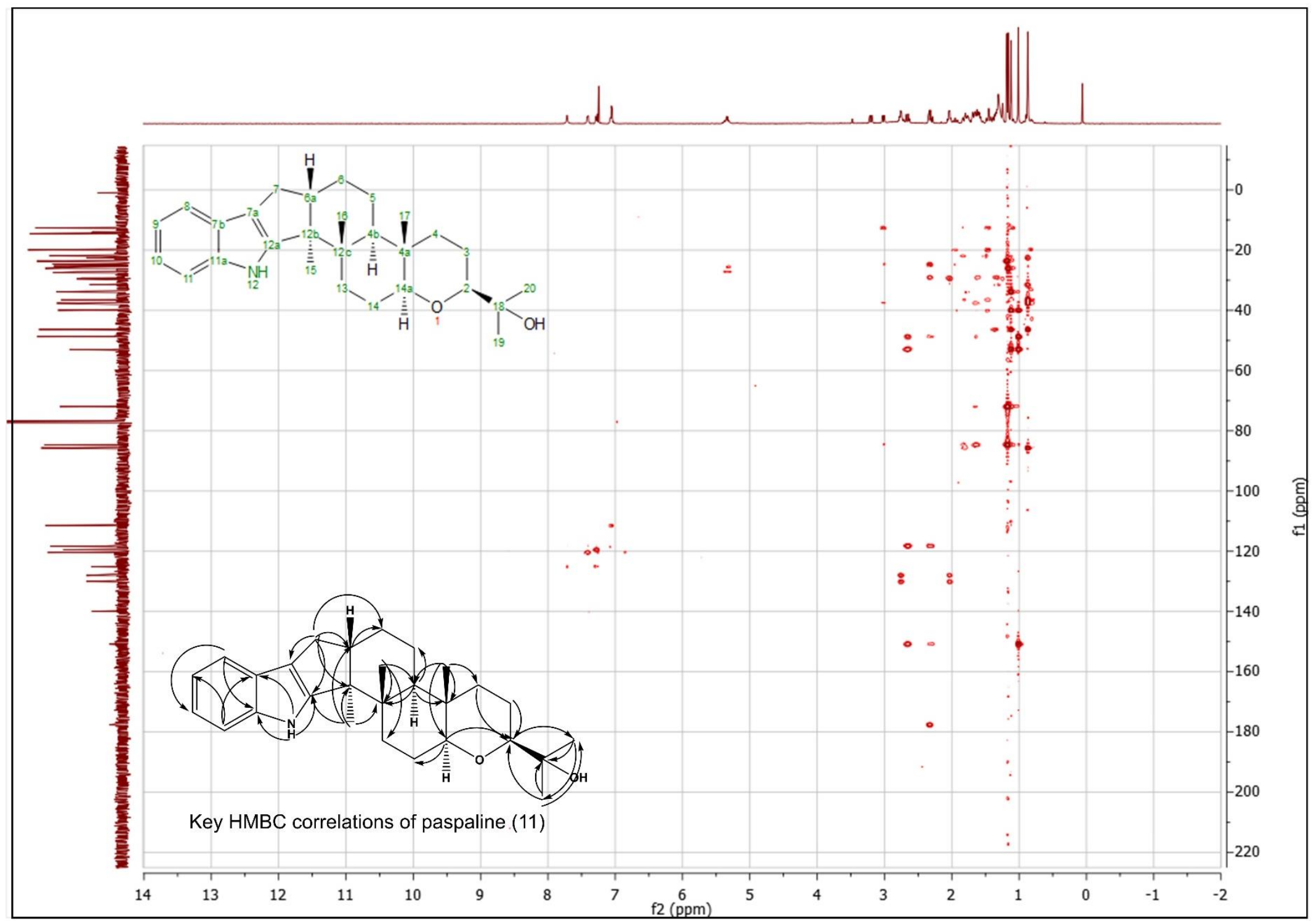

Figure 9.15. HMBC-NMR spectra for paspaline (11) standard in $\mathrm{CDCl}_{3}$ at $400 \mathrm{MHz}$. 


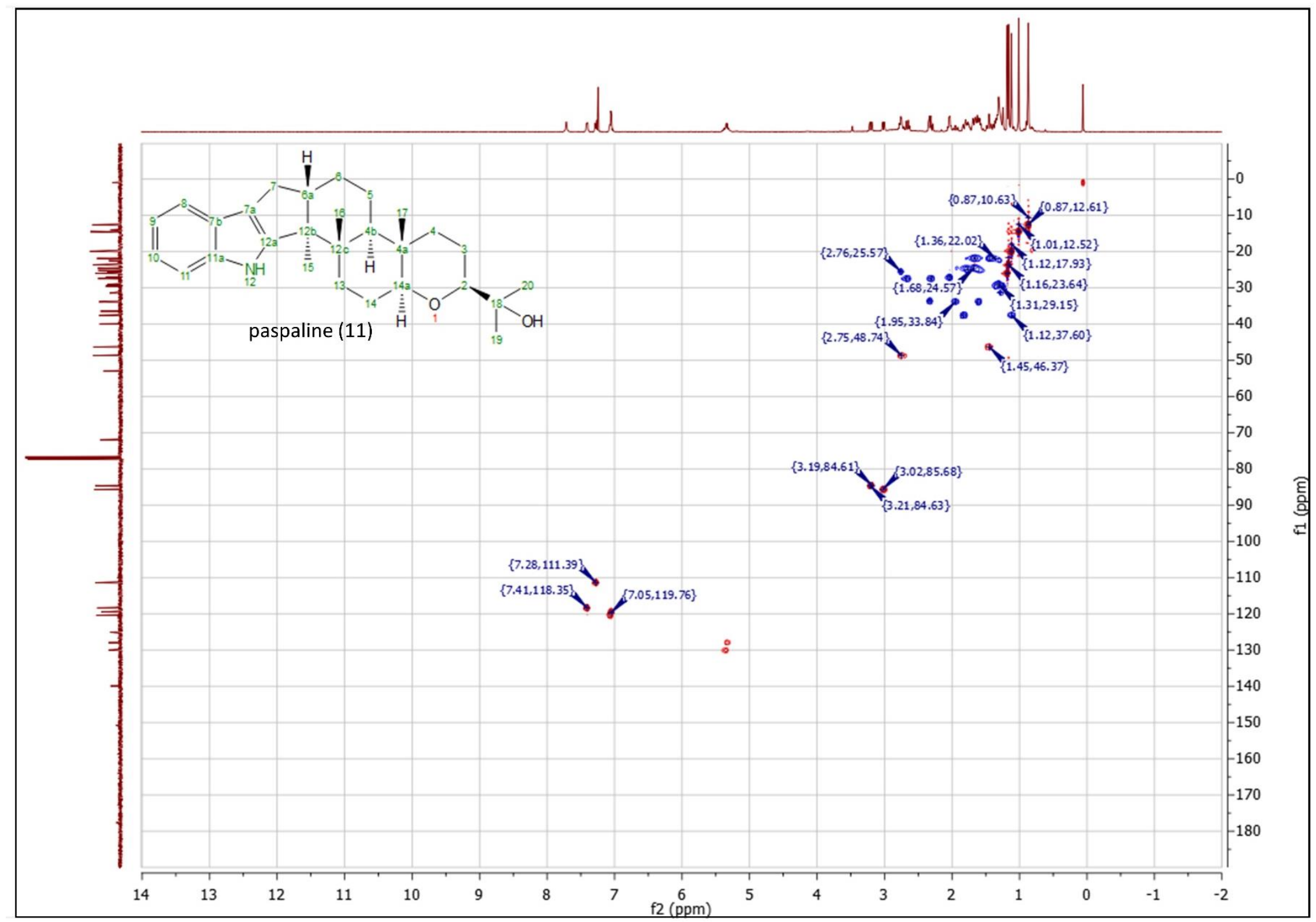

Figure 9.16. HSQC-NMR spectra for paspaline (11) standard in $\mathrm{CDCl}_{3}$ at $400 \mathrm{MHz}$. Blue correlations represent secondary carbons and red correlations represent primary and tertiary carbons. 
Table 9.8. ${ }^{1} \mathrm{H}$ and ${ }^{13} \mathrm{C}$ NMR assignment of paxilline (2) in $\mathrm{CDCl}_{3}$. Paxilline (2) spectra are depicted in Figure 9.17 to 9.21.

\begin{tabular}{|c|c|c|c|}
\hline \multirow{2}{*}{ Position } & \multicolumn{2}{|c|}{ Paxilline (2) } & \multirow[b]{2}{*}{ COSY } \\
\hline & ${ }^{1} \mathrm{H}$ & ${ }^{13} \mathrm{C}$ & \\
\hline 1 & - & - & - \\
\hline 2 & $3.69, \mathrm{~s}$ & $83.16, \mathrm{CH}$ & \\
\hline 3 & & $199.41, \mathrm{C}$ & \\
\hline 4 & $5.85, \mathrm{~s}$ & $\begin{array}{l}119.46, \\
\mathrm{CH}\end{array}$ & \\
\hline $4 a$ & & 168.64, C & \\
\hline $4 b$ & & $77.20, \mathrm{C}$ & \\
\hline 5 & $1.64 \mathrm{~d}, 2.03 \mathrm{~m}$ & $34.13, \mathrm{CH}_{2}$ & \\
\hline 6 & $1.76 \mathrm{~m}, 2.03 \mathrm{~m}$ & $20.87, \mathrm{CH}_{2}$ & $\mathrm{H} 5$ \\
\hline $6 a$ & $2.82, \mathrm{~m}$ & $49.47, \mathrm{CH}$ & $\mathrm{H} 6, \mathrm{H} 7$ \\
\hline 7 & $2.44 \mathrm{dd}, 2.75 \mathrm{dd}$ & $27.17, \mathrm{CH}_{2}$ & \\
\hline $7 a$ & & $117.45, \mathrm{C}$ & \\
\hline $7 b$ & & 125.03, C & \\
\hline 8 & $7.42, \mathrm{~m}$ & $\begin{array}{l}118.51, \\
\mathrm{CH}\end{array}$ & H9 \\
\hline 9 & 7.07, m & $\begin{array}{l}119.70, \\
\mathrm{CH}\end{array}$ & \\
\hline 10 & 7.07, m & $\begin{array}{l}\text { 120.60, } \\
\mathrm{CH}\end{array}$ & \\
\hline 11 & 7.30, m & $\begin{array}{l}111.50, \\
\mathrm{CH}\end{array}$ & $\mathrm{H} 10$ \\
\hline $11 a$ & & $139.73, \mathrm{C}$ & \\
\hline 12 & 7.82, brs & & \\
\hline $12 a$ & & 151.64, C & \\
\hline $12 b$ & & $50.80, \mathrm{C}$ & \\
\hline $12 c$ & & $43.12, \mathrm{C}$ & \\
\hline 13 & $1.45, \mathrm{dd} 2.80, \mathrm{~m}$ & $27.92, \mathrm{CH}_{2}$ & \\
\hline 14 & $2.32 \mathrm{~m}, 1.90 \mathrm{~m}$ & $28.47, \mathrm{CH}_{2}$ & \\
\hline $14 a$ & $4.84, \mathrm{t}$ & $72.67, \mathrm{CH}$ & \\
\hline 15 & $1.32, \mathrm{~s}$ & $16.24, \mathrm{CH}_{3}$ & \\
\hline 16 & $1.01, \mathrm{~s}$ & $19.53, \mathrm{CH}_{3}$ & \\
\hline 17 & - & - & - \\
\hline 18 & & $72.66, \mathrm{C}$ & \\
\hline 19 & $1.29, \mathrm{~s}$ & $26.56, \mathrm{CH}_{3}$ & \\
\hline 20 & $1.27, \mathrm{~s}$ & $24.06, \mathrm{CH}_{3}$ & \\
\hline
\end{tabular}

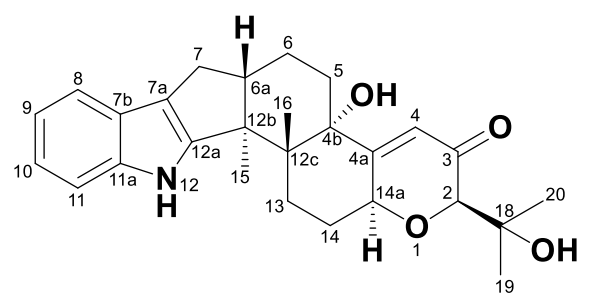

paxilline (2)

$\mathrm{C}_{27} \mathrm{H}_{33} \mathrm{NO}_{4}$

Exact Mass: 435.24

calc. $[\mathrm{M}+\mathrm{H}]+436.2482 \mathrm{~m} / \mathrm{z}$

obs. [M+H]+ $436.2485 \mathrm{~m} / \mathrm{z}$

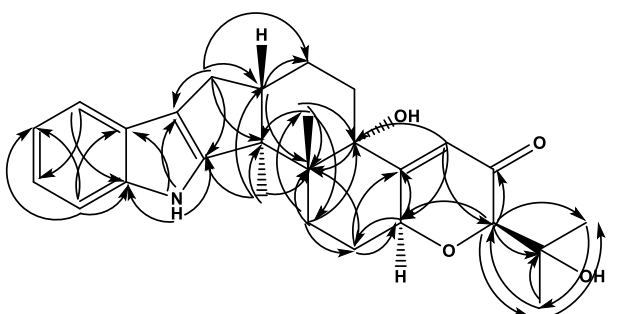

Key $\mathrm{HMBC}$ correlations of paxilline, (2) 


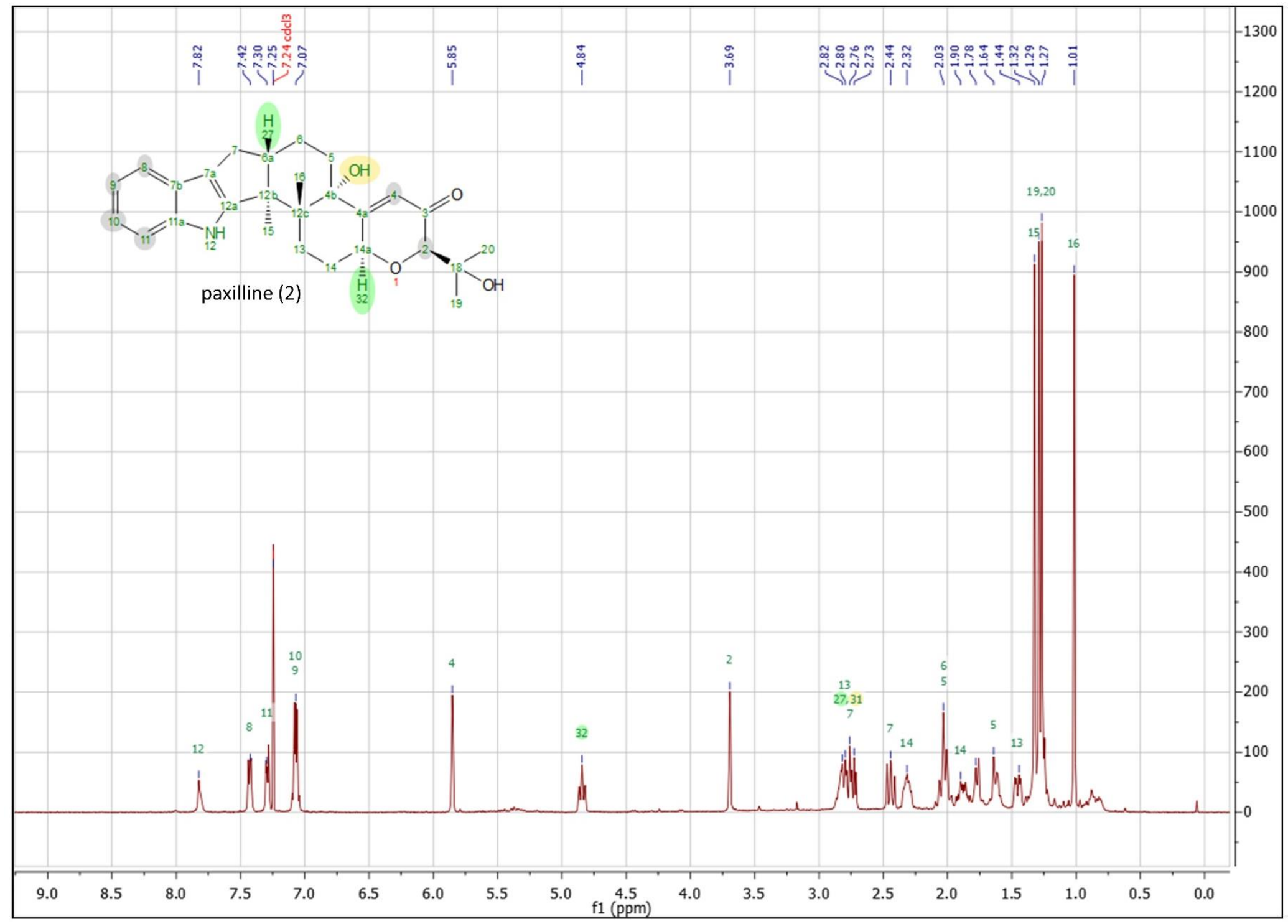

Figure 9.17. ${ }^{1} \mathrm{H}-\mathrm{NMR}$ spectra for paxilline (2) standard in $\mathrm{CDCl}_{3}$ at $400 \mathrm{MHz}$. 


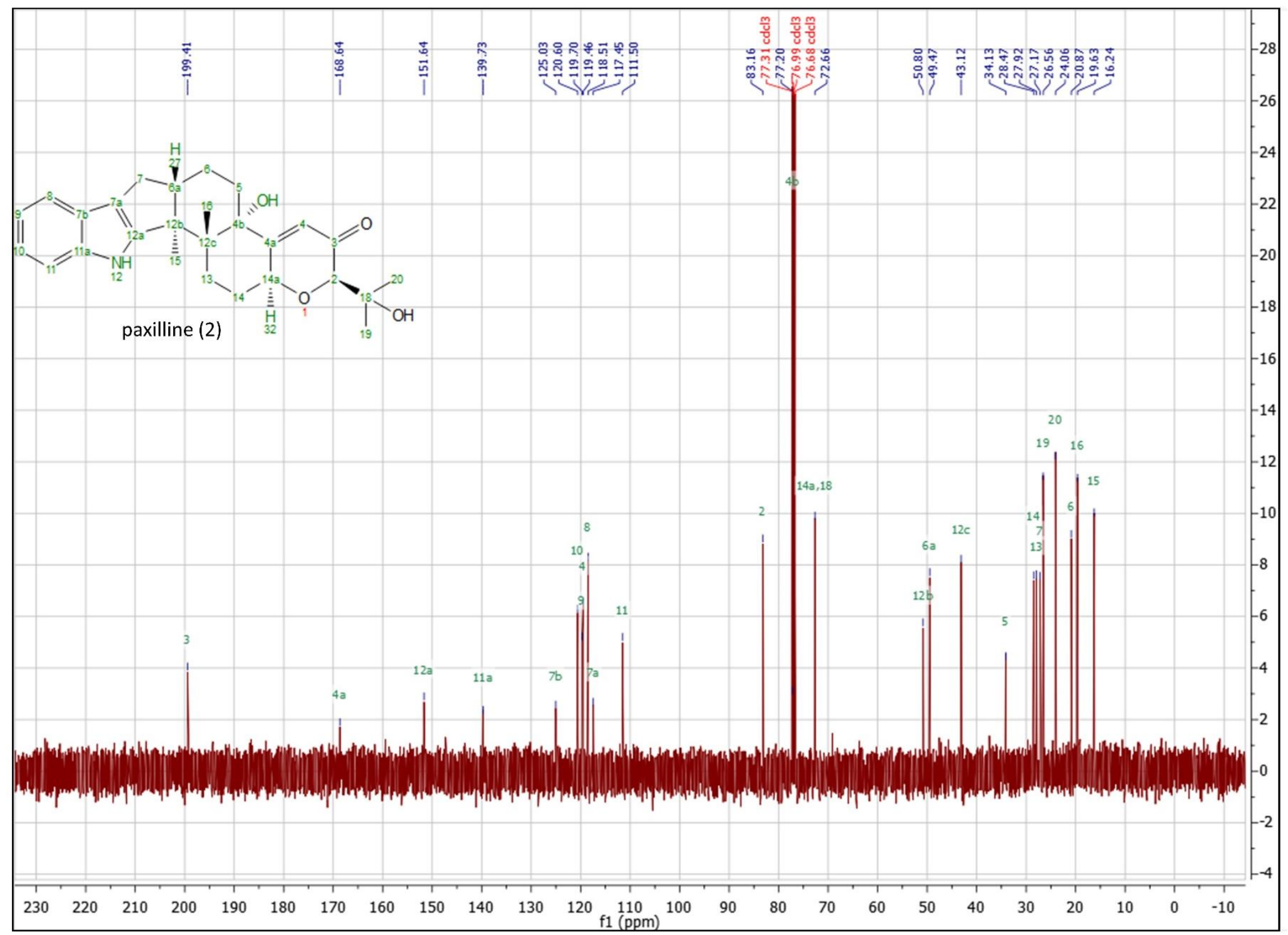

Figure 9.18. ${ }^{13} \mathrm{C}-\mathrm{NMR}$ spectra for paxilline (2) standard in $\mathrm{CDCl}_{3}$ at $100 \mathrm{MHz}$. 


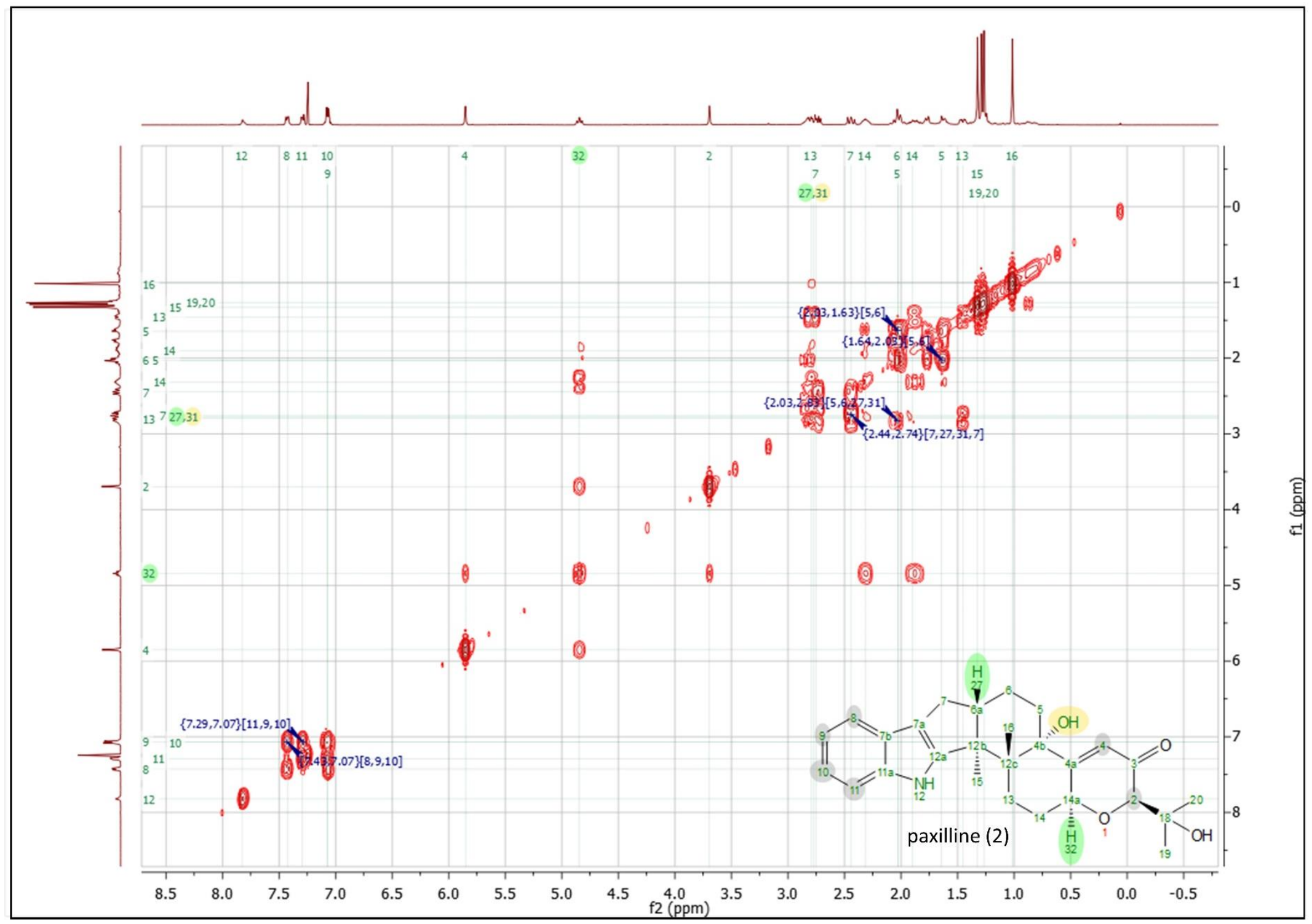

Figure 9 19. ${ }^{1} \mathrm{H}-{ }^{1} \mathrm{H}$ COSY-NMR spectra for paxilline (2) standard in $\mathrm{CDCl}_{3}$ at $400 \mathrm{MHz}$. 


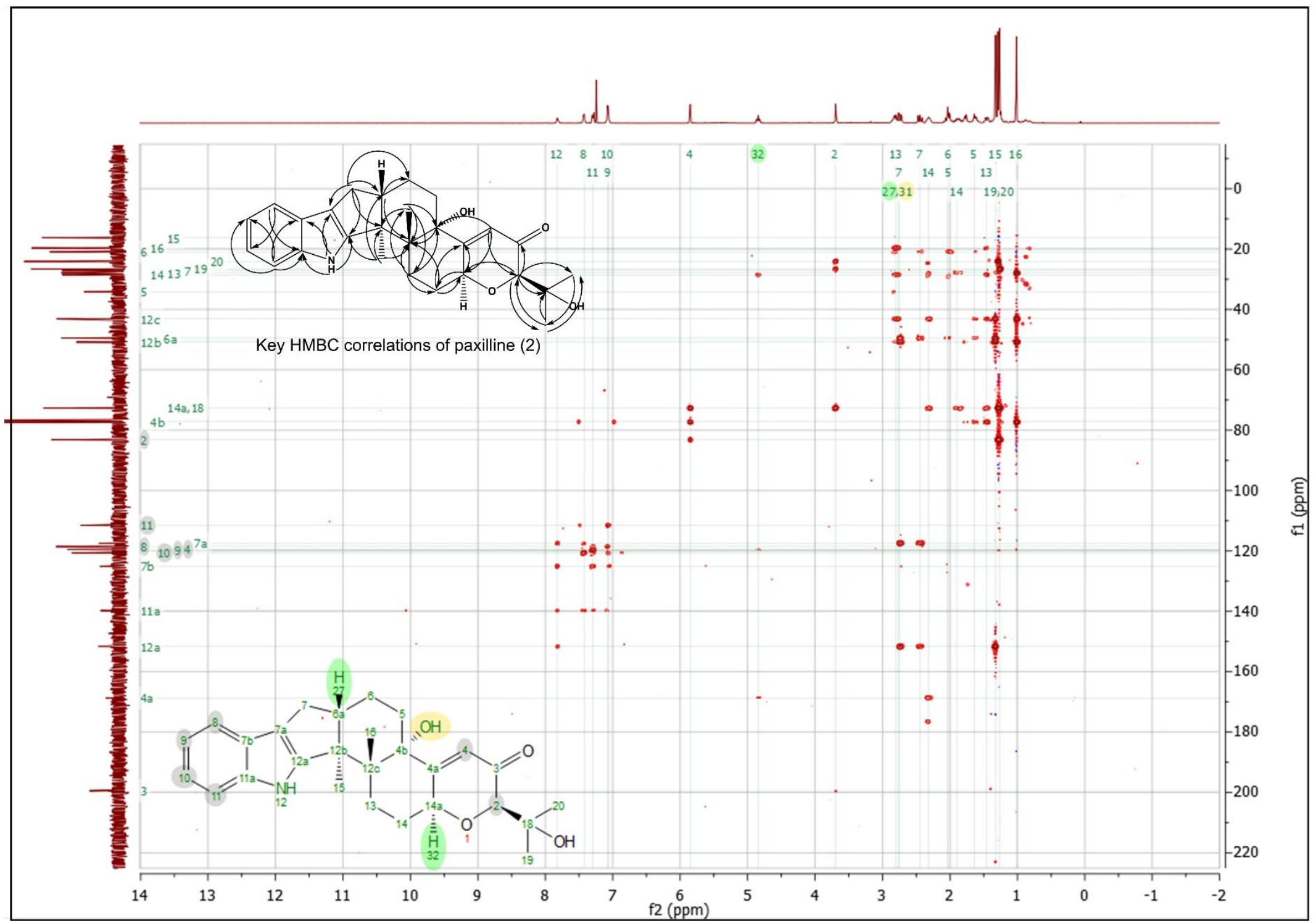

Figure 9.20. $\mathrm{HMBC}-\mathrm{NMR}$ spectra for paxilline (2) standard in $\mathrm{CDCl}_{3}$ at $400 \mathrm{MHz}$. 


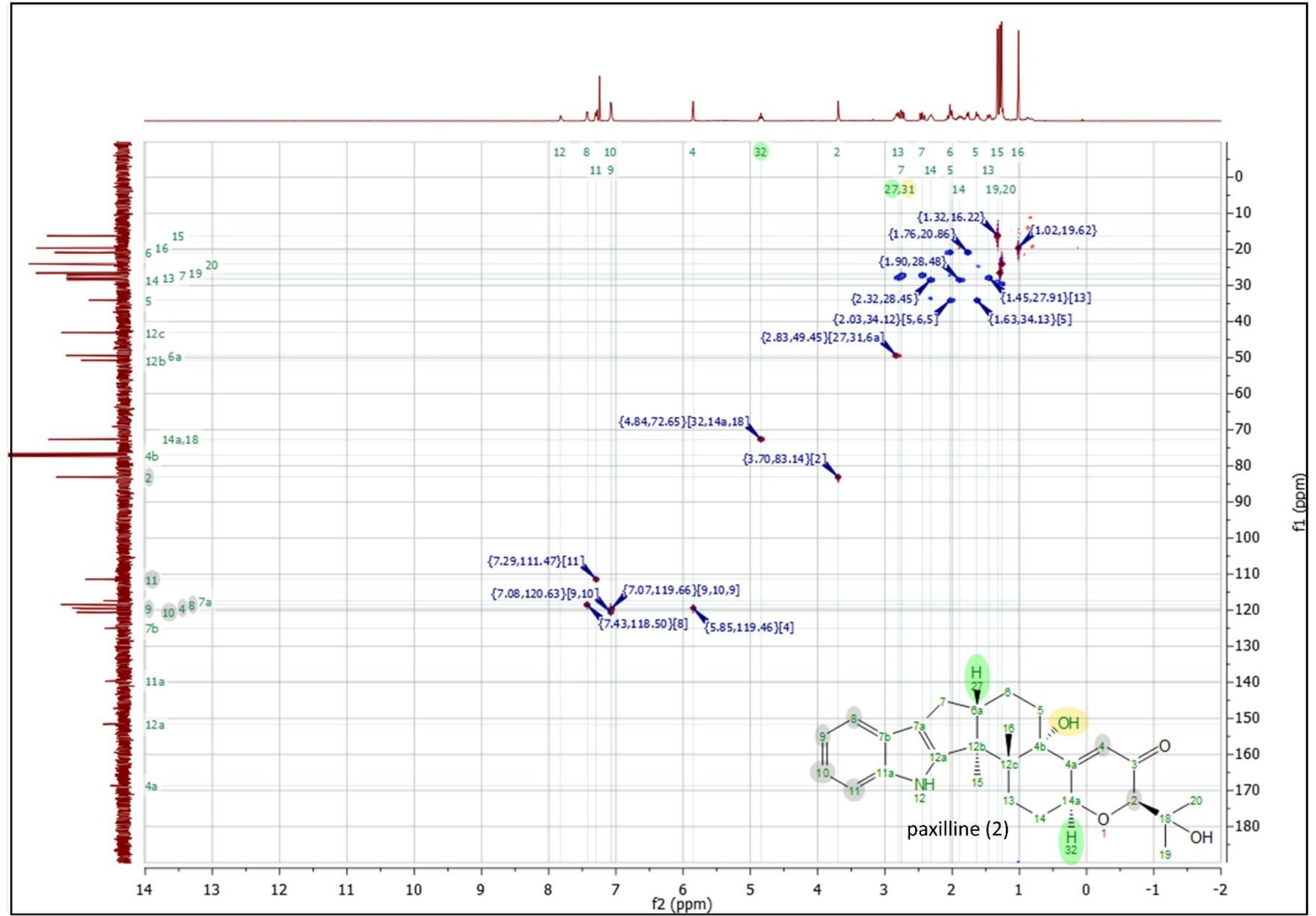

Figure 9.21. HSQC-NMR spectra for paxilline (2) standard in $\mathrm{CDCl}_{3}$ at $400 \mathrm{MHz}$. Blue correlations represent secondary carbons and red correlations represent primary and tertiary carbons. 
Table 9.9. ${ }^{1} \mathrm{H}$ and ${ }^{13} \mathrm{C}$ NMR assignment of nodulisporic acid $\mathrm{F}$ (NAF) (82) in $\mathrm{CD}_{3} \mathrm{OD}$. Spectra depicted in Figure 9.22 to 9.25.

\begin{tabular}{|c|c|c|}
\hline \multirow{2}{*}{ Position } & \multicolumn{2}{|c|}{ nodulisporic acid F (82) } \\
\hline & ${ }^{1} \mathbf{H}$ & ${ }^{13} \mathrm{C}$ \\
\hline 1 & - & - \\
\hline 2 & & $150.78, \mathrm{C}$ \\
\hline 3 & & $52.90, \mathrm{C}$ \\
\hline 4 & & $39.00, C$ \\
\hline 5 & $1.84 \mathrm{~m}, 1.86 \mathrm{~m}$ & $32.31, \mathrm{CH}_{2}$ \\
\hline 6 & $1.77 \mathrm{~m}, 1.86 \mathrm{~m}$ & $26.93, \mathrm{CH}_{2}$ \\
\hline 7 & $3.53, \mathrm{dd}$ & $72.51, \mathrm{CH}$ \\
\hline 8 & & $41.13, \mathrm{C}$ \\
\hline 9 & $1.76, \mathrm{t}$ & $39.94, \mathrm{CH}$ \\
\hline 10 & $1.49 \mathrm{~d}, 1.65 \mathrm{~m}$ & $22.62, \mathrm{CH}_{2}$ \\
\hline 11 & $1.65 \mathrm{~m}, 1.77 \mathrm{~m}$ & $24.90, \mathrm{CH}_{2}$ \\
\hline 12 & $2.75, \mathrm{~m}$ & $48.84, \mathrm{CH}$ \\
\hline 13 & $2.29 \mathrm{dd}, 2.61 \mathrm{dd}$ & $26.85, \mathrm{CH}_{2}$ \\
\hline 14 & & $116.55, \mathrm{C}$ \\
\hline 15 & & $124.82, \mathrm{C}$ \\
\hline 16 & $7.27, \mathrm{brd}$ & $117.27, \mathrm{CH}$ \\
\hline 17 & $6.94, \mathrm{dt}$ & $119.27, \mathrm{CH}$ \\
\hline 18 & $6.90, \mathrm{dt}$ & $118.26, \mathrm{CH}$ \\
\hline 19 & $7.27, \mathrm{brd}$ & $111.20, \mathrm{CH}$ \\
\hline 20 & & $140.64, \mathrm{C}$ \\
\hline 21 & $1.01, \mathrm{~s}$ & $13.46, \mathrm{CH}_{3}$ \\
\hline 22 & $1.12, \mathrm{~s}$ & $18.02, \mathrm{CH}_{3}$ \\
\hline 23 & $0.84, \mathrm{~s}$ & $15.84, \mathrm{CH}_{3}$ \\
\hline 24 & $1.70 \mathrm{~m}, 1.36 \mathrm{~m}$ & $35.61, \mathrm{CH}_{2}$ \\
\hline 25 & $2.13 \mathrm{~m}, 2.20 \mathrm{~m}$ & $22.02, \mathrm{CH}_{2}$ \\
\hline 26 & $6.79, \mathrm{t}$ & $142.60, \mathrm{CH}$ \\
\hline 27 & & $127.45, \mathrm{C}$ \\
\hline 28 & & $170.73, \mathrm{C}$ \\
\hline 29 & $1.85, \mathrm{~s}$ & $11.09, \mathrm{CH}_{3}$ \\
\hline
\end{tabular}

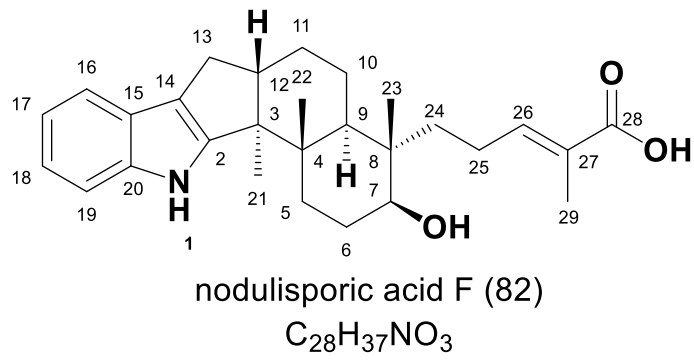

Exact Mass: 435.28

calc. $[\mathrm{M}+\mathrm{H}]+436.2846 \mathrm{~m} / \mathrm{z}$

obs. $[\mathrm{M}+\mathrm{H}]+436.2870 \mathrm{~m} / \mathrm{z}$

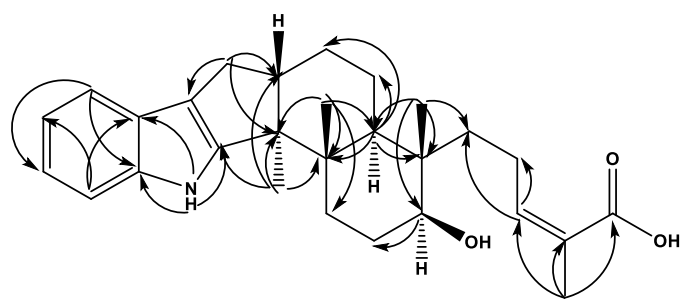

Key HMBC correlations of nodulisporic acid F (82) 


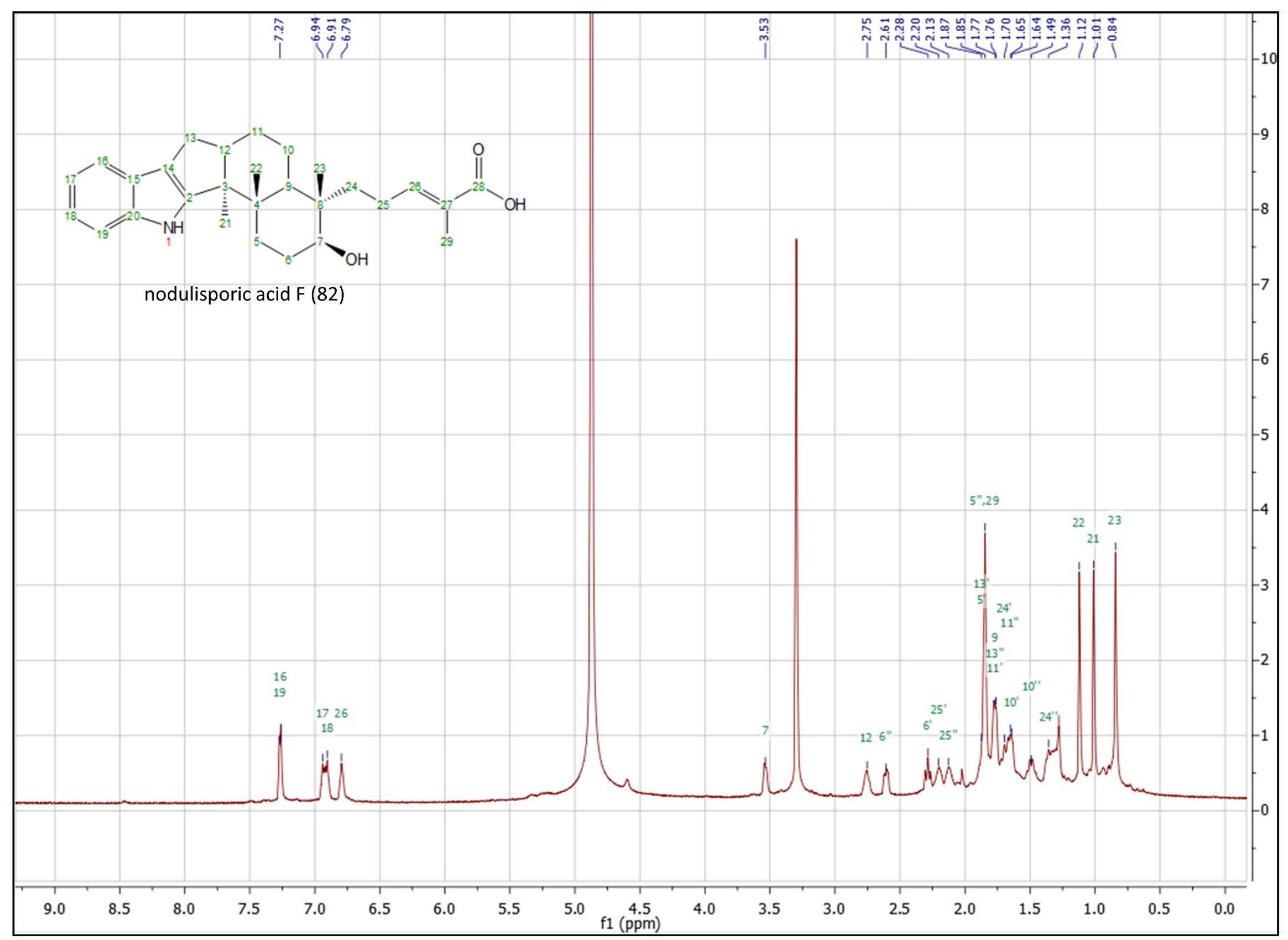

Figure 9.22. ${ }^{1} \mathrm{H}-\mathrm{NMR}$ spectra for nodulisporic acid $\mathrm{F}(82)$ standard in $\mathrm{CD}_{3} \mathrm{OD}$ at $400 \mathrm{MHz}$. 


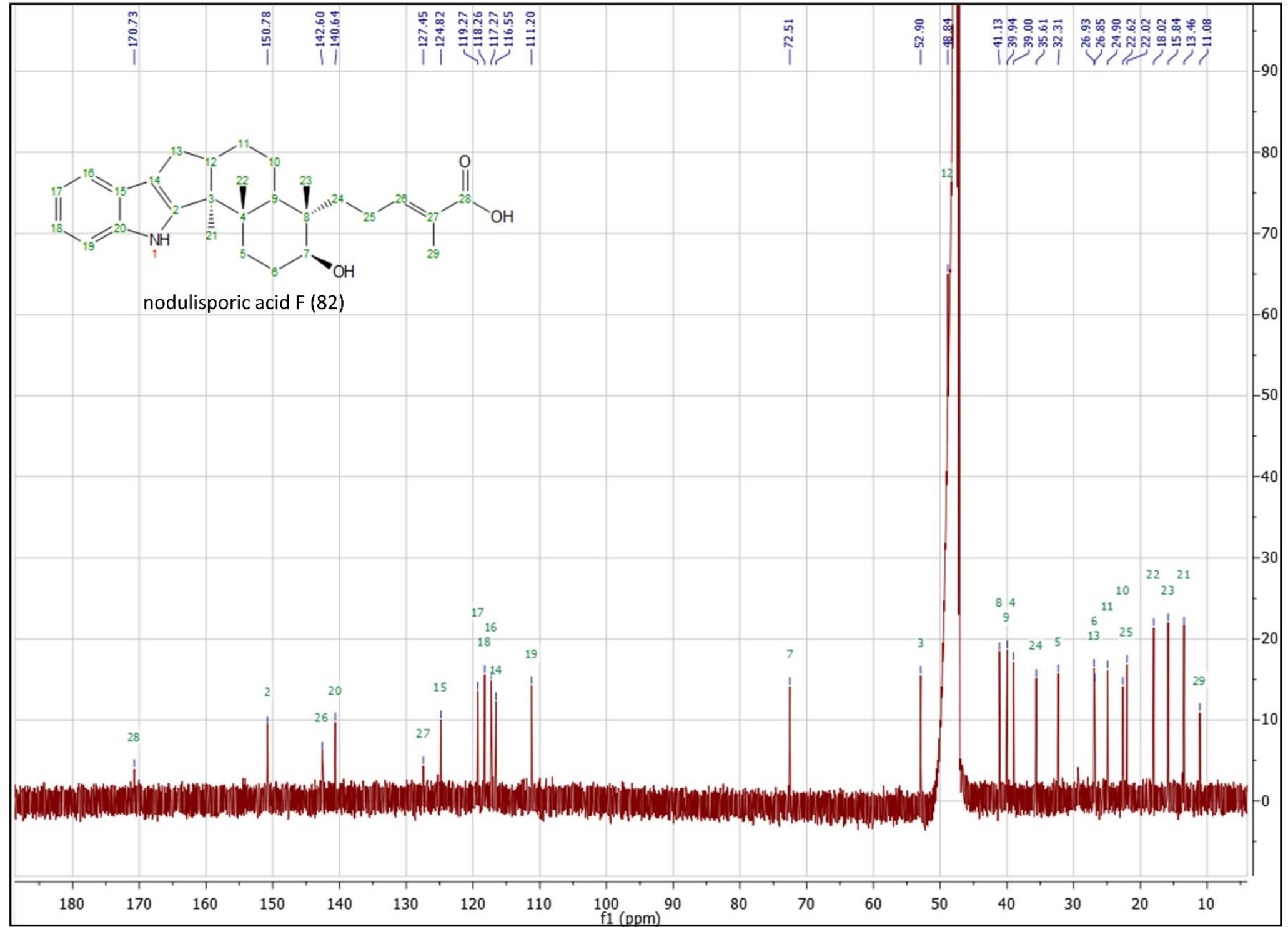

Figure 9.23. ${ }^{13} \mathrm{C}-\mathrm{NMR}$ spectra for nodulisporic acid $\mathrm{F}(82)$ standard in $\mathrm{CD}_{3} \mathrm{OD}$ at $100 \mathrm{MHz}$. 


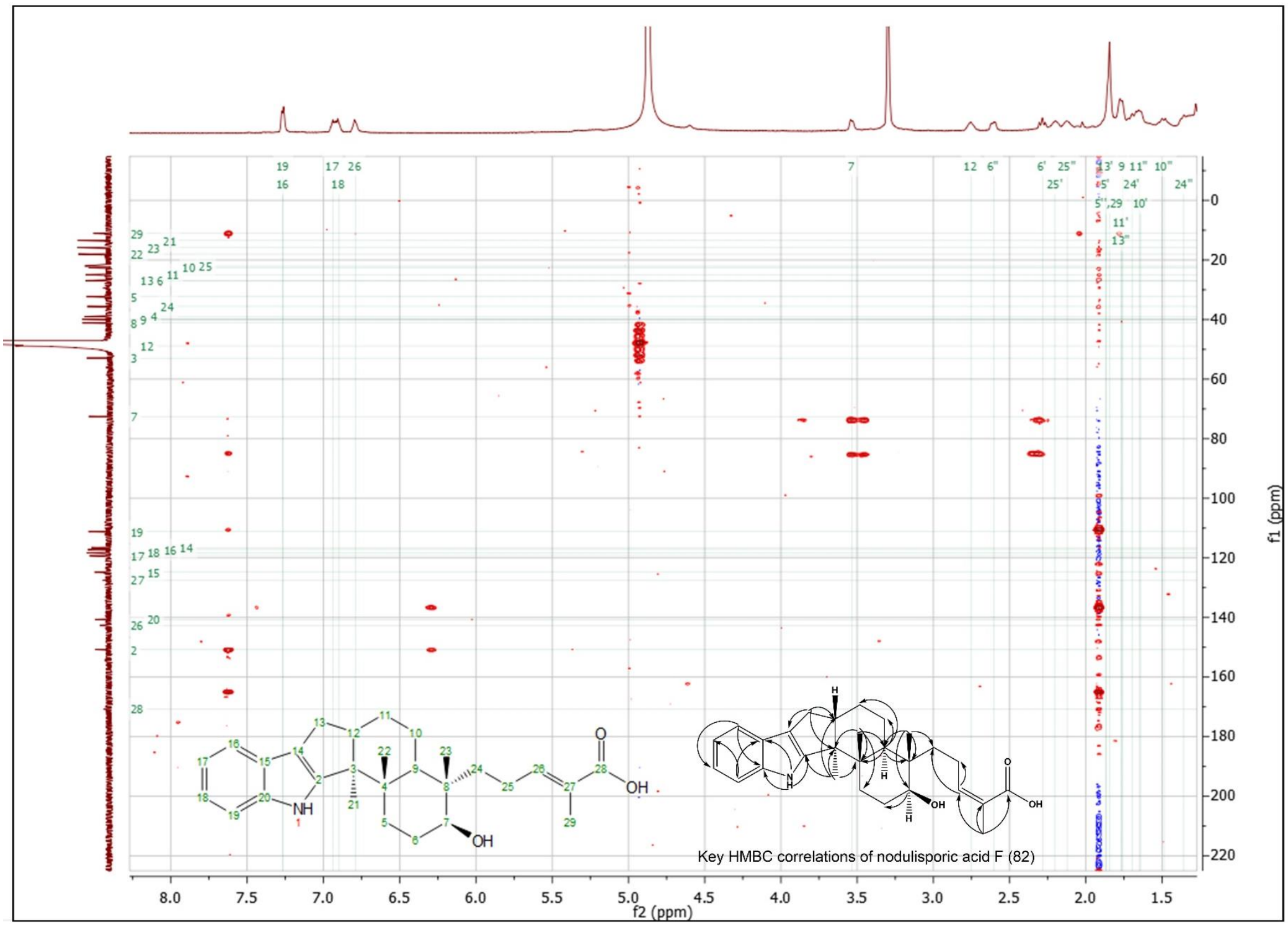

Figure 9.24. HMBC-NMR spectra for nodulisporic acid $\mathrm{F}(82)$ standard in $\mathrm{CD}_{3} \mathrm{OD}$ at $600 \mathrm{MHz}$. 


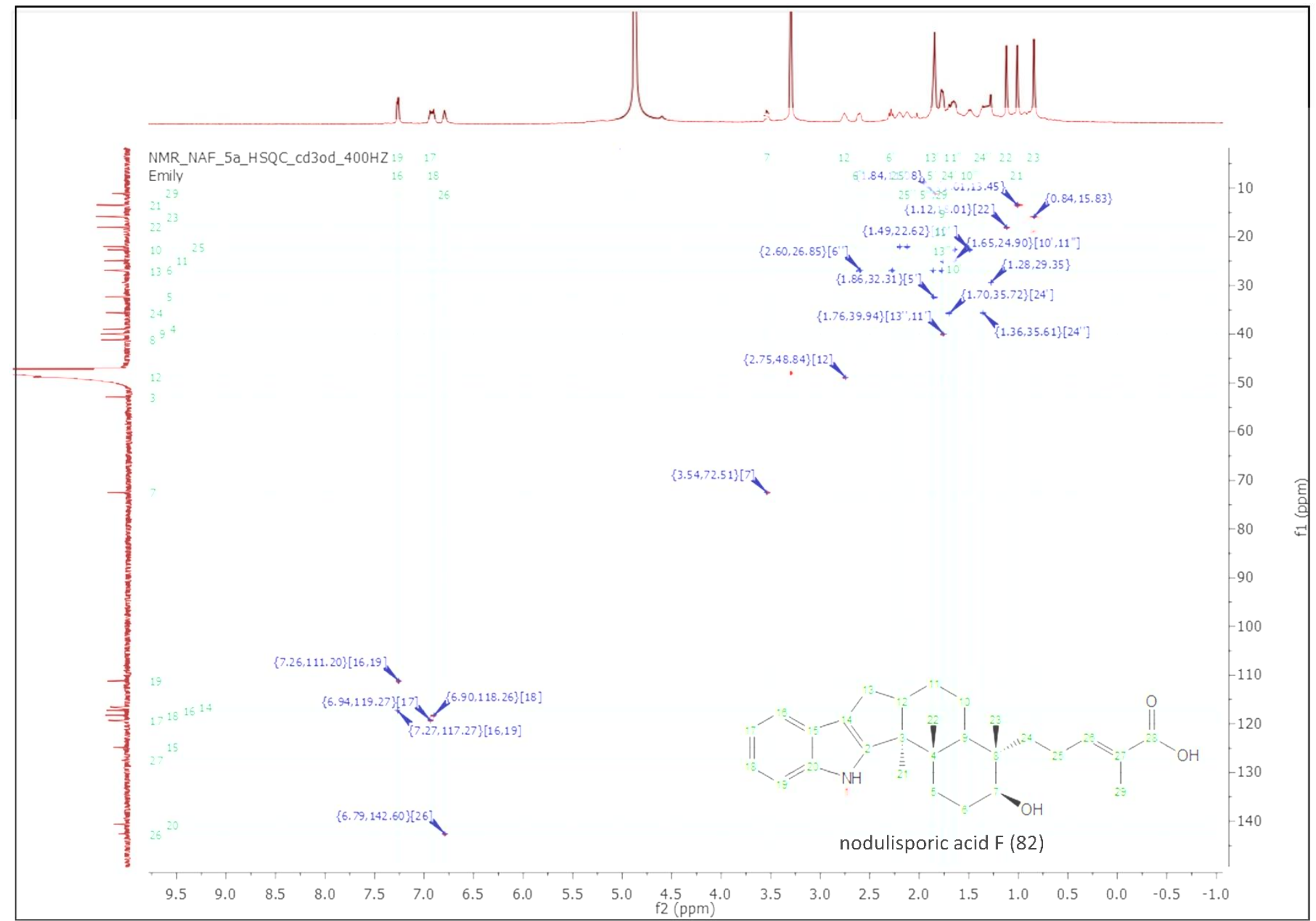

Figure 9.25. HSQC-NMR spectra for nodulisporic acid F (82) standard in $\mathrm{CD}_{3} \mathrm{OD}$ at $400 \mathrm{MHz}$. Blue correlations represent secondary carbons and red correlations represent primary and tertiary carbons. 
Table 9.10. Table of numbered compounds in this thesis.

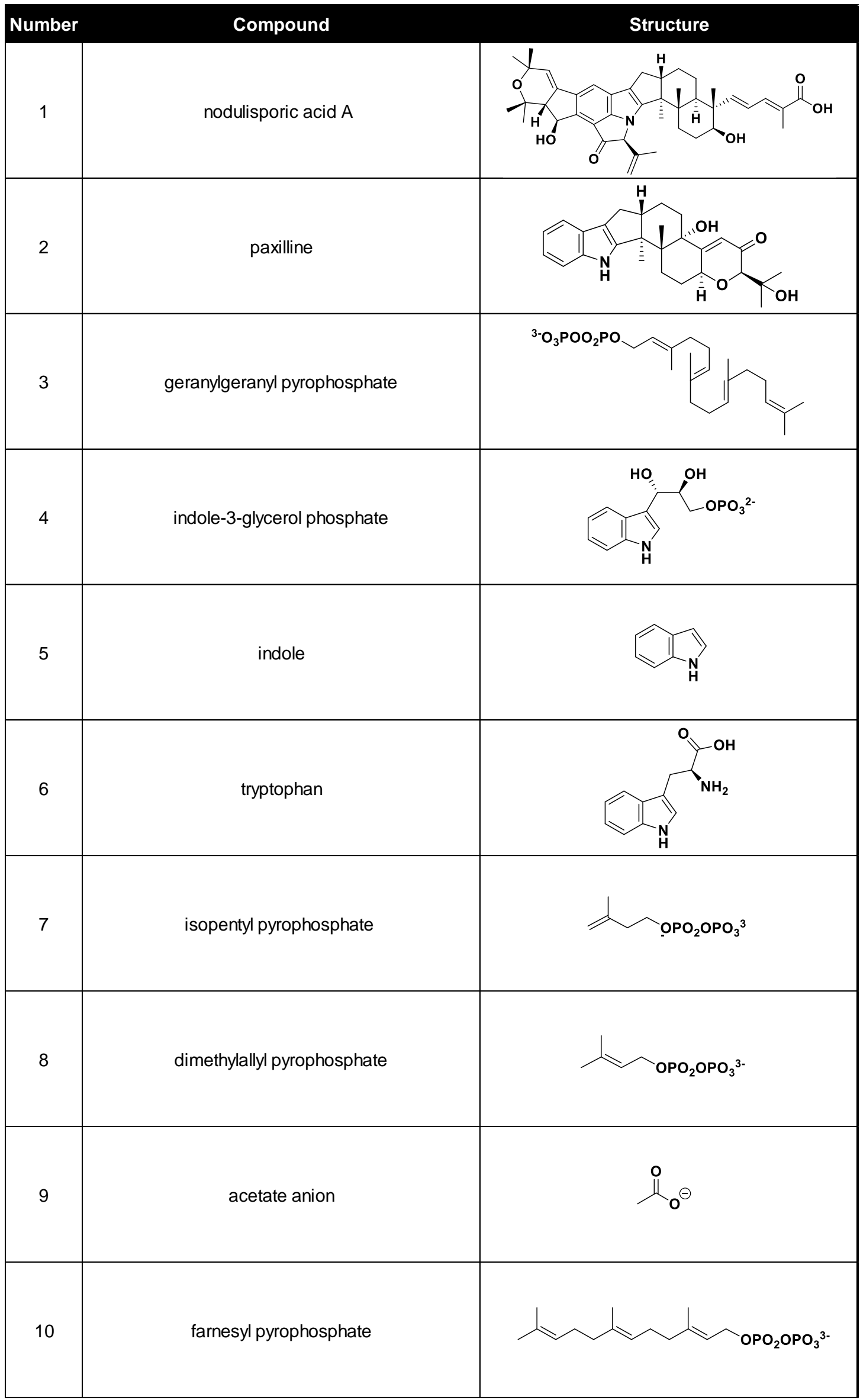




\begin{tabular}{|c|c|c|}
\hline Number & Compound & Structure \\
\hline 11 & paspaline & \\
\hline 12 & penitrem $\mathrm{A}$ & \\
\hline 13 & 3-geranylgeranylindole & \\
\hline 14 & farnesylindole & \\
\hline 15 & 17,18-epoxy-3-geranylgeranylindole & \\
\hline 16 & 13,14-epoxy-3-geranylgeranylindole & \\
\hline 17 & bisepoxy-3-geranylgeranylindole & \\
\hline 18 & emindole SB & \\
\hline 19 & emindole DB & \\
\hline 20 & petromindole & \\
\hline
\end{tabular}




\begin{tabular}{|c|c|c|}
\hline Number & Compound & Structure \\
\hline 21 & anominine & \\
\hline 22 & 10,23-dihydro-24,25-dehydroaflavinine & \\
\hline 23 & aflavinine & \\
\hline 24 & aflavinine congener & \\
\hline 25 & emindole PA precursor & \\
\hline 26 & emeniveol & \\
\hline 27 & 9-desoxypaxilline & \\
\hline 28 & $\beta-P C-M 6$ & \\
\hline 29 & a-PC-M6 & \\
\hline 30 & a-paxitriol & \\
\hline
\end{tabular}




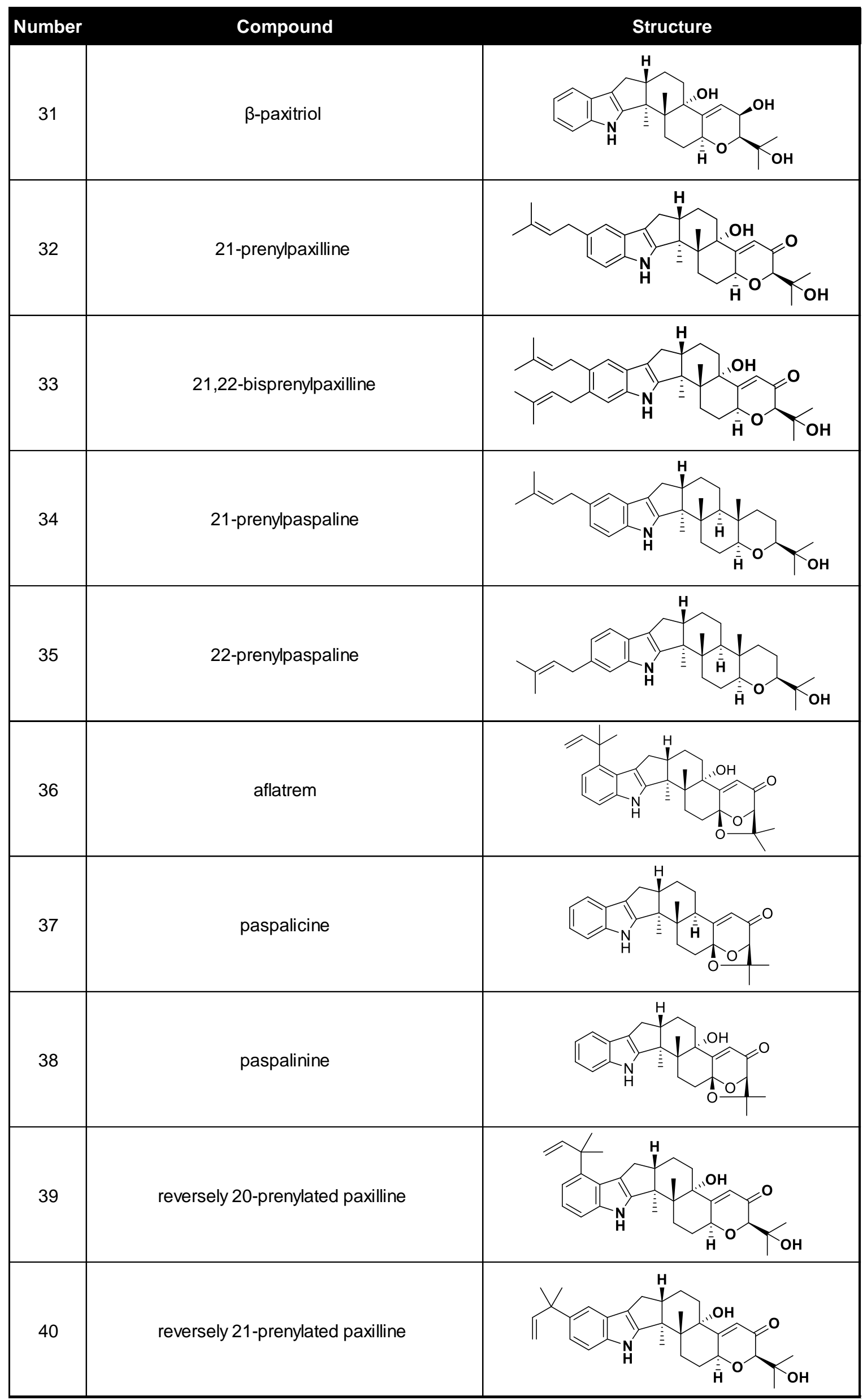




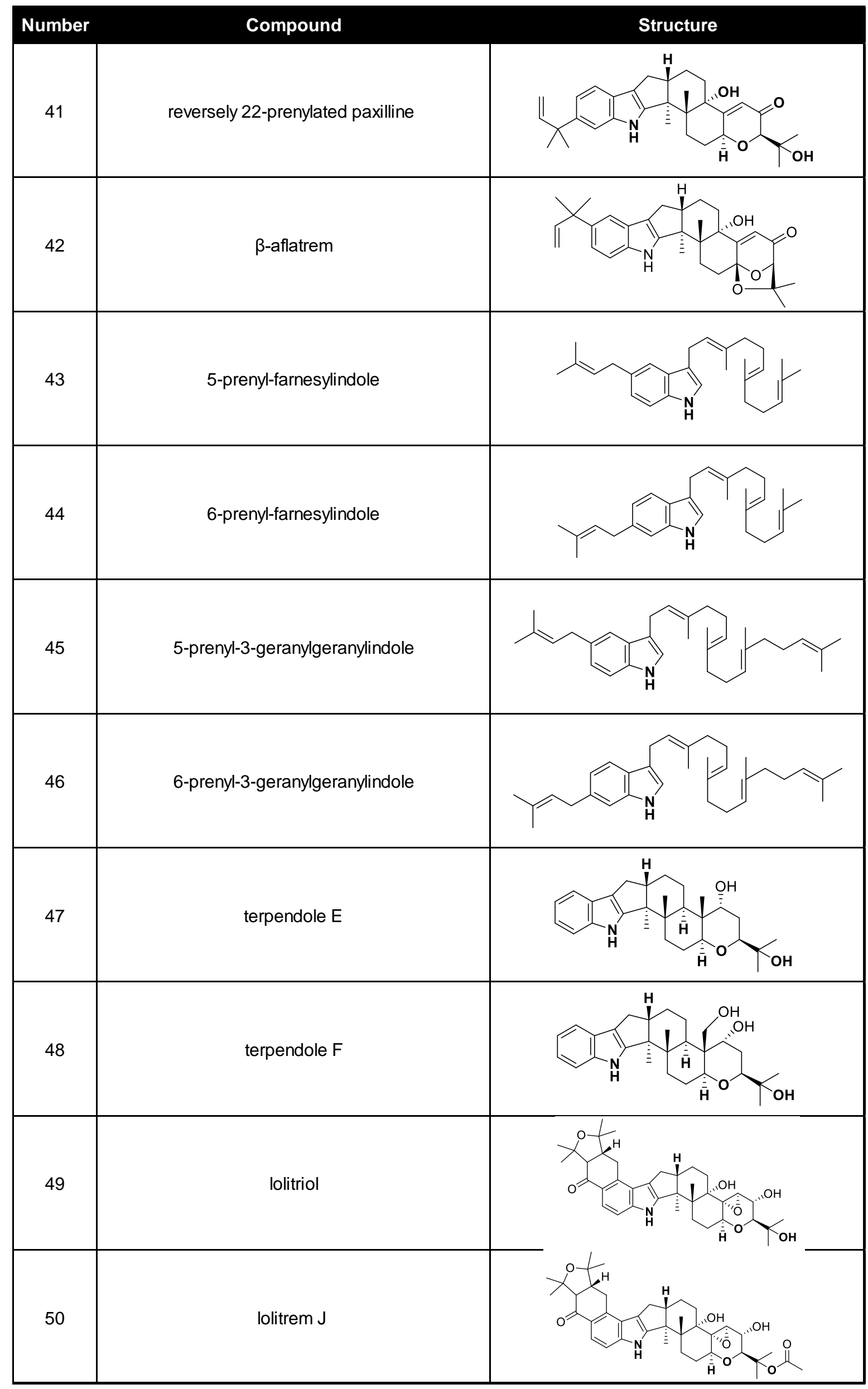




\begin{tabular}{|c|c|c|}
\hline Number & Compound & Structure \\
\hline 51 & Iolitrem B & \\
\hline 52 & lolitrem E & \\
\hline 53 & terpendole C & \\
\hline 54 & terpendole $\mathrm{M}$ & \\
\hline 55 & 9-desoxyterpendole I & \\
\hline 56 & terpendole I & \\
\hline 57 & 20 -prenyl- $\beta$-paxitriol & \\
\hline 58 & 20-prenylpenijanthine A & \\
\hline 59 & PC-M4 & \\
\hline 60 & PC-M5 & \\
\hline
\end{tabular}




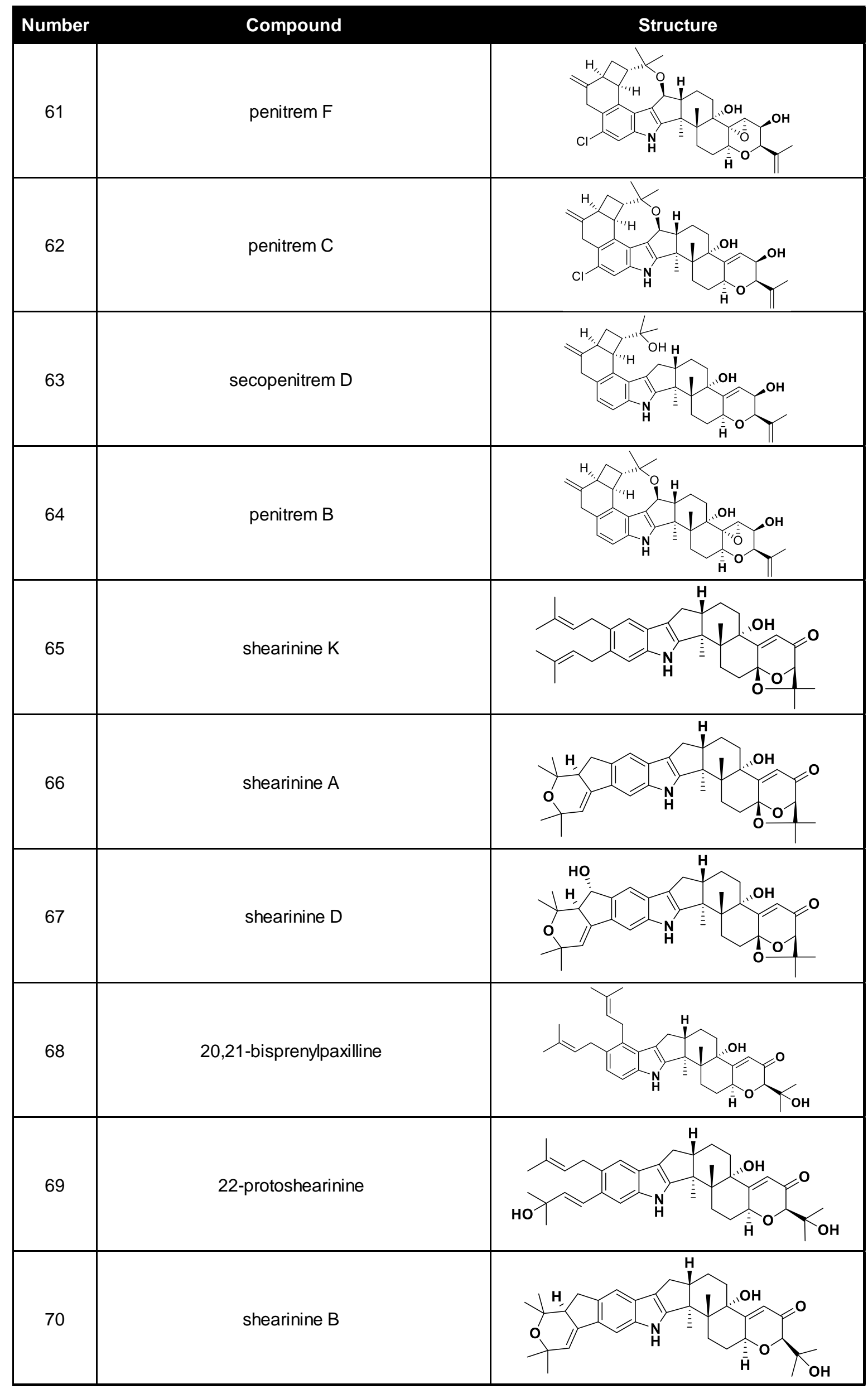




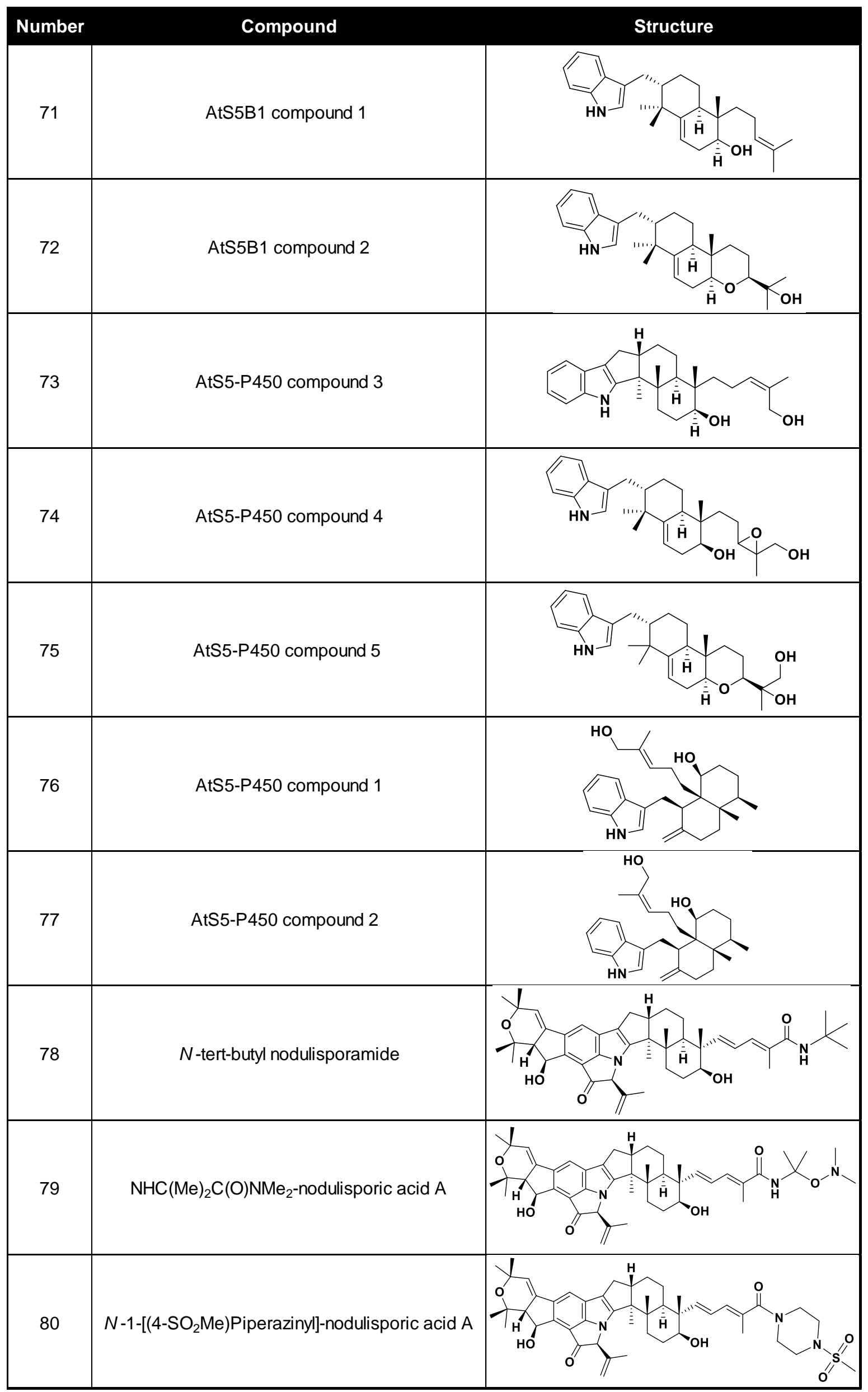




\begin{tabular}{|c|c|c|}
\hline \multicolumn{2}{|r|}{ Compound } & Structure \\
\hline 81 & dimethylamino oxazole-nodulisporic acid A & \\
\hline 82 & nodulisporic acid $\mathrm{F}$ & \\
\hline
\end{tabular}

\section{REFERENCES}

1. Van de Bittner, K. C.; Nicholson, M. J.; Bustamante, L. Y.; Kessans, S. A.; Ram, A.; van Dolleweerd, C. J.; Scott, B.; Parker, E. J., Heterologous biosynthesis of nodulisporic acid F. J. Am. Chem. Soc. 2018, 140 (2), 582-585.

2. Nicholson, M. J.; Van de Bittner, K. C.; Ram, A.; Bustamante, L. Y.; Parker, E. J., Draft Genome Sequence of the Filamentous Fungus Hypoxylon pulicicidum ATCC 74245. Genome A. 2018, 6 (2).

3. van Dolleweerd, C. J.; Kessans, S. A.; Van de Bittner, K. C.; Bustamante, L. Y.; Bundela, R.; Scott, B.; Nicholson, M. J.; Parker, E. J., MIDAS: A modular DNA assembly system for synthetic biology. ACS Synth. Biol. 2018, 7 (4), 1018-1029.

4. Raistrick, H., Bakerian lecture: a region of biosynthesis. P. Roy. Soc, Lond. B. Bio. 1950, 136 (885), 481-508.

5. Keller, N. P.; Turner, G.; Bennett, J. W., Fungal secondary metabolism -from biochemistry to genomics. Nat. Rev. Microbiol. 2005, 3 (12), 937.

6. Peldáez, F., Biological activities of fungal metabolites. In Handbook of industrial mycology, CRC Press: 2004; pp 71-121.

7. Gloer, J. B.; TePaske, M. R.; Sima, J. S.; Wicklow, D. T.; Dowd, P. F., Antiinsectan aflavinine derivatives from the sclerotia of Aspergillus flavus. J. Org. Chem. 1988, 53 (23), 5457-5460.

8. Wicklow, D. T.; Dowd, P. F.; Tepaske, M. R.; Gloer, J. B., Sclerotial metabolites of Aspergillus flavus toxic to a detritivorous maize insect (Carpophilus hemipterus, Nitidulidae). T. Brit. Mycol. Soc. 1988, 91 (3), 433-438.

9. Laakso, J. A.; Dowd, P. F.; Gloer, J. B.; Wicklow, D. T. Radarin antiinsectan metabolites. WO1993002049 A1, 1993/02/04/, 1993.

10. Li, C.; Gloer, J. B.; Wicklow, D. T.; Dowd, P. F., Thiersinines A and B: novel antiinsectan indole diterpenoids from a new fungicolous Penicillium species (NRRL 28147). Org. Lett. 2002, 4 (18), 30953098.

11. Meinke, P. T.; Smith, M. M.; Shoop, W. L., Nodulisporic acid: its chemistry and biology. Curr. Top. Med. Chem. 2002, 2 (7), 655-674.

12. Nakazawa, J.; Yajima, J.; Usui, T.; Ueki, M.; Takatsuki, A.; Imoto, M.; Toyoshima, Y. Y.; Osada, H., A Novel Action of Terpendole E on the Motor Activity of Mitotic Kinesin Eg5. Chem. Biol. 2003, 10 (2), 131-137.

13. Sallam, A. A.; Ayoub, N. M.; Foudah, A. I.; Gissendanner, C. R.; Meyer, S. A.; El Sayed, K. A., Indole diterpene alkaloids as novel inhibitors of the $\mathrm{Wnt} / \beta$-catenin pathway in breast cancer cells. Eur. J. Med. Chem. 2013, 70, 594-606. 
14. Ogata, M.; Ueda, J.-y.; Hoshi, M.; Hashimoto, J.; Nakashima, T.; Anzai, K.; Takagi, M.; Shin-ya, K., A Novel Indole-diterpenoid, JBIR-03 with Anti-MRSA Activity from Dichotomomyces cejpii var. cejpii NBRC 103559. J. Antibiot. 2007, 60 (10), 645-648.

15. Fan, Y.; Wang, Y.; Liu, P.; Fu, P.; Zhu, T.; Wang, W.; Zhu, W., Indole-diterpenoids with anti-H1N1 activity from the aciduric fungus Penicillium camemberti OUCMDZ-1492. J. Nat. Prod. 2013, 76 (7), 1328-1336.

16. Knaus, H.-G.; McManus, O. B.; Lee, S. H.; Schmalhofer, W. A.; Garcia-Calvo, M.; Helms, L. M. H.; Sanchez, M.; Giangiacomo, K.; Reuben, J. P., Tremorgenic Indole Alkaloids Potently Inhibit Smooth Muscle High-Conductance Calcium-Activated Potassium Channels. Biochemistry 1994, 33 (19), 58195828.

17. Bills, G. F.; González-Menéndez, V.; Martín, J.; Platas, G.; Fournier, J.; Peršoh, D.; Stadler, M., Hypoxylon pulicicidum sp. nov. (Ascomycota, Xylariales), a Pantropical Insecticide-Producing Endophyte. PLOS ONE 2012, 7 (10), e46687.

18. Acklin, W.; Weibel, F.; Arigoni, D., On the biosynthesis of paspalin and related metabolites from Claviceps paspali. Chimia 1977, 31 (2), 63.

19. De Jesus, A. E.; Steyn, P. S.; Van Heerden, F. R.; Vleggaar, R.; Wessels, P. L.; Hull, W. E., Structure and biosynthesis of the penitrems A-F, six novel tremorgenic mycotoxins from Penicillium crustosum. J. Am. Chem. Soc. 1981, (6), 289-291.

20. de Jesus, A. E.; Hull, W. E.; Steyn, P. S.; van Heerden, F. R.; Vleggaar, R.; Wessels, P. L., Highfield ${ }^{13} \mathrm{C}$ n.m.r. evidence for the formation of $\left[1,2-{ }^{13} \mathrm{C}\right]$ acetate from $\left[2-{ }^{13} \mathrm{C}\right]$ acetate during the biosynthesis of penitrem A by Penicillium crustosum. J. Chem. Soc. Chem. Comm. 1982, 0, 837-838.

21. de Jesus, A. E.; Gorst-Allman, C. P.; Steyn, P. S.; van Heerden, F. R.; Vleggaar, R.; Wessels, P. L.; Hull, W. E., Tremorgenic mycotoxins from Penicillium crustosum. Biosynthesis of penitrem A. J. Am. Chem. Soc. 1983, 1863-1868.

22. Mantle, P. G.; Weedon, C. M., Biosynthesis and transformation of tremorgenic indolediterpenoids by Penicillium paxilli and Acremonium lolii. Phytochemistry 1994, 36 (5), 12091217.

23. Young, C.; Itoh, Y.; Johnson, R.; Garthwaite, I.; Miles, C. O.; Munday-Finch, S. C.; Scott, B., Paxilline-negative mutants of Penicillium paxilli generated by heterologous and homologous plasmid integration. Curr. Genet. 1998, 33 (5), 368-377.

24. Young, C.; McMillan, L.; Telfer, E.; Scott, B., Molecular cloning and genetic analysis of an indolediterpene gene cluster from Penicillium paxilli. Mol. Microbiol. 2001, 39 (3), 754-764.

25. Zhang, S.; Monahan, B. J.; Tkacz, J. S.; Scott, B., Indole-Diterpene Gene Cluster from Aspergillus flavus. Appl. Environ. Microb. 2004, 70 (11), 6875-6883.

26. Young, C. A.; Bryant, M. K.; Christensen, M. J.; Tapper, B. A.; Bryan, G. T.; Scott, B., Molecular cloning and genetic analysis of a symbiosis-expressed gene cluster for lolitrem biosynthesis from a mutualistic endophyte of perennial ryegrass. Mol. Genet. Genomics 2005, 274 (1), 13-29.

27. Young, C. A.; Felitti, S.; Shields, K.; Spangenberg, G.; Johnson, R. D.; Bryan, G. T.; Saikia, S.; Scott, B., A complex gene cluster for indole-diterpene biosynthesis in the grass endophyte Neotyphodium lolii. Fungal Genet. Biol. 2006, 43 (10), 679-693.

28. Nicholson, M. J.; Koulman, A.; Monahan, B. J.; Pritchard, B. L.; Payne, G. A.; Scott, B., Identification of Two Aflatrem Biosynthesis Gene Loci in Aspergillus flavus and Metabolic Engineering of Penicillium paxilli To Elucidate Their Function. Appl. Environ. Microb. 2009, 75 (23), 7469-7481.

29. Saikia, S.; Takemoto, D.; Tapper, B. A.; Lane, G. A.; Fraser, K.; Scott, B., Functional analysis of an indole-diterpene gene cluster for lolitrem $B$ biosynthesis in the grass endosymbiont Epichloë festucae. FEBS Lett. 2012, 586 (16), 2563-2569.

30. Motoyama, T.; Hayashi, T.; Hirota, H.; Ueki, M.; Osada, H., Terpendole E, a Kinesin Eg5 Inhibitor, Is a Key Biosynthetic Intermediate of Indole-Diterpenes in the Producing Fungus Chaunopycnis alba. Chem. Biol. 2012, 19 (12), 1611-1619.

31. Liu, C.; Tagami, K.; Minami, A.; Matsumoto, T.; Frisvad, J. C.; Suzuki, H.; Ishikawa, J.; Gomi, K.; Oikawa, H., Reconstitution of Biosynthetic Machinery for the Synthesis of the Highly Elaborated Indole Diterpene Penitrem. Angew. Chem. Int. Edit. 2015, 54 (19), 5748-5752. 
32. Nicholson, M. J.; Eaton, C. J.; Stärkel, C.; Tapper, B. A.; Cox, M. P.; Scott, B., Molecular Cloning and Functional Analysis of Gene Clusters for the Biosynthesis of Indole-Diterpenes in Penicillium crustosum and $P$. janthinellum. Toxins 2015, 7 (8), 2701-2722.

33. Tang, M.-C.; Lin, H.-C.; Li, D.; Zou, Y.; Li, J.; Xu, W.; Cacho, R. A.; Hillenmeyer, M. E.; Garg, N. K.; Tang, Y., Discovery of Unclustered Fungal Indole Diterpene Biosynthetic Pathways through Combinatorial Pathway Reassembly in Engineered Yeast. J. Am. Chem. Soc. 2015, 137 (43), 1372413727.

34. McMillan, L. K.; Carr, R. L.; Young, C. A.; Astin, J. W.; Lowe, R. G. T.; Parker, E. J.; Jameson, G. B.; Finch, S. C.; Miles, C. O.; McManus, O. B.; Schmalhofer, W. A.; Garcia, M. L.; Kaczorowski, G. J.; Goetz, M.; Tkacz, J. S.; Scott, B., Molecular analysis of two cytochrome P450 monooxygenase genes required for paxilline biosynthesis in Penicillium paxilli, and effects of paxilline intermediates on mammalian maxi-K ion channels. Mol. Genet. Genomics 2003, 270 (1), 9-23.

35. Saikia, S.; Parker, E. J.; Koulman, A.; Scott, B., Four gene products are required for the fungal synthesis of the indole-diterpene, paspaline. FEBS Lett. 2006, 580 (6), 1625-1630.

36. Saikia, S.; Parker, E. J.; Koulman, A.; Scott, B., Defining Paxilline Biosynthesis in Penicillium paxilli FUNCTIONAL CHARACTERIZATION OF TWO CYTOCHROME P450 MONOOXYGENASES. J. Biol. Chem 2007, 282 (23), 16829-16837.

37. Saikia, S.; Scott, B., Functional analysis and subcellular localization of two geranylgeranyl diphosphate synthases from Penicillium paxilli. Mol. Genet. Genomics 2009, 282 (3), 257-271.

38. Scott, B.; Young, C. A.; Saikia, S.; McMillan, L. K.; Monahan, B. J.; Koulman, A.; Astin, J.; Eaton, C. J.; Bryant, A.; Wrenn, R. E.; Finch, S. C.; Tapper, B. A.; Parker, E. J.; Jameson, G. B., Deletion and Gene Expression Analyses Define the Paxilline Biosynthetic Gene Cluster in Penicillium paxilli. Toxins 2013, 5 (8), 1422-1446.

39. Liu, C.; Minami, A.; Noike, M.; Toshima, H.; Oikawa, H.; Dairi, T., Regiospecificities and prenylation mode specificities of the fungal indole diterpene prenyltransferases AtmD and PaxD. Appl. Environ. Microb. 2013, 79 (23), 7298-7304.

40. Tagami, K.; Liu, C.; Minami, A.; Noike, M.; Isaka, T.; Fueki, S.; Shichijo, Y.; Toshima, H.; Gomi, K.; Dairi, T.; Oikawa, H., Reconstitution of Biosynthetic Machinery for Indole-Diterpene Paxilline in Aspergillus oryzae. J. Am. Chem. Soc. 2013, 135 (4), 1260-1263.

41. Tagami, K.; Minami, A.; Fujii, R.; Liu, C.; Tanaka, M.; Gomi, K.; Dairi, T.; Oikawa, H., Rapid Reconstitution of Biosynthetic Machinery for Fungal Metabolites in Aspergillus oryzae: Total Biosynthesis of Aflatrem. ChemBioChem 2014, 15 (14), 2076-2080.

42. Liu, C.; Noike, M.; Minami, A.; Oikawa, H.; Dairi, T., Functional analysis of a prenyltransferase gene (paxD) in the paxilline biosynthetic gene cluster. Appl. Microbiol. Biot. 2014, 98 (1), 199-206.

43. Liu, C.; Minami, A.; Dairi, T.; Gomi, K.; Scott, B.; Oikawa, H., Biosynthesis of Shearinine: Diversification of a Tandem Prenyl Moiety of Fungal Indole Diterpenes. Org. Lett. 2016, 18 (19), 50265029.

44. Byrne, K. M.; Smith, S. K.; Ondeyka, J. G., Biosynthesis of Nodulisporic Acid A: Precursor Studies. J. Am. Chem. Soc. 2002, 124 (24), 7055-7060.

45. Fueki, S.; Tokiwano, T.; Toshima, H.; Oikawa, H., Biosynthesis of Indole Diterpenes, Emindole, and Paxilline: Involvement of a Common Intermediate. Org. Lett. 2004, 6 (16), 2697-2700.

46. Uchida, R.; Tomoda, H.; Ōmura, S., Biosynthesis of sespendole. J. Antibiot. 2006, 59 (5), 298302.

47. Zhao, L.; Chang, W.-C.; Xiao, Y.; Liu, H.-W.; Liu, P., Methylerythritol phosphate pathway of isoprenoid biosynthesis. Annual review of biochemistry 2013, 82, 497-530.

48. Nozawa, K.; Yuyama, M.; Nakajima, S.; Kawai, K.-i.; Udagawa, S.-i., Studies on fungal products. Part 19. Isolation and structure of a novel indoloditerpene, emindole SA, from Emericella striata. J. Am. Chem. Soc. 1988, (8), 2155-2160.

49. Xiong, Q.; Zhu, X.; Wilson, W. K.; Ganesan, A.; Matsuda, S. P. T., Enzymatic Synthesis of an Indole Diterpene by an Oxidosqualene Cyclase: Mechanistic, Biosynthetic, and Phylogenetic Implications. J. Am. Chem. Soc. 2003, 125 (30), 9002-9003. 
50. Singh, S. B.; Ondeyka, J. G.; Jayasuriya, H.; Zink, D. L.; Ha, S. N.; Dahl-Roshak, A.; Greene, J.; Kim, J. A.; Smith, M. M.; Shoop, W.; Tkacz, J. S., Nodulisporic acids DF: structure, biological activities, and biogenetic relationships. J. Nat. Prod. 2004, 67 (9), 1496-1506.

51. Ondeyka, J. G.; Byrne, K.; Vesey, D.; Zink, D. L.; Shoop, W. L.; Goetz, M. A.; Singh, S. B., Nodulisporic Acids C, C1, and C2: A Series of D-Ring-Opened Nodulisporic Acids from the Fungus Nodulisporium sp. J. Nat. Prod. 2003, 66 (1), 121-124.

52. Ondeyka, J. G.; Dahl-Roshak, A. M.; Tkacz, J. S.; Zink, D. L.; Zakson-Aiken, M.; Shoop, W. L.; Goetz, M. A.; Singh, S. B., Nodulisporic acid B, B1, and B2: a series of 1'-deoxy-nodulisporic acids from Nodulisporium sp. Bioorg. Med. Chem. Lett. 2002, 12 (20), 2941-2944.

53. Hensens, O. D.; Ondeyka, J. G.; Dombrowski, A. W.; Ostlind, D. A.; Zink, D. L., Isolation and structure of nodulisporic acid A1 and A2, novel insecticides from a Nodulisporium sp. Tetrahedron Lett. 1999, 40 (30), 5455-5458.

54. Meinke, P. T.; Ayer, M. B.; Colletti, S. L.; Li, C.; Lim, J.; Ok, D.; Salva, S.; Schmatz, D. M.; Shih, T. L.; Shoop, W. L.; Warmke, L. M.; Wyvratt, M. J.; Zakson-Aiken, M.; Fisher, M. H., Chemical modification of nodulisporic acid A: preliminary structure-activity relationships. Bioorg. Med. Chem. Lett. 2000, 10 (20), 2371-2374.

55. Meinke, P. T.; Colletti, S. L.; Fisher, M. H.; Wyvratt, M. J.; Shih, T. L.; Ayer, M. B.; Li, C.; Lim, J.; Ok, D.; Salva, S.; Warmke, L. M.; Zakson, M.; Michael, B. F.; Demontigny, P.; Ostlind, D. A.; Fink, D.; Drag, M.; Schmatz, D. M.; Shoop, W. L., Discovery of the development candidate N-tert-butyl nodulisporamide: a safe and efficacious once monthly oral agent for the control of fleas and ticks on companion animals. J. Med. Chem. 2009, 52 (11), 3505-3515.

56. Ostlind, D. A.; Felcetto, T.; Misura, A.; Ondeyka, J.; Smith, S.; Goetz, M.; Shoop, W.; Mickle, W., Discovery of a novel indole diterpene insecticide using first instars of Lucilia sericata. Med. Vet. Entomol. 1997, 11 (4), 407-408.

57. Ostlind, D. A.; Cifelli, S.; Conroy, J. A.; Mickle, W. G.; Ewanciw, D. V.; Andriuli, F. J.; Ho, P., A novel Cimex lectularius - Rodent assay for the detection of systemic ectoparasiticide activity. 2001; Vol. 26, $\mathrm{p}$ 181-186.

58. Ondeyka, J. G.; Helms, G. L.; Hensens, O. D.; Goetz, M. A.; Zink, D. L.; Tsipouras, A.; Shoop, W. L.; Slayton, L.; Dombrowski, A. W.; Polishook, J. D.; others, Nodulisporic acid A, a novel and potent insecticide from a Nodulisporium sp. Isolation, structure determination, and chemical transformations. J. Am. Chem. Soc. 1997, 119 (38), 8809-8816.

59. Shoop, W. L.; Gregory, L. M.; Zakson-Aiken, M.; Michael, B. F.; Haines, H. W.; Ondeyka, J. G.; Meinke, P. T.; Schmatz, D. M., Systemic efficacy of nodulisporic acid against fleas on dogs. J. Parasitol. 2001, 87 (2), 419-423.

60. Berger, R.; Shoop, W. L.; Pivnichny, J. V.; Warmke, L. M.; Zakson-Aiken, M.; Owens, K. A.; deMontigny, P.; Schmatz, D. M.; Wyvratt, M. J.; Fisher, M. H., Synthesis of Nodulisporic Acid 2 "Oxazoles and 2 "-Thiazoles. Org. Lett. 2001, 3 (23), 3715-3718.

61. Ludmerer, S. W.; Warren, V. A.; Williams, B. S.; Zheng, Y.; Hunt, D. C.; Ayer, M. B.; Wallace, M. A.; Chaudhary, A. G.; Egan, M. A.; Meinke, P. T., Ivermectin and nodulisporic acid receptors in Drosophila melanogaster contain both $\gamma$-aminobutyric acid-gated $\mathrm{Rdl}$ and glutamate-gated GluCla chloride channel subunits. Biochemistry 2002, 41 (20), 6548-6560.

62. Smith, M. M.; Warren, V. A.; Thomas, B. S.; Brochu, R. M.; Ertel, E. A.; Rohrer, S.; Schaeffer, J.; Schmatz, D.; Petuch, B. R.; Tang, Y. S.; Meinke, P. T.; Kaczorowski, G. J.; Cohen, C. J., Nodulisporic Acid Opens Insect Glutamate-Gated Chloride Channels: Identification of a New High Affinity Modulator. Biochemistry 2000, 39 (18), 5543-5554.

63. Davies, T.; Field, L.; Usherwood, P.; Williamson, M., DDT, pyrethrins, pyrethroids and insect sodium channels. IUBMB life 2007, 59 (3), 151-162.

64. Bass, C.; Schroeder, I.; Turberg, A.; Field, L. M.; Williamson, M. S., Identification of mutations associated with pyrethroid resistance in the para-type sodium channel of the cat flea, Ctenocephalides felis. Insect Biochem. Molec. 2004, 34 (12), 1305-1313. 
65. Saleem, M. A.; Ahmad, M.; Ahmad, M.; Aslam, M.; Sayyed, A. H., Resistance to selected organochlorin, organophosphate, carbamate and pyrethroid, in Spodoptera litura (Lepidoptera: Noctuidae) from Pakistan. J. Econ. Entomol. 2008, 101 (5), 1667-1675.

66. Shad, S. A.; Sayyed, A. H.; Fazal, S.; Saleem, M. A.; Zaka, S. M.; Ali, M., Field evolved resistance to carbamates, organophosphates, pyrethroids, and new chemistry insecticides in Spodoptera litura Fab.(Lepidoptera: Noctuidae). J. Pest Sci. 2012, 85 (1), 153-162.

67. Tong, H.; Su, Q.; Zhou, X.; Bai, L., Field resistance of Spodoptera litura (Lepidoptera: Noctuidae) to organophosphates, pyrethroids, carbamates and four newer chemistry insecticides in Hunan, China. J. Pest Sci. 2013, 86 (3), 599-609.

68. Smith, A. B.; Davulcu, A. H.; Cho, Y. S.; Ohmoto, K.; Kürti, L.; Ishiyama, H., Indole Diterpene Synthetic Studies. Total Synthesis of (+)-Nodulisporic Acid F and Construction of the Heptacyclic Cores of (+)-Nodulisporic Acids A and B and (-)-Nodulisporic Acid D. J. Org. Chem. 2007, 72 (13), 4596-4610. 69. Zou, Y.; Melvin, J. E.; Gonzales, S. S.; Spafford, M. J.; Smith, A. B., Total Synthesis of (-)Nodulisporic Acid D. J. Am. Chem. Soc. 2015, 137 (22), 7095-7098.

70. Melvin, J. E., Studies toward the synthesis of nodulisporic acids A and D. University of Pennsylvania 2014, PhD (PN, USA), 1-273.

71. Wu, W.; Davis, R. W.; Tran-Gyamfi, M. B.; Kuo, A.; LaButti, K.; Mihaltcheva, S.; Hundley, H.; Chovatia, M.; Lindquist, E.; Barry, K., Characterization of four endophytic fungi as potential consolidated bioprocessing hosts for conversion of lignocellulose into advanced biofuels. Appl. Microbiol. Biot. 2017, 101 (6), 2603-2618.

72. Itoh, Y.; Johnson, R.; Scott, B., Integrative transformation of the mycotoxin-producing fungus, Penicillium paxilli. Curr. Genet. 1994, 25 (6), 508-513.

73. Terpe, K., Overview of bacterial expression systems for heterologous protein production: from molecular and biochemical fundamentals to commercial systems. Appl. Microbiol. Biot. 2006, 72 (2), 211.

74. Belofsky, G. N.; Gloer, J. B.; Wicklow, D. T.; Dowd, P. F., Antiinsectan alkaloids: Shearinines A$\mathrm{C}$ and a new paxilline derivative from the ascostromata of Eupenicillium shearii. Tetrahedron 1995, 51 (14), 3959-3968.

75. Belofsky, G. N.; Gloer, J. B.; Wicklow, D. T.; Dowd, P. F. Indole antiinsectan metabolites from the ascostromata of Eupenicillium shearii. US5492902 A, 1996/02/20/, 1996.

76. Gloer, J. B.; Rinderknecht, B. L.; Wicklow, D. T.; Dowd, P. F., Nominine: a new insecticidal indole diterpene from the sclerotia of Aspergillus nomius. J. Org. Chem. 1989, 54 (11), 2530-2532.

77. Laakso, J. A.; Gloer, J. B.; Wicklow, D. T.; Dowd, P. F., Sulpinines A-C and secopenitrem B: new antiinsectan metabolites from the sclerotia of Aspergillus sulphureus. J. Org. Chem. 1992, 57 (7), 20662071.

78. Laakso, J. A.; TePaske, M. R.; Dowd, P. F.; Gloer, J. B.; Wicklow, D. T.; Staub, G. M. Indole antiinsectan metabolites. US5227396 A, 1993/07/13/, 1993.

79. Sallam, A. A.; Houssen, W. E.; Gissendanner, C. R.; Orabi, K. Y.; Foudah, A. I.; El Sayed, K. A., Bioguided discovery and pharmacophore modeling of the mycotoxic indole diterpene alkaloids penitrems as breast cancer proliferation, migration, and invasion inhibitors. Med. Chem. Comm. 2013, $4(10), 1360$.

80. Gao, S.-S.; Li, X.-M.; Williams, K.; Proksch, P.; Ji, N.-Y.; Wang, B.-G., Rhizovarins A-F, IndoleDiterpenes from the Mangrove-Derived Endophytic Fungus Mucor irregularis QEN-189. J. Nat. Prod. 2016, 79 (8), 2066-2074.

81. Nagumo, Y.; Motoyama, T.; Hayashi, T.; Hirota, H.; Aono, H.; Kawatani, M.; Osada, H.; Usui, T., Structure-Activity Relationships of Terpendole E and Its Natural Derivatives. ChemistrySelect 2017, 2 (4), 1533-1536.

82. Tarui, Y.; Chinen, T.; Nagumo, Y.; Motoyama, T.; Hayashi, T.; Hirota, H.; Muroi, M.; Ishii, Y.; Kondo, H.; Osada, H., Terpendole E and its Derivative Inhibit STLC-and GSK-1-Resistant Eg5. ChemBioChem 2014, 15 (7), 934-938.

83. Sun, X.; Shen, X.; Jain, R.; Lin, Y.; Wang, J.; Sun, J.; Wang, J.; Yan, Y.; Yuan, Q., Synthesis of chemicals by metabolic engineering of microbes. Chem. Soc. Rev. 2015, 44 (11), 3760-3785. 
84. Dombrowski, A. W.; Endris, R. G.; Helms, G. L.; Hensens, O. D.; Ondeyka, J. G.; Ostlind, D. A.; Polishook, J. D.; Zink, D. L. United States Patent: 5399582 - Antiparasitic agents. 5399582, 1995/03/21/, 1995.

85. Byrd, A. D.; Schardl, C. L.; Songlin, P. J.; Mogen, K. L.; Siegel, M. R., The B-tubulin gene of Epichloe typhina from perennial ryegrass (Lolium perenne). Curr. Genet. 1990, 18 (4), 347-354.

86. Solovyev, V.; Kosarev, P.; Seledsov, I.; Vorobyev, D., Automatic annotation of eukaryotic genes, pseudogenes and promoters. Genome Biol. 2006, 7 (1), S10.

87. de Marco, A.; Deuerling, E.; Mogk, A.; Tomoyasu, T.; Bukau, B., Chaperone-based procedure to increase yields of soluble recombinant proteins produced in E. coli. BMC biotechnology 2007, 7, 32.

88. Miyazaki, K., Molecular engineering of a PheS counterselection marker for improved operating efficiency in Escherichia coli. BioTechniques 2015, 58 (2), 86-8.

89. Patron, N. J.; Orzaez, D.; Marillonnet, S.; Warzecha, H.; Matthewman, C.; Youles, M.; Raitskin, O.; Leveau, A.; Farre, G.; Rogers, C.; Smith, A.; Hibberd, J.; Webb, A. A.; Locke, J.; Schornack, S.; Ajioka, J.; Baulcombe, D. C.; Zipfel, C.; Kamoun, S.; Jones, J. D.; Kuhn, H.; Robatzek, S.; Van Esse, H. P.; Sanders, D.; Oldroyd, G.; Martin, C.; Field, R.; O'Connor, S.; Fox, S.; Wulff, B.; Miller, B.; Breakspear, A.; Radhakrishnan, G.; Delaux, P. M.; Loque, D.; Granell, A.; Tissier, A.; Shih, P.; Brutnell, T. P.; Quick, W. P.; Rischer, H.; Fraser, P. D.; Aharoni, A.; Raines, C.; South, P. F.; Ane, J. M.; Hamberger, B. R.; Langdale, J.; Stougaard, J.; Bouwmeester, H.; Udvardi, M.; Murray, J. A.; Ntoukakis, V.; Schafer, P.; Denby, K.; Edwards, K. J.; Osbourn, A.; Haseloff, J., Standards for plant synthetic biology: a common syntax for exchange of DNA parts. The New phytologist 2015, 208 (1), 13-9.

90. Yelton, M. M.; Hamer, J. E.; Timberlake, W. E., Transformation of Aspergillus nidulans by using a trpC plasmid. PNAS 1984, 81 (5), 1470-1474.

91. Vollmer, S. J.; Yanofsky, C., Efficient cloning of genes of Neurospora crassa. PNAS 1986, 83 (13), 4869-4873.

92. Oliver, R. P.; Roberts, I. N.; Harling, R.; Kenyon, L.; Punt, P. J.; Dingemanse, M. A.; van den Hondel, C. A. M. J. J., Transformation of Fulvia fulva, a fungal pathogen of tomato, to hygromycin B resistance. Curr. Genet. 1987, 12 (3), 231-233.

93. Penn, J.; Mantle, P. G., Biosynthetic intermediates of indole-diterpenoid mycotoxins from selected transformations at C-10 of paxilline. Phytochemistry 1994, 35 (4), 921-926.

94. Penn, J.; Swift, R.; Wigley, L. J.; Mantle, P. G.; Bilton, J. N.; Sheppard, R. N., Janthitrems B and C, two principal indole-diterpenoids produced by Penicillium janthinellum. Phytochemistry 1993, 32 (6), 1431-1434.

95. Namiki, F.; Matsunaga, M.; Okuda, M.; Inoue, I.; Nishi, K.; Fujita, Y.; Tsuge, T., Mutation of an Arginine Biosynthesis Gene Causes Reduced Pathogenicity in Fusarium oxysporum f. sp. melonis. Mol. Plant Microbe In. 2001, 14 (4), 580-584. 